\title{
E-Poster Viewing
}

\section{E-Poster Viewing - 7-9 April: Anxiety Disorders and Somatoform Disorders}

\section{E-PV0002}

\section{"Premenstrual dysphoric disorder: a} case report"

C. Alario Ruiz ${ }^{1 *}$, C. Rodríguez Fernández ${ }^{1}$, E. Castaño García ${ }^{2}$

${ }^{1}$ Psychiatry, Psychiatry, Palencia, Spain; ${ }^{2}$ Psychiatry, Psychology, Palencia, Spain

${ }^{*}$ Corresponding author.

Background and aims.- The mood in women is cyclic due to the influence of hormonal systems on neurotransmission and neuropeptidic systems at the diencephalic and cortical levels. $75 \%$ of women during reproductive age have mood variations, but without requiring any specific treatment. But those women whose symptoms cause them disability in psychosocial functioning require early diagnosis and treatment since this pathology can disable them intermittently for a third of their lives. Premenstrual Dysphoric Disorder (PMDD) is the pathological exacerbation of the somatic and psychic changes that occur during the end of the luteal phase in the normal feminine cycle and produces physical and psychic disabilities and problems in family, work and social relationships. The latter is an essential condition for the woman to meet DSM-5 clinical criteria.

Objective.- Establish differences between Premenstrual Dysphoric Disorder and other Mood Disorders.

Bibliographic review of the treatments accepted for Premenstrual Dysphoric Disorder.

Methods.- We present a clinical case of a patient referred to the Mental Health Unit, who was considered to suffer from this disorder.

Results.-

- The Premenstrual Syndrome (75-80\% of women) differs from Premenstrual Dysphoric Disorder (5-8\% of women), which involves somatic/ affective symptoms that produces dysfunction or significant disability.

Conclusions.- PMDD is a well-defined clinical entity with an important impact on the lives of affected women. - It is necessary to consider PMDD in the differential diagnosis of Affective and Anxious Syndromes in women of childbearing age. - Treatment with SSRIs is effective - If there is no response to SSRI, it could benefit from association with progestagens.

Disclosure of interest.- The authors have not supplied a conflict of interest statement.

\section{E-PV0003}

\section{Dissociative pseudodementia and electroconvulsive therapy: a series of two cases and review of literature}

G. Anmella Diaz ${ }^{1 *}$, P.J. Sanchez ${ }^{2}$, A. Lladó ${ }^{2}$, L. Pintor ${ }^{1}$, V. Navarro ${ }^{1}$, A. Gimenez ${ }^{1}$, J. Pinzon ${ }^{1}$, M. Sagué ${ }^{1}$, S. Madero ${ }^{1}$, M.T. Pons ${ }^{1}$, A. Stoppa $^{3}$, R. De la Mata ${ }^{4}$, C. Llach ${ }^{1}$, A. Benabarre ${ }^{1}$

${ }^{1}$ Hospital Clínic of Barcelona, Barcelona- Catalonia- Spain, Department of Psychiatry and Psychology, Barcelona, Spain; ${ }^{2}$ Hospital Clínic of Barcelona, Barcelona- Catalonia- Spain, Department of Neurology, Barcelona, Spain; ${ }^{3}$ Hospital Consorci de Terrassa, Terrassa- Catalonia- Spain, Department of Psychiatry and Psychology, Terrassa, Spain; ${ }^{4}$ Hospital de Salamanca, SalamancaSpain, Department of Psychiatry and Psychology, Salamanca, Spain * Corresponding author.

Background and aims.-

Introduction.- Very-few literature has been published about the use of ECT in dissociative-disorders, mostly on dissociative-identity-

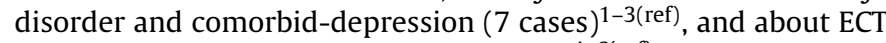
in depressive-pseudodementia (16 cases $)^{4-6(\text { ref })}$. We did not find literature about ECT in dissociative-pseudodementia.

Aims.- To examine the utility of ECT in dissociativepseudodementia.

Methods.- We present a series of 2-cases of dissociativepseudodementia and discuss the potential uses of ECT:

Case 1: 63-year-old-woman without relevant-psychiatric/ medical-record presented rapidly-progressive cognitive impairment presenting with absolute mutism in 1.5-years-evolution.

Case 2: 46-year-old-man, without relevant-psychiatric/medicalrecord presented with insidious 5-year progressive psychomotorinhibition, apathic-behaviour and progressive speech decrease till absolute mutism.

In both cases, organic etiologies were discarded after several tests. Antipsychotic, antidepressant and anxiolytic treatments were essayed without response. Both cases were admitted in psychiatryhospitalization-unit for ex-juvantibus ECT.

Results.-

Case 1: after the first-ECT-session the patient presented with spontaneous coherent speech, preserved orientation and functional recovery, MMSE $=24 / 30$, approximate-answers, highsuggestibility and dissociative-amnesia.

Case 2: after 16-ECT-sessions and tryciclic-antidepressant the patient presented spontaneous speech, active-behaviour and 
MMSE $=29 / 30$. After 3-days of recovery there was a regression to the initial clinical-state, presenting fluctuations afterwards.

Both cases were diagnosed with dissociative-pseudodementia due to the dissociative features and unpredictable-situation-dependent fluctuating clinical evolution. Outpatient follow-up supported the diagnostic-orientation.

Conclusions.- Both cases showed an unexpected, rapid and clinically-substantial response after ECT. Thus, ECT permitted a diagnostic-orientation and showed therapeutic potential in these two complex cases, in line with literature reports. Therefore, in selected cases with dissociative-disorders and pseudodementia, ECT should be considered as a valid diagnostic and therapeutic tool. Disclosure of interest.- The authors have not supplied a conflict of interest statement.

\section{E-PV0010}

\section{Analysis of actual psychological condition in patients with functional intestinal disorders}

N. Chernus ${ }^{*}$, R. Gorenkov ${ }^{2}$, S. Sivkova ${ }^{3}$, A. Sivkov ${ }^{3}$, A.

Zolotovickaja $^{1}$, T. Savina ${ }^{1}$

${ }^{1}$ I.M. Sechenov First Moscow State Medical University, Outpatient Care Department, Moscow, Russia; ${ }^{2}$ I.M. Sechenov First Moscow State Medical University, Higher Healthcare Organization Management, Moscow, Russia; ${ }^{3}$ I.M. Sechenov First Moscow State Medical University, Department of Clinical Pharmacology and Internal Diseases Propaedeutics of the Medical Faculty, Moscow, Russia

* Corresponding author.

Background and aims.- Phenomenon of psychological mechanisms specific gravity/significance determination in the pathologic behavior of functional intestinal disorders of various forms has claimed attention from physicians and psychologists for the more than 40 years.

Methods.- The study enrolled 114 patients (26 male and 88 female) with irritable bowel syndrome (IBS-D, IBS-C, IBS-M and IBS-U), functional constipation (FC) and functional diarrhea (FD). The average subject age was $43,6 \pm 11$ years. In order to achieve the study objective, MMPI method was used, where the range between 30 and $70 \mathrm{~T}$-scores indicated normal psychological condition, while increased score by one or several clinical scales were deemed to signal the pathological profile.

Results.- Analysis of averaged subject profiles showed that in most subjects the average profile score exceeded $80 \mathrm{~T}$-scores, and namely in $64 \%$ FC patients, 60\% IBS-M patients and 56\% IBS-C patients vs. $44 \%$ IBS-U patients, $43 \%$ IBS-D patients and $33 \%$ FD patients, $p<0,01$. The average profile above $70 \mathrm{~T}$-scores, but below $80 \mathrm{~T}$-scores by one or several clinical scales was observed in 50\% for FD vs. $32 \%$ for IBS-D; $p<0,01$; and twice as often for IBS-C: $33 \%$ vs. $16 \%$ for FC. In the lower part of continuum, where the psychological factor influence is relatively low, the profile distribution was as follows: $25 \%$ for IBS-D, 20\% for FC and IBS-M, 17\% for FD and IBS-M, 11\% for IBS-C.

Conclusions.- The data obtained demonstrate the significant specific gravity of psychosomatic component in disease development, progression and chronization in patients with FC, IBS-M.

Disclosure of interest.- The authors have not supplied a conflict of interest statement.

\section{E-PV0011}

\section{Pain and depression in rheumatic patients}

N. Chernus ${ }^{1 *}$, R. Gorenkov ${ }^{2}$, S. Sivkova ${ }^{1}$, A. Sivkov ${ }^{3}$, A.

Zolotovickaja $^{1}$, T. Savina ${ }^{1}$

${ }^{1}$ I.M. Sechenov First Moscow State Medical University, Outpatient

Care Department, Moscow, Russia; ${ }^{2}$ I.M. Sechenov First Moscow State Medical University, Higher Healthcare Organization

Management, Moscow, Russia; ${ }^{3}$ I.M. Sechenov First Moscow State

Medical University, Department of Clinical Pharmacology and

Internal Diseases Propaedeutics of the Medical Faculty, Moscow,

Russia

* Corresponding author.

Background and aims.- Chronic recurrent pain is a clinical symptom of rheumatic diseases with primary joint damage. The mechanisms of chronic pain syndrome development are often associated with depression.

Methods.- The study enrolled 67 patients with ostheoarthrosis (OA), rheumatoid arthritis (RA) and lower back pain. The average subject age was $59,5 \pm 5,3 ; 24$ were males and 43 were females. In order to achieve the study objective, Luscher test, CES-D; TAS; Spielberg-Hanin's scale; MMPI; VAS scale and McGill Pain Questionnaire were used.

Results.- Following the testing results, 32 (47,8\%) of 67 patients were diagnosed with chronic pain syndrome associated with depression respectively $p=0,002$. The average pain syndrome (PS) level by VAS scale in patients with depression was almost 1,7 times higher than in patients without psychological disorders, 8,6 $\pm 0,9$ vs. $5,2 \pm 1,09 ; p<0,001$. Under adaptive tension conditions, when the MMPI profile exceeded $70 \mathrm{~T}$-scores, PS tended to exacerbate $(p<0,001)$, while manifestation of depression symptoms correlated with PS $(\Gamma=0,627)$ and the presence of personality traits accentuation $(r=0,531)$, along with increased anxiety $(\Gamma=0,425)$, asthenic condition $(\Gamma=0,392)$ and general TAS alexithymia score $(r=0,473)$. After the course of therapy involving antidepressant drugs, PS decreased by 2,9 times as compared to the baseline level, $p<0,001$.

Conclusions.- Indications for antidepressant use in patients with PS include the chronic pain syndrome associated with depression at the background of basic anti-inflammatory therapy.

Disclosure of interest.- The authors have not supplied a conflict of interest statement.

\section{E-PV0012}

\section{Psychosomatic aspects of adaptation development in gastroenterological patients. Prospective study}

N. Chernus ${ }^{1 *}$, R. Gorenkov ${ }^{2}$, S. Sivkova ${ }^{1}$, A. Sivkov $^{3}$, A.

Zolotovickaja $^{1}$, T. Savina ${ }^{1}$

${ }^{1}$ I.M. Sechenov First Moscow State Medical University, Outpatient

Care Department, Moscow, Russia; ${ }^{2}$ I.M. Sechenov First Moscow

State Medical University, Higher Healthcare Organization

Management, Moscow, Russia; ${ }^{3}$ I.M. Sechenov First Moscow State

Medical University, Department of Clinical Pharmacology and Internal Diseases Propaedeutics of the Medical Faculty, Moscow, Russia

" Corresponding author.

Background and aims.- Studying the clinical significance and interrelations of psychovegetative, immunological micro-ecological system disorders as different levels of disturbance of normal body adaptive response to disease is the relevant research topic.

Methods.- The study included 674 patients with various gastroenterological pathologies (average age 38,5: 136 males, 517 females), who had been followed-up from 2,5 to 15 years. All the subjects underwent standard gastroenterological examination. In order to 
achieve the study objective, the patients were also subject to microbiological, immunological and psychovegetative tests, and namely: Spielberg-Hanin's scale, Lusher test, CES-D, TAS, MMPI; diagnostic criteria for panic attacks and chronic pains, VAS scale and McGill Pain Questionnaire.

Results.- Obtained results allowed to identify the stages of disease recovery and early rehabilitation period, where psychovegetative condition of patient was the basic system with correction period of 2,5-6 months; at this background, the pain syndrome management duration tends to decrease $r=0,672, p<0,01$; while the microbiological system recovery is determined by the correlation between the psychovegetative, large intestine movement $r=0,459, p<0,05$, as well as pre- and probiotic therapy within the period from 7 months to 1,2 years; immunological status correlated with psychovegetative and micro-ecological status $r=0,498, p<0,01$, and did not require any correction.

Conclusions.- The study conducted identified the specific gravity of each system influence during disease recovery and early rehabilitations as part of the body adaptive response to disease.

Disclosure of interest.- The authors have not supplied a conflict of interest statement.

\section{E-PV0015 \\ Assesment of the quality of treatment in patients with somatoform disorders at the prehospital level}

E. Navasardyan ${ }^{1}$, M. Artemieva ${ }^{2}$, I. Danilin ${ }^{2}$, R. Suleymanov ${ }^{2}$, A. Lazukova $^{2}$, I. Belokrylov ${ }^{2}$, V. Sokolov ${ }^{2}$, Z. Niewozinska ${ }^{3}$

${ }^{1}$ Z. P. Solovyov Scientific and Practical Psychoneurological Center, Moscow Healthcare Department, Moscow, Russia; ${ }^{2}$ Peoples' Friendship University of Russia RUDN University, Department of Psychiatry and Medical Psychology, Moscow, Russia; ${ }^{3}$ N.I. Pirogov Russian National Research Medical University, Moscow Scientific and Practical Center of Dermatovenereology and Cosmetology, Moscow, Russia

* Corresponding author.

Background and aims.- To evaluate the quality of treatment of patients with somatoform disorders at the prehospital level.

Methods.- The questionnaire revealed the duration and quality of treatment of patients with somatoform disorder at the prehospital level. It consisted of questions assessing: the duration of treatment by doctors of other specialties, frequency of appointment of psychotropic therapy by doctors of other specialties, quality of appointment of psychotropic therapy, and revealed the frequency of doctors of other specialties refer patients with somatoform disorder to a psychiatrist. 110 people (from 20 to 55 years) were included, including 67 women and 43 men with somatoform disorders. Results.- 49 patients were prescribed psychotropic drugs by doctors of other specialties (44.5\%), 61 patients were not prescribed psychotropic drugs (55.5\%). 41 patients out of 49 patients psychotropic drugs were prescribed by neurologist $(83,7 \%), 8$ - by therapist (16,3\%). 37 patients did not get effect of therapy (75.5\%), 12 patients got insignificant effect of therapy (24.5\%). Doctors of other specialties recommended to consult with the psychiatrist to 84 patients $(76,4 \%)$.

Conclusions.- 56,4\% treated their somatoform disease at the doctor of other specialty from 1 to 3 months. Most often psychotropic drugs were prescribed by a neurologist (83,7\%). $16.3 \%$ patients psychotropic drugs were prescribed by the therapist. Most patients (75.5\%) did not get the effect of the prescribed psychotropic therapy. $75 \%$ of patients were recommended by neurologist to consult with a psychiatrist, as a rule, after the appointment of ineffective therapy.

The publication was prepared with the support of the "RUDN University Program 5-100".

Disclosure of interest.- The authors have not supplied a conflict of interest statement.

\section{E-PV0016 \\ Dismorphophobia: between neurosis and psychosis}

L. Gallardo Borge*, E. Rodríguez Vázquez, C. Capella Meseguer, M. Gómez García, M. De Lorenzo Calzón, N. De Uribe Viloria, P.

Marqués Cabezas

Hospital Clínico Universitario, Psychiatry, Valladolid, Spain

* Corresponding author.

Background and aims.- Dismorphophobia is the dissatisfaction with one's body, feeling it as anomalous or deformed in one of its features. This generates a deep discomfort, even when there are no objective nor observable reasons to assess their physical appearance in those terms.

Methods.- Descriptive study of a clinical case and literature review. Results.- A 31-year-old woman, single. With no psychiatric history. Initiates referral from primary care for anxiety. She related her hair loss two years ago after a treatment for acne and ferropenia, starting to think later that she has baldness, even though Endocrinology and Dermatology indicate that there's no alopecia. She says that she can not stop thinking about this defect and does not believe that she has normal hair. This fact generates a lot of anxiety and discomfort. Maintains work life, few social relations. The examination shows a normal hair without referred alopecia areas. Mild cognitive and somatic anxiety. Isolation. Insomnia for early awakening. Clinical judgment: Overrated idea that is near delirious in an obsessive personality. Body dysmorphic disorder.

Conclusions.- According to the ICD-10, body dysmorphic disorder is classified into somatoform disorders. On the other hand, the DSM-V classifies it as a type of disorder within the obsessive spectrum, with a psychotic variant classified as a somatic delusion. Dysmorphophobia is a sensoperceptive disorder in which the patient strongly believes in the physical nature of the disorder, although it is still considered mostly within the neurotic spectrum of diseases.

Disclosure of interest.- The authors have not supplied a conflict of interest statement.

\section{E-PV0017}

\section{THe impact of anxiety on the course of hypertension}

S. Ellini ${ }^{1}$, N. Ghazouani ${ }^{2}$, Y. Jemli ${ }^{1}$, A. Rebai ${ }^{2}$, M. Cheour ${ }^{1}$

${ }^{1}$ Razi University Hospital, Psychiatry, Manouba, Tunisia; ${ }^{2}$ Medical

School of Tunis, Psychiatry, Tunis, Tunisia

* Corresponding author.

Background and aims.- High blood pressure is a chronic disease that constitutes a public health problem. In spite of the high prevalence of anxiety in patients with hypertension, screening for anxiety in patients with hypertension is not yet systematic.

This study aims to find out whether the co-occurring of hypertension and anxiety worsens the course of hypertension.

Methods.- This was a cross-sectional, descriptive and analytical study among patients diagnosed with hypertension consulting a basic health center in the governorate of Manouba in Tunisia. The assessment of anxiety was done using the Hospital Anxiety and Depression Scale (HAD-S).

Results.- One hundred patients diagnosed with hypertension were included in the study. The prevalence of anxiety in patients with hypertension was 58\%. Anxiety was significantly associated with poor adherence to treatment with $p$ value inferior to 0.01 . The use of polytherapy for hypertension was also associated with anxiety with $p$ value of 0.06 .

Neither of the following factors was associated with anxiety: the quality of follow up $(p=0.124)$ treatment resistance $(p=0.97)$, the change of the therapeutic class during the course of the disease $(p=0.088)$, the presence of adverse affects related to current antihypertensive treatment $(p=0.9)$, the occurrence of adverse events 
that motivated the change of treatment in the past $(p=0.45)$ and the therapeutic response $(p=0.68)$.

Conclusions.- The results of our study put under a spot of light the need for a multidisciplinary management of hypertension taking into account the role of psychological distress as a worsening factor of the course of this disease.

Disclosure of interest.- The authors have not supplied a conflict of interest statement.

\section{E-PV0018 \\ Quality of life in panic disorder: the effect of clinical features and personality traits}

C. Gitahy Falcao Faria*, R. Christophe Freire, M. Fidry, M. Costa do Cabo, A. Egidio Nardi

Federal University of Rio de Janeiro, Laboratory of Panic and

Respiration, Institute of Psychiatry, Rio de Janeiro, Brazil

Corresponding author.

Background and aims.- Panic Disorder (PD) is an anxiety disorder, known for its negative impact on patients' quality of life (QoL), while some personality traits may also have influence on QoL. The QoL measurement has been increasingly used as an outcome measure in clinical trials, in health technology assessment, and in epidemiological surveys to assess the subjective health and wellbeing of the population. The aim of the study is to identify which clinical features and personality traits are associated to the QoL in PD patients.

Methods.- We recruited 98 patients with the diagnosis of PD (72 women). The mean age was 38.4 (SD: 11.6) years. Most of the patients had one or more previous treatments (53.6\%). The brief version of the World Health Organization Quality of Life Questionnaire (WHOQOL-BREF) and Big Five Inventory were used respectively to access QoL and personality traits. The correlation strength was measured with Spearman's correlation. We also performed multiple linear regressions, considering demographic data, scores from clinical scales as independent variables and QoL scores as dependent variables.

Results.- WHOQOL-BREF scale means were: physical (mean: 37; SD: 17), psychological (mean: 41; SD:19), social (mean: 47; SD:22), environmental (mean: 45; SD:14) General perception of QoL (mean:49; SD:26) and general satisfaction with personal health. (mean: 32; SD: 23).

Conclusions.- Depressive symptoms had a strong negative correlation with QoL, and to a lesser extent, panic and anxiety symptoms were also negatively correlated to the QoL. While consciousness, extraversion and agreeableness had a mild positive correlation with QoL, neuroticism had a strong negative correlation with QoL. Disclosure of interest.- The authors have not supplied a conflict of interest statement.

\section{E-PV0020}

\section{The concept of "psychological mediation" \\ (Vygotsky-Lurya-Zeigarnik School) in respiration regulation in hyperventilation patients and healthy persons}

J. Koniukhovskaia*, E. Pervichko

Lomonosov Moscow State University, Psychology, Moscow, Russia

* Corresponding author.

Background and aims.- The Clinical Psychology of Corporeality (Tkhostov, 2002; Nikolaeva, Arina, 1998, 2009) suggests viewing the body not exclusively as a 'natural' biological function, but also as a culturally mediated and regulated process similar to the higher mental functions in Cultural-Historical Approach Vygotsky-LuryaZeigarnikSchool.

The regulation of breathing is both an autonomous and a voluntary function, what permits to research respiratory regulation through psychological mediation.

Aims.- To show the role of psychological mediation at the dysfunction of respiration regulation.

Methods.- The psychological mediation is assessed through: a) the respiratory experience interviews, b) the anxiety level, c) psychosemantic techniques.

The capability of voluntary respiration regulation is examined through breath holding.

The study involves 20 patients with hyperventilation syndrome (HVS) and 20 healthy participants.

Results.- The patients, on the contrary of the healthy participants, have less ability to hold breath. The breath holding provokes the hyperventilation symptoms at $55 \%$ of patients. The interview about the respiratory experience revealed that the patients have more chronic disorders, panic attacks, phobias and smoking habit in comparison to the healthy volunteers. The short breath holding is correlated with a higher anxiety level, a larger vocabulary of negative breathing experience and the fixation on present unpleasant sensations, described by emotional, but not bodily words.

Conclusions.- The negative interpretation of breathing sensation at patients, who have HVS, leads to less breathing control. Thus, the psychological mediation is connected with respiration regulation and allows to identify the altered breath meaning and formulate the goals of psychotherapy.

Disclosure of interest.- The authors have not supplied a conflict of interest statement.

\section{E-PV0021}

\section{Psychological factors of provoking} somatic sensations in the real and false biofeedback experiment

M. Kovyazina ${ }^{*}$, E. Rasskazova ${ }^{2}$, J. Migunova ${ }^{2}$, N. Varako ${ }^{2}$

${ }^{1}$ Moscow State University, Psychology, Moscow, Russia; ${ }^{2}$ Moscow

State University, Clinical psychology, Moscow, Russia

Corresponding author.

Background and aims.- Cognitive factors of somatoform disorders include attention to bodily sensations, catastrophizing and somatosensory amplification (Rief et al., 1997, Martinez et al., 1999). Biofeedback experiment provokes attention to the body whereas false biofeedback models a situation where external information and introspective sensations are mismatched, so bodily sensations in a false biofeedback can indicate difficulties in introceptive sensitivity.

The aim was to reveal differences between real and false biofeedback in provoking bodily sensations in the head and neck in healthy participants as well as cognitive factors of such provocation.

Methods.- 33 subjects (12 males, 18-25 years old) undergoing biofeedback experiment including randomized false and real conditions were instructed that "typically people in experiment have sensations in head and neck" to provoke bodily attention, then filled Screening for somatoform symptoms (Rief, Hiller, 2003), Cognitions About Body and Health Questionnaire (Rief et al., 1998).

Results.- Bodily sensations were more likely to occur in a true biofeedback (58,3\%) and less likely - in a false biofeedback only $(18,8 \%)$ but didn't depend on cognitive factors. Subjective appraisal of success in regulation of bodily functions in false BFB was higher in those who reported sensations in the experiment $(F=5,34, p<0,05$, $\eta^{2}=0,28$ ).

Conclusions.- Attention to bodily sensations accompanied by external proof for such sensations (real biofeedback) are factors provoking sensations in more than half of healthy participants regardless their cognitive vulnerability to somatoform disorders. Discrepancy between external and internal stimulation attenuate 
this effect. Research is supported by the Russian Foundation for Basic Research, project No. 17-29-02169.

Disclosure of interest.- The authors have not supplied a conflict of interest statement.

\section{E-PV0022}

\section{Cognitive factors of bodily sensations: priming in provocation of sensations in healthy subjects}

M. Kovyazina ${ }^{1}$, E. Rasskazova ${ }^{2}$, J. Migunova ${ }^{2}$, N. Varako ${ }^{2}$

${ }^{1}$ Moscow State University, Psychology, Moscow, Russia; ${ }^{2}$ Moscow

State University, Clinical Psychology, Moscow, Russia

${ }^{*}$ Corresponding author.

Background and aims.- Psychosomatics suggest two mechanisms for epidemiology of unexplained bodily complaints in somatoform disorders: according to "general sensitivity" the key role in somatization is played by the attention to sensations whereas in bodily function regulation model (Tkhostov, 2002) sensations depend on their subjective meaning.

The aim was to reveal cognitive factors of positive and negative priming of somatic sensations that are typical for patients with somatoform disorders.

Methods.- In 36 healthy controls (15 men, 18-25 years old) during the task of self-regulation using the biofeedback method, attention was drawn to sensations in the head and neck with one of three instructions (emphasizing the neutral, positive and negative personal meaning of sensations). For example, positive instruction included description of people having sensations as "open, conscious, empathetic and intellectual". They filled Screening for somatoform symptoms (Rief, Hiller, 2003), Cognitions About Body and Health Questionnaire (Rief et al., 1998).

Results.- In $50 \%$ of respondents general attention led to sensations in a head and a neck. The risk was higher at the persons inclined to somatization $\left(F=4,27, p<0,05, \eta^{2}=0,12\right)$ and considering their body as weak and vulnerable $\left(F=3,32, p<0,08, \eta^{2}=0,10\right)$. Both positive and negative personal meaning increased the probability of provocation $\left(\chi^{2}=9,52, p<0,05\right.$, Cramer's $\left.V=0,36\right)$, especially in persons with a tendency to catastrophize bodily sensations $(F=4,18$, $\left.p<0,05, \eta^{2}=0,22\right)$.

Conclusions.- Any personal meaning of sensations increased the probability of provocation, especially in persons with a tendency to catastrophize bodily sensations. Research is supported by the Russian Foundation for Basic Research, project No. 17-29-02169. Disclosure of interest.- The authors have not supplied a conflict of interest statement.

\section{E-PV0023}

\section{Psychotherapy anxiety and phobic \\ disorders}

\section{O. Kudinova*, T. Chorna}

Kharkiv Medical Academy of Postgraduate Education, Psychotherapy, Kharkiv, Ukraine

${ }^{*}$ Corresponding author.

Background and aims.- The aim of the study: the development of a comprehensive system of psychotherapy and psychocorrection of anxiety disorders of neurotic case, based on the study of their clinical structure and peculiarities of emotional damages.

Scope and contributing research: 100 patients with diagnostic categories: F41.0 - Panic disorder (episodic paroxysmal anxiety, F41.1-Generalized anxiety disorder, F41.2-mixed anxietydepressive disorder who are on the examination and treatment at the psychiatric unit. A control group included 40 patients.
Methods.- Spielberger, Eysenck Personality Questionnaire (EPQ), The Hamilton Rating Scale for Depression (HRSD), The study of accentuation of personality by K. Leonhard.

Results.- We performed the exploration of the peculiarities of clinical anxiety and patterns of therapeutic effect, based on the influence of short-term group and individual psychotherapy in the treatment of anxiety disorders, and evaluation the effectiveness of its reconition. The development of the methodology of applying a short-term group and individual psychotherapy in the treatment of anxiety disorders based on combination relaxation, hypnosis, cognitive-behavioral teqniques in combination on with short-term group therapy. In fact, this is a new real model psychotherapy based on integrative principles. The higt efficacy was shown in $82 \%$ patients, compared with 54\% efficacy in control group patients. Conclusions.- We will offer a new comprehensive methodology in the treatment of anxiety disorders of neurotic case that will improve the therapeutic efficacy of the treatment process, reduce the time of treatment, reduce the period of drug therapy.

Disclosure of interest.- The authors have not supplied a conflict of interest statement.

\section{E-PV0024 \\ Group psychoeducation 'drop it' decreases repetitive negative thinking in major depression and generalized anxiety disorder}

R. Rogiers ${ }^{1}$, C. Baeken ${ }^{1}$, D. Van den Abbeele ${ }^{2}$, R. De Raedt ${ }^{3}$, E. Watkins ${ }^{4}$, J. Remue ${ }^{5}$, R. Colman ${ }^{6}$, G. Lemmens ${ }^{5}$

${ }^{1}$ Ghent University, Dept. of Psychiatry, Ghent, Belgium; ${ }^{2}$ Psychiatric Center Ghent-Sleidinge, Depression and Anxiety Unit, Ghent, Belgium; ${ }^{3}$ Ghent University, dDepartment of Experimental Clinical and Health Psychology, Ghent, Belgium; ${ }^{4}$ University of Exeter, College of Life and Environmental Sciences, Exeter, United Kingdom; ${ }^{5}$ Ghent University Hospital, Dept. of Psychiatry, Ghent, Belgium; ${ }^{6}$ Ghent University, Department of Biostatistics, Faculty of Medicine and Health Sciences, Ghent, Belgium

" Corresponding author.

Background and aims.- Repetitive negative thinking (RNT) is considered as an important transdiagnostic factor in the onset, course and recurrence of both depressive and anxiety disorders. This study aimed to investigate whether a group psychoeducation (GP) would improve RNT, anxiety and depressive symptoms, quality of life and self-esteem of patients with Major Depressive Disorder (MDD) and/or Generalized Anxiety Disorder (GAD).

Methods.- Eighty patients were randomized to group psychoeducation (GP; $n=45$ ) or waiting list control (WLC; $n=35$ ). Assessments took place before randomization and 12 weeks later (after treatment). Multiple linear regression analyses adjusted for baseline scores were conducted.

Results.- Although all outcomes improved after participation in GP, only the uncontrollability of rumination $(p=0.03)$, worrying $(p=0.002)$, distancing oneself from one's thoughts $(p=0.003)$ and quality of life $(p=0.02)$ remained statistically significant after Bonferroni correction compared to the WLC. Limitations of the study included the small sample size, the lack of a long-term follow-up and the inclusion of highly educated patients with mainly high comorbid GAD and depression.

Conclusions.- Group psychoeducation improves RNT and quality of life of patients with MDD and GAD. Improvements remain stable until 9 months after treatment.

Disclosure of interest.- The authors have not supplied a conflict of interest statement. 
E-PV0025

\section{Somatization disorder - a frequent association with school phobia in children and adolescents}

M.M. Leti ${ }^{1}$, R. Grozavescu ${ }^{2}$, M. Hățis ${ }^{1}$, I. Moraru1 ${ }^{1}$, A. Popa ${ }^{1}$, A. Anton ${ }^{1}$

1 "Prof. Dr. Alexandru Obregia" Psychiatric Hospital- Bucharest, Child and Adolescent Psychiatry, Bucharest, Romania; 2 "Prof. Dr. Alexandru Obregia" Psychiatric Hospital- University of Medicine "Carol Davila"- Bucharest, Child and Adolescent Psychiatry, Bucharest, Romania

* Corresponding author.

Background and aims.- In the literature there are several studies that indicate a frequent association among children and adolescents between school phobia and somatization disorder [1]. Comorbidity is more common in patients with a history of chronic illness, including allergies, asthma, recurrent migraines. Unfortunately, this type of patients have multiple presentations in pediatric services, where all paraclinical diagnostic methods are depleted, without a favorable result. Thus, the addressability to pediatric psychiatry and the correct diagnosis are delayed [2].

[1] Inglés et. all (2015). Current status of research on school refusal. [2] Malas, N.et. all (2017) Pediatric Somatic Symptom Disorders. Methods.- In this context, we present three clinical cases admitted in the Child and Adolescent Clinic from "Prof. Dr. Alexandru Obregia" Psychiatric Hospital, Bucharest.

Results.- After applying the specific tests (projective tests, assessment scales, the genogram) and finding the history of the disease, the patients were diagnosed and included in a suitable therapeutic program. The evolution was favorable, so the children resumed their school activity and learned how to manage their phobia.

Conclusions.- There is a need for awareness programs among pediatricians and general practitioners about the frequent association between child and adolescent somatization disorder and school phobia. Patients should be guided as early as possible to pediatric psychiatric services to avoid unnecessary admissions in other pediatric services, the increase of parental frustration due to the uncertainty of diagnosis, taking into consideration that these delays in diagnosis and intervention may cause worsening of symptoms. Disclosure of interest.- The authors have not supplied a conflict of interest statement.

\section{E-PV0027}

\section{Psychopathological and sociodemographic risk factor for persistent somatoform pain disorder}

T. López-Arteaga ${ }^{*}$, P. Padilla-Romero ${ }^{2}$, C. Romero-Martin³ ${ }^{3}$, J.M. Gallego-Bellido $^{4}$, C. Moreno-Rubio ${ }^{2}$

${ }^{1}$ Psychiatrist, Department of Psychiatry- Hospital Ntra. Sra. del Prado, Talavera de la Reina TO- C.P. 45600, Spain; ${ }^{2}$ Hospital Ntra. Sra. del Prado, Psychiatry, Talavera de la Reina, Spain; ${ }^{3}$ Hospital Ntra. Sra. del Prado, Hospital Pharmacy, Talavera de la Reina, Spain; ${ }^{4}$ Hospital Ntra. Sra. Del Prado, Unit Addictive Behaviors, Talavera de la Reina, Spain

* Corresponding author.

Background and aims.- The best-suited approach of persistent somatoform pain disorder (DSM-5 300.82) (PSPD) is stepped care with cooperation operating on the basis of a biopsychosocial model of integrating somatic as well as psychosocial determinants of distress and therapeutic factors.

Objetives.- Determine the psychopathologic and sociodemographic risk factor for PSPD in our area and determine percentage of patients have it associated to chronic non cancer pain (CNCP).
Methods.- Prospective descriptive study. Sample: 187 patients evaluated by the department of Psychiatry in the year 2017 derived from Pain Unit (PU) for psychopathological assessment. Inclusion criteria: patients with CNCP and poor response to treatment and/or torpid evolution. Exclusion criteria: $<18$ years old.

Results.- Patients with PSPD 51\%.

Gender female: OR 8'50 (IC 95\%: 4'161-17'36, z stadist: 5'87, $p<0^{\prime} 0001$ )

Personal psychiatric history: OR 4'00 (IC 95\%: 2'45-7'13, z stadist: $4^{\prime} 704, p<0^{\prime} 0001$ )

Family substance abuse history OR $0^{\prime} 32$ (IC 95\%: 0'184-0'55, z stadist: $4^{\prime} 075, p<0^{\prime} 0001$ )

Considering having suffered a traumatic event in the biography OR 3'32 (IC 95\%: 1'907-5'78, z stadist: 4'235, $p<0^{\prime} 0001$ )

Not being the oldest of the siblings OR 6'92 (IC 95\%: $3^{\prime} 54-13^{\prime} 50, z$ stadist: 5'668, $\left.p<0^{\prime} 0001\right)$

Fibromyalgia OR $4^{\prime} 00$ (IC 95\%: 2'24-7'13, z stadist: $4^{\prime} 704$, $p<0^{\prime} 0001$ )

Conclusions.- Half of patients with chronic non-cancer pain and poor evolution have a pain disorder associated. The risk factors for presenting pain disorder according to their statistical weight are: being a woman, not being the oldest of the siblings, having a personal psychiatric history, having fibromyalgia, considering having suffered a traumatic event in the biography and not having a psychiatric family history.

Disclosure of interest.- The authors have not supplied a conflict of interest statement.

\section{E-PV0028}

\section{Anxiety disorders in Southern Tunisia: prevalence and associated factors}

M. Ben Jmaa ${ }^{1}$, Y. Mejdoub ${ }^{1}$, R. Boukhchina ${ }^{2}$, L. Ghanmi ${ }^{2}$, M. Maalej $^{3^{*}}$, J. Jdidi ${ }^{1}$, M. Ben Hmida ${ }^{1}$, M. Trigui ${ }^{1}$, M. Abbes ${ }^{2}$, S. Yaich ${ }^{1}$, L. Zouari ${ }^{3}$, J. Damak ${ }^{1}$, M. Maalej ${ }^{3}$

${ }^{1}$ Hedi Chaker University Hospital, Department of Community Medicine, Sfax, Tunisia; ${ }^{2}$ Regional Hospital of Gabes, Department of Psychiatry, Gabes, Tunisia; ${ }^{3}$ Hedi Chaker University Hospital, Psychiatry « C » Department, Sfax, Tunisia

${ }^{*}$ Corresponding author.

Background and aims.- Anxiety disorders (AD) are the most prevalent psychiatric disorders and represent an emerging public health threat in low income countries such as Tunisia. Our study aimed to assess the epidemiology of $\mathrm{AD}$ and their associated factors among psychiatric patients in Southern Tunisia.

Methods.- We conducted a descriptive cross-sectional study. We included all patients with AD whose first consultation in the psychiatric department of Gabes in Southern Tunisia occurred between 2010 and 2013. We collected data using a pre-established anonymous questionnaire exploring the patient's data.

Results.- We registered 147 patients with AD (9.1\%) among all patients consulting in our department. The sex ratio was 0.75 . The mean age was 32 years (IQR $=[22-44$ years $]$ ). Most of the cases were with low socioeconomic status (81 cases; $55.9 \%$ ) and high educational attainment ( 82 cases; $56.6 \%$ ). Forty seven (32.4\%) were unemployed. Seventeen patients $(11.7 \%)$ had addictive behaviors. Principal reasons for medical consultation were anxiety $(n=59$; $40.7 \%)$, somatic complaints $(n=31 ; 21.4 \%)$, and sleep disorders $(n=21 ; 14.5 \%)$. The median time from the onset of symptoms to consultation was 6 months (IQR $=[1-18$ months]). AD were significantly less frequent among elderly ( $O R=0.23 ; p=0.007)$, unemployed patients $(\mathrm{OR}=0.44 ; p<0.001)$ and patients with addictive behaviors $(\mathrm{OR}=0.6 ; p=0.04)$. Patients with high educational attainment and medium to high socio-economic status had two times higher risk for $\mathrm{AD}$ with respectively $(\mathrm{OR}=2.2 ; p<0.001)$ and $(\mathrm{OR}=2.7 ; p<0.001)$. Patients with AD consulted earlier compared to the other psychiatric patients (12.8 monthsV 24.3 months; $p<0.001$ ). 
Conclusions.- In our study, the burden of AD was found to be significant. Having high educational attainment or medium to high socio-economic status were predictors of $\mathrm{AD}$, while joblessness, addictive behaviours and advanced age were associated with a lower risk of $\mathrm{AD}$. Therefore, it is recommended to design preventive strategies that target these associated factors to minimize the disease burden.

Disclosure of interest.- The authors have not supplied a conflict of interest statement.

\section{E-PV0030}

\section{Psychological determinants of psychosomatic disorders in gastroenterology}

S. Moroz , V. Semenikhina, I. Makarova, R. Khaitov, N. Turishcheva CI "Dnipropetrovsk Region Clinical Hospital Named after I.I. Mechnikov", Psychoneurology Department, Dnipro, Ukraine ${ }^{*}$ Corresponding author.

Background and aims.- The question of psychological factors of the risks of development of somatic diseases is a relevant and pressing issue.

Despite these, depression and anxiety often remain unrecognised in such patients. We examined 139 patients with peptic ulcer disease. Methods.- To assess the psychological status, we performed a clinicpsychopathological examination, Locus of Control based on J.B. Rotter, the picture-association Method and its Application in a Study of Reactions to Frustration by Rosenzweig.

Results.- Depression $(33,1 \%, n=46)$ and anxiety $(43,9 \%, n=61)$ are the most common psychiatric comorbidity. The results of the picture-association Method and its Application in a Study of Reactions to Frustration by Rosenzweig revealed the predominance of extrapunitiveness $(58,3 \%, n=81)$ and obstacle-dominance $(46,0 \%, n=64)$. The scores on Rotter's Internal-External Control Scale significantly correlated with psychiatric comorbidity disorders. Identified.

Conclusions.- Psychiatric comorbidity is common in theGastrointestinal diseases and psychological phenomena arenonspecific risk factors for development and enhancement of a somaticpathology. These results should be taken into account in the therapeuticprocess.

Disclosure of interest.- The authors have not supplied a conflict of interest statement.

\section{E-PV0031}

\section{The impact of sleep disorders on the quality of life of patients with somatoform disorders}

\section{S. Moroz , I. Makarova}

CI "Dnipropetrovsk Region Clinical Hospital Named after I.I.

Mechnikov", Psychoneurology Department, Dnipro, Ukraine

${ }^{*}$ Corresponding author.

Background and aims.- The impact of sleep disorders on the quality of life of patients with somatoform disorders is often underestimated. To assess the insomnia, its connection with somatovegetative disorders, contribution to the deterioration of the quality of life in patients with somatoform disorders.

Methods.- 124 (69 women and 55 men) patients diagnosed with somatoform disorders were examined. The average age is $52.16 \pm 0.3$ years. Questioning conducted using the scale PSQA (Pittsburgh Sleep Quality Assessment), Epworth Sleepiness Scales, The Short Form (36) Health Survey were applied.

Results.- We have determined that Violations of falling asleep were observed in $34 \%$ of patients, awakening with sleep fragmentation in $86 \%$, early morning awakenings in $48 \%$ of patients. Greater fre- quency of sleep disturbances, nighttime awakenings, unpleasant dreams were noted in women. There is a tendency to the progression of manifestations of insomnia as the somatoform disorder increases. Clarified the impact of insomnia manifestations on the quality of life parameters.

Conclusions.- Sleep disorders occur in most patients with somatoform disorders, worsening the quality of life of patients. Given the heterogenety of sleep disorders, a differentiated approach to therapy is required, taking into account the nature of the dissomnic disorders and the leading pathophysiological factors.

Disclosure of interest.- The authors have not supplied a conflict of interest statement.

\section{E-PV0032 \\ Practical observations on virtual reality (VR) exposure in the treatment of social phobia}

S. Murawiec ${ }^{1^{*}}$, P. Mierzejewski ${ }^{2}$, T. Parnowski ${ }^{3}$, P. Bienkowski ${ }^{4}, \mathrm{~K}$. Hanusz $^{5}$, D. Draczynska ${ }^{6}$

${ }^{1}$ Scientific Association of Psychodynamic Psychotherapy, Tomorrow Ltd., Warsaw, Poland; ${ }^{2}$ Institute of Psychiatry and NeurologyTomorrow Ltd, Department of Pharmacology, Warsaw, Poland; 3 Polish Geriatric Psychiatry Association, Polish Language Council of Polish Academy of Science, Warsaw, Poland; ${ }^{4}$ Warsaw Medical University, Department of Psychiatry, Warsaw, Poland; ${ }^{5}$ Tomorrow Ltd., RED Department, Warsaw, Poland; ${ }^{6}$ Tomorrow Ltd., PM Director, Warsaw, Poland

* Corresponding author.

Background and aims.- Social phobia occurs with a lifetime prevalence of $2-5 \%$ in adults and is a serious mental health problem limiting daily life functioning of affected patients.

Methods.- Virtual reality (VR) is a modern technique that allows direct (non-imaginative) exposure of patients with social phobia to phobic situations. Four patients were exposed to VR session (giving a talk in front of an audience) in a pilot study on the feasibility of VR in the treatment of social phobia.

Results.- The VR exposure to the situation of giving a talk in front of an audience elicited high levels of anxiety. Thus it was confirmed that this technique allows to induce a social fear in vulnerable people exposed to VR. The anxiety in this situation was so intense that 2 of 4 persons ended the exposure without any verbal expression. Two persons reported that anxiety was elicited both by exposure to VR as well as the presence of the researcher as a person who could judge their performance during the task. The patient's prior knowledge of the researcher may have a significant effect on the level of anxiety. Social phobia patients reported that during the exposure to VR they experienced loss of possibility to observe and control the communication with the researcher, which was an additional source of distress.

Conclusions.- VR may be an effective method of exposure to anxiety situations in social phobia. The specific features of this type of exposure should be carefully monitored and taken into account in planning of therapy with VR.

Disclosure of interest.- The authors have not supplied a conflict of interest statement.

\section{E-PV0035}

\section{Anxiety, looking forward. state of the \\ art}

V. Rosello Molina*, M. Simon Blanes, C. Ribera, E. Moreno Soldado, J. Tejera

Hospital Universitario La Ribera, Psiquiatría, Valencia, Spain

* Corresponding author.

Background and aims.- Anxiety Disorders (AD) (DSM-V) are a group of prevalent disorders affecting globally around $5 \%$ of the popula- 
tion. In US and Western Europe, its incidence is higher reaching 4\% of adult population, with increasing annual costs.

The aim of this study was to assess the current situation and perspectives concerning the treatment of AD worldwide.

Methods.- We reviewed the existing literature in Pubmed Database, MESH terms "Anxiety Disorders"/“Drug Therapy", English and including humans, during the last 5 years, RCT, Meta-analysis and Systematic Reviews were evaluated. Obsessive Compulsive Disorder and not anxiety related disorders were excluded.

Results.- The recommended first-line pharmacological treatments were SSRIS and IRSNS, with no significant differences. Previous patient response, patient treatment preferences and clinical experience were considered as well. Intensity of baseline symptoms and treatment response were not directly related. Regarding lack of response or intolerance, second-line pharmacological treatments were:

- Benzodiazepines: with or without SSRIS / IRSNS combined.

- Antipsychotics: Quetiapine.

- Other antidepressant: Vortioxetine, Bupropion, Vilazidone.

- Pregabaline is more likely to be chosen rather than Gabapentine as a second line of treatment.

- Upcoming therapies: Chamomile and Cannabidiol derivatives.

CBT appears as the ultimate cost effective therapy. Furthermore, CBT lasts over time more than drugs, being the most preferable choice.

Conclusions.- We evidenced a global lack of consensus in the treatment of AD. The role of the Psychiatrist and the screening of comorbidities is relevant to assess an adequate clinical response. Long-term independent studies with a more representative sample are needed to improve the outcomes in the treatment of $\mathrm{AD}$.

Disclosure of interest.- The authors have not supplied a conflict of interest statement.

\section{E-PV0037}

\section{Anxiety in somatic clinics}

A. Todorov ${ }^{1}{ }^{*}$, P. Chumpalova $^{1}$, M. Stoimenova ${ }^{1}$, I. Veleva ${ }^{1}$, L. Tumbev $^{1}$, G. Georgiev ${ }^{1}$, D. Todorieva ${ }^{2}$, P. Vladova ${ }^{3}$, E. Dimitrova ${ }^{4}$

${ }^{1}$ Medical University - Pleven, Psychiatry and Medical Psychology, Pleven, Bulgaria; ${ }^{2}$ Medical University - Pleven, NephrologyHaematology and Gastroenteology, Pleven, Bulgaria; ${ }^{3}$ Medical University - Pleven, Surgical Propaedeutics, Pleven, Bulgaria; ${ }^{4}$ UMHAT “D-r Georgi Stranski”, First Psychiatry Cinic, Pleven, Bulgaria ${ }^{*}$ Corresponding author.

Background and aims.- Anxiety is a psychological term to define an emotional state, characterized by an insecure feeling and worry. Anxiety appears and increases, regardless of local environment changes. It is one of the mechanisms for the organism to point out the necessity for reaction preparation, when a situation changes and extreme physical and psychological tension is present, the socalled normal anxiety. It is connected to past or present experience and is formed by one's view and unconscious internal conflicts; stereotyped and repeated.

Aim.- To investigate and compare level of anxiety in patients in surgical department and oncohematology department.

Methods.- We investigated 100 patients - 50 from general surgery clinic and 50 from oncohematology clinic. For assessment of anxiety we use The State-Trait Anxiety Inventory (STAI) to assess both state and trait anxiety.

Results.- During the first day of hospitalization all patients showed increased level of situational and other types of anxiety. At the end of in-patient time situational anxiety level decreased significantly. Most of the surgery patients reduced their level of anxiety. On the other hand, we noticed decrease in anxiety among oncohematological patients, but the level remained higher than those in surgery patients. About $20 \%$ of the patients were found to have extremely higher anxiety levels - situational and other types. They were referred for further psychiatrist consults.
Conclusions.- Anxiety is an important symptom that is commonly found in somatic clinics. Bound to the fear, unknown and unexplained, of a serious somatic problem, it could turn into an anxiety disorder.

Disclosure of interest.- The authors have not supplied a conflict of interest statement.

\section{E-PV0038}

\section{A curious case of an oscillating boy}

S.D. Vanniasingham ${ }^{1 *}, \mathrm{~J} \mathrm{Ng}^{2}$

${ }^{1}$ Institute of Mental Health, Department of Psychosis, Singapore,

Singapore; ${ }^{2}$ Institute of Mental Health, Department of

Developmental Psychiatry, Singapore, Singapore

* Corresponding author.

Background and aims.- The patient is a 23 year old male who had just enlisted into the army 2 months prior to presenting to mental health services. He reported experiencing vertiginous giddiness episodes for 5 years, associated with a sensation of disequilibrium. He also had a 4 months history of involuntary movements of his body, rocking side to side in an oscillatory fashion. He had difficulty functioning at home and in camp. This resulted in depressed mood, low self-esteem and suicidal ideation. His Neurologist opined that this was a case of "Hysterical Vertigo", the differential being Mal De Debarquement Syndrome. His Otolaryngologist believed that his clinical presentation was on the spectrum of Persistent Postural Perceptual Dizziness. A psychiatric diagnostic dilemma ensued and differentials such as Conversion Disorder and Factitious Disorder were considered. The aim was to treat his co-morbid mood symptoms and restore his functioning in society.

Methods.- A review of the existing literature on somatoform disorders and perusal of the patient's clinical notes was carried out. Liaison with his primary treating Psychiatrist, Psychologist, Neurologist and Otolaryngologist took place.

Results.- Clinical impression was Conversion Disorder. He was on anti-depressant medication and received regular psychology therapy sessions. With resolution of his stressors, there was good improvement in his symptoms, mood and functioning.

Conclusions.- The complex interaction between one's physical symptoms, mental psyche and social circumstances needs to be evaluated and appreciated in great depth, to develop effective treatment strategies for Somatoform Disorders. There is a scope for further research in this area of medicine.

Disclosure of interest.- The authors have not supplied a conflict of interest statement.

\section{E-PV0039}

\section{Cognitive behavioral therapy in social anxiety disorder- a case series \\ O. Vasiliu}

"Dr. Carol Davila" University Central Military Hospital, Psychiatry, Bucharest, Romania

* Corresponding author.

Background and aims.- Cognitive behavioral therapy (CBT) is considered one of the most evidence-based treatments for improving SAD patients clinical status and overall functionality.

Methods.- Three patients diagnosed with SAD according to the DSM-5 criteria have been included in an individual CBT programme focused on cognitive restructuring and imaginative exposure training.

Results.- The first patient was a female, age 35, without psychiatric or somatic co-morbidity, which responded well after 3 months of weekly CBT sessions, but relapsed soon and needed a new course of 12 -week sessions. The main trigger of relapse was found in her familial environment, where the relationship with 
her husband was dysfunctional, and family therapy sessions were recommended. The second patient was 24 -year old, unemployed, severely impaired by $\mathrm{SAD}$, and the $\mathrm{CBT}$ was combined with fluoxetine $40 \mathrm{mg}$ daily. He responded relatively well in therapy, the Liebowitz Social Anxiety Scale (LSAS) "anxiety/fear" decreased with $50 \%$ and "avoidance" with $40 \%$ after 16 weeks, but avoidant personality features slowed the evolution toward recovery. The third patient, a 21-year old female student, was also diagnosed with alcohol and marijuana use disorder, which necessitated a more complex CBT approach, with techniques focused on both social avoidance and drug craving. Her evolution was oscillating and she discontinued therapy after 10 weeks.

Conclusions.- CBT could improve SAD symptoms, but the response may be delayed by comorbid personality disorders, drug use, depressive and anxiety disorders, various psychological and social factors with negative impact (e.g. familial relationships, burn-out, reduced self esteem, lack of therapeutic adherence).

Disclosure of interest.- The authors have not supplied a conflict of interest statement.

\section{E-Poster Viewing - 7-9 April: Bipolar Disorders}

\section{E-PV0040 \\ Evaluation of clinical features between bipolar patients remitted with mood stabilizer monotherapy or antipsychotic combination \\ K. Altinbas*, B. Sen \\ Selcuk University Faculty of Medicine, Psychiatry-Mazhar Osman \\ Mood Clinic, Konya, Turkey \\ ${ }^{*}$ Corresponding author.}

Background and aims.- Bipolar disorder (BD) is a chronic mood disorder with recurrent mood episodes and remission periods between acute episodes. Morbidity and general functioning of the patients are strongly associated with effective long-term treatment. Therefore, we aim to compare the demographic and clinical characteristics of bipolar patients on single/dual mood stabilizer (MS) and/or mood stabilizer plus antipsychotic (MS + AP) treatment.

Methods.- Remitted bipolar patients $(n=100)$ who are followed in Mazhar Osman Mood Clinic of Selcuk University Medical Faculty were recruited for the study. Demographic and clinical features of patients on single/dual MS and MS plus antipsychotic (AP) treatment were compared.

Results.- There was no significant difference between groups in terms of gender $\left(p: 0.75, x^{2}: 0.11\right)$, family history of illness ( $p: 0.69$, $\left.x^{2}: 1.44\right)$, age ( $\left.p: 0.94, z: 0.08\right)$, education $(p: 0.08, z:-1.72)$, age at onset ( $p: 0.74, z: 0.33$ ), total number of hospitalization and mood episodes ( $p: 0.62, z: 0.49 ; p: 0.93, z: 0.08)$ and duration of illness ( $p: 0.82, z: 0.22$ ). However, the rate of psychosis in the first episode was significantly higher in MS + AP group than MS group $\left(p: 0.02, x^{2}\right.$ : 0.93 ). Total number of mania and proportion of manic first episode were significantly higher in the MS + AP group while proportion of depressive first episode was higher in MS group ( $\left.p: 0.02, x^{2}: 5.35\right)$. Conclusions.- Preference of antipsychotics for long-term treatment is consistent with the literature that psychotic bipolar patients were more difficult to treat. In Turkey, only quetiapine is available among approved treatment options for bipolar depression. Therefore, clinicians might prefer MS for the patients whose first episode is depression which also means that these patients would experience more depressive recurrence.

Disclosure of interest.- The authors have not supplied a conflict of interest statement.

\section{E-PV0041}

Neutrophil-lymphocyte ratio, platelet-lymphocyte ratio and C-reactive protein predict metabolic syndrome in patients with a bipolar

\section{disorder}

B. Arranz ${ }^{1^{*}}$, M. Sanchez-Autet ${ }^{2}$, G. Safont ${ }^{3}$, P. Sierra ${ }^{4}$, A. Garcia-Blanco $^{5}$, L. De la Fuente ${ }^{6}$, M. Garriga ${ }^{7}$, G.P. Maria Paz

${ }^{1}$ Parc Sanitari Sant Joan de Deu- CIBERSAM, Department of Psychiatry, Cornella de Llobregat-Barcelona, Spain; ${ }^{2}$ Parc Sanitari Sant Joan de Deu- CIBERSAM, Department of Psychiatry, Barcelona, Spain; ${ }^{3}$ Hospital Universitari Mutua de Terrassa- Universitat de Barcelona- CIBERSAM, Department of Psychiatry, TerrassaBarcelona, Spain; ${ }^{4}$ Health Research Institute La Fe - Valencia, Department of Psychiatry, Valencia, Spain; ${ }^{5}$ Health Research Institute La Fe-Valencia, Department of Psychiatry, Valencia, Spain; 6 University of Oviedo- Mental Health Servives of Principado de Asturias SESPA- CIBERSAM, Department of Psychiatry, Oviedo, Spain; ${ }^{7}$ Hospital Clinic de Barcelona- CIBERSAM, Programa de Trastorno Bipolar, Barcelona, Spain

* Corresponding author.

Background and aims.- Neutrophil-lymphocyte ratio (NLR) and platelet-lymphocyte ratio (PLR) are inexpensive markers of inflammation which seem to be increased in patients with mood disorders. NLR and PLR are also related to a higher risk of metabolic syndrome (MetS), and to cardiovascular (CV) risk factors in the general population, although this relationship has not been studied in BD.

Methods.- Our aim was to determine the association between the inflammatory biomarkers NLR, PLR and C-reactive protein (CRP) and the metabolic status (number of metabolic risk factors, presence of MetS, insulin sensitivity: QUICKI index and insulin resistance: HOMA index, in a sample of 219 outpatients with a bipolar disorder (77 men, 142 women). Linear and logistic regression models were used.

Results.- Older age $(\beta=0.290, p<0.001)$, low PLR ratio $(\beta=-0.276$, $p=0.001)$, high NLR ratio $(\beta=0.234, p=0.003)$ and high CRP levels $(\beta=0.141, p=0.040)$ significantly predicted a higher number of MetS risk factors. The multiple linear regression model explained $20.6 \%$ of the variance $(F=7.656, p<0.001)$.

Older age $(\mathrm{OR}=1.035)$ and low PLR ratio $(\mathrm{OR}=0.988)$ were associated to a greater likelihood of presenting a MetS $\left(\chi^{2}\right.$ $(6, N=194)=17.541, p=0.007)$. The logistic regression model explained between $8.6 \%$ (Cox and Snell $\mathrm{R}$ square) and $12.6 \%$ (Nagelkerke $R$ squared) of the variance, and correctly classified $72.2 \%$ of the individuals.

Conclusions.- We propose that monitoring PLR and NLR ratios in patients with bipolar disorder may help to identify those patients with a higher risk of presenting the MetS, who might benefit from healthy lifestyle counselling and early intervention.

Study supported by grant PI11/02493.

Disclosure of interest.- The authors have not supplied a conflict of interest statement.

\section{E-PV0042}

\section{The psychosocial adaptation and quality of life in patients with bipolar disorder I type in remission}

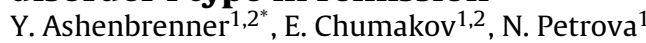

${ }^{1}$ Saint-Petersburg State University, Department of Psychiatry and Addictions, Saint-Petersburg, Russia; ${ }^{2}$ Saint-Petersburg Psychiatric Hospital №1 Named after P.P. Kaschenko, Day in-Patient Department, Saint-Petersburg, Russia

* Corresponding author. 
Background and aims.- Bipolar disorder (BD) is characterized not only by recurrent mood disorders but also by impairment in psychosocial functioning and adaptation, which affects patient's quality of life (QOL). Aims: to assess psychosocial adaptation and QOL in patients with BD I in remission.

Methods.- The sample included 60 patients with BD I in remission (according to the DSM-V criteria), who received outpatient treatment in 2017-2018. The onset of the disorder - 27.92 \pm 4.09 years, the average duration of the disorder $-8.08 \pm 4.53$ years. We applied a questionnaire to collect sociodemographic characteristics. Patient's QOL was assessed with WHOQOL-100 scale.

Results.- It was revealed that 14 patients were unemployed, of whom 6 people were dismissed from work due to the onset of the next episode. Allowance for disability was received by 14 patients (23.3\%), these patients had strong difficulties in implementing even low-skilled labor $(R=-0.413 ; p=0.001)$. Only $55.0 \%(n=33)$ of patients were in serious relationship or married, of those $30.0 \%$ rated their relationship as conflict. Patients on disability benefits were less likely to have a harmonious relationship in the family $(R=-0.413 ; p=0.001) .31 .7 \%(n=19)$ of patients committed a suicide in the anamnesis. The average WHOQOL-100 score was $191.8 \pm 17.7$ (the moderate QOL level).

Conclusions.- Sociodemographic characteristics in the examined patients indicate a low level of objective indicators of adaptation. Patients with BD I in remission have many adaptation problems (high level of disability, violations in the family relationships). It is necessary to conduct psychoeducational work with patients and their relatives, to increase the level of compliance between the patient and the doctor to more carefully monitoring of the patient's condition.

Disclosure of interest.- The authors have not supplied a conflict of interest statement.

\section{E-PV0044 \\ Offspring of mothers with bipolar disorder: systematic review on psychological characteristics}

R. Bastos*, L.K.S. Campos, D.B. Faria-Schützer, M.E. Brito, D.A.R. Silva, A.D.S. Junior, E.R. Turato

State University of Campinas, Laboratory of Clinical-Qualitative Research LPCQ Campinas, Brazil

${ }^{*}$ Corresponding author.

Background and aims.- Bipolar affective disorder (BAD) is a psychiatric disorder with high heritability, widely demonstrated. In this context, the offspring of patients with BAD are interesting ways to evaluate the natural history of the disease and its prodromal symptoms. Systematic review about psychological characteristics of these offspring would be a way of knowing the characteristics already described in the literature and analyzing them. This study aims to identify in the scientific literature the psychological characteristics of the offspring of women with BAD.

Methods.- A systematic review made from the prior consultation to the databases The Center for Reviews and Dissemination (CRD) and the Cochrane Database of Systematic Reviews. The study was then recorded in the International Prospective Register of Systematic Review (PROSPERO) under the number CRD42017039010.

Results.- We found 10 articles that suggest vulnerability factors in the offspring of mothers with $\mathrm{BD}$; high rates of axis 1 psychiatric disorders; as well as behavioral and temperament problems.

Conclusions.- There is a complex family context to which the offspring of mothers with BD that expose psychosocial difficulties and losses in their lives are exposed. This context indicates the need for family interventions, including early interventions.

Disclosure of interest.- The authors have not supplied a conflict of interest statement.

\section{E-PV0045}

\section{Delirious mania: a debut of late-onset bipolar disorder}

H. Becerra Darriba

USM Hospital de Alcañiz, Psychiatry, Alcañiz, Spain

Corresponding author.

Background and aims.- Bipolar Disorder is a chronic disease characterized by cycling episodes of (hypo)mania and depression, with or without psychotic features. About $10 \%$ of patients are reported to have a late-onset of illness after age 50. Developing a first manic episode as a delirious mania in the fifth decade of life is considered a rare clinical presentation.

Objective.- To describe a clinical case reflecting the diagnostic difficulty of atypical new-onset delirious mania as a debut of late-onset bipolar disorder.

Methods.- Qualitative design. A case report study of a 56 year-old female without psychiatric history is presented and discussed. Psychopathological data were collected through in-depth interviews. She was admitted to an outpatient service with a state of variable erratic behavior (hiding the earrings under the bed, disrobing), restlessness, hyperactivity with purposeless movements during the night, mutism or stereotyped speech with repetitive bizarre comments such as claiming to be "under magnetic waves or sleeping", thought blocking, insomnia and inconsistent disorientation. These symptoms began one week after the hospitalization of her mother with bipolar disorder.

Results.- Neuroimaging, urinalysis, encephalopathy, autoimmune and other laboratory test results were normal. After taking Olanzapine $15 \mathrm{mg} /$ day for 20 days with poor clinical response, we considered non-malignant delirious mania as a late onset of bipolar disorder. Treatment was supplemented with lorazepam $4 \mathrm{mg}$ /day and lithium was added to $800 \mathrm{mg} /$ day. On day 40 , the patient became stable with improvement of speech and behavior.

Conclusions.- The prevalence of delirious mania in uncertain. Antipsychotics and mood stabilizers are useful psychotropic medications.

Disclosure of interest.- The authors have not supplied a conflict of interest statement.

\section{E-PV0046 \\ Clinical practice in prescribing lithium in Tunisia \\ I. Ben Abid ${ }^{*}$, H. Mammi ${ }^{1}$, H. Baccari ${ }^{2}$ \\ ${ }^{1}$ Mohamed Taher Maamouri University Hospital, Mental Health, Nabeul, Tunisia; ${ }^{2}$ Mohamed Taher Maamouri University Hospital, Menatl Health, Nabeul, Tunisia \\ Corresponding author.}

Background and aims.- Lithium is recommended as the first-choice mood-stabilizer (MS) for maintenance treatment of bipolar disorder.

Despite this fact, the use of lithium in mood disorders has been shown to be decreasing at the expense of other MS.

There are hence substantial differences among guidelines indications and clinical practice for prescribing and monitoring lithium.

The aim of this study is to investigate the determinants of lithium prescription.

Methods.- This study was performed over the course of 4 months among psychiatrists.

An anonymous self-report survey was conducted among health care professionals prescribing lithium.

The items were grouped in different sections ranging from prescription pattern to therapeutic drug monitoring. Other items explore the perception of the prescriber toward patients and treatments in bipolar disorders.

Results.- A preliminary analysis of the very first data collected suggested that overall knowledge about lithium prescription and 
monitoring were satisfying, even though still need to be improved. Lithium is mostly used as second-line treatment and is still being perceived as a toxic medication. Patients are most of the time considered as not being good candidates for a lithium therapy.

In this study, we assessed the attitude of psychiatrists toward lithium prescription but not their behavior. A "know-do" gap has not been explored.

Conclusions.- Tunisian psychiatrists' knowledge and perception of lithium are the most important determinants of its prescription. Further studies are needed to investigate the differences between psychiatrist's' knowledge and their real clinical practice. Hence, the general refrain from lithium could be adressed.

Disclosure of interest.- The authors have not supplied a conflict of interest statement.

\section{E-PV0047}

\section{Therapeutic actualities in the management of bipolar disorder in the depressive phase}

M. Bitar ${ }^{1 *}$, L. Achour Achour ${ }^{2}$, F. Laboudi Laboudi ${ }^{2}$, A. Ouanass Ouanass $^{2}$

${ }^{1}$ Dr, Hopital Arrazi, Rabat, Morocco; ${ }^{2}$ Arrazi, Emergency, Rabat, Morocco

* Corresponding author.

Background and aims.- Bipolar depression, still poorly understood, represents 30 to $50 \%$ of depressed patients and is the main source of morbidity in bipolar disorder. The current therapeutic modalities proposed in the management of this depressive phase are in perpetual controversy so various algorithms are regularly proposed and new molecules are tested. Thymoregulators are currently recommended in first intention however the place of antipsychotics and antidepressants remains unclear.

The objective of our study is to update the different modes of management of bipolar disorder in the depressive phase.

Methods.- This is a descriptive study carried out on medical files aimed at describing how to treat patients who are consultants or hospitalized at Ar-razi de Salé hospital with bipolar disorder in the depressive phase. This is done using a heterogeneous questionnaire that surveys the different data and assesses therapeutic attitudes in the management of bipolar disorder in the depressive phase.

Results.- In our sample of 45 patients, the average age is 34.6 years. An age range between 18 and 63 years old. All our patients are under a combination of antipsychotics and mood stabilizers.

$44.4 \%$ of our patients were placed on sodium valproate and $28.9 \%$ on lamotrigine, $15.6 \%$ on carbamazepine and $11.1 \%$ on lithium. $51.1 \%$ of our patients were on olanzapine and $20 \%$ on quetiapine. In our sample, 37 patients (80\%) were put on antidepressant. $46.7 \%$ of our patients were put on Sertraline, $15.6 \%$ on Escitalopram, $15.6 \%$ on Fluoxetine and $4.4 \%$ on Paroxetine.

Conclusions.- The management of bipolar depressions remains complex and a major therapeutic challenge for the practitioner. Disclosure of interest.- The authors have not supplied a conflict of interest statement.

\section{E-PV0048 \\ Dysthyroidism in bipolar patients treated with lithium}

A. Haouala, S. Abid, I. Chaari*, B. Amamou, A. Mechri, L. Gaha Fattouma Bourguiba University Hospital, Psychiatry, Monastir, Tunisia

* Corresponding author.

Background and aims.- Bipolar disorder is the main indication of lithium. In all cases, the adverse effects of lithiotherapy such as disturbances in thyroid functioning should be taken into consideration. The objectives of our study are to determine the frequency of dysthyroid diseases in patients under long-term lithiotherapy, to seek correlations between these dysthyroidism and some characteristics of lithiotherapy, and to develop recommendations for the monitoring and Practice of lithiotherapy in patients at risk for dysthyroidism.

Methods.- Retrospective study performed on the records of 60 patients attending the psychiatry-department at the FattoumaBourguiba-hospital in Monastir for bipolar disorders and treated long-term by lithium salts (theralite*).

Results.- A global percentage of hypothyroidism is $39.6 \%$. This is mainly subclinical hypothyroidism in $31.4 \%$ of cases. The study of the age at onset of lithium-therapy as a risk factor in the dysthyroid population showed a significant correlation between this factor and thyroid disturbances. As a result, the earlier the age of initiation of lithium therapy, the greater the risk of developing dysthyroidism. The study of the duration of treatment and of lithemia as risk factors showed the absence of significant correlations between these factors and the dysthyroids. Regular monitoring of the thyroid is necessary because if hypothyroidism occurs and is untreated, it may result in resistance to treatment and worsen the prognosis of the disease.

Conclusions.- We recommend these patients should be made aware of the need for regular monitoring of their treatment and compliance with thyroid control and avoid as far as possible the introduction of lithium-therapy at an advanced age.

Disclosure of interest.- The authors have not supplied a conflict of interest statement.

\section{E-PV0050 \\ Diagnostic shift from schizophrenia to bipolar disorder: retrospective chart review}

A. Chrobak ${ }^{1}$, W. Janeczko ${ }^{2}$, M. Żmudka ${ }^{3}$, D. Storman ${ }^{3}$, A. Arciszewska ${ }^{1}$, M. Siwek ${ }^{4}$, D. Dudek ${ }^{1}$

${ }^{1}$ Jagiellonian University Medical College, Department of Adult Psychiatry, Krakow, Poland; ${ }^{2}$ Jagiellonian University Medical College, Students' Scientific Association of Affective Disorders, Kraków, Poland; ' 3 Jagiellonian University Medical College, Students' Scientific Association of Affective Disorders, Krakow, Poland; ${ }^{4}$ Jagiellonian University Medical College, Department of Affective Disorders, Krakow, Poland

* Corresponding author.

Background and aims.- Modern studies undermine Kraepelinian dichotomous subtyping of psychosis into schizophrenic and affective ones. The diagnostic shift between those diagnostic entities is not an unusual phenomenon and clinicians should be aware of eventuality of the change of it. Our objective is to evaluate the rate and the clinical characteristic of patients presenting diagnostic shift from schizophrenia (SZ) to bipolar disorder (BD).

Methods.- A retrospective chart review was performed. 420 charts of the patients hospitalized with the diagnosis of BD consistent with ICD-9 or ICD-10 criteria were analysed. The group enrolled in this study were admitted to the Department of Adult, Child and Adolescent Psychiatry in Kraków between 1980 and 2012. Data regarding age at the diagnosis, type of the diseases, number of affective episodes, and the dominant polarity have been collected. Results.- One of thirteen BD patients (33 out of 420) received initial diagnosis of SZ. Mean time to the diagnostic shift was 7.4 yrs. BD patients which converted from SZ presented higher number of hospitalizations due to psychotic depressive episodes $(p=0.002)$, psychotic manic episodes $(p=0.03)$, non-psychotic manic episodes $(p=0.03)$, and more frequent occurrence of type I of BD $(p<0.001)$, manic and depressive dominant polarities $(p=0.01)$ that nonconverting BD patients.

Conclusions.- Diagnostic shift from SZ to BD is not uncommon, and it is associated with higher number of psychotic depressive episodes, psychotic and non-psychotic manic episodes, dominant polarities and type I BD. 
Disclosure of interest.- The authors have not supplied a conflict of interest statement.

\section{E-PV0052 \\ Diagnosis of posible multiple sclerosis in a patient with bipolar disorder: case report}

P. Del Sol*, A. Izquierdo, L. Gayubo

Hospital Universitario Puerta de Hierro Majadahonda, Psychiatric

Acute Hospitalization Unit, Madrid, Spain

* Corresponding author.

Background and aims.- The aim of this case is to show the possible comorbidity between bipolar disease and multiple scerosis.

Methods.- The patient is a 43 years old women who was attended at the emergency room due to heavy disorganized behaviour during the previous days. She was diagnosed with bipolar disorder in 2001 (previously she was diagnosed with brief acute psychotic episode and dissociative psychosis). She was treated with lithium $1000 \mathrm{mg} /$ day, carbamacepine $1000 \mathrm{mg} /$ day, risperidone $3 \mathrm{mg} /$ day and clonazepam $2 \mathrm{mg} /$ day. For the 10 past days she barely slept, she presented high psychomotor restlessness and pressured speech, and she was fired from her job (pharmacy) because of inappropiate behaviour. Risperidone dose was increased to $6 \mathrm{mg}$ without improvement. During the admission she presented irritability, childish speech and low collaboration which were alternated with polite and gentle behaviour other days. A MRI was made with the result of demyelinating lesions.

Results.- The finding of demyelinating lesions is a typical sign of multiple sclerosis. In this patient who didn't responded to antipsychotic treatment and who also presented a fluctuating symptomatology it was thought that she might be suffering a neuropsychiatric comorbidity so that a MRI test was made.

Conclusions.- More studies are needed to prove the relationship between multiple sclerosis (ME) and bipolar disorder. Cognitive impairment can be found in both. It is known that patients diagnosed with ME are more likely to present psychiatric symptoms. Depression is presented in 50\% of ME. Also bipolar disorder is twice as common as in the general population.

Disclosure of interest.- The authors have not supplied a conflict of interest statement.

\section{E-PV0055}

\section{Bipolarity traits among backpackers}

M. Gąsior

University Clinical Centre in Gdańsk, Psychiatry Department, Gdańsk, Poland

* Corresponding author.

Background and aims.- Introduction: Backpacking is a form of lowcost, independent travel. Most backpackers are in their 20s (even though the average age of backpackers has gradually increased over time, and it is now more common to see people in their 30s, 40s, or even older, travelling this way). The mean age of onset for bipolar I disorder is 18 years and for bipolar disorder 20 years. Taking into consideration the common opinion about the nature of backpacking (spontaneous, risky, etc.), clinical features of hypomania, and above-mentioned age characteristics, it is only natural to wonder whether the prevalence of mood disorders (specifically: hypomania episodes) among backpackers is higher than in the general population.

Objectives.- To establish whether the prevalence of hypomania episodes among backpackers exceeds that in general population.
Methods.- Methods: An anonymous online questionnaire consisting of 3 parts: 1) 2 introductory questions: a screening question about the participant's current emotional state and a question about the person's typical temperament, both excerpted from the HCL-32 questionnaire, 2) 32 close-ended questions excerpted from the HCL-32 questionnaire, and 3) demographic data and psychiatric history.

Results.- Results: A total of 36 people took part in the study. In 29 cases the total score in the second part was 14 or more positive ("agree") answers. Only 2 participants admitted having been treated for psychiatric disorders and taking medication - mood stabilizers in both cases, combined with antidepressants in one case.

Conclusions.- Conclusions: The results suggest a correlation between backpacking and traits of bipolarity. However, further investigation is required.

Disclosure of interest.- The authors have not supplied a conflict of interest statement.

\section{E-PV0056}

\section{Vortioxetine for bipolar depression, effectiveness and safety. A clinical experience from a serie of cases}

S.P. Gomes Da Costa ${ }^{*}$, E. Araúz Cedeño ${ }^{2}$, N. Arbelo ${ }^{1}$, A. Gimenez ${ }^{1}$, L. Ilzarbe ${ }^{1}$, C. Llach $^{1}$, P. Bruguera ${ }^{3}$, A. Benabarre ${ }^{3}$, M. Valentî́ ${ }^{3}$

${ }^{1}$ Hospital Clinic, Psychiatry - Clinical Institute of Neuroscience, Barcelona, Spain; ${ }^{2}$ National Institute of Mental Health of Panama, Psychiatry, Panama, Panama; ${ }^{3}$ Hospital Clinic, Psychiatry - Bipolar Disorder Unit - Clinical Institute of Neuroscience, Barcelona, Spain * Corresponding author.

Background and aims.- Bipolar Depression remains an unresolved challenge for psychiatric therapeutics. It is associated with significant disability and mortality, and represents a major proportion of follow-up time spent in morbid states despite use of available treatments. Evidence regarding effectiveness of standard treatments, particularly antidepressants, remains limited and inconsistent. Vortioxetine is a novel antidepressant, acting as a multimodal serotoninergic agent with good tolerability. Data on the safety of vortioxetine for treatment of Bipolar Depression is still lacking. The objective of the study is to evaluate the clinical efficacy and safety profile of vortioxetine in patients with Bipolar Depression.

Methods.- It is an observational, retrospective study. Data collection was performed after the introduction of vortioxetine as an adjunctive treatment in depressive patients with Bipolar Disorder (BD) I and II (DSM-V criteria), followed at external consultation. Sociodemographic data, medical history, and clinical and psychometric variables were collected.

Results.- The sample includes 3 men and 4 women with a mean age of 51'7 years old. Four (4) patients were diagnosed with BD I and 3 patients with BD II. The introduction of vortioxetine led to a clinical improvement with a mean reduction in Hamilton Depression Scale of 12,86 points. The prescribed doses ranged between 10 and $25 \mathrm{mg}$ with a mean length of treatment of 13,57 months. Only 2 patients presented mild side effects.

Conclusions.- The use of vortioxetine in patients with BD I and II has meant a clinical improvement, with a favourable profile of tolerability. More studies are needed to evaluate effectiveness and safety of this promising antidepressant alternative.

Disclosure of interest.- The authors have not supplied a conflict of interest statement. 


\section{E-PV0057}

\section{Investigation of clinical factors associated with the prediction of manic symptom course in the hospitalized manic subjects with bipolar disorder \\ S.W. Hahn}

Soonchunhyang University Seoul Hospital, Psychiatry, Seoul, Republic of Korea

${ }^{*}$ Corresponding author.

Background and aims.- This study was aimed to investigate clinical factors associated with the prediction of clinical course in hospitalized patients with bipolar disorder, manic episode.

Methods.- We performed a retrospective observational study based on the medical records review of 53 bipolar disorder manic patients, who had been hospitalized in the psychiatric ward. During the hospitalization, Young Mania Rating Scales (YMRS) have been measured periodically. Demographic information and clinical characteristics including medications and history of prior hospitalization have been collected in each patient. Linear mixed effect model has been used to assess the effect of clinical factors on the changes of YMRS over time. Selection of clinical factors was conducted using backward elimination with the minimization of Akaike Information Criterion.

Results.- Mean days of hospitalization were $29.74 \pm 16.96$. Mean YMRS at the admission was $33.64 \pm 7.57$. Effective factors for the model included YMRS at baseline, combination of mood stabilizer, and the history of prior hospitalization. Predicted YMRS at the discharge was 10.43 (95\% confidence interval 7.13-13.72).

Conclusions.- The current findings suggest the model which may predict the duration of hospitalization of the bipolar disorder manic patients. It would be useful to establish the treatment plan for the patients.

Disclosure of interest.- The authors have not supplied a conflict of interest statement.

\section{E-PV0058}

\section{Self-management disease skills in people with severe neuro-cognitive disorders: a myth or matter of systematic education?}

\section{A. Hatzioannou ${ }^{1 *}$, E. Papastayrou ${ }^{1}$, A. Chatzittofi $^{2}$, S. Sokratous ${ }^{1}$,} M. Karanikola ${ }^{1}$

${ }^{1}$ Cyprus University of Technology, Nursing Department, Limassol, Cyprus; ${ }^{2}$ University of Cyprus, Medical School, Nicosia, Cyprus * Corresponding author.

Background and aims.- Effective treatment of chronic, severe neuro-biological disturbances, including Bipolar Disorder (BD), is a challenge for healthcare professionals, given the increased prevalence of these disorders.

To investigate, in people diagnosed with $\mathrm{BD}$, the effectiveness of standardised educational interventions in enhancing selfmanagement skills regarding: (i) relapse prevention, (ii) adherence to pharmacotherapy, (iii) improvement of quality of life.

Methods.- Systematic review of available literature on quantitative studies published between 2012 and 2018 in English or Greek languages. Included studies were evaluated for the quality of their methodology with the "Health Evidence-Quality Assessment Tool. Results.- Standardised educational interventions were found to be associated with: a) early identification of relapse symptoms by the patients themselves, b) reduction in the number of relapses, c) reduction in the frequency and length of hospitalization, and d) improved adherence to pharmacotherapy. Moreover, participants in the studies reviewed reported alleviation of the perceived social stigma related to mental illness, as well as an improvement in their overall functioning and quality of life. No studies were found that explore the association between standardised educational programmes and impulse control changes or improvement of cognitive impairment in people diagnosed with severe mental illness.

Conclusions.- There is evidence that participation in standardised educational programs is associated with a strengthening of disease self-management skills in people diagnosed with $\mathrm{BD}$, and an improvement of the overall quality of life. Further studies are warranted on the effectiveness of training programs in other clinically relevant disease parameters, such as impulsivity and cognitive decline.

Disclosure of interest.- The authors have not supplied a conflict of interest statement.

\section{E-PV0061}

\section{Bipolar disease onset in patients undergoing immunomodulatory treatments, a case study}

M. Khatib ${ }^{*}$, I. Ibañez Plans ${ }^{2}$, G. Prat Vigué ${ }^{1}$, E. Nieto Rodriguez ${ }^{1}$, C. Isern Tena ${ }^{1}$

${ }^{1}$ Mental Health, Psychiatry, Manresa, Spain; ${ }^{2}$ Mental Health, Psychiatry Child and Adolestent Department, Manresa, Spain

* Corresponding author.

Background and aims.- It has been reported that inflammatory pathways may have an implications in understanding mental illness. Reported studies suggest refractive depresion could be treated with antiinflammatory treatments. In this case report we report onset of bipolar disease in a 34 year old patient with history of non treated ADHD, undergoing immunomodulatory treatment due to severe spondyloarthropathy.

Methods.- Review in recient publications, mesh words used: psychiatry, inflammtion, tnf a antagonist, bipolar switch, resistent depresion.

Results.- Consistent information was found on antidepresant proporties of inmunomodulatory treatments, inflammation as part of the physiopatology in depresion.

Conclusions.- There are few reports published which to implicate a TNF-a antagonist in the development of manic switches. Although the role of TNF-a in the pathophysiology of depressive and manic episodes is still unclear, TNF-a antagonists, as well as other immunomodulatory medications, have been studied as reasonable alternatives in the treatment of depressive symptoms in recent years. Reports of manic switch induction associated with these medications suggest that infliximab may have an antidepressant effect.

Disclosure of interest.- The authors have not supplied a conflict of interest statement.

\section{E-PV0064}

\section{Depot neuroleptics in bipolar disorder}

T. López-Arteaga ${ }^{1^{*}}$, P. Padilla-Romero ${ }^{2}$, C. Romero-Martín ${ }^{3}$

${ }^{1}$ Psychiatrist, Department of Psychiatry- Hospital Ntra. Sra. del Prado, Talavera de la Reina TO- C.P. 45600, Spain; ${ }^{2}$ Hospital Ntra. Sra. del Prado, Psychiatry, Talavera de la Reina, Spain; ${ }^{3}$ Hospital Ntra. Sra. del Prado, Hospital Pharmacy, Talavera de la Reina, Spain * Corresponding author.

Background and aims.-

- Introduction.- Bipolar disorder (BD) is a complex pathology, which not only associates clinical affect, but also psychotic and high rates of comorbidity. There is not enough evidence in the literature about the use of depot in bipolar disorder for both acute and maintenance phases.

- Objetives.- We assess the effectiveness of paliperidone palmitate once monthly (PP1 M) for the psychopathological stabilization of patients with BD. 
Methods.-

- Retrospective study of a series of patients with bipolar disorder: women aged 24 to 50 years old, admitted to Mental Health Hospital from January to June 2018. Substance use disorder (SUD), both bipolar disorder subtype and patients family psychiatric history are not factors of exclusion (table 1).

Results.- iology of bipolar disorders and encourage further research on the place of anti-inflammatory drugs in the treatment of bipolar disorders.

Disclosure of interest.- The authors have not supplied a conflict of interest statement.

Table 1 Retrospective study of a series of patients with bipolar disorder

\begin{tabular}{|c|c|c|c|c|c|c|c|c|}
\hline & Age & SUD & $\begin{array}{l}\text { Family } \\
\text { history }\end{array}$ & $\begin{array}{l}\text { Time of disease } \\
\text { evolution }\end{array}$ & $\begin{array}{l}\text { Last } \\
\text { episode }\end{array}$ & $\begin{array}{l}\text { Time for } \\
\text { euthymia }\end{array}$ & Treatment & $\begin{array}{l}\text { Criteria for choice } \\
\text { of treatment }\end{array}$ \\
\hline Patient 1 & 24 & yes & no & 3 years & $\begin{array}{l}\text { F31.2; F12.20; } \\
\text { F61 }\end{array}$ & 8 weeks & $\begin{array}{l}\text { Valproic } 1.500 \mathrm{mg} / \text { day } \\
\text { PP1M 100mg }\end{array}$ & $\begin{array}{l}\text { SUD, without } \\
\text { conscience of disease }\end{array}$ \\
\hline Patient 3 & 50 & no & yes & 33 years & F31.71 & 4 weeks & $\begin{array}{l}\text { Valproic } 1.500 \mathrm{mg} / \text { day } \\
\text { PP1M } 150 \mathrm{mg}\end{array}$ & $\begin{array}{l}\text { Side effects to } \\
\text { lithium: tremor, } \\
\text { diplopia. Clinical } \\
\text { instability }\end{array}$ \\
\hline
\end{tabular}

Conclusions.- The combination of valproic acid and PP1M is effective in the acute phase of BD. It is useful for long-term adherence and maintenance, has no side effects such as lithium and achieves improvement in substance abstinence.

Disclosure of interest.- The authors have not supplied a conflict of interest statement.

\section{E-PV0065}

\section{Relevance of the dosage of high sensitivity C-reactive protein in the investigation of bipolar disorders}

M. Maalej ${ }^{1}$, M. Naifar ${ }^{2}$, A.S. Ellouze ${ }^{1}$, W. Guidara ${ }^{3}$, S. Omri ${ }^{1}$, M. Maalej $^{1}$, F. Ayadi ${ }^{2}$, N. Charfi ${ }^{1}$, J. Ben Thabet ${ }^{1}$

${ }^{1}$ Hedi Chaker University Hospital, Psychiatry "C" Department, Sfax, Tunisia; ${ }^{2}$ Habib Bourguiba University Hospital, Laboratory of Biochemistry, Sfax, Tunisia; ${ }^{3}$ Sfax Medical School- University of Sfax, Research Unit "Molecular Basis of Human Pathology", Sfax, Tunisia * Corresponding author.

Background and aims.- Several studies suggest the involvement of inflammatory and immunological processes in the pathophysiology of psychiatric disorders.

The objective of this work was to study the relevance of the dosage of high sensitivity C-reactive protein (hsCRP) in the investigation of bipolar disorders in comparison with control subjects.

Methods.- We carried out a case-control study, from June 2016 to December 2017, at the psychiatry "C" department of Hedi Chaker University Hospital. Patients with bipolar disorder (BD), who had not taken antipsychotic medication for at least three months before admission, were included in the study. The diagnosis of BD was made according to the DSM-5 criteria. Each of the patients and the controls had a clinical examination, an assessment by the positive and negative symptoms scale (PANSS) and inflammatory blood tests.

Results.- Thirty two patients and 90 controls were included in the study. The hsCRP level was significantly higher in patients than in controls $(3.28 \pm 3.48 \mathrm{mg} / \mathrm{L}$ versus $1.82 \pm 2.06 \mathrm{mg} / \mathrm{L}, p=0.012)$. The inflammatory state was significantly correlated to the PANSS score of positive symptoms $(p=0.043)$. Patients whose state required the association of many psychotropic medications had a hSCRP level on admission significantly higher than those who were treated by a monotherapy $(3.32 \pm 2.94 \mathrm{mg} / \mathrm{L}$ vs $0.65 \pm 0.07 \mathrm{mg} / \mathrm{L}$, $p=0.02$ ).

Conclusions.- Our results are consistent with the hypothesis of the involvement of inflammation processes in the pathophys-

\section{E-PV0068}

\section{Impulsiveness and disorder of cannabis use in bipolar patients}

M. Mlika*, A. Bouallagui, O. Moula, A. Ferchichi, R. Ghachem Razi Hospital, Psychiatry, Tunis, Tunisia

${ }^{*}$ Corresponding author.

Background and aims.- In the bipolar patient, the passage to the act can be prepared and premeditated, but it can also be impulsive and brutal, in the form of drive discharge and of psychic economy.

Describe the socio-demographic and clinical characteristics and different impulsiveness scores of bipolar patients with cannabis use disorder.

Methods.- This is a descriptive cross-sectional and analytical study conducted on patients followed for bipolar disorder at Razi La Mannouba hospital.

The patients recruited had a disorder of Cannabis use according to DSM 5.

Impulsivity was evaluated by the BIS 11 scale.

Results.- We recruited 19 patients all of them male. The average age of the patients was around 34, 11 years old.

Single persons were in the majority with a percentage of 73.7 The majority of patients were of urban origin (84.2\%).

The secondary level was the majority in this group with a percentage of 84.2 .

The presence of a family history was found in $26.9 \%$ of the cases whose bipolar disorders represented $40.8 \%$ The antecedent of attempted suicide were reported by 8 patients $(42,1 \%)$. Thirteen out of nineteen patients had daily cannabis use More than half of these patients consumed more than 4 joints a day Sixteen out of 19 patients (84.2\%) had a high level of impulsivity.

No significant relationship was found between impulsivity and cannabis use.

Conclusions.- Abuse of substance and especially cannabis, although it shares a high impulsivity with Bipolar Disorder, could not fully explain the elevation of the BIS 11 score in patients with this psychiatric disorder.

Disclosure of interest.- The authors have not supplied a conflict of interest statement. 


\section{E-PV0069}

\section{A case of comorbid posttraumatic stress disorder in bipolar disorder in military patient \\ D. Mnif}

Psychatric Resident, Psychatric Department of Military Hospital of

Tunis, Tunis, Tunisia

${ }^{*}$ Corresponding author.

\section{Background and aims.-}

Introduction.- Studies provide evidence for elevated rates of posttraumatic stress disorder (PTSD) in patients with bipolar disorder (BD). The coexistence of PTSD is a particularly difficult challenge in the treatment of BD with antidepressants (AD).

Aims.- Description of clinical presentation and treatment strategies for a better recovery.

Methods.- A case report of a military patient in treatment for comorbid PTSD in BD type 1.

Results.- A 41-year- old patient with a family history of mood disorder. His mother was treated for bipolar depression. He was initially treated, at the age of 33 , for depression with resistance for different $\mathrm{AD}$ (paroxetin, anafranil) and persistant low global functioning. He was diagnosed with Comorbid PTSD and substance use disorder (SUD) after 7 years of treatment. The stressor event happend five years before the first episod. He received then venlafaxine for one year without getting better. He was hopitalized for maniac episod. We decided to stop AD and to put him under valproate (1500 mg). We observed improvement in impulsivity and intrusive symptoms with mood stabilizer.

Conclusions.- PTSD occurs at elevated rates in patients with BD, and is associated with a more severe course of disorder. Clinicians should think about monitoring symptoms of PTSD in patients with BD. Some mood stabilizers appear to be effective for the treatment of PTSD.

Disclosure of interest.- The authors have not supplied a conflict of interest statement.

\section{E-PV0070}

\section{Bipolar affective disorder in multiple sclerosis: a review}

\section{R. Mota Freitas*, A. Boavida Guerra}

Espírito Santo Hospital, Department of Psychiatry and Mental Health, Évora, Portugal

${ }^{*}$ Corresponding author.

Background and aims.- The presence of psychiatric manifestations in multiple sclerosis (MS) is well established and they may be the presenting symptoms of the disease. Bipolar affective disorder (BD) is estimated to be twice as common in MS as in the general population. The effects of steroid treatment alone cannot explain this association and the link between MS and BD is not fully understood. In this review, we aimed to summarize the current understanding of BD in MS patients and the most recent perspectives in its management.

Methods.- We searched the PubMed database for reviews, systematic reviews, meta-analyses and case reports on bipolar disorder in multiple sclerosis in humans, published in the last 10 years. Titles and abstracts were screened for relevance, followed by full-text review. The search retrieved 65 results. Following title, abstract and full-text reading, 13 papers were included.

Results.- Etiopathogenic bases explaining the association between $\mathrm{BD}$ and MS remain unclear. The effects of corticosteroid therapy and the psychological distress caused by the diagnosis of MS cannot fully explain it. The role of genetic vulnerability and a common neurological substrate have been suggested, but evidence to support these hypotheses is scarce and contradictory. Other factors, such as shared autoimmune and infectious mechanisms are likely involved.

Conclusions.- Management of BD in MS is not yet solidly established. Additional research in this area must be conducted to further assess the association of BD and MS so that appropriate management strategies can be developed.

Disclosure of interest.- The authors have not supplied a conflict of interest statement.

\section{E-PV0072}

\section{Post stroke mania - a rare complication not to forget}

R. Saraiva ${ }^{1 *}$, F. Proença ${ }^{2}$, M.J. Gonçalves ${ }^{1}$, A.C. Sereijo ${ }^{1}$, R.

Barandas $^{1}$, R. Coentre ${ }^{1}$

${ }^{1}$ Hospital Santa Maria Centro Hospitalar Lisboa Norte, Psiquiatria, Lisboa, Portugal; ${ }^{2}$ Hospital Santa Maria Centro Hospitalar Lisboa Norte, Neuroradiologia, Lisboa, Portugal

"Corresponding author.

Background and aims.- Various organic diseases can trigger a manic episode. Stroke is often associated with psychiatric symptoms, particularly depressive, and more rarely manic.

We aim to report a case of recurrent mania after a stroke, in a patient without previous history of psychiatric illness and to do a brief review about post stroke mania.

Methods.- Literature review on mania and bipolar disorder after stroke (MEDLINE literature review) and description of the clinical case with information provided by the patient or by his medical records.

Results.- Post stroke mania is rare and reports of the evolution after acute phase of the manic episode or reports of recurrence are even rarer. We describe a 67-year-old man with no personal or family history of psychiatric illness, with hypertension, dyslipidemia and diabetes, who at age 64 had a bilateral ischemic stroke in the middle cerebral artery territory. About twenty days after this stroke he developed a manic episode with hospitalization in a psychiatric ward. In the following years, he was clinically stable under $1000 \mathrm{mg}$ sodium valproate per day, being totally independent in the activities of daily living. Three years after this episode he developed a new manic episode.

Conclusions.- This case shows that it is possible for an organic disease to cause a syndrome with an evolution overlapping a primary psychiatric illness even in individuals without history of psychiatric disorders. Although rare, the diagnosis of mania after stroke should not be forgotten, and most important, one should be aware the a recurrence mays occur.

Disclosure of interest.- The authors have not supplied a conflict of interest statement.

\section{E-PV0073}

\section{The longitudinal outcome of bipolar disorder}

R. Paunescu, A.C. Serban*, B. Suciu, I. Miclutia

University of Medicine and Pharmacy Cluj Napoca, Psychiatry, Cluj

Napoca, Romania

* Corresponding author.

Background and aims.- Bipolar disorder is a chronic psychiatric illness with compresses affective episodes of different polarities. Its outcome involves relapses, complete and partial recoveries and euthymic periods.

Methods.- We analyzed the medical files of 70 patients diagnosed with bipolar disorder for a period of 5 years since the onset of illness. The aim of the study was to assess the number of episodes/hospitalizations, types of mood episodes, the presence of psychotic features and psychiatric and non psychiatric comorbities, 
Results.- The patients included cumulated a number of 217 hospitalizations over the 5 years following the onset of bipolar disorder. Most admissions to the hospital were due to manic episodes $(55,1 \%)$, followed by depressive episodes $(37,9 \%)$ and episodes with mixed features (7\%). The number of hospitalizations ranged from 1 to 11 for the mentioned time period. The mean duration between hospitalizations was 11,8 months varying from 2,74 to 41,16 months. The mean number of admissions was higher in females patients compared to male patients. Psychotic symptoms occurred in $24,3 \%$ of depressive patients and in $51,2 \%$ of manic patients. $32 \%$ of the patients had psychiatric comorbidite. Medical comorbidities were seen in $43,69 \%$ of the patients.

Conclusions.- For a five years period from the onset of bipolar disorder, most patients presented more than one admission to the hospital. The duration of the hospitalization is correlated with the severity of the mood episode and the presence of psychotic features. Disclosure of interest.- The authors have not supplied a conflict of interest statement.

\section{E-Poster Viewing - 7-9 April: Child and Adolescent Psychiatry}

\section{E-PV0077}

\section{Adolescence and self-injuries: characteristics of current society}

Y. Quevedo, S. Viani, A. Dörr

University of Chile, Psychiatric and Mental Health, Santiago, Chile

Corresponding author.

Background and aims.- This research aims to enrich the reflection on the current affairs and an invitation to think about worrying phenomena in youngsters: self-injuries or cuts in the arms in eight young adolescent patients. We try to figure out the underlying cause of such behavior, which is more common every day and reveals some characteristics of society. Sociocultural, individual and family changes are analyzed, showing possible connections among these instances, identity and self-injuries.

Methods.- Clinical interview and questionnaire:

With a qualitative methodology, descriptive, exploratory design, we try to find the constitutive elements of phenomena about the self-injuries or cuts in the arms in young adolescent patients. Three levels are analyzed in ten young adolescent to know if there is some possible connections among these instances and the development of identity and self-injuries:

- Sociocultural,

- individual

- family changes

Results.-

The analysis of the questionnaire applied during the clinical interview, the results show a possible connection between the three levels analyzed (sociocultural, individual; family changes) and practices such as self-injury, cuts, tattoos, branding and piercing in different parts of the body.

Conclusions.- We suggest that the difficulties in identity development may be related to practices such as self-injury or others, which are related to mentalization problems. In the sociocultural level we find a way to socialize in which subjective discomfort has increased, there is overabundance, mass consumption, and the traditional social institutions (family, school and religion) have failed. Youngsters do not know themselves nor their projects

Disclosure of interest.- The authors have not supplied a conflict of interest statement.
E-PV0079

\section{Neuropsychiatric disorders in children and adolescents with klinefelter syndrome $(47, X X Y)$ the importance of social cognition: a case report}

N. Fernández Gómez ${ }^{*}$, M. Alcamí Pertejo, M.A. Ortiz Villalobos, L. Mellado Cuerno, V. Romero Pardo, J.M. Pastor Haro, J. Curto

Ramos, M.F. Bravo Ortiz

Hospital Universitario La Paz, Psychiatry and Mental Health, Madrid, Spain

Corresponding author.

Background and aims.- Klinefelter syndrome (KS) is a genetic disorder characterized by the presence of an extra $X$ chromosome (47, XXY). Despite of the fact that endocrinologic and physical features are the most described, KS also involves increased risk of developing psychiatric disorders (autism, schizofrenia, schizoid personality) which are related to social cognition and verbal impairments. Our aim is to describe the clinical case of a 15 years old boy with KS, behavioural disturbances and poor social interaction. We also aimed to reach a well defined psychiatric diagnosis of these clinical symptoms.

Methods.- We describe and analyse the clinical case of a 15 years old boy with KS and behavioural disturbances. Autism Diagnosis Observation Schedule-2 (ADOS-2) Module 4 was administered and results of Wechsler Intelligence Scale for Children- Fouth Edition (WISC-IV) were included. We also conduct a literature review about the neuropsychiatric disorders in this syndrome.

Results.- WISC-IV score was 100 (average intelligence quotient -IQ). Impairments in verbal skills were not detected. Resuts of ADOS-2 Module 4 showed an Autism Spectrum Disorder (ASD) diagnosis. Most remarkable impairments were detected in communication and social reciprocity areas.

Conclusions.- KS may involve neuropsychological impairments. However, they are rarely intelectually disabled. Language disabilitys are the most common identified in children with KS. They also show impairments in social and emotional cognition, with difficulties in expressing emotions and decoding and interpreting another's emotions and facial expressions. Psychiatric disorders related to dysfunctions in social cognition, such as ADS, should be considered in differential diagnosis in order to improve the clinical and psychopedagogical management. These disorders may be the underlying cause of behavioral disturbances, as in the clinical case that we describe.

Disclosure of interest.- The authors have not supplied a conflict of interest statement.

\section{E-PV0080}

Trends and outcomes in child and adolescent mental health emergencies: a 7-year follow-up study

Á. Fernández-Quintana ${ }^{1}$, A. Novo-Ponte ${ }^{2}$, C. Quiroga-Fernández ${ }^{2}$, R. Mendez-Iglesias ${ }^{2}$

${ }^{1}$ Clinical Hospital of Västmanland, Psychiatry, Västerås, Sweden; ${ }^{2}$ Clinical University Hospital of La Coruña, Psychiatry, La Coruña, Spain ${ }^{*}$ Corresponding author.

Background and aims.- Children and adolescents require increasing numbers of visits to psychiatry emergency departments (ED).

Aims.- To underpin sociodemographic and clinical variables associated with an upward trend in ED visits. To analyse diagnostic stability over the follow-up.

Methods.- Retrospective study of 160 patients aged under 17 years who attended ED due to psychiatric concerns over a 7-year period (2010-2017).

Results.- Mean age 14,62 years (SD: 2,15). 52,5\% females. Prevalence of ED visits spanning from $14,4 \%$ in 2010 to $25 \%$ in $2016.71,3 \%$ lived 
in urban settings, $51 \%$ of parents were divorced. Most prevalent causes of ED visits: aggressive behaviour in males (56\%), self-harm in females (40\%). Personal history of suicide attempt was significantly more prevalent among females $(p<0.001)$. Severe problems of primary support group detected in $25,8 \%$ of subjects. Somatic history: epilepsy $(10,1 \%)$, coeliac disease $(6,3 \%)$, diabetes $(5,7 \%)$, heart disease $(1,9 \%)$, thyroid disease $(1,9 \%)$, cerebral palsy $(1,3 \%)$. $89,9 \%$ had a prior psychiatric diagnosis: Conduct Disorders (40,6\%), ADHD (16,3\%), Major Depression (11,9\%).73\% received psychopharmacological treatment prior to ED admission (44\% antipsychotics, $43 \%$ benzodiacepines, $30 \%$ antidepressants, $14 \%$ mood stabilizers, $11 \%$ stimulants). $12 \%$ abused drugs (THC being the most prevalent). Alcohol abuse was significantly more prevalent among females $(p=0,026)$. Psychiatric family history positive in $42 \%$ of cases. Inpatient admission rates: $13,2 \%$. Attendance to first follow-up appointment: $78,1 \% .57,8 \%$ collected ED prescriptions. Diagnostic stability over 7 years: $73 \%$.

Conclusions.- Significant gender differences were noted among children and adolescents who attend ED. Low rates of compliance to ED prescriptions and deficits in follow-up care need to be addressed.

Disclosure of interest.- The authors have not supplied a conflict of interest statement.

\section{E-PV0082}

\section{The validity of child and youth depression diagnoses in the danish central psychiatric research register}

L.H. Frederiksen ${ }^{*}$, N. Bilenberg ${ }^{1}$, L. Andersen ${ }^{2}$, N. Henriksen ${ }^{2}$, B. Lyng-Rasmussen $^{3}$, R. Wesselhöft ${ }^{1}$

${ }^{1}$ University of Southern Denmark, Research Unit of Child and Adolescent Mental Health- Department of Clinical Research, Odense, Denmark; ${ }^{2}$ Mental Health Services in the Region of Southern Denmark, Department of Child and Adolescent Psychiatry Odense, Odense, Denmark; ${ }^{3}$ Hospital Lillebælt, Department of Paediatrics, Kolding, Denmark

* Corresponding author.

Background and aims.- All psychiatric diagnoses assigned for inpatients and outpatients in Denmark are recorded in the Danish Central Psychiatric Research Register (DCPRR). Danish registerbased studies have contributed significantly to international psychiatric research, however to be able to use registries for observational studies it is imperative that the clinical diagnoses recorded are valid. Validation studies of the depression diagnosis have been carried out for adults, supporting that the depression diagnosis is valid in the DCPRR. However, no studies have so far examined the validity of the depression diagnoses assigned for children and adolescents.

The objective of this study is to investigate the validity of first onset depressive episode (DE) diagnoses in the PCPRR for children aged 6-17 years. Furthermore, the study will examine if there is a difference in validity between the different severities of DE, and if there is a higher validity among those patients who are prescribed antidepressant medication.

Methods.- The study is a population-based study using a random sample nested within a cohort of all Danish children and adolescents diagnosed with DE. The PCPRR is used to identify a cohort of 500 children and adolescents assigned with a first onset DE between 1996-2016. The validity is determined by reviewing hospital charts to see if the ICD-10 diagnostic criteria are met.

Results.- Data collection will start November 2018. Preliminary results will be presented at EPA April 2019 in Warsaw.

Conclusions.- The study can provide important information about the usefulness of the DCPRR in child and youth depression research. Disclosure of interest.- The authors have not supplied a conflict of interest statement.

\section{E-PV0083}

\section{PRE-validation of a battery of} Tunisian social cognition tests

M. Ghazzai*, A. Taamallah, O. Rajhi, M. Moussa, S. Halayem, Z. Abbes Ghorbel Salma, M. Hajri, A. Bouden

Razi Hospital, Department of Child and Adolescent Psychiatry,

Mannouba, Tunisia

* Corresponding author.

Background and aims.- To better understand and assess the development of social skills among Tunisian children with typical development, we created a battery of assessment tools for emotions' recognition, mentalization and empathy.

Objectives.- Validate each test in children population to establish normative references.

Methods.- The Participants are Tunisian children without mental retardation and psychiatric disorders aged between 7 and 11 years old.

Recognition of Facial Emotions: Tunisian Test for Children (FETTC): consists of photographs and videos representing the 6 basic emotions and neutrality.

Tunisian Emapthy Scale for Children (TESC): measures the components of Empathy. Tunisian Social Situations Instrument (TSSI): a verbal test for theory of mind,

Picture Sequencing: a non-verbal test for theory of mind.

Results.-

FETTC: There was a positive correlation between empathic attitude and age, $r=0.423, n=46, p=0.003$.

TESC: The total score of empathy was significantly higher among girls. There was a positive correlation between between the cognitive empathy attitude and mental age, $r=0.486, n=60, p=0.025$. TSSI: There was an improvement in the global score with mental age, and school level without an identified celling effect.

Picture Sequencing: There was an improvement in the global score with age, and a ceiling effect around 9 years old.Conclusions.- These results are encouraging and will be discussed in the light of literature data.

* Corresponding author.

Disclosure of interest.- The authors have not supplied a conflict of interest statement.

\section{E-PV0084}

\section{Body mass index and their changes among child and adolescent patients with psychotic versus non-psychotic disorders}

S.P. Gomes Da Costa ${ }^{1}$, A. Gomez ${ }^{2}$, A. Gimenez ${ }^{1}$, G. Anmella ${ }^{1}, \mathrm{~J}$. Pinzon ${ }^{1}$, P. Guzman ${ }^{1}$, A. Morer ${ }^{3}$, I. Baeza ${ }^{3}$

${ }^{1}$ Hospital Clinic, Psychiatry - Clinical Institute of Neuroscience, Barcelona, Spain; ${ }^{2}$ Universidad de Antioquia, Psychiatry, Medellin, Colombia; ${ }^{3}$ Hospital Clinic, Child and Adolescent Psychiatry - Clinical Institute of Neuroscience, Barcelona, Spain

${ }^{*}$ Corresponding author.

Background and aims.- Despite the increasing use of psychotropic medications in children and adolescents, and the concerns about their metabolic side effects, data regarding their safety are limited and mostly focused on psychotic disorders. There are few studies evaluating metabolic repercussions of psychotropic medications in other psychiatric disorders, where the use of antipsychotics is widespread. The aim of this study is to analyse the differences in BMI (Body Mass Index) and weight change between patients with psychotic and non-psychotic disorders during their attendance to a day hospital. 
Methods.- A retrospective analysis of the clinical records of all patients between 12 and 18 years old, who were admitted to our Child and Adolescent Psychiatry Day Hospital during 2016 was carried out. Patients with eating disorders were excluded.

Results.- From the initial sample of 48 patients, BMI was recorded in 36 patients. Fifteen patients $(41,6 \%)$ had psychotic disorders (Schizoprenia, Bipolar, Schizoaffective or non-specified psychosis) and 21 had other mental disorders, showing a mean BMI, adjusted by age and gender, of 23,75 and 23,68 respectively, with a mean increase of BMI during hospitalization of 1,42 and 1,38 respectively. The percentage of patients receiving antipsychotic medications were $93.3 \%$ in psychotic disorders group and $85,7 \%$ in the nonpsychotic group.

Conclusions.- Our study didn't find significant differences between BMI or BMI change among the diagnostic subgroups, with a high prevalence of antipsychotic utilization in non-psychotic group. The metabolic concerns established for psychotic patients should not be undervalued in non-psychotic patients, and longitudinal studies evaluating the metabolic disturbances in non-psychotic patients are needed.

Disclosure of interest.- The authors have not supplied a conflict of interest statement.

\section{E-PV0086}

\section{Psychiatric morbidity among the patient of first ever ishchemic stroke}

M. Inam

BMDC, Psychiatry, Kulaura, Bangladesh

Corresponding author.

Background and aims.- Stroke is the most common cause of mortality worldwide and a serious cause of disability in the community. Stroke affects not only physical but also emotional, psychological, cognitive, and social aspects of patients. Some of the neuropsychiatric disorders associated with stroke include post stroke depression (PSD), bipolar disorder, anxiety disorder, apathy without depression, psychotic disorder and catastrophic reaction. Aims.- To evaluate psychiatric morbidity among the patients of first ever ischemic stroke.

Methods.- This cross sectional comparative study was carried out in the Department of Psychiatry, Sylhet MAG Osmani Medical College Hospital. 66 ischaemic stroke patients of first attack between 2 weeks to 2 years of stroke, aged above 18 years irrespective of sex and 66 accompanying healthy person of the patients and other patients without any kind of stroke matching age and sex fulfilling inclusion and exclusion criteria were taken in Group-A and Group-B respectively. Exclusion criteria were patients with transient ischaemic attack, haemorrhagic stroke, previous stroke, head injury, known psychiatric disorder, serious cognitive impairment and other chronic diseases that may cause psychiatric morbidity.

Results.- Co-morbid specific psychiatric disorders were generalized anxiety disorder in $13.6 \%$ and major depressive disorder in $21.2 \%$ in stroke group; while co-morbid specific psychiatric disorders were generalized anxiety disorder in 3.0\% and major depressive disorder in $10.6 \%$ respondents in control group.

Conclusions.- Co-morbid psychiatric disorders are quite common among patients with first ever ischaemic stroke in the form of major depressive disorder and generalized anxiety disorder. Therefore, attention should be paid to the anxiety and depressive symptoms in stroke units.

Disclosure of interest.- The authors have not supplied a conflict of interest statement.

\section{E-PV0087}

\section{Psychosis in autism spectrum disease: report a case}

A. Izquierdo De La Puente*, P. del Sol Calderón

Universitario Puerta de Hierro de Majadahonda, Psychiatry, Madrid, Spain

Corresponding author.

Background and aims.- The aim of this case is to perform a review of psychosis in autisim spectrum disease (ASD).

Methods.- 8-year-old woman with a previous diagnosis of ASD, attended for presenting delusional and hallucinations symptons. The patient reported that there was a fantasy world, with imaginary beings, with whom she was able to communicate. She said that she felt how those beings touched her and talked with them. She described that when she was alone, she heard voices inside her head that she related to these beings. He also explained that she had a certain function in that fantasy world. In addition to ASD, she was diagnosed with psychotic symptoms. was treated with antipsychotics, achieving complete remission of symptoms.

Results.- In the psychiatry of the 70s, no distinction was made between psychosis and ASD. It is a very controversial topic, because many of the symptoms of ASD can be confused with psychotic symptoms. Especially, there is a difference in boys with ASD and girls. The latter present a fantasy world much more structured than children with ASD and have a lower degree of difficulty for social interaction. For this reason, in girls, it is difficult to make the diagnosis of psychosis and in addition, ASD is diagnosed later.

Conclusions.- It is difficult to make a differential diagnosis between both pathologies. However, early diagnosis of psychotic symptoms is essential to establish the antipsychotic treatment in the earliest way, to improve the prognosis.

Disclosure of interest.- The authors have not supplied a conflict of interest statement.

\section{E-PV0088}

\section{Early onset psychosis in a pre-adolescent male with a history of exposure to pre-natal maternal infection and hydronephrosis}

M. James", U. Mihir, M. Jennings, P. Korenis, S. Gunturu Bronx Care Hospital System, Psychiatry, New York, USA

Corresponding author.

Background and aims.- Maternal infection during pregnancy can be associated with a number of adverse post-natal outcomes. These complications are attributable to an ever-growing list of bacteria and viruses responsible for vertical transmission of infection in utero, during delivery, or while breastfeeding. Even with advancements in prevention, diagnosis, and treatment of maternal infections, the limited epidemiology and etiology data is a barrier to implementing effective public health measures.

Methods.- We present the case of a 10-year-old African American male born to an African mother who migrated from Ghana shortly after she realized she was pregnant with the patient. In utero, patient was exposed to maternal infections that included Toxoplasmosis and Cytomegalovirus. He was brought to the hospital after experiencing command auditory and visual hallucinations for six months to stab his younger sister with a knife.

Results.- With appropriate measures, there has been a substantial reduction in neonatal morbidity and mortality, and better mitigation of adverse effects to the newborn. However, the impact of maternal infection can linger long after the infection has resolved, and consequences may arise years later, including psychiatric complications. Among the after-effects is the potential for psychosis, a broadly-defined psychiatric term for symptoms that cause an indi- 
vidual to have sensory experiences without stimuli or beliefs not based in reality.

Conclusions.- In studying this case, health care practitioners may better recognize the prodromal symptoms of psychosis in patients with a history of exposure to maternal infection, allowing for potential control of symptoms to avoid exacerbation leading to debilitation.

Disclosure of interest.- The authors have not supplied a conflict of interest statement.

\section{E-PV0089}

\section{FASD - the impact on a patient's life and his/her social and family environment}

P.S. Katarzyna*, D. Puzio, A. Gmitrowicz, I. Makowska

Medical Uniwersity of Lodz, Clinic of Adolescent Psychiatry, Łódź,

Poland

${ }^{*}$ Corresponding author.

Background and aims.- Fetal Alcohol Spectrum Disorder (FASD) is a broad term which encompasses neurodevelopmental abnormalities caused by maternal alcohol intake during pregnancy. It comprises Fetal Alcohol Syndrome (FAS), Partial Fetal Alcohol Syndrome (pFAS) and Alcohol Related Neurodevelopmental Disorder (ARND). A full-blown FAS is characterised by facial dysmorphology, growth retardation, low body weight, microcephaly and specific central nervous system (CNS) damage. Prenatal alcohol exposure may also impair organogenesis of organs other than brain causing their structural and functional anomalies. Since other FASD conditions have a range of presentations, diagnostic process in these cases requires professionals' cooperation in a multidisciplinary team.

Methods.- We would like to present common problems experienced by patients diagnosed with FASD and their families based on a case study. Family, educational, legal and occupational aspects of patient's life will be discussed from birth, throughout early childhood and adolescence to adulthood. We will demonstrate the role of early diagnosis for better future functioning.

Results. - Patients with FASD might not be understood by others and might show impairments in social functioning. The burden stemming from physical malformations may hamper educational and employment activity. CNS damage may cause poor understanding of social rules, disability to foresee consequences and poor judgement. This in turn leads to secondary disabilities such as impaired social bonding and conflicts.

Conclusions.- Poster underscores the significance of early and adequate diagnosis of FASD in order to improve health condition as well as social, educational and occupational abilities of affected individuals.

Disclosure of interest.- The authors have not supplied a conflict of interest statement.

\section{E-PV0090 \\ Analysis of factors that influence the young children's internalized and externalized behaviors}

S. Kim ${ }^{*}$, S. Cho ${ }^{1}$, M. Jang ${ }^{2}$, H. Han ${ }^{1}$

${ }^{1}$ Ajou University School of Medicine, Department of Psychiatry and Behavioral science, Suwon, Republic of Korea; ${ }^{2}$ National Center for Mental Health, Department of Psychiatry, Seoul, Republic of Korea

${ }^{*}$ Corresponding author.

Background and aims.- Multiple factors including Parental factors, children's factors and environmental factors has known to influence the various behavioral problems of the young children. But There have been continuing concerns about the extent to which factors may influence maternal reports of children's behavior.

The aim of the present study was to identify parental, children's and environmental factors that influence the internalized and externalized behavior among Korean children from ages 3-5.

Methods.- In 2014-2015, the parents of 400 children aged 3 to 5 years (207 boys and 193 girls, mean age $3.86 \pm 0.8$ years) were surveyed using a questionnaire on the family life style, children's behaviors and temperaments and the parental mental health including parenting stress index, BDI, BAI, internet \& smartphone addiction scale at 3 community based children's mental health centers. Statistical analyses including the Pearson's correlation test and multiple linear regressions were performed in this study.

Results.- Maternal depression were most highly associated with the young children's behavioral problems (OR 6.422, $p<.001$ ). The externalized behavioral problems (OR 6.336, $p<.001$ ) were much associated with the maternal depression than the internalized behavioral problems (OR 5.261, $p<.001$ ). Maternal smartphone addiction (OR 1.628, $p=0.11$ ) and maternal anxiety (OR 2.778, $p=0.08$ ) were associated with the children's internalized behavioral problems, but it was not significant.

Conclusions.- Maternal depression has a greater impact on the children's internalized and externalized behavioral problems than any other factors. Therefore, prevention and treatment of maternal depression are the most important for the mental health of the young children.

Disclosure of interest.- The authors have not supplied a conflict of interest statement.

\section{E-PV0092}

\section{Is own body really aversive stimulus in eating disorder? is their subjective and physical reaction similar?}

T. Knejzlikova*, M. Svetlak

Faculty of Medicine- Masaryk University Brno- Czech Republic, Department of Psychology and Psychosomatic, Brno, Czech Republic * Corresponding author.

Background and aims.- Disturbance perception of body shape and body dissatisfaction are one of the main clinical symptoms of anorexia nervosa. Subjective perception of their own body is nonrealistic and often is very different from ideal body, which eating disorder (ED) patients want to have. Disturbed body image play an important role in development and maintenance of eating disorder. During therapy, they slowly become accustomed to their own self-image, accepting it more. In general, a view of the body, either supposed, or real in the mirror, represents one of the most aversive stimulus for girls with eating disorders.

Methods.- Thirty-two adolescent girls with restrictive type of anorexia nervosa and thirty-three health controls (HC) were repeatedly exposed to own body in the mirror and physiological activity (ECG, skin conductance) was measured during experiment. Subjects were rating their emotional reaction on SAM scale after mirror exposure.

Results.- We hypothesize, that girls with ED will have increased stress reactivity indexed to mirror exposure than $\mathrm{HC}$. We also expected significant discrepancy between subjective evaluation of exposure indexed by SAM and objective physiological reactivity in ED group. The results of the present study demonstrate that ED group experienced higher negative and intensive emotional response to body exposure compare to $\mathrm{HC}$ and showed higher physiological arousal in skin conductance. We could not confirm the paradoxical relationship between the subjective and objective response to mirror exposure in patients.

Conclusions.- This report was written with the support of the Specific University Research provided by MŠMT, MUNI/A/0976/2017. 
Disclosure of interest.- The authors have not supplied a conflict of interest statement.

\section{E-PV0093 \\ Differences in sociodemographgic and clinical characteristics between kids and adolescents diagnosed with social (pragmatic) communication dissorder and autisim spectrum disorder acroding to DSM-V criteria}

S. Madero ${ }^{1 *}$, A. Blazquez $^{2}$, A. Ortiz Encarnacion ${ }^{2}$, E. Varela $^{2}, \mathrm{R}$. Calvo $^{2}$, O. Puig ${ }^{2}$, L. Lazaro ${ }^{2}$

${ }^{1}$ Hospital Clinic of Barcelona, Department of Psychiatry and Psychology, Barcelona, Spain; ${ }^{2}$ Hospital Clinic of Barcelona, Department of Child and Adolescent Psychiatry and Psychology Hospital Clinic of Barcelona, Barcelona, Spain

* Corresponding author.

Background and aims.- Introduction: The recently introduced social (pragmatic) comunication dissorder (SCD) differentiatates from autism spectrum disorder (ASD) in both the restricted interests and repetitive conducts [1], absent in SCD. Its phenomenological base is widely controverted and requires further research [2,3].

Objectives.- Analyse the clinical and sociodemographical characteristics between patients diagnosed of ASD and SCD according to DSM-V criteria.

Methods.- A retrospective analysis of database information including 83 clinical records that fulfilled criteria for ASD and SCD diagnosis acording to DSM-V and the autism diagnostic interview revised (ADI-R). The neuropshycological battery were also included (WISC-IV). Individuals with a below normal inteligence quotient (IQ) were exculuded. Sociodemographical and clinical variables were collected in order to study posible short term pronostic differences between groups (comorbid diagnosis, treatment, follow-up, academic performance and social functioning with peers).

Results.- A total of 68 patients diagnosed with ASD and 15 with SCD were included. No gender or age, and family socioeconomical level differences were found between groups. Differences in IQ were found, being higher statiscitaly significant in the $\operatorname{SCD}(p=0.039)$. Furthermore, ASD showed higher scores in the ADI-R B $(p=0.24)$ domain when compared to the SCD. No statisticaly significant differences were found regarding comorbidity, follow-up, academic performance and social functioning. Analysis showeds ASD patients were more likely to have psychofarmacologic treatment $(p=0.045)$. Conclusions.- Our findings suggest that the clinical differences observed between ASD and SCD may account, at least in part, to between group differences in psychopharmacologic treatment. No differences in the proposed pronostic outcomes were found between ASD and SCD.

Disclosure of interest.- The authors have not supplied a conflict of interest statement.

\section{E-PV0094}

\section{Treatment of ADHD in children with congenital heart diseases: a literature review}

P. Manocha ${ }^{1}$, D. Bhullar ${ }^{1}$, H. Raai ${ }^{2}$, M. Zeshan ${ }^{3}$

${ }^{1}$ BronxCare Health System, Psychiatry, Bronx, USA; ${ }^{2}$ Montefiore Medical Center, Psychiatry, Bronx, USA ${ }^{3}$ Boston Children HospitalHarvard Medical School, Psychiatry, Boston, USA

* Corresponding author.

Background and aims.- With recent advances in medicine, mortality rates in children and adolescents with complex congenital heart disease (CHD) have gone down, which has lead to increased incidence of complex neurodevelopmental (ND) and behavior pattern such as learning disabilities, impairments in core communication skills, as well as inattention, impulsive behavior, and impaired executive functions. Since treatment of ADHD requires medications such as amphetamine based stimulants, alpha 2 adrenergic agonist, as well as noradrenergic agents all known to cause cardiac effects, the safety of these medications use in children with underlying cardiovascular abnormalities has been concerning.

Methods.- Literature was searched using key terms "Treatment of ADHD" and "Congenital Heart Disease" for articles indexed in PubMed and Google Scholar in last 10 years.

Results.- Literature suggests that early identification of these ND impairments and intervention by treating these symptoms is the better approach in minimizing future disabilities. ADHD medications increase the dopamine level and thus improving neurological functioning. They also affect cardiac functioning by affecting heart rate and blood pressure. There has been consensus that all patients treated with ADHD medications require continued surveillance of heart rate and blood pressure, but there are no practice parameters for risk assessment before initiation of pharmacologic therapy for ADHD.

Conclusions.- None of the studies have shown any evidence of increased risk of sudden death in-patient receiving stimulants or any other ADHD drugs. Coordination of care between the primary care physician, the ADHD medication-prescribing physician, and the pediatric cardiologist may be an important component of treatment.

Disclosure of interest.- The authors have not supplied a conflict of interest statement.

\section{E-PV0095}

\section{The use of transcranial magnetic stimulation in autistic spectrum disorders with predominance of speech impairment}

M. Marachev*, A. Grigorieva, M. Sheina, I. Kazachenko, A. Kostileva Centre for Medico-Psychological Correction and Rehabilitation

"Neurocentre"- Moscow-Russian Federation, Centre for

Medico-Psychological Correction and Rehabilitation "Neurocentre"Moscow- Russian Federation, Moscow, Russia

* Corresponding author.

Background and aims.- The worldwide population prevalence Autism spectrum disorders (ASD) is about 1\% (Meng-Chuan Lai et al., 2013). Symptoms of ASD include a violation of social interaction, interpersonal functions and atypical perception of information. Thus, a possible correction includes a set of measures aimed at the maximum number of brain functions affected in the pathological process.

To assess the effectiveness of TMS when combined with standard therapy of ASD.

Methods.- Children diagnosed with ASD $(N=8$ mean age $7.25 \pm 0.8)$, the clinical evaluation method and the Childhood Autism Rating Scale (CARS) scale were used. All children underwent a course of correction therapy with Speech-language pathologists and a development specialist (20 sessions per month), 4 children underwent 15 additional procedures rTMS (DLPFC, $10 \mathrm{~Hz}, 30 \mathrm{imp}$ ( $3 \mathrm{sec}$ ), 50 train, 27 sec int).

Results.- CARS in the standard therapy group + TMS was significantly better (mean score at the beginning of therapy was $46.7 \pm 6.4$ compared to $38.7 \pm 3.3$ at the end), compared with the group where only standard correction methods were used (mean score at the beginning of therapy is $45.7 \pm 4.9$ vs. $42.2 \pm 3.9$ )Subjective assessment of improvement in the standard therapy group + TMS by the parents was noted at 5-7 sessions, while in the other group (standard therapy) not earlier than the 15 th session. 
Conclusions.- The joint application of rTMS and standart therapy leads to a more rapid dynamics in the state of children with ASD. Mainly due to the improvement of speech function, greater concentration and ordering of behavior.

Disclosure of interest.- The authors have not supplied a conflict of interest statement.

\section{E-PV0096}

\section{Children and adolescents affected by psychiatric disorders exhibit lower HPA axis reactivity: the influence of sex and pubertal stage}

L. Marques-Feixa ${ }^{{ }^{*}}$, H. Palma-Gudiel ${ }^{1}$, Á. Castro-Quinto ${ }^{2}$, M. Martín $^{3}$, M. Rapado-Castro ${ }^{4}$, M. Mayoral ${ }^{4}$, I. Zorrilla ${ }^{5}$, M. Ramirez $^{6}$, S. Romero ${ }^{7}$, I. Mendez ${ }^{7}$, H. Blasco ${ }^{8}$, M.J. Lobato ${ }^{8}$, L. Fañanás ${ }^{1}$ ${ }^{1}$ University of Barcelona and CIBERSAM Mental Health Networking Biomedical Research Centre, Department of Evolutionary BiologyEcology and Environmental Sciences, Barcelona, Spain; ${ }^{2}$ University of Barcelona, Department of Evolutionary Biology-Ecology and Environmental Sciences, Barcelona, Spain; ${ }^{3}$ Hospital Benito Menni CASM, Unidad de Crisis del Adolescente UCA, Sant Boi de Llobregat, Spain; ${ }^{4}$ Hospital General Universitario Gregorio Marañón and CIBERSAM Mental Health Networking Biomedical Research Centre, Child and Adolescent Psychiatry, Madrid, Spain; ${ }^{5}$ Hospital Santiago Apostol and CIBERSAM Mental Health Networking Biomedical Research Centre, Department of Psychiatry, Vitoria-Gasteiz, Spain; 6 Mental Health Services Galdakao, Child and Adolescent Mental Health, Galdakao, Spain; ${ }^{7}$ Hospital Clinic of Barcelona and CIBERSAM Mental Health Networking Biomedical Research Centre, Child and Adolescent Mental Health Services, Barcelona, Spain; 8 Hospital Universitario Puerta de Hierro-Majadahonda, Department of Psychiatry, Majadahonda, Spain

${ }^{*}$ Corresponding author.

Background and aims.- The hypothalamic-pituitary-adrenal (HPA) axis is the main regulator of the stress response and its malfunction may underlie the etiology of mental disorders. The role of HPA axis in psychopathology has been scarcely studied in child and adolescent populations. The aim of this study is describe the HPA axis reactivity - assessed by Trier Social Stress Test (TSST)- in a representative sample of children and adolescents (affected and non-affected by psychiatric disorders) from the Spanish_multicentric_EPI_Young_Stress_Project.

Methods.- We analyzed a preliminary sample of 135 children from 7 to 17 years old: 84 with psychiatric diagnosis (K-SADS-PL) and 50 healthy stratified controls. Pubertal stage was assessed by Tanner stage: Children (Tanner stages I and II) and adolescents (Tanner stages III, IV and V). All subjects were exposed to the TSST, a psychosocial stress task which ensures the activation of the HPA axis. Saliva samples were collected at five time points during the procedure to analyze the variability of cortisol as a proxy for HPA axis activation.

Results.- HPA axis reactivity increased according to Tanner stage. However, while pre-pubertal subjects displayed comparable levels of HPA axis reactivity during TSST, adolescent females exhibited higher reactivity of HPA axis when compared with males. Affected subjects exhibited lower HPA axis reactivity after TSST when compared to controls.

Conclusions.- HPA axis reactivity in front of a psychological stressor was lower in subjects with psychiatric disorder. Both the pubertal stage and sex influence the variability of HPA axis reactivity and have to be taken into account to understand the stress response. Acknowledgment:FIS_PI15/00097.

Disclosure of interest.- The authors have not supplied a conflict of interest statement.

\section{E-PV0100}

Differential diagnosis program and early diagnosis of patients with suspected autism spectrum disorders at the hospital 12 de octubre of Madrid

N. Martinez-Martin", N. Gutiérrez Fernández, C.N. Acosta Velázquez, E. Pérez Valiente, J.C. Espín Jaime

Hospital 12 de Octubre, Child and Adolescent Mental Health Department, Madrid, Spain

* Corresponding author.

Background and aims.- Autism Spectrum Disorder (ASD) is a neurodevelopmental disorder defined by certain behavioral features. Although the symptoms occur in the early period of development, these may not be fully manifested until social demands exceed the limited capabilities of the child or may be masked by learned strategies. Our aims is the early detection and intervention in order to improve the prognosis of the patients.

Aims.- Descriptive evaluation of the current status of the autism program.

Methods.- Descriptive study of patients treated in the program from May 2014 until October 2018.

- Inclusion criteria: children between 12 months old and $18 \mathrm{y}$-o, previously assessed by Mental Health specialists and with diagnostic doubts or specific tests for its diagnosis.

- Exclusion criteria: children diagnosed with ASD.

The tests performed for the assessment are the observation scale for the diagnosis of autism spectrum disorder (ADOS-2), the diagnostic interview for autism reviewed (ADI-R), mentalization tasks (storytelling, gaze test of Baron-Cohen for children, social stories of F. Hapé).

Results.- $N=309$; Boys: 267; Girls: 41; Tests performed: ADOS$2=150$ (Module1 =79, Module2 =44, Module3 =56, Module4=5, Module $T=2$ ); $A D I R=68 ;$ Diagnoses: No $T E A=72 ; \quad P D D=24$; $\mathrm{TEA}=197$.

Conclusions.- The implementation of early detection programs of serious pathologies such as ASD is an important advance that allows an early intervention, improving the prognosis in a significant proportion of children and also clarifies the doubts and distress of the parents

Disclosure of interest.- The authors have not supplied a conflict of interest statement.

\section{E-PV0101}

\section{Genetic factors of the anxiety disorders formation in children}

T. Matkovska1 ${ }^{*}$, E. Mykhailova Matkovska ${ }^{2}$, N. Bagatska ${ }^{3}$, D. Mitelov Matkovska ${ }^{2}$, A. Goloborodko Matkovska ${ }^{4}$

${ }_{1}^{1}$ SI «Institute for Children and Adolescents Health Care of the NAMS of Ukraine»- Ukraine- 2. V. N. Karazin Kharkiv National UniversityUkraine, psychiatry, Kharkov, Ukraine; ${ }^{2}$ SI «Institute for Children and Adolescents Health Care of the NAMS of Ukraine, Psychiatry, Kharkov, Ukraine; ${ }^{3}$ SI «Institute for Children and Adolescents Health Care of the NAMS of Ukraine»- Ukraine 2V. N. Karazin Kharkiv National University- Ukraine, Genetic, Kharkov, Ukraine; ${ }^{4}$ V. N. Karazin Kharkiv National University- Ukraine, 4 year, Kharkov, Ukraine * Corresponding author.

Background and aims.- At present, the concept of the role of the somatic mosaicism mechanisms (activation of the movement of mobile genetic elements in the pathogenesis of mental disorders) is suggested; it is supposed that one of the main causes of the genome destabilization and changes in the system for maintaining the genome stability is the group of RNA-editing proteins AID / APOBEC.

Methods.- our study included 90 families of children with the anxiety disorders (AD) and 75 families with healthy children. Family 
tree analysis was carried out using the method of P. Harper. The cytogenetic analysis was performed in 50 children of 6-14 years with AD. The comparison group comprised 50 conditionally healthy age-matched children.

Results.- $72.2 \%$ of families have a hereditary load of mental illness. Mental, neurological, cardiovascular, oncologic diseases prevail in the relatives of the probands. The coefficient of heritability is $\mathrm{h} 2=0.23$, that is, the contribution of the hereditary component is $23.2 \%$, and the environmental component comes to $76.8 \%$. The level of spontaneous frequency of chromosomal aberrations in blood lymphocytes of our patients is $5.31 \%$, which is higher than that of the healthy peers $(1.59 \%, p<0.001)$. Dicentric chromosomes have been revealed in $14 \%$ of children with $\mathrm{AD}$, and chromosomal exchange has been established in $4 \%$ of such children, in the absence of these disorders in healthy children.

Conclusions.- The performed study confirms the role of heredity in the formation of AD and the need for differentiated treatment and prophylactic measures, taking into account the above-mentioned disorders.

Disclosure of interest.- The authors have not supplied a conflict of interest statement.

\section{E-PV0102}

\section{Estradiol and aggressive behaviour in boys and girls: a systematic review}

P. Michielsen

GGZ Westelijk Noord Brabant, Adult Outpatients Clinic, Halsteren, The Netherlands

* Corresponding author.

Background and aims.- Disruptive behaviour (DBD) and aggression related disorders have been studied from a neurobiological and hormonal perspectieve. If any contributing effects to psychopathology were found most notably adrenal hormones (DHEA, Cortisol) and gonadal hormones (Testosterone) were mentioned. Less is known about the role of Estradiol in the onset and maintenance of aggression and DBD in both boys and girls.

Methods.- A systematic review was conducted using Ovid Medline, Psycinfo and Embase databases (up to 30 July 2018), using [estradiol] and [aggression] as search terms. Within the search results only studies in humans and minors (age $0-18$ ) were included.

Results.- In total 9 studies were found in which Estradiol and aggressive behaviour were used as (in)dependent variables. Only one study tested hormone concentrations in a clinical sample of conduct disorder girls. In some studies no association was found between Estradiol concentrations and aggression, in some an association was found only when personality traits or low Cortisol concentrations were equally present. Corrections for menstrual cycle phase were lacking. Another limitation is that not every study corrected for effects of Testosterone as gonadal precursor.

Conclusions.- Studies on Estradiol and aggressive behaviour or DBD produce mixed results, with some studies showing no associations, some only in boys or girls, or only when a mediating effect of emotional instability or negative life events occurred.

Future studies should control for effects of Cortisol and Testosterone and use mixed gender samples, preferably after puberty onset as results of Estradiol assays are more reliable when taken after gonadarche.

Disclosure of interest.- The authors have not supplied a conflict of interest statement.

\section{E-PV0103}

\section{Sexuality on asperger adolescents: a critical interaction solution and the risk behind it \\ M.M. Mihai ${ }^{*}$, D. Iuliana ${ }^{2}$}

${ }^{1}$ University of Medicine and Pharmacy "Carol Davila" Bucharest, General Medicine, Bucharest, Romania; ${ }^{2}$ University of Medicine and Pharmacy "Carol Davila" Bucharest, Child and Adolescent Psychiatry, Bucharest, Romania

* Corresponding author.

Background and aims.-

Introduction.- The issues regarding sexuality on adolescents diagnosed with Asperger's syndrome usually revolve more around inappropriate sexual behaviors and paraphilias and less around the consequences of augmented sexual interest combined with the lack of social understanding. Furthermore, these two elements put together (limited sexual knowledge and experience, and social deficits) may place the patients at high risk of sexual victimization. Objective.- The purpose of this study is to reveal a different side of sexuality in these patients, not as a part of the disorder, but as a dysfunctional mean to achieve social awareness and ultimately acceptance.

Methods.- This case report was based on a close monitoring and frequent clinical and psychological tests performed on a singular female patient, spanning from age 11 to 14 , diagnosed with Asperger's syndrome, temporary obsessive-compulsive behavior and transitory psychotic episodes. Other underlying conditions of the patient also include family history of mental illness and difficult upbringing.

Results.- As a result of the inappropriate sexual behavior employed by the patient to gain social awareness from her peers, there were instances in which the adolescent was exposed to a high risk of sexual abuse from an early age.

Conclusions.- The patient's sexual behavior was only a part of the cumulative obscene pattern of manifestations utilized in order to achieve desired social interaction and integration, and could not be solely attributed to the underlining syndrome.

Disclosure of interest.- The authors have not supplied a conflict of interest statement.

\section{E-PV0104}

\section{The role of mirror neurons in terms of neurodevelopmental theory of autism spectrum disorder (ASD)-preliminary research}

M. Zietal ${ }^{1}$, M. Mirek ${ }^{2}$, A. Gmitrowicz ${ }^{3}$, A. Wyderka ${ }^{1}$

${ }^{1}$ Psychologist, Daily Rehabilitation Psychiatric Unit for Children and Youth in Clinic JiM, Lodz, Poland; ${ }^{2}$ Psychiatrist, Daily Rehabilitation Psychiatric Unit for Children and Youth in Clinic JiM, Lodz, Poland; ${ }^{3}$ Psychiatrist, Department of Adolescent Psychiatry- Medical

University of Lodz, Lodz, Poland

* Corresponding author.

Background and aims.- In the 1990s Giacomo Rizzolatti and colleagues discovered mirror neurons. Researchers confirmed this finding, clarifying their role in neurodevelopmental diseases (i.e. schizophrenia, ASD). Some studies indicate that dysfunction of mirror neurons has an effect on abnormal visual communication in patients with ASD.

This study is to examine the qualitative and quantitative differences in the response of children with ASD and healthy children to the reflection in the mirror they perceive.

Methods.- Based on the literature review, it was assumed that children with ASD are different from healthy children in terms of visual perception disorders. The study group - 35 patients undergoing rehabilitation at the Clinic JiM with the diagnosis of ASD according to the DSM 5 classification confirmed additionally with ADOS 2 and the control group - 35 healthy children, selected in terms of sex and age. A detailed examination protocol was developed, including the approval of guardians. A ten-minute, non-invasive observational study was conducted using a questionnaire, taking into account variables relevant to the study (i.e. fun time, way of play, child's 
reaction). The researcher will be a specially prepared psychologist and doctor. Two educational tables covered with unbreakable mirror will be used, including one of them will be matted. The examined child voluntarily chooses at which table they will play and how.

Results.-

Conclusions.- We assume that the dysfunction of mirror neurons in children with ASD will cause them to choose fun at a table with a matte mirror. Obtained results may have diagnostic and practical implications in planning rehabilitation activities.

Disclosure of interest.- The authors have not supplied a conflict of interest statement.

\section{E-PV0108 \\ Prevalence of depression and anxiety in a sample of normal iq adolescents with autism}

V. Muñoz Martinez ${ }^{1 *}$, B. Mata-Saenz ${ }^{1}$, L. Asensio-Aguerri ${ }^{2}$, L. Nuevo-Fernández ${ }^{3}$, L. Beato-Fernández ${ }^{4}$

${ }^{1}$ Hospital General Universitario de Ciudad Real, Psychiatry, Ciudad Real, Spain; ${ }^{2}$ Hospital General Universitario de Ciudad Real, Ciudad Real, Spain; ${ }^{3}$ Hospital General Universitario de Ciudad Real, Ciudad Real, Spain; ${ }^{4}$ Hospital General Universitario de Ciudad Real., Ciudad Real, Spain

${ }^{*}$ Corresponding author.

Background and aims.- Anxiety and depression is frequent in Autism during adolescence. The reason of that is because they perceived their difficulties in social competence, which increase the risk of depression and anxiety. The objective of this study is to analyze the relationship between anxiety and depression symptoms in individuals with autism and normal IQ.

Methods.- 50 patients were recruited. The range of age was from 13 to 17 years old. The diagnosis was made using the ADOS-2. The IQ were measured using the Wechsler Intelligence Scale for Children4th Edition (WISC-IV) and the depression and anxiety symptoms with the Child Behavior Checklist (CBCL).

Results.- The mean age was 14.2 years old; men were the $92 \%$ of the sample and the women the $8 \%$. The mean of the IQ was 109.1 . The mean scores from the ADOS-2 were 12.3 (autism diagnosis). The mean age in atients with high scores in depression (CBCL > 64) was 15.7 years, the male had higher scores than girls ( $84 \%$ vs $16 \%$ ), IQ mean score was 103.2 and the ADOS-2 mean scores were 11.5. Those individuals with high scores in anxiety, their mean age was 13.2 , the men was the $73 \%$ of the sample and the women $27 \%$. When we made the media scores of the IQ the found that it was 110.1. Finally, in this group the averages scores for the ADOS-2 were 12.3 .

Conclusions.- We can say that emotional problem is a common problem in ASD so we need to explore this symptoms because their difficulties to recognize their emotions.

Disclosure of interest.- The authors have not supplied a conflict of interest statement.

\section{E-PV0109}

\section{The alteration of the sensory consciousness of the self in children with developmental disorders. a preliminary study}

A. Bernardini, O. Orsini* , D. Montanaro

${ }^{1}$ Freelance, Freelance, Rome, Italy

${ }^{*}$ Corresponding author.

Background and aims.- We assume that a relationship with a nonresponsive caregiver could lead to an impairment of psychophysic sensitivity fundamental to develop the "internal image of the Self" (M. Fagioli, 2017).

Neurobiology studies indicate that the first years of life is characterized by intense neuronal plasticity, modulated by the developmental environment of children, with consequential modification of the Nervous System (Giovanelli, 1997).

Clinical studies (Atzori, 2017; 2018) highlight a correlation between a difficulty to constitute a mental configuration of a self 'body scheme' and an 'impairment of the first internal image of the Self' (M. Fagioli, cit.), that originates at birth. This first internal image represents a "Primal Self" that gives the newborn the certainty to exist and the possibility to recreate homeostatics sensations and good feelings in human relationships (M. Fagioli, 1971).

Methods.- This study examines the factors at the base of a developmental alteration in a 5 year old girl with a mixed developmental disorder in psychomotor treatment, by structured clinical observation, drawing tests and praxic and motor activities.

Results.- Observation and assessment aimed to evaluate the impairment of the mental configuration of the self body scheme at the base of motor difficulties, corroborate the hypothesis that it is linked to an alteration of the "internal image" of the body impaired by non responsive relationship with the caregiver.

Conclusions.- We propose that these difficulties can be gradually overcome in treatment based on a responsive relationship in which the child could recreate an inner image of the Self and body, through a constant stimulation of the psychophysical sensitivity.

Disclosure of interest.- The authors have not supplied a conflict of interest statement.

\section{E-PV0110 \\ Mental health problems among Polish children and adolescents: preliminary results of the MINI Kid survey}

\section{K. Ostaszewski ${ }^{*}$, J. Moskalewicz ${ }^{2}$, M. Kucharski ${ }^{2}$}

${ }^{1}$ Institute of Psychiatry and Neurology, Department of Public Health, Warsaw, Poland; ${ }^{2}$ Institute of Psychiatry and Neurology, Department of Studies on Alcoholism and Drug Dependence, Warsaw, Poland ${ }^{*}$ Corresponding author.

Background and aims.- This study was the first national-wide survey in Poland utilizing the Mini International Neuropsychiatry Interview for Children and Adolescent (MINI Kid). It was a part of a wider project called EZOP II that aimed at assessment of mental health of the general population in Poland, including children, adolescents and adult population. The study aim was to estimate the prevalence and determinants of mental and behavioral disorders in children aged 7-11 years and adolescents aged 12-17 years.

Methods.- The latest version of the instrument based on classification system DSM-V (MINI Kid 7.0.2) was translated into Polish and used in the study. Computer-assisted personal interviewing (CAPI) was conducted by trained interviewers among 2000 children and adolescents randomly sampled from the population register. Interviews with children were administrated with parental assistance, while adolescents were answered the questions alone. Data collection was completed in September 2018.

Results.- Preliminary results show a good response rate and suggest a very high feasibility of MINI Kid instrument in the national epidemiological research. The analyses are in the process and will be presented in the Congress.

Conclusions.- The use of the clinical instruments in population surveys is a challenging task and requires a very good preparation both in terms of interviewers training and logistics of the study.

Disclosure of interest.- The authors have not supplied a conflict of interest statement. 
E-PV0113

\section{A developmental perspective on Grief: death concept and reactions to loss}

\section{A. Salgueiro de Lima ${ }^{1}$, C. Pires De Lima ${ }^{1,2^{*}}$, S. Gonçalves ${ }^{1}$}

${ }^{1}$ ForAll - Desenvolvimento Pessoal e Bem-Estar- Unipessoal- Lda., Psychology, Paredes de Coura, Portugal; ${ }^{2}$ CINTESIS - Center for Health Technology and Services Research- Porto., Psychology, Porto, Portugal * Corresponding author.

Background and aims.- During development, children often experience the loss of someone or something important to them (Corr, 1996). Although their grieving process is similar to that of the adult (Worden, 1998), in this young age grieving is affected by prior experiences and the level of development (Speece \& Brent, 1984; Speece \& Brent, 1996). Children's understanding of death is strongly related to the cognitive development process (Speece \& Brent, 1984).

Methods.- Literature review.

Results.- In the initial stages of development children interact with the world through their senses, acquiring a concrete understanding of their surroundings hence their death concept is based on physical and functional aspects (Himelstein, Hilden, Boldt \& Weissman, 2004). They progressively develop new skills and attain a more complex concept of death. This understanding of death implies the following dimensions: irreversibility, finality or non-functionality, universality and causality (Speece \& Brent, 1996; Schonfeld \& Kappelman, 1990).

Conclusions.- If we take the developmental perspective into consideration it becomes possible to anticipate typical reactions to loss in each developmental stage which allows us to adjust the intervention to each child (Silva, 2009). The children's grieving process shouldn't be underestimated, for it can negatively impact their development. Therefore adults should include the children in the family's grieving process, be supportive and take care of them. Disclosure of interest.- The authors have not supplied a conflict of interest statement.

\section{E-PV0114}

\section{'School refusal' in the emergency room}

F. Reis*, T. Cartaxo

Centro Hospitalar e Universitário de Coimbra, Serviço de Psiquiatria da Infância e da Adolescência, Coimbra, Portugal

${ }^{*}$ Corresponding author.

Background and aims.- School refusal occurs when a student rejects to go to school or regularly experiences severe distress related to school attendance, which can lead to school abstinence. It is important to address this problem, not only because of its consequences, but mainly because of the diagnosis that it may be concealing.

The aim of this study is to characterize the patients observed for 'school refusal' in the child and adolescent psychiatric emergency room throughout the year of 2017.

Methods.- We compiled the episodes of observation for 'school refusal' in the child and adolescent psychiatric emergency room, during 2017, in the Centro Hospitalar e Universitário de Coimbra, Portugal. We characterized the sample in terms of final diagnosis, taking psychiatric medications, attending psychiatric consultations and referral after the discharge.

Results.- There were 29 episodes of observation for 'school refusal'. The observed population was mainly masculine (58,6\%), with an average age of 13,7 years old. The majority of these patients were diagnosed with adjustment (34,5\%), anxiety (31\%), or depressive disorders (24,1\%). Also, 51,7\% had no previous psychiatric consultations, and $75,9 \%$ were not under medication. After the discharge, $93,2 \%$ had a follow-up consultation.

Conclusions.- The results showed that school refusing was related to important psychiatric diagnoses, most of the times. These diagnoses included anxiety and/or depressive disorders that need accurate interventions.

Therefore, it is especially important to correctly evaluate these situations, and inform caregivers this is not a behavior problem, but an important warning of distress which needs to be properly assessed, treated and followed.

Disclosure of interest.- The authors have not supplied a conflict of interest statement.

\section{E-PV0115 \\ Incoherence in attitudes and representations of self, adults and peers in adolescents with eating disorders}

A. Ryzhov ${ }^{1}$, E.T. Sokolova ${ }^{1}$, L. Pechnikova ${ }^{1}$, A. Tkhostov ${ }^{1}$, A.P. Zaharova $^{2}$, A.A. Korneev ${ }^{1}$

${ }^{1}$ Lomonosov MSU, Psychology, Moscow, Russia; ${ }^{2}$ Research Centre of Eating Behaviour Disorders, Stationary Department, Moscow, Russia ${ }^{*}$ Corresponding author.

Background and aims.- Body-image and self-concept distortions, reliance on social ideals are related to the struggle for autonomy from parental figures in eating disorders and the reduction of incoherence of representations could be an important intervention target.

Objectives.- To compare incoherence manifestations in representations and attitudes of self, parental figures and peers in patients with eating disorders.

Methods.- Complementary measures of incoherence in representations and attitudes were used: a modified repertory grid technique with assessment of mean distances between self-aspects, adults and peers elements; "Splitting Index" questionnaire (Gould et al., 1996), adapted version of Affective scale for TAT (Thomas, 2008). 23 impatient adolescent girls with eating disorders participated; Splitting Index scores were compared with a control group of 54 adolescents.

Results.- Mean distances in repertory grids were significantly highest for self-aspects (Wicoxon-test, $p<.01$ ), and non-significantly lowest for adult elements. On self-report questionnaire patients scored significantly higher than controls (Mann-Whitney, $p<.05$ ) on Overall, Self and Friend scales, but not on Family Splitting scale. In contrast, on TAT Affective scale the scores indicate more negative and ambivalent attitudes towards both self and parental figures then towards peers/siblings (Wilcoxon-test, $p<.05$ ). No significant correlations between indexes from different instruments were found. Qualitative phenomena of self non-integration (MakhloufNorris, 1976) were found in $35 \%$ of repertory grids.

Conclusions.- While self-representation is least coherent in eating disorders, there is contrast between conformity of representations of parents and adults in cognitive tasks and ambivalent attitudes manifested in projective assessment. Further study with qualitative analysis of thematic content is suggested.

Disclosure of interest.- The authors have not supplied a conflict of interest statement.

\section{E-PV0121 \\ Recognition of facial emotions test among tunisian children: a pilot study}

A. Taamallah ${ }^{*}$, S. Halayem ${ }^{2}$, Z. Abbes ${ }^{3}$, M. Hajri ${ }^{4}$, A. Bouden ${ }^{5}$

${ }^{1}$ Resident, Child and Adolescent Department/ Razi Hospital,

Manouba, Tunisia; ${ }^{2}$ Doctor, Child and Adolescent Departement/ Razi

Hospital, Manouba, Tunisia; ${ }^{3}$ Doctor, Child and Adolescent

Department/Razi Hospital, Manouba, Tunisia; ${ }^{4}$ Doctor, Child and

Adolescent Department/ Razi Hospital, Manouba, Tunisia; ${ }^{5}$ Professor, Child and Adolescent Department/Razi Hospital, Manouba, Tunisia

Corresponding author. 
Background and aims.- Facial emotions' recognition is an essential social skill. It enables to detect another person's emotional status and reaction, to respond appropriately in different social situations. A new tool adapted to Tunisian children has been developed to explore the development of facial emotions' recognition.

The aim is to validate the test in children from 7 to 12 years old, in order to establish normative references.

Methods.- The test is composed of photographs and videos, performed by six amateur actors of different ages and gender, representing the six basic emotions (joy, sadness, fear, disgust, anger and surprise) and neutrality.

It contains 114 photographs (19 per actor). Three photographs of increasing intensity, for the same actor, represent each emotion. 36 videos were edited, with a maximal duration of five minutes. Data was presented to the medical staff for a first selection. Final selection was based on The Facial Action Coding System.

We conducted a pilot study, in order to initiate subsequently the validation.

Results.- Sixteen children were included. Seven of them were male (44\%), and nine were female (56\%). The average age was 9 years. The average rate of correct answers was 78 out of $114(68-96 / 114)$. Age was significantly correlated to the number of correct answers $(p=0.035)$. The recognition of fear and neutrality was also significantly correlated to the age $(p=0.01 ; p=0.03)$. Gender didn't appear to affect the recogniton's ability among participants. The emotions' recognition was higher for videos than in photographs.

Conclusions.- the preliminary results are encouraging and they will be discussed according to the results of the literature.

Disclosure of interest.- The authors have not supplied a conflict of interest statement.

\section{E-PV0122}

\section{Psychiatric manifestations in auto-immune diseases: a case report}

A. Taamallah ${ }^{1 *}$, S. Taleb ${ }^{2}$, H. Ben mustapha ${ }^{2}$, Z. Abbes ${ }^{3}$, S. Halayem $^{4}$, M. Hajri ${ }^{5}$, A. Bouden ${ }^{6}$

${ }^{1}$ Resident, Child and Adolescent Department/ Razi Hospital, Manouba, Tunisia; ${ }^{2}$ Resident, Child and Adolescent Department/Razi Hospital, Manouba, Tunisia; ${ }^{3}$ Doctor, Child and Adolescent Department Razi Hospital, Manouba, Tunisia; ${ }^{4}$ Doctor, Child and Adolescent Department, Manouba, Tunisia; ${ }^{5}$ Doctor, Child and Children Department Razi Hospital, Manouba, Tunisia; ${ }^{6}$ Professor, Child ans Adolescent Depatment Razi Hospital, Manouba, Tunisia ${ }^{*}$ Corresponding author.

Background and aims.- Autoimmune diseases have a chronic evolution. Patients with chronic condition suffer from depression and/or anxiety. The relationshipbetween depression and immunity has been widely discussed but few studies have treated anxious symptoms and behavior change. The aim of our work is to discuss the link between autoimmune diseases and psychiatric manifestations, with a review of the current literature.

Methods.- A case report discussed with a literature review, based in Pubmed, using " autoimmunediseases » " depression " " anxiety " « immunity " " psychiatric manifestations " as key words.

Results.- Female 14 years old college student, without personal or familial history of psychiatric or auto-immune diseases. Two years ago, a partial epilepsy was diagnosed, unbalanced despite the changing of antiepileptic medication. The patient was referred to child and adolescent psychiatry department for drug suicide attempt. Three months ago, she becamesad, cried easily, had headaches, lost interest in the daily activities, presented heart palpitations and disturbance in sleep and appetite. The symptoms were gradually worsening. She was sad and anxious. She had anhedonia, suicidal ideation, low self-esteem and insomnia. Shepresented headache and hand tremor. After a year of follow up, only the depressive have improved. Six months later, she was diagnosed with autoimmune pancreatitis and epilepsy and a treatement with prednisone $40 \mathrm{mg}$ /day was started. Currently, she takes $5 \mathrm{mg}$ /day. The depressive symptoms disapeared. The psychatric examination reveal anxious symptoms, evoking social anxiety disorder.

Conclusions.- Distinction between psychiatric manifestations of autoimmune disease and comorbidity remains difficult. It requires a knowledge of the symptoms' chronology and a long-term followup.

Disclosure of interest.- The authors have not supplied a conflict of interest statement.

\section{E-PV0123 \\ Management of hypersomnia in child psychiatry: a case report}

R. Khemakhem, M. Hajri, A. Taamallah*, Z. Abbes, S. Halayem, A. Bouden

Razi Hospital, Child and Adolescent Psychiatry, Manouba, Tunisia

* Corresponding author.

Background and aims.- Daytime sleepiness is a common subjective experience: 17 to $21 \%$ of school-age children suffer from it.

To illustrate the diagnosis and therapeutic difficulties faced by the child psychiatrist with excessive daytime sleepiness.

Methods.- Illustration by a case report then review of the literature by research carried out on the Pubmed and Sciencedirect databases using the following key words "excessive daytime hypersomnia", "narcolepsy", "central hypersomnia" and "idiopathic hypersomnia".

Results.- M F, 8 years-old with normal psychomotor and language developments, had recurrent depressive episods in mother's history and an encephalopathy in the younger brother. She presented excessive daytime sleepiness (EDS) since the age of 4 . It hinders learning and family interactions. She slept 14 to 15 hours at day ( 12 hours of nocturnal sleep and 2 to 3 hours of nap) to which are added frequent daytime sleep in class noted during moments of monotony. Episodes of cataplexy unrelated to strong emotions are inconsistently associated with it. Note the absence of nocturnal sleep disorders, automatic behaviors, hallucinations (hypnagogic or hypnopompic) or sleep paralysis. We noted in our psychiatric exam an eutrophic girl, immature emotionally and with moderate psychomotor instability. Different explorations (EEG, brain MRI, Holter monitoring) did not reveal any abnormalities. A Multiple Sleep Latency Test (MSLT) confirmed the excessive daytime sleepiness. We diagnosed a central diurnal hypersomnia. But is it narcolepsy or idiopathic diurnal hypersomnia? We have provided parental guidance and we discuss the methylphenidate's use.

Conclusions.- Despite advances in understanding the pathophysiology of central hypersomnia, there is currently no specific treatment. Disclosure of interest.- The authors have not supplied a conflict of interest statement.

\section{E-PV0124}

\section{A systematic review of autism spectrum in children and adolescents with 22q11.DS: prevalence, clinical significance and relationship with onset of psychotic symptoms}

M. Pontillo, S. Guerrera, R. Averna, F. Demaria, P. Gargiullo, M.C. Tata*, O. Santonastaso, S. Vicari

Paediatric Hospital Bambino Gesù, Department of Neuroscience Child Neuropsychiatry, Rome, Italy

* Corresponding author.

Background and aims.- 22q11.2 deletion syndrome (22q11DS) is a genetic syndrome associated with different neurodevelopmental and psychiatric disorders. Several studies reported contrasting data 
about prevalence of autism spectrum disorder (ASD) in 22q11DS. Aim of our review was to summarize the evidence in literature on the prevalence and clinical significance of autism spectrum disorders (ASD) in children and adolescents with 22q11DS. Subsequently, we also investigate the association between autism spectrum disorders and onset of psychotic symptoms in this clinical population.

Methods.- A systematic review of the literature published from 1970 to March 2018 was conducted. Nine studies are included. Results.- Four of nine studies included reported elevated (10-40\%) rates of autism spectrum disorders, including autism. However, in these studies the use of only parental report, not associated with the clinical direct observation, tends to overestimate the prevalence of ASD in children and adolescents with 22q11DS. Studies included on the relationship between ASD and psychosis in 22q11DS showed that early childhood autistic features (both ASD diagnosis and only presence of autistic symptoms) are not predictive of subsequent onset of psychotic symptoms.

Conclusions.- Based on findings of this review, ASD-like behaviors are present in children and adolescents with 22q11DS and they are similar at the symptom level of idiophatic ASD. However, the social impairments in 22q11DS and idiophatic ASD could be expression of different neuropsychiatric picture. This should be considered in the planning of the treatment of the social impairments of 22q11DS. Disclosure of interest.- The authors have not supplied a conflict of interest statement.

\section{E-PV0125}

\section{Association between polymorphisms in oxtr and avpr 1a genes and social deficits in autism spectrum disorder}

K.M. Wilczyński ${ }^{1,2}{ }^{*}$, M. Janas-Kozik ${ }^{1,2}$

${ }^{1}$ Medical University of Silesia, Deparment of Psychiatry and Psychotherapy of Developmental Age, Sosnowiec, Poland; ${ }^{2}$ Pediatric Centre of John Paul II in Sosnowiec Sp.zo.o, Sosnowiec, Poland * Corresponding author.

Background and aims.- One of the defining features of autism spectrum disorder (ASD) are deficits in social interaction and communication. Although their etiology is poorly understood, several lines of evidence from studies on humans and rodents suggest that neuropeptides such as oxytocine and vasopressine might play a crucial role in their development. Single nucleotide polymorphisms of the oxytocine and vasopressine receptors genes have been tested for correlations with social cognition deficits in course of ASD in various populations, yielding contradictory results.

Methods.- We have conducted a systematic review of literature, published between january 2003 and august 2018, on associations of single nucleotide polymorphisms in oxytocynergic and vasopressynergic systems with social cognition in ASD. Literature search was performed with utilization of MEDLINE/PubMed, Cochrane Library and APA psycNET databases, as well as Google Scholar search engine.

Results.- The review yielded 29 original publications, in most of them association between social cognition deficits in ASD and single nucleotide polymorphisms in oxytocine and vasopressin receptor genes was statistically significant $(p<0.05)$.

Conclusions.- Our findings seem to support the hypothesis that single nucleotide polymorphisms in oxytocynergic and vasopressynergic systems may be associated with social cognition deficits in ASD. Most studies presented statistically significant correlations between various single nucleotide polymorphisms and features of ASD, however different authors pointed out different polymorphisms, frequently contradicting preceding reports. This may be caused by significant heterogenity of study groups between publications, which were recruited across mainfold ethnicities and age groups, as well as lack of gender stratification in all analyzed reports.
Disclosure of interest.- The authors have not supplied a conflict of interest statement.

\section{E-PV0127}

\section{Clinical and psychological evaluation of children and adolescents with obsessive-compulsive disorders}

N. Zvereva ${ }^{*}$, T. Blinova ${ }^{2}$, E. Balakireva ${ }^{2}$, M. Zvereva $^{3}$, N. Simashkova ${ }^{2}$

${ }^{1}$ Mental Health Research Center- MSUPE, Department of Clinical Psychology, Moscow, Russia; ${ }^{2}$ Mental Health Research Center, Department of Child Psychiatry, Moscow, Russia; ${ }^{3}$ Mental Health Research Center, Department of Clinical Psychology, Moscow, Russia Corresponding author.

Background and aims.- Systematics of obsessive-compulsive disorders of childhood is one of the important problems in the developmental psychopathology. We don't know too much about the difference between children with autistic and schizotypal spectrum. It concerns clinical and psychological assessment. This investigation is a pilot one in the field of differential diagnostics.

Aim.- Assessment clinical and psychological area of patients with obsessive-compulsive disorders in child psychiatric hospital

Methods.- 22 patients (16 males) 4-15 years old with schizotypal (F21), and autistic spectrum disorder - ASD (F84) were observed during hospitalization in child psychiatric clinic of MHRC. Mean age was $9.9 \pm 4.9$ years. Children $\&$ adolescents were assessed by doctors and clinical psychologists. We use brief assessment according to psychologist conclusion concerned cognitive and personality state ( $0-3$ points in 4 intellectual domain and 4 personality domain, common score).

Results.- Patients were divorced to groups: children up to 8,5 -CG and adolescents upper then 11,5 years - AG There was difference between patients. In clinical symptoms: CG -patients with ASD (F84) demonstrate obsessive movements; and AG - patients with schizotypal (F21)demonstrate motor-ideator disturbances. In psychological assessment: common score in CG $(13,4)$ is larger than in AG (9). We have found dissociation between intellectual and personality domain in CG and haven't found it in AG.

Conclusions.- Pilot investigation children and adolescents with obsessive-compulsive disorders show the difference in clinical symptoms and psychological evaluation children with this disorders suffering from autism and schizotypal. Next stage will be sample expansion and analyzing clinical factors (diagnosis, age of onset etc.)

Disclosure of interest.- The authors have not supplied a conflict of interest statement.

\section{E-PV0128}

\section{Cognitive defect in children with endogenous mental illnesses}

N. Zvereva ${ }^{*}$, S. Strogova ${ }^{2}$

${ }^{1}$ FBSI MHRC-MSUPE, Clinical Psychology, Moscow, Russia; ${ }^{2}$ Mental Health Research Center, Department of Clinical Psychology, Moscow, Russia

* Corresponding author.

Background and aims.- Researching of the cognitive defect among children and adolescents with endogenous mental illnesses (schizophrenia spectrum, severe forms - SS) as a basic violation related to this pathology demands analysis and psychological verification (lagging behind normative adolescents, special age dynamics).

Aim.- Estimation of emerging (formed) cognitive defect among children and adolescents with SS using the psychometric method 
an d taking into account the age of onset of the disease and sex of patients.

Methods.- 74 (22 girls) children and adolescents with SS (avg = 11 y.o.) were observed. Assessment was carried out by psychometric test WISC2 during the period of stability of the patient's condition. We use a special scale for cognitive defect assessment according to results of WISC.

Results.- According to research data, we found 31 patients with reduced intelligence that is cognitive defect $(I Q<70)$ It is important that 21 of them have very early onset of the disease near 3 years. We obtained two groups. First "total cognitive defect" - 14 patients ( 11 boys, 3 girls) have a total low intelligence and it is impossible to calculate the total score. Second; "partial cognitive defect"- 17 subjects ( 12 boys, 5 girls) have an intellectual decline in combination with uneven productivity of the accomplishing tasks of the test. Conclusions.- Age of onset of the disease has a significant importance for children with SS in case of the formation of a cognitive defect of total type (the sooner disease begins, the more severe tdefect). Partial cognitive defect is more often observed in girls with SS.

Disclosure of interest.- The authors have not supplied a conflict of interest statement.

\section{E-Poster Viewing - 7-9 April: Classification of mental disorders}

\section{E-PV0132}

\section{Albanian public attitudes to mental health \\ A. Hashorva ${ }^{1 *}$, T. Pengili ${ }^{2}$ \\ ${ }^{1}$ Taulant Pengili, Neuroscience, Tirana, Albania; ${ }^{2}$ UHC Mother \\ Teresa- Tirana, Neuroscience, Tirana, Albania \\ * Corresponding author.}

Background and aims.- To obtain a comprehensive view of attitudes to mental health among the Albanian adult population, ultimately to give support to mental health awareness comparing. a) raising the awareness of individuals of the mental health and wellbeing. b) increasing the public knowledge of mental health and problems positively influencing attitudes and behaviors toward people with mental health problems. c) encouraging people with mental health problems to recognize the importance of seeking professional and social support and empowering them to take on responsibility for their therapy and treatment.

Methods.- This survey and the questioner used for the data collection where partly based on survey used for the same purpose by the Health Service Executive-Mental Health Ireland. Adults aged $18+$, sample size 520 , from 10 cities of the countries. September-December 2015.

Results.- $8 \%$ of people studied reported to have personally experienced a mental health problem. main sources of information of mental health are television-radio programs and technology. $75 \%$ of people studied that Anyone can experience mental health problems and about $90 \%$ of them accept it that in our society many stereotypes exist about people with mental health problems.

Conclusions.- $30 \%$ of the respondents could successfully recognize the symptoms of depression and only $15 \%$ of the symptoms of schizophrenia. 9 in 10 people think that people with mental health problems should not be allowed to be employed in jobs directly with people. More than one in 2 respondents think that people with mental health problems should not have the right to vote.

Disclosure of interest.- The authors have not supplied a conflict of interest statement.

\section{E-PV0134 \\ Psychiatrists' opinion on mental disorder classifications in Poland}

A. Dudek, M. Dec, M. Buras, K. Nowak, K. Krysta*

Medical University of Silesia, Department of Rehabilitation

Psychiatry, Katowice, Poland

"Corresponding author.

Background and aims.- The necessity for a fundamental revision of the current versions of international classifications of diseases is widely discussed in the community of psychiatrists. Clinicians call for changes in specific diagnostic areas. DSM-5 has already been published and the works on the 11th edition of the International Classification of Diseases (ICD-11) are ongoing. Our research aims to systematize the knowledge about the expectations of Polish psychiatrists regarding the upcoming changes.

Methods.- The author's questionnaire was prepared. The questions regarded the respondents' opinion on classification systems. 102 psychiatrists were interviewed during a national psychiatric conference. Responses were collected and analyzed.

Results.- All respondents take advantage of the ICD classification on a daily basis, $22.5 \%$ of them also use DSM. The majority of polish psychiatrists are interested in the development of ICD 11. Criteria for mood disorders and schizophrenia were considered as the most useful in the diagnostic process, whereas the least useful were criteria for anxiety, and somatoform disorders. Respondents support the replacement of older categories like: "alcohol abuse" and "alcohol dependence" with "substance use disorder" in DSM-5. In general psychiatrists are satisfied with the elimination of the traditional division of schizophrenia into subtypes.

Conclusions.- Current revision of the ICD classification is important to improve its clinical utility.

Disclosure of interest.- The authors have not supplied a conflict of interest statement.

\section{E-PV0135}

\section{A metacognitive group-concept based on psychiatric network approach}

\section{S. Paulsen*, P. Greve}

Helios Klinikum Bad Saarow, Psychiatry, Bad Saarow, Germany

* Corresponding author.

Background and aims.- The network approach (Borsboom) is one of the most discussed innovations in psychiatry. The benefits of the implications were explored in a metacognitive group.

Objectives.- Explorative group, to find out helpful interventions and implications from the network approach to psychiatric symptoms. Methods.- The network approach to psychiatric symptoms was focused in a psychoeducative group with 5 outpatients. The group was running over 6 sessions in 2018. The patients had to name their symptoms and to estimate the severity in a scale (1 to 10 points). The effect of the psychoeducation was measured in a timeline ( 2 weeks before start, at the second and the last session). The concept of the psychiatric network approach was explained to the participants. Specific, helpful interventions, focusing on isolated symptoms were discriminated.

Results.- Severity of specific symptoms was reduced during the measured timeline. Anxiety decreased for nearly all patients (about 1 to 5 points). Also related symptoms like social phobia and negative cognitions decreased. Other symptoms stayed stable (e.g. sleep disorder, somatic symptoms). For all participants helplessness decreased. In the last sessions patients felt more competent to influence their symptoms in a positive way.

Conclusions.- The network-approach of psychiatric symptoms can be used for metacognitive therapy, e.g. in a psychoeducative group-concept. The approach tends to focus on specific helpful interventions, which are individual and may reduce severity of 
focused and related symptoms. The approach seems to reduce helplessness of psychiatric patients.

Disclosure of interest.- The authors have not supplied a conflict of interest statement.

\section{E-Poster Viewing - 7-9 April: Comorbidity - Dual Pathologies}

\author{
E-PV0137 \\ Psychosis and substances use; what do \\ we know? \\ F.F. Alharbi \\ King Abdulaziz Medical City, Mental Health Department, Riyadh, \\ Saudi Arabia \\ Corresponding author.
}

Background and aims.-

Background.- Prospective studies clearly demonstrate a temporal relationship between exposure to substances and psychosis albeit more accurate details on causality remain to be assessed.

Objective.- This review focus on cannabis and amphetamine-type stimulant (ATS) because of their high prevalence. We reviewed each substance group and its association with the risk for psychosis, psychosis and risk for substance use, age of onset of psychosis, and development of schizophrenia.

Methods.- A systematic literature search was conducted from 1980 to date in the following databases: MEDLINE, PsycINFO and PubMed. Articles were included if they were highlighting substances induced psychoses, with particular emphasis on stimulants/amphetamine/methamphetamine and cannabis/marijuana induced psychoses, schizophrenia-spectrum disorder or schizophrenia.

Results.- There are many differences between these two substances regarding neurobiological processes, average latency periods before developing psychosis, clinical features as compared to schizophrenia, risk of using drugs and developing psychosis and drugs use \& development of schizophrenia.

Conclusions.- The comparative studies across substances and its association with psychosis could shed further insight into the development of psychotic features. The relative prominence of paranoid features of ATS-induced psychosis could lead to further insight into the study of this prototypical delusional system. Disclosure of interest.- The authors have not supplied a conflict of interest statement.

\section{E-PV0138}

\section{An interesting case of a post epileptic psychotic episode:a case report}

P. Argitis ${ }^{1 *}$, A. Kourti ${ }^{1}$, M. Poulou ${ }^{2}$, K. Paschalidis ${ }^{3}$, K. Charisiou ${ }^{4}$, A. Karampas $^{5}$, C.M. Platsa ${ }^{1}$

${ }^{1}$ General Hospital Of Corfu, Psychiatric, Corfu, Greece; ${ }^{2}$ General Hospital Of Kymi, Anesthesiologyst, Kymi, Greece; ${ }^{3}$ Psychiatric Hospital of Thessaloniki, Psychiatric, Thessaloniki, Greece; ${ }^{4}$ University Hospital Of Ioannina, Neurologist, Ioannina, Greece; 5 University Hospital of Ioannina, Psychiatric, Ioannina, Greece * Corresponding author.

Background and aims.- Epilepsy and multiple sclerosis comorbidity, is a often mentioned condition in the international bibliography. $2-7 \%$ of patients suffering from temporal lobe epilepsy, also present psychotic symptomatology.

Methods.- We present a Case of a 28-year-old patient without previous psychiatric symptoms, was addressed to our emergency department with persecutory delusions, delusions of reference and auditory hallucinations. The person had a history of multiple sclerosis treated with Dimethylfumarate. The necessary laboratory tests were conducted, with no specific pathologyconfirmed, followed by the tests required for a first psychotic episode. For the acutephase the patient was treated with olanzapine $20 \mathrm{mg}$ and lorazepam $2.5 \mathrm{mg}$ three timesdaily. The next day, the patient seemed to respond well in treatment, not presentingany signs of acute psychopathology. We did not perform a drug test, but since thepatient confessed that he was using cannaboid, we suspected that it might have been adrug-induced brief psychotic episode. Due to excessive sedation we decreased thebenzodiazepine dosage. After 8 hours, the patient appeared confused, agitated anddelusional. An one minute lasting complex focal epilepsy episode was observed, followed by the same symptomatology. The incidence reoccurred six times during an hour, taking the form of nonconvulsive status epilepticus. Since no medication was effective for the seizures, the episode was addressed by intubation.

Results.- An MRI was performed which revealed a new active T2 lesion on the cortex of the temporal lobe. The patient was eventually stabilized with anti-epileptic medication, presenting no signs of psychotic symptomatology for the following fifteen months. Conclusions.- There is a need for extensive laboratory tests and imaging assessment in first psychotic episodes, something that is often overlooked.

Disclosure of interest.- The authors have not supplied a conflict of interest statement.

\section{E-PV0140}

\section{Stress urinary incontinence and depression - a place for duloxetine - literature based analysis of efficacy and safety of treatment}

D. Berent ${ }^{1,2 *}$, M. Skoneczny ${ }^{3}$

${ }^{1}$ Masovian Regional Hospital "Drewnica”, Adult Psychiatry, Zabki/Warsaw, Poland; ${ }^{2}$ PsychoMedic.pl, Adult Psychiatry, Warsaw, Poland; ${ }^{3}$ Pirogow Regional Hospital in Łódź, Urology and Renal Transplantology, Łódź, Poland

* Corresponding author.

Background and aims.- Duloxetine, a serotonin and noradrenalinereuptake inhibitor, increases rhabdosphincter contractility via the stimulation of pudendal motor neuron $\alpha-1$ adrenergic and 5hydroxytryptamine- 2 receptors. Wide literature supports use of duloxetine in patients with stress urinary incontinence (SUI). Patients with SUI are known to be at greater risk of depression than the general population. This is to analyse safety and efficacy of duloxetine treatment in patients with SUI and to shed light on the subpopulation of patients with depression and SUI and/or patients with SUI and threatening depression as a noteworthy treatment target for duloxetine.

Methods.- A literature search of the Science Direct database was done up to September 2018, restricted to English-language articles, using terms related to duloxetine, depression, stress urinary incontinence. Original articles, reviews and meta-analyses were included.

Results.- European Association of Urology suggests to offer duloxetine in selected patients with symptoms of SUI when surgery is not indicated. In women and in men with SUI after prostate surgery, duloxetine $80 \mathrm{mg}$ may be superior to placebo and improves quality of life. Nausea is the most common treatment adverse event, was mostly experienced within the first few days and resolved within 1 to 4 weeks. Depression can result from incontinence and also, when it is already present, can influence adversely motivation to stay continent.

Conclusions.- A unique mechanism of action on rhabdosphincter contractility and documented improvement of the quality of life 
in patients with SUI suggest that patients with SUI and concomitant/threatening depression may gain from the treatment with duloxetine.

Disclosure of interest.- The authors have not supplied a conflict of interest statement.

\section{E-PV0142}

\section{Psychiatric comorbidity in patients with posttraumatic stress disorder and enduring personality change after catastrophic experience}

\section{Bradic ${ }^{1 *}$, D. Begic ${ }^{2}$, I. Lokmic-Pekic ${ }^{2}$, A. Hrnjica ${ }^{3}$, M. Ahmic ${ }^{4}$}

${ }^{1}$ Psychiatric Hospital of Canton Sarajev, Men's Psychatric department, Sarajevo, Bosnia - Herzegovina; ${ }^{2}$ Psychiatric Hospital of Canton Sarajevo, Intensive Care Unit, Sarajevo, Bosnia - Herzegovina; ${ }^{3}$ Psychiatric Hospital of Canton Sarajevo, Woman's Department, Sarajevo, Bosnia - Herzegovina; ${ }^{4}$ Psychiatric Hospital of Canton Sarajevo, Head of hospital, Sarajevo, Bosnia - Herzegovina * Corresponding author.

Background and aims.- Post-Traumatic Stress Disorder (PTSD) is a trauma and stress related disorder that may develop after exposure to an event in which death, severe physical harm or violence occurred or was threatened (1). Enduring personality change after catastrophic experience is characterized by personality changes after a significant event or events. This condition may be preceded by PTSD (2).

Aim of this research was to examine prevalence of psychiatric comorbidity and to analyze sociodemographic characteristics in patients with PTSD and enduring personality change after catastrophic experience.

Methods.- This retrospective study included 20 patients that were admitted in our hospital in one year with diagnosis of PTSD and Enduring personality change after catastrophic event.

Results.- Among all patient, there were 12 men and 8 women. There were 11 patients with PTSD diagnosis. Enduring personality change after catastrophic experience diagnosis had 9 patients. Comorbidity was present in 17 patients. The most common comorbidities in women were F 33.2 and F 32.1; in men F 10.2 and F 10.1. We had 15 married patients. Twelve patients had high-school level of education. Unemployed were 7 women and 7 men.

Conclusions.- Results implied that in women with PTSD and Enduring personality change after catastrophic experience diagnosis, the most common comorbidities were depressive disorders, while in men were Mental and behavioral disorders due to use of alcohol. Analysis of sociodemographic characteristics demonstrated that unemployment was present in high percentage equally distributed among both sex, and that most of the patients were married. Disclosure of interest.- The authors have not supplied a conflict of interest statement.

\section{E-PV0144}

\section{Opioid use: an update and the situation of the opioid consumption in the unit of dual pathology and the unit of detoxification of salamanca, \\ Spain}

N.M. Casado Espada*, R. De Alarcón Gómez, J.I. De La Iglesia Larrad, M.T. Lozano López, S.R. Gamonal Limcaoco, I. Valriberas Herrero, A. Bullón, A.I. Alvarez Navares, M.A. Garzón de Paz University of Salamanca Health Care Complex, Psychiatry, Salamanca, Spain

${ }^{*}$ Corresponding author.

Background and aims.- The prescription of opiates has increased significantly. Moreover, the consumption of opiates and the num- ber of deaths caused by an overdose from these substances has also increased.

Reviewing, collecting and presenting up-to-date information on a very important public health problem. Assessing the situation of the UDT AND UDP of Salamanca in the years 2016 to 2018 with regard to the consumption of opiates.

Methods.- Retrospective data collection from patients treated in the years 2016 to 2018 in the Unit of Dual Pathology and the Unit of Detoxification of Salamanca. Literature review in the main search engines (PUBMED, COCHRANE).

Results.- In our study, we collect, according to the literature review, the role of the different substances in the opioid crisis and the proposals aimed at reducing the mortality rates as a consequence of opioid overdose. Moreover we show the situation of our region in the opioid crisis.

Conclusions.- FENTANYL has been suggested as one of the main agents responsible for de opioid epidemics.

Another fundamental factor is the new marked of drugs ${ }^{1}$, whose characteristics help buyers and sellers alike and make it difficult for public security forces to track them.

With regard to the situation of our region and the consumption of opiates, according to the data collected and analyzed by the UDP and UDT of Salamanca in the years 2016-2018, we may conclude that there has also been an increase in the proportion of opiate users among the patients treated in our center, mainly associated to cases of consumption of methadone and to polydrug use. Disclosure of interest.- The authors have not supplied a conflict of interest statement.

\section{E-PV0145}

\section{A behcet's disease with a bipolar mood disorder: a case report}

A. Haouala ${ }^{1}$, M. Guedria ${ }^{1}$, N. Hamza ${ }^{1}$, I. Chaari ${ }^{*}$, R. Machraoui ${ }^{1}$, S. Younes ${ }^{1}$

${ }^{1}$ Taher Sfar Hospital, Neurology-Endocrinology Department, Mahdia, Tunisia; ${ }^{2}$ Fattouma Bourguiba University Hospital, Psychiatry Department, Monastir, Tunisia

* Corresponding author.

Background and aims.- Behcet's disease is a chronic, relapsing multisystem-vascular-inflammatory disorder. Vasculitis is its major pathological-feature. Psychiatric manifestations have been described, not necessarily attributable to cerebral lesions.

Methods.- We report a case of Behcet's-syndrome, in which BipolarDisorder revealed during the first physical manifestations Results.- A 44-year-old right-handed male, with previously healthy status, was admitted with complaints of acute, generalized headache associated with left-arm-and-leg weakness. He had weakness, loss of appetite and a progressive worsening of communication with family members for 3-4 days before the admission. Physical examination revealed oral aphthous ulcers. A thorough neurological examination revealed a quadripyramidal syndrome with predominant left motor deficit. Psychiatric interview showed a sad mood (HAD-D =17), excessive anxiety (HAD-A=12) and visual-hallucinations. The diagnosis of Behcet's-disease was made based on the presence of recurrent oral-aphthous ulcers, vascularinvolvement, a positive Pathergy-test and positive HLA-B51 in the absence of evidence of other diseases. Since then, he was treated with Methylprednisolone-pulses linked with a high-dose regimen of prednisone during 2 months, daily Azathioprine, and physical rehabilitation was started. Psychiatric treatment included an antidepressant (escitalopram $10 \mathrm{mg} /$ day) and an antipsychotic (risperidone $2 \mathrm{mg} /$ day). Two years after discharge, despite marked regression of physical impairment, he presented a depressive relapse followed by a manic episode induced by tricyclic-antidepressant (amitriptyline $50 \mathrm{mg}$ /day). He was diagnosed with a Bipolar disorder type-I according to DSM IV-TR. Mood stabilizer was introduced (Sodium-valproate $1500 \mathrm{mg} /$ day). 
During the following 6years he had two brief recurrent episodes in a context of poor-therapeutic-compliance.

Conclusions.- This association was previously reported. Data suggest a common neurobiological substrate at least in certain cases. That may present an attractive therapeutic target.

Disclosure of interest.- The authors have not supplied a conflict of interest statement.

\section{E-PV0146}

\section{Congenital hypopituitarism and psychosis symptoms: a case report}

S. Smaoui ${ }^{1}$, R. Damak ${ }^{1}$, F. Cherif ${ }^{*}$, F. Fekih Romdhane ${ }^{1}$, W. Cherif ${ }^{1}$, S. Ellini ${ }^{1}$, M. Cheour ${ }^{1}$

${ }^{1}$ Razi Hospital, Ibn Oumrane Psychiatry Department, la Manouba,

Tunisia; ${ }^{2}$ Hedi Chaker University Hospital, Psychiatry "C"

Department, Sfax, Tunisia

Corresponding author.

Background and aims.- The number of cases in the literature linking psychosis to hypopituitarism is limited.

Methods.- We report a case of a 48 year-old female patient who was referred to our department of psychiatry for psychotic symptoms. Results.- The patient was followed since she was young for congenital hypopituitarism, and genetics studies revealed mutation R73C in PROP1 gene. She interrupted follow-up since 11 years but she kept on medical treatment with only hydrocortisone. She was admitted to the psychiatry department because of behavioral disorder. She presented auditory hallucinations with delusional interpretation and persecution delirium which appeared since 18 months. Basic studies revealed anemia $(10,2 \mathrm{~g} / \mathrm{dl})$ and thyrotropic insufficiency $(\mathrm{FT} 4=6,39 \mathrm{pmol} / \mathrm{l} ; \mathrm{TSH}=1,77 \mathrm{uUI} / \mathrm{ml})$. Initially, neuroleptic treatment did not improve psychotic symptoms until we corrected biological disturbances especially thyrotropic insufficiency with thyroxin.

Conclusions.- The causal relationship between hypopituitarism and psychosis is not clear. Prospective follow-up of patients with pituitary dysfunction would clarify prevalence rates.

Disclosure of interest.- The authors have not supplied a conflict of interest statement.

\section{E-PV0147}

\section{Low dose chlorprothixene administration in patients with atopic dermatitis}

I. Danilin ${ }^{1 *}$, Z. Ziewozinska ${ }^{2}$, I. Korsunskaya ${ }^{3}$, M. Artemieva $^{1}$, R. Suleymanov ${ }^{1}$, V. Sokolov ${ }^{1}$, V. Karnozov ${ }^{1}$

1 Peoples' Friendship University of Russia RUDN University, Department of Psychiatry and Medical Psychology, Moscow, Russia; ${ }^{2}$ Pirogov Russian National Research Medical University, Moscow Scientific-and-Practical Center for Dermatovenereology and Cosmetology, Moscow, Russia; ${ }^{3}$ Moscow Scientific and Practical Center of Dermatovenereology and Cosmetolog, Moscow Helthcare Department, Moscow, Russia

${ }^{*}$ Corresponding author.

Background and aims.- Atopic dermatitis is a chronic skin disease, affecting up to $12 \%$ of population. Most scientists attribute atopic dermatitis to combine genetic, allergenic and emotional etiologic factors. There is definitely more than one trigger modality that can lead to the manifestation or exacerbation of symptoms. Psychogenic factors, often take a leading position in this index. Some personality traits may lead to instability of the emotional sphere that can act alternately as a cause, then as an aftereffect of exacerbation of the chronic process, forming a kind of psychosomatic "vicious circle", which requires exploration and treatment.
Methods.- Working together with dermatologist, we examined 30 patients with diagnosis of atopic dermatitis. All patients underwent psychiatric interviewing and psychological testing (STAI). The results revealed increased trait anxiety levels among 23 of them with moderate severity of the process according to SCORAD index. Chlorprothixene in dosage of 7,5 to $15 \mathrm{mg}$. daily was added to the common dermatologic therapy in this group.

Results.- Patients noted not only improvement of general condition, reduction of anxiety, sleep improvement, but also much more rapid and pronounced decrease of skin symptoms. 8 patients noted a marked improvement even in the absence of a previous history of good remission.

Conclusions.- This modest result may aim further clinical research on psychopharmacological treatment of some skin diseases in close cooperation with dermatologists. Finding intercommunications in pathogenesis of skin and emotional disorders could significantly optimize the treatment and enhance patient's quality of life. The publication was prepared with the support of the "RUDN University Program 5-100".

Disclosure of interest.- The authors have not supplied a conflict of interest statement.

\section{E-PV0149 \\ Clinical aspects and therapeutic approaches in bipolar disorder with pseudo-obsessional onset}

I.A. Georgescu ${ }^{1,2^{*}}$, M.M. Leți ${ }^{1}$, M. Hățiș ${ }^{1}$, R. Grozăvescu ${ }^{1,3}$

${ }^{1}$ Prof. Dr. Alexandru Obregia Clinical Psychiatry Hospital, Child and Adolescent Psychiatry, Bucharest, Romania; ${ }^{2}$ Carol Davila University of Medicine and Pharmacy- Faculty of Medicine, Physiology and Neuroscience, Bucharest, Romania; ${ }^{3}$ Carol Davila University of Medicine and Pharmacy- Faculty of Medicine, Child and Adolescent Psychiatry, Bucharest, Romania

${ }^{*}$ Corresponding author.

Background and aims.- Bipolar Disorder - Obsessive Compulsive Disorder comorbidity is frequent among adolescents, with a higher incidence than adults (23.2\% versus $13.56 \%$ ) [1]. However, there is still no official protocol for treating patients with this kind of pathology. Standard obsessive compulsive disorder treatment (SSRI) can worsen bipolar disorder symptoms, turning to mania, therefore mood stabilizers are most commonly administrated and, if the patients are refractory to treatment, they can be associated with atypical antipsychotics (Risperidone, Quetiapine, Aripiprazole) [2].

The main purpose of our study was to analyse in detail the case of a 16-year-old patient with this comorbidity, hospitalised at "Prof. Dr. Alexandru Obregia" Clinical Psychiatry Hospital in Bucharest. Methods.- The patient was psychologically assessed and the results were correlated with his personal history. We examined the therapeutic approach and the periods of remission in order to better understand the pathology and increase the quality of his life.

Results.- The particularity of the case is represented by the therapeutic resistance of the disease, even though multiple intervention schemes were tested. Also, even if the patient had an increased insight of the disease and was receptive to therapy, he still had obsessive thoughts and felt insecure, which significantly reduced the quality of his life.

Conclusions.- In conclusion, the pharmacological treatment should be associated with cognitive-behavioral therapy, important parental support and constant counselling to help these patients integrate into their age group [3]. In this way they will better manage their human interactions and increase their life quality.

Disclosure of interest.- The authors have not supplied a conflict of interest statement. 
E-PV0152

\section{Acute intermittent porphyria in a psychiatric patient - the wolf in sheep's clothing: a case report and literature review}

K. Hofert ${ }^{*}$, J. Baek

Greater Manchester Mental Health NHS Trust, Psychiatry,

Manchester, United Kingdom

${ }^{*}$ Corresponding author.

Background and aims.- Acute Intermittent Porphyria (AIP) is an autosomal dominant disorder in the haem synthesis pathway. AIP can have a variable presentation but psychiatric symptoms such as psychosis form part of the classical triad alongside abdominal pain and impaired cognitive function. The prevalence of AIP in psychiatric patients is higher than the typical population (Elder et al., 2013).

Methods.- Here we report a case of psychiatric inpatient who presented with an acute attack of porphyria whose diagnosis of AIP was clouded by complex psychiatric co-morbidity. We also reviewed the current literatures focusing on patient diagnosed with AIP in the psychiatric health setting.

Results.- Purely psychiatric manifestations of AIP are reported in the literature (Ellencweig et al., 2006), but there is no current consensus on prevalence. This creates a clinical quagmire for patients with pre-existing psychiatric conditions as this has the potential to masque psychiatric symptoms of AIP.

Conclusions.- This diagnostic difficulty may lead to under diagnosis of AIP. Therefore, we encourage consideration of AIP in the differential diagnosis in any acute psychiatric presentation. Furthermore, by reviewing the literature we hope to shed light on the prevalence of psychiatric manifestations of AIP.

Disclosure of interest.- The authors have not supplied a conflict of interest statement.

\section{E-PV0153}

\section{Clinical and therapeutic aspects of PMS comorbid with depressive disorder}

R. Iakimova ${ }^{1}$, M. Stoianova ${ }^{1}$, M. Stoimenova-Popova ${ }^{2}$, P.

Chumpalova $^{2}$, I. Veleva ${ }^{2}$, L. Tumbev ${ }^{2}$

${ }^{1}$ Medical University, Psychiatry and Medical Psychology, Sofia, Bulgaria; ${ }^{2}$ Medical University, Psychiatry and Medical Psychology, Pleven, Bulgaria

* Corresponding author.

Background and aims.- Premenstrual syndrome (PMS) is intimately related to major depressive disorder (MDD). In DSM-5 premenstrual dysphoric disorder (PMDD), a subtype of PMS, is even classified in the chapter Depressive disorders. Selective serotonin re-uptake inhibitors (SSRIs) are treatment of choice in the management of PMS. Objectives: To examine the clinical symptoms of PMS in women with current depressive episode before and after 6-month course of SSRI treatment.

Methods.- Longitudinal interventional study on the clinical picture of PMS and comorbid MDD before nd after 6-month SSRIs intake. We examined 31 women (mean age $39.4 \pm 7.21$ ) suffering from PMS and a current episode of MDD eligible to SSRI treatment. PMS and MDD were verified by means of PSST and MINI, respectively.

Results.- According to our results the most prevalent symptoms in comorbid patients are mood lability, anxiety, fatigue (100\%), irritability and sleep changes (96.8\%), breast tension and tenderness (100\%), abdominal bloating (93.5\%), headaches (93.1\%). Psychological symptoms dominate the syndrome being around three times more common than somatic. PMS is mostly moderately severe. After 6-month SSRI treatment a statistically significant $(p<0.001)$ effect on both symptom types was noticeable, although certain somatic symptoms still persisted. At the end of the follow up severe cases were completely resolved and mild cases prevailed.

Conclusions.- 1. PMS with comorbid MDD is dominated by mood lability, fatigue, anxiety, breast tension and tenderness. 2. Psychological symptoms prevail. 3. SSRIs are helpful in treating both symptom types, but still more pronouncedly psychological ones. Disclosure of interest.- The authors have not supplied a conflict of interest statement.

\section{E-PV0155}

\section{A depressive disorder masking a gender dysphoria: a case report}

W. Kabtni*, C. Bencheikh, O. Zeriaa, M. Hsayri, A. Baatout, I. Bouzouita, M.W. Krir, H. El Kefi, A. Oumaya Military Hospital, Psychiatry Department, Tunis, Tunisia * Corresponding author.

Background and aims.- Gender dysphoria is a new entity introduced in the Diagnostic and Statistical Manual of Mental Disorder V that often starts in the childhood. Despite the psychological distress and the significant alteration of social and academic life that it causes, it remains misdiagnosed.

This case report aims to describe a case of gender dysphoria masked by depressive disorder.

Methods.- A patient case is presented with associated literature review.

Results.- Mr. NL, aged 17, an assigned male at birth, with no medical history, is a student in the third year of secondary school.

The patient was referred by his parents initially for a decline in school results with a tendency to isolation evolving since one year. At the interview, the depressive symptomatology was in the first place. The patient was put on antidepressant treatment combined with supportive psychotherapy.

Through the psychotherapy sessions, it was possible to emphasize the presence of a strong desire to be a girl, he would prefer to dress like a girl, playing often the stereotyped "girlish" games along with girls' group. In addition, he tells with great suffering and sadness that he "is trapped in the wrong body" and he expresses an intense desire to acquire feminine physical forms.

The diagnosis of gender dysphoria was retained. The patient is still going through psychotherapy sessions to help him make the right decision.

Conclusions.- An early positive and etiological diagnosis of gender dysphoria will prevent a psychiatric complication that may hinder therapeutic management.

Disclosure of interest.- The authors have not supplied a conflict of interest statement.

\section{E-PV0159 \\ Psoriasis exacerbation in bipolar disorder relapse: a case report}

R. Maamouri ${ }^{1}$, N. Bram² ${ }^{2}$ C. Ben Said ${ }^{1}$, I. Ben Romdhane ${ }^{2}$, W. Homri $^{2}$, R. Labbane ${ }^{2}$

${ }^{1}$ Medical School of Tunis, Psychiatry, Tunis, Tunisia; ${ }^{2}$ Hospital Razi, Psychiatry ' $C$ ' department, Mannouba, Tunisia

"Corresponding author.

Background and aims.- Psoriasis is a chronic and systematic disease that equally affect men and women in $1.5 \%$ to $2 \%$ of the population, it's a frequent skin disorder that needs a long-term pharmacological treatment and may have a profound impact on the quality of life. Otherwise, psychiatric disorders and especially bipolar disorder are some of the associated problems that can be observed in patients with psoriasis.

Methods.- We will discuss a clinical case and will eventually proceed to a literature review about this subject. 
Results.- A60 years old man suffering since four years from psoriasis and stabilized under a local medical treatment, was hospitalized for psycho-motor instability and logorrhea. The diagnosis of a maniac episode was assessed by the fifth edition of the diagnostic and statistical manual of mental disorders (DSM -5). The patient has many stress factors: he was about to retire, he got removed from his usual work spot and he has a conflict with his brother. Two months before the maniac episode, the patient had an itching thick red scaly patch of skin. During his hospitalization, he got risperidone and chlorpromazine and was examined by a dermatologist who assessed an exacerbation of psoriasis and prescribed adequate local treatment. The evolution was favorable, both disorders was managed.

Conclusions.- The association between psychiatric disorders and dermatological conditions is well known, but the dermatologist has often a difficulty to identify the psychological distress that's why we need to enhance the knowledge of such comorbidities to a better quality of medical care.

Disclosure of interest.- The authors have not supplied a conflict of interest statement.

\section{E-PV0161}

\section{The relationship between impulsivity and alexithymia in a sample of strong nicotine addicted. a preliminary study} D. Montanaro ${ }^{1 *}$, C. Medici $^{2}$

${ }^{1}$ Freelance, Freelance, Rome, Italy; ${ }^{2}$ Specializing in Psychiatry, University of Rome La Sapienza, Rome, Italy

Corresponding author.

Background and aims.- Given the correlation between tobacco addiction and impulsivity, this study means to evaluate the role of alexithymia in the relationship between impulsivity and tobacco addiction. Alexithymia is defined as a difficulty in the mental representation of emotions due to a loss of integration between physiological and cognitive component of emotions. Alexithymia can be characterized by an operative kind of thought, lacking in imagination, fantasy or oneiric activity that, according to the "Human Birth Theory" by Massimo Fagioli, are fundamental to ensure the possibility to mentally elaborate psychic and physical sensation. Consequently, a lack of psychophysical sensibility could prejudice the imaginative thinking process, mystifying our experience awearness (Atzori, 2017), and making emotional experience less comprehensible and intense.

Methods.- This preliminary study examines the correlation between the dimensions of impulsivity and alexithymia in a sample of 30 help-seekers related to a Service for Addictions, diagnosed with Tobacco Addiction, through the analysis of the results of a test battery distributed at the admission.

Results.- Test results suggest that alexithymia has a role in leading to impulsive action. The greatest correlation was found between alexithymia and the impulsivity sub-factor called "lack of reflection and behavioral planning".

Conclusions.- The difficulties in mentalization, due to the lack of psychophysical sensitivity, would expose to the possibility of acting-out and the search for sensory stimulation, aimed at overcoming these deficiencies and maintaining the integrity of the self (Atzori 2017, 2018). The tendency to impulsive action could, therefore, be sustained by a lack of psychophysical sensitivity that alters the process of mentalization.

Disclosure of interest.- The authors have not supplied a conflict of interest statement.

\section{E-PV0162}

\section{Why and how to create "identified beds" in palliative care for psychiatric patients in long term unit?}

A. Nasfi

Usld la Roseraie EPS Maison Blanche, Seine Saint Denis, Neuilly Sur

Marne, France

Corresponding author.

Background and aims.- Our Long stay Unit take in charge psychiatric patients. Most of them harbore many co-morbidities and die in the structure. Because of their psychotic disorders, the approach of pain and end of life is complex and requires a specific approach.

Methods.- Progressive implementation of a palliative approach to accompany the end of life.

Results.-

1) Creation of an evaluation of professional practice, working group on end of life.

2) Conducting an audit on end-of-life support practices. This audit highlighted the previous experience of the caregivers in this field but also the need for a better support of the professionals, a mobile team of palliative care, and training.

3) A study carried out on the psychiatric patients who died in the structure for 10 years showed that despite a lower age than the geriatric population, $26 \%$ died during this period mainly due to tumors. The accompaniment was carried out for $90 \%$ of people in our unit.

4) Establishment of a medical and paramedical training plan, including a university diploma of palliative care program.

5) Establishment of specific support for caregivers by a psychologist.

6) Creation of specific care protocols

7) Information for patients and their family

8) Convention with a mobile team of palliative care

Conclusions.- This innovative project to create" Identified Beds "Palliative Care, currently submitted to the Health Agency, will allow an effective recognition of the work done by caregivers and the means necessary for its operation. It will allow to offer psychiatric patients better end-of-life care without breaking the psychiatric care in their unit.

Disclosure of interest.- The authors have not supplied a conflict of interest statement.

\section{E-PV0163}

\section{Psychiatric disorders in cancer patients}

S. Omri", M. Daoud, W. Bouattour, M. Maalej, R. Feki, L. Zouari, N. Charfi, J. Ben Thabet, M. Maalej

Psychiatry "C" Department at Hedi Chaker University Hospital- in Sfax-Tunisia, Psychiatry "C" Department at Hedi Chaker University Hospital-in Sfax -Tunisia, Sfax, Tunisia

* Corresponding author.

Background and aims.- Although a diagnosis of cancer today may no longer be considered to be the equivalent of a death sentence, many previous studies have revealed that such a diagnosis places many kinds of emotional burden on a patient. The study aims to investigate the characteristics, reason for psychiatric consultation and psychiatric diagnosis of patients with cancer.

Methods.- A retrospective study, involving 50 outpatients diagnosed with cancer and followed in the psychiatry " $C$ " department at Hedi Chaker university Hospital, in Sfax -Tunisia, between January 2016 and September 2018. Sociodemographic, clinical and therapeutic data was collected from their medical records. The statistical study was carried out using the SPSS software (18.0).

Results.- The obtained results showed that $1.75 \%$ (50/2851) of outpatients had a cancer (average age $=32$ years; sex-ratio $=1.8$ ). The most common types of cancer were especially colon cancer 
(30\%) and breast cancer (28\%). Thirty-five percent of patients had metastatic cancer. The major grounds for consultation were depressive and anxious symptoms (53\%) and sleep disturbance $(28,2 \%)$. The time elapsing between cancer diagnosis and the psychiatric consultation was five months. The most frequent psychiatric diagnoses were depression (33\%) and anxiety disorders (31\%). Poor adherence and irregular follow-up were noted in $82 \%$ of patients. Conclusions.- Psychiatric disorders represent a significant comorbidity in oncology and their neglect may have significant negative consequences on the quality of life of patients. Consideration should be given to include a psychologist in the multidisciplinary evaluation of the cancer patient.

Disclosure of interest.- The authors have not supplied a conflict of interest statement.

\section{E-PV0164 \\ Refraktarity in a clinical case with BPD and OCD co-morbidity}

T. Pengili ${ }^{*}$, I. Prifti ${ }^{2}$, M. Lici ${ }^{3}$, E. Murati ${ }^{3}$, A. Hashorva $^{3}$

${ }^{1}$ University Medical Center "Mother Teresa", Psychiatry, Tirana,

Albania; ${ }^{2}$ University Medical Center of "Mother Theresa", Psychiatry, Tirana, Albania; ${ }^{3}$ University Medical Center "Mother Theresa", Psychiatry, Tirana, Albania

* Corresponding author.

Background and aims.- Studies show that these disorders coexist in $18-25 \%$ of cases where $50-60 \%$ of them are treated efficiently. We are going to discuss from our clinical experience a difficult case of a 23-year-old patient with a three-year history of the disease.

Methods.- The initial symptoms were disorientation and amnesia and as a result we suspected for an organic condition. The patient took a brain MRI, which resulted with asequential lacunar hypersignal in flair positioned on the 4th ventricle, but according to the neurologist this did not affect the condition of the patient. He was treated for over 2 years with various treatment schemes. During this time he displayed therapeutic resistance and he did notremission. The first hospitalization was back in 2015 and at the time he came as a suicidal attempt and was diagnosed with OCD. According to his family, the suicidal attempt was a kind of a demonstrative action.

Results.- The psychosis was dominated within a few days in the clinic but we had remaining symptoms such as: obsessive thinking, compulsive behavior, depersonalization, derealysis, tenseness of confidentiality with other medical staff, ecopraxis, strongly denying the hallucinations and delusions, which excluded a diagnose of schizophrenia and reinforced the theory of a BPD diagnose.

Conclusions.- He is now being treated with Olanzapine $20 \mathrm{mg}$, Venlafaxine $75 \mathrm{mg}$, Lorazepam $5 \mathrm{mg}$, CarbonatLithiumi $500 \mathrm{mg}$ but his condition continues to be not good.

Disclosure of interest.- The authors have not supplied a conflict of interest statement.

\section{E-PV0165}

\section{Moving towards personalized medicine in psychiatry: a value of genotyping in assessing of risks and benefits in patients receiving SSRI'S and cardiovascular medication}

\section{S. Petrykiv ${ }^{1 *}$, M. Arts ${ }^{2}$, L. de Jonge ${ }^{3}$}

${ }^{1}$ Maastricht University Medical Center, Department of Clinical Pharmacy and Pharmacology, Maastricht, The Netherlands; ${ }^{2}$ Mental Health Care -Western North Brabant GGZ-WNB, Department of Old Age Psychiatry and Neuropsychiatry, Halsteren, The Netherlands; ${ }^{3}$ Leonardo Scientific Research Institute, Department of

Neuropsychiatry, Groningen, The Netherlands

${ }^{*}$ Corresponding author.
Background and aims.- A high prevalence of poor metabolizers $(6-10 \%)$ in western population is a potential source of drug-drug interactions. To date, the human pharmacokinetic data on SSRi's and cardioprotective drugs are inconclusive because of the wide interpersonal variation in outcomes, a limitation which can be eliminated by a personalized approach through genotyping to identify patients at risk (slow metabolizers).

Methods.- First, we performed a meta-analysis to assess the effect of SSRI's on percentage change in AUC of statins in general population. Secondly, we reviewed the neuropsychiatric effects of antihypertensive drugs when co-administrated with SSRI's. Finally, we speculated on the value of genotyping as a potential tool to identify patients at risk.

Results.- Fluvoxamine increased the mean substrate AUC 0[infinity] by $112.47 \%$ (CI: 21.08; 203.86). The AUC 0-[infinity] levels of CYP3A4 substrates were marginally increased by $11.1 \%$ in citalopram treated subjects ( $\mathrm{CI}:-20.63 ; 42.86)$, by $10.8 \%$ in fluoxetine treated group ( $\mathrm{CI}:-25.53 ; 47.16)$, by $8.18 \%$ in those who received paroxetine $(\mathrm{CI}:-3.49 ; 19.86)$ and by $-3.3 \%$ in venlafaxine treated group (CI: $-5.85 ;-0.75)$. Based on literature search, clinically important links may exist between SSRI's with propranolol, metoprolol and amlodipine.

Conclusions.- Citalopram, fluoxetine, paroxetine and venlafaxine are almost certain to be safe with all statins. Fluvoxamine inhibits CYP3A4 metabolic pathway and is potentially unsafe in cotherapy with atorvastatin, simvastatin and lovastatin. Co-administration of lipophilic $\beta$-blokkers are thought to be associated with higher rates of neuropsychiatric consequences. We advocate for genotyping in patients experiencing adverse events and in those receiving polypharmacy.

Disclosure of interest.- The authors have not supplied a conflict of interest statement.

\section{E-PV0166}

\section{Eating disorder risk in overweight and obese polycystic ovary syndrome patients}

D. Rodriguez-Paris ${ }^{1 *}$, B. Banaszewska ${ }^{2}$, A. Remlinger-Molenda ${ }^{3}$ ${ }^{1}$ Provincial Neuropsychiatric Hospital in Kościan, Psychiatry, Kościan, Poland; ${ }^{2}$ Poznań University of Medical Sciences, Department of Reproductive Endocrinology, Poznań, Poland; ${ }^{3}$ Poznań University of Medical Sciences, Department of Adult Psychiatry, Poznań, Poland * Corresponding author.

Background and aims.- A study in progress assessing eating disorder risk (EDR) among overweight and obese PCOS patients of reproductive age and a matched control group. The assessment and comparison of EDR among the PCOS and control group as well as potentially finding a correlation between specific biochemical and hormonal levels and the risk of eating disorder development.

Methods.- The study is expected to involve 200 women of reproductive age with a body mass index $\geq 25$. They are qualified as PCOS or control utilizing the Rotterdam criteria. They subsequently undergo a series of biochemical and hormonal tests as well as a psychiatric assessment utilizing a structured interview and a series of questionnaires. Some of the diagnostic tools include: Mini-International Neuropsychiatric Interview, Bulimic Investigatory Test Edinburgh (BITE), Binge Eating Scale (BES) and the Eating Disorder Examination Questionnaire 6.0 (EDE-Q).

Results.- The study currently includes 51 patients (31 PCOS, 20 control). In x (\%) PCOS vs. $\mathrm{x}(\%)$ controls increased EDR was noted. Utilizing BITE $45.2 \%$ vs. $40 \%$. Utilizing BES $32.3 \%$ vs. $25 \%$. The subject groups are as of yet too small to draw statistically relevant conclusions or attempt to correlate eating disorder risk to specific hormonal and biochemical imbalances.

Conclusions.- Both patient groups demonstrate increased overall risk for developing eating disorders as compared to the general population. The PCOS group appears to display a higher percent- 
age of subjects at significant risk of having or developing an eating disorder. No statistically significant correlation has yet been found between hormonal and biochemical levels and eating disorder risk. Disclosure of interest.- The authors have not supplied a conflict of interest statement.

\section{E-PV0167}

\section{HCV+ among drug-dependent patients admitted to the detoxification/dual disorder unit of salamanca in the period 2014 to 2017}

C. Roncero ${ }^{1,2,3^{*}}$, C. Llanes ${ }^{4}$, N. Casado ${ }^{1}$, J. de la Iglesia ${ }^{1}$, M.T. Lozano $^{1}$, M.Á. Garzón ${ }^{5}$, M.P. Ándres ${ }^{1}$, E. Alvarez-Lamas ${ }^{5}$, A. Pérez-Madruga ${ }^{1}$, M. Rodriguez ${ }^{5}$, E. Dominguez ${ }^{5}, C$. Gamonal-Limcaoco ${ }^{1}$, M.V. Martín ${ }^{5}$, E. Valades ${ }^{5}$, A. Álvarez ${ }^{5}$ ${ }^{1}$ University of Salamanca Health Care Complex, Psychiatry, Salamanca, Spain; ${ }^{2}$ University of Salamanca, Psychiatry, Salamanca, Spain; ${ }^{3}$ University of Salamanca, Institute of Biomedicine of Salamanca, Salamanca, Spain; ${ }^{4}$ Zamora Health Care Complex, Psychiatry, Zamora, Spain; ${ }^{5}$ University of Salamanca Health Care Complex, Dual Diagnosis Unit. Psychiatry, Salamanca, Spain ${ }^{*}$ Corresponding author.

Background and aims.- Hepatitis $\mathrm{C}$ is a disease which can be transmitted through blood or sexual intercourse. It is very common in addicted and Dual Disorder (DD) patients (those who present an addiction and another mental disorder).

Objective.- To analyze the characteristics of patients with HCV infection admitted during 2014-2017 to the Detoxification/Dual disorder Unit of the Psychiatric Service.

Methods.- Descriptive, observational, retrospective study of 830 inpatients, among which 34\% (279) were infected by HCV.

Results.- Of the patients HCV+:80\% were male, 46 years old on average. $30 \%$ were as well infected with HIV. $69 \%$ of them had DD, with the following concurrent diagnoses: personality disorders (45\%), affective disorders (35\%), anxiety disorders (13\%) and psychotic disorders (10\%). Additionally, $70 \%$ of the patients presented opiate dependence, $35 \%$ cocaine dependence and $16 \%$ alcohol dependence. The main route of consumption before the hospitalization was oral in $64 \%$, pulmonary in $30 \%$, injected in $4 \%$ and snorted in $2 \%$. In many cases they had used the intravenous route in the past. In all the 279 cases the detection of anti-HCV antibodies was confirmed. Only $13 \%$ had recived treatment, 23 patients had completed treatment with Interferon and 14 had been treated with direct-acting antivirals (DAAs). Patients with positive anti-HCV antibodies who had not received treatment were referred to specialized services to complete the study and assess the need for treatment.

Conclusions.- Patients with SUD and DD are more at risk of getting HCV infection. Access to HCV treatment is improving, but until recently the need to adequately inform treatment options for this group has been neglected.

Disclosure of interest.- The authors have not supplied a conflict of interest statement.

\section{E-PV0168}

\section{Alcohol and drug abuse in psychiatric patients and healthy controls}

M. Pacetti ${ }^{1}$, F. Ambrosini ${ }^{2}$, M. Sanza ${ }^{3}$, R.P. Sant'angelo ${ }^{4}$

${ }^{1}$ Ausl Romagna, Mental Health- CSM, Forli, Italy; ${ }^{2}$ University of Bologna, Faculty of Psychology, Cesena, Italy; ${ }^{3}$ Ausl Romagna, Italy; ${ }^{4}$ Ausl Romagna, Mental Health, Cesena, Italy

* Corresponding author.

Background and aims.- Alcohol and drug addictions have a high incidence in Borderline Personality Disorder (BPD). However, the presence of addiction is rarely examined comparing an heterogeneous sample of psychiatric disorders and controls (HC).

Our aim is to compare the incidence of alcohol and drug abuse in patients with BPD, Affective disorders (AD), Psychosis (P) and HC. Methods.- 112 individuals were assessed using SCID I and II. The final sample was divided in BPD (24,1\%), P (24.1\%), AD (22.3\%) and HC (29.5\%). A self-report questionnaire was used to collect data on demographics, lifetime drug abuse and injection drug use. As screening the TWEAK test was administered, considering a score higher than 3 as indicative of alcohol abuse or addiction. The four groups were compared using chi-squared test.

Results.- BPD has higher score at TWEAK than P and HC, exhibiting a score similar to that of AD. Drug abuse in BPD is more frequent than in HC, P and AD. However, the lifetime injection drug use is similar in the three patient groups.

Conclusions.- BPD seems to behave similarly to AD for alcohol abuse. However, the incidence of druga ddiction is higher in BPD than in the other groups, confirming the literature.

Disclosure of interest.- The authors have not supplied a conflict of interest statement.

\section{E-PV0171 \\ A case of Niemman-Pick's disease in psychiatry}

M. Stoimenova-Popova*, E. Dimitrova, A. Todorov, G. Georgiev, P. Chumpalova

Medical University, Psychiatry and Medical Psychology, Pleven, Bulgaria

* Corresponding author.

Background and aims.- The patient with Niemann-Pick disease type B usually presents with hepatosplenomegaly, pathologic alterations on lungs, pancytopenia, caused by splenomegaly, sometimes there are eye abnormalities (cherry-red spot), neurological symptoms, most often mental retardation, peripheral neuropathy and seizures, bones also may be affected.

Methods.- A 35-year-old woman is hospitalized in a psychiatric clinic. About a month before hospitalization, she became tense, psychomotor agitated, with lively speech, mentally torn, unable to cope with her daily activities. She has psychotic symptoms since the age of 17. Till now she was treated in different psychiatric departments for affective and psychotic symptoms. In 2015 she was diagnosed with Niemann-Pick Type B disease - an intermediate form, because of neurological symptoms that she has.

Results.- After reviewing the medical history our team decided to start treating the patient with Ziprasidone $40 \mathrm{mg} /$ daily, Quetiapine $100 \mathrm{mg} /$ daily and Valproic acid $1000 \mathrm{mg} /$ daily. Diagnosis was defined as Organic Mood Disorder. At the first week of the treatment we observed improvement in psychotic symptoms. Gradually there was a reduction in the psychomotor agitation, she aligned mentally, despite of discrete flight of ideas, perceptual disturbances were decasualized. The patient became notably autonomous to self-care and move independently.

Conclusions.- Although the psychotic symptoms of Niemann-Pick type B disease - intermediate form are uncommon, in the case presented, they determine the poor quality of life and lead to a more severe course of the disease.

Disclosure of interest.- The authors have not supplied a conflict of interest statement.

\section{E-PV0172}

\section{A case of ekbom syndrome recurrent in patient with dual diagnosis and chronic insomnia}

E. Suarez 
Hospital Infanta Sofía, CSM de Alcobendas, Psychiatry, SAN Sebastian de los Reyes, Spain

${ }^{*}$ Corresponding author.

Background and aims.- Since the middle of the 19th century reports have appeared in the scientific literature of patients who claimed to be infested with parasites without medical evidence to prove it. It was first described by Thibierge in 1894, calling it "Acarophobia", and then by Ekbom in 1938, in his work Der praesenile dermatozoenwhan. Ekbom syndrome is a mental disorder in which the patient has the monothematic delusion of being infected by parasites. It is an uncommon condition that was initially studied by dermatologists. The exactly etiology is unknown to date, though several causes have been proposed, including metabolic diseases (among other physical causes), psychiatric disorders, drugs, etc.

Methods.- Case report To analyse the behavioral changes in a 48 year-old patient, with the diagnosis of dual disorder (affective diseases + drug dependence) who had a delusion of infestation and insomnia. The patient was treated with antipsycotic depot drugs. He responds effectively but insomnia remains. After 1 year, the patient decided to refused the antipsycotic treatment. 8 months later he relapse. I begun with the antipsycotic depot treatment and he responds effectively less the insomnia I treated with some combination of drugs without results. The combination of the Perampanel with other hipnotic drugs inprove the quality of de sleep.

Results.- The Ekbom syndrome is a rare subtype of delusional disorder that usually responds to antipsychotic drugs effectively. Conclusions.- The síndrom can be recurrent specialy if the period of treatment is short. The syndrom are often associate with chronic insomnia. Perampanel inprove the quality of de sleep.

Disclosure of interest.- The authors have not supplied a conflict of interest statement.

\section{E-PV0173}

\section{Comorbidities in patients diagnosed with major depressive disorder}

B.D. Suciu ${ }^{1 *}$, R. Păunescu ${ }^{2}$, I.V. Micluția ${ }^{2}$

${ }^{1}$ Children's Emergency Hospital, Psychiatry Clinic I, Cluj Napoca, Romania; ${ }^{2}$ Iuliu Hațieganu University of Medicine and Pharmacy, Neurosciences Department, Cluj Napoca, Romania

${ }^{*}$ Corresponding author.

Background and aims.- Studies reveal the presence of many psychiatric and somatic comorbidities in depressed patients. It is known that major depressive disorder has a high prevalence, a chronic course and it is often associated with numerous comorbidities which have a considerable impact on the patient's quality of life. Our aim was to evaluate the comorbid conditions of patients diagnosed with major depressive disorder that were admitted to the Psychiatry Clinic in Cluj Napoca.

Methods.- The study included 40 patients (aged between 23 and 65) diagnosed with recurrent major depressive disorder (according to ICD-10 and DSM-V), that were evaluated during their hospital admission. The severity of depression was quantified clinically with the help of the Hamilton Depression Rating Scale -17 items, while the comorbid conditions were identified using the Mini International Neuropsychiatric Interview and also by consulting the patients' charts.

Results.- The group had similar gender distribution, different levels of education and married patients were predominant. $77 \%$ of patients had a medical comorbidity, represented by: cardiovascular disorders, diabetes, chronic obstructive pulmonary disease, hepatitis and anaemia. In the same time, $63 \%$ of patients had another psychiatric condition, represented mostly by anxiety disorders or alcohol problems.
Conclusions.- These results are in accordance with the majority of studies and due to the fact that these disorders coexist with major depressive disorder, they could be considered an important source of mortality.

Disclosure of interest.- The authors have not supplied a conflict of interest statement.

\section{E-PV0174}

\section{Usher syndrome and psychiatric symptoms: a case report}

A. Taamallah ${ }^{*}$, M. Daoud ${ }^{2}$, S. Taleb ${ }^{3}$, H. Ben Ammar ${ }^{4}$, Z. El

Hechmi $^{5}$

${ }^{1}$ Resident, Psychiatric Department "F" /Razi Hospital, Manouba,

Tunisia; ${ }^{2}$ Resident, Psychiatric Department/Razi Hospital, Manouba, Tunisia; ${ }^{3}$ Resident, Psychiatric Department/ Razi Hospital, Manouba, Tunisia; ${ }^{4}$ Doctor, Psychiatric Department "F"/ Razi Hospital, Manouba, Tunisia; 5 Professor, Psychiatric Department "F"/ Razi Hospital, Manouba, Tunisia

* Corresponding author.

Background and aims.- Usher syndrome is an autosomal recessive genetic disorder manifested by hearing impairment, retinitis pigmentosa and variable vestibular deficit. Few studies suggest possible mechanisms of association between mental disorders and Usher syndrom. The most common manifestations are psychotic symptoms. Mood and behavioral disorders are rarely described.

The aim of our work is to discuss the link between Usher syndrom and psychiatric manifestations, with a review of the current literature.

Methods.- A case report discussed with a literature review, based in Pubmed, using "Usher syndrom", "psychiatric symptoms" as key words.

Results.- IM, a 40 years old female, was born full term with congenital hypoacusis. His language were delayed. She had sub-average intellectual and social functioning since childhood. She developed decreased vision at night. She is able to perform simple household tasks and self-care activities. His older brother and her father also have congenital deaf-blindness and mental retardation. There was a known history family of bipolar disorder. She was hospitalized for change in mood, psychomotor agitation, decreased sleep and strangulation suicide attempt.

She was sad and anxious. She had anhedonia, suicidal ideation, low self-esteem and delusional ideas, treated with olanzapine $20 \mathrm{mg} /$ day, fluoxetine $20 \mathrm{mg} /$ day and anxiolytics. The symptoms partly remitted. Ophthalmological examination showed pigmentary changes in both retinas and high myopia. Audiometric evaluation were indicated.

Laboratory investigations, brain scan and electroencephalogram were normal.

Conclusions.- Access to mental health services is particularly difficult for deaf and deaf-blind people. The difficulties in communication are a challenge for patients and doctors to understand symptoms. Further studies are needed to understand the link between mental disorders and Usher syndrome.

Disclosure of interest.- The authors have not supplied a conflict of interest statement.

\section{E-PV0175}

\section{Treatment challenges in nonepileptic psychogenic seizures}

J. Milczarek, A. Skiba, M. Łukasik, A. Orzechowska, M. Kowalczyk, M. Talarowska*

Medical University of Lodz, Department of Adult Psychiatry, Łódź, Poland

${ }^{*}$ Corresponding author. 
Background and aims.-

Introduction.- Psychogenic non-epileptic seizures (PNES) are timelimited, paroxysmal disturbances movement, sensation, behavior and consciousness that may resemble epileptic seizures. However they are not the result of epileptic discharges in the central nervous system. Differentiating epileptic seizures (ES) from psychogenic nonepileptic seizures (PNES) represents a challenging differential diagnosis. An accurate recognition is important in order to receive the appropriate treatment but it is only the beginning of a difficult road. Psychological intervention are favorable treatment offered to people with PNES.

Objectives.- The aim of the study was to present a case report of patient diagnosed for organic mood disorder (F06.3) suffering from nonelpileptic psychogenic seizures.

Methods.- Case report.

Results.- Patient aged 55. Hospitalized for the first time in 2017, in ambulatory care since 2003, firstly diagnosed with recurrent depressive disorders (F33) and mistakenly treated for epileptic seizures. During second hospitality patient experience a high level of anxiety, cannot cope with stressful situations. The behavior is described as neurotic, disinhibited, emotionally labile and unconventional. Psychological tests indicate cognitive deficits - CNS damage characteristic for changes in the frontal and cortical structure.

Conclusions.- PNES is one of the most common medically unexplained neurological symptoms. Patient diagnose for organic mood disorder with accompanying PNES seems to be a group of very limited treatment option. Strong mechanism of repression, low level of motivation, lack of insight and co-occurring cognitive deficits are insufficient psychological resources for effective therapeutic effect in the treatment of PNES in this case.

Disclosure of interest.- The authors have not supplied a conflict of interest statement.

\section{E-PV0176}

\section{Paliperidone palmitate for a dual diagnosis forensic psychiatric patient}

G. Tzeferakos ${ }^{1 *}$, A. Apostolopoulos ${ }^{1}$, O. Vamvakouri ${ }^{2}$, A. Douzenis ${ }^{1}$ ${ }^{1}$ University of Athens - Medical School, 2nd Department of

Psychiatry, Athens, Greece; ${ }^{2}$ Hellenic Agency Against Drugs, OST Unit - Atticon Hospital, Athens, Greece

* Corresponding author.

Background and aims.- Almost 50\% of patients with schizophrenia have a life time history of substance use disorder. These patients are characterized by: poor adherence to medication, multiple relapses and hospitalizations (involuntary and voluntary), increased victimization, violence, suicidality, and risk of HIV, HCV and HBV infections. Quite often they are involvemed with the Criminal Justice System.

Methods.- We present a case of a young female dual diagnosis patient (schizophrenia and polytoxicomania), enrolled in an OST program with methadone, with a long history of multiple hospitalizations (mainly involuntary) and violent outbursts/arrests, extremely poor adherence to medication, continuous abuse of several psychoactive substances (BZD, MDMA, Heroin) and active psychotic symptomatology.

Results.- Upon her admission to our OST unit, the patient suffered an acute psychotic exacerbation, after inhalation of methamphetamine, which led to her involuntary admission. During her hospitalization, the patient was initiated PP $1 \mathrm{M} 75 \mathrm{mg}$, which was gradually titrated to $150 \mathrm{mg} /$ monthly.

We present her clinical course, as depicted by different scales (PANSS, PSP, CGI-C and CGI-C, every 3 months) (table 1).
Table 1 Clinical course depicted by different scales

\begin{tabular}{llll}
\hline Scale & Admission & 3rd month & 6th month \\
\hline PANSS & 121 & 92 & 60 \\
CGI-S & 7 & 6 & 4 \\
CGI-C & N/A & 2 & 2 \\
PSP & 25 & 40 & 50
\end{tabular}

Conclusions.- PP $1 \mathrm{M}$ seems to have helped stabilize the patient. Disclosure of interest.- The authors have not supplied a conflict of interest statement.

\section{E-PV0177}

\section{Addiction \& ASD}

P.J.M. Van Wijngaarden-Cremer

Dimence, Centre for Developmental Disorders, Zwolle, The Netherlands

* Corresponding author.

Background and aims.- Dopamine plays a key role in a series of seemly-unrelated conditions such as Parkinson's disease, ADHD and addiction. Autism spectrum disorders (ASD) are so to say at the intersection of these developmental disorders in the sense that individuals with ASD present with cognitive problems especially in the area of executive functions, motor oddities and preoccupations and stereotypies. The obsessive-compulsive preoccupations, the motor stereotypies are functional in young children as they help them cope with the stimuli overload that they experience. As development progresses these behaviours can evolve into addictive like behaviours and substance abuse.

Aim.- Can this be confirmed in a clinical population?

Methods. - A two year cohort of patients admitted both to the outpatient and inpatient services of our department for developmental disorders was thoroughly assessed. Data from development, somatic and psychiatric appraisal, substance and behavioural addictions were gathered.

Results.- It appeared that in $80 \%$ of the cases in this adult ASS sample some behavioural and/or substance related addiction was found. There were now significant differences with regard to gender in terms of prevalence but clearly in the type of addiction. In this presentation the detailed outcomes will be presented and discussed. Conclusions.- It is clear that in adults with ASD addiction is an important issue to take into consideration. But instead of aiming straight away at detoxification and behaviour modification, it appears to be crucial to make a functional analysis taking all contextual features into consideration in order to understand and address the underlying dynamics of the addictive behaviour as such. Disclosure of interest.- The authors have not supplied a conflict of interest statement.

\section{E-PV0178}

\section{Neuropsychiatric symptoms in huntington's disease- 5 case series}

M. Vedean ${ }^{1 *}$, A. Oleleu ${ }^{1}$, I. Dragomir ${ }^{2}$, G. Scarisoreanu ${ }^{1}$, C. Marginean ${ }^{1}$

${ }^{1}$ Emergency County Hospital Cluj Napoca Romania, Psychiatry I Ward, Cluj Napoca, Romania; ${ }^{2}$ Private Practice, Psychiatry, Cluj Napoca, Romania

${ }^{*}$ Corresponding author.

Background and aims.- Huntington's disease is an autosomal dominant neurodegenerative disorder characterized by a triad of symptoms consisting of cognitive impairment, motor disturbances and psychiatric features. Studies have shown that psychiatric manifestations often precede the motor signs of the disease by sometimes even more than a decade. 
This study aims to report a series of 5 patients with genetic diagnosis of Huntington's disease and to describe preclinical and clinical neuropsychiatric manifestations.

Methods.- The authors reviewed medical records of 5 patients seen in the psychiatric departments, and have used PubMed for a systematic literature review on the topic.

Results.- The 5 patients studied consisted of 2 males and 3 females all with a family history of Huntington's two of the patients being relatives (father and daughter). Aside from one patient who presented with juvenile Huntington's disease and early onset of choreiform movements and cognitive impairment all other 4 patients presented with psychiatric pre-clinical manifestations consisting of: insomnia, anxiety, depression, restlessness, irritable mood and mild psychotic symptoms that much preceded the motor involvement of the disorder.

Conclusions.- Psychiatric symptoms are prevalent in Huntington's and are relatively independent of the motor and cognitive symptoms of the disease and their progression. In the majority of the cases psychiatric symptoms preceded the onset of the other symptoms by years. Gaining better understanding of the symptoms is essential as these have strong implications for disease management, prognosis and quality of life.

Disclosure of interest.- The authors have not supplied a conflict of interest statement.

\section{E-Poster Viewing - 7-9 April: Consultation Liaison Psychiatry and Psychosomatics}

\author{
E-PV0180 \\ Physical restraints in acute \\ confusional syndrome patients \\ A. Alonso Sanchez , H. De la Red, A. Álvarez, M. Gómez, M. De \\ Lorenzo, N. De Uribe, C. Capella, E.M. Rodriguez, I. De la Montaña, \\ J. Gonçalves, A. San Roman \\ Hospital Clínico Universitario de Valladolid, Psychiatry, Valladolid, \\ Spain
}

${ }^{*}$ Corresponding author.

Background and aims.- Physical restraints are still used in nonpsychiatric wards in some countries. We have tried to identify de possible causes with the aim of reducing these techniques.

Methods.- Case report and bibliographic review.

Results.- Liaison psychiatric service attention was required to evaluate a 75-year old patient in the general surgery ward. He had been operated for cholecystitis 24 hours before and at that moment he was suffering from restlessness, aggressivity towards the staff, anxiety and delusions. He was staying in a 3-patient bedroom, he had no family with him and he did not understand how to ask for help. He was physically restrained to avoid self-harm and harm to others. He was diagnosed with Acute Confusional Syndrome.

We prescribed pharmacological treatment with a low dose of haloperidol and a non-pharmacological intervention consisting of close supervision by the staff, single bed bedroom and adequate ambience light. With this new approach, the patient quickly improved and no other physical restraints were needed.

Conclusions.- Physical restraints are generally overused in some countries due to the lack of an adequate training for the acute confusional syndrome and lack of resources.

Health staff should always consider alternative approaches such us specialised psychopharmacological treatment and some nonpharmacological interventions with the aim to avoid the use of physical restraints in a general hospital.

Disclosure of interest.- The authors have not supplied a conflict of interest statement.

\section{E-PV0181 \\ Hereditary metabolic disorders causing psychiatric symptoms, a diagnostic challenge}

A. Antunes ${ }^{*}$, D. Telles Correia

Centro Hospitalar Lisboa Norte, Psychiatry, Lisboa, Portugal

"Corresponding author.

Background and aims.- A number of hereditary metabolic disorders (HMD) that affect the central nervous system can present in childhood or adulthood as a phenocopy of major psychiatric syndromes, leading to incorrect primary psychiatric diagnosis.

The main objective of this work is to provide a better understanding and awareness of secondary syndromes in metabolic disorders, which we considerer of great importance for the psychiatrists as it can lead to an early diagnosis of HMD, with great impact in the course and prognosis, preventing permanent neurologic damage and allowing for genetic counseling.

Methods.- A literature review was conducted through the PubMed database examining all the systematic reviews published in the last five years, which include all the clinical cases of HMD presenting with significant psychiatric symptoms that have been reported to the date.

Results.- Twenty HMD causing psychiatric symptoms were identified. We highlight the ones that are potentially treatable: Urea cycle disorders, Homocysteine remethylation defects and Cystathionine-synthase deficiency, presenting typically with confusion, behavioral changes and psychotic symptoms; NiemannPick disease type $C$ and Cerebrotendinous Xantomatosis and Wilson disease which main features are impulsivity and irritability. A simple algorithm for the screening of metabolic disorders in cases with atypical psychiatric presentations was also obtained.

Conclusions.- There is a group of metabolic disorders presenting with isolated neuropsychiatric symptoms, currently not identified by most diagnostic paradigms, that may be far more common than we are aware. It is importante to be able to recognize them in cases with acute onset and atypical psychiatric presentations or with poor response to standard treatments.

Disclosure of interest.- The authors have not supplied a conflict of interest statement.

\section{E-PV0182}

\section{Tramadol-induced delirium}

M.H.L. Arts ${ }^{1 *}$, S. Petrykiv ${ }^{2}$, L. de Jonge ${ }^{3}$

${ }^{1}$ Mental Health Care -Western North Brabant GGZ-WNB,

Department of Old Age Psychiatry and Neuropsychiatry, Halsteren,

The Netherlands; ${ }^{2}$ MUMC, Department of Clinical Pharmacy and Pharmacology, Maastricht, The Netherlands; ${ }^{3}$ Leonardo Scientific Research Institute, Department of Neuropsychiatry, Groningen, The Netherlands

${ }^{*}$ Corresponding author.

Background and aims.-

Background.- Tramadol is a widely used centrally acting synthetic opioid analgesic for the treatment of acute or chronic, moderate to severe pain. It has been associate with a wide range of side effects, including mood changes, confusion, and occasionally psychosis. Cases of tramadol-induced delirium have been reported rarely.

Aims.- To present a case report of tramadol-induced delirium, followed by a literature review.

Methods.- An English language literature search was conducted using Pubmed and EMBASE searching for case reports and observational studies reporting delirium due to treatment with tramadol. Results.- A 76-year-old female was admitted to hospital with acute lower back pain. For pain management she received two injections of tramadol $100 \mathrm{mg}$ intramuscular (I.M.), with an interval of 6 hours. After the second injection, the patient suddenly developed an acute confusion, auditory hallucinations, delusions, and violent 
behaviour. She was sedated with lorazepam $2 \mathrm{mg}$ I.M. and haloperidol $2 \mathrm{mg}$ I.M. There was no personal or family history of psychiatric disorder or mental illness. Blood laboratory testing excluded any abnormalities. Neurological examination revealed no significant results. After psychiatric examination she was diagnosed with tramadol induced delirium. Confusion, psychotic symptoms and violent behavior resolved after discontinuation of tramadol.

Conclusions.- Although tramadol is a widely used analgesic for the treatment of moderate to severe pain, it should only be used after careful consideration in older adults.

Disclosure of interest.- The authors have not supplied a conflict of interest statement.

\section{E-PV0183}

\section{Charles bonnet syndrome: a prodromal stage of lewy body dementia?}

M.H.L. Arts ${ }^{1 *}$, S. Petrykiv ${ }^{2}$, L. de Jonge ${ }^{3}$

${ }^{1}$ Mental Health Care -Western North Brabant GGZ-WNB, Department of Old Age Psychiatry and Neuropsychiatry, Halsteren, The Netherlands; ${ }^{2}$ MUMC, Department of Clinical Pharmacy and Pharmacology, Maastricht, The Netherlands; ${ }^{3}$ Leonardo Scientific Research Institute, Department of Neuropsychiatry, Groningen, The Netherlands

* Corresponding author.

Background and aims.-

Background.- Charles Bonnet Syndrome (CBS) is a disorder in older adults, and is characterized by a triad of recurrent vivid visual hallucinations, ocular pathology causing visual impairment due to lesions in central or peripheral visual pathways, and normal cognitive status. It is often misdiagnosed as a psychosis or a drug related condition. CBS was anecdotally reported as a prodromal stage of early dementia.

Aims.- To present a case report, describing CBS as a prodromal stage of LBD.

Methods.- A case report is presented and discussed, followed by a literature review.

Results.- An 68-year old female was admitted to hospital for persistent vivid visual hallucinations. There was no past personal or family history of mental illness. On neurological examination we observed discrete balance problems and rigidity, the patients MMSE score was 25/30. Ophthalmological examination showed visual impairment due to age related macular degeneration. Treatment with antipsychotics proved not to be effective and instead increased muscle rigidity. During the next months, we noticed progressive cognitive decline with other core features of LBS including fluctuating cognition with pronounced variations in attention and alertness, recurrent visual hallucinations, and spontaneous motor features of parkinsonism.

Conclusions.- CBS is a rare disorder that may represent a prodromal stage of LBD. However, there is some overlap in symptoms between these two disorders, making a correct diagnosis difficult, particularly in early stages of dementia. In this study we describe that CBS may be a marker of a prodromal stage of LBD.

Disclosure of interest.- The authors have not supplied a conflict of interest statement.

\section{E-PV0185}

\section{Team work to better health - consultative psychiatry}

I. Ljutica, Z. Barac Otasevic*, S. Kalac

Clinical Center of Montenegro, Psychiatric Clinic, Podgorica,

Montenegro

* Corresponding author.
Background and aims.- Liaison psychiatry or consultative psychiatry is the sub-specialty which provides psychiatric treatment to patients attending general hospitals, whether they attend out-patient clinics, emergency departments or are admitted to in-patient wards. Therefore it deals with the interface between physical and psychological health.

Consultative psychiatry is an important part of the treatment of patients in non-psychiatric departments.

The goal of this paper was to determine the departments where the most frequent psychiatric consultations were in order to improve the holistic approach by using consultative psychiatric examinations.

Psychiatric consultation is intended to improve the quality of patients care, and also to bring about an ethical imbalance in the treatment of psychiatric patients.

Methods.- Clinical study.

Results.- The survey included patients who were examined by the psychiatrist during year of 2016. There were 1420 consultations. In the period from 01.01. 2017 until 01.09.2017 there were 1023 consultations. During the first eight months of this year, actually, in the period from 01.01.2018-01.09.2018 there were 890 consultative reviews were carried out, at the other Clinics of the Clinical Center of Montenegro. Medical documentation was used to monitor the consultations, as well as the ICD 10 and WHO.

Conclusions.- There were an increase in the number of consultative examinations on some non-psychiatric departments. We can conclude that the holistic approach to the patient is not neglected, and that the colleagues in somatic departments pay more attention to the role of psychological factors in genesis and the resolution of somatic diseases.

Disclosure of interest.- The authors have not supplied a conflict of interest statement.

\section{E-PV0186}

\section{Transplantation and psychopharmacology - what should we do when our patients are transplanted?}

A.M. Bernardo ${ }^{1^{*}}$, I. Pinto ${ }^{2}$

${ }^{1}$ Hospital Garcia de Orta, Serviço de Psiquiatria e Saúde Mental, Almada, Portugal; ${ }^{2}$ Centro Hospitalar Psiquiátrico de Lisboa, Psiquiatria, Lisboa, Portugal

Corresponding author.

Background and aims.-

Introduction.- Patients with mental health disorders are a risk group of multiple organic diseases, associated to high prevalence of nonhealthy behaviors and late search for medical care. Mental health patients represent a population with increased risk of organ transplantation. In case of an organ transplant, we could expect that psychopharmacs need a medical review, due to hight probability of iatrogenic, pharmacokinetic and pharmakodinamic issues.

Objectives.- The purpose of this work is to review psychopharmacology in patients with mental health disorders, when submitted to organ transplantation.

Methods.- The authors revised the published literature about this topic, including the topic words "organ transplant", "antidepressant", "antipsychotics", "anxiolytics" and "mood stabilizer" in scientific date base. The relevant articles were selected and complemented with information from medical books, non available on-line.

Results.- There is a heterogeneous approach to those patients and most of the information available is due to clinical cases. Some of the challenging topics identified in clinical management relate to changes in the metabolism and elimination of drugs during organ insufficiency, drug interactions, and overlapping side effects between psychopharmacological and immunosuppressive drugs. One of the limitations found was the exclusion criteria of some 
organ transplant centers, which consider the presence of a mental health disorder a total or partial contra-indication for treatment. Conclusions.- Organ transplant is still a recent medical procedure in constant upgrade, with limited information about psychopharmacology in mental health patients.

Disclosure of interest.- The authors have not supplied a conflict of interest statement.

\section{E-PV0188}

\section{A manic episode with psychotic features following corticotherapy for dermatomyositis}

\section{Bonea ${ }^{1 *}$, I.M. Parau ${ }^{2}$, C.A. Crisan ${ }^{1}$}

${ }^{1}$ Iuliu Hatieganu University of Medicine and Pharmacy, Psychiatry, Cluj-Napoca, Romania; ${ }^{2}$ Cluj County Clinical Emergency Hospital, Psychiatry, Cluj-Napoca, Romania

${ }^{*}$ Corresponding author.

\section{Background and aims.-}

Introduction.- Dermatomyositis is an idiopathic inflammatory disorder of the connective tissue which affects the cutaneous and the striated muscle cells. Systemic inflammation was correlated with a high risk of psychosis and affective disorders, especially depression. Corticotherapy used for the treatment of rheumatologic disorders can induce psychiatric symptoms ranging from severe depression to euphoria and psychosis.

Objectives.- To present the case of a 49 years old man, with no previous psychiatric history, admitted to the Psychiatry Clinic for a manic episode after being diagnosed with dermatomyositis

Methods.- A case report is discussed.

Results.- The rheumatologic symptoms (proximal muscle weakness of the lower limbs, with difficulties in climbing stairs, dermatomyositis "glasses", Gottron's papules and "machinist's hand") first appeared a year before. From the heteroanamnesis, we learned that the first psychiatric symptoms appeared simultaneously with the inflammatory ones, with mild disinhibited behavior, slightly elevated mood and self-esteem, logorrhea episodes, without affecting the quality of life of the patient or of his family. Nevertheless, after methylprednisolone $16 \mathrm{mg} /$ day initiation, they reached the intensity of a severe manic episode with psychotic features. The psychological exam showed: severe mania (YMRS $=42$ ), $\mathrm{QI}=98$ (Raven test), cognitive dysfunction and schizotypal personality disorder (SCID-II). During the admission, the rheumatologist decided the reduction of the dose of methylprednisolone to a minimum of $8 \mathrm{mg}$ /day and augmentation with azathioprine $100 \mathrm{mg} /$ day, to favor the effect of the psychotropic therapy with quetiapine $800 \mathrm{mg} /$ day and valproate $1 \mathrm{~g} /$ day.

Conclusions.- Both corticotherapy and systemic inflammation can induce affective episodes, thus interdisciplinary communication is essential

Disclosure of interest.- The authors have not supplied a conflict of interest statement.

\section{E-PV0189}

\section{Mindfulness moderates severity of anxiety symptoms in psoriasis patients}

E. Parlapani ${ }^{1}$, E. Mitsiou ${ }^{2}$, D. Kirla ${ }^{1}$, A. Patsatsi ${ }^{2}$, G. Floros $^{3}$, I. Diakogiannis $^{1}$, V. Bozikas ${ }^{3^{*}}$

${ }^{1}$ Faculty of Medicine, Aristotle University of Thessaloniki, 1st Department of Psychiatry, Thessaloniki, Greece; ${ }^{2}$ Faculty of Medicine, Aristotle University of Thessaloniki, 2nd Department of Dermatology and Venereology, Thessaloniki, Greece; ${ }^{3}$ Faculty of Medicine, Aristotle University of Thessaloniki, 2nd Department of Psychiatry, Thessaloniki, Greece

* Corresponding author.
Background and aims.- Mindfulness is defined as "the state of being attentive to and aware of what is taking place in the present". Higher mindfulness levels have been associated with less distress in patients suffering from different dermatologic conditions. Psoriasis patients show a high prevalence of anxiety symptoms, associated with the severity of skin lesions. The present study focused on the association between mindfulness and severity of anxiety symptoms in psoriasis patients.

Methods.- Fifty-eight study participants were recruited from the Psoriasis Outpatient Clinic of the $2^{\text {nd }}$ Department of Dermatology and Venereology, Aristotle University of Thessaloniki, during a 14-month period. Sociodemographic data, psychiatric and medical history were recorded based on a semi-structured interview and patients' medical records. Psoriasis severity was evaluated by the Psoriasis Area and Severity Index, the Physician Global Assessment and the Body Surface Area. Psychometric assessment included the Beck Anxiety Inventory (BAI) and the Mindful Attention Awareness Scale (MAAS). Participants were divided into two subgroups based on the optimal BAI cut-off score.

Results.- Psoriasis patients with more severe anxiety symptoms displayed significantly lower mindfulness levels. In addition, anxiety symptoms were significantly correlated with mindfulness levels, a negative correlation. A stepwise regression analysis revealed that mindfulness contributed significantly to the calculation of BAI score, explaining $23.4 \%$ of the variance.

Conclusions.- There is a bidirectional relation between anxiety symptoms and severity of psoriasis. As a result, a psychodermatologic approach is more beneficial for psoriasis patients. Mindfulness may moderate severity of anxiety symptoms. Therefore, mindfulness-based interventions could improve psoriasis global outcome.

Disclosure of interest.- The authors have not supplied a conflict of interest statement.

\section{E-PV0191}

\section{Depression, anxiety and somatization}

M. Daoud ${ }^{1 *}$, M. Hamza ${ }^{2}$, S. Bourgou ${ }^{1}$, F. Charfi ${ }^{1}$, A. Belhadj ${ }^{1}$

${ }^{1}$ Mongi Slim Hospital, Department of Child and Adolescent

Psychiatry, Tunis, Tunisia; ${ }^{2}$ Mongi Slim Hospital, Department of

Child and Adolescent Psychiatry, Tuins, Tunisia

* Corresponding author.

Background and aims.- Somatization is the tendency to experience and communicate somatic symptoms that are unaccounted for by pathological findings but certain somatic symptoms can be part of anxiety and depression disorders.

Objectives.- to explore somatization in a sample of female adults in relation to anxiety and depression.

Methods.- Mothers attending with their child, the child and adolescent psychiatry department were recruited. Somatic complaints were assessed via the Patient Health Questionnaire 15 (PHQ 15). The Hospital Anxiety and Depression Scale (HADS) was used to evaluate anxiety and depression.

Results.- Seventy-three mothers were selected for the study. A psychiatric history was found in more than half of the cases with mainly post-traumatic stress disorder $(26 \%)$. The mean score on the HADS was 17,7 with an average of 9,3 on the anxiety subscale and 8,3 on the depression subscale. Mean score of the PHQ15 was 9,3 with $75,3 \%$ of the sample meeting criteria for moderate somatization disorder and for severe somatization disorder in $24,7 \%$ of cases. PHQ15 scores positively and significantly correlated with the total HADS score as well as the anxiety and depression subscale scores ( $p=0,000$ for all). When considering each PHQ15 item separately, scores on the "Fainting spells" weren't associated neither with depression nor anxiety scores and "Shortness of breath" was found to be associated only with depression subscale scores $(p=0,028)$. 
Conclusions.- Somatization is more likely to be found in depressed and/or anxious people and could have a screening value for these disorders. This however may be subject to the influence of cultural environment.

Disclosure of interest.- The authors have not supplied a conflict of interest statement.

\section{E-PV0192}

\section{Chest pain in childhood- when to stop testing and start listening?}

S. Chakrabarti, A. Datta*

Child and Adolescent Psychiatry, Paediatric Liaison Team, London, United Kingdom

* Corresponding author.

\section{Background and aims.-}

Introduction.- 12-year child, experiencing episodic (2-10) daily sharp central chest pain for 3 months presenting to the Emergency Department of a multispecialty hospital. Numerous investigations done revealed no physical cause. He was referred to the paediatric psychiatry department for exploration of his symptoms.

Aims.- We demonstrate the value of child psychiatry service with young people afflicted with similar problems. This includes understanding the physical health findings and incorporating them with pre-existing core beliefs about illness within the ecology of the child.

Methods.- This child had a thorough psychiatric assessment.

Results.- Physical health examination revealed morbid obesity.

Psychiatric assessment revealed a history of bullying in primary school; family history of nonspecific back pain in mother, multiple medical conditions in father who is wheelchair bound and also awaiting bariatric surgery. In the session the child experienced chest pain which started abruptly when talking about his school and abated with maternal comfort and after the assessing child psychiatrist palpated his apex beat.

Conclusions.- From the history, medical workup and psychiatric assessment, it was deemed that this chest pain was psychogenic in origin.

With ongoing therapy and school liaison, he experienced far fewer episodes with a reduction in the intensity of pain alongside losing $5 \mathrm{~kg}$ weight.

Research suggests a higher level of somatic symptoms in obesity; highlighting this might lead to a more interdisciplinary approach in assessment, reducing risk of over- investigation and validating the psychosocial component of illness for the families and young people.

Disclosure of interest.- The authors have not supplied a conflict of interest statement.

\section{E-PV0194}

\section{Frequent attenders in the general practice in Italy: a preliminary report on clinical variables related to low functioning}

V. Dell'oste ${ }^{1}$, C. Carmassi ${ }^{1}$, D. Ceresoli ${ }^{1}$, E. Diadema ${ }^{1}$, A. Cordone ${ }^{1}$, S. Moscardini ${ }^{2}$, E. Bianchi ${ }^{3}$, R. Landi ${ }^{4}$, G. Massimetti ${ }^{1}$, C. Nisita ${ }^{1}$, L. Dell'Osso ${ }^{1}$

${ }^{1}$ University of Pisa, Department of Clinical and Experimental Medicine, Pisa, Italy; ${ }^{2}$ General Medicine Outpatient Clinic, Asl 5 Tuscany, Pisa, Italy; ${ }^{3}$ General Medicine Outpatient Clinic, Asl 6 Tuscany, Livorno, Italy; ${ }^{4}$ General Medicine Outpatient Clinic, Asl 2 Tuscany, Lucca, Italy

* Corresponding author.

Background and aims.- Frequent Attenders (FA), defined as patients reporting a disproportionate number of visits to General Praction- ers (GP), may represent up to a third of GP patients responsible for a high burden of care not always justified by the severity of the medical condition.

The aim of this study was to explore socio-demographic and clinical characteristics of FA of GP in Italy with particular attention to functional impairment.

Methods.- 75 FA (defined as individuals who had consulted GP 15 times or more per year) of Italian primary care centers were enrolled and assessed by socio-demographic scale, SCID-5, global functioning (GAF), illness behavior and perceived health (IBI), somatic comorbidity (CIRS).

Results.- Most of the sample were females, middle aged, married or cohabiting, with low levels of education. One third of FA was low-functioning (GAF score <70), with no differences in the socio-demographic variables. About $70.3 \%$ reported a current SCID diagnosis, in particular major depressive disorder, somatic symptom disorders, and panic disorder, all being more frequent in low-functioning (LF) patients. Half were taking a psychopharmacological therapy, mostly benzodiazepines.

Conclusions.- The surprisingly high rate of psychopathology may allow us to classify FA as a special population affected by a mild-moderate severity of mental illness, and treated chronically with benzodiazepines. Low-functioning FA perceived themselves as more impaired regarding the health perception and social role. In the light of these findings, educational initiatives should be undertaken, aiming to increase doctors' and patients' confidence in psychological support, as well as to sustain cooperation and the referral system between general practitioners and psychiatrists. Disclosure of interest.- The authors have not supplied a conflict of interest statement.

\section{E-PV0195}

\section{Relation of creatinine level to depression in hemodialysis patients}

O. Farajli

Ministry of Health, Male Clinic, Baku, Azerbaijan

* Corresponding author.

Background and aims.- This study aims to investigate the relationship between increase in creatinine levels of patients receiving hemodialysis and emergence of depressive symptoms in these patients

Methods.- The DASS 21 is a 21 item self report questionnaire designed to measure the severity of a range of symptoms common to both Depression and Anxiety. In completing the DASS, the individual is required to indicate the presence of a symptom over the previous week.

Results.- As demonstrated by our findings, among 40 hemodialysis patients involved in the study, a relationship between levels of creatinine and severity of depressive symptoms exists. In other words, it was observed that the higher the level of creatinine, the higher the severity of depressive symptoms (table 1).

Table 1 The study on relationship between levels of creatinine and severity of depressive symptoms

\begin{tabular}{llllll}
\hline $\begin{array}{l}\text { Cretaine } \\
\text { level }\end{array}$ & Number & Normal & Mild Moderate & Severe & $\begin{array}{l}\text { Extremely } \\
\text { severe }\end{array}$ \\
\hline $400-600$ & 10 & 8 & 1 & & 1 \\
$600-800$ & 21 & & 13 & 6 & 2 \\
$800-1200$ & 9 & & 2 & 4 & 3 \\
\hline
\end{tabular}

Conclusions.- The reason for this research is to investigate depressive symptoms in hemodialysis patients. Other similar studies on this topic have identified that severity of depression among such patients is high. The main challenges for this study are low number of participating patients and lack of information on monthly cre- 
atinine levels. Although these limitations influence our findings, calculated figures lead to us conclude that the results are reliable. Disclosure of interest.- The authors have not supplied a conflict of interest statement.

\section{E-PV0196}

\section{Sexual functioning in a sample of obese and overweight Tunisian women}

R. Feki ${ }^{1}$, R. Damak ${ }^{2}$, T. Naceri ${ }^{2}$, S. Smaoui ${ }^{1}$, A. Feki ${ }^{2}{ }^{*}$, M. Cheour ${ }^{2}$, H. Jamoussi ${ }^{3}$

${ }^{1}$ Medical School, Psychiatry, Sfax, Tunisia; ${ }^{2}$ University Tunis El

Manar, Psychiatry, tunis, Tunisia; ${ }^{3}$ University Tunis El Manar,

Nutrition, Tunis, Tunisia

${ }^{*}$ Corresponding author.

Background and aims.- Background: Obesity is a major public health in Tunisia. Prevalences of obesity and overweight are respectively $12.3 \%$ and $21.8 \%$ ( $8.9 \%$ in men, $12.8 \%$ in women). Studies in women assessing female sexual function index (FSFI) and body weight are limited.

Aims: We aimed to assess sexual function in obese and overweight female women.

Methods.- We conducted a cross sectional study from Mars to Septembre 2018 among outpatients referring to the centre for the care of obesity in the national institute of nutrition. Fifty female participants with a body mass index $(\mathrm{BMI}) \geq 25$ were recruited. Medical history, obesity-related complications and medication use, as well as demographic, social information were obtained. We used The FSFI to assess sexual function.

Results.- The mean age was 38.5 years. Among participants $12 \%$ were illiterate, $46 \%$ had low socio-economic level, $94 \%$ were married, $64 \%$ had no chronic illness and $72 \%$ wasn't under any medication. Six women suffered from amenorrhea. We noted a smonking habit in $10 \%$ of participants. Eleven women were overweight and 39 were obese. The mean BMI was $37.02 \pm 8.75$. The mean FSFI score was $27.41 \pm 6.18$. There was no correlation between FSFI score and the BMI.

Conclusions.- Obesity and overweight seemed to be not a brake on sexuality in our context.

Disclosure of interest.- The authors have not supplied a conflict of interest statement.

\section{E-PV0198}

\section{Skin stress? - A psychocutaneous disease case report}

B. Guedes* , A. Silva, E. Monteiro, A.L. Costa

Tondela-Viseu Hospital Centre, Department of Psychiatry, Viseu, Portugal

${ }^{*}$ Corresponding author.

Background and aims.- Psychocutaneous diseases can be described as primary psychiatric diseases with cutaneous manifestations, but it is well known that a psychiatric illness may either be the cause or the consequence of a dermatologic disease. Underlying psychiatric conditions are estimated to occur in up to one third of dermatologic patients. On the other hand, dermatological adverse effects of psychiatric medications are known; despite this, the anti-inflammatory properties of antidepressants are increasingly recognized and can be used with benefits in dermatological conditions.

Our aim was to review the literature on the relationship between psychiatric dermatological pathologies, to discuss the potential mechanisms of stress induced dermatological disease. To present a brief clinical case of alopecia areata associated with an adjustment reaction.
Methods.- A research of the literature on the subject was conducted using Pubmed ${ }^{\circledR}$ online search engine taking into account the keywords: Psychodermatology, psychocutaneous disorders.

Results.- Case Report: Male, aged 34, referred to the psychiatry consultation due to alopecia areata refractory to all dermatological treatments. From the organic point of view, no infectious, inflammatory or degenerative causes were found. Psychiatric evaluation was characterized by anxious and depressive symptomatology, reactive to an affective rupture and mother's prolonged illness. An SSRI was started. Current investigations actually show that skin is a target of distress, as well as an active participant of stress response, and both pharmacological and psychological interventions can be used to help treating psychocutaneous disorders.

Conclusions.- Given the difficulty in characterizing skin lesions and the subjectivity of psychopathology, psychocutaneous diseases can be difficult to recognize and interpret.

Disclosure of interest.- The authors have not supplied a conflict of interest statement.

\section{E-PV0201 \\ Do alexithymia and anxiety increase the risk of the heart diseases?}

M. Janowska ${ }^{1 *}$, A. Czernikiewicz ${ }^{2}$, H. Karakuła-Juchnowicz ${ }^{1}$

${ }^{1}$ Medical University, I Department of Psychiatry-Psychotherapy and

Early Intervention, Lublin, Poland; ${ }^{2}$ Marie Curie Skłodowska

University, Department of Speech and Language Therapy and Applied Linguistics, Lublin, Poland

* Corresponding author.

Background and aims.-

Introduction.- According to the M. Bleuler's classification, coronary heart disease and hypertension are defined as the psychosomatic disorders. Apart from genetic and environmental factors which lead to development of the cardiovascular diseases, psychological causes (including anxiety and alexithymia) also play a crucial role. The aim of the research.- The aim of the research was to evaluate the influence of the experienced level of alexithymia and co-ocurrence of alexithymia and anxiety of the heart diseases.

Methods.- The research involved 140 adolescents, with heart diseases: hypertension and hypertension with stable or unstable angina. Questionaires TAS-20, STAI were used to collect data.

Results.- The average level of alexithymia in population was 55,06 while the group of patients with hypertension scored 54,88 ( $p$ value $=0,767$ ). Patiens with heart disease scored 56,24 and 54,26 (stable vs. unstable angina). During the assesment of occurence of alexithymia and the "anxiety as a state" and "anxiety as a trait" statistically significant positive linear correlation was found (for both $p$ value $<0,0001, r=0,484$ and $r=0,563$ ). In both analyses the strongest correlation was found among the patients with hypertension.

Conclusions.- No link between alexithymia level and development of heart disease was noted. Coincidence of alexithymia and anxiety was related to the severity of symptoms of cardiovascular diseases. Disclosure of interest.- The authors have not supplied a conflict of interest statement.

\section{E-PV0202}

\section{Aspirin abuse (salicylism) - case analysis}

K. Kaufman", A. Rehan, D. Goldrich, A. Sreedhar, A. Tobia, A. Trenton

Rutgers Robert Wood Johnson Medical School, Psychiatry, New Brunswick, USA

${ }^{*}$ Corresponding author.

Background and aims.- Over-the-counter (OTC) medication abuse is a prevalent problem which increases the risk of unintentional 
overdose, medical complications, emergency department (ED) presentation, and hospitalization. This case describes a patient with chronic aspirin abuse who presented to the ED with salicylate intoxication (salicylism) and endorsed elation secondary to deliberate aspirin consumption.

Aim.- Expanding clinicians' index of suspicion regarding potential OTC medication abuse, including aspirin, with psychoactive effects. Methods.- Case analysis.

Results.- 49-year-old male with no significant past medical history presented with ataxia/lethargy/confusion to the ED after ingesting unknown quantity of aspirin. Past psychiatric history included schizoaffective disorder, Tourette's syndrome, multiple suicide attempts (overdoses), and polysubstance dependence. All standard laboratories were within normal limits except for CO2 $19.2 \mathrm{mmol} / \mathrm{L}$, anion gap $13 \mathrm{mEq} / \mathrm{L}$, and blood salicylate level $43 \mathrm{mg} / \mathrm{dL}$. Lithium level was $0.84 \mathrm{mEq} / \mathrm{L}$; acetaminophen level and urine toxicology were negative. Psychiatric consultation noted delirium (Folstein =19/30) without depressive/manic/psychotic features. He acknowledged receiving OTC tablets from another patient at his day program "for a buzz." Within 48-hours, his symptoms, including delirium and elation, had resolved. Prior medical records noted a similar admission 5-months earlier for salicylism (blood salicylate level $59 \mathrm{mg} / \mathrm{dL}, \mathrm{CO} 218.3 \mathrm{mmol} / \mathrm{L}$, anion gap $18 \mathrm{mEq} / \mathrm{L}$ ) in which he admitted taking OTC medication because it made him "feel good."

Conclusions.- OTC medication abuse may occur in monitored settings, in this instance intentional salicylate intoxication. Standardized/comprehensive screening for OTC medication abuse should lead to early treatment, minimize analgesic abuse complications, reduce ED presentations and hospitalizations, and decrease morbidity and mortality. Clinician/patient education is required. Disclosure of interest.- The authors have not supplied a conflict of interest statement.

\section{E-PV0203}

\section{Consequences of psychosocial factors on the treatment efficiency of diabetes mellitus type 1 and type 2}

\section{O. Khaustova*, A. Kogut}

Bogomolets National Medical University, Medical Psychology-

Psychosomatic Medicine and Psychotherapy, Kiyv, Ukraine

Corresponding author.

Background and aims.- The most common problem in patients with diabetes mellitus (DM) type 1 (DM1) and type 2 (DM2) is lack of compliance. Therefore, glycemic control largely depends on treatment adherence.

The factors of improvement of DM patients' treatment efficiency was studied.

Methods.- Study population ( $n=60)$ consists of: comparable groups (CG) with DM1 $(n=16)$, DM2 $(n=33)$ and newly diagnosed DM. Study data consists of demographic data, including: Medication Compliance Scale (MCS), Holmes and Rahe Stress Scale (HRSS), Dysfunctional attitudes Scale (DAS), Toronto Alexithymia Scale (TAS-20), The Depression, Anxiety and Stress Scale (DASS-21) and Chaban Quality of Life Scale (CQLS). Glycemic control was assessed by glycosylated hemoglobin (HbA1c) results. The statistics analysis has been performed using descriptive statistics and Pearson's correlation with SPSS Statistics 22.0.

Results.- Statistically significant difference was found, according to MCS: in patients with high (HC), middle (MC) and low level of compliance (LC). HC had higher rates according to CQLS on the level $(p=0,004)$ and low rates of the DAS, TAS-20, DASS-21 and HRSS results $(p=0,0001)$. LC had a higher level of HbA1c $(M=13$ SD: 1 vs. $M C M=9.83$, SD:1.4 vs. HC M =9, SD:1.4). The average values according to MCS: DM1 $(M=19), D M 2(M=18)$ present MC; by HbA1c: DM1 $(M=10), D M 2(M=10)$ have identical indicators.
Therefore, no statistically significant difference was found between CG.

Conclusions.- The level of glycosylated hemoglobin is a sensitive marker of adherence to the treatment of patients with diabetes, regardless of its type.

Disclosure of interest.- The authors have not supplied a conflict of interest statement.

\section{E-PV0204 \\ Chaban's quality of life scale in \\ psychosomatic patients with anxiety-depressive disorders}

O. Khaustova*, O. Chaban

Bogomolets National Medical University, Medical Psychology,

Psychosomatic Medicine and Psychotherapy, Kiyv, Ukraine

* Corresponding author.

Background and aims.- The quality of life of psychosomatic patients is the most sensual marker of the cure effectiveness, which combines physical, mental and social components. The new Chaban's Quality of Life Scale (CQLS) been developed and validated in the healthy volunteers. We evaluated the quality of life of psychosomatic patients with anxiety-depressive disorders on a scale (CQLS). Methods.- 63 patients (mean age 46.7 years) with psychosomatic diseases, arterial hypertension, coronary heart disease) and anxiety-depressive disorders (F41.2) were twice assessed on the 10 -items scale CQLS (at the baseline and at the end of treatment 21 day), with an interval of $0-100$ points.

Results.- At the beginning of the study, quality of life was 57.3 points, which corresponded to a low level of quality of life. After treatment, the quality of life was 74.5 points (average level). The most sensitive items were physical condition, mood, everyday activity and general satisfaction with life. Items of social activity, financial well-being, living conditions remained unchanged. The Cronbach's $\alpha$ coefficient was 0.903 in CQLS. The correlation coefficient between tests and repeated tests confirmed the reliability of $\operatorname{CQLS}(r=0,918 ; p<0,001)$.

Conclusions.- Short-term treatment of psychosomatic patients with anxiety-depressive disorders improves their quality of life $(p<0.005)$, but changes relate only to individual items. The CQLS scale has proven acceptable reliability and validity, which allows us to recommend it for further use.

Disclosure of interest.- The authors have not supplied a conflict of interest statement.

\section{E-PV0206}

\section{Clinical profile of inpatients referred to a consultation-liaison psychiatry service in a general hospital in Chile \\ J. Libuy \\ Pontificia Universidad Católica, Psiquiatría, Santiago, Chile \\ "Corresponding author.}

Background and aims.- Specific models of cooperation in the form of consultation-liaison service between specialised psychiatry departments and other medical departments play a crucial role in providing care to patients. The aim of these work is described the characteristics of consultation-liaison psychiatry (CLP) services in a general hospital in Chile, and to determine the possible changes of the clinical practice.

Methods.- Data were collected retrospectively with standardized operating procedures on consecutive inpatient consultation requests to the Dra. Eloisa Díaz Hospital in La Florida CLP service in Chile in 4-year-period.

Subsequently, the relevance of these was reviewed and a model of inter-consultation management was proposed. 
Results.- The most frequent psychiatric diagnoses were suicide attempts, delirium and substance use disorder. The majority of patients were treated with psychopharmacologic drugs. There has been an increase in the number of CLP per year, mainly due to substance use disorders. In order to improve the cost-effectiveness of the service, was decided to propose inclusion criteria to determine the need for a psychiatric evaluation of the patient.

Inclusion criteria:

- Severe decompensated psychiatric disorder

- Psychotic episode

- Manic / hypomanic episode

- Catatonia

- Severe withdrawal syndrome

- High risk of heteroaggression

- Neuroleptic malignant syndrome or serotoninergic syndrome

- Intoxication by psychotropic drugs without altered mental state

A referral model is also proposed for those patients who do not meet these criteria.

Conclusions.- It is important to optimize human resources in the public health service. To improve the cost-effectiveness of liaison psychiatry, we have to make an adequate prioritization and, in this way, provide better care to patients who require who require psychiatric help more urgently.

Disclosure of interest.- The authors have not supplied a conflict of interest statement.

\section{E-PV0208}

\section{Prevalence of long qtc in acute inpatients treated with psychiatric drugs}

G. Simon ${ }^{1}$, A. Alomar ${ }^{1}$, I. González ${ }^{1}$, X. Elizondo ${ }^{1}$, M. Madre ${ }^{1,2}{ }^{*}$

${ }^{1}$ Hospital Benito Menni CASM, Psychiatry, Sant Boi de Llobregat,

Spain; ${ }^{2}$ FIDMAG Research Foundation, Psychiatry, Sant Boi de

Llobregat, Spain

${ }^{*}$ Corresponding author.

Background and aims.- QT interval prolongation in the electrocardiogram (EKG) is considered a cardiac risk factor. Long QT syndrome can be caused by most antipsychotics and some antidepressants.

The aim is to investigte the prevalence of long QT in inpatients admitted to the acute unit of Benito Menni Complejo Asistencia en Salut Mental (Sant Boi de Llobregat, Barcelona).

We hypothesized that acute inpatients are treated with higher doses of psychiatric drugs and risk of suffering cardiac side effects increases.

Methods.- In a cross-sectional study we investigate the prevalence of long QT in inpatients treated with psychiatric drugs during 7 months (February -August 2018).

Two trained medical doctors calculated the QTc using the Bazett formula $(\mathrm{QTC}=\mathrm{QT} /$ square root of RR). A QTc value $>450 \mathrm{~ms}$ was considered a potential risk of developing arrhythmias, with the risk being elevated when QTc is $>500 \mathrm{~ms}$.

We identified patients with a QTc $>440 \mathrm{~ms}$.

Results.- 325 inpatients were studied: 139 women and 186 men, mean age was 42 years (18-89, SD:14).

$19(5,8 \%)$ had a QTc $>440 \mathrm{~ms}$. No patients had a QTc superior of $500 \mathrm{~ms}$.

Patients with long QTc were treated with: Venlafaxine (N:9), Olanzapine (N:6), Quetiapine (N:3) and Clomipramine (N:1).

Preventive measures to avoid arrhythmias were: dose drug reduction, change of treatment, frequent EKGs assessments.

Conclusions.- The prevalence of long QTc in our sample was similar to the general population.

Baseline EKG and medical history can help doctor to identify patients with long QT and undertake preventive measures.

Disclosure of interest.- The authors have not supplied a conflict of interest statement.

\section{E-PV0209}

\section{Perceived burden and quality of life for family caregivers of patients with type 2 diabetes}

N. Messeddi ${ }^{*}$, A. Bejar ${ }^{1}$, F. Hadj Kacem ${ }^{2}$, F. Charfeddine ${ }^{1}$, L. Aribi ${ }^{1}$, M. Abid ${ }^{2}$, O. Amami ${ }^{1}$

${ }^{1}$ Hédi Chaker university Hospital, department of psychiatry B, sfax,

Tunisia; ${ }^{2}$ Hédi Chaker University Hospital, Department of

Endocrinology, Sfax, Tunisia

* Corresponding author.

Background and aims.- Diabetes has an impact on the patient's life, but how can it affect the quality of life of their caregivers?

Objectives: To evaluate the burden among family caregivers of patients with type 2 diabetes, and their quality of life (QOL) while highlighting the factors associated.

Methods.- a cross-sectional, analytical study of 30 caregivers of type 2 diabetic patients hospitalized in the endocrinology department during the month of August 2018.

Zarit scale: to assess the level of burden and SF36 questionnaire: to evaluate the QOL.

Results.- the average age of diabetic patients was 59.83 years old The average age of their caregivers was 44.83 years. Their sex ratio was $0.3(8 \mathrm{M} / 22 \mathrm{~W})$. These latter were unemployed in $46.7 \%$ and have a moderate socio-economic level in $60 \%$. We find a conflictual marital relationship in $27.3 \%$. They presented a chronic somatic diseases in $50 \%$ The average burden score was $37.30 \pm 16.39$. The daily load was moderate in $30 \%$ of cases. The burden was perceived as severe among $10 \%$ of caregivers. The mean QOL score was $65.21 \pm 21.62,40 \%$ of caregivers had an impaired QOL. The factors correlated with the burden: moderate socio-economic level $(p=0.041)$ and conflictual marital relationship $(p=0.039)$. The factors correlated with the impaired QOL: moderate burden $(p=0.019)$ and a chronic somatic diseases $(p=0.015)$.

Conclusions.- Our study shows that diabetes affects the quality of life of caregivers, especially if it is perceived as a burden. Better support is necessary of the latter.

Disclosure of interest.- The authors have not supplied a conflict of interest statement.

\section{E-PV0210}

\section{Conversion disorder after general anaesthetic - a critical review}

D. Mirfin, A. Shuman

Surrey and Borders Partnership NHS Foundation Trust, Liaison Psychiatry, Chertsey, United Kingdom

* Corresponding author.

Background and aims.- General anaesthetic is known to immediately precede Conversion Disorder. We describe a clinical case vignette to illustrate this and then reviewed the literature to evaluate the evidence with the aim of developing a better understanding of the underlying aetiology and frequency of these events.

Methods.- We conducted a literature search using electronic databases and reference list exploring the associations between conversion disorders or functional neurological disorders and general anaesthetics.

Results.- There was limited literature exploring this relationship despite general anaesthetic being known to be a common trigger to an episode of a functional neurological disorder. The publications that were present seemed to focus on specific medications or the use of therapeutic sedation as a method to resolve a functional episode. There were many case reports discussing this association however limited larger studies which explored the underlying aetiology and those who were more at risk of developing this. This is a rare outcome and those with a history of anxiety and/or depression appear to be at increased risk of developing these disorders. 
Conclusions.- There is currently limited literature which explores this association with most publications limited to case reports on individuals. This is a rare outcome of a general anaesthetic however we are able to suggest some risk factors which may increase the risk of developing a functional neurological disorder. There is a need for larger scale studies within this area to elucidate this association further and understand the aetiology and ability to predict these episodes.

Disclosure of interest.- The authors have not supplied a conflict of interest statement.

\section{E-PV0212}

\section{Central coherence in eating disorders}

V. Muñoz Martinez ${ }^{*}$, B. Mata-Saenz, T. Rodriguez-Cano, L. Beato-Fernández

Hospital General Universitario de Ciudad Real, Psychiatry, Ciudad Real, Spain

${ }^{*}$ Corresponding author.

Background and aims.- We can divide central coherence into a cognitive component (metaphor comprehension and general information memory) and another perceptive (visual global prosecution). Global processing has been affected in different disorders as eating disorders with inconsistency in these results according to studies.

The objective of this study is to analyse central coherence with the The Rey-Osterrieth complex figure test (ROCF) in an eating disorder-sample.

Methods.- A sample of patients was recruited in our ED unit. They completed several ED-questionnaires and were evaluated by a Psychiatrist specialist in Neuropsychology. Sociodemographical, clinical, psychopathological and neuropsychological variables were collected. Data were analysed using SPSS 19.0.

Results.- A total of 81 patients were analyzed. All of them were females with average age of 29.1 years (standard deviation, SD, 9.8). IMC was on average 21.8 (SD 6.9). The distribution of diagnoses was: $58 \%$ anorexia nervosa (AN), 39\% bulimia nervosa (BN), $10 \%$ binge eating disorder (BED) and $18 \%$ other eating disorders. To measure the accuracy of figure, the quantitative index of the copy of ROCF was 34.5 on average (SD 2.3) and of the recall trial was 19.2 (SD 6.1). The order of construction index was 2.2 (SD 0.6), style index 1.5 (SD 0.3) and coherence index 1.4 (SD 0.3).

Conclusions.- In comparison with Spanish samples, quantitative index of accuracy was above the 80th percentile for the copy and bellow the 40th one for the recall. No significant differences were found between different ED in order or style index.

Disclosure of interest.- The authors have not supplied a conflict of interest statement.

\section{E-PV0213}

\section{The role psychotherapy in somatic medicine}

B. Mykhaylov*, B. Fedak, V. Zabashta, O. Kudinova, T. Alieva

Kharkiv Medical Academy of Postgraduate Education, Psychotherapy, Kharkiv, Ukraine

* Corresponding author.

Background and aims.- Over the last years there is a worldwide steady growth of somatic diseases, in aetiopathogenesis of which a psychogenic factor plays a significant role. The same trend is representative to Ukraine. Provided that in the clinical pattern of somatic diseases are observed states posing a vital threat, such as Coronary Heart Disease (CHD), Myocardial infarction (MI), Transient Ischaemic Attacks (TIA), Celebral Stroke (CS) patients. The main goal of our research were observed the psychosomatic relationships in theese patients and established the psychotherapy oriented sup- port system. Thas the 480 patients, were investigated 165 CHD, 90 TIA, 105 CS, 110 MI.

Methods.- A formation of inadequate internal disease pattern, fear of oneselves future promote generation and progression of maladaptive psychological responses that are unfavourable for disease prognosis and decrease a level of patients' social function and life quality the all groups patients.

Results.- In most cases, an efficiency of treatment products depends on both adequacy of therapeutic methods choice and numerous psychological factors. A formation of motivation, greater patient's involvement in treatment process is the philosophy of our psychotherapy system, multimodal based. All patients were separated on randomized intervened and control groups. The more high efficacy were observed in intervened groups $(p<0,05)$.

Conclusions.- It also requires an introduction of new organizational forms of medical and psychological and psychotherapeutic activity's implementation on all levels of health care system. Provided of importance is a development of new programs for medical and psychological follow-up of such patients on different treatment stages.

Disclosure of interest.- The authors have not supplied a conflict of interest statement.

\section{E-PV0215}

\section{When the sensation of shortness of breath is more than a symptom of anxiety: pulmonary toxicity induced by maprotiline}

M. Oscoz Irurozqui ${ }^{*}$, O. Alcoverro-Fortuny ${ }^{2}$, Y. Galea-Colón ${ }^{3}$, A. Mola Ausiró $^{3}$, L. Biosca-López ${ }^{3}$, F. Viñas-Usán ${ }^{1}$

${ }^{1}$ Benito Menni CASM, Consultation-Liaison Psychiatry, Granollers, Spain; ${ }^{2}$ Benito Menni CASM, Acute Psychiatric Inpatient Hospitalization, Granollers, Spain; ${ }^{3}$ Hospital General de Granollers, Pneumology, Granollers, Spain

Corresponding author.

Background and aims.- Maprotiline is a tetracyclic noradrenergic antidepressant.

Drugs are a frequent cause of iatrogenesis and cause morbidity and mortality in up to $5 \%$ of hospitalized patients.

We report a case of lung disease possibly due to maprotiline.

Methods.- A 59-year-old woman on antidepressant treatment with maprotiline consults for dyspnea of 5 days of evolution; severe respiratory insufficiency is confirmed and a ground-glass pattern and bilateral alveolar condensations are found in the chest X-ray, that were also visible on a radiograph a month ago. After discarding pulmonary thromboembolism, heart failure and infectious process, and given the suspicion of diffuse interstitial lung disease (DILD) due to maprotiline, the drug, introduced 3 months earlier, is removed and systemic corticosteroids are initiated. Clinical and analytical screening is performed, and DILD associated with connective tissue disease and inhalation of organic and inorganic powders is discarded. Pulmonary functionalism reveals a parenchymal restrictive disorder and stress desaturation.

Results.- A week later the patient is discharged. After one month, the patient is asymptomatic, and functional improvement is observed.

Conclusions.- Clinical presentation, radiological alterations, resolution of the picture with the drug withdrawal and exclusion of other causes, support that maprotiline may be the cause of it; however, only a few similar cases are reported in the literature.

Exact mechanism of injury induced by maprotiline is unknown and withdrawal is usually enough for resolution.

Disclosure of interest.- The authors have not supplied a conflict of interest statement. 


\section{E-PV0216}

\section{Self-perception, depression and} anxiety in end-stage renal disease

\section{patients}

A. Papanastasiou ${ }^{1}$, D. Vlachakos ${ }^{3}$, P. Ferentinos ${ }^{2}$, K. Tournikioti $^{2}$, A. Douzenis ${ }^{2}$, I. Michopoulos ${ }^{2}$

${ }^{1}$ Attikon University Hospital, 2nd Department of Psychiatry, Athens, Greece; ${ }^{2}$ Medical School- National and Kapodistrian University of Athens- Attikon University Hospital, 2nd Department of Psychiatry, Athens, Greece; ${ }^{3}$ National and Kapodistrian University of AthensAttikon University Hospital, Nephrology-Renal Unit Department, Athens, Greece

${ }^{*}$ Corresponding author.

Background and aims.- Depression and Anxiety are important clinical issues for the quality of life and outcome of end-stage renal disease (ESRD) patients. Self-perception has been shown to be linked to life expectancy and adaptability in many chronic diseases. Objective.- To investigate the role of various aspects of selfperception with respect to depression and anxiety rates.

Methods.- Thirty-two ESRD patients undergoing hemodialysis and 33 controls were recruited for the study. The Hospital Anxiety and Depression Scale (HADS) and the Self-Perception Profile for Adults were used to assess anxiety, depression and self-perception respectively.

Results.- No differences were found between patients and controls in anxiety and depression. Regarding self-perception, patients scored worse than controls in athletic competence $(p=0,043)$, especially male patients and those living in rural areas. Anxiety and depression levels were negatively correlated with self-assessment in sociability $(p=0.016)$ and interpersonal relationships $(p=.020)$ only in patients. Female patients rated themselves lower than their male counterparts in terms of sociability $(p=0.026)$ and intelligence $(p=0.016)$. Age was negatively correlated with self-image for sense of humor $(p=0.022)$ and intelligence $(p=0.032)$, whereas years of education were positively correlated with sense of humor $(p=0.030)$ in the ESRD group.

Conclusions.- Self-image of athletic competence is compromised and self-perception of sociability and interpersonal relationships correlates with significant clinical aspects in ESRD. Patients should be encouraged to take regular exercise and be more supported to engage in social activities.

Disclosure of interest.- The authors have not supplied a conflict of interest statement.

\section{E-PV0218}

\section{Combined treatment of non-psychotic mental disorders in patients with hyperthyroidism}

O. Pityk ${ }^{1}$, I. Kuzhda ${ }^{2}$, N. Karbovskyi ${ }^{1}$, L. Tkachuk ${ }^{1}$

${ }^{1}$ Ivano-Frankivsk National Medical University, PsychiatryNarcology and Medical Psychology, Ivano-Frankivsk, Ukraine; ${ }^{2}$ Ivano-Frankivsk Regional Children Hospital, Ophtalmological Department, Ivano-Frankivsk, Ukraine

${ }^{*}$ Corresponding author.

Background and aims.- The challenges for psychiatry in the $21^{\text {st }}$ century includes the promotion of mental health and prevention mental disorders. It can be done due to integration between somatic medicine and psychiatry mere. Mental disorder is a serious medical and social problem, significantly affecting the quality of life of patients with somatic profile. The valid assessment of the health and the effectiveness of treatment is quality of life, defined by WHO as "individuals perception of their position in life, including physical, mental and social well-being, independence and quality of environment context of culture and value systems among whom they live, and according to their own objectives, expectations, stan- dards and concerns". The aim was to investigate the influence of combined treatment which included psychotropic and psychotherapeutic methods. 125 patients were examined. Non-psychotic mental disorders with different syndromologic structure were found in 95 patients (main group), among which anxious-asthenic, anxious-depressive and asthenic psychopathological patterns were dominant. Significant difference was found in quality of life in patients of main and comparison groups.

Methods.- Quality of life was assessed using a questionnaire developed Mezzich, Cohen, Ruiperez, Liu \& Yoon (1999).

Results.- Thus, mental disorders in hyperthyroidism require appropriate correction that must take into account both biological and socio -psychological basis of specified disease and psychopathological patterns which strongly affect the quality of life of such people.

Conclusions.- The average in the overall perception of life (sense of satisfaction and happiness in general) in the main group was 5.19 in the control group - 6.50 .

Disclosure of interest.- The authors have not supplied a conflict of interest statement.

\section{E-PV0219 \\ Depression as a major issue in chronic heart failure}

O. Pityk $^{1^{*}}$, A. Sikora ${ }^{1}$, S. Fedorov ${ }^{2}$, N. Karbovskyi ${ }^{1}$

${ }^{1}$ Ivano-Frankivsk National Medical University, Psychiatry- Narcology and Medical Psychology, Ivano-Frankivsk, Ukraine; ${ }^{2}$ Ivano-Frankivsk National Medical University, Department of Therapy and Family Medicine of Postgraduate Faculty, Ivano-Frankivsk, Ukraine * Corresponding author.

Background and aims.- Depression is a major issue in chronic heart failure (CHF) and its prevalence is about $20-40 \%$, which is $4-5 \%$ higher than in the normal population. The prevalence of depression increases with New York Heart Association (NYHA) functional class, with the biggest difference seen between NYHA classes II and III. The purpose of study was evaluation of correlations between depression and main hemodynamic and lipids' metabolism parameters in patients with chronic heart failure. 80 patients with $\mathrm{CHF}$ II-III NYHA classes caused by chronic coronary artery diseases (CAD) were observed. All patients were divided into two groups: 1-20 persons without signs of depression and 2-60 persons with depression.

Methods.- Diagnosis of CHF was confirmed based on ESC guidelines (2016). Depression was diagnosed by some questionnaires (Zung Self-Rating Depression Scale, Beck Depression Inventory, Hamilton's Depression Scale).

Results.- Using Hamilton's Depression Scale, it was established that mild depressive disorders are mostly observed in patients with CHF - 71.6\% of patients. However, the average expression of depression was observed in $23.4 \%$ of patients studied, severe one - in $3.3 \%$, and extreme hard depression - in $1.7 \%$ of cases. It was reported about the reciprocal medium strength relationship between the severity of depression and the level of blood pressure: both systolic (SBP): $r=-0.30$ and diastolic (DBP): $r=-0.35$.

Conclusions.- Therefore, patients with chronic heart failure and depression have more strong central hemodynamic and lipid metabolism disorders versus without depression.

Disclosure of interest.- The authors have not supplied a conflict of interest statement.

\section{E-PV0220}

Body dysmorphic disorder: a literature review and case study H. Raai ${ }^{*}$, P. Manocha ${ }^{2}$, A. Martin ${ }^{2}$, L. Gonzalez $^{3}$, A. Khadivi $^{4}$ 
${ }^{1}$ Montefiore Medical Center- Albert Einstein College of Medicine, Department of Psychiatry and Behavioral Sciences, New York, USA; ${ }^{2}$ BronxCare Health System, Department of Psychiatry and Behavioral Sciences, New York, USA; ${ }^{3}$ James J. Peters VA Medical Center, Psychiatry, New York, USA; ${ }^{4}$ BronxCare Health System- and Albert Einstein College of Medicine, Department of Psychiatry and Behavioral Sciences, New York, USA

${ }^{*}$ Corresponding author.

Background and aims.- The aim of this study is twofold. First; to critically review literature of body dysmorphic disorder (BDD). Second; to examine three cases of body dysmorphic disorder; a male patient with a perceived defect of his face, a female patient with a perceived defect in her right side of her body, and, a male patient who presented with a perceived defect of his chest as well as face. In addition, we will explore prevalence, clinical features, comorbidities, and, association with suicidal ideations and attempts. Methods.- Case study and literature search of the term "Body dysmorphic disorder" using PubMed, selected relevant articles published in last 5 years in peer-reviewed journals.

Results.- The review of the literature has shown that body dysmorphic disorder has a high level of comorbidity with other psychiatric disorders, such as anxiety disorder and depression. Patients with BDD commonly have varied presentations and have high rate of psychiatric admissions, in addition to high rate of suicidal ideations and attempts. Recent evidence suggested that selective serotonin inhibitors and cognitive-behavioral therapy are often effective treatment for BDD. All the three cases had comorbid depressive disorders, had suicidal ideations and had varied fixed belief with regard to their perceived physical defect.

Conclusions.- Individuals with BDD usually consult dermatologists and cosmetic surgeons rather than psychiatrists. They often have variable presentations, and frequently referred to psychosomatic medicine. Collaboration between different specialties would optimize treatment outcome. Psychiatrist need to be diligent in evaluating patients with BDD for other comorbid psychiatric conditions and conduct a thorough suicide risk assessment.

Disclosure of interest.- The authors have not supplied a conflict of interest statement.

\section{E-PV0221}

\section{Are psychogenic non epileptic seizures being erroneously treated as epilepsy?: a review about the implications of the problem}

V. Romero Pardo ${ }^{1 *}$, L. Mellado Cuerno ${ }^{1}$, N. Fernandez Gomez ${ }^{1}$, J.M. Pastor Haro ${ }^{1}$, I.I. Louzao Rojas ${ }^{1}$, R. Gallego Estebanez ${ }^{1}$, J. Curto Ramos $^{1}$, B. Rodriguez Vega ${ }^{1}$, M.J. Aguilar-Amat Prior ${ }^{2}$, P. Alonso Singer ${ }^{2}$

${ }^{1}$ Hospital La Paz, Psychiatry, Madrid, Spain; ${ }^{2}$ Hospital La Paz, Neurology, Madrid, Spain

* Corresponding author.

Background and aims.- Our objective is to review the evidence about the main therapeutic interventions in non-psychogenic non epileptic seizures (PNES) and examine the consequences of treating it erroneously as epilepsy.

Methods.- For this aim, we have reviewed the therapeutic choices - pharmacologic and notpharmacologic- in patients with PNES and the information about prevalence, cost and security of treating the patients as epileptics.

Results.- High doses of antiepileptic drugs (AED) are sometimes used among patients diagnosed withPNES. Those kind of errors in treatment assignation are associated with more economic cost, pharmacologic iatrogenia and worse prognosis. A well assigned treatment focused on psychoeducation -Cognitive Behaviour Teraphy (CBT), Interpersonal Theraphy (IPT)- has evi- denced effectiveness decreasing the number of crisis. Diagnosis acceptation predicts a better quality of life.

Conclusions.- Despite the availability of accurate methods to diagnose PNES, there are still many patients treated as epileptics. The acceptation and understanding about PNES diagnosis are essential for a correct treatment assignation that implicates different consequences. Therapies that focus on disease explanation have shown to be effective. It is necessary to learn more about the PNES diagnosiscommunicating process in order to improve management.

Disclosure of interest.- The authors have not supplied a conflict of interest statement.

\section{E-PV0222}

\section{HCV among patients with chronic mental disorders: the relevance of dual-diagnosis}

C. Roncero ${ }^{1,2,3^{*}}$, Á. Martín-Sánchez ${ }^{1}$, A.I. Alvarez ${ }^{1}$, M.P. Andrés ${ }^{1}, \mathrm{~B}$. Vicente $^{1}$, C. Gamonal-Limcaoco ${ }^{1}$, R. de la Mata ${ }^{1}$, M.L. Aguilar ${ }^{1,2,3}$, F. Sanchez-Casado ${ }^{1}$, J.L. Villegas ${ }^{1}$, I. Valriberas ${ }^{1}$, R. Alarcon ${ }^{1}$, L. García-Ullán $1,2,3$

${ }^{1}$ University of Salamanca Health Care Complex Salamanca, Psychiatry, Salamanca, Spain; ${ }^{2}$ University of Salamanca, Psychiatry, Salamanca, Spain; ${ }^{3}$ University of Salamanca, Institute of

Biomedicine of Salamanca, Salamanca, Spain

${ }^{*}$ Corresponding author.

Background and aims.- Data on HCV prevalence among inpatients with chronic mental disorders are scare. Even Dual Disorders patients have not been well studied.

Methods.- 311 patients were admitted to a mid-long term psychiatric inpatient unit from 2007 to 2018 (45.66\% man, 43.32 mean age). Patients were referred from short psychiatric unit because there has been hospitalized more than one o because they need a mid term and intensive treatment. The main diagnosis were $52.73 \%$ Schizophrenic or psychotic disorders, $13.83 \%$ bipolar, $18.65 \%$ depressive, OCD $3.22 \%$ and $11.57 \%$ others disorders.

Results.- $36.33 \%$ were dual diagnosis patients suffering an addition (excluding tobacco) and other mental disorders. 63.72\% have alcohol dependence, $25.66 \%$ cocaine dependence, $37.17 \%$ cannabis dependence, $7.96 \%$ heroine dependence and $21.23 \%$ other drugs such as caffeine and benzodiazepines. The prevalence of HCV C was 3.22\%: $7.08 \%$ in the DD -group and $0.64 \%$ in the non-DD group.

Discussion.- The prevalence is higher than the general population. Especially the prevalence among DD patients is higher than the non-DD patients.

Conclusions.- Although we study a sample mainly of nonintravenous drug users the prevalence of CHV is higher that the prevalence on general population, especially among the dual diagnosis patients. Patients suffering mental disorders different than drug-dependence should be the next step in HCV the elimination process.

Disclosure of interest.- The authors have not supplied a conflict of interest statement.

\section{E-PV0223}

Reversing opioid-induced constipation in methadone users with oral naloxone: clinically meaningful placebo effect?

A. Samokhvalov ${ }^{1 *}$, J. Rehm²

${ }^{1}$ University of Toronto, Department of Psychiatry, Toronto, Canada; ${ }^{2}$ Centre for Addiction and Mental Health, Institute for Mental Health Policy Research, Toronto, Canada

* Corresponding author. 
Background and aims.- Opioid-induced constipation (OIC) is a severe and persistent side effect of methadone maintenance therapy. The effectiveness of conventional laxatives is questionable, whereas the use of oral naloxone might be a promising and physiologically justified solution.

Aim.- To test the effectiveness of oral naloxone for treatment of OIC.

Methods.- Triple-blind randomized placebo-controlled clinical trial $(n=20)$. Crossover design. Mixed effects.

Results.- Twenty subjects were enrolled, 10 women and 10 men, $46.0 \pm 11.6$ years old, MMT duration of $6.6 \pm 6.7$, Bowel function index (BFI) of $74.4 \pm 20.8$ and number of bowel movements (BM) of $2.2 \pm 1.2$ at baseline. Subjects were randomized into two groups: one received naloxone treatment for 1 week followed by 1 week of placebo and another received treatments in reverse order. There were no statistically significant differences between the two groups at baseline. As a result of the treatment the BFI has decreased in both groups and both cohorts of patients reported increased number of bowel movements $(5.3 \pm 2.9$ and $5.0 \pm 3.0$ after weeks 1 and 2 , respectively, see Fig. 1). There was no significant association between BFI and treatment sequence.

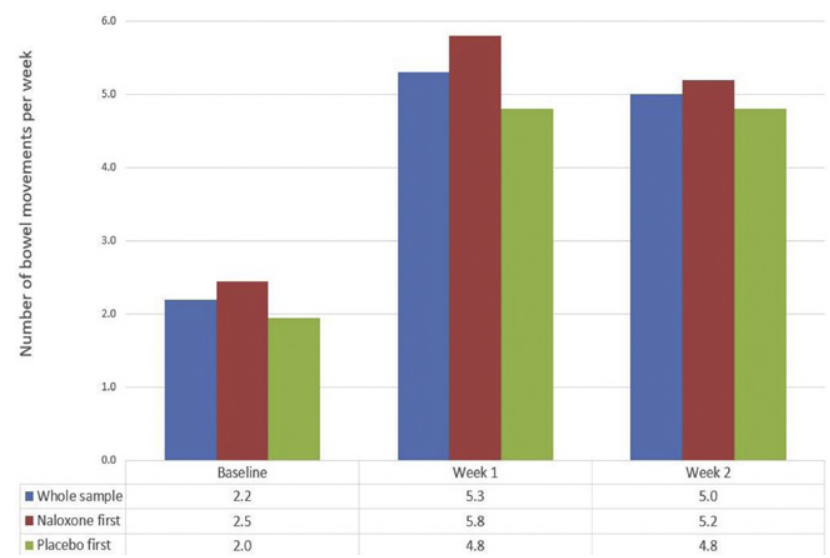

Figure 1. Changes in the number of bowel movements per week.

Conclusions.- The use of oral naloxone for OIC had no effect on BFI, but the objective improvement in bowel functioning was observed in both groups, which might be attributed to the placebo effect. Disclosure of interest.- The authors have not supplied a conflict of interest statement.

\section{E-PV0224}

\section{Differences in psychometric profile between malingerers and factitious disorders}

A. Serrano García $1^{*}$, I. González Rodríguez ${ }^{2}$, P. García Vázquez ${ }^{2}$, S. Núñez Sevillano ${ }^{2}$, M.L. Suárez Huerta ${ }^{3}$, J. Valdés Valdazo², M.Á. Heredero Sanz ${ }^{2}$

${ }^{1}$ Complejo Asistencial Universitario de León. Universidad de León, Psychiatry, León, Spain; ${ }^{2}$ Complejo Asistencial Universitario de León, Psychiatry, León, Spain; ${ }^{3}$ Hospital Universitario de Cabueñes, Orthopedics, Gijón, Spain

* Corresponding author.

Background and aims.- The main difference between Malingering (M) and Factitious Disorders (FD) is that the first one is not considered a mental disorder but a fraud behaviour. FD are a psychopathological disorder in which the patient freight his symptoms in order to be treated as an ill person, not in order to achieve an economic or social benefit.
Objectives.- Explore the differences in the psychometric profile between patients with FD and $\mathrm{M}$.

Methods.- After a multidisciplinary approach patients were classified as M or FD. All of them completed a standard psychometric protocol which included the following test: Structured Inventory of Malingered Symptoms (SIMS), Sensitization Central Inventory (SCI), Familiar Functioning Inventory (FF-SIL), Hospital Anxiety and Depression Scale (HADS) and Sheehan Disability Inventory (SDI). Also sociodemographic data were collected. Statistical analysis was performed in order to find psychometric differences between the groups.

Results.- The sample after the evaluation of 287 patients was of $39 \mathrm{M}$ and $45 \mathrm{~F}$. Differences were found in sex $(p=0.019)$, work status $(p<0.001)$ and work coalification $(p=0.047)$. No differences were found in academic status $(p=0.347)$ neither in marital status $(p=0.173)$. Psychometry showed differences in Total SIMS $(p=0.008)$, Total SCI $(\mathrm{P}=0.005)$, Total FF-SIL $(p=0.001)$. No differences were found in Total HADS $(p=0.842)$.

Conclusions.-FD show a different psychometric profile than M. They score significantly higher for simulation test, for somatization test and show a worse familiar functioning than $\mathrm{M}$.

Disclosure of interest.- The authors have not supplied a conflict of interest statement.

\section{E-PV0227 \\ Levetiracetam-induced psychosis, a review of literature}

A. Stoppa ${ }^{1 *}, \mathrm{G}$. Anmella ${ }^{2}$

${ }^{1}$ Hospital de Terrassa- Consorci Sanitari de Terrassa, Department of

Psychiatry, Terrassa, Spain; ${ }^{2}$ Hospital Clinic i Provincial, Department of Psychiatry and Psychology-Institut Clínic de Neurociències, Barcelona, Spain

* Corresponding author.

Background and aims.- Levetiracetam (LEV) is an antiepileptic drug approved for less than 20 years but with increasing prescription due to good tolerability. There are very few case reports of psychosis with LEV. Our purpose is to identify all case reports about psychosis related to LEV.

Methods.- From a bibliographical research using MEDLINE (Pubmed) and key words "Levetiracetam" and "psychosis", we identified all case reports of LEV-induced psychosis.

Results.- 56 articles were identified, with a total of 10 reported probable cases of psychosis induced by LEV, in which total remission of psychosis was reached when LEV was discontinued. LEV psychiatric most common side effects are change in mood as depression (7,3\%), irritability (12,5\%) and anxiety (2,5\%).

Conclusions.- LEV psychosis is very rarely reported $(<1 \%)$. Nevertheless, it is not to be underestimated due to increasing LEV presence. Since Epilepsy is itself related to psychosis, it is quite difficult to distinguish whether psychosis after seizures is due to drug side effects or consequence of epilepsy itself. Several factors for differential diagnosis can be considered, such as time between epileptic crisis and psychosis and type of antiepileptic drug. Antiepileptic dosing and psychosis characteristics have not been identified to play a role in LEV induced diagnosis. Visual hallucinations, however, may be considered as key symptom in LEV psychosis (present in 3/10 case reports). No studies have shown individual characteristics that make individuals prone to LEV adverse effects. More studies are needed in this line.

Disclosure of interest.- The authors have not supplied a conflict of interest statement. 
E-PV0228

\section{Psychiatric presentation of a patient with a falco-tentorial meningioma}

C. Tan, M.J. Yong

Khoo Teck Puat Hospital, Psychological Medicine, Singapore, Singapore

${ }^{*}$ Corresponding author.

Background and aims.- Organic causes such as brain tumours can sometimes present as psychiatric symptoms. This study describes a case of falcotentorial meningioma presenting with psychiatric symptoms and discusses the challenges of identifying psychiatric symptoms secondary to an intracranial mass and its subsequent management issues.

Methods.- Information was obtained from the patient's medical records and direct interviews.

Results.- Mdm.L, a 71-year-old lady first presented with memory loss and low mood. MRI brain revealed a right-sided mass suggestive of a falcotentorial meningioma, for which she opted for conservative management. Over the next four months, she developed hostility and aggression toward her family for which she was commenced on valproate. She reported improvements in her mood prior to defaulting her appointments. She presented again after six months following an attempt to jump from her apartment window. On arrival she appeared manic with overfamiliarity and flight of ideas. She thereafter became agitated requiring restraints. A short course of dexamethasone, together with haloperidol 0.5 to $1 \mathrm{mg}$ thrice daily, valproate $500 \mathrm{mg} \mathrm{ON}$ and clonazepam $0.25 \mathrm{mg}$ ON as required was effective in reducing her agitation so that an MRI brain could be repeated. This showed interval enlargement of the tumour. Conclusions.- Her previously expressed wishes for conservative management were honoured. Optimization of care involved management of her medical comorbidities, regulating psychotropics to avoid excessive sedation and enhancing her environment to reduce confusion- such as positioning her utensils on the right of her tray after she was found to have developed a left homonymous hemianopia. She was eventually discharged to a nursing home.

Disclosure of interest.- The authors have not supplied a conflict of interest statement.

\section{E-PV0229}

\section{Adjustment disorder - regarding the retrospective analysis of requests for psychiatric collaboration at the inpatient services}

D. Teixeira ${ }^{1}$, E. Palha ${ }^{2}$, D. Faria ${ }^{3}$, R. Quelhas ${ }^{4}$, F. Ferreira ${ }^{5}$

${ }^{1}$ Centro Hospitalar Tondela-Viseu, Departamento Psiquiatria e Saúde Mental, São Pedro do Sul, Portugal; ${ }^{2}$ Unidade Local de Saúde do Alto Minho, Departamento Psiquiatria, Viana do Castelo, Portugal; ${ }^{3}$

Unidade Local de Saúde de Matosinhos, Departamento Saúde Mental, Matosinhos, Portugal; ${ }^{4}$ Unidade Local Saúde de Matosinhos, Departamento Saúde Mental, Matosinhos, Portugal; ${ }^{5}$ Unidade local saude matosinhos, Departamento Saúde Mental, Matosinhos, Portugal

* Corresponding author.

Background and aims.- Adjustment Disorder (AD) is considered a situational disorder that occurs after a stressfull event and usually resolves within a time period. Classified midway between the normal and the pathologic, it is a subtreshold diagnostic and psychiatric drugs are generally not recommended as the first treatment. Although is one of the most diagnosed conditions by Psychiatry Liaison at inpatient services (around 13\%), AD remains poorly studied. To review the scientific literature regarding $A D$, to analise our data considering the first trimester of 2018 about psychiatric collaborations at inpatient services, and to build guidelines for the evaluation and treatment of depressive-anxiety symptoms for all inpatient services at Hospital Pedro Hispano (HPH).

Methods.- Using the terms "Adjustment Disorder" and "Adjustment Disorder in Psychiatry Liaison" we searched Pubmed ${ }^{\circledR}$, then analised and discussed the selected articles; on a second fase we analised and compared our data with the scientific literature; finally, we built the guidelines and established them at HPH.

Results.- 13 out of 52 inpatients (25\%) were diagnosed with AD, from which $79 \%$ were secondary to a medical-cirurgical condition. Only $31 \%$ have had a previous contact with a Psychiatric setting, and $85 \%$ were medicated or had their pharmacological prescriptions changed.

Conclusions.- AD was the number one condition diagnosed at our study. While the criteria for AD are still controverse, and thus difficulting its use amongst other medical specialities, its clinical importance is undeniable. More studies are needed to understand $A D$ real prevalence and undiagnosed morbidity. The guidelines we built based on this study are now being applied.

Disclosure of interest.- The authors have not supplied a conflict of interest statement.

\section{E-PV0230 \\ Adjustment disorder - a scientific review}

D. Teixeira ${ }^{*}$, E. Palha ${ }^{2}$, D. Faria ${ }^{3}$, R. Quelhas ${ }^{3}$, F. Ferreira ${ }^{3}$

${ }^{1}$ Centro Hospitalar Tondela-Viseu, Departamento Psiquiatria e Saúde Mental, Viseu, Portugal; ${ }^{2}$ Unidade Local Saúde Alto Minho,

Departamento Psiquiatria e Saúde Mental, Viana do Castelo, Portugal; ${ }^{3}$ Unidade Local Saúde Matosinhos, Departamento Saúde Mental, Matosinhos, Portugal

Corresponding author.

Background and aims.- Adjustment Disorder (AD) is considered a situational disorder that occurs after a stressfull event and usually resolves within a time period. Classified midway between the normal and the pathologic, it is a subtreshold diagnostic. Although is one of the most diagnosed conditions at Psychiatry Liaison consultations (around 12\%), AD remains poorly studied partially because the criteria have changed dramatically over the years, in part due to a lack of pathognomonic symptoms.

Our aim was to review the scientific literature regarding AD on a non systematic way.

Methods.- Using the terms "Adjustment Disorder" and "Adjustment Disorder in Psychiatry Liaison" we searched Pubmed ${ }^{\circledR}$ and analised the selected articles.

Results.- Suicidality occurs earlier in AD than in Major Depression and early intervention may prevent an evolution to another mental major disease. The diagnosis cannot simply rely on a group of symptoms, but depends on the clinical judgement, the cultural context, the person's vulnerability and coping mechanisms, and on the presumption of which course the disease will take. While the criteria for $\mathrm{AD}$ remains controverse and thus difficulting its use amongst other medical specialities, its clinical importance is undeniable. Adjustment Disorder New Module is a recent screening test and might help uniformize the criteria in the future.

Conclusions.- Although some argue that $\mathrm{AD}$ is a way of making normal experiencies pathologic, in our opinion $\mathrm{AD}$ is a mental disease that needs to be diagnosed and treated just like the others. More studies are needed to understand AD real prevalence and undiagnosed morbidity.

Disclosure of interest.- The authors have not supplied a conflict of interest statement. 
E-PV0231

\section{Assessment of temperament and personality traits of male patients diagnosed with FMS \\ C. Tuz \\ Erenköy FTR Hospital, Psychiatry, Istanbul, Turkey \\ ${ }^{*}$ Corresponding author.}

\section{Background and aims.-}

Objective.- Physical and psychological symptoms of FMS are important in the clinical course of the disease. In this study, temperament and personality traits of male patients diagnosed with FMS were investigated.

Methods.- 62 male patients who were examined at Erenköy FTR Hospital, FTR polyclinics in 2017 and diagnosed with FMS using Modified 2010 ACR FMS Diagnostic Scale and 65 healthy male control groups were included in this study. Subjects were evaluated with SCID-I, SCID-II, TPS, Beck Depression Scale, Fibromyalgia Effect Questionnaire and HAM-D scales. Socio-demographic characteristics were detected. Whether there is difference between the groups was analyzed by t test on parametric conditions and Mann-Whitney $\mathrm{U}$ test on nonparametric conditions.

Results.- FMS group and the control group were found to have significant differences in avoidance of harm, shyness and anxious approach to foreigners, loss of self, and spiritual acceptance scores in terms of subscales of the TPS $(p<0.005)$. According to this, male patients diagnosed with FMS were more pessimistic, self-confident, diffident, introverted, weaker and get tired more quickly compared to the control group.

Conclusions.-

Discussion.- As a result of the study, there were differences in the parameters of the TPS in FMS diagnosed male patients compared to the control group. In the study, all values are given in the tables. Comments will be made using the tables.

Disclosure of interest.- The authors have not supplied a conflict of interest statement.

\section{E-PV0232}

\section{Short psychiatric interventions in primary care - a model of consultation-liaison psychiatry}

K. Tzartzas*, T.S. Goncalves Alvane, C. Derbel, R. Marion-Veyron University of Lausanne, Department of Ambulatory Care and Community Medicine, Lausanne, Switzerland

${ }^{*}$ Corresponding author.

Background and aims.- Mental and substance use disorders are the leading cause of non-fatal burden of disease. Different ways of working at the interface between primary care and psychiatrists are described, but psychiatry is yet to consider how to develop this way of working. Consultation-liaison (CL) psychiatry could deliver high-quality integrated mental health care, when correctly adapted to specific local primary care settings. The aim of this study was to demonstrate the feasibility and the effectiveness of CL for patients consulting a primary care physician (PCP).

Methods.- An observational study was conducted in the Department of Ambulatory Care and Community Medicine at the University of Lausanne (Switzerland). The CL psychiatrist provided consulting, tripartite sessions and brief psychiatric/psychotherapeutic interventions.

Results.- The first 100 inquires were collected and analyzed. In 36 cases, consulting (without meeting the patient) was enough to manage the situation; 64 patients were seen by the psychiatrist (at least one session). In 58/64 (91\%) interventions a first joint consultation was conducted (CL-PCP-patient) and in 51 (80\%) a brief psychiatric and psychotherapeutic intervention was carried out. Only in one case a psychiatric hospitalization was necessary. PCPs unanimously felt that the presence of psychiatrists into their working environment and their direct participation in their consultations improved collaboration, helped decision-making and treatment planning, especially in complex cases.

Conclusions.- There is an opportunity for CL psychiatrists to develop primary care interfaces. Short CL intervention could be a model, which can reinforce the collaboration with PCPs and promote early detection and efficient treatment of mental disorders in primary care.

Disclosure of interest.- The authors have not supplied a conflict of interest statement.

\section{E-Poster Viewing - 7-9 April: Cultural Psychiatry}

\section{E-PV0234 \\ Parents' representations and expectations from the child in different historical conditions and their influence on the emotional and personal development of the children}

N. Burlakova*, P. Davidovich

Lomonosov Moscow State University- Faculty of Psychology, Department of Neuro- and Pathopsychology, Moscow, Russia * Corresponding author.

Background and aims.- The study explores a subjective image attributed by parents to their child that was shaped under the influence of different historical conditions: in the period of the crisis of 1992-2000 and the stabilization period of 2012-2015 in Russia. Methods.- The study focuses on two groups of families (group 1, $n=24$, children born in 1992-1993, group $2, n=31$, children born in 2006-2009) at the time of the study they were 5,5-7 years old, attended additional developmental activities.

The research involved qualitative analysis of objective historical and socio-demographic data, results of interviews with parents, long-term observations in children institutions.

Results.- The following areas of risk in intergenerational transmission are identified:

1) discrepancy of ambitions and claims among parents in the 1st group with the real level of development of the child; conflicting parental aspirations in the 2nd group;

2) various risky accents in the same qualities encouraged by parents of different generations:

a. the encouragement of "independence" with a focus on the need to rely only on themselves in the 1 st group, and individualistic nonconformism in the 2 nd group;

b. emphasis on "thrusting, leadership qualities" in the view of parents, in the 1st group understood as activity, defensive aggression, in the 2nd group, as an explanation of difficulties of targeting at the other.

Conclusions.- Representations and expectations posed by a specific family, cohort and more broadly - an entire generation that determine the emotional-personal risks and adaptive potential of the child are shown.

Disclosure of interest.- The authors have not supplied a conflict of interest statement.

\section{E-PV0235}

\section{"Types of delusions in psychiatric population comparing with general population"}

E.M. Ciorabai

Universitatea Ovidius Constanta, Psychiatric Clinic, Constanta, Romania

* Corresponding author. 
Background and aims.- Abstract: Abnormal paranoid ideation is a fixed, false belief, based on incorrect inferences about social reality, external to, or about oneself, starting with overvalued ideas contrasting with delusional ideas. The occurrence of these types of paranoid ideas were assessed in patients hospitalized in Psychiatric Clinic and two control samples of students in first and sixth year in Medical School, and a sample of patients with neurological conditions.

Cultural formulation, cultural dynamics and treatment outcome was assessed, False belief is viewed as a scientific puzzle to be solved comparing with the view as a chronic illness.

Methods.- We carried out serial interviews with patients hospitalized in Psychiatric clinic in emergency room comparing with interviews with students in medical school and with neurological patients. We used items from "Green at al Paranoid Thought Scale" Results.- The most common ideas include delusion of control, value judgement, when the value judgment made is so extreme that it is not credible. Delusional misidentification syndromes associated with doubt, need to verify, to control occur commonly in the context of neurological conditions affecting the right hemisphere. the presence of these types of delusions should determine a neurological evaluation for potentially aethiologically treatable conditions.

Conclusions.- A continuum from dominant ideas, overvalued ideas, delusional ideas affect the clinical consequences of trust, particularly how and when the diagnosis is delvered. whether pathological belief is viewed as a scientific puzzle to be solved or is seen as a chronic illness significantly influences the organizational processes of clinical assessment.

Disclosure of interest.- The authors have not supplied a conflict of interest statement.

\section{E-PV0236 \\ Management of obsessive compulsive disorder in three muslim refugee patients in the German context: a case series \\ N. Elnahrawy ${ }^{1 *}$, M. Elsheikh ${ }^{2}$ \\ ${ }^{1}$ Mainz University Hospital, Department of Psychiatry and Psychotherapy, Mainz, Germany; ${ }^{2}$ Al-Azhar University Hospital, Department of Psychiatry and Psychotherapy, Cairo, Egypt ${ }^{*}$ Corresponding author.}

Background and aims.- Obsessive Compulsive Disorder (OCD) is the fourth most common mental disorder. A delay in help-seeking behaviour as well as the associated feelings of shame and guilt make OCD difficult to diagnose. In the arab muslim culture, contamination and religious obsessions have been found to be the commonest kind of obsessive thoughts. The availability of culturally sensitive mental health services for refugee populations in Germany is limited. Here we aim at (1) Explaining obstacles to diagnosing and treating OCD among arab muslim patients in the German context and (2) Suggesting a set of culturally relevant clinical questions that lead to correct diagnosis of OCD in these patients.

Methods.- A case series of three asylum seekers: two females (25 Arab, 37 Kurdish) and one male (27 Arab) from middle-eastern background is presented. The difficulties to correctly diagnose OCD in the primary health care system is explained. The course of treatment is highlighted and a set of clinical questions that enable the diagnosis of OCD in Muslim patients is presented. Methods to overcome incompliance and treatment resistance are discussed.

Results.- In the presented case series, use of the proposed culturally relevant set of clinical questions enabled the correct diagnosis of OCD in muslim patients within a German context.

Conclusions.- OCD in refugee muslim patients is difficult to diagnose for many reasons. Culturally sensitive assessment is crucial to avoid under- or misdiagnosis of OCD. Attention to key motives regularly found in Arab muslims with OCD can enable correct diagnosis.

Disclosure of interest.- The authors have not supplied a conflict of interest statement.

\section{E-PV0237}

\section{The role of mass media in mental health stigmatization: a double-edged Weapon}

N. Ghazouani ${ }^{*}$, S. Ellouze ${ }^{2}$, Z. Boudeli ${ }^{1}$, O. Moula ${ }^{2}$, R. Ghachem ${ }^{2}$ ${ }^{1}$ Medical school of Tunis, psychiatry, tunis, Tunisia; ${ }^{2}$ Razi university hospital, psychiatry, Manouba, Tunisia

Corresponding author.

Background and aims.- Mental health stigma is the false association of patients with mental illness, their families, friends and health care providers with something shameful. The role of media as an anti-stigma tool has not been unanimously advocated.

The aim of this study was to expose both the negative and positive effects of mass media on psychiatric stigma with a highlight on changing the media's approach to serve as an anti-stigma tool.

Methods.- A literature review using the subsequent databases: Pubmed, Sciencedirect, Embase and the keywords: mass media, social media, mental illness, and stigmatization.

Results.- The negative impact of social media consists in the inaccurate representations of mental illness in the mass media, the use of stigmatizing language in relation to the mentally ill, the depiction of mental illnesses as unsurmountable, and the identification of mental illness with violence and criminality in popular film and literature. Meanwhile, if used properly, media can serve as one of the most important anti-stigma tools through the embracing of an anti-stigma discourse, the promotion of a new approach based on a less sensational media reporting and awareness examples of campaigns that focus directly on the stigma of mental illness with the aim of changing public attitudes to mental illness on a broader level. Conclusions.- If used in a goal directed approach, mass media is a powerful tool to address the stigma surrounding mental health, raising the awareness about the mental health patients suffering and struggle and not pinpointing their difference as a source of violence and social unrest.

Disclosure of interest.- The authors have not supplied a conflict of interest statement.

\section{E-PV0238}

\section{Transcultural medicine, cultural knowledge in the clinical work}

S. Golsabahi-Broclawski

MITK, Psychiatry, Bielefeld, Germany

* Corresponding author.

Background and aims.- The interaction of care providers and patients or their relatives is culturally shaped by different norms, values and everyday-world ideas. For example, the understanding of health, illness and death, as well as the demands and expectations of care, can be intercultural. Cultural and linguistic barriers as well as cultural stereotypes can make a goal-oriented treatment more difficult.

Methods.- The Academy for Continuing Medical Education offers the curricular training "Transcultural Medicine - Cultural Competences in Clinical Everyday Life in Cooperation with Medical Transcultural Institute in Bielefeld.

Modules are:

1 Introduction to transcultural medicine

2 Culturespecific specificities of the diagnostics, taking into account cultural-specific examination methods

3 Medical Specialties in the Transcultural Context: GENERAL MEDICINE /SURGERY 
4 FOCUS - NEUROLOGY / PSYCHIATRY

5 FOCUS - PSYCHOSOMATIC

6 FOCUS - WOMEN'S CURE AND GE-BURTLEHILFE / CHILDREN AND YOUTH MEDICINE

Results.- The Transcultural Medicine is part of the Study of German physicians and it is settled as part of the medical training.

Conclusions.- Transcultural competence is increasingly important in everyday clinical practice. A stress factor because the usual techniques and procedures of the service providers do not reach or even miss their goal and lead to misunderstandings and injuries on both sides. This can lead to misunderstandings in the communication and the psychosocial care of migrants, as well as to problems in diagnostics, therapy and care. We offer since 2015 the Transcultural Training to improve the Treatment of Migrants.

Disclosure of interest.- The authors have not supplied a conflict of interest statement.

\section{E-PV0239}

\section{Relationship between cultural intelligence and emotional creativity with learning english in students}

M.S. Hosseini ${ }^{1}$, A. Homayouni ${ }^{2}$, S. Shahriary ${ }^{2}$

${ }_{1}$ Department of Psychology- Bandargaz Branch-Islamic Azad

University- Bandargaz-Iran, I, I, Iran; ${ }^{2}$ Department of Psychology-

Bandargaz Branch- Islamic Azad University- Bandargaz- Iran, l, l, Iran

* Corresponding author.

Background and aims.- The aim of this study was to investigate the relationship between cultural intelligence and emotional creativity with learning English in students.

Methods.- The research method was correlational. The statistical population of this study was high school schools that 150 students were selected and responded to Ang et al's cultural intelligence questionnaire, Averill's emotional creativity inventory and learning English scores. The data were analyzed by using Pearson correlation formula.

Results.- The findings of this study showed that there is a positive and significant correlation between cultural intelligence and emotional creativity with learning English.

Conclusions.- Based on the findings, cultural intelligence and emotional creativity are very important and influential factors on learning English language, and also in intercultural relations with people from different languages and cultures.

Disclosure of interest.- The authors have not supplied a conflict of interest statement.

\section{E-PV0240}

\section{Psychometric properties of the urdu translation of the dissociative experiences scale among a non-clinical sample of Pakistani university students}

C.A. Lewis ${ }^{1,2^{*}}$, S. Musharraf ${ }^{3}$, J.A. Malik ${ }^{4}$, M.J. Dorahy ${ }^{5}$, M.J. Lewis ${ }^{6}$

${ }^{1}$ Bishop Grosseteste University, School of Social Sciences, Lincoln, United Kingdom; ${ }^{2}$ University of Warwick, Centre for Education Studies, Coventry, United Kingdom; ${ }^{3}$ The Women University, Applied Psychology, Multan, Pakistan; ${ }^{4}$ Quaid-i-Azam University-, National Institute of Psychology, Islamabad, Pakistan; ${ }^{5}$ University of Canterbury, Psychology, Christchurch, New Zealand; ${ }^{6}$ Glyndw $r$ University, Psychology, Wrexham, United Kingdom

${ }^{*}$ Corresponding author.

Background and aims.- Dissociative disorders are common in Pakistan, accounting for over $10 \%$ of admissions in psychiatric wards. Despite this, little attention has been given to the suitability of established psychometric measures of dissociation in this context. The Dissociative Experiences Scale (DES) is a widely used 28-item self-report measure of dissociation that has been translated into a number of languages. The aim of the present study was to examine the psychometric properties of the recently translated Urdu version of the DES among a non-clinical sample of university students in Pakistan.

Methods.- A convenience sample of 253 university students in Pakistan completed a short survey comprising of the Urdu version of the DES and the English version of the DES, along with the Urdu version of the Eysenck Personality Questionnaire (EPQ).

Results.- The Urdu version of the DES demonstrated high internal consistency (alpha $=.91)$, cross-language validity ( $r s=.89$ to .93 ), satisfactory temporal stability over four weeks $(r=.89)$, and good convergent validity with the Urdu version of the EPQ's Extraversion, Neuroticism, Psychoticism, and Lie subscales. Exploratory factor analysis of the Urdu version of the DES suggested a single factor model was the best description of the data.

Conclusions.- These findings provide satisfactory evidence for the reliability and validity of the Urdu version of the DES among a non-clinical sample of university students in Pakistan and it is recommend for further use among Urdu speakers.

Disclosure of interest.- The authors have not supplied a conflict of interest statement.

\section{E-PV0241 \\ Exploratory factor analysis of the Urdu translation of the Ottawa self-injury inventory version $\mathbf{3 . 1}$} among a sample of Pakistan students

C.A. Lewis ${ }^{1,2^{*}}$, S. Davis ${ }^{3}$, T. Sohail ${ }^{4}$, A.O. Khawaja ${ }^{4}$, J.A. Malik ${ }^{5}$, S. Musharraf ${ }^{6}$

${ }^{1}$ Bishop Grosseteste University, School of Social Sciences, Lincoln, United Kingdom; ${ }^{2}$ University of Warwick, Centre for Education Studies, Coventry, United Kingdom; ${ }^{3}$ Glyndwr University, Psychology, Wrexham, United Kingdom; ${ }^{4}$ Lahore College for Women University, Applied Psychology, Lahore, Pakistan; ${ }^{5}$ Quaid-i-Azam University, National Institute of Psycholog, Islamabad, Pakistan; 6 The Women University, Applied Psychology, Multan, Pakistan * Corresponding author.

Background and aims.- The growing interest in self-harm (nonsuicidal self-injury) has been accompanied with the development of a number of self-report measures of self-harm. One such measure of self-harm is the Ottawa Self-injury Inventory Version 3.1 (OSI 3.1), which measures both the functions and potential addictive features of self-harm, and has been translated into a number of foreign languages. However, as yet, the Urdu translation has not been subjected to any empirical analysis. The aim of the present study was to examine the factor structure of the Urdu translation of the OSI 3.1 and four additional items in the Addictive Features section among a non-clinical sample of young female Urdu speakers in Pakistan.

Methods.- Data was collected from a non-clinical sample of 200 female Pakistani university students who completed the Urdu version of the OSI 3.1 and four additional items in the Addictive Features section. Data was subsequently subjected to exploratory factor analysis.

Results.- Exploratory factor analysis revealed four functions factors (internal emotion regulation, social influence, external emotion regulation, and sensation seeking) and one addictive features factor. Each of the four functions factors was significantly positively associated with each other. These findings are comparable to those reported previously among other samples.

Conclusions.- These findings provide preliminary evidence for the factor structure of the Urdu version of the OSI 3.1 and four additional items in the Addictive Features section among a non-clinical sample of university students in Pakistan and it is recommended for 
further use among Urdu speakers. Suggestions for further research are provided.

Disclosure of interest.- The authors have not supplied a conflict of interest statement.

\section{E-PV0242}

\section{Cross-language validity of the Russian translation of the Ottawa self-injury inventory version 3.1 among a sample of bilingual Russian students}

C.A. Lewis ${ }^{1,2,3^{*}}$, S. Davis ${ }^{4}$, E. Filimonova ${ }^{5}$, Z. Safina $^{5}$, S. Galyautdinova $^{3}$, M. Khukhrin ${ }^{3}$

${ }^{1}$ Bishop Grosseteste University, School of Social Sciences, Lincoln, United Kingdom; ${ }^{2}$ University of Warwick, Centre for Education Studies, Coventry, United Kingdom; ${ }^{3}$ Bashkir State University, Psychology, Ufa, Russia; ${ }^{4}$ Glyndŵw University, Psychology, Wrexham, United Kingdom; ${ }^{5}$ Bashkir State University, Translatology, Ufa, Russia

* Corresponding author.

Background and aims.- As a first step toward establishing a coherent research programme examining the mental health correlates of self-harm (non-suicidal self-injury) in Russia, the aim of the present study was to develop a Russian translation of the Ottawa Self-injury Inventory Version 3.1 (OSI 3.1) and four additional items foe the Addictive Features section.

Methods.- The OSI 3.1 and four additional items for the Addictive Features section were translated into Russian and back-translated into English by two experienced translators and one experienced psychologist familiar with health-related research. Subsequently, a sample of 20 bilingual Russian students completed the English version of the OSI 3.1 and four additional items for the Addictive Features section. Two weeks later, the respondents completed the Russian version. In contrast, this procedure was counter-balanced by a further sample of 20 bilingual Russian students who completed the Russian version first, and then two weeks later the English version.

Results.- No matters of concern with the translation process were identified by the three translators. Moreover, in terms of crosslanguage validity, a very strong positive association was found between responses to the English version of the OSI 3.1 and the four additional items in the Addictive Features section with responses to the Russian version.

Conclusions.- These findings provide preliminary evidence for the cross-language validity of the Russian version of the OSI 3.1 and the four additional items in the Addictive Features section among a non-clinical sample of bilingual university students in Russia. Suggestions for further research among Russian speakers are provided. Disclosure of interest.- The authors have not supplied a conflict of interest statement.

\section{E-PV0244}

\section{What patients think about quality of psychiatric care in different countries}

A. Schröder ${ }^{1 *}$, L.O. Lundqvist ${ }^{2}$

${ }^{1}$ Norwegian University of Science and Technology NTNU-Gjövik, Department of Nursing- Faculty of Health-Care and NursingNorwegian University of Science and Technology NTNU- Gjövik., Gjövik, Norway; ${ }^{2}$ University Health Care Research Center, Faculty of Medicine and Health- Örebro University, Örebro, Sweden

* Corresponding author.

Background and aims.- The international project Quality in Psychiatric Care $(\mathrm{QPC})$ is a large research programme aiming at adapting the versions of the QPC instrument for patients and staff to different international settings.
The aim is to describe the quality of care among patients and staff as measured by this instrument in different countries and to test the psychometric properties of the different language versions of QPC.

Methods.- The QPC has been developed on the basis of interviews with patients where they were asked what they perceived as quality of care. In this project we used the instrument versions for patient and staff in Forensic in-patient care, In-patient care and Out-patient care.

Results.- So far in this international project it shows that patients and staff in different cultures and health care systems may have different views on some aspects of quality of care, but most of all that they have many views in common, particularly the low ratings on participation.

Conclusions.- The psychometric test and validations of the instrument QPC in different language and country versions will assist countries to compare quality of care, quality improvement and permits benchmarking.

Disclosure of interest.- The authors have not supplied a conflict of interest statement.

\section{E-PV0245 \\ Subjective well-being of bilinguals and monolinguals living in the multilanguage regions of Russia: a pilot study}

L. Shaigerova, Y. Zinchenko, R. Shilko", A. Raevskiy

Lomonosov Moscow State University, Psychology, Moscow, Russia

Corresponding author.

Background and aims.- The rising scientific and practical interest to the problem of bilingualism resulted in the studies of the influence of bilingualism on the cognitive processes (memory, attention, perception and thinking) and on the cultural identity formation. The connection between bilingualism/monolingualism and the mental health and subjective well-being has not been studied enough.

The aim of the research is to study connection between bilingualism/monolingualism and subjective well-being.

Methods.- Respondents $(N=120)$ from three multilanguage and multicultural regions of Russia (Respublic of Crimea, Respublic of Tatarstan, Respublic of Dagestan), which have their own official languages except Russian, took part in the pilot study. The respondents were divided into two parts: (1) - people who speak only Russian, (2) - people who speak both Russian and other official languages in the regions of Russia, where the study was conducted. The respondents answered the question, how often they feel happy, according to the Likert scale from 1 to 5 ( 1 - always, 5 - never).

Results.- Independently of the ethnicity, age and region, monolinguals feel significantly less happy than bilinguals and multilinguals. It is true even in the case when the absolute majority of population in a certain region (for example, according to the 2014 population census, $84 \%$ in Respublic of Crimea) considers Russian as a mother tongue.

Conclusions.- Thus, the results have indicated a positive correlation between bilingualism/multilingualism and subjective well-being in the regions where several languages are considered official.

The reported study was funded by RFBR according to the research project No. 17-29-09167.

Disclosure of interest.- The authors have not supplied a conflict of interest statement. 


\section{E-PV0248}

\section{Description of the content and \\ characteristics of auditory \\ hallucinations: phenomenological aspects in Pakistani patients}

S. Vargas*, F. Collazos

Hospital Vall d'Hebron, Psychiatry Unit, Barcelona, Spain

${ }^{*}$ Corresponding author.

Background and aims.- Hallucinations are a universal human experience, present in all cultures, already described since Ancient Greece. In contrast to the pathological vision of Western society, non-Western cultures often attribute them to spiritual phenomena. Both aspects of the immediate environment and of the culture itself can have a relevant impact, determining the characteristics and content of delusions and hallucinations.

The present article aims to describe the content of auditory hallucinations in Pakistani patients with psychosis.

Methods.- We describe the content of auditory hallucinations through internationally validated instruments in three Pakistani patients with chronic psychosis.

Results.- The three patients reported auditory hallucinations in the form of animal noises (birds, barking dogs ...), in contrast to the usual third-person auditory hallucinations of the first-rank symptoms described by Kurt Schneider, so common in Western countries.

Conclusions.- To date, articles on hallucinations in different ethnic groups have focused mainly on the study of its prevalence and type. Regarding auditory hallucinations, they tend to be cited as "listening to voices", according to Schneider description. Nevertheless, a phenomenological description shows different contents, such as animal sounds in this case, that may have a relevant symbolic weight in patient's religious world, questioning the supposed universality of Schneider's first-rank criteria. Western-based therapies may ignore the meaning of patients' experiences in the treatment of hallucinations in other cultures.

Disclosure of interest.- The authors have not supplied a conflict of interest statement.

\section{E-PV0249}

\section{'Pasung' and other forms of physical restraint: a critical review of the treatment gap for severe mental illness in low-resource settings}

\section{A. Wijayanti}

University of Glasgow, Institute of Health and Wellbeing, Glasgow,

United Kingdom

${ }^{*}$ Corresponding author.

Background and aims.- Physical restraint has been used widely in institutions or by caregiver worldwide. The caregivers in some countries use pasung, which is a form of confinement with chain or another medium to restrict mobilisation toward mentally ill people. This practice associated with stigma or as an effort to ensure the safety. However, neither type of explanation can relate this case to the treatment gap issue. This article critically reviews the current studies that have investigated the physical restraint characteristics and scarce-resource in mental health services, particularly in the case of pasung. The analysis aims at developing recommendations for optimising community resources to minimise treatment gap and reduce the rate of physical restraint.

Methods.- The review identified the report discrepancies from articles published between 2008 and 2018 with quantitative and qualitative studies in the field.
Results.- Results demonstrate various motives and strategies in low-resource settings to deal with physical restraint. The treatment gap moderated by the lack of cost-effective services in local context, minimum treatment coverage and the absence of monitoring after patients discharged from mental health hospitals. Conclusions.- This study contributes towards understanding of treatment gap within physical restraint for severely mentally ill people. Using the existing analysis, stakeholder can create evidence-informed policy. Future studies and scope of intervention should consider an iterative process of involving communities in capacity development to prevent pasung or other forms of physical restraint.

Disclosure of interest.- The authors have not supplied a conflict of interest statement.

\section{E-Poster Viewing - 7-9 April: Depression}

\author{
E-PV0250 \\ Depression in the elderly. A series of 9 \\ patients \\ N. Arbelo ${ }^{1}$, G. Anmella ${ }^{1}$, S. Gomes da Costa ${ }^{1}$, C. Llach ${ }^{1}$, L. Ilzarbe ${ }^{1}$, \\ E. Arauz ${ }^{2}, A$. Benabarre ${ }^{3}$ \\ ${ }^{1}$ Hospital Clinic de Barcelona, Department of Psychiatry and \\ Psychology, Barcelona, Spain; ${ }^{2}$ National Institute of Mental Health, \\ Department of Psychiatry, Panama, Panama; ${ }^{3}$ Bipolar Disorder \\ Program- Hospital Clinic- University of Barcelona- IDIBAPS- \\ CIBERSAM, Department of Psychiatry and Psychology, Barcelona, \\ Spain \\ Corresponding author.
}

Background and aims.- Depression is one of the most frequent mental disorders in adults aged over 60 . In view of the rapid aging of the population, it is clear how depression in the elderly will be soon a public health challenge.

The objective of the study is to evaluate the social situation, comorbidity and clinical course of depression in older adults.

Methods.- It is a case series of the patients aged $\geq 65$ admitted for depressive disorder in a psychiatric ward during a period of 3 months. Socio-demographic information, medical history and clinical variables were studied.

Results.- 9 patients were included (67\% women) with a mean age of $78( \pm 8,53) .100 \%$ were widow or single, and $89 \%$ lived alone. $67 \%$ $(n=6)$ were diagnosed with major depressive disorder which first episode had happened before age 65 in $67 \%(n=4)$.

Hypertension (55\%), dyslipidemia (37,5\%) and hypothyroidism (37,5\%) were usual comorbidities and a $78 \%$ had altered laboratory markers of malnutrition. Complications like infections or delirium were common $(66,6 \%)$.

The mean duration of admission among these patients was larger than the mean duration in the ward ( 28,6 vs 18 days). A $55 \%(n=5)$ stayed $\geq 3$ weeks because of poor response to medication (100\%), complications ( $40 \%$ ) or lack of family support (40\%).

Conclusions.- Older adults hospitalized for depression usually have a lack of family support and suffer complications, which may lead to a larger time of admission. It is unclear if malnutrition is only a consequence or may be also a cause of depression, but its elevated prevalence highlights the need of performing an accurate nutritional assessment.

Disclosure of interest.- The authors have not supplied a conflict of interest statement. 
E-PV0251

\section{Peripheral blood expression of ogg1, mutyh, neil1 and apex 1 in the chronic mild stress model of depression and during pharmacotherapy using venlafaxine}

P. Czarny ${ }^{1 *}$, P. Wigner ${ }^{2}$, E. Synowiec ${ }^{2}$, J. Strycharz ${ }^{1}$, J. Szemraj ${ }^{1}$, M. Papp $^{3}$, T. Sliwinski ${ }^{2}$

${ }^{1}$ Medical University of Lodz, Department of Medical Biochemistry, Lodz, Poland; ${ }^{2}$ University of Lodz, Laboratory of Medical GeneticsFaculty of Biology and Environmental Protection, Lodz, Poland; 3 Polish Academy of Sciences, Institute of Pharmacology, Krakow, Poland

Corresponding author.

Background and aims.- Recently, it was shown that depression is accompanied by increased levels of 8-oxoguanine - a marker of DNA damage. Over the past three years, our team has published several papers in which we confirmed: (i) the presence of increased DNA lesions in depressed patients; (ii) impairments of DNA damage repair, (iii) modulation of depression occurrence caused by the single-nucleotide polymorphisms (SNPs) of genes involved in base excision repair (BER), (iv) that those SNPs may contribute to less efficient DNA repair in the patients. In current study, we used chronic mild stress (CMS) model, to elucidate, whether peripheral blood expression of genes involved in the first steps of BER, i.e. Ogg1, Mutyh, Neil1 and Apex1, changes during depression and after the use of venlafaxine.

Methods.- 24 Wistar rats were divided into 4 groups $(n=6)$, i.e. controls, CMS rats, CMS rats administered with vehicle and those treated with venlafaxine $(10 \mathrm{mg} / \mathrm{kg}$, IP). After experiments, animals were decapitated and RNA was isolated from their peripheral blood mononuclear cells (PBMCs). Expression changes were estimated using TaqMan probes, $2^{-\Delta \mathrm{Ct}}$ method and $18 \mathrm{~S}$ rRNA gene as an endogenous control.

Results.- CMS procedure caused decreased expression of Ogg1, Mutyh and Apex1 when compared to control rats. Interestingly, while pharmacotherapy did not affect expression of Ogg1 and Mutyh, it triggered further downregulation of Apex1. Neil1 was expressed at lower level only in rats administrated with venlafaxine.

Conclusions.- Expression of BER genes may be affected by depression and its pharmacotherapy.

Disclosure of interest.- The authors have not supplied a conflict of interest statement.

\section{E-PV0252}

\section{The relationship between depression and percevied social support among university students}

R. Damak ${ }^{*}$, F. Fekih-Romdhane, R. Feki, A. Feki, M. Cheour

Razi HOspital, Ibn Oumarne, Manouba, Tunisia

Corresponding author.

Background and aims.-

Background.- University students are a special group of people that are enduring a transitory period in which they are going from adolescence to adulthood. Depression among students have significant impact on quality of life and academic performances.

Aims.- The aims of the study were to estimate the prevalence of depression among university students and to examine the relationship between social support and depression.

Methods.- A cross-sectional survey was conducted among students of human and social sciences faculty and faculty of medicine of Tunis, between the $1^{\text {st }}$ February and 31 may 2017. We assessed depression by using the Depression Beck inventory. The perceived social support was evaluated by the Multidimensional Scale of Perceived Social Support.

Results.- The sample included 206 students. Median age was 23 years with quartiles 21 and 26 years. Among the sample, 62.6\% was female and $37.4 \%$ was male. One hundred thirty five students (65.5\%) had a low perceived social support. Depression prevalence was $41.7 \%$ among students. Results showed that $9.2 \%$ of students had a severe depression. Twelve students (5.8\%) reported previous suicide attempts. We found a strongly negative and significant association between depression and perceived social support $(p=0.001)$ and a positive association between depression and suicide attempts $(p=0.000)$. No associations were found between depression and gender $(p=0.47)$, socioeconomic level $(p=0.068)$ and marital status $(p=0.27)$.

Conclusions.- Our results highlighted the need for early screening and management of depression among students. Preventive strategies to enrich the social network of students are needed.

Disclosure of interest.- The authors have not supplied a conflict of interest statement.

\section{E-PV0253}

\section{Cognitive impairment in patients with depressive disorder}

S. Fedorová*, R. BartečekBartecek.Richard@fnbrno.cz

Faculty of Medicine Masaryk University and University Hospital Brno, Department of Psychiatry FN Brno and LF MU, Brno, Czech Republic * Corresponding author.

Background and aims.-

Introduction.- Cognitive impairment in patients with depressive disorder is a subject of intensive research.

Objectives.- This study deals with the cognitive impairment in patients with unipolar major depressive episode and patients with recurrent depressive disorder during the acute state of illness.

Aims.- The aim was to define main variables which influence cognitive performace during the acute state of depressive episode.

Methods.- We have used neuropsychological test battery (Auditory-Verbal Learning Test, Rey-Osterrieth Complex Figure Test, Digit span test, Trail making test, Verbal Fluency Test and Block Design) for the evaluation of the cognitive functions in patients with unipolar major depressive episode $(n=14)$ and patients with recurrent depressive disorder $(n=7)$.

Results.- We found cognitive impairment in all examined domains in both groups of patients. More profound cognitive impairment was found in patients with recurrent depressive disorder, particularly in visuo-constructive abilities, executive functions and verbal memory in relation to higher age of the patient.

Conclusions.- Our findings suggest higher age of the patient and repeated depressive phase as the most important variables which affects cognitive impairment.

Disclosure of interest.- The authors have not supplied a conflict of interest statement.

\section{E-PV0255}

\section{Postnatal depression in fathers. Systematic review of recent clinical}

\section{trials}

G. Hernandez-Santillan ${ }^{1 *}$, G. Lahera-Forteza ${ }^{2}$, M.F. Bravo-Ortiz ${ }^{3}$

${ }^{1}$ University Hospital Principe de Asturias, Psychiatry Unit, Madrid, Spain; ${ }^{2}$ University of Alcala, School of Medicine, Madrid, Spain; ${ }^{3}$ University Hospital La Paz, Psychiatry Unit, Madrid, Spain ${ }^{*}$ Corresponding author.

Background and aims.- Introduction: In contrast to the vast knowledge in mothers, postpartum depression in parents is still a "spot blind". However, its approach is gaining more interest at the 
scientific and clinical level given the reversible effects of its timely intervention.

Objectives.- To analyze clinical trials about postnatal depression in fathers.

Methods.- Systematic search of clinical trials in the last 5 years published in Pubmed and Embase. Keywords: men, father, depressive, depression, postpartum, postnatal.

Results.- 11 clinical trials were found in Pubmed and 12 in Embase. It has been found that, after childbirth, almost continuous skinto-skin contact decreases infants cortisol reactivity in response to handling and improves the concordance between mothers and infants 'salivary cortisol levels, and decreases fathers' experiences of spouse relationship problems. Likewise, it has been described that sex hormone fluctuations may contribute to heightened risk for developing depressive symptoms. Also, psychoeducation for parents through booklets in the perinatal care rooms is giving good results. Delivery of coparenting interventions (compared to relationship interventions) seem to attract more interest from couples and are likely to be easier to integrate into existing services. Psychoeducational interventions in primary care for couples of primitive women optimizes family functioning and reduces the risk of common mental disorders in the postpartum period. Finally, the postpartum screening intervention for fathers could be costeffective compared to no screening.

Conclusions.- The inclusion of parents in interventions for prevention, promotion and treatment of mental disorders in the postpartum period is giving good clinical, social and economic results.

Disclosure of interest.- The authors have not supplied a conflict of interest statement.

\section{E-PV0256}

\section{The impact of psychosocial factors on the evolution of major depressive disorder}

S. Ben Ali, Y. Zgueb, R. Jomli*, U. Ouali, F. Nacef

Razi Hospital, Psychiatric Departement, Tunis, Tunisia

${ }^{*}$ Corresponding author.

\section{Background and aims.-}

Background.- The evolution of major depressive disorder (MDD) is influenced by several meteorological, biological, psychological and social factors. Thus, life events have an important role in relapsing during MDD.

Aims.- Identify pychosocial and environmental factors associated with relapse and recurrence.

Methods.- We realize a retrospective study at the psychiatric departement $A$ in the Razi universal hospital.

A data sheet was used to collecte socio-demographic and clinical characteristics of 34 patients who was hospitalsed for a depressive episode.

Results.- The average age was 47.58. The sex ratio was 0.03 . The unemployment rate was $61 \%$. The main psychosocial and environmental problems found were problems with the main support group (85.66\%) economic problems (17.33\%) and professional problems (13.2\%). The average of hospitalization for the patients presenting these problems was 6.9 against 3.1 for the patients without this type of problems.

Conclusions.- Life events have a definite impact on the evolution and management of depressive disorder. their identifications is necessary.

Disclosure of interest.- The authors have not supplied a conflict of interest statement.

\section{E-PV0257}

\section{Suicidility features among Tunisian patients with mood disorders}

L. Jouini", U. Ouali, Y. Zgueb, R. Jomli, F. Nacef

Razi Hospital- La Mannouba- Tunisia, Psychiatry Department "A", Tunis, Tunisia

* Corresponding author.

Background and aims.- Suicidality is common among psychiatric patients suffering from mood disorders. Its severity can vary from a simple desire to die to repetitive suicidal attempts. The aim of our study was to explore the different features of suicidality among Tunisian patients with mood disorders and to determine associated factors.

Methods.- Patients with a diagnosis of depression $(n=150)$ were interviewed using the Structured Clinical Interview for DSM IV for mood disorders. Patients then received a diagnosis of Major Depressive Disorder (MDD) or Bipolar Disorder (BD). Patients were asked about their suicidality features during their depressive episodes: their desire to die, their suicidal thoughts, their suicidal attempts and if they had a precise suicide project or not.

Results.- Patients were diagnosed with MDD (58.7\%, $n=88)$, BD $(39.3 \%, n=59)$ and NOS $(2 \%, n=3)$. A history of suicide attempt was reported by $25.3 \%(n=11)$ of patients. Suicidality features during depressive episodes were as follows: desire to die $(36 \%, n=54)$, suicidal thoughts $(19.3 \%, n=29)$, suicidal attempts $(11.3 \%, n=17)$, having a precise project $(8 \%, n=12)$. Men had significantly more suicidal thoughts than women $(p=.015)$. The diagnosis of BD was more predictive of suicidal attempts than MDD and DD $(p=0.022$, $p=0.017$ ). The presence of mixed features was significantly associated with less suicidal thoughts $(p=0.041)$. A history of suicide attempt had a strong association with current suicide attempts $(p=10-3)$ and with a precise project $(p=0.08)$.

Conclusions.- Identifying the factors related to suicidality among Tunisian patients with mood disorders may improve the clinical assessment of suicide risk in people with depression.

Disclosure of interest.- The authors have not supplied a conflict of interest statement.

\section{E-PV0260 \\ Effect of treatment with escitalopram on the composition of intestinal flora in patients diagnosed with depression}

P. Liśkiewicz ${ }^{1^{*}}$, J. Pełka-Wysiecka ${ }^{1}$, M. Wroński ${ }^{1}$, A. Bąba-Kubiśs ${ }^{1}$, I. Łoniewski $^{2}$, J. Samochowiec ${ }^{1}$

${ }^{1}$ Pomeranian Medical University in Szczecin, Department of Psychiatry, Szczecin, Poland; ${ }^{2}$ Pomeranian Medical University in Szczecin, Departament of Biochemistry and Human Nutrition,

Szczecin, Poland

* Corresponding author.

Background and aims.- Despite the broad spectrum of pharmacological and non-pharmacological treatment methods for depression, a significant percentage of patients are drug-resistant or present insufficient therapeutic response. This indicates that the pathomechanism of the disease is not fully understood. It needs to be analyzed at the microbiological, immunological and endocrine level. Recent reports suggest that abnormalities of the microbiome and malfunctioning of the intestinal barrier are involved in the pathogenesis of depression. Findings so far have proved insufficient. New biological facts could therefore lay the groundwork for innovative disease concepts and creation of more effective treatment methods.

The aim of the study was to demonstrate that therapy with an antidepressant in patients suffering from depression leads to changes in the bacterial composition of the intestinal flora.

Methods.- The study included 20 somatically healthy patients diagnosed with depressive episode according to the ICD-10. Psy- 
chometric tests were performed before and after 2, 4, 6 weeks of taking escitalopram, the composition of bacterial intestinal flora was assessed before and after 6 weeks of pharmacotherapy.

Results.- The study confirmed shifts in bacterial composition of the intestinal microflora in patients diagnosed with depressive episode after treatment with escitalopram.

Conclusions.- The results have been promising so far and they need further research.

Disclosure of interest.- The authors have not supplied a conflict of interest statement.

\section{E-PV0261}

\section{Thyroid hormone treatment in the management of treatment-resistant unipolar depression: a systematic review and meta-analysis}

R. Lorentzen ${ }^{*}$, J.N. Kjær ${ }^{2}$, S.D. Østergaard ${ }^{3}$, M.M. Madsen ${ }^{2}$

${ }^{1}$ Aarhus University, Faculty of Health, Aarhus, Denmark; ${ }^{2}$ Aarhus University Hospital, Risskov, Aarhus, Denmark; ${ }^{3}$ Aarhus University Hospital and Aarhus University, Department of Clinical Medicine, Aarhus, Denmark

* Corresponding author.

Background and aims.- Treatment-resistance is of significant clinical concern, affecting approximately $40 \%$ of patients with unipolar depression. Treatment with thyroid hormones is occasionally used in the management of treatment-resistant or refractory depression, however, the evidence for the effect of this pharmacological intervention is not fully established. In this study, we aim to systematically review the literature on the effect of thyroid hormone treatment in the management of treatment-resistant unipolar depression.

Methods.- The study is registered at PROSPERO and we follow the PRISMA guideline for systematic reviews and meta analyses. Searches were carried out on the $27^{\text {th }}$ of August 2018 in the MEDLINE (PubMed), EMBASE and PsycINFO databases. No restrictions on language or publication date were applied. Each record is screened independently by at least two authors. In the case of discrepancies, consensus will be reached upon discussion. Data extraction will be performed independently by at least two authors. Again, in the case of discrepancies, consensus will be reached upon discussion.

Results.- The search yielded 1323 records, of which 419 were duplicates. Abstract and full text screening of the 904 remaining records is currently underway and will be followed by data extraction. Preliminary results will be presented at the EPA Congress 2019.

Conclusions.- We expect that the findings of this study will be of relevance for psychiatrists managing treatment-resistant unipolar depression.

Disclosure of interest.- The authors have not supplied a conflict of interest statement.

\section{E-PV0263 \\ Depression in elderly hemodialysed patients: multicentric study in the South of Tunisia}

M. Trigui ${ }^{1}$, Y. Mejdoub ${ }^{1}$, L. Ghanmi ${ }^{2}$, M. Maalej ${ }^{3}{ }^{*}$, J. Jdidi ${ }^{1}$, M. Ben Hmida $^{1}$, R. Boukhchina ${ }^{2}$, S. Deymi ${ }^{4}$, A. Abdennour ${ }^{5}$, M. Abbes ${ }^{2}$, S. Yaich $^{1}$, L. Zouari ${ }^{3}$, J. Damak ${ }^{1}$, M. Maalej ${ }^{3}$

${ }^{1}$ Hédi Chaker University Hospital, Department of Community Medicine, Sfax, Tunisia; ${ }^{2}$ Regional Hospital of Gabes, Department of Psychiatry, Gabes, Tunisia; ${ }^{3}$ Hédi Chaker University Hospital, Department of Psychiatry “C”, Sfax, Tunisia; ${ }^{4}$ University Hospital of Medenine, Department of hemodialysis, Medenine, Tunisia; ${ }^{5}$ National Health Insurance Fund, National Health Insurance Fund, Tataouine, Tunisia

* Corresponding author.
Background and aims.- Depression is commonly noted in elderly hemodialysed patients (EHP) and is associated with both an impaired quality of life and increased morbidity and mortality. We aimed to assess the frequency of depression in EHP and to identify the factors associated with depression among this population.

Methods.- We conducted a multicenter cross-sectional study in the South of Tunisia, during the month of October 2017. Were included all patients older than 65 years, with a chronic renal failure undergoing periodic hemodialysis. We collected data by a semi-directive interview of the patient and a member of his family.

Results.- A total of 81 patients were included. At the time of the survey $67 \%$ of the patients had a depression. Depression was significantly associated with: illiteracy $(p=0.02)$, the lack of physical activity $(p=10-3)$, the absence of recreational activity $(p=0.003)$, the presence of a stressor in the last 6 months $(p=0.035)$, the lack of autonomy $(p=0.002)$, the presence of a motor deficit $(p<10-3)$, the presence of a physical comorbidity $(p=0.003)$, predialytic creatinine $\leqslant 800 \mu \mathrm{mol} / \mathrm{l}(p=0.018)$, hypoprotidemia $(p=0.002)$ and the presence of cognitive disorders $(p<10-3)$.

Conclusions.- We found that depression was common in our population and we identified several risk factors. It is, therefore, important to develop systematic approaches to detecting depression in hemodialysed patients, and to develop strategies, taking into account risk factors for the proper management of these patients. Disclosure of interest.- The authors have not supplied a conflict of interest statement.

\section{E-PV0265}

\section{Clinical and psychopathological features of early and late dysthymia}

N. Maruta*, V. Fedchenko

"Institute of Neurology- Psychiatry and Narcology of the NAMS of

Ukraine" SI, borderline mental pathology, Kharkiv, Ukraine

${ }^{*}$ Corresponding author.

Background and aims.- Depressive disorders are the most common forms of mental illness with significant medical and social consequences and require adequate and timely medical care. An important aim on this path is to study their peculiarities of the course.

Methods.- To realize the goals and objectives of the study, an integrated approach including clinical and psychopathological (study of complaints, clinical history, psychopathological condition and its dynamics), psychometric (Hamilton's scale - HDRS) methods was used. 38 patients with dysthymia were examined (F 34.1).

Results.- The study of the effect of the onset of the disorder on the structure of clinical symptoms showed that the early onset of dystymia (up to 30 years) leads to the formation of hypochondria $(r=0,387)$, early insomnia $(r=0,369)$, and disorders in the field of work and activity $(r=0,327)$. While the later onset of dysthymia (after 30 years of age) affects the formation of common somatic symptoms (in particular, a sense of energy loss or loss of strength $(r=0.428)$, insomnia of the middle (restless night sleep) $(r=0.405)$, mental anxiety $(r=0.363)$. It has been established that atypical dystymia is more likely to be characterized by apatitic and hypochondriaal syndrome complexes $(p<0,05)$, while distalphobic and somato-vegetative syndrome complexes $(p<0,05)$ with a later onset.

Conclusions.- The influence of the age of the onset of dystymia on the structure of clinical symptoms is revealed and individual leading syndromes are identified, which has a prognostic significance for the selection of adequate therapeutic tactics.

Disclosure of interest.- The authors have not supplied a conflict of interest statement. 


\section{E-PV0268}

\section{The role of anhedonia in depressive disorder}

A. Pérez Morenilla ${ }^{*}$, A. Salaza Couso ${ }^{2}$, I. Failde Martínez ${ }^{2}$, J.A. Micó Segura ${ }^{3}$

${ }^{1}$ Psychiatrist, Psychiatry and Mental Health, Cádiz, Spain; ${ }^{2}$ University of Cádiz, Department of Epidemiology and Public Health, Cádiz, Spain; ${ }^{3}$ University of Cádiz, Department of Neuroscience Pharmacology and Psychiatry- CIBERSAM Group G18- INIBICA Instituto Investigación Biomédica de Cádiz, Cádiz, Spain ${ }^{*}$ Corresponding author.

Background and aims.- One every four has a diagnosable mental disorder, in which Depression is the most frequent. The importance of Depressive Disorder (DD) lies in the high disability and impact on the quality of life that it causes on these patients, greater economic costs and use of health resources and important morbidity and mortality; all of which makes it a public health problem. DD is characterized by changes in affect (depressed mood and/or loss of interest or anhedonia), as well as impairments in cognition and neurovegetative functions (somatic symptoms).

Methods.- Anhedonia, defined as "inability to experience pleasure," is a symptom present in patients with depressive disorder. Somatic symptoms also appear as fundamental symptoms in patients with DD. From a neurobiological point of view, both share multitude of brain structures and neurotransmission systems, particularly Monoaminergic System.

Results.- The presence of both symptoms has been related to a worse prognosis of DD, lower remission rates, higher recurrence, longer duration of the depressive episode, worse antidepressant treatment results, greater use of Health Services and worse quality of life related to health.

Conclusions.- Anhedonia seems to be undoubtedly a nuclear symptom in DD, as well as somatic symptoms, without knowing how they relate to each other until now. Based on these data, it would be recommended to carry out researches to elucidate the implication of the presence or absence of anhedonia in the rest of the dimensions (affective, cognitive and somatic, especially pain) in depressed patients, as well as their involvement on the quality of life related to health.

Disclosure of interest.- The authors have not supplied a conflict of interest statement.

\section{E-PV0269}

\section{Postpartum depression in males: a case report}

M.T. Ponte Lopez ${ }^{*}$, S. Boi, J. Herranz Herrer, M. Martín García, E. Gil Benito, B. Sanz-Aránguez Ávila

Hospital Universitario Puerta de Hierro, Psiquiatría, Madrid, Spain

* Corresponding author.

Background and aims.- Puerperium women's mental health has drawn research attention in the late years. However there are few parenthood mental health trials available.

Methods.- We present a case report of a 41 years old father of a 4 years old and a newborn girl. He showed low and unstable mood, insomnia and elevated anguish during the last weeks that increased after the birth, a week ago, adding then harm, ruin and guilt delusional ideas, related mostly to the newborn.

In psicopathologic examination perplexity, suspicion, hypomimia, sparing languaje with increased response latency and moodcoherent delusions was outlined.

Results.- Negative organic screening was shown. Diagnosis was severe depressive episode with psychotic symptoms on the basis of wife's postpartum. There were coincidental precarious labour and economic situation and anancastic personality traits, high selfdemand and sense of responsibility that triggered the mentioned symptomatology.
Conclusions.- Postpartum depression can happen to one in ten men, being mother's depression a major risk factor according to several studies. There are some differences regarding symptomatology and causes. It would be valuable to consider both parents mental healt state during pregnancy and postpartum, specially in previous social or economic lack situations and other vulnerability factors. This could allow to pick patients subsidiary to pharmacological or psycological treatment as well as to diminish the sickness comorbidity risks in the child's development.

Disclosure of interest.- The authors have not supplied a conflict of interest statement.

\section{E-PV0270 \\ Depression, search for new challenges in treatment}

M. Soto Laguna*, G. Belmonte Garcia, M. Fernández-Torija Daza, A. Sanchez Cabezudo, F.J. Gonzalez Diaz

Complejo Hospitalario de Toledo, Psiquiatria, Toledo, Spain

* Corresponding author.

Background and aims.-

Introduction.- Depression is one of the most disabling diseases of the 21 st century.

Early recovery avoids chronic symptoms.

Currently the patient not only seeks an improvement of mood but also the social aspect, work, relationship and sexual area.

Goals.- In the Hospital of Toledo (Spain), a study was carried out to check the improvements in affective, anxiety and cognitive status in patients with 3 types of antidepressants: selective serotonin reuptake inhibitors, antidepressants dual and a multimodal antidepressant (vortioxetine).

Methods.- Our inclusion criteria were: being over 18 years of age, diagnosis of depression or Adaptive Disorder according to DSM-V criteria, not having received antidepressant treatment in the last year, not presenting intellectual disability, signing the informed consent.

The Montreal Cognitive Assessment (MOCA) scales for cognitive aspects, the Montgomery-Asberg Depression Rating Scale (MADRS) for depressive symptoms and the Hamilton scale for measuring anxiety have been used.

Results.- The data analysis was through the SPSS program the data analysis was carried out.

Similar results were obtained in treatment with any of the three antidepressants in anxiety symptoms, the depressive symptoms improved more significantly with dual antidepressants and with the multimodal antidepressant and the cognition improved with the multimodal antidepressant

Conclusions.- Currently the treatment of depression implies a greater ambition in the recovery of all areas of life of the patient in the shortest time in the search for complete recovery.

Our results indicate better functionality with dual antidepressants and vortioxetine.

Disclosure of interest.- The authors have not supplied a conflict of interest statement.

\section{E-PV0271}

\section{Use of aripiperazole in late life depression: case note review}

S. Srivastava*, A. Sonal, P.K. Chaubey, A. Singh

King George's Medical University, Geriatric Mental Health, Lucknow, India

${ }^{*}$ Corresponding author.

Background and aims.- The response rates in depression are as low as $30 \%$ to first line antidepressants. Thus, there is need for newer pharamacological agents to be used either as monotherapy, or in conjunction with traditional antidepressants to achieve remission 
earlier. Aripiperazole is one such drug which has been used as augmentation therapy in treatment resistant cases. However, its use from the beginning of the treatment - either in conjunction with another antidepressant or on its own, has not been studied.

Methods.- In the outpatient walk-in clinic, patients over the age of 60 years are referred from other departments of the University, psychiatrists practicing elsewhere, or by themselves. In additionto the usual history, cognitive screening on Hindi Mental State Examination is done on all patients, the depressed patients are also evaluated on Hamilton Rating Scale for Depression (HAMD) and Geriatric Depression Scale (GDS). On subsequent visits, the patients are rated on HAMD and GDS.

Results.- Over 2 years, 53 patients (37 male, mean age 68 years) were prescribed aripiperazole - either given alone or in combination with an antidepressant from the first visit onwards, or as add-on medication if first line medication was not effective. The followup period varied between 1 week to 1 year for all the patients, but in the present report only the data for the first 12 weeks will be presented.

Conclusions.- The efficacy of aripiperazole in a clinical setting is reported here as it can be a useful adjunct to first line antidepressants.

Disclosure of interest.- The authors have not supplied a conflict of interest statement.

\section{E-PV0272}

\section{Depression and negative emotional interference in working memory}

F. Sultanova*, B.B. Velichkovsky, A.A. Kachina, G.E. Rupchev Lomonosov Moscow State University, Psychology, Moscow, Russia ${ }^{*}$ Corresponding author.

Background and aims.- Depression is an important negative condition and may significantly affect the life of people. As depression has a significant cognitive component, a specific focus on the cognitive mechanisms of depression is warranted (Snyder, 2013). We replicated the study of De Lissnyder et al. (2012) to check whether there is an association between the sensitivity to negative emotional interference in working memory and depression level in a non-clinical sample.

Methods.- $N=60$ (44 female, mean age $27 \pm 7$ years). The participants completed a Russian version of Beck Depression Inventory (BDI-II) with scores ranging from 0 to $26(\mathrm{M}=7.5, \mathrm{SD}=6.6)$ and performed the Internal Shift Task. To assess the effects of sub-clinical depression, we median-splitted $(\mathrm{Me}=5.5)$ the sample giving a euphoric and a non-euphoric group.

Results. - We found a Group $\times$ Valence interaction, $F(1,58)=4.48$, $p<0.05$, confirming the result of the previous analyses - the euphoric group was characterized by reduced RTs during the processing of angry faces.

Conclusions.- We found typical effects of stimuli emotionality (emotional materials being processed more slowly) and switching (the change of a stimulus category leading to slower processing). More importantly, we found no signs that presenting a negative stimulus in the previous probe selectively impairs processing in the subsequent probe in subjects with more depressive symptoms. However, we found that non-dysphoric subjects exhibit a protective bias away from negative information processing the negative stimuli more quickly than neutral stimuli.

Disclosure of interest.- The authors have not supplied a conflict of interest statement.

\section{E-PV0273}

\section{Postnatal depression among tunisian mothers of newborns hospitalized in the neonatal intensive care unit}

S. Taleb, S. Bourgou, A. Taamallah*, M. Hamza, F. Charfi, A. Belhadj Mongi Slim Hospital, Child and adolescent Psychiatry, Tunis, Tunisia "Corresponding author.

Background and aims.- The separation of mother -infant dyad at birth, because of the newborn baby's need for intensive medical attention, is an unexpected and traumatic event for the mother. It may affect maternal mental health and so the infant's development. The study aimed to investigate levels of postpartum depression among mothers whose babies were hospitalized at birth in the neonatal intensive care unit (NICU) of the Mongi Slim hospital in Tunisia. We also tried to determine maternal/infant characteristics that predict risk for developing postpartum depression in these mothers.

Methods.- It was conducted by the Child psychiatry department of Mongi Slim hospital (Tunisia). Socio-biographical and gynaecological characteristics were collected from Medical Files. Mothers completed the validated Arabic version Edinburgh Post-Natal Depression Scale (EPDS).

Results.- We included 22 mothers. The mean age of participants was 30, 45 years. Most of them have an average socioeconomic conditions. Pregnancy was induced for three mothers. Six of them have presented complications during pregnancy and four required hospitalization in a gynecology ward. Delivery was by caesarean section in the majority of cases (15). Caesarean section was urgently needed for 10 of them. The average duration of hospitalization of the newborn is 10.6 days. The mean EPDS score was 17, 2. Nineteen mothers had scores equal or superior to 11 , which is an indicator of postnatal depression.

Conclusions.- Mothers of NICU-hospitalized infants are emotionally vulnerable. Determining risk factors can Help Therapists detect and manage early the postpartum depression and help mothers to establish a healthy relationship with their babies.

Disclosure of interest.- The authors have not supplied a conflict of interest statement.

\section{E-PV0274}

\section{Anxiety as a state and as a trait in people diagnosed with a depressive episode}

P. Maruszewska, A. Skiba, J. Jasionowska, M. Talarowska*, P. Galecki

Medical University of Lodz, Department of Adult Psychiatry, Lodz, Poland

* Corresponding author.

Background and aims.- Anxiety is one of the most common symptoms accompanying depression. The aim of the study was to check whether variables such as occurrence of depression, severity of depression symptoms and gender differentiate the subjects in the scope of anxiety understood as a situational state of the individual and in the scope of anxiety as a permanent personality trait.

Methods.- Thirty-nine people ( 31 women and 8 men) took part in the experiment. Twenty of them met the criteria of a depressive episode, while 19 individuals not treated psychiatrically were qualified to the control group. Hamilton Depression Rating Scale was used to assess the severity of depression symptoms, while State-Trait Anxiety Inventory was applied to investigate the level of anxiety.

Results.- Analyses revealed statistically significant differences between the individuals with diagnosed depression and the healthy subjects in terms of the level of anxiety as a state $(p=0.00)$ and as a trait $(p=0.00)$. No statistically significant differences were found 
related to gender and the level of anxiety as a state $(p=0.7)$ or the level of anxiety as a trait $(p=0.54)$. There were also no significant differences between the severity of depression and the level of anxiety as a state $(p=0.57)$ and as a trait $(p=0.37)$.

Conclusions.- The level of anxiety both as a trait and as a state is higher among people with diagnosed depression than among healthy individuals. Neither the severity of depression symptoms nor gender is associated with the level of anxiety as a state and as a trait.

Disclosure of interest.- The authors have not supplied a conflict of interest statement.

\section{E-PV0275}

\section{Particularities of the productive phenomenology in the major depressive episode fregoli delusions and olfactory hallucinations}

\section{S. Trifu ${ }^{1}$, E.G. Carp ${ }^{2}$, M. Dragoi ${ }^{3}$}

${ }^{1}$ University of Medicine and Pharmacy "Carol Davila”, Neurosciences - Psychiatry, Bucharest, Romania; ${ }^{2}$ Hospital for Psychiatry, Psychiatry, Sapunari, Romania; ${ }^{3}$ Hospital for Psyhciatyr "Alex. Obregia", Psychology, Bucharest, Romania

* Corresponding author.

Background and aims.- The trigger is represented by the death of her father six months before, the case particularity consisting in the association with psychotic phenomena of delusional interpretativity, with Fregoli delusions and olfactory hallucinations, which imposes a first differential diagnosis with cerebral organicity.

Methods.- The predominantly delusional ideation, the interpretativity, auditory, olfactory and visual hallucinations and delusions dictated the hospitalization. Combined antidepressant and antipsychotic treatment has been initiated. Personality has been evaluated as regards deep functioning, being highlighted the dependency and existential insecurity area. Both psychological and projective investigation has been used, as well as the psychometric one, by clinical scales. She has been psychiatrically monitored and emotional support has been offered following psychodynamic exploration.

Results.- She has a coherent speech, against the concentration difficulties, attention stability and selectivity, with psychic focus on delusion area, on xenopathy and delusional interpretativity of daily life phenomena. Events chronology (initial mood change in the depressive sense, of endo-reactive colours, undetected and untreated, followed by the association of psychotic elements) dictates the affective colours of disorder, in the detriment of delusional disorder diagnosis, even if the age can facilitate the evolution in any of the two zones. The alteration of social functioning would be the second criteria, in longitudinal follow-up the patient's evolution highlighting the resumption of daily activities at the same sociooccupational level reached previously, after mood normalization. Conclusions.- As princeps psychological elements, we encounter the fear of loneliness, the need for affection and listening, various pains with migratory character at somatic level, the need for emotional support.

Disclosure of interest.- The authors have not supplied a conflict of interest statement.

\section{E-PV0276}

\section{Nicotine as augmentation agent in depression and anxiety}

G. Tzeferakos ${ }^{1}$, M. Dimitraka ${ }^{2}$, D. Petsas ${ }^{3}$, P. Papdimitriou ${ }^{4}$, A. Gatos- Gatopoulos ${ }^{4}$, C. Tsopelas ${ }^{5^{*}}$

${ }^{1}$ University of Athens- Medical School- 2nd Dept. of Psychiatry,

University of Athens- Medical School-2nd Dept. of Psychiatry, athens, Greece; ${ }^{2}$ Psychiatric Hospital of Attica, 5th Psychiatric Dept., Athens, Greece; ${ }^{3}$ Psychiatric Hospital of Attica, 4th Psychiatric Dept., Athens,
Greece; ${ }^{4}$ 5th Psychiatric Dept. Psychiatric Hospital of Attica, 5th Psychiatric Dept. Psychiatric Hospital of Attica, Athens, Greece; ${ }^{5}$ Psychiatric Hsopital of Attica, 5th Psychiatric Dept., Athens, Greece * Corresponding author.

Background and aims.- Current evidence suggests that Nicotine can be used as augmentation in depression and anxiety. Acetylcholine nicotinic systems and serotonergic systems are known to interact. In this review we are trying to explore the possibilities of nicotine use as a therapeutic agent in depression and anxiety.

Methods.- Thorough research of the main databases, and web search engines such as Goggle, for relevant studies, using appropriate keywords. We scrutinize them independently, before reaching consensus about appropriateness.

Results.- In non smokers, drug naïve patients diadermal administration of $17.5 \mathrm{mg}$ nicotine for 4 days reduced depressive symptoms; $3.5 \mathrm{mg}$ nicotine TTS for 8 days reduced depressive symptoms in other study. But other studies reported reverse of these positive results after cessation of nicotine. Chronic nicotine or cigarette use results in decreased monoamine oxidase $A$ and $B$ activity and a substantial reduction in alpha -sub-4beta -sub-2 nicotinic acetylcholine receptor ( $\mathrm{nAChR}$ ) availability in thalamus and putamen. The desensitization of DRN 5-HT neurons after chronic single daily injections of $1 \mathrm{mg} / \mathrm{kg}$ of nicotine suggests an antidepressant-like effect of chronic nicotine. Antidepresant effect was quick in onset of action, but requires continous use.

Conclusions.- Nicotine has complex interactions with other neurotransmitters in the brain. Investigations into the neurobiology, and biophysical and pharmacological properties of nicotinic acetylcholine receptors (nAChRs), have led to an improved understanding of their role in a variety of neuropsychiatric disorders for the treatment of depressive and anxiety symptoms not only to smokers after abstinence but also to non smokers

Disclosure of interest.- The authors have not supplied a conflict of interest statement.

\section{E-PV0277}

\section{Patient reports about emotional meanings on refractory pharmacological treatments in depressive disorders: a clinical-qualitative study in a Brazilian specialized adult outpatient service}

L.C. Vieira, R.A. Bastos, E.R. Turato*

State University of Campinas, Laboratory of Clinical-Qualitative Research, Campinas, Brazil

${ }^{*}$ Corresponding author.

Background and aims.- Psychosocial aspects and life experiences related to the treatment of depression are important to take into account by psychiatry team, mainly in case of refractoriness. Resistant treatment depression is considered by poor response to two antidepressants in dosage and adequate duration. Beliefs, disease representations, or the doctor-patient relationship, are important aspects that go beyond pharmacologic treatment.

To explore emotional meanings attributed by patients with resistant depression to medication in follow up at the outpatient service in General Hospital, at the State University of Campinas, South eastern Brazil.

Methods.- A clinical-qualitative study using semi-directed interviews with open-ended questions, in-depth. Eight patients participated of the investigation in a sample closed by information saturation. The interviews were audio recorded, fully transcribed and categorized by Qualitative Content Analysis. The results were peer-reviewed in several meetings in the Laboratory of ClinicalQualitative Research. 
Results.- Emerging categories: (1) Perception of refractoriness in the understanding of the illness symptoms permanence; (2) refractory depression from a profound melancholy perspective of life; (3) Personal impotence in the perception of the refractoriness complexity phenomenon; (4) Good treatment adherence face to a fear on relapse.

Conclusions.- Meanings attributed by patients to refractory depression come from the persistence of symptoms due to the continued use of the medication, appearing to be 'not taking'. Despite the complete remission, there are reports of the symbolic of adhering to care, threatened by fear of worsening without the remedy. This context generates anguish, especially by the fears associated with existential and poorly elaborated life experiences.

Disclosure of interest.- The authors have not supplied a conflict of interest statement.

\section{E-PV0279}

\section{Time resolved tryptophan fluorescence as a new approach for investigation of serum albumin disturbances in melancholic depression}

M. Uzbekov ${ }^{1 *}$, T. Syrejshchikova ${ }^{2}$, N. Smolina ${ }^{1}$, E. Syromyatnikova $^{2}$, V. Brilliantova ${ }^{1}$, G. Dobretsov ${ }^{3}$

${ }^{1}$ Moscow Research Institute of Psychiatry, Brain Pathology, Moscow, Russia; ${ }^{2}$ Lebedev Physical Institute, High Energy, Moscow, Russia; ${ }^{3}$ Research and Clinical Center of Physic-Chemical Medicine, medical biophysics, Moscow, Russia

${ }^{*}$ Corresponding author.

Background and aims.- Psychiatry.

Objectives.- Fluorescence of amino acid Tryptophan-214 residues (Trp-214-R) as inner probe of albumin molecules can give information about protein conformational changes. In human serum albumin excitation wavelength at $295-305 \mathrm{~nm}$ is mainly absorbed by Trp-214-R that gives possibility of selective observation of state of albumin molecule.

Aim.- Investigate of kinetics of tryptophan fluorescence decay in serum albumin of patients with melancholic depression (MD) using subnanosecond fluorescent spectroscopy.

Material and Methods.- There were investigated 14 patients with MD and 14 healthy volunteers. Patient's state was defined as a depressive episode in frame of bipolar depressive disorder (type 2) (F32) and in structure of recurrent depressive disorder (F33). Fluorescence decay of serum albumin Trp-214-R was measured in subnanosecond range using laser device (LED, Pico-Quant). Excitation wavelength $-290 \pm 10 \mathrm{~nm}$; samples were excited by laser flash $\left(7 \times 10^{-10} \mathrm{sec}\right)$.

Results.- Results. The form of decay kinetics was represented as a sum of three exponentials, described by three decay times $\tau i$ (in 6.5 , 2.8 and 1 ns region) and three amplitudes $A \mathrm{i}$. There were received significant differences for amplitudes $A 1$ and $A 3(p<0.01)$ and relationship of $A 1 / A 3$ и $A 1 / A 2$ ( $p=0.01$ ) between MD and control.

Conclusions.- Conclusion. It was shown the first time in literature that conformational changes of albumin in mental disorders can be detected by Trp-214-R fluorescence. Fluorescence of Trp-214-R can serve as potential biomarker of albumin conformation disturbances.

Disclosure of interest.- The authors have not supplied a conflict of interest statement.
E-PV0281

\section{Have treatment studies of depression become even less generalizable?}

M. Zimmerman ${ }^{*}$, C. Balling ${ }^{2}$, I. Chelminski ${ }^{2}$, K. Dalrymple $^{2}$

${ }^{1}$ Brown University, Psychiatry and Human Behavior, Providence, USA ${ }^{2}$ Rhode Island Hospital, Psychiatry, Providence, USA

Corresponding author.

Background and aims.- Antidepressants are amongst the most frequently prescribed medications. More than a decade ago our clinical research group applied a prototypic set of inclusion/exclusion criteria used in an antidepressant efficacy trial (AET) to patients presenting for treatment in our outpatient practice and found that most patients would not qualify for the trial. In the present report from the Rhode Island Methods to Improve Diagnostic Assessment and Services (MIDAS) project we apply the psychiatric inclusion/exclusion criteria used in 158 placebocontrolled studies to a large sample of depressed patients who presented for outpatient treatment to determine the range and extent of the representativeness of samples treated in AETs and whether this has changed over time.

Methods.- We applied the inclusion and exclusion criteria used in 158 AETs to 1,271 patients presenting to an outpatient practice who received a principal diagnosis of major depressive disorder. The patients underwent a thorough diagnostic evaluation.

Results.- Across all 158 studies, the percentage of patients that would have been excluded ranged from $44.4 \%$ to $99.8 \%$ (mean $=86.1 \%$ ). The percentage of patients that would have been excluded was significantly higher in the studies published in 2010 through 2014 compared to the studies published from 1995 to 2009 $(91.4 \%$ vs. $83.8 \%, t(156)=3.74, p<.001)$.

Conclusions.- Only a minority of depressed patients seen in clinical practice are likely to be eligible for most AETs. The generalizability of AETs has decreased over time. It is unclear how generalizable the results of AETs are to patients treated in real world clinical practice. Disclosure of interest.- The authors have not supplied a conflict of interest statement.

\section{E-Poster Viewing - 7-9 April: E-mental Health}

\section{E-PV0283 \\ Development and usability test in a primary care centre of an app for the needle exchange programs}

F. Calvo ${ }^{*}$, X. Carbonell ${ }^{2}$, C. Mundet ${ }^{3}$, C. Giralt ${ }^{4}$, S. Castillejos ${ }^{5}$, L. Cabanillas $^{6}$, S. Saura ${ }^{6}$

${ }^{1}$ Institut d'Assistència Sanitària, Health, Girona, Spain; ${ }^{2}$ FPCEE Blanquerna, Universitat Ramon Llull, Barcelona, Spain; ${ }^{3}$ Servei de Promoció de la Salut a Girona, Agència de Salut pública de Catalunya, Girona, Spain; ${ }^{4}$ CAS Girona, Institut d'Assistència Sanitària, Girona, Spain; ${ }^{5}$ FPE, Universitat de Girona, Girona, Spain; ${ }^{6}$ CAP Montilivi-Vila Roja, Institut Català de la Salut, Girona, Spain * Corresponding author.

Background and aims.- Usability tests are essentials before to offer to general population health apps. Needle Exchange Programs needs new interventions to improve its results. The aim of this study is to analyse the process of development and test of usability of a mobile device application designed to complement the Needle Exchange Program.

Methods.- A transversal and observational design was conducted in a Primary Care Centre and in a street drug-consumption zone, both in the city of Girona.

Main measures: Sex, age and personal use of ICT was compared with the acceptance of the app. Central tendency and dispersion 
measures, U-Mann Whitney test and cross tables was used for the statistical analysis.

A literature review of mHealth and drug dependence was developed and a usability test for each target group was performed.

Results.- Participants were 13 members of a multidisciplinary medical team and 9 people who inject drugs. No differences were found between the analysed variables and the opinion of the app. Participants proposed improvements about the prototype that will be apply in the definitive interface.

Conclusions.- Professionals and potential users share similar opinion about the app and its uses. The participation of professionals and patients in usability test of mobile applications facilitates the detection of particular needs.

Disclosure of interest.- The authors have not supplied a conflict of interest statement.

\section{E-PV0285}

\section{Association of life orientation index and parameters of emotional intelligence in university students: a data mining approach}

E. Gkintoni ${ }^{*}$, C. Halkiopoulos ${ }^{2}$, H. Antonopoulou ${ }^{2}$, M. Georgiadi ${ }^{3}$, S. Plexousakis ${ }^{3}$

${ }^{1}$ Technological Institute of Western Greece, Entrepreneurship \& Digital Innovation Laboratory, Patras, Greece; ${ }^{2}$ Technological Institute of Western Greece, Entrepreneurship \& Digital Innovation Laboratory, Patras, Greece; ${ }^{3}$ University of Crete, Psychology, Rethymno- Crete, Greece

${ }^{*}$ Corresponding author.

Background and aims.- Life Orientation is aimed at developing and engaging young adults in personal, psychological, neurocognitive, motor, physical, moral, spiritual, cultural and socio-economic areas, so that they can achieve their full potential in order to success in future life. The aim of the present study is to assess individual differences in generalized optimism versus pessimism in the index of life orientation and parameters of emotional intelligence in a sample of university students through application of data mining methods. Methods.- The sample comprised 300 university students, males and females, aged 18-30 years and assessed using self-administered questionnaires. The 10-item version of Life Orientation Test Revised (LOT-10), and the Trait Emotional Intelligence Questionnaire (TEIQue). The methodology adopted, in first phase consists of electronic questionnaires, which were created and posted through the website http://www.cicos.gr. Subsequently data were collected and preprocessed from the questionnaires and then introduced into the $\mathrm{R}$ (Programming Language and Machine Learning Platform) for analysis and extraction of useful knowledge.

Results.- More specifically, through using classification algorithms (C4.5) there was a production of prospectively decision trees. Furthermore, clustering technique (K-Means algorithm), was applied and the parameters of the algorithm were set, depending on the application cases and the respondents were classified into clusters based on the items of the two scales.

Conclusions.- The results indicate among others, that the use of Data Mining methods is an important tool to export and receive the conclusions and decisions especially in the field of psychological assessment and in mental health.

Disclosure of interest.- The authors have not supplied a conflict of interest statement.
E-PV0287

\section{Virtual reality in psychiatry: is it not too early to announce a shift in the paradigm?}

A. Karlińska*, S. Mandes

University of Warsaw, Institute of Sociology, Warsaw, Poland

* Corresponding author.

Background and aims.- First attempts to use virtual reality (VR) for diagnostic and therapeutic purposes in mental health $(\mathrm{MH})$ dates back to 1990s. Recent technical developments made VR feasible and raised expectations about its usefulness for clinical practice. Our aim is to provide a methodological critique of research on VR technology conducted by MH professionals, especially concerning the issue of ecological validity.

Methods.- The study will be based on a systematic review of the literature performed in compliance with the Preferred Reporting Items for Systematic Reviews and Meta-Analyses statement. Two researchers will independently search MEDLINE, PsycINFO and EMBASE from the years 1990-2018 to identify studies evaluating the use of VR for diagnostic or therapeutic purposes in $\mathrm{MH}$.

Results.- Few papers indicate what makes virtual environment an effective diagnostic and therapeutic tool. $\mathrm{MH}$ professionals very rarely address the issue of ecological validity of their research and limit themselves to stating that the VR therapy can result in significant real-world outcomes. The results of previous studies do not allow for such generalizations due to small sample sizes and methodological limitations.

Conclusions.- MH professionals tend to view VR as a harbinger of a shift in the paradigm of diagnosing and treating psychiatric disorders. However, there is still large discrepancy between the expected and proved usefulness of VR. Further research may also bring ambiguous results unless the conceptual apparatus becomes clearly defined, including uniform methodological approach and measures of outcome.

Disclosure of interest.- The authors have not supplied a conflict of interest statement.

\section{E-PV0288}

\section{Efficient use of micro-services} architecture for emental health care development in the Czech Republic

\section{T. Knejzlikova ${ }^{1^{*}}$, M. Svetlak ${ }^{1}$, P. Linhartova ${ }^{2}$}

${ }^{1}$ Faculty of medicine- Masaryk University Brno- Czech Republic, Department of psychology and psychosomatic, Brno, Czech Republic;

${ }^{2}$ Faculty of medicine- Masaryk University Brno-Czech Republic, Department of psychiatry, Brno, Czech Republic

"Corresponding author.

Background and aims.- The aim of the project is to create a group of services, which, with their interconnection and implementation into existing or emerging systems and mobile devices support, will be used to develop eMental health care in the Czech Republic.

The main innovation and contribution is the usage of potential of the micro-services architecture from the world of informatics into preventive and interventional medicine with automatic setting of all services according to GDPR principles. By creating the individual services necessary for each new eMental health care application, we build the foundation stone for implementing specific implementations such as intervention and education programs, screening programs and more.

Methods.- Our current practice with developed services and their applications for the end user is currently in the scope of developing basic tools and implementation in the Mindfulness intervention program.

To our program "The eight-week on-line program to promote mental and physical health based on mindfulness for students and 
employees" more than 800 people have signed up, which exceeds the share of all published similar programs (Fish, Brimson, \& Lynch, 2016).

Results.- The program was fully automated using Slack. The Slack application is more compatible with our system and gives us space to get statistics about program efficiency and users adherence.

Conclusions.- One part of the system presentation will be about analysed data from the currently running Mindfulness program and adherence.

This project was supported by VaV program from MUNI, 2018.

Disclosure of interest.- The authors have not supplied a conflict of interest statement.

\section{E-PV0290}

\section{An exploratory analysis of the uses and representations shaping French schizophrenia groups on facebook}

D. Lampropoulos*, A. Thémis

Aix-Marseille University, Laboratoire de Psychologie Sociale EA849,

Aix-en-Provence, France

* Corresponding author.

Background and aims.- Health-related groups on Facebook are being increasingly used for peer support and information exchange. However, research on their functions and the representations that constitute them is still quite limited and specifically, there is no study on schizophrenia groups on Facebook. The current study, drawing from the Social Representations approach, aimed to explore the uses and functions of French-speaking Facebook groups related to schizophrenia, as well as the representations that underly them.

Methods.- Two main schizophrenia groups have been identified through Facebook's own search function, the first being administered by professionals, the second by peers. After data extraction, initial posts were submitted to an inductive thematic analysis in order to identify the purpose of communication and both posts and comments were submitted to a content analysis concerning their representational content.

Results.- Thematic analysis indicated that while the main function of the first group was to answer to members' demand for support and knowledge, through experience sharing and advice seeking, the second focused on the offer of support and knowledge, though advice giving and information sharing. The first group explicitly promotes evidence-based practices and is pro-psychiatry, while the second group is encompassed by tensions concerning traditional structures, such as psychiatry that is often seen as repressing, family that is often seen as a cause of suffering and medication that is presented as harmful.

Conclusions.- Research on health-related Facebook groups allows exploration of representational contents and tensions that shape one's relation to the disorder and citizenship.

Disclosure of interest.- The authors have not supplied a conflict of interest statement.

\section{E-PV0291}

\section{E-health: serving rural, remote and institutional transgender populations} - a review

\author{
M. Marshall ${ }^{1 *}$, G. Knudson ${ }^{2}$, S. Leibel ${ }^{3}$ \\ ${ }^{1}$ University of Alberta, Psychiatry, Edmonton, Canada; ${ }^{2}$ University of \\ British Columbia, Psychiatry, Victoria, Canada; ${ }^{3}$ University of \\ Calgary, Social Work, Edmonton, Canada \\ * Corresponding author.
}

Background and aims.- Persons in Canada who are transgender and gender diverse face barriers to accessing good, compassionate and timely physical and mental health care. This is more so for individuals who live in rural or remote Canadian communities and in institutional settings. It has been shown that transgender individuals have higher rates of mental health morbidity than the general population. This is coupled with the stigma and isolation of remote and rural communities.

Telemental health has been successful in providing psychiatric healthcare to remote communities throughout Canada. This paper examines whether telemental health has also been used to remove the barriers to care for the transgender and gender diverse population.

Methods.- The authors reviewed published literature on the use of e-health (telemental health, tele psychiatry, and others) to remote, rural, institutional and minority communities in Canada, to find data about its effectiveness in these communities and whether or not the transgender community was represented.

Results.- Telemental health is used widely in many populations but there is little published data on its use and effectiveness in the transgender and gender diverse populations in Canada.

Conclusions.- Telemental health's use and effectiveness has been demonstrated in various rural, remote and minority populations throughout Canada. There is little published data on its use in bridging the gap in the lack of access to transgender individuals in remote and rural areas. There is a need for more research in the use in the transgender population.

Disclosure of interest.- The authors have not supplied a conflict of interest statement.

\section{E-PV0293}

\section{E - sapiens sapiens the next evolutionary step?}

G. Hesles Sanchez ${ }^{1}$, L. Moron Nozaleda ${ }^{2 *}$

${ }^{1}$ Universidad Miguel de Cervantes. Universidad Villanueva., Social sciences, Madrid, Spain; ${ }^{2}$ Pofessor Dr, Neuroscience, Madrid, Spain Corresponding author.

Background and aims.- We live with cyborgs, with humans repaired or restored thanks to pacemakers, automatic defibrillators, auditory transducers (cloquear implants), organs or bionic limbs, antennas to hear the colors (eyeborg), and there is great hope in the graphene retinas to recover vision.

After the repair or restoration, it usually comes the improvement. As in the intellectual field we achieved hundreds of years ago with pencils and papers- to increase our memory and collective memory: what can we achieve with AI applied exclusively to the neurophysiology of human being?

Our intention is to demonstrate that AI has no path in neurophysiology without natural intelligence. While the second is optimal to get answers, the first is vital to raise the questions that really allow us to advance in this man-machine symbiosis. Our study raises the need to generate the right questions, that is, to formulate a projection of the possible applications of AI to neurophysiology.

Methods.- General research. Specialized publicatios, journals, websites.

Results.- The objective is to add the best of human abilities, intuition, creativity, empathy, with computational skills, which we will call e-sapiens.

Conclusions.- Natural intelligence has allowed - for good, for evil in some cases - to end natural selection. And in full artificial selection we face the improved human, with an increased intelligence that thanks to implanted brain-machine interfaces will connect people to computers that will allow the former to process more data (big data) at a higher speed, discarding failed roads before traveling over them, and also, of course, detect, diagnose and eradicate mental illness.

Disclosure of interest.- The authors have not supplied a conflict of interest statement. 


\section{E-PV0294}

\section{Teleconsultation in major depressive} disorder

J. Mota-Pereira ${ }^{1 *}$, D. Fonte ${ }^{1}$, R.J. Teixeira ${ }^{2}$, S. Carvalho ${ }^{3}$

${ }^{1}$ Clínica Médico-Psiquiátrica da Ordem, Psychiatry, Porto, Portugal; ${ }^{2}$ Clínica Médico-Psiquiátrica da Ordem, Psychology, Porto, Portugal; ${ }^{3}$ Hospital de Magalhães Lemos, Porto Psychiatric Department, Porto, Portugal

${ }^{*}$ Corresponding author.

Background and aims.-

Introduction.- Due to the severity of symptoms associated with Major Depressive Disorder (MDD), some patients have difficulties in attending the appointments with their psychiatrist, such as the distance to the clinic, financial difficulties or agoraphobia. Skype removes all these barriers, and could promote not only more regular medical appointments but also, and not less important, allow for patients with agoraphobia to have appointments, since some of these patients refuse to leave home. The understanding of the impact of psychiatry consultations by videoconference platforms on clinical results and wellbeing of the patients is important for the adaptation and modification of the psychiatric clinical practice. Objectives.- The aim of this work was to assess the efficacy of Skype in patients with treatment-resistant major depressive disorder (TRMDD) with or without other psychiatric co-morbidities, such as agoraphobia or generalized anxiety disorder.

Methods.- One-hundred and seventy patients were divided into two groups, one office consultation group $(n=85)$ and a Skype consultation group $(n=85)$. All patients maintained their usual pharmacotherapy. All participants were evaluated at baseline, 6 and 12 months for depressive symptoms (HAMD17), disease severity (CGI-S) and function (GAF).

Results.- There were no differences in these three parameters among the two groups, over time. However, the percentage of patients in remission after 12 months was higher in the Skype group.

Conclusions.- Psychiatric online consultations are more effective than office (face-to-face) consultations regarding remission of patients with major depressive disorder, allowing them to overcome the barriers caused by the distance or some co-morbidities. Disclosure of interest.- The authors have not supplied a conflict of interest statement.

\section{E-PV0295}

\section{Present-day scope for psychological internet counseling in mental health problems}

\section{E. Nikolaev", V. Ivanov}

Ulianov Chuvash State University, Social and Clinical Psychology

Department, Cheboksary, Russia

* Corresponding author.

Background and aims.- Information and communication technologies have gradually entered nearly all spheres of life bringing in each something novel. Internet-based psychological counseling for mental health problems has also joined the cause. Originally, professional psychological help had been available only face-toface during a patient's personal visit to a psychologist. Steady development of the Internet technologies opened new ways of psychological services. More people with mental disorders seek help on the Internet, which is more advantageous.

Methods.- Based on the analysis of both the authors' personal practical experience and a wide range of offers of psychological counseling available today on the Internet, below, we describe the potentials and restrictions of online psychological counseling. Results.- The advantages of online psychological counseling include absence of boundaries, saving a patient's time and a psycholo- gist's costs, a patient's possibility to choose a specialist, possibility of urgent assistance, location of a patient in their personal comfortable zone, availability of assistance for disabled people. Online counseling can be carried out in the form of text messages via email, forums, chatting, SMS, or audio or video counseling via Skype, Webinar Rooms, Viber, WhatsApp, etc. It can address one individual or a group in a real-time or delayed-response mode. The main restrictions are ethical and judicial, psychological.

Conclusions.- The described potentials open good prospects for the development of both mental health services and psychologists. Psychological Internet counseling may be used as a separate measure or may be combined with face-to-face practices of psychological interventions in case of mental health problems.

Disclosure of interest.- The authors have not supplied a conflict of interest statement.

\section{E-PV0296}

\section{Mental health mobile apps: a new therapeutic tool}

S. Petrykiv ${ }^{1^{*}}$, L. de Jonge ${ }^{2}$, M. Arts ${ }^{3}$

${ }^{1}$ Maastricht University Medical Center, Department of Clinical Pharmacy and Pharmacology, Maastricht, The Netherlands; ${ }^{2}$ Leonardo Scientific Research Institute, Department of Neuropsychiatry, Groningen, The Netherlands; ${ }^{3}$ GGZ WNB Mental Health Care, Department of Old Age Psychiatry and Neuropsychiatry, Halsteren, The Netherlands

"Corresponding author.

\section{Background and aims.-}

Background.- Mental health mobile apps represent a new alternative to enlarge the quality and availability of psychiatric treatment. Although these types of treatment seem to be cost-effective, it's efficacy remains questioned. In this study, we provide an overview of apps that are applicable for mental health.

Aims.- To present the efficacy of evidence-based mobile apps for the treatment of mood disorders, anxiety, and psychotic disorders. Methods.- An English language literature search was conducted using Pubmed and EMBASE searching for reporting the efficacy of mental health software applications.

Results.- Preliminary evidence indicates that mental health mobile apps show promise in improving medication adherence, reduce anxiety symptoms, decreasing psychotic and depressive symptoms.

Conclusions.- Mobile apps for the treatment of psychiatric disorders have expanded rapidly and have a considerable potential. However, the heterogeneity and often poor quality still hinder any explicit conclusions.

Disclosure of interest.- The authors have not supplied a conflict of interest statement.

\section{E-Poster Viewing - 7-9 April: Eating Disorders}

\section{E-PV0298 \\ Variants of dysmorphophobic disorders in children and adolescents with anorexia nervosa and bulimia \\ nervosa}

A. Bryukhin, I. Belokrylov, T. Lineva, I. Danilin*, E. Okonishnikova, G. Kirsanova

Peoples' Friendship University of Russia RUDN University, Department of psychiatry and medical psychology, Moscow, Russia " Corresponding author. 
Background and aims.- Previously, the occurrence of anorexia nervosa (AN) and bulimia nervosa (BN) in childhood and adolescence was considered as casuistry. In recent decades, we have observed a clear pathomorphosis - typical anorectic and bulimic symptoms that formed already in childhood, Identification, classification, study of pathogenesis and dynamics of dysmorphophobia in childhood and adolescence in patients with $\mathrm{AN}$ and $\mathrm{BN}$ were the aims of the study.

Methods.- 65 patients with AN and BN aged from 12 to 18 years were examined. The duration of the disease is from 3 months to 7 years. Methods of research: anamnesis, clinicalpsychopathological, anthropometric, catamnestic, psychometric scales - Questionnaire image of one's own body, the Scale of satisfaction with one's body.

Results.- Dissatisfaction with their body in $43 \%$ was severe, $25 \%$ moderate, $32 \%$ light. $42 \%$ were not satisfied with the head, $54 \%$ not satisfied with the body and $57 \%$ with the lower part of the body. In $40 \%$ of patients was observed polydysmorphophobia. In $30 \%$ of patients dysmorphophobia occurred in childhood, 70\% - in early adolescence.

Conclusions.- In most patients with $\mathrm{AN}$ and BN, dysmorphophobia occurred at an early age, was often polythematic, determined the quality of life, influenced the course, outcomes and prognosis of the disease.

The publication was prepared with the support of the "RUDN University Program 5-100".

Disclosure of interest.- The authors have not supplied a conflict of interest statement.

\section{E-PV0299}

\section{Representatiom of anorexia nervosa in caregivers, working with children and adolescents in a mental healthcare centre}

D. Dovbysh ${ }^{1,2}$, E. Podurova $^{1}$

${ }^{1}$ Scientific-Practical Children's and Adolescents Mental Health Center n.a. G. Sukhareva- Moscow Department of Health Care-MoscowRussia, Psychiatry, Moscow, Russia; ${ }^{2}$ Federal State Autonomous Educational Institution of Higher Education I.M. Sechenov First Moscow State Medical University of the Ministry of Health of the Russian Federation Sechenov University, Faculty of Higher Nursing Training- Psychology and Social Work, Moscow, Russia Corresponding author.

Background and aims.- Despite the fact that anorexia nervosa officially belongs to DSM since the first edition (1952) and has long been considered as a mental disorder, in modern society it is often not even nowadays considered to be a serious, life threatening disease.

Assess the idea about anorexia nervosa after undergoing an educational destigmatizing program.

Methods.- A training seminar about eating disorders in advanced training course; structured interview, built around the analysis of cases of anorexia nervosa in adult and adolescent patients.

Results.- $97 \%$ of participants consider anorexia nervosa a mental disorder, but in only $73 \%$ of the cases psychiatrist was recommend. $42 \%$ of the participants on the direct question of the parents' fault in the formation of anorexia nervosa answer "yes», $33 \%$ would blame the person for illness. $80 \%$ of the respondents would dissuade relatives from getting married with an adult with symptoms of for work in the absence of doubt in his professional skills. There are no statistically significant differences between groups of participants with different experience in psychiatry. A separate result of the work is the selection of the most popular symptoms of anorexia in terms of employees.

Conclusions.- Lack of professional knowledge about the specific features of eating disorders among professionals working in psy- chiatry potentially reduces quality of care and contributes to the formation of distorted ideas about anorexia nervosa in patients and in society.

Disclosure of interest.- The authors have not supplied a conflict of interest statement.

\section{E-PV0301 \\ Protocol for investigating the cardiometabolic profile of individuals with anorexia nervosa}

Z. Jenkins ${ }^{1 *}$, A. Phillipou ${ }^{2}$, D. Castle ${ }^{3}$, E. Lambert ${ }^{4}$

${ }^{1}$ St. Vincent's Hospital, Mental Health, Melbourne, Australia; ${ }^{2}$

Swinburne University of Technology, Centre for Mental Health,

Melbourne, Australia; ${ }^{3}$ St. Vincent's Hospital \& the University of

Melbourne, Psychiatry, Melbourne, Australia; ${ }^{4}$ Swinburne University

of Technology, Iverson Health Innovation Research Institute,

Melbourne, Australia

Corresponding author.

Background and aims.- The energy deprivation and malnutrition associated with Anorexia Nervosa (AN) places immense pressure on the cardiovascular system, with up to $80 \%$ of patients suffering from cardiovascular or metabolic complications, of which the aetiology remains poorly understood. Research suggests that cardiovascular and metabolic abnormalities are linked to disturbances in nerve activity called autonomic dysfunction. This research will specifically investigate sympathetic nervous system aberration in AN and its relationship to organ damage and metabolic abnormalities by measuring autonomic function in individuals with AN. It is hypothesised that the AN group will demonstrate atypical sympathetic nervous system compared to the control group, and this will be related to increased cardio-metabolic disturbances.

Methods.- Participants will include 30 individuals with AN, 30 recovered from AN and 30 matched controls who will be assessed on measures of autonomic nervous system function including; nerve activity, endothelial function, arterial stiffness and sudomotor function. Participants will provide blood and urine samples which will undergo lipidomic and metabolomic analysis.

Results.- All assessment variables will be compared between the two AN and the control group with analysis of variance (ANOVAs). Pearson's and Spearman's correlations will be used to evaluate the relationships among different variables.

Conclusions.- It is hypothesised that the AN group will demonstrate atypical ANS activity compared to the control group, and this will be related to increased cardiometabolic disturbances. Understanding the underlying mechanisms of organ and metabolic dysfunction associated with AN will allow clinicians to establish effective prevention and treatments to prevent long term organ damage in AN. Disclosure of interest.- The authors have not supplied a conflict of interest statement.

\section{E-PV0302}

\section{Patients with eating disorders:} outcome inpatient care

J. Jerónimo*, J. Rema, T. Cavaco, E. Reis, J. Santos

Centro Hospitalar Lisboa Norte, Serviço de Psiquiatria e Saúde Mental, Lisboa, Portugal

* Corresponding author.

Background and aims.- Eating disorders (ED) are characterized by a persistent disturbance of eating behavior that results in altered consumption of food and significantly impairs physical health or psychosocial functioning.

ED classified in DSM-5 are anorexia nervosa (AN), bulimia nervosa (BN), binge eating disorder, pica, rumination disorder, avoidant/restrictive food intake disorder (ARFID), other specified 
feeding or eating disorder (OSFED) and unspecified feeding or eating disorder (UFED).

ED' treatment is complex and challenging and sometimes hospitalization is needed. Criteria for hospitalization in ED are defined and NICE clinical guidelines are among the most frequently used.

To present and discuss our units results of the inpatient treatment program.

Methods.- Review of clinical files of all patients hospitalized at our unit from 1 January 2013 to 31 August 2018 followed by statistical analysis of data. Treatment outcome was assessed by body mass index (BMI) variation. BMI was determined at baseline and discharged.

Results.- Anorexia nervosa was the most frequent diagnosis. A remarkable high medical and/or psychiatric comorbidity was presented. When looking for compensatory behaviors presented by inpatients we notice the major predominance of vomiting, either isolated, or in association with misuse of laxatives.

Conclusions.- Inpatient treatment for patients with eating disorders in our unit is considered only for those whose disorder has not improved with appropriate outpatient treatment, associated with high or moderate physical risk, or for whom there is a significant risk of suicide or severe self-harm. So, most inpatients at have disorders of high severity.

Disclosure of interest.- The authors have not supplied a conflict of interest statement.

\section{E-PV0303}

\section{Anorexia nervosa and mesenteric superior artery syndrome}

\section{J. Jerónimo*, T. Cavaco, E. Reis, J. Rema, J. Santos}

Centro Hospitalar Lisboa Norte, Serviço de Psiquiatria e Saúde

Mental, Lisboa, Portugal

${ }^{*}$ Corresponding author.

Background and aims.- The superior mesenteric artery syndrome (SAMS) results from compression of the third portion of the duodenum, limited posteriorly by the aorta and anteriorly by the superior mesenteric artery. The simultaneous diagnosis of SAMS and anorexia nervosa (AN) is rare, and there are only a few cases in the literature.

The relationship between these pathologies, with common clinical manifestations, is not consensual. Presentation of a literature review of simultaneous diagnosis of SAMS and AN.

Methods.- Bibliographical search in the PubMed database, using as keywords "anorexia nervosa" and "mesenteric superior artery".

Results.- Rapid weight loss increases the likelihood of duodenal compression by reducing the fat layer that protects the artery, which is associated with nausea, vomiting, epigastric pain and gastric distension, that may be perpetuators of food restriction. Delayed diagnosis implies an increased risk of complications.

In AN, patients with aorto-mesenteric angle narrowing may develop multiple complications during food reintroduction and episodes of food voracity.

Conclusions.- The complications of SAMS are life-threatening, and its early recognition and the adoption of preventive therapeutic strategies are important. The progressive weight gain has been shown to be essential as a therapeutic intervention. However, evaluation by Gastroenterology/General Surgery must not be dispensed.

Disclosure of interest.- The authors have not supplied a conflict of interest statement.

\section{E-PV0306}

\section{Trauma, emotion regulation}

difficulties and binge eating

C. Alves ${ }^{1}$, M. Marques ${ }^{2 *}$, A.C. Ribeiro ${ }^{1}$, P. Correia ${ }^{1}$, S. Soares ${ }^{1}$, P. Silva $^{1}$, L. Lemos ${ }^{3}$, S. Simões ${ }^{3}$, B.R. Maia ${ }^{4}$

${ }^{1}$ Miguel Torga Higher Institute, Research and Development Department, Coimbra, Portugal; ${ }^{2}$ Miguel Torga Higher Institute ISMT, Research and Development Department ISMT-Clinical Psychology Unit CHUC, Coimbra, Portugal; ${ }^{3}$ Miguel Torga Higher Institute, Research and Development Department, Coimbra, Portugal;

${ }^{4}$ Faculty of Philosophy and Social Sciences, The Catholic University of Portugal- Regional Centre, Braga, Portugal

* Corresponding author.

Background and aims.- The growing research interest in binge eating symptoms is associated to its high comorbidity with obesity and psychiatric symptoms, its related health complications and growing prevalence rates. As a multidetermined phenomenon, it is crucial to continue exploring its correlates, particularly in the general population (not only in clinical samples). Our main goals are: to explore if traumatic experiences ocurrence and emotional regulation difficulties are associated with (and predict) binge eating symptoms; to verify if emotional regulation difficulties mediate the relation between having experienced traumatic experiences and binge eating symptoms.

Methods.- 421 university students and adults from the general population (women, $n=300,71,3 \%$ ) answered a sociodemographic questionnaire, the Traumatic Events Checklist (TEC), the Difficulties in Emotion Regulation Scale (DERS) and the Binge Eating Scale (BES).

Results.- $5.9 \%$ of the sample presented mild to moderate binge eating symptoms and $2.6 \%$ severe binge eating symptoms, similar values to those found in Portuguese studies using the BES. Binge eating total score positively correlated with family trauma and traumatic experiences total score (TEC). It also positively correlated with impulses, non-acceptance, objetives, strategies and lack of emotional clarity (DERS), and with Body Mass Index (BMI). The binge eating total score, reported weight at childhood, BMI, traumatic experiences total score and strategies (DERS) significantly predicted binge eating total score. We did not found any mediation effect.

Conclusions.- These results show the importance of traumatic life experiences and of emotion regulation difficulties as correlates of binge eating symptoms, in a sample of university students and people from the general population.

Disclosure of interest.- The authors have not supplied a conflict of interest statement.

\section{E-PV0307 \\ Orthorexia nervosa: when healthy eating becames a problem}

F. Martins Alves ${ }^{1^{*}}$, A. Fornelos ${ }^{2}$, I. Brandão ${ }^{3}$

${ }^{1}$ Local Health Unit of Alto Minho- EPE, Department of Psychiatry and Mental Health, Viana do Castelo, Portugal; ${ }^{2}$ Hospital Center of Trás-os-Montes e Alto Douro- EPE, Department of Psychiatry and Mental Health, Vila Real, Portugal; ${ }^{3}$ Hospital Center of São João, Psychiatry and Mental Health Clinic, Porto, Portugal

* Corresponding author.

Background and aims.- Food is essential to life. Globalization has led to excessive worship for a "perfect" body image and the valuing of healthy lifestyles as a way to prevent chronic and potentially disabling diseases.

With this work, we pretend to disseminate the concept of Orthorexia Nervosa, understand its characteristics and possibly harmful consequences.

Methods.- A literature review using the MEDLINE ${ }^{\circledR}$ database. The keywords "orthorexia nervosa" and "eating disorders" were used. 
Selected articles based on the content and relevance of the abstract and date of publication (from 2015 to present).

Results.- Orthorexia Nervosa was first described in 1997. Currently, it is estimated that its prevalence varies between $6.9 \%$ in the general population and $57.8 \%$ in populations at risk. It is characterized by a pathological obsession directed at the quality of food in order to optimize physical health and well-being. Instead of changing the quantities of food, there is a demand for healthier, pure and natural foods. However, an extreme preoccupation with food, time spent in planning and obtaining it, and restricting food variety can lead to nutritional deficits, severe weight loss, and organic complications. Conclusions.- The change in eating patterns has created an environment conducive to the emergence of new pathologies of eating behavior. Adherence to a rigorous and healthy diet is seen in the Western world as acceptable and desired, generating feelings of admiration for others. It is important to become more aware that the behaviors characteristic of orthorexics, performed in an obsessive, rigid and repetitive way can lead to physical and psychosocial dysfunction.

Disclosure of interest.- The authors have not supplied a conflict of interest statement.

\section{E-PV0310}

\section{Excessive physical exercise in eating disorders: a conceptual discussion}

M. Ouellet", J. Monthuy-Blanc

University of Québec at Trois-Rivières, 3351, Boul. des Forges, Trois-Rivières, Québec (Canada) G8Z 4M3

* Corresponding author.

Excessive physical exercise (EPE) is a common inappropriate compensatory behavior, as induced vomiting or use of laxative, often employed by patients with an eating disorder. The lifetime prevalence of EPE varies between $26 \%$ and $80 \%$ depending on the samples and measures (Davis \& Kaptein, 2006; Levallius, Collin \& Birgegard, 2017). EPE is defined as an abnormal amount of physical activity combined with a compulsion to exercise. This behavior leads to harmful psychosocial and physical consequences such as impaired social relations, injuries, potassium drops risk and death often involve cardiac arrest or suicide. This symptom is very worrisome for clinical teams because exerciser patients have poorer prognoses than non-exerciser patients (Stiles-Shields et al., 2015). Although most inappropriate compensatory behaviors are well controlled during hospitalization, the use of EPE remains frequent and persistent during intervention programs, as well as after recovery, thereby leading to a higher risk of relapse (Meyer, Taranis, \& Touyz, 2008). However, it is difficult for clinical teams to assume care for this symptom because of the lack of empirical knowledge about EPE. This could be explained by the polysemy leading to a multitude of definitions in the literature referring to EPE (Probst et al., 2014). If some authors consider only the quantitative component of EPE in their studies, others use only the compulsive component, while still others consider both. This problem could lead to biased results. The necessity to consider both the quantitative and compulsive components of EPE in researches will be discussed.

Disclosure of interest.- The authors have not supplied a conflict of interest statement.

\section{E-PV0311}

\section{Relation between physical self-concept and excessive physical exercise in eating disorders: preliminary results}

M. Ouellet", J. Monthuy-Blanc

University of Québec at Trois-Rivières, 3351, boul. des Forges, Trois-Rivières, Québec (Canada) G8Z 4M3

* Corresponding author.
Anorexia nervosa and bulimia nervosa are among the most common chronic diseases in adolescents and young adults (Garner, 2004). Among inappropriate compensatory behaviors such as induced vomiting or laxative abuse, excessive physical exercise (EPE) is very worrisome for clinical teams since exerciser patients often have poorer prognoses than non-exerciser patients (StilesShields et al., 2015). EPE is defined as an abnormal amount of physical activity combined with a compulsion to exercise. Although the relation between poor physical self-concept and ED is well established (APA, 2013), to our knowledge the relation between physical self-concept and EPE has never been studied. This research aims to document the relation between physical self-concept and EPE. The sample of this cross-sectional study comprises ED patients between the ages of 14 and 26 years who participate in a ED intervention program. The Physical Self-Inventory (Maiano et al., 2008) and the Exercise and Eating Disorder test (Danielson et al., 2014) are used to measure variables. Preliminary results based on 15 patients indicate that there is a positive relation between sport competence and EPE quantity $(r=0,650, p$.

Disclosure of interest.- The authors have not supplied a conflict of interest statement.

\section{E-PV0312 \\ The role of rumination in the relationship between perfectionistic self-presentation and eating psychopathology}

R. Silva1 ${ }^{1}$, C. Marques ${ }^{2}$, A.T. Pereira ${ }^{2 *}$, A. Macedo ${ }^{2,3}$

${ }^{1}$ University of Coimbra, Faculty of Medicine, Coimbra, Portugal; 2

Faculty of Medicine - University of Coimbra, Institute of Psychological Medicine, Coimbra, Portugal; ${ }^{3}$ Hospital and University Centre,

Psychiatry Department, Coimbra, Portugal

* Corresponding author.

Background and aims.- Rumination is defined as a maladaptive emotion regulation strategy where the individual has compulsive and repetitive thoughts on the meaning, causes and consequences of his distress. It is generally accepted a bidimensional model of rumination - brooding and reflection. Specific rumination about eating, weight and shape has been consolidated in the literature as a core feature in eating psychopathology. Perfectionistic selfpresentation, as a personality trait concerning the need to appear perfect to others, has also shown a particular association with eating psychopathology, and ruminative thoughts on eating, weight and shape may have an important role in the maintenance of that relation.

Objectives.- The present study analyses the mediating role of brooding and reflection in the relationship between perfectionistic self-presentation and eating psychopathology, controlling for gender.

Methods.- A total of 98 university students ( 57 females and 41 males) completed a battery of self-report scales measuring perfectionistic self-presentation, rumination and eating psychopathology.

Results.- All the variables showed significant and positive correlations (0.26-0.78) between them. Moreover, the mediation analyses revealed that both ruminative reflection and brooding on eating, weight and shape concerns are mediators of the relationship between perfectionistic self-presentation and eating psychopathology.

Conclusions.- These findings seem to show that perfectionistic self-presentation may impact on eating psychopathology through rumination (both brooding and reflection). Also, therapeutic and prevention strategies can target perfectionistic self-presentation and rumination on eating, weight and shape concerns to diminish eating psychopathology. 
Disclosure of interest.- The authors have not supplied a conflict of interest statement.

\section{E-PV0313 \\ Eating disorders: a literature review and case study}

H. Raai*, M. Rovner, H. Forman, M.A. O’Dowd

Montefiore Medical Center / Albert Einstein College of Medicine, Department of Psychiatry and Behavioral Sciences, New York, USA

* Corresponding author.

Background and aims.- The aim of this study is to critically review the literature of eating disorders, exploring prevalence, clinical features, comorbidities, and, association of eating disorders with suicidal ideation and attempts. We will also discuss the case of a 23-year-old woman with anorexia nervosa, who was admitted to the medical unit due to a hypoglycemic episode in the context of refusing to eat or drink for several days and excessive exercise.

Methods.- Case study and literature search of the terms "Anorexia Nervosa", "Bulimia Nervosa" and "Eating Disorders" using PubMed and selected relevant articles published in last 10 years in peerreviewed journals.

Results.- The etiology of eating disorders is unknown and probably multifactorial. Environmental influences, such as societal idealization about weight and body shape, play a major role. The review of the literature has shown that eating disorders have a high level of comorbidity with other psychiatric disorders, such as anxiety disorder and depression. Patients with eating disorders have a high rate of suicidal ideations and attempts and have the highest mortality rate of any mental disorder. Recent evidence suggests that cognitive-behavioral therapy is often effective treatment for eating disorders. Antidepressants, including SSRIs, may help mitigate symptoms of depression and suicidal ideations in these patients. Collaboration between different specialties may optimize treatment outcome.

Conclusions.- Physicians need to be diligent in screening patients for eating disorders, evaluate for other comorbid psychiatric conditions and conduct a thorough suicide risk assessment.

Disclosure of interest.- The authors have not supplied a conflict of interest statement.

\section{E-PV0314 \\ Fatal anorexia nervosa case with extremely low BMI - an important correlation between clinical pathology and forensic medicine}

R. Skowronek ${ }^{1 *}$, I. Jelonek ${ }^{2}$, A. Skowronek ${ }^{3}$, A. Pałasz ${ }^{4}$, P. Paleń ${ }^{5}$, R. Wojnicz $^{6}$

${ }^{1}$ Medical University of Silesia in Katowice, Department of Forensic Medicine and Forensic Toxicology, Katowice, Poland; ${ }^{2}$ Medical University of Silesia in Katowice, Developmental Age Clinical WardDepartment of Psychiatry and Psychotherapy, Sosnowiec, Poland; ${ }^{3}$ Outpatient Clinic for Mentally and Nervous Sick NZOZ "Integritas", Outpatient Clinic for Mentally and Nervous Sick NZOZ "Integritas", Oświęcim, Poland; ${ }^{4}$ Medical University of Silesia in Katowice, Department of Histology, Katowice, Poland; ${ }^{5}$ Medical University of Silesia in Katowice, Department of Pathomorphology and Molecular Diagnostics, Katowice, Poland; ${ }^{6}$ Medical University of Silesia in Katowice, Department of Histology and Cell Pathology, Zabrze, Poland ${ }^{*}$ Corresponding author.

Background and aims.- Anorexia nervosa (AN) is considered the most lethal among all psychiatric disorders. It is relatively rarely seen in forensic medical practice, but sometimes sudden death of the young people raises suspicions of the prosecutor regarding the circumstances and mechanism of death. The objective was presentation of rare fatal anorexia nervosa case with extremely low BMI with particular attention paid to the histopathological changes in the heart.

Methods.- Analysis of available medical records and post-mortem findings.

Results.- The body of a 24-year-old girl was disclosed by her sister in their flat. The prosecutor decided to order the medicolegal autopsy. Post mortem BMI was $9.3(157 \mathrm{~cm}, 23 \mathrm{~kg})$. Except the features of extreme cachexia and typical symptoms of sudden death there were no other lesions. The weight of the heart was $93 \mathrm{~g}$, while in the woman's age it should be about $160 \mathrm{~g}$. Detailed cardiopathological examination revealed mainly the features of severe atrophic changes in all microstructures: cardiomyocytes, parasympathetic ganglia, vascular endothelial and smooth muscle cells. In general, the histopathological image of the heart analyzed under smaller magnification resembled intriguingly a morphology typical for neonatal organ. In the course of the investigation, it turned out that woman had been suffering from AN for 9 years. At that time, she was in the outpatient clinic only twice and once she was hospitalized in a psychiatric ward for several days.

Conclusions.- If left untreated, AN causes serious, life-threatening atrophic changes in the myocardium which may often result in sudden functional death.

Disclosure of interest.- The authors have not supplied a conflict of interest statement.

\section{E-PV0315 \\ Learning problems in adolescent girls with anorexia nervosa}

N. Zvereva ${ }^{1}$, S. Voronova ${ }^{2}$

${ }^{1}$ FBSI MHRC-MSUPE, Clinical Psychology, Moscow, Russia; ${ }^{2}$ Mental Health Research Center, Department of Clinical Psychology, Moscow, Russia

* Corresponding author.

Background and aims.- Now days the number of adolescents with eating disorders increases, anorexia nervosa (NA) is one of them. Having psychiatric diagnosis adversely affects the education of girls. We know specific features of patients with NA, found mainly in adult patients: perfectionism, social desirability, desire to show better results. Due to traumatic situation, activities aimed at learning and social activity are shifting to desire to correct real or imaginary shortcoming. The motive shifts to the goal, and leading activity is replaced. Psychological studies of girls with NA showed the presence of high motivation for achievements, and distortion of self-perception.

Aim Evaluation characteristics of educational trajectory of patients with NA in child psychiatry department.

Methods.- 27 girls (10-16 years old) with NA treated at child psychiatry department of Mental Health Research Center were quality assessed in anamnesis including learning problems and educational trajectory.

Results.- Data on the analysis of anamnestic materials related to the learning process are presented. Patients were divided into 2 groups: first (56\%) - patients without learning problem, second (44\%) - patients experience learning difficulties. Girls in first group were younger than in second, and did not have such a long illness. Conclusions.- NA in adolescents is often referred to as "excellent pupil disease", our empirical and clinical data do not confirm this NA characteristic completely, especially considering the different stages of the disease development and the age of onset. The question of the fate of a girl with NA and the presentation of adaptive or non-adaptive behaviors requires further research.

Disclosure of interest.- The authors have not supplied a conflict of interest statement. 


\section{E-Poster Viewing - 7-9 April: Emergency Psychiatry}

\section{E-PV0316}

\section{Psychiatric disorders revealing neurobrucellosis: about a case report}

M.H. Aoun", S. Zouari, H. Hadj Kacem, O. Hdiji, N. Farhat, S. Sakka, M. Damak, C. Mhiri

Habib Bourguiba, Neurology, Sfax, Tunisia

* Corresponding author.

Background and aims.- Brucellosis is caused by a gram-negative aerobic bacteria belonging to the Brucella genus. This disease is endemic in Tunisia. Its neuropsychiatric presentation accounts for 1.7 to $10 \%$ of cases. Here we describe a case with a twelve month history of psychiatric impairment, who was later diagnosed with neurobrucellosis.

Methods.- This is a 48-year-old male patient, with a history of raw milk consumption, who presented at our department for psychiatric disorders that have been evolving for an year. We reviewed his clinical and paraclinical data.

Results.- At questionnaire, the patient's relatives reported recurrent transient confusional states associated with psychomotor agitation and insomnia during last year. Few months before his admission, he became displaying atypical absences. Later, our patient suffered from severe headache and vomiting, which motivated his admission. At clinical examination, we found a conscious patient but reluctant, disoriented, agitated without delusions nor hallucination. He also exhibited hypoacusis and a stiffness of the neck. Cerebrospinal fluid analysis showed hyperalbuminorrachia, hypoglycorrachia and elevated white elements counts with isolation of Brucella SPP species on cultures. Wright serology was positive at $1 / 320$. Brain MRI showed sub-cortical white matter hyperintensities without gadolinium enhancement. Our patient was kept on antibiotic with a good clinical outcome.

Conclusions.- This case raises the importance of considering neurobrucellosis among patients with psychiatric disorders originating from endemic regions.

Disclosure of interest.- The authors have not supplied a conflict of interest statement.

\section{E-PV0317}

\section{Cerebral sinus venous thrombosis revealed by psychiatric disorders:} about a clinical case

M.H. Aoun", O. Hdiji, N. Farhat, S. Sakka, H. Hadj Kacem, M. Damak, C. Mhiri

Habib Bourguiba, Neurology, Sfax, Tunisia

Corresponding author.

Background and aims.- Cerebral sinus venous thrombosis (CSVT) is a rare phenomenon that can be seen among old and young patients. CSVT is a multifactorial condition with gender-related specific causes, with a wide clinical presentation. The leading causes differ between developed and developing countries, converting CSVT in a condition characterized by a highly variable clinical spectra, difficult diagnosis, variable etiologies and prognosis that requires fine medical skills and a high suspicious index. Here we describe a case with one month history of psychiatric impairment, who was later diagnosed with cerebral sinus venous thrombosis.

Methods.- This is a 64-year-old female patient, with a history of atrial fibrillation and phlebitis, who presented at our department for psychiatric disorders that have been evolving for one month. We reviewed his clinical and paraclinical data.

Results.- At questionnaire, the patient's relatives reported confusion, delirium, disrupted attention, having visual delusions, agitation during last month. At clinical examination, we found a conscious patient but reluctant, disoriented, agitated with delusions and visual hallucinations. Neurological exam was normal. All her biological tests were normal. Cerebral CT-Scan and MRI showed venous thrombosis of the left lateral sinus and left jugular vein. Our patient was kept on heparin then switched by vitamin K antagonist with a good clinical outcome.

Conclusions.- This case raises the importance of considering cerebral sinus venous thrombosis among patients with confusion status associated with psychiatric disorders evolving for a short period. Disclosure of interest.- The authors have not supplied a conflict of interest statement.

\section{E-PV0318}

\section{A resistant depression revealing neuropsychiatric Behçet disease: about a case report}

M.H. Aoun*, O. Hdiji, H. Hadj Kacem, S. Sakka, N. Farhat, M. Damak, C. Mhiri

Habib Bourguiba, Neurology, Sfax, Tunisia

* Corresponding author.

Background and aims.- Behçet's disease is a multisystem panvasculitis of unknown etiology originally described as a syndrome of oral ulcers, genital ulcers, and iritis. Neuropsychiatric involvement occurs in about $20 \%$ of the cases, usually as a transient but sometimes fatal episode of acute illness. Psychiatric presentation has not been well described. Dementia has been reported occasionally. Here we describe a case with a twelve month history of psychiatric impairment, who was later diagnosed with Neuropsychiatric Behçet Disease.

Methods.- This is a 50-year-old male patient, without any medical history, who presented at our department for psychiatric disorders that have been evolving for a year. We reviewed his clinical and paraclinical data.

Results.- The patient was a father of four children. His wife committed suicide. Then he developed sadness, anorexia, asthenia, weight loss. He consulted a psychiatrist. At examination, the patient had depressed mood, anhedonia, psychomotor slowdown and loss of vital momentum. He was prescribed antidepressants. His depression was resistant against three types of antidepressants for eight months. Later, the patient developed progressive worsening memory loss, disabling tremor and urinary signs. At clinical examination we found a conscious patient but disoriented. The patient had Dementia Syndrome, Parkinson's syndrome, oculomotor disorders and vesico-sphincteric disorders. Brain MRI showed demyelinating lesions of basal ganglia. Cerebrospinal analysis revealed a meningitis and ophthalmological examination showed uveitis. The patient was diagnosed with Neuropsychiatric Behçet Disease and he was kept on immunosuppressors (Cyclophosphamide). Sixth months later, the patient died.

Conclusions.- This case raises the importance of considering organic causes among patients with resistant depression.

Disclosure of interest.- The authors have not supplied a conflict of interest statement.

\section{E-PV0319}

\section{A case of paraneoplastic limbic encephalitis during the course of depression}

M.H. Aoun*, S. Sakka, N. Farhat, H. Hadj Kacem, O. Hdiji, M. Damak, C. Mhiri

Habib Bourguiba, Neurology, Sfax, Tunisia

* Corresponding author. 
Background and aims.- Limbic encephalitis is characterized by irritability, depression, sleeping disorders, seizure, hallucination and short-term memory loss. Most of the patients evolve with confusion and epileptic temporal lobe seizures. Previously associated with cancer, recent evidence suggests that limbic encephalitis in fact more often occurs independently of cancer, as an autoimmune phenomenon.

Methods.- Here we describe a first case report with a two-week history of psychiatric impairment, who was later diagnosed with Limbic Encephalitis.

Results.- This is a 32-year-old female patient, with a history of Thyroid Papillary carcinoma. She had a total thyroidectomy in 2015 with Iodotherapy. She had been taking since Levothyroxin. She presented apathy, anorexia and adynamia with memory loss. Two weeks later, she presented an epileptic seizure. She was started on carbamazepine $400 \mathrm{mg} /$ day. Laboratory assessment and Cerebrospinal fluid (CSF) were normal. CSF was negative for herpes simplex virus via PCR. CSF cytology was negative, and CSF protein electrophoresis did not identify any oligoclonal bands. MRI of the brain showed hyperintense signals from both hippocampi, highly suggestive of limbic encephalitis presenting as a paraneoplastic manifestation of papillary carcinoma. EEG showed a disorganized background with bitemporal slowing consistent with encephalopathy.

Conclusions.- Paraneoplastic neurological syndromes are rare occurring in less than $1 \%$ of patients with malignancies. It is important to consider paraneoplastic syndromes such as limbic encephalitis early on when incongruous neuropsychiatric symptoms are present, as early diagnosis of malignancy and prompt initiation of treatment can lead to improvement in the neuropsychiatric manifestations.

Disclosure of interest.- The authors have not supplied a conflict of interest statement.

\section{E-PV0320}

\section{Disaster management and psychiatry: a review \\ J. Fonseca*, E. Machado, R. Rodrigues \\ Hospital Senhora da Oliveira- Guimarães, Psychiatry and Mental \\ Health, Guimarães, Portugal \\ ${ }^{*}$ Corresponding author.}

Background and aims.- Everyday people are exposed to disasters and traumas that strike unexpectedly and bring devastating physical and psychological consequences for the communities facing them. Disasters derive from disruptive events, such as natural calamities or human destructive acts, overcoming the adaptive capacity of a social group.

This scenario is very different from the regular clinical activities psychiatrists have to do, and faces them with challenges when an intervention is suddenly needed.

It is known that early interventions are important to help survivors mitigating suffering and to detect psychiatric symptoms in those affected before some chronic condition develops.

The aim of this work is to review the role of psychiatry in emergencies management.

Methods.- We proceeded to an electronic research of the latest articles in "Pubmed" as well as of consensus regarding disaster psychiatry.

Results.- In the aftermath of a disaster people tend to react in a particular pattern divided into a heroic phase, honeymoon phase, disillusionment phase and restoration phase.

The disillusionment phase can last from 3 to 36 months and it is the most critical period for an individual to develop a psychiatric disorder which is estimated to range from 8.6 to 57.3 percent. Although the risk is higher, not every people will be affected by a psychiatric disorder, but sub-syndromal signs of distress may appear, mostly somatic.
Post traumatic stress disorder, depressive and anxiety disorders, and substance abuse are the most reported in survivors.

Conclusions.- Psychiatrists play a pivotal role in a disaster setting and contact with this kind of interventions should be promoted. Disclosure of interest.- The authors have not supplied a conflict of interest statement.

\section{E-PV0321}

\section{The acute psychotic disorders in patients with alcoholic dependence in withdrawal state of alcohol}

\section{Kuzminov}

SI «Institute of Neurology- Psychiatry and Narcology of NAMS of

Ukraine», Department of Emergency Psychiatry and Narcology,

Kharkiv, Ukraine

* Corresponding author.

Background and aims.- The abstract presents the results of longterm studies of patients with acute psychotic disorder in 560 patients with alcohol dependence that has emerged in the state of alcohol withdrawal (primary hospitalization in the framework of this study).

The most of them were examined in a dynamics after a rehospitalization after 5 years due to acute psychosis.

The purpose of the study was to study the factors affecting the severity and clinical picture of acute psychotic disorders in the state of alcohol withdrawal.

Methods.- Clinical psychopathological, electrophysiological, statistical, catamnestic.

Results.- The polyetology of acute psychotic disorders in patients with alcohol dependence in the state of withdrawal was shown. At the same time it has been proved that in repeated hospitalizations, met alcoholic psychosis in most cases repeats the clinical picture of the primary clinical episode. The influence of the factors of permanent and temporary on the clinical picture and the pathology of alcoholic delirium have been studied. The impact of acute psychotic disorders on the course of alcohol dependence, including the formation of cognitive deficits was estimated. However, the reverse effect cognitive deficits on the severity of the psychotic disorder (assessed by the duration of the psychotic disorder and the threat to life) were significantly less.

Conclusions.- The severity of the acute psychotic disorder in the state of alcohol withdrawal mostly depended situational factors such as the number of days of severe drinking before a psychotic disorder, the pattern of nutrition, the quality and quantity of alcohol consumed, the presence of acute somatic diseases.

Disclosure of interest.- The authors have not supplied a conflict of interest statement.

\section{E-PV0322 \\ Wernicke's encephalopathy and bipolar disorder at the emergency unit: a case report}

D. Rentero*, I. Torío, M. Caballero-González, E.M. Sánchez-Morla, M. Aragües, R. Rodríguez-Jiménez

Hospital Universitario 12 de Octubre, Psychiatry, Madrid, Spain

* Corresponding author.

Background and aims.- Wernicke's encephalopathy is the presence of neurological symptoms caused by biochemical lesions of the central nervous system after exhaustion of B-vitamin reserves, in particular thiamine (vitamin B1). The prevalence is around $2 \%$, and is considered underdiagnosed. Probably, many cases are in patients who do not have commonly-associated symptoms, as occur in patients with comorbid psychiatric diagnosis. 
Methods.- We present a case of 57 years old man who arrived to the emergency unit because of behavior disorders.

When we evaluated the patient, he had low level of consciousness. He presented myoclonus in all extremities and bilateral multidirectional nystagmus. The patient had a previous diagnosis of bipolar disorder and in recent months he had been living in a caravan in unhealthy conditions, with a diet based on water intake exclusively. Results.- Admission to the general hospital was carried out. After carrying out complementary tests, the patient had vitamin B1 deficiency as well as other vitamins such as folic acid. After resolving organic syndrome, a psychotic depression episode was diagnosed. Probably, this episode led to the state of isolation and malnutrition that the patient presented from the last weeks before arriving at the emergency unit.

Conclusions.- The coexistence of somatic and psychiatric diseases is not uncommon. As the case presented, when the symptoms are not completely clear and there are data of a certain organicity, it is important to work as a team with the rest of medical specialities ensuring an integral attention of our patients, always prioritizing the most serious pathology.

Disclosure of interest.- The authors have not supplied a conflict of interest statement.

\section{E-PV0323}

\section{Adult and pediatric frequent attenders in the psychiatric emergency department: impact and characteristics in a tertiary referral hospital}

M. Sagué Vilavella*, G. Anmella, S. Madero, J.E. Pinzón, A. Giménez, M.T. Pons, M. Vázquez, V. Soler, I. Méndez, E. Solé

Clínic Institute of Neurosciences- Hospital Clínic of Barcelona,

Department of Psychiatry and Psychology, Barcelona, Spain

* Corresponding author.

Background and aims.-

Introduction.- Frequent attenders (FA) to psychiatric emergency departments affect quality of care and imply resource overuse. Prior studies suggest they are a growing phenomenon.

Objectives.- To determine the current impact of FA in the psychiatric emergency department. To characterize adult and pediatric FA and identify differences compared to non-FA.

Methods.- Year 2017 psychiatric emergency department attendance from Hospital Clínic of Barcelona (Spain) was analyzed, separately for adult and pediatric population. Frequent attendance was established as $\geq 5$ visits/year. Comparison between FA and nonFA was restricted to patients belonging to the hospital service area. Trend analysis included the 2012-2017 period.

Results. $-2.5 \%(n=78)$ of adults and $3.2 \%(n=22)$ of minors were FA in 2017, generating $14.8 \%(n=653)$ and $14.4 \%(n=152)$ of the annual visits respectively. The number of FA showed a significant increasing trend between 2012 and 2017. In adults, FA were significantly younger than non-FA, whereas in minors there were more foreigners. Among FA, the chief complaint was anxiety (42.2\%) in adults and behavioral disturbance $(72.7 \%)$ in children; the most prevalent main diagnosis was psychotic disorder in adults (25,6\%) and behavior disorder in minors (40,9\%); $96,2 \%$ and $100 \%$ were on psychopharmacological treatment respectively (antipsychotics in $78.2 \%$ and $95.5 \%$; $\geq 3$ psychotropics in $55 \%$ and $22.7 \%$; $18 \%$ and $50 \%$ lived in residential facilities; and social/family problems were identified in $21,8 \%$ of adults and $63,6 \%$ of minors.

Conclusions.- In our hospital, FA have increased in recent years and generate substantial workload. Both adult and minor FA are highly-medicated and socially-disadvantaged groups, although their diagnoses and social problems differ.

Disclosure of interest.- The authors have not supplied a conflict of interest statement.
E-PV0325

\section{Borderline personality disorder (BPD) patients as frequent patients in emergency psychiatry}

S.S. Sanchez*, L. Soldado R., A. Alvarado D., M.O. Solis, F. Vilchez E., C. Coca C., G.M. Ruiz M.

Complejo Hospitalario de Jaen, UGC Salud Mental, Jaen, Spain

Corresponding author.

Background and aims.- Borderline Personality Disorder (BPD) is one of the most common mental disorder in Emergency Psychiatry, nowadays. Symptoms usually appear as a critical situation, personal problems and a conflictive environment.

- To objectively describe the mention of BPD patients as frequent users, due to repetitive medical attention, similar reasons for consultation and a typical symptomatology in emergency areas.

Methods.- A follow-up of 6 months study for 10 patients diagnosed as BPD, aged between 19 and 38, who come to the hospital repeatedly, from 2 to 12 times per semester, with a total of 61 emergency medical care.

Results.-

- Average value of 6,1 urgent consultations of each patient during 6 months.

- The average age on emergency room is 28,5 years-old and there are gender differences.

- The main problems are conjugal (50\%) and parental (50\%) relationships.

- Other personal problems such as: laboral and economic issues (30\%) and substance abuse (30\%) specially.

- Prevalence of self-harm in women (66.6\%) and suicidal behaviour in men (50\%), related with impulsivity.

- Anxiety, impulsivity and depression are the main symptoms observed: $80 \%, 70 \%$ and $60 \%$, respectively.

Conclusions.- High demand of these patients, usually in non-BPDspecialized services, means a greater caregiver burden (Zarit Scale) and difficulties for psychiatrist and medical personnel in urgency room. It is estimated that BPD patients use to consult another doctors more than two to three times, during the follow-up as outpatients care. Psychosocial and educational approach to care is justified in the clinical units, where the objective is the clinical habilitation and the treatment of social skills, which can contribute to reduce urgent consultations.

Disclosure of interest.- The authors have not supplied a conflict of interest statement.

\section{E-Poster Viewing - 7-9 April: Epidemiology and Social Psychiatry}

\section{E-PV0329}

\section{Revolving doors}

M.J. Gordillo Montaño*, S.V. Boned Torres, M. de Amuedo Rincon, E. Sanchez Navarrete, M. Guisado Rico, J. Iglesias Lopez

Hospital Can Misses, Psychiatry, Eivissa, Spain

Corresponding author.

Background and aims.- There is a tendency of making short incomes, appearing the revolving door phenomenon. There is no consensus in the literature to refer to a re-admitted patient. One of the criteria is to have three or more income in one year. We have different predictors of re-entry on which we use for taking the variables of the study. We hypothesized whether the patients with multiple admissions present a common pattern.

Methods.- A quantitative, observational, descriptive, transversal and retrospective study of a population compounded of patients admitted in 2017 in the Brief Hospitalization Unit. Including those 
admitted more than 2 times. Collecting these variables; number of readmissions, age, sex, employment status, diagnosis, family support, housing, therapeutic compliance and substance abuse. Results.- We obtained the following data: There were 380 admissions, 68 of them were re-admissions. 43 patients re-admitted twice and eleven patients three or more times. We selected the eleven patients with three or more admissions, assuming a total of 36 admissions. Three people had four incomes and eight, three. Four women and seven men. Ages between 24 and 60 years. Only two were working. Diagnoses: Bipolar disorder, BPD, Schizoaffective and cognitive impairment. Five without family support. Five homeless. Five with good therapeutic compliance. Eight with substance abuse.

Conclusions.- We did not find a common pattern in the different patients, being the most notorious the substance abuse. In future studies we will increase the sample.

Disclosure of interest.- The authors have not supplied a conflict of interest statement.

\section{E-PV0330}

\section{How does age at onset of mental illness associate to later educational and employment outcomes?}

J. Immonen*, E. Jääskeläinen, T. Nordström, L. Ala-Mursula, N. Rautio, J. Miettunen

University of Oulu, Center for Life Course Health Research, Oulu, Finland

${ }^{*}$ Corresponding author.

Background and aims.- Mental illness often begins in the foundational years of education and may disrupt it. Later on, this can lead to social exclusion and unemployment. We investigated how age at onset of mental illness affects the long-term educational and employment outcomes in prospective general population based Northern Finland Birth Cohort $1966(n=11234)$.

Methods.- For people with narrow schizophrenia ( $n=137)$, affective psychoses $(n=100)$, and non-psychotic affective disorders $(n=659)$ onset age was analyzed (quartiles for each diagnose) from register data. A yearly employment rate for years 1979-2015 and educational outcome by year 2014 (completion of basic level, upper secondary or tertiary education) was compared between diagnostic groups and between those having earlier versus later age of illness onset by using regression analysis. Cohort members without psychiatric diagnosis were the control group.

Results.- For education, individuals with an early onset schizophrenia had 6-fold odds and affective psychosis 5-fold odds for achieving only basic level education compared to peers. Figure 1 depicts the results for yearly mean employment rate per diagnoses and onset age groups. In psychotic disorders, especially in narrow schizophrenia, the employment rate is low compared to other diagnoses and peers. People with an earlier onset tend have poorer employment rates than the ones with a later onset.
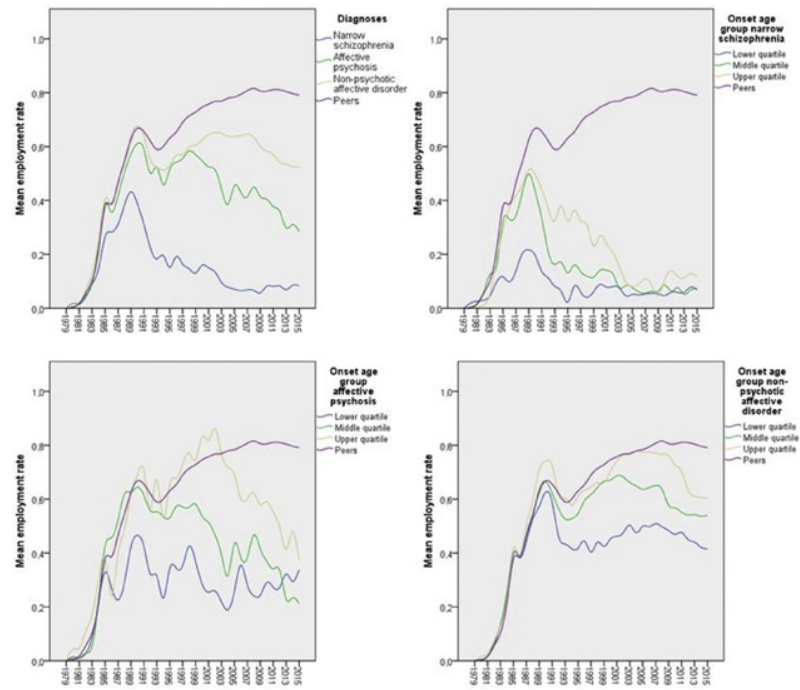

Figure 1. Results for yearly mean employment rate per diagnoses and onset age groups.

Conclusions.- Early onset mental illness hinders education and results in poorer employment especially in psychotic disorders. Disclosure of interest.- The authors have not supplied a conflict of interest statement.

\section{E-PV0331}

\section{Epidemiological profile of newly admitted psychotic patients in psychiatry}

R. Jouini ${ }^{1}$, M. EL Karoui ${ }^{2}$, L. Robbana ${ }^{1}$, S. Ben Ali ${ }^{3}$, F. Ellouze ${ }^{1}$, F. Mrad $^{1}$

${ }^{1}$ Razi Hospital, Psychiatry G Department, Tunis, Tunisia; ${ }^{2}$ Razi Hospital, Psychiatry G DepartmentTu, Tunis, Tunisia; ${ }^{3}$ Razi Hospital, Psychaitry G Department, Tunis, Tunisia

"Corresponding author.

Background and aims.- Psychiatric hospitalization, when indicated, provides a safe environment for patients with acute mental disturbance. This study aims to describe the epidemiological profile of patients with schizophrenia and other psychotic disorders (DSM 5) and to set up a comparison between their characteristics and the rest of the patients.

Methods.- A retrospective descriptive and comparative study including all newly admitted patients $(n=240)$. We examined demographic and clinical characteristics and we established a comparison based on the psychiatric diagnosis.

Results.- The average age of patients with psychotic disorder was 34 years. The majority of patients with psychotic disorder were male $(61,6 \%)$, single $(72,1 \%)$ and unemployed $(59,1 \%)$. The mean duration of untreated illness was more important in patients with psychotic disorder. The mean duration between the onset of the illness and the hospitalization was more important in patients with psychotic disorder. Hospitalization modality was in $93,6 \%$ of the cases an involuntary confinement in patients with psychotic disorder. Physical restraint was used in $21,1 \%$ of patients with psychotic disorder and only in $8,1 \%$ of the rest of the patients. Antipsychotics were more prescribed in the group of patients with psychotic disorder. The duration of parenteral treatment was longer in patients with psychotic disorder and the mean duration of hospitalization was more important in the same group. Almost $85 \%$ of the psychotic patients attended the first follow up visit.

Conclusions.- Analyzing clinical and socio-demographic characteristics of newly admitted patients enables the improvement of medical care and thus prognosis optimization. 
Disclosure of interest.- The authors have not supplied a conflict of interest statement.

\section{E-PV0333}

\section{Paranoia and risk of personality disorder in the general population}

J.E. Muñoz Negro ${ }^{*}$, C. Prudent ${ }^{2}$, J. Cervilla ${ }^{3}$

${ }_{1}^{1}$ Servicio Andaluz de Salud, UGC Salud Mental Hospital Universitario San Cecilio, Granada, Spain; ${ }^{2}$ Université de Lorraine, Laboratoire INTERPSY, Nancy, France; ${ }^{3}$ Universidad de Granada, Psiquiatría, Granada, Spain

* Corresponding author.

Background and aims.- We hypothesized that paranoia is associated to personality disorder (PD) in the general population. The aim is to explore this relationship between both entities.

Methods.- This was a population-based cross-sectional survey carried out in Andalusia (Spain) using a representative sample of 4507 participants. Paranoia was measured using the Green Paranoid Thought Scale and risk of PD was screened using the Standardized Assessment of Personality Abbreviated Scale whilst Borderline PD was measured with the CEPER-III Exploratory Interview of PD. Adjusted Pearsons' correlations between paranoia and PD/BPD were calculated.

Results.- Paranoia was associated with the risk of having PD and, more robustly, with BPD. Both associations held true when tested for both GPTS Paranoia subtypes (i.e., persecutory and reference) for PD and for BPD and after accounting for the effects of age, sex and child abuse.

Conclusions.- Paranoia seems to either augment the risk for, or be part of, PD/BPD.

Disclosure of interest.- The authors have not supplied a conflict of interest statement.

\section{E-PV0335}

\section{Re-admission over four years in our psychiatric units}

M.T. Perez Castellano ${ }^{1^{*}}$, C. Romero Martin², P. Padilla Romero ${ }^{3}$, G. Sanchez Amador ${ }^{1}$

${ }^{1}$ Psychiatrist. Hospital Ntra, Sra. del Prado., Unit of Psychiatric Hospitalization, Talavera de la Reina Toledo, Spain; ${ }^{2}$ Hospital Ntra. Sra. del Prado, Pharmacology, Talavera de la Reina Toledo, Spain; ${ }^{3}$ Hospital Ntra. Sra. del Prado, Psychiatry, Talavera de la Reina Toledo, Spain

Corresponding author.

Background and aims.- In our Unit 35\% of the patients are First time admission and 65\%Readmissions. It is vitally important to find out the main qualities of our patients. The characteristics of the patients will help to implement prevention plans for early detection and intervention in order to stop the readmission.

Methods.- Descriptive analysis of the patients characteristics during their admission in the Unit. The study was done from 2014 to 2017 and the categories that the study design were the discharge of the patients, according to the criteria included in the CIE 10. Results.- A significance increase in the neurosis diagnose (F40F49) was founded along the years, the $35 \%$ of the new admissions. Although the Psychotic disorders (F20-F29) were the main cause for First time admissions, their tendency was to be stable and unchanging during the four years; something very similar happened with the mood disorders (F30-F39).

Conclusions.- The Psychotic and Mood disorders are the patients with the highest number of admissions (75\% of the Psicosis cases) what drived to conclusion that action plans were needed to be created with the purpose to stop the elevate number of readmissions in this group of patients. On the contrary $75 \%$ of organic disorders (F0-F09) are the First time admissions and the Neurotic disorders
(F40-F49) had lower readmissions rate, in these case is necessary to prevent and early diagnostic to avoid admissions.

Disclosure of interest.- The authors have not supplied a conflict of interest statement.

\section{E-PV0336 \\ Involuntary admission to our psychiatric units over four years (2014 to 2017)}

M.T. Perez Castellano ${ }^{1}$, P. Padilla Romero ${ }^{2}$, C. Romero Martin ${ }^{3}$, G. Sanchez Amador ${ }^{1}$

${ }^{1}$ Psychiatrist. Hospital Ntra. Sra. del Prado., Unit of Psychiatric Hospitalization, Talavera de la Reina Toledo, Spain; ${ }^{2}$ Hospital Ntra. Sra. del Prado, Psychiatry, Talavera de la Reina Toledo, Spain; ${ }^{3}$ Hospital Ntra. Sra. del Prado, Farmacology, Talavera de la Reina Toledo, Spain

* Corresponding author.

Background and aims.- In Spain the Involuntary Psychiatric admission is regulated by Civil trial law $1 / 2000$, 7th January, article 763. Develop a study about involuntary admissions during the years 2014 to 2017 . The aim of this research is to determine the effects of the admissions in the patient's pathologies.

Methods.- Descriptive research of patients divided into two groups; diagnosis categories and discharges (according to the criteria of the CIE 10) with the purpose of compare and contrast the results obtained.

Results.- The average of all involuntary admissions is 25 '3\% of the whole number of patients. The highest porcentage was found in patients with psychotic disorders (F20-F29), 42\% of the total involuntary admissions. Althought only $30 \%$ of patients were admitted as a consequence of this disorder. The mood disorders (F30-F39) are $26 \%$ of the involuntary in-patient population. As a result of this research we found that organic disorders (F70-F79) are the main patients with involuntary admissions. The $80 \%$ of the people associated with these disorders are patients whom had a direct relation with their legal status of incapacitated situation.

Conclusions.-

Disclosure of interest.- The authors have not supplied a conflict of interest statement.

\section{E-PV0337}

\section{Burnout and cognitive-affective empathy in health professionals in monteria (Colombia)}

E. Ruiz ${ }^{1}$, A.M. Romero ${ }^{1}$, M. Crespi ${ }^{2}$, I. Ramos-Vidal ${ }^{3 *}$

${ }^{1}$ Universidad Pontificia Bolivariana, Psychology, Monteria, Colombia; 2 Universidad de Buenos Aires, Psychology, Buenos Aires, Argentina; ${ }^{3}$ University of Seville and Universidad Pontificia Bolivariana

Montería- Colombia, Department of Social Psychology, Seville, Spain ${ }^{*}$ Corresponding author.

Background and aims.- The Burnout syndrome is considered one of the psychosocial work harms with greater impact on the physical and mental health. So it has become necessary to evaluate factors which may triggering this syndrome. On the other hand, empathy is defined as the ability of the clinician to put himself in the place of the patient, considering himself as a valued medical attribute. However, some studies indicate that it may also constitute a risk factor for Burnout development. This study sought to determine the existence of Burnout syndrome in health professionals in the city of Monteria and analyze their possible relationship with empathy. Methods.- A quantitative cross-sectional and correlational study was carried out in 201 general practitioners, with an equilibrate distribution of men and women. The (a) Cognitive and Affective Empathy Test (TECA) was used to evaluate the emphaty dimen- 
sion; and (b) the Maslach Burnout Inventory (MBI) was applied to evaluate the Burnout syndrome.

Results.- The results shows high levels of Burnout in the participants evaluated (66\%) and the existence of a statistically significant negative correlation between the variables of emotional exhaustion of MBI and cognitive empathy $(p<.01)$. The same tendency is observed in the case of the depersonalization domain $(p<.01)$.

Conclusions.- The main conclusion of this preliminary study is that the lower the Empathy of the doctors evaluated, the greater the Burnout syndrome. Further research is needed to understand and disentangle the mediating mechanims between empathy and burnout in health proffesionals.

Disclosure of interest.- The authors have not supplied a conflict of interest statement.

\section{E-PV0339}

\section{The mental health risks of economic crisis in spain: evidence from primary care centres, 2006, 2010 and 2017}

M. Roca ${ }^{1}$, M. Gili ${ }^{2}$, A. Seguí ${ }^{3}$, A. Castro ${ }^{3}$, M.Á. Pérez-Ara ${ }^{3}$, M. Garcia-Toro ${ }^{1}$, I. Ricci ${ }^{4}$, A. Riera ${ }^{3}$

${ }^{1}$ University of Balearic Islands, Department of Medicine, Palma de Mallorca, Spain; ${ }^{2}$ University of Balearic Islands, IUNICS-Idisba, Palma de Mallorca, Spain; ${ }^{3}$ University of Balearic Islands, IUNICS University of Balearic Islands, Palma de Mallorca, Spain; ${ }^{4}$ Ib-Salut, Gerencia de Atención Primaria Mallorca., Palma de Mallorca, Spain ${ }^{*}$ Corresponding author.

Background and aims.- Spain was one of the countries most affected by economic crisis that began in 2007 . We investigated the relationship between the frequency of mood, anxiety, somatoform, alcohol-related and eating disorders during the different stages of the economic crisis $(2006,2010,2017)$ among population Spanish primary care settings. The aim of the study was estimate the changes of mental health in primary care before financial crisis, during this period and at the current situation.

Methods.- Patients attending Spanish primary care centres were chosen randomly by physicians 7940 patients in 2006, 5876 were selected in 2010 and 1090 in 2017. We used an own-elaborated questionnaire (data on demographic features, socio-economic and work- related variables and clinical characteristics), questionnaire of external stressful factors (Holmes and Rahe Scale) and a structured psychiatric interview for Primary Care (PRIME-MD) for the mental health diagnosis.

Results.- Compared with the pre-crisis period of 2006, the 2010 survey revealed substantial and significant increases in the proportion of patients with mood (19.4\% in major depression), anxiety (8.4\% in generalized anxiety disorder), somatoform (7.3\%), alcoholrelated disorders (4.6\% in alcohol dependence) and eating disorders $(0.15 \%)$. In the third wave (2017) the prevalence of mental health disorders has decreased to similar rates before economic crisis. Conclusions.- Past global economic downturn seems to not have long effects on the mental health of the population.

Disclosure of interest.- The authors have not supplied a conflict of interest statement.

\section{E-PV0340}

\section{Employees' knowledge, attitudes and intended behavior regarding coworkers with mental health problems}

G. Sampogna $1^{*}$, E. Brouwers ${ }^{2}$, C. Dewa ${ }^{3}$, C. Henderson ${ }^{4}$, J. Van Weeghel ${ }^{2}$

${ }^{1}$ University of Campania “Luigi Vanvitelli", Department of Psychiatry, Naples, Italy; ${ }^{2}$ Tilburg University, Tilburg School of Social and Behavioral Sciences- Department Tranzo- Tilburg University, Tilburg,
The Netherlands; ${ }^{3}$ University of California, Department of Psychiatry and Behavioral Sciences, Sacramento, USA; ${ }^{4}$ King's College, Institute of Psychiatry- Psychology and Neuroscience, London, United Kingdom Corresponding author.

Background and aims.- In many countries, participation of people with mental health problems (MHP) in the workforce is problematic. Employees with MHP have a higher risk for sick leave, early retirement, and unemployment than other workers. The extent to which workplace stigma plays a role in this problem has been under researched. The main aims of this work are: 1) How do employees think about coworkers with MHP?; 2) What is their willingness to spend time to support them?; 3 ) which variables explain the social distance to coworkers with MHP in the workplace?

Methods.- In February 2018, a cross-sectional survey was carried out among a nationally representative internet panel of Dutch employees. A total of 1246 respondents filled out the questionnaire (response rate 74,6\%). Concepts measured were knowledge, attitude and (intended) behavior.

Results.- A total of $39,9 \%$ preferred not to have a close colleague with MHP, and 61,2\% preferred not to work for a manager MHP. The extent to which they were willing to spend extra time on a coworker with MHP depended on whether they liked the coworker (78,3\% agreed). Main concerns were that the coworker could not handle work $(44,7 \%)$, that the employee would have to take over the coworkers' work (32,9\%), or would not know how to help the coworker (37,6\%). Factors that explain social distance will be presented at the conference.

Conclusions.- Negative attitudes towards coworkers with MHP are highly prevalent. The present findings are relevant for future studies on sustainable employability of workers with MHE.

Disclosure of interest.- The authors have not supplied a conflict of interest statement.

\section{E-PV0341 \\ Quality of life, chronic pain and medical conditions in different psychiatric disorders: a cluster analytic approach}

M. Pacetti ${ }^{1}$, R.P. Sant'angelo ${ }^{2 *}$, M. Sanza ${ }^{2}$, F. Ambrosini ${ }^{3}$

${ }^{1}$ Ausl Romagna, Mental Health, Forli, Italy; ${ }^{2}$ Ausl Romagna, Mental

Health, Cesena, Italy; ${ }^{3}$ University of Bologna, Faculty of Psychology,

Cesena, Italy

* Corresponding author.

Background and aims.- Psychiatric patients compared to healthy controls tend to show a worse quality of life and worse medical conditions. However, only few studies used a cluster analytic approach to stratify these aspects in a cross-diagnostic sample.

Our aim is to cluster a group of psychiatric patients based on a set of variables concerning quality of life and other medical conditions. Methods.- 27 patients (34.6\%) with Borderline Personality disorder (BPD), 24 with Affective Disorders (AD,30.8\%) and 27 with Psychosis (P,34.6\%) were recruited. A two-step cluster analysis was conducted based on: drug and anti-inflammatory abuse, sexually transmitted diseases, voluntary termination of pregnancy, parasuicidal behaviors, suicide attempts, fractures; BMI; chronic pain and internal medicine comorbidities; scores to SCOFF, TWEAK, WHOQoL; scores to a VAS about the experience of physical pain.

Results.- Two clusters were obtained. Cluster 1 (C1) (31 subjects, $39.7 \%$ ) and Cluster 2 (C2) (47 individuals, 60.3\%) differed mostly on WHOQoL, severity of pain experienced, occurrence of fractures and score to the SCOFF test. A MANOVA confirmed that C2 has better quality of life, whereas chi-squared tests confirmed that $\mathrm{C} 1$ tend to show more frequently anti-inflammatory abuse, severe pain, fractures and higher scores to SCOFF. Interestingly, the clusters differed in diagnosis $(p=.000)$, with BPD individuals over-represented in C1. 
Conclusions.- The detection of groups with similar internal medical conditions and quality of life can be useful to tailor specific interventions on psychiatric patients. Attention must be paid to BPD as it seems to be highly vulnerable on these aspects.

Disclosure of interest.- The authors have not supplied a conflict of interest statement.

\section{E-PV0343}

\section{Demographics, clinical characterization, and length of hospitalization of patients admitted to general psychiatry unit of multidisciplinary province hospital in tarnowskie góry, Poland in 2017.}

A.R. Szczegielniak ${ }^{1,2}$, P. Ścisło ${ }^{2}$, M. Błachut ${ }^{2,3}$, P. Gorczyca ${ }^{2,3}$

${ }^{1}$ Medical University of Silesia, Department of Psychiatry and Psychotherapy-School of Medicine in Katowice, Katowice, Poland; ${ }^{2}$ Multidisciplinary District Hospital, General Psychiatry Unit, Tarnowskie Góry, Poland; ${ }^{3}$ Medical University of Silesia, Department of Psychiatry in Tarnowskie Gorry-School of Medicine with the Division of Dentistry in Zabrze, Katowice, Poland

Corresponding author.

Background and aims.- Mental healthcare across European region is very diverse in terms of availability of treatment, models of psychiatric care, and ways of professional training. Thus, in the light of challenges that modern psychiatry faces resulting from high levels of mental disorders, limited financial resources, insufficient facilities for people suffering from mental health outside hospitals and deep-rooted stigma surrounding psychiatric treatment, regular assessment of patients' profile is vital. Polish psychiatry requires urgent systemic and mental changes, while the demand for psychiatric treatment in the country is growing. The aim of this research is to describe the clinical and demographic characteristics of patients referred to a General Psychiatric Units of Multidisciplinary Province Hospital in Tarnowskie Góry, Poland over a one year period (2017) to describe a profile of patients visiting the facility with insight into interlinkages between given diagnoses, length of stay, prescribed medications, available reimbursement of medical expenditures.

Methods.- Retrospective data from the patients' records was extracted and modalities analyzed.

Results.- This is a first phase of a multicenter study that would focus on characterization of patients admitted to general psychiatry departments across Europe, gathering data from Hospitals in Poland, Turkey, Romania, Albania, Ireland, Spain and Portugal to define demographics, characterize profile of the patients and define common trends in treatment.

Conclusions.- A better understanding of patients' needs can be the basis for effective initiatives related to the prevention of mental illness and the improvement of the structure of existing healthcare facilities.

Disclosure of interest.- The authors have not supplied a conflict of interest statement.

\section{E-PV0344 \\ Psychiatric disorders among patients with multiple sclerosis in Poland}

A.R. Szczegielniak ${ }^{1,2^{*}}$, M. Zając ${ }^{2}$, K. Swierzy ${ }^{2}$, M. Błachut ${ }^{2,3}$, K. Kubicka-Bączyk $^{4}$, M. Adamczyk-Sowa ${ }^{4}$, P. Gorczyca ${ }^{2,3}$ ${ }^{1}$ Medical University of Silesia, Department of Psychiatry and Psychotherapy-School of Medicine in Katowice, Katowice, Poland; ${ }^{2}$ Multidisciplinary District Hospital, General Psychiatry, Tarnowskie Góry, Poland; ${ }^{3}$ Medical University of Silesia, Department of Psychiatry in Tarnowskie Góry-School of Medicine with the Division of Dentistry in Zabrze, Katowice, Poland; ${ }^{4}$ Medical University of
Silesia, Department and Clinic of Neurology-School of Medicine with the Division of Dentistry in Zabrze, Katowice, Poland

"Corresponding author.

Background and aims.- Multiple Sclerosis (MS) is one of the most common cause of a nontraumatic neurologic disability worldwide, especially among young adults. Mental health challenges significantly affect the quality of life of patients in addition to the neurological burden of MS. Epidemiological data on the occurence of mental disorders in MS patients in Poland is deficient, and the results of other studies globally are divergent. The aim of the study was to determine prevalence of mental disorders in a sample of Polish MS patients.

Methods.- The study took place in 2017-2018 and consisted of 103 MS patients treated in the Neurological Outpatient Clinic of the Medical University of Silesia Hospital No. 1 in Zabrze, Poland. Data on sociodemographics, type and course of the underlying disease, comorbidities and used medicines were collected. MINI-international neuropsychiatric interview and psychiatric examination were utilized to assess the occurrence of mental disorders.

Results.- All the patients were examined by a qualified psychiatrist. Among them 68\% presented psychiatric diagnosis at some point in their life with only $4 \%$ hospitalized before; $49,5 \%$ met diagnostic criteria of different psychiatric disorders $(8,7 \%$ depressive episode; $6,7 \%$ anxiety disorder; $36,8 \%$ other disorders including mixed anxiety and depressive disorder), and 17,5\% presented cognitive disorders. $6,8 \%$ of all patients had at the time of the study suicidal thoughts (including 4 people with a high risk of suicidal attempt according to MINI).

Conclusions.- Significant prevalence of mental disorders among MS patients has been confirmed however further studies should be continued as presented results may reflect several factors.

Disclosure of interest.- The authors have not supplied a conflict of interest statement.

\section{E-PV0346 \\ Parkinson's disease and psychiatric comorbidity: a register-generated nationwide cohort \\ C.C. Vasarhelyi \\ University of Southern Denmark, Fokuseret Forskningsenhed \\ Aabenraa, Odense, Denmark \\ * Corresponding author.}

Background and aims.- Parkinsonian diseases are still today incurable degenerative diseases of the brain, and a reality for millions. With no curing treatment, extra effort should be made to treat symptoms and comorbidities. These individuals are estimated to be at an increased risk for psychiatric disease, but little mapping of psychiatric comorbidity exists.

The Aim of this study: To determine prevalence and onset of psychiatric comorbidity in patients suffering from Parkinsons Disease or Atypical Parkinson syndrome, before and after time of diagnosis. Methods.- This is a cohort study using the Danish National Patient Registry and the Danish Psychiatric Central Register. The patient group is compared to a control group of people matched on age and sex, in the number of 10 controls to 1 case. Inclusion criteria: Parkinson or Atypical Parkinson Syndrome patients younger than 65 years are all included. Patients must have their diagnosis for 5 years, to minimize the risk of misdiagnosis. The psychiatric diseases are grouped in the ICD-categories F1, F2, F3, F4 and F6; relating to substance abuse, schizophrenia spectrum disorders, mood affective disorders, anxiety related disorders and disorders of personality and behavior, respectively.

Results.- The results are under conduction. 
Conclusions.- Perspective: We expect to find higher rates of psychiatric diseases in Parkinsonian patients compared to the general population.

Disclosure of interest.- The authors have not supplied a conflict of interest statement.

\section{E-PV0347}

\section{First episode psychosis in}

schizophrenia: internalized stigma

and social adjustment. study on a

number of patients of the omsk

region (Russia)

K. Vasilchenko*, Y. Drozdovsky

Omsk State Medical University, Department of Psychiatry and

Medical Psychology, Omsk, Russia

* Corresponding author.

Background and aims.- The issue of treating patients with a firstepisode psychosis in schizophrenia (FEPS) remains highly relevant. To sustain a proper level of social functioning, the rehabilitation measures are needed to be implemented at the early stages of the disease, when psychopathological processes retain their plasticity. The number of studies of internalized stigma (IS) in patients with FEPS for the purposes of their social and clinical adjustment has been limited.

Objective.- to study the specificity for patient adjustment with FEPS, taking into consideration the IS-level for the follow-on elaboration of rehabilitation programs.

Methods.- The sample consisted of patients with FEPS $(n=134)$. The diagnosis of schizophrenia was established based on the criteria of the ICD-10. Adjustment of patients summing up of one of 7 levels of social and clinical adaptation was investigated. The levels 1 to 4 were considered as compensated, and 5 to 7 were decompensated. The ISMI scale has been used as a measure of IS. To explore the distribution of values of the quantitative variables Kolmogorov-Smirnov and Shapiro-Wilk tests have been used.

Results.- All the patients corresponding to four types of adjustment were distributed into 4 groups: integrative $(n=112)$, destructive $(n=6)$, extravert $(n=6)$ and introvert $(n=10)$. The highest level of IS was detected in destructive and introvert groups in comparison with others $(p<0.01)$.

Conclusions.- The interrelation of IS and social adjustment is significantly higher than the interrelation of IS and clinical adjustment. The data obtained allowed to develop new rehabilitation programs for patients with FEPS based on the organization of destigma measures.

Disclosure of interest.- The authors have not supplied a conflict of interest statement.

\section{E-PV0348}

\section{Renaming schizoprenia in Russia. Will it destigmatize? a study on sample of medical students \\ K. Vasilchenko*, V. Ismagilova, Y. Drozdovsky \\ Omsk State Medical University, Psychiatry and medical psychology, Omsk, Russia \\ ${ }^{*}$ Corresponding author.}

Background and aims.- To determine the perception of the term schizophrenia among medical university students.

Methods.- The cross-sectional study was performed in October 2018 with a sample of 164 medical students from Omsk State medical University (Omsk, Russia). One of three patient histories was shown to students randomly before participants started to fill the questionnaire form. The questionnaires were administered in a class environment.
Results.- There were no statistically significant differences determined between the forms differed only in diagnosis term $(p=0,91)$. The Kruskal-Wallis test was used to compare statements. No statistically significant difference was found between all of the statements $(p>0,05)$.

Conclusions.- There was no difference determined between medical students' attitudes toward three different terms of diagnoses. Nevertheless, the limitations of the study should be considered. Thus, stigma of schizophrenia in a population's mind is suggested not to depend on a name of the illness but on another factors that are needed to be investigated in further studies.

Disclosure of interest.- The authors have not supplied a conflict of interest statement.

\section{E-Poster Viewing - 7-9 April: Ethics and Psychiatry}

\section{E-PV0349}

\section{Involuntary psychiatric commitment in a terminally ill patient: a case report} P. Argitis ${ }^{1 *}$, C.M. Platsa ${ }^{1}$, S. Karavia ${ }^{1}$, A. Kourti ${ }^{1}$, L. Gkeldis ${ }^{1}$, P. Bouras $^{2}$, K. Gatsiou ${ }^{1}$, P. Portokalakis ${ }^{1}$, Z. Chaviaras ${ }^{1}$

${ }^{1}$ General Hospital of Corfu, Psychiatric, Corfu, Greece; ${ }^{2}$ General Hospital of Corfu, Internal Medicine, Corfu, Greece

${ }^{*}$ Corresponding author.

Background and aims.- Terminally ill, with advanced cancer patients experience a lot of distress, as a result of their disease and preparatory grief. We present a case of a 53-year-old woman, that was transferred to the Psychiatric Department of the General Hospital of Corfu through a Public Prosecutor's request, with a refusal of nutrition.

Methods.- The patient's assessment did not present any major psychopathology, or a previous psychiatric history. A Mini Mental State Evaluation (MMSE) test was performed in which she scored 30. She was referred to the Internal Medicine Department.

Results.- The laboratory tests and the imaging assessments revealed a primary tumor of the left lung with esophageal and pericardial infiltrations, as well as two metastatic tumors of cerebellum. During the patient's admission, we were asked to assess her condition many times, due to her refusal for further painful examinations and treatment by the doctors. She did not present any major psychopathology, or cognitive impairments. She wished to be discharged and die at home.

Conclusions.- As psychiatrists, it is crucial to distinguish symptoms of grief and depression in terminally ill patients, in order to make the correct intervention. As doctors, however, we must ask ourselves, where lies the fine line between arrogance and respect to another human being's will?

Disclosure of interest.- The authors have not supplied a conflict of interest statement.

\section{E-PV0350}

\section{Assisted suicide for psychiatric patients: current status in switzerland} S. De Lucia ${ }^{{ }^{*}}$, G. Bondolfi ${ }^{1}$, E. Girard ${ }^{1}$, C. Marti², O. Boillat ${ }^{2}$, S. Hurst $^{3}$

${ }^{1}$ Liaison Psychiatry SPLIC- Geneva University Hospital, Psychiatry DSMP, Geneva, Switzerland; ${ }^{2}$ Internal Medicine SMIG- Geneva University Hospital, Internal medicine- geriatrics and rehabilitation DMIRG, Geneva, Switzerland; ${ }^{3}$ Université de Genève, Institut Ethique- Histoire- Humanités, Geneva, Switzerland * Corresponding author.

Background and aims.- In most European countries assisted suicide is not legal. Switzerland, uniquely, allows suicide assistance 
by non-physicians. Long considered exclusively for terminally ill patients, suicide assistance is increasingly considered for chronically ill patients, including those suffering from moral distress related to a mental condition. Up to $3 \%$ of assisted suicide have been performed for psychological suffering.

To discuss legal and ethical issues in providing assisted suicide to psychiatric patients.

Methods.- We report the case of an older patient who was admitted to our hospital with a stuporous state after having ingested phenobarbital. A letter from her GP was found, stating that the patient was in such a psychological distress that the GP supported the patient's project to kill herself. Based on this situation we discuss the legal and ethical issues raised by patients who ask their doctors to provide them with suicide assistance for psychological suffering.

Results.- Assisted suicide for psychiatric patients -with or without somatic comorbidities-is legal in Switzerland provided that the patient is competent and the doctor providing assistance has no self-serving purpose. Case law has confirmed that a blanket exclusion of psychiatric patients from suicide assistance would constitute discrimination. However, providing such assistance requires a psychiatric evaluation and failure (or competent refusal) of all available alternatives.

Conclusions.- Psychological suffering is very common and can lead people to ask for assisted suicide. There are various policies and laws about this topic in European countries. In our opinion, Switzerland is a good example of a country which has made efforts in this purpose.

Disclosure of interest.- The authors have not supplied a conflict of interest statement.

\section{E-PV0352}

\section{Meningioma related psychosis - an ethical reflection on therapeutic decision}

\author{
T. Ferreira ${ }^{1 *}$, F. Godinho ${ }^{2}$, L. Ferreira ${ }^{3}$, A. Luís ${ }^{1}$, T. Maia ${ }^{1}$ \\ ${ }^{1}$ Hospital Prof. Doutor Fernando da Fonseca, Department of \\ Psychiatry, Amadora, Portugal; ${ }^{2}$ Hospital do Espírito Santo, \\ Department of Psychiatry, Évora, Portugal; ${ }^{3}$ Hospital Distrital de \\ Santarém, Department of Psychiatry, Santarém, Portugal \\ * Corresponding author.
}

Background and aims.- Neuropsychiatric manifestations of central nervous system tumors are common. Although tumor removal can resolve this symptomatology, offering treatment may be impossible when there's lack of insight, such as when psychotic symptoms are present. Our aim was to develop an ethical reflection about a clinical case.

Methods.- Review of the clinical case and literature.

Results.- A 40-year-old female with no previous psychiatric history was taken to the emergency department due to psychomotor agitation. In the initial evaluation, persecutory delusions and two previous episodes of seizures were detected. Head Computed Tomography revealed an expansive bilateral frontal lesion suggestive of meningioma, confirmed by Magnetic Resonance Imaging. Psychotic symptomatology responded inconspicuously to risperidone. Due to characteristics of the lesion and the chronological relationship with the psychotic symptoms, surgery was proposed; however, the patient refused it as part of her persecutory delusion, denying the tumor presence. Due to the lack of insight for the disease or need of treatment, and following the beneficence principle, surgery was pondered. The ethics committee was consulted, as the autonomy principle was compromised since psychotic symptoms distorted the appreciation of the disease and the autonomy of thought when making her decisions. Because of increasing psychotic symptoms and agitation, compulsive admission on a psychiatric ward was considered prior to the surgery. When informed of this hypothesis, the patient agreed to the neurosurgical treatment.

Conclusions.- Although the dilemma has been resolved spontaneously, it is worth reviewing this case and ethical principles involved in light of relevance to clinical practice.

Disclosure of interest.- The authors have not supplied a conflict of interest statement.

\section{E-PV0354}

\section{The role of ethics in global mental health}

S.O. Okpaku

Center for Health-Culture and Society, Center for Health-Culture and Society, Nashville, USA

Corresponding author.

Background and aims.- Aim: Exploration of essential ethical issues in foreign aid projects \& global mental health.

Global Mental Health implies a bilateral or multilateral engagement usually between a donor (from a developed country) and a recipient organization or institution from a developing country. These relationships are often asymmetrical as the donor institutions country the purse strings. In 2005 the Organization for Economic Cooperation and Development enacted a declaration aimed at accountability e.g. developed and developing countries for developing and managing and in terms of 5 principles. In 2013 John Sopko suggested a template of seven questions that are critical in the planning and evaluative plan of reconstruction. Although he was referring specifically to Afghanistan, this template may also apply to other foreign and projects. Okpaku on defining the criteria for global mental health emphasized the need for the recipient countries to the problem. This presentation will explore the ethical issues of foreign and projects and discuss the implications for research and practice. The presentation will expose the limitation of what may initially be seen as a war on war situation.

Methods.- Literature review.

Results.- Identification of ethical issues in foreign aid projects. Conclusions.- A consideration of ethical issues in foreign aid projects is critical. The opportunities for improvement in this field of activity.

Disclosure of interest.- The authors have not supplied a conflict of interest statement.

\section{E-PV0355}

Knowledge of the fundamental principles of bioethics among healthcare professionals

B.O. Plasencia García De Diego ${ }^{1 *}$, S.L. Romero Guillena ${ }^{2}$, F. Gotor Sanchez-Luengo ${ }^{3}$, O. Santamaría Gómez ${ }^{3}$, R. Navarro Pablo ${ }^{3}$

${ }^{1}$ Hospital de la Merced, Psychiatry, OSUNA, Spain; ${ }^{2}$ Hospital Universitario Virgen de la Macarena, Psychiatry, Sevilla, Spain; ${ }^{3}$ Hospital Nuestra Señora de Valme, Psychiatry, Sevilla, Spain * Corresponding author.

Background and aims.- Bioethics is born with the intention of becoming a guide for action, such as using knowledge for the good and the future of the human condition.

Objectives.- Analyse the knowledge of healthcare professionals in the basic principles of bioethics.

Methods.- Observational study carried out through an anonymous survey of health professionals (Medicine and Nursing).

The survey collects socio-demographic variables: age, gender and professional category; questions related to knowledge and training in bioethics.

Results.- 96 subjects ( 44 men and 52 women). 63 doctors and 33 nurses. Average age of 46.31 years (D. S. 10.6). 
Knowledge of the principles of bioethics:

${ }^{*} 45.8 \%$ of the sample knows some principle of bioethics; the most mentioned is that of charity (39.6\%), non-deficiency (33.3\%), autonomy (24\%) and justice (17.7\%).

${ }^{*}$ A $13.5 \%$, knows the 4 principles

*Age. $<45$ years old, has a higher level of knowledge vs. $>45$ years (60\% versus $33.3 \%, p<0.001, \chi^{2}: 6.84$ ).

Academic training in bioethics: $32.2 \%$ during university. Some $6.3 \%$ had completed some form of bioethics training in the last two years. The group with academic education has more knowledge than those without it ( $71 \%$ versus $33.8 \%, p<0.001, \chi^{2}: 11.64$ )

There are no differences according to gender or professional category.

Conclusions.- Knowledge of the general principles of bioethics in the health professionals in our study is insufficient. Less than $50 \%$ of respondents know of any principle of beginner bioethics. The importance of university academic education should encourage the strengthening of curricular formation

Disclosure of interest.- The authors have not supplied a conflict of interest statement.

\section{E-Poster Viewing - 7-9 April: Forensic Psychiatry}

\section{E-PV0356}

\section{Huntington's disease or schizophrenia- does it really matter? /in forensic evaluations/}

D. Bosnjak Kuharic ${ }^{*}$, N. Buzina ${ }^{2}$, G. Arbanas ${ }^{2}$

${ }^{1}$ University Psychiatric Hospital Vrapce, Department for Diagnostics and Intensive Care, Zagreb, Croatia; ${ }^{2}$ University Psychiatric Hospital Vrapce, Department for Forensic Psychiatry, Zagreb, Croatia

${ }^{*}$ Corresponding author.

Background and aims.- Huntington's disease (HD) is a neurodegenerative disease characterized by motor, psychiatric and cognitive symptoms. Although the main criteria for HD diagnosis include positive genetic test and involuntary choreiform movements, "premotor" symptoms like depression, psychosis, impulsivity, hostility or cognitive impairment can present years or even decades before the motor abnormalities. Our aim was to discuss potential differential diagnosis in the case of a forensic patient previously diagnosed with schizophrenia who developed HD with progressive cognitive decline later-on.

Methods.- We present relevant clinical data including history, psychiatric evaluation and complete diagnostic work-up.

Results.- A 50-year-old male patient diagnosed and treated for paranoid schizophrenia since 2012 was admitted to the Forensics department after he was assessed not guilty for the reason of insanity for murder attempt of a passer-by in 2017 due to acute psychosis. During 8 months of hospital treatment his state has worsen rapidly with disorganized behavior, visual hallucinations, cognitive deterioration, weight loss, inability to control sphincters or to take care of himself. Due to these specific symptoms and positive psychiatric heredity for both schizophrenia and dementia, a wide range of diagnostic work-up was done revealing CAG triplets and positive genotype with CAG-expanded allele gene characteristic for HD.

Conclusions.- Although the patient fulfilled diagnostic criteria for schizophrenia confirmed by multiple psychiatrists and forensic evaluation, later course of illness questions the nature of his psychotic decompensations: were they relapses of schizophrenia or early, non-specific representations of HD? But, whatever the case, the forensic evaluation of his criminal responsibility would be the same.

Disclosure of interest.- The authors have not supplied a conflict of interest statement.

\section{E-PV0359}

\section{Autoimmune encephalitis: diagnose difficulties and forensic implications}

\section{A.L. Costa ${ }^{*}$, A.I. Oliveira ${ }^{2}$, C.A. Pereira ${ }^{1}$, S. Caetano ${ }^{3}$, T. Casanova ${ }^{2}$}

${ }^{1}$ Resident of Psychiatry, Legal medical and Forensic Science Office

Dão-Lafões - Viseu of National Institute of Legal Medicine and

Forensic Science of Portugal, Viseu, Portugal; ${ }^{2}$ Graduate Assistent of Psychiatry, Legal medical and Forensic Science Office Dão-Lafões -

Viseu of National Institute of Legal Medicine and Forensic Science of Portugal, Viseu, Portugal; ${ }^{3}$ Assistent of Psychiatry, Legal medical and Forensic Science Office Dão-Lafões - Viseu of National Institute of Legal Medicine and Forensic Science of Portugal, Viseu, Portugal * Corresponding author.

Background and aims.- Autoimmune encephalitis (AE) is a rare and heterogeneous disease that can be manifested by neurologic and psychiatric symptoms.

It causes subacute deficits of memory and cognition often followed by a suppressed level of consciousness or coma. Often psychiatric symptoms also appear subacutely, in patients without any psychiatric history.

Because the presence of psychiatric symptoms can hinder the diagnosis, the aim of this report is to provide clues to help earlier detection, to describe difficulties in diagnosing and managing a case of $\mathrm{AE}$ and its forensic implications.

Methods.- Case report and non-systematic review of the literature. Results.- We reported a case of a 43-year-old man who was admitted on an inpatiente unit after being agressive to an old coffeeshop owner. He exhibited bizarre behaviour, agitation, aggressiveness, confusion, disorientation and memory loss. There was no other relevant psychiatric or medical history. A medical work up was completed with several blood investigations, electroencephalogram (EEG), cerebrospinal fluid (CSF), and cranial magnetic resonance imaging (MRI) and he was diagnosed with autoimmune encephalitis. Nowadays he still doesn't remember anything from that day and he was considered unimputable after a criminal procedure has been opened.

Conclusions.- AE diagnosis is difficult and it has to be considered in the differential diagnosis by psychiatrists because it often has primary psychiatric presentations. In neurological investigations, CSF and EEG should be considered, especially when the patient has no history of psychiatric manifestations. In the context of Legal Medicine and Forensic Science this patient is considered unimputable and shouldn't be criminally liable for his actions.

Disclosure of interest.- The authors have not supplied a conflict of interest statement.

\section{E-PV0360}

\section{Partialist paedophiilia: a report of a dangerous association}

M.L. De Falco ${ }^{*}$, T.E. Abreu ${ }^{2}$, J. Rodrigues ${ }^{2}$, N. Klein ${ }^{2}$, M.C. Lippe ${ }^{2}$, D. Baltieri ${ }^{2}$

${ }^{1}$ FMABC BRAZIL, Psychiatry, São Paulo, Brazil; ${ }^{2}$ Fmabc, Psychiatry, São Paulo, Brazil

"Corresponding author.

Background and aims.- Foot partialism is a type of paraphilia where the main source of sexual attraction is to manipulate, kiss, smell, bite or massage the feet of another person. The combination of partialism with paedophilia can go unnoticed by other persons and consequently be highly harmful to the victims.

Methods.- Intensive medical revision under the supervision of the staff of the Sexual Disorders Outpatient Clinic of ABC Medical School (ABSex)

Results.- We are reporting the case of a 48 year-old male children molester, married, with two sons, and without apparent psychiatry background. This patient turned to the ABSex after his wife and son having caught images of naked children on the patient's computer. 
Since his adolescence, he has reported erotic fantasies about having sex with children's feet. He has already manipulated feet of familial children followed by masturbation, denying any act of sexual penetration or genital manipulation of children. His medical treatment has been done weekly and only after his written agreement with ethical standards was signed. Sertraline was introduced $(100 \mathrm{mg} /$ day). He has participated in a psychotherapeutic group where some topics are coped with, such as: control of intrusive thoughts, masturbation redirection, modification of cognitive distortions and perceptive errors, empathy training, among others. Conclusions.- Paedophilia has a complex diagnosis process and a challenging treatment. Paedophiles may have a reasonably good therapeutic result when a specialized treatment and a juridical supervision are associated. A special care must be given to paedophiles with comorbidities or with other paraphilias, given that the recidivism risk tend to be greater.

Disclosure of interest.- The authors have not supplied a conflict of interest statement.

\section{E-PV0361}

\section{The arousal of a project for mentally disordered offenders in a Portuguese forensic psychiatric unit (hospital senhora da Oliveira - Guimarães) \\ M. Esteves Pereira ${ }^{1}$, S. Ramos ${ }^{2}$ \\ ${ }^{1}$ MD Forensic Psychiatry Unit, Department of Psychiatry and Mental Health of Hospital Senhora da Oliveira - Guimarães, Guimarães, Portugal; ${ }^{2}$ Phd- MD Forensic Psychiatry Unit, Department of Psychiatry and Mental Health of Hospital Senhora da Oliveira - Guimarães, Guimarães, Portugal \\ * Corresponding author.}

Background and aims.- Forensic psychiatry is the branch of psychiatry that deals with issues arising in the interface between psychiatry and the law, and with the flow of mentally disordered offenders (MDOs) along the continuum of the law system. These MDOs are often cared for in secure psychiatric environments or prisons. One of the problems is that these inmate patients may be discharged at the end of their security measure if the Judicial System so considers or, in advance, assume a figure of freedom for evidence. It is known that some of these patients will never leave the judicial system becoming social cases and others, who are discharged, may become dilute and out of the medical system with the risk of recidivism of the criminal behavior if not treated.

Methods.- Our Forensic Psychiatry Unit, within the framework of the National Health Service, aims fundamentally to guarantee a program for treatment and rehabilitation of citizens considered insane, according to their needs, individual aptitudes and risk assessment, aiming their clinical stabilization and social reinsertion.

Results.- We created a program that articulates the corners of the triangle Health (at the level of Primary Health and Hospital Care), Justice (Prison Services and Judicial Court) and Reinsertion (Social Services) so that we can identify, monitor and rehabilitate these patients. This allowed us to identify and closely monitor 53 patients. Conclusions.- Our project aims to provide our Forensic Psychiatry Unit with a specialized structure with a multidisciplinary approach for MDOs and this attitude constitutes a policy of respect for the human dignity.

Disclosure of interest.- The authors have not supplied a conflict of interest statement.

\section{E-PV0362}

\section{Capacity assessment and refusal to treatment in liaison psychiatry}

A. Fernandez-Ribas ${ }^{1 *}$, V. Perez-Rodriguez ${ }^{1}$, M. Bove-Lascurain ${ }^{1}$, M. Robles-Martínez ${ }^{2}$, J.A. Ramos-Quiroga ${ }^{1}$
${ }^{1}$ Vall d'Hebron University Hospital, Psychiatry, Barcelona, Spain; ${ }^{2}$ Institut de Neuropsiquiatria i Addiccions- Hospital del mar-

Barcelona- Spain- IMIM Institut Hospital del Mar d'Investigacions Mèdiques- Barcelona- Spain, Psychiatry, Barcelona, Spain

Corresponding author.

Background and aims.- The assessment of capacity to decide is one of the main daily tasks in liaison psychiatry. When it involves the decision of a patient to refuse treatment, the clinician faces ethical and legal dilemmas he cannot ignore. The Law on the Autonomy of Patients recognises the right to refuse a treatment.

Aims.- To review concepts and laws related to decision making and capacity to refuse treatment.

Methods.- The Liaison Team was consulted regarding a patient who refused medical treatment. According to the standing legislation, a patient has the right to refuse medical treatment at any point. This can apply as long as he or she has capacity to decide and does not present with psychopathology that affects that capacity.

Results.- Our team assessed a 61 year-old male admitted at a medical ward with a bad prognosis infective endocarditis.

Medical history: Bilateral leg ischemia having required right limb amputation. Chronic kidney failure having required kidney transplantation with subsequent transplant rejection. He is currently in dialysis treatment.

Psychiatric history: Nil. He denies drug use.

The patient has been informed about the potential consequences of his decision. He does not present with affective or psychotic symptoms. He elaborates an adequate speech exposing his decision coherently. Capacity to decide and consent is present.

Conclusions.- According to Law 41/2002, art. 2 apt. 4, the patient did not present psychopathology altering his perception. He preserved his capacity to decide and consent. Therefore I respected his decision keeping written acknowledgement of it.

Disclosure of interest.- The authors have not supplied a conflict of interest statement.

\section{E-PV0368}

\section{Psychoeducation as a part of the rehabilitation system in compulsory treatment for mentally ills}

M. Kulygina*, O. Makushkina, E. Panchenko

V. Serbsky National Medical Research Centre on Psychiatry and

Narcology, Department of prevention in forensic psychiatry, Moscow, Russia

"Corresponding author.

Background and aims.- To prevent socially dangerous behaviour and repeated criminalization of mentally ills the psychoeducational programme in the frames of compulsory treatment has been developed. It was aimed to promotion of social functioning, better compliance and prosocial behaviour of the patients with severe mental disorders.

Methods.- The programme consists of 3 blocks intended to be used not only for the patients, but also for their families and personnel. Differentiated approach to the content of tasks and trainings depending on the psychopathology type and mechanisms of committed illegal acts has proved to be most effective. Awareness-raising work, self-motivation improvement, trainings of social, cognitive and emotional skills have been in the focus of psychoeducation.

Results.- The protective role of the family regarding the reduction of the public danger of the mentally ills can be achieved by encouraging the relatives to form a more tolerant attitude to the patient and to their delicts, as well as by giving information about the initial signs of mental disorders and teaching them how to recognize a relapse timely. The medical treatment control provided by relatives and the development of strategies of non-confrontational 
family interaction can also prevent repetitive socially dangerous behavior.

Conclusions.- Psychoeducational program based on multiprofessional principles, team-work, system of psychosocial interventions constitutes a key part of psychorehabilitation in forensic psychiatry. Disclosure of interest.- The authors have not supplied a conflict of interest statement.

\section{E-PV0370}

\section{Psychiatry of the radicalizing family}

\section{Lazzari*, M. Rabottini}

International Centre for Healthcare and Medical Education, Psychiatry, Wakefield, United Kingdom

* Corresponding author.

Background and aims.- The current study aims to highlight the role of community psychiatry in identifying radicalizing families which can lead to radicalization and terrorism in childhood.

Methods.- The authors work with population vulnerable to radicalization. An ethnographic approach helped in the collection of the evidence. Perry dualistic stage helped explain the findings.

Results.- Dualistic thinking belongs to people who had problematic upbringing. In radicalizing families, children are dualistically taught that only some people are always respectable while others are evil. Radicalized children might hence develop out of dualistic upbringing and thinking. In some instance, children's acceptance of family radicalization is instinctively accepted if the dualistic ideology does not endanger or contradict the pathological role models to whom the child is exposed. Besides, a radicalized child might remain in the dualistic stage also during adolescence and adulthood. This event occurs because the dualistic stage also allows a paranoid projection and cognitive distortion of the identity of the potential victims located outside the family ethos. Hence, when a radicalized child and adolescent start to endorse family's negative qualifications of distinct others, these last can become easy victims of retaliation and terrorist attacks presently by the child or adolescent (diagnosed with challenging behaviors), or when the child has grown up into an adult (diagnosed with a dissocial personality disorder).

Conclusions.- Mental-health professionals working in the community can identify the early signs of dualistic thinking in radicalizing families. These last require more attention to avoid pathological upbringing of young people.

Disclosure of interest.- The authors have not supplied a conflict of interest statement.

\section{E-PV0371}

\section{To be or not to be criminally responsible \\ P. Makaric ${ }^{*}$, G. Arbanas ${ }^{2}$, N. Buzina ${ }^{2}$ \\ ${ }^{1}$ University Psychiatric Hospital Vrapce, Affective Disorders, Zagreb, Croatia; ${ }^{2}$ University Psychiatric Hospital Vrapce, Department for Forensic Psychiatry, Zagreb, Croatia \\ ${ }^{*}$ Corresponding author.}

Background and aims.- In Croatia in cases when a person is not able to stand trial for the reason of mental disorder and was not yet assessed as not-guilty-for the reason of insanity, the court has to wait until the person becomes able to stand trial again, before continuing the trial. Our aim was to discuss a possibility of developing a psychotic mental disorder after committing a crime and not being able to stand trial.

Methods.- We present a case report with relevant clinical data. Results.- A 25-year old man, with no history of mental disorder prior to the offence committed a violent crime - murder of his girlfriend. Soon after the event he started talking about a friend of his who travels around the world and kills people and who was the murderer of his girlfriend. All data were taken from a video game Grand Theft Auto. There was a lack of appropriate affect, along with the periods when he was not able to verbalize his thoughts. He was diagnosed with Munchausen's syndrome (dissociative disorder) of a psychotic level. He shielded himself from the painful truth by developing dissociative symptoms. In his current state he was assessed as not able to participate at the court.

Conclusions.- Developing symptoms of a psychiatric disorder in traumatic situations can have the effect of protecting a person from painful emotions, but in a forensic situation can protect a person from being able to stand trial.

Disclosure of interest.- The authors have not supplied a conflict of interest statement.

\section{E-PV0372}

\section{The role of police referral: differences in patients compulsorily admitted}

S. Martinho*, A.M. Carvalheiro, M. Simões

Leiria Hospital Centre, Psychiatry and Mental Health, Leiria, Portugal * Corresponding author.

Background and aims.- As psychiatric services move to a more community-based model, mental illness might be responsible for public behavioral changes that will motivate recognition and intervention by police forces. Given the lack of insight into their condition, compulsory admitted patients are often led to the emergency room following police referral. We aimed to evaluated differences between patients referred by the police and those who weren't, in a sample of compulsorily admitted individuals.

Methods.- The discharge notes of patients admitted between January 2011 and December 2017, were retrospectively reviewed. We divided individuals into two groups, patients hospitalized following police referral and those who were not. Differences were ascertain using Chi-square test for categorical variables and the Mann-Whitney test for numerical ones. Statistical significance was set at $p<0.05$.

Results.- Of 404 individuals, patients hospitalized following police referral were more likely to be male $(p=.04)$, older $(p<.01)$, to have an ICD's F20.x diagnosis $(p<.01)$ and longer hospitalization $(p<.01)$. These patients were more likely to be discharged with higher antipsychotic doses $(p<.01)$, with long-acting antipsychotics, but not with mood-stabilizers $(p=.11)$ or benzodiazepines $(p=.23)$. They were also more likely to be discharged in involuntary outpatient treatment $(p=.02)$.

Conclusions.- Patients compulsorily admitted following police referral might represent a group of patients with greater illness severity and less prone to regain insight. Moreover, since the police may be called to intervene more often, perhaps it would be pertinent to consider some form of training in this professional class. Disclosure of interest.- The authors have not supplied a conflict of interest statement.

\section{E-PV0373 \\ Delinquency and psychopathology: between delictual and psychopathological acting out}

F. Nyegue ${ }^{1^{*}}$, A. Fraud ${ }^{2}$

${ }^{1}$ Lirces/Universite de Nice Sophia-Antipolis,

Psychologie/Psychanalyse, Nice, France; ${ }^{2}$ Lash, Nice, France

"Corresponding author.

Background and aims.- To refer to the french Penal Code, "a person who, at the time of his acts had a psychic or neuropsychic disorder that abolished his or her judgment or control is not criminally responsible for this act. The question is to know how to discern what belongs to a particular pathology of a properly speaking delinquent 
act. The diagnosis of the expert results in a focus of the person (from hospitalization to the prison environment). The confusion between the criminalization of mental pathologies and the pathologization of delinquency is more and more incresing. From this moment, comes the question of the consequences of the impacts of a bad diagnostics on the patient?

If "deviant or delinquent behavior is not necessarily pathological and vice versa", the aim will be to confront the conceptual ambiguity related to the notion of delinquency, with the consequences that legal decisions specific to the act can have in this young public. Methods.- Our project is principally based on theoretical concepts of the clinic of the child and the adolescent. We use clinical interviews, but also psychoanalysis theories.

Results.- In progress.

Conclusions.- Although psychopathy disappeared from child and adolescent psychiatry textbooks during the 1980s, the rate of youths aged between 10 and 24 involved in criminal cases in 2014 was $5.2 \%$ of their age class. It seems necessary to identify in the young adult what, from this act, is an intentional or, on the contrary, what would be revealing the subjective position of this young person.

Disclosure of interest.- The authors have not supplied a conflict of interest statement.

\section{E-PV0375}

\section{Psychiatric aspects of burnout}

P. Padilla Romero ${ }^{1 *}$, C. Romeromartin ${ }^{2}$, T. López-Arteaga ${ }^{1}$, M.T. Perez Castellano ${ }^{1}$

${ }^{1}$ Hospital Nuestra Señora Del Prado, Psychiatry, Talavera de la Reina, Spain; ${ }^{2}$ Hospital Nuestra Señora Del Prado, Hospital Pharmacy, Talavera de la Reina, Spain

* Corresponding author.

Background and aims.- Introduction: In the current clinical practice, it is frequent to apply for psychiatric evaluation of people who presents Burnout.

Objectives.- To collect methodology and diagnosis criteria specific for Burnout, in the context of Legal Psychiatry.

Methods.- Case-series descriptive study. Clinical data were collected from 2 patients: 1 male aged 45 , and 1 female aged 53, both mediacl doctors without psychiatric background, who check with the Occupational Risks department about clinical affective compatible with Burnout. Review of existing literature about legal and forensic psychiatry of burnout in PubMed and Cochrane.

Results.- Both patients presented the same diagnosis: adaptive disorder (F43.20) and Problems related to employment (Z56). The temporary withdrawal of the stressing factor facilitated recovery, but in one of case the return to work triggered exacerbation.

Conclusions.- Recently, several sentences which recognize Burnout as a working-related disease have occurred. This composes expert medical evidence of significant importance.

The most likely professionals to suffer from it are: health professionals, educational professionals, police, public servants and social workers. There are 3 distinctive symptoms: emotional exhaustion; depersonalization and failure of expectations and personal achievements. The variables related to the risk to suffer from it are: age, (the older you get, the more you're at risk for burnt out); gender; females have more risk; features as stiffness thinking and emotional instability; and professional training, people with lower profesional training are more vulnerable.

Disclosure of interest.- The authors have not supplied a conflict of interest statement.
E-PV0376

\section{A new psychiatric and juristic definition is needed for sexual harassment to reduce legal and political abuse}

K. Pirkalani ${ }^{*}$, Z. Talaeerad ${ }^{1}$, R. Khodabakhsh Pirkalani ${ }^{2}$

${ }^{1}$ Mehr Medical Group, Internal Medicine/Psychiatry, Tehran, Iran; ${ }^{2}$

Alzahra University Tehran, Psychology, Tehran, Iran

* Corresponding author.

Background and aims.- Recently, there appears lots of allegations and reports containing sexual harassment. The two major component of annoying content and discriminating or ridiculing nature are neither well explained nor detailed reported.

Objectives.- To verify deep differences between normal and abnormal behavior of the offender.

Methods.- Forty five young girls who described themselves as victim in the workplace were interviewed. Additionally they were tested by the MCMI-III.

Results.- The first contacts seemed very faint and of low sexual potential in 39 of 45 cases. The nature of the offense became more severe with time in the range of 3 weeks to 6 months as the response was not compliant. The activity of the offender reduced only if there were alternatives in the workplace or legal threats. With interview, it was indirectly clarified that the six persons had antisocial and/or sadistic traits. Besides, by way of the interview we concluded that most "victims" are shy and of low sociability. Report of fourteen victims could not completely been attributed to harassment and in another ten it showed only mild landmarks.

Conclusions.- We conclude, that although harassment is a serious event in the workplace the time to report might be a good marker for its realistic value. About $5-10 \%$ of the offenders have antisocial traits. About $25 \%$ of the reports seem to have low realistic value. The fear of being fired is of low value in report compared to the personality of the offended.

Disclosure of interest.- The authors have not supplied a conflict of interest statement.

\section{E-PV0377 \\ A contribution the italian validation of the reflective functioning questionnaire}

G. Rogier ${ }^{*}$, S. Beomonte Zobel ${ }^{1}$, P. Velotti ${ }^{2}$

${ }^{1}$ University of Rome-Sapienza, Department of Dynamic and Clinical Psychology, Rome, Italy; ${ }^{2}$ Educational Sciences Unit, Genoa, Italy

* Corresponding author.

Background and aims.- As described within the psychoanalytic framework (Fonagy \& Target, 1998), one crucial aspect of human functioning consists in the Mentalization capacity corresponding to the efforts to understand ourselves and an others. Assessing mentalizing abilities is a complex issue. Only recently (Fonagy et al., 2016), an instrument assessing mentalization has been developed: The Reflective Functioning Questionnaire (RFQ). However, this instrument has not yet been validated in its Italian version, limiting the range of existing studies on Mentalization.

Objective.- To provide a preliminary contribution to the Italian Validation of the RFQ.

Methods.- A sample of community participants $(N=130)$ and a sample of violent offenders $(N=39)$ fulfilled the Italian version of the Reflective Functioning Questionnaire (RFQ, Fonagy et al., 2016), the Aggression Questionnaire (AQ, Buss \& Perry, 1992) and the Personality Inventory for DSM-5 (PID-5; Krueger et al., 2011).

Results.- Confirmatory Factor Analysis supported the original bidimensional model of the RFQ. Analyses evidenced a good reliability of both subscales. In the offender sample, the "Certainty about Mental States" subscale of the RFQ was negatively related to measures 
of pathological personality and aggression. In contrast, the "Uncertainty about Mental States" subscale correlated positively with the PID-5 and the AQ scores.

Conclusions.- These preliminary results suggest that the Italian version of the RFQ shows adequate psychometric properties and can be used in Italian studies. Furthermore, the study highlights the proficiency of the RFQ which provides additional explanation of aggressive behavior in addition to others central variables as such as pathological personality.

Disclosure of interest.- The authors have not supplied a conflict of interest statement.

\section{E-PV0378}

\section{Involuntary psychiatric admissions: a review}

E. Segura ${ }^{*}$, F. Martínez Criado ${ }^{2}$, C. Rodríguez Gómez Carreño ${ }^{3}$

${ }_{1}$ Associate prof, general Hospital Ciudad Real, Ciudad real, Spain; ${ }^{2}$

University Castilla La Mancha, Psychiatry, Ciudad Real, Spain; ${ }^{3}$

General Hospital Ciudad Real, Psychiatry, ciudad real, Spain

${ }^{*}$ Corresponding author.

Background and aims.- In Spain, and in our region, there are few studies of voluntary/involuntary admissions. even involuntary admission is regulated by specifics laws, this procedure is coerce and supposed to be traumatic for patient and family.

To regulate and minimize this kind of restraint will be important to reduce the impact in the patient's life and in prognosis.

Aim.- The aim of the study is to analyze the characteristics of involuntary admissions during a period, and compare the results with voluntary admissions.

Methods.- We analyzed admissions during a the first the months of the year.

Results.- Involuntary psychiatric holds are the $60,9 \%$ of total admissions, and twice are men, single and without differences in education or rural or urban environment. The most frequent diagnosis is Paranoid Schizophrenia and personality disorders.

Conclusions.- The characteristics of involuntary admissions are similar to other studies in literature, even with different laws and medical practice. This knowledge will help to promote prevention of coerce treatments and relapse.

Disclosure of interest.- The authors have not supplied a conflict of interest statement.

\section{E-PV0379}

\section{Violence in cyberspace - medical and legal perspectives in the Republic of Croatia}

\section{Sendula Jengic ${ }^{1 *}$, M. Sendula Pavelic ${ }^{2}$}

${ }^{1}$ Psihijatrijska bolnica Rab, Department for Forensic Psychiatry, Rab, Croatia; ${ }^{2}$ Faculty of Medicine-University of Rijeka, Social and humanistic sciences in medicine, Rijeka, Croatia

${ }^{*}$ Corresponding author.

Background and aims.- Violence in society is not a new problem. However, with the emergence of new communication media it has gained a new tool, giving the perpetrator the benefit of anonymity as well as a vast field and a diverse selection of social communities in cyberspace from which they can choose a victim. Cyberbullying and trolling are a relatively new problem in Croatia and are slowly gaining media space. Research on the incidence of offenses, impact on society and ultimately seriousness of the problem is scarce. Recently there have been some changes made in the legislation to meet the needs that the new phenomena have posed, but the question remains whether they can keep up with the fast changing times and customs. Two case reports will be presented. Methods.-

Results.-
Conclusions.-

Disclosure of interest.- The authors have not supplied a conflict of interest statement.

\section{E-PV0380 \\ PSP (primary care suport program). Implementation of a new model of mental health primary care for prisoners}

V. Tort Herrando ${ }^{1^{*}}$, A. Muro ${ }^{2}$, A.E. Olive ${ }^{3}$, E. Infantes ${ }^{4}$, M. Rovira ${ }^{5}$

${ }^{1}$ Psychiatrist, Penitentiary Psychiatry, Barcelona, Spain; ${ }^{2}$ Parc

Sanitari Sant Joan de Deu, Penitentiary Psychiatry, Sant Boi de LLobregat . Barcelona, Spain; ${ }^{3}$ Parc Sanitari Sant Joan de Deu, Penitentiary Psychiatry, Sant Boi de LLobregat. Barcelona, Spain; 4 Parc Sanitari Sant Joan de Deu, Penitentiary Psychiatry, Sant Boi de LLobregat, Spain; ${ }^{5}$ Parc Sanitari Sant Joan de Deu, Penitentiary Psychiatry, Sant Boid e LLobregat. Barcelona, Spain

* Corresponding author.

Background and aims.- The prevalence of the mental disorders in prison is higher than in general population. Psychiatric primary care of prisoners is of the paramount importance in health care in prison. There are different models of primary mental health for mentally ill offenders in prison. We present a new model than has been implemented in catalan prisons during 2018.

Methods.- We reviewed all the visits done by multidisciplinary teams (psychiatrists, psychologists, nurses and occupational therapist) and compare with the previous year (2017) when the new model was not implemented.

Also we look if the new interventions, re/admissions were found in our psychiatric acute unit and new cases had a better detection and more adequate treatment.

Results.- With the new aproach we found that we have more than $50 \%$ visits of previous year. Better detection of new cases and wider coordination with prison services. As well a higher support to primary care doctors.

Conclusions.- New models of mental health care are needed to give an adequate care for prisoners with a mental disoder. Our model seems to fullfil of criteria for thsi kind of care trying to do an equivalent care to those in the community.

Disclosure of interest.- The authors have not supplied a conflict of interest statement.

\section{E-PV0381 \\ Innovative daily multidisciplinary team handover utilising words on a secure unit}

N. Zaman ", K. Matiwa, A. Bashier, V. Nagy, L. Roberts, M. Benson Essex Partnership University Trust, Robin Pinto Unit, Luton, United Kingdom

* Corresponding author.

Background and aims.- The WORDS Daily Handover is an innovation in multidisciplinary communication within a secure unit. Communication within multi-disciplinary teams working across various health settings can be suboptimal and variable. One of the leading causes of adverse events leading to harm is miscommunication. As such better communication has been demonstrated to lead to improved patient outcomes. On secure wards traditional handovers were considered to be hierarchical, of variable relevance, of limited accountability and variable.

Methods.- The presentation demonstrates perspectives from the multi-disciplinary team of an innovative approach and development of a multidisciplinary handover procedure on a low secure unit. The words handover framework achieved initial acceptance by the multi-disciplinary team and was embedded into the ward rou- 
tine and culture. The words mnemonic incorporates development of a welcoming culture, reporting of observations, encouraging appropriate recording, cultivating team discussion and subsequent appropriate sharing outside of the immediate MDT.

Results.- Initial feedback of staff acceptance was significantly positive.

Conclusions.- The words multi-disciplinary daily handover has scope for wider acceptance within secure care and other healthcare settings supporting multi-disciplinary communication and potential for reducing harm to patients.

Disclosure of interest.- The authors have not supplied a conflict of interest statement.

\section{E-Poster Viewing - 7-9 April: Genetics \& Molecular Neurobiology}

E-PV0382

\section{Towards an endophenotype for behavioral addictions: is it impulsivity our target?}

Í. Alberdi-Páramo*, G. Montero-Hernández, L. Niell-Galmés, J. Rodríguez-Quijano, R.Á. Baena-Mures

Clinico San Carlos Hospital, Psychiatry, Madrid, Spain

${ }^{*}$ Corresponding author.

Background and aims.- There exists a body of evidence on the importance of impulsivity as a personality trait in behavioral addictions. It could be included in the current drug addiction models, where impulsivity is an important core trait. Elsewhere, there is some evidence of heritability and genetic association for behavioral measures of impulsivity.

The aim of this work is to do a systematic review of impulsivity as a endophenotype candidate for behavioral addictions.

Methods.- We have done a systematic review in PubMed aboput this topic.

Results.- Impulsivity plays an important role as a core factor in behavioral addictions. Every day appear an increasing number of publications and researches analyzing the extent of impulsivity to behavioral addictions. Behavioral dysregulation is thought to characterize suicidal behavior, with impulsivity trait related with it. There is some evidence of heritability and genetic association for behavioral measures of impulsivity. Whilst there are very few publications about genetic studies of behavioral addictions, impulsivity endophenotype model could be suitable for behavioral addictions. Conclusions.-

- Very few articles talk about genetic in behavioral addictions.

- Impulsivity is a core factor in behavioral addictions.

- Impulsivity endophenotype model could be interesting to define the role of genetic in behavioral addictions.

Disclosure of interest.- The authors have not supplied a conflict of interest statement.

\section{E-PV0383}

\section{Dysfunction of melatonin expression in depression - pilot study}

M. Dmitrzak-Weglarz ${ }^{1}$, J. Pawlak ${ }^{1}$, E. Reszka², A. Szczepankiewicz ${ }^{1}$, P. Kapelski ${ }^{1}$, S. Dziuda ${ }^{1}$, A. Rajewska-Rager ${ }^{1}$, J. Duda $^{1}$, P. Czerski ${ }^{1}$, J. Hauser ${ }^{1}$

1 Poznan University of Medical Sciences- Poland, Department of Psychiatric Genetics- Department of Psychiatry, Poznan, Poland; 2 Nofer Institute of Occupational Medicine, Department of Molecular Genetics and Epigenetics, Lodz, Poland

${ }^{*}$ Corresponding author.
Background and aims.- Circadian rhythm alterations resulting in disturbed sleep and disturbed melatonin secretion are flagship features of depression. In shift workers, an abnormal melatonin secretion has been reported to cause several psychiatric diseases including depression.

Despite a well-established mechanism of melatonin synthesis in the brain, there is no comprehensive study of this pathway and its changes in the periphery. Therefore, the aim of this study is to assess the level of serotonin, ANAAT, ASMT and melatonin in the serum of patients with depression in the course of unipolar (UD) and bipolar (BD) disorders.

Methods.- For our study we included 50 in-patients with depression episode in course of unipolar (UP, $n=25$ ) and bipolar disorder (BP, $n=25$ ) in the age range $18-65$ as defined by research criteria of ICD-10 (WHO, 1993). Clinical evaluation and sample collection were performed twice: at baseline and at time point $\mathrm{K}=$ remission - less than 8 points in Hamilton (17 item HDRS). Patients taking melatonin medication or undergoing chronotherapeutic intervention were not included into the study.

Results.- Data are presented as means \pm standard deviations, medians, minimum and maximum values or percentage, as appropriate. All results were considered significant at $p<0.05$. Statistical analyses were performed with STATISTICA 12.0 (StatSoft Inc.).

Conclusions.- The presented results make a comprehensive analysis of the level of melatonin and key enzymes involved in its synthesis on the periphery. The observed differences prove the necessity of conducting further studies on larger groups using healthy volunteers as a control group.

NCN Grant no. 2016/23/B/NZ5/02634.

Disclosure of interest.- The authors have not supplied a conflict of interest statement.

\section{E-PV0384}

\section{Orphan nuclear receptor as candidate} biomarker in depression - pilot study

J. Chaberska, K. Kwiatkowska, J. Duda, B. Narożna, J. Pawlak, A. Szczepankiewicz, P. Kapelski, S. Dziuda*, A. Rajewska-Rager, M. Dmitrzak-Weglarz

Poznan University of Medical Sciences- Poland, Department of Psychiatric Genetics- Department of Psychiatry, Poznan, Poland Corresponding author.

Background and aims.- Discovery of useful biomarkers in depression may facilitate diagnosis and optimal treatment. According to global transcriptomic profiles defined by using the Human microarray, we selected NR4A1 (Nuclear Receptor Subfamily4GroupAMember1) as a candidate to further molecular analysis. NR4A1 acted as a transcription factor for changing the expression of target genes. Animal studies indicated that NR4A1 is a stress-inducible modifier of mitochondrial energetic competence and dendritic spine number in the prefrontal cortex.

Methods.- In the pilot study, we included 205 patients with the current episode of depression (moderate and severe) and 359 healthy controls. We provided genotyping of rs2242107 C/T reported as potentially connected with insulin sensitivity and we also measured changes of serum level in the acute state of illness and after remission.

Results.- We observed that serum level was slightly higher during depression episode $(17.24 \mathrm{pg} / \mathrm{ml})$ than in remission $(14.43 \mathrm{pg} / \mathrm{ml})$, however without statistical significance. We did not find any significant differences in genotyping distribution between patients and controls even after the division due to gender.

Conclusions.- In light that NR4A1 may participate in mitochondrial function and thus in synaptogenesis it appears to be an attractive biomarker in depression and other psychiatric disorders, partially confirmed by our results obtained from the serum level test. Our data suggest that common variation within the NR4A1 gene locus may not play a major role in the depression. However, we cannot 
exclude that rarer variants may be associated with depression what should be a check on a larger cohort. Grant founded by NCN no. 2016/23/B/NZ5/02634.

Disclosure of interest.- The authors have not supplied a conflict of interest statement.

\section{E-PV0385}

\section{Effects of fluoxetine on nuclear parameters of gray level co-occurrence matrix in saccharomyces cerevisiae}

\section{Pantic, E. Kadric , A. Milanovic}

University of Belgrade, Faculty of Medicine- Institute of medical physiology, Belgrade, Serbia

* Corresponding author.

Background and aims.- In recent years, many new mathematical and computational methods have been suggested for structural analysis of cells and subcellular components. One such method is Gray level co-occurrence matrix (GLCM) analysis, which is capable of detecting discrete changes in cell nuclei during optical and electron microscopy. Effects of antidepressants on nuclear GLCM parameters are unknown. The aim of our research was to investigate potential dose-dependent effects of fluoxetine on GLCM parameters in Saccharomyces cerevisiae yeast.

Methods.- Saccharomyces cerevisiae cells were exposed to 3 different doses of fluoxetine after which digital micrographs of cell nuclei were obtained. A total 400 nuclei (100 cells per sample, 3 experimental samples, 1 control sample) were analyzed using GLCM method. For each nucleus, values of angular second moment (indicator of textural uniformity), inverse difference moment (indicator of textural homogeneity) and GLCM entropy (indicator of textural chaos and disorder) were calculated using second order statistical algorithm.

Results.- Exposure to fluoxetine was associated to dose-dependent changes in nuclear GLCM parameters. Values of angular second moment and inverse difference moment were increased $(p<0.01)$ after the treatment. Nuclear entropy, on the other hand, significantly $(p<0.01)$ decreased. These results indicate that fluoxetine increases nuclear textural homogeneity and uniformity while at the same time decreases nuclear level of textural disorder.

Conclusions.- Exposure to fluoxetine in Saccharomyces cerevisiae yeast leads to discrete morphological changes in nuclear architecture quantifiable using gray level co-occurrence matrix algorithm. Disclosure of interest.- The authors have not supplied a conflict of interest statement.

\section{E-Poster Viewing - 7-9 April: Intellectual Disability}

\section{E-PV0390 \\ Mental development of children suffering from opsoclonus myoclonus syndrome: factors of age and number of relapses}

N. Burlakova ${ }^{1^{*}}$, Y. Fedorova ${ }^{1}$, Y. Mikadze ${ }^{1}$, R. Bembeeva ${ }^{2}$, E. Ilyina $^{3}$, N. Zavadenko ${ }^{2}$

${ }^{1}$ Lomonosov Moscow State University- Faculty of Psychology, Department of Neuro- and Pathopsychology, Moscow, Russia; ${ }^{2}$ Pirogov Russian National Research Medical University- Ministry of Health of Russia- Faculty of Pediatrics, Department of NeurologyNeurosurgery and Medical Genetics, Moscow, Russia; ${ }^{3}$ Russian Children's Clinical Hospital- Ministry of Health of Russia,

Psychoneurology Department, Moscow, Russia

${ }^{*}$ Corresponding author.
Background and aims.- Opsoclonus-myoclonus syndrome (OMS) severity is determined by the first episode and the number of relapses. The influence of different factors on the risk of relapses as well as connection between number of relapses and mental development remain unexplored.

Methods.- The aim of the research was to explore dependence of cognitive and psychological development on different number of relapses. The research included 26 children with OMS aged from 1 year 7 months to 13 years. The following methods were used: analysis of patient's development and medical record, observation of child's psychological condition during psychological examination, neuropsychological and pathopsychological assessment.

Results.- The risk of disease relapses (from 2 to 5) is high for children aged from 1 to 7 years and for children with early disease onset time (from 1 to 3 years -11 of $26,42 \%$ ). Multiple relapses (more than 5) lead to intellectual disability. Behavioral changes (general passivity of children; low level of initiative in communication with an adult; poor differentiated emotional states) as well as cognitive impairment (disturbances of visual-spatial functions, speech, memory, thought processes) were diagnosed. Individual variations were indicated: 1$)$ intellectual disability is determined by small number of relapses (from 1 to 3 ( 3 of $26,11 \%$ ));2) children with multiple relapses (from 4 to 5 ) demonstrated potentially compensated delay in cognitive development ( 3 из 26, 11\%).

Conclusions.- Intellectual disability correlates with multiple relapses (more than 5). Analysis of individual cases indicates indirect connection between multiple relapses and severity of developmental delay. Other factors require further research. Disclosure of interest.- The authors have not supplied a conflict of interest statement.

\section{E-PV0391 \\ Neuro-cognitive functioning in children with autism spectrum disorders}

D. Galletta ${ }^{1}$, A.I. Califano ${ }^{1}$, A. Bruno ${ }^{2}$, M. D'Amaro ${ }^{2}$, S. Celentano ${ }^{2}$ ${ }^{1}$ University of Naples Federico II, Department of Neurosciences and Reproductive and Odontostomatological Sciences, Naples, Italy; ${ }^{2}$ Sanitary Pole La Filanda lars, Sanitary Pole La Filanda Lars, Sarno, Italy

${ }^{*}$ Corresponding author.

Background and aims.-

Introduction.- Autism spectrum disorder (ASD) is a neurodevelopmental disorder that begins early in childhood and lasts throughout a person's life. It is characterized by persistent deficits in the communication, social interaction and behavioral areas (DSM 5, 2013). ASD includes a wide range, "a spectrum" of symptoms, skills, and levels of disability. The aim of neuropsychological assessment is to identify cognitive strengths and weaknesses, to assess their consequences in everyday life, and to organize appropriate support. Regarding the neuropsychological profile of subjects with autism, the international scientific literature has highlighted the presence of deficits in the following domains: attention, executive functions, language, learning and Memory, Sensorimotor Processing (Molly Losh et al., 2009; Narzisi et al., 2013; Fjola Hyseni 2018) Objectives: to compare the neuropsychological profile of 4 school-age children diagnosed with autism.

Methods.- The sample consists of 4 male children aged between 6 and 9 years. Tools used are Wisc-IV and BVN scale (battery for neuropsychological assessment in childhood).

Results.- All children with low functioning presented different cognitive profiles. More impairments were found in working memory, processing speed, executive functions, visual-spatial memory. Strength is represented by short term verbal memory.

Conclusions.- A comprehensive investigation of the neuropsychological strengths and weaknesses of children with autism may help to better describe their cognitive abilities and to design appropri- 
ate interventions, in order to improve the quality of life of these subjects.

Disclosure of interest.- The authors have not supplied a conflict of interest statement.

\section{E-PV0394}

\section{“Don't eliminate me!" - ethical considerations in intellectual disabilities research}

B. Kowalewska ${ }^{1,2^{*}}$, W. Drozdz ${ }^{1}$, L. Kowalewski ${ }^{2}$, M. Skibicka $^{3}$, L. ${\text { Gizinski }{ }^{1}, \text { R. Ceannt }}^{4}$

${ }^{1}$ Ind Clinic of Psychiatry-Department of Psychiatry Collegium Medicum in Bydgoszcz- Nicolaus Copernicus University in TorunPoland, Department of Psychiatry Collegium Medicum in BydgoszczNicolaus Copernicus University in Torun, Torun, Poland; ${ }^{2}$ Institute Psychiatry- Psychology and Neuroscience, Forensic and Neurosciences, London, United Kingdom; ${ }^{3}$ Ind Psychiatric WardBieganski Regional Hospital in Grudziadz- Poland, Ind Psychiatric Ward-Bieganski Regional Hospital in Grudziadz- Poland, Grudziadz, Poland; ${ }^{4}$ Public Health Medicine, Lacken, Ireland

* Corresponding author.

Background and aims.- Studies suggest that between 27\% and 41\% of adults and about $40 \%$ of children with intellectual disability (ID), suffer from mental health problems. There are important social and scientific benefits to including the individuals in research, including opportunities for socially valued contributions, and the reduction of persistent health, economic, and social disparities. Researchers play a gatekeeper function with respect to certain forms of knowledge generated about intellectual disability. Over 90 percent of medical research trials are designed to automatically exclude persons with ID from participation. Ethical and legal difficulties are one of main obstacles to research within the ID field.

Methods.- Studies identified in a current comprehensive literature review discuss subjects worth to considering while conducting a study with ID participants based.

Results.- Frequently decisions about research participationincluding discussions related to coercion, capacity, consent, assent, and promoting understanding-constitute the bulk of discourse in ID ethics. Some of the studies' authors suggest that it must not be assumed that individuals with ID are uniform in their abilities to understand research and offer informed consent or assent.

Conclusions.- It is crucial to take into account the individual factors of each participant, and to be open to creative and novel approaches that enable communication and active participation. This requires an inclusive approach to research that affirms the importance of engaging and respecting the voices and perspectives of people with ID and, when appropriate, their advocates, surrogates and/or families.

Disclosure of interest.- The authors have not supplied a conflict of interest statement.

\section{E-PV0395 \\ “He doesn't know how to cry!" - mood disorders in intellectually disabled patients \\ L. Lopes*, S. Pereira \\ Centro Hospitalar de Vila Nova de Gaia/Espinho, Psiquiatria, Vila Nova de Gaia, Portugal \\ * Corresponding author.}

Background and aims.- Mental health problems can affect any individual and do not exclude intellectual disabled people. In fact, psychiatric disorders are more prevalent among this population, albeit it is usually difficult to accurately identify the specific disorder.
We present the clinical case of a 25 years old male gender patient diagnosed with Neuhauser syndrome that presented to a first psychiatry consultation. Based on the mother and the family doctor information's, he started presenting mood swings some months before, ranging from crying to uncontrolled laughter with no apparent cause. We review the syndrome characteristics and the specificities of mental disorders presentation in intellectual disabled patients.

Methods.- This work consists of a clinical case and a non-systematic review of the literature on clinical presentation of mood disorders in patients with intellectual disabilities.

Results.- Neuhauser syndrome is an extremely rare genetic disorder, characterized by abnormalities of the eye (primary megalocornea) and cognitive impairment of varying degrees. Intellectual disabled patients seem to be more susceptible to environmental influences given their less developed coping skills. For instance, literature on the topic emphasizes that new settings or carer's changes may trigger depressive episodes. These patients are more prone to mental disorders, and depression seems to be more prevalent among patients with mild disability. Clinical identification of specific mental disorders is often challenged by communication limitations and symptoms presentation variance with developmental level.

Conclusions.- The carer's information and the structured assessment of symptoms are vital to guide the interpretation of behavioural changes in patients with mental health disorders and intellectual disabilities.

Disclosure of interest.- The authors have not supplied a conflict of interest statement.

\section{E-PV0396}

\section{The complicated relationship between psychosis and intellectual disability. A case report}

I.I. Louzao Rojas*, J. Curto Ramos, V. Romero Pardo, Á. García Cardenal, M.D. Trigo Varela, G. Kollias, C. Casado San Martín, B. Rodríguez Vega, M.F. Bravo Ortiz

University Hospital La Paz, Mental Health and Psychiatry

Department, Madrid, Spain

${ }^{*}$ Corresponding author.

Background and aims.- From Kraepelin, the relation between cognitive deficits and psychotic symptoms is discussed. To date, it seems that prevalence of psychotic disorders is higher in people with intellectual disabilities, although the form of such symptoms varies depending on IQ, underlying genetic alterations, associated neurological pathology ... and delusions are usually less systematized

Methods.- We report the case of a 29-year-old man with mildmoderate intellectual disability due to a neurosurgical intervention at the age of 5 years -not congenital-, with no history of taking psychotropic drugs, which presented with an episode of behaviour disorders after an episode of frustration and several social stressors. The clinical picture is completed with the belief that his parents have been replaced by doubles (Capgras syndrome), with suspicion and refusal to return home with them.

Results.- The patient was treated with low doses of risperidone, with partial outcome, without delusion disappearing but allowing an incorporation to his normal life several weeks after the onset of symptoms

Conclusions.- It is difficult to establish the etiology of psychosis in patients with intellectual disability, being multifactorial in most cases. Antipsychotic treatments have been shown to be less effective compare to people with normal IQ as our case evidences. More research (RCTs) is needed to understand the relation between psychosis and cognitive disability in order to develop clinical guidelines for the identification and management of psychotic symptoms in people with these features. 
Disclosure of interest.- The authors have not supplied a conflict of interest statement.

\section{E-PV0397}

\section{Service evaluation looking at transition of young people with intellectual disability into adult neurodevelopmental services in kingston and richmond}

\section{P. Nagasinghe ${ }^{1^{*}}$, A. Sahni ${ }^{2}$, R. Patel ${ }^{3}$}

${ }^{1}$ South West London \& St George's Mental Health NHS Trust, Liaison Psychiatry, London, United Kingdom; ${ }^{2}$ Surrey and Borders Mental Heath NHS Trust, Child \& Adolescent Intellectual Disability, London, United Kingdom; ${ }^{3}$ Your Healtcare CIC, Richmond

Neurodevelopmental Services, London, United Kingdom

${ }^{*}$ Corresponding author.

Background and aims.- Transition to adulthood is a complex process. Our service had been rated 'red' following completion of the Self Assessment Checklist from Senate Evaluation Tool. There were significant gaps in the availability to work with people with an intellectual disability (ID) from the age of 14 .

Aim.- Assess the quality of transition of young people with ID into adult services and to identify any service gaps.

Methods.- Data was collected on all who transitioned into our service between 06/2016-06/2018. A questionnaire was developed using; Green Light Tool Kit 2013 (National Development Team for Inclusion), A transition guide for all services (DoH) and draft NICE guidelines on Mental health problems in people with learning disabilities: prevention, assessment and management. Due to the small sample size, descriptive statistics were applied.

Results.- Nine people were transitioned into our service (33\% female, $66 \%$ male). Majority had moderate or severe ID and all except one had autism. Three referrals came from CAMHS. We identified that $33 \%$ of people had joint transition meetings with the referring team. Median age at time of referral to our service was 17 years and 10 months. All young people had a named transition worker and no one missed their first appointment.

Conclusions.- Joint transition meetings with services were held in only $33 \%$ of people referred and referrals are not being made until close to the age of 18 . There is clearly a need to improve the pathway of transition to adult ID services from all referral sources and for the process to start earlier.

Disclosure of interest.- The authors have not supplied a conflict of interest statement.

\section{E-PV0400 \\ Psychiatric and psychosocial interventions in two adults with 22Q11.2 deletion syndrome and comorbid mental illnesses}

M.M. Theil $1,2^{*}$, C. Krzoska ${ }^{1,2}$, B. Perera ${ }^{3}$, E. Boot ${ }^{4,5,6,7}$, F. Colmegna ${ }^{8}$, A. Merra ${ }^{8,9}$, M. Clerici $^{8,10}$, M.O. Bertelli ${ }^{11}$, J. Santambrogio ${ }^{10,12}$

${ }^{1}$ Evangelische Stiftung Neuerkerode, Integrierter Gesundheitsdienst Neuerkerode- Lukas-Werk Gesundheitsdienste $\mathrm{GmbH}$,

Sickte-Neuerkerode, Germany; ${ }^{2}$ Evangelische Stiftung Neuerkerode, MZEB Braunschweig- Lukas-Werk Gesundheitsdienste GmbH, Braunschweig, Germany; ${ }^{3}$ Barnet- Enfield and Haringey Mental Health Trust- Haringey LD partnership, River Park House, London, United Kingdom; ${ }^{4}$ Heeren Loo Zorggroep, Heeren Loo Zorggroep, Amersfoort, The Netherlands, ${ }^{5}$ Academic Medical Center, Department of Nuclear Medicine, Amsterdam, The Netherlands; ${ }^{6}$ Maastricht University Medical Centre, Department of Psychiatry and Psychology-School for Mental Health and Neuroscience, Maastricht, The Netherlands; ${ }^{7}$ University Health Network, The Dalglish Family 22q Clinic for Adults and Department of Psychiatry, Toronto, Canada; ${ }^{8}$ Azienda Socio Sanitaria Territoriale Monza- ASST Monza, Mental Health and Substance Dependence Department, Monza, Italy; ${ }^{9}$ Azienda Socio Sanitaria Territoriale Monza- ASST Monza, Clinical Psychology Unit, Monza, Italy; ${ }^{10}$ University of Milano Bicocca, Department of Medicine and Surgery, Monza, Italy; ${ }^{11}$ San Sebastiano Foundation, CREA - Research and Clinical Center, Florence, Italy; ${ }^{12}$ AS.FRA., Fondazione Onlus, Vedano al Lambro MB, Italy * Corresponding author.

Background and aims.-

Introduction.- 22q11.2 deletion syndrome (22q11.2DS) is a genetic condition associated with multiple physical and mental health manifestations such as congenital heart defects, hypocalcemia, intellectual disability (ID) and psychotic disorders. Despite the high risk for developing psychiatric illnesses, there is scarce literature on the response to standard treatment in this patient population.

Objectives.- To present the clinical course of two female patients with 22q11.2DS, who received psychiatric and psychosocial interventions for mental illnesses.

Methods.- In this case report, we discuss the clinical course in two patients with 22q11.2DS and comorbid mental illnesses: patient one, a 37-year-old woman with moderate ID and comorbid schizoaffective disorder who presented with acute psychotic symptoms, patient two, a 26-year-old woman with mild ID who presented with subthreshold psychotic symptoms and obsessivecompulsive disorder with trichotillomania.

Results.- Patient one was treated initially with escitalopram (10 mg) and risperidone $(6 \mathrm{mg}$ ), later risperidone was switched to paliperidone $(263 \mathrm{mg}$ ) depot injection to avoid dose fluctuations. Patient two was initially treated with sertraline $(100 \mathrm{mg})$ and haloperidol (1 mg), and switched to venlafaxine $(150 \mathrm{mg})$ and aripiprazole (15 mg) because of side effects. Both patients received long-term behavioural therapy and psychoeducation aimed at improving perception of personal needs, social skills and acceptance of their conditions. Both patients showed a substantial reduction of their (sub-threshold) psychotic symptoms and improved social skills. The interventions empowered the patients to make their own decisions.

Conclusions.- These two cases show how patients with 22q11.2DS comorbid mental illnesses may benefit from standard pharmacological treatment in combination with individualized behavioural therapy.

Disclosure of interest.- The authors have not supplied a conflict of interest statement.

\section{E-PV0401 \\ Intervention in patients with intellectual disability and psychogenic non-epileptic seizure}

S. Trincado ${ }^{1^{*}}$, L. Gonzalez ${ }^{1}$, H. Saiz ${ }^{2}$, J. Rodriguez ${ }^{1}$, T. Ruiz de Azua $^{1}$, O. Uriarte ${ }^{1}$, D.J. Perez ${ }^{1}$, E. Perez ${ }^{3}$, J. Perez-Agote ${ }^{4}$ ${ }^{1}$ Psychiatry, Red de Salud Mental de Bizkaia, Bilbao, Spain; ${ }^{2}$ Psychiatry, Complejo Hospitalario de Navarra, Pamplona, Spain; ${ }^{3}$ Psychology, Red de salud mental de Bizkaia, Bilbao, Spain; ${ }^{4}$ Psychiatry, Asociación Gorabide, Bilbao, Spain * Corresponding author.

Background and aims.- The semiology of conversion disorder in intellectual disabilited patients is scarcely studied. Although the incidence of the symptoms is frequent in this group, they tend to be excluded in most of the researches in this field.

The sharing of the therapeutic intervention carried out in a patient with this disorder since there are not many cases described, as well as, a bibliographic review of studies concerning this field. Methods.- An extensive review of the bibliography concerning this disorder has been made, just as a review of the clinic record. Results.- They correspond to a clinical impression. 
Conclusions.- The case could match with a psychogenic non epileptic seizure, and in that case, the therapeutic intervention applied, without aiming to be a tool reference, could be orientative in cases with a comparable profile.

Disclosure of interest.- The authors have not supplied a conflict of interest statement.

\section{E-Poster Viewing - 7-9 April: Mental Health Care}

\section{E-PV0402 \\ Depression and trauma as drawbacks of identification processes within buddhist groups \\ A.I.M. Anders \\ Institute for Social and Cultural Anthropology- Munich University, Faculty for the Study of Culture, Munich, Germany \\ * Corresponding author.}

Background and aims.- Introduction: During the past years several scandals have struck Buddhist communities. One recent example is the Open Letter (July 2017) within the organisation Rigpa and the Lewis Silkin Report (August 2018). Even though the victims being asked in this case, a lack of investigation in the many others who have been left unheard and of treatment for those who were left back traumatised or depressed can be seen. A research project funded by German Federal Ministry of Education and Research covers that topic since August 2018.

Objectives: Following an analysis of terminological traps and indoctrination, of psychological issues and diagnoses, the objective is to develop a structure of treatment and care for the people concerned. Methods.- Methods: The investigation is conducted with a questionnaire designed for people studying in Buddhist centers, standardized psychological questionnaires and interviews.

Results.- Results: The results will encompass not only the amount of people with trauma, depression or other clinical diagnoses, but analyze effects of multi-level messages and identification processes. The complexity of treatment of these diseases after years, sometimes even decades, of indoctrination and the resulting loss of confidence should not be underestimated. This complexity hinders the prosecution authorities in their efforts to roll up the cases as well as individual therapy processes.

Conclusions.- Conclusions: Even though Buddhism and Tibet in general are still attributed with high idealization, the identification processes involved lead to risks, not only for vulnerable personalities, and there is a need for treatment of those who have been damaged by personality cults.

Disclosure of interest.- The authors have not supplied a conflict of interest statement.

\section{E-PV0403}

\section{Confirmatory factor analysis and concurrent validity of the Portuguese version of the patient perception of patient-centeredness (PPCD-16)}

A. Macedo ${ }^{1}$, A. Araújo ${ }^{*}$, D. Moura ${ }^{1}$, M.J. Soares ${ }^{1}$, C. Marques ${ }^{1}$, A. Pereira $^{1}$

${ }^{1}$ Institute of Psychological Medicine, Faculty of Medicine- Coimbra University- Portugal, Coimbra, Portugal; ${ }^{2}$ Faculty of MedicineUniversity of Coimbra, Institute of Psychological Medicine, Coimbra, Portugal

* Corresponding author.

Background and aims.- Patient-centeredness is widely acknowledged as a core value in medicine (WHO 2010). The Patient
Perception of Patient-Centeredness (PPCD; Stewart et al., 2000) is a 14-item self-report questionnaire to measure patient perceptions' of patient-centered care during the last visit with a family physician. We developed the Portuguese version of the PPCD (PPCD-16) by adding two items (15 and 16) based on Mead and Bower (2000) biopsychosocial perspective of patient-centeredness, to the original scale. In our preliminary psychometric analysis, the PPCD-16 (Macedo et al., 2012) showed good reliability and validity (Exploratory Factor Analysis) resulting in a two factors structure (F1: Empathy; F2: Active Patient Involvement).

Aims.- To re-examine the factor structure (using Confirmatory Factor Analysis, CFA) and the concurrent validity of the PPCD-16 in a different sample.

Methods.- A sample of 244 adults [162 (66,4\%) women; mean age $=33.67 \pm 13.478$ years] completed the preliminary version of PPCD-16 and the Portuguese Communication Assessment Tool (CAT). The CFA was obtained using the AMOS 23 software.

Results.- CFA indicated a good fit for the second-order factor with two factors $(\mathrm{X} 2 / \mathrm{df}=2.331 ; \mathrm{CFI}=.935 ; \mathrm{GFI}=.891 ; \mathrm{TLI}=.906$; $\mathrm{P}[\mathrm{rmsea} \leq .01]=.058)$. The PPCD-16 revealed good internal consistency $(\alpha=.92)$, as revealed by Cronbach's alpha. Both factors presented good reliability: Empathy $(\alpha=.91)$; Active Patient Involvement $(\alpha=.79)$. PPCD-16 $\left(r=.758^{* *}\right)$, Empathy $\left(r=.750^{* *}\right)$ and Active Patient Involvement $\left(r=.621^{* *}\right)$ highly correlated with the CAT.

Conclusions.- The Portuguese adapted version of the PPCD (PPCD16 ) is highly reliable and valid to assess the patient perception of patient-centeredness.

Disclosure of interest.- The authors have not supplied a conflict of interest statement.

\section{E-PV0404 \\ Outpatient clozapine monitoring at primary health care units}

A. Kourti ${ }^{1}$, P. Argitis ${ }^{1}$, C.M. Platsa ${ }^{1}$, N. Pagkratis ${ }^{1}$, P. Voukia ${ }^{1}$, P. Dalli $^{1}$, S. Karavia ${ }^{1}$, K. Paschalidis ${ }^{2}$, A. Karampas ${ }^{3}$, F. Kakavitsas ${ }^{4}$, Z. Chaviaras $^{1}$

${ }^{1}$ General Hospital Of Corfu, Psychiatric, Corfu, Greece; ${ }^{2}$ Psychatric Hospital of Thessaloniki, Psichiatric, Thessaloniki, Greece; ${ }^{3}$

University Hospital of Ioannina, Psychatrit, Ioannina, Greece; ${ }^{4}$ General Hospital of Corfu, GP-Manager of General Hospital Of Corfu, Corfu, Greece

"Corresponding author.

Background and aims.- Clozapine is a second-generation antipsychotic, reaching a response rate of $30-60 \%$ in treatment refractory schizophrenia. In Greece, the introduction of clozapine in patients' medication is permittable only during hospitalization, due to its hazardous adverse effects (neutropenia, agranulocytosis, myocarditis, seizures). In the General Hospital of Corfu, with starting point the end of 2017,we apply a protocol of outpatient introduction of clozapine in patients' treatment, which resulted in adequate response in treatment as well as satisfactory cost effectiveness.

Methods.- During the application of the protocol, most patients admitted they were reluctant or even distressed to visit weekly our Psychiatric clinic's outpatient units for the necessary regular monitoring (blood tests and vital sign measurements) throughout the titration period. For the facilitation of the patients, we tried to find a more suitable solution for them, one that is accompanied by an even better compliance ratio and a corresponding cost effectiveness. Results.- Primary Health Care practitioners is possible to be involved in the monitoring of clozapine treatment. GPs are able to conduct all the required tests and trained to identify pathological results in patients receiving clozapine treatment. Furthermore, PHC units are more accessible to patients due to their working policy. GPs will be in contact with the attending psychiatrist to inform 
them of the patient's well-being and test results, as well as the possibility of a transportation to the Hospital if the patient's condition requires so.

Conclusions.- The abovementioned proposal is possible to be implemented and have a positive effect on the patients' quality of life while their safety is ensured. It will be on a trial at our Hospital and the results will subsequently be publicized.

Disclosure of interest.- The authors have not supplied a conflict of interest statement.

\section{E-PV0405}

\section{Efficacy of different stress management interventions}

B. Barmi

Fortis Escort, Psychology, New Delhi, India

${ }^{*}$ Corresponding author.

Background and aims.- To study the effectiveness of relaxation and workshop module in the management of stress and enhancement of well being in executives.

To study the effectives of cognitive appraisal along with relaxation in the management of stress and enhancement of well being in executives.

To compare the relative efficacy of Relaxation and Cognitive Apprasial with Relaxation.

To compare the relative efficacy of Cognitive of Relaxation and Workshop Module.

To compare the relative efficacy of Cognitive Appraisal with Relaxation and Workshop Module.

Methods.- The sample comprised of executives both male and female ranging from 25-45 years were randomly allocated to three groups of equal size viz Relaxation Training (RT), Cognitive Appraisal with Relaxation (CA and RT) and Group 3 (G3) Workshop Group and Control Group. Pre, post and Follow up assessment was done and data was analyzed using qualitative and quantitative methods.

Results.- Relaxation training was found to be effective in significantly reducing somatic stress symptoms and enhancing the subjective well-being. Cognitive Appraisal with Relaxation was found to be effective in significantly reducing the total, somatic and cognitive stress symptoms. Relaxation training was found to be more effective than cognitive appraisal with relaxation in significantly reducing the somatic stress symptoms. Relaxation training was found to be equally effective to the workshop module. Cognitive Appraisal with Relaxation was found to be equally effective to the Workshop module.

Conclusions.- Stress can be thought of as resulting from an "imbalance between demands and resources" thus stress management interventions helps in building about the resources over the stress enhancing well-being.

Disclosure of interest.- The authors have not supplied a conflict of interest statement.

\section{E-PV0406 \\ Creating the orientation on addictions service for undergraduate students of the university of Girona. \\ F. Calvo ${ }^{1 *}$, C. Giralt ${ }^{2}$, X. Carbonell ${ }^{3}$ \\ ${ }^{1}$ Institut d'Assistència Sanitària, Health, Girona, Spain; ${ }^{2}$ CAS Girona, Institut d'Assistència Sanitària, Girona, Spain; ${ }^{3}$ FPCEE Blanquerna, Universitat Ramon Llull, Barcelona, Spain \\ ${ }^{*}$ Corresponding author.}

Background and aims.- A team of different professionals working in the University of Girona realize that, at the end of seminaries about drug addiction, a significative number of students consulted about their own problems with drugs. This experience motivated the creation of a specialized service about orientation on addictions for students. (resources of the community, possibility of treatment). The objective of this study is to describe the pilot intervention of the Addictions Orientation Service (AOS) of the University of Girona (Catalonia).

Methods.- The responsible of the service recruit the population apply for orientation about addiction. The analysed variables were sex, age, the reason to attend the service and how we could help them.

Results.- The pilot test starts 2017 October 19 and finalized 2017 December 21. In two months we attend 9 students, 6 women and 3 men. The medium age was $23,2(\mathrm{SD}=8,7)$. Seven cases were interested about how to manage the addiction of parents, brothers and sisters or other familiars. They described difficulties of communication with care professionals and providers with sons of addicts as them. Two cases present substance dependence criteria and one was transferred to a treatment public centre.

Conclusions.- There are gaps in the drug attention in Catalonia and is a requirement to cover the needs of students and other young people about treatment and information. AOS activity demonstrated the need to incorporate addiction orientation in the Catalan universities.

Disclosure of interest.- The authors have not supplied a conflict of interest statement.

\section{E-PV0409}

\section{Tackling social inclusion in mental health through social farming}

F. Cirulli ${ }^{*}$, M. Borgi ${ }^{1}$, C. Correale ${ }^{1}$, R. Orlich ${ }^{2}$, A. Grizzo ${ }^{2}$

${ }^{1}$ Istituto Superiore di Sanità, Center for Behavioral Sciences and Mental Health, Rome, Italy; ${ }^{2}$ Healthcare Authority of Western Friuli, Social and Health Services, Pordenone, Italy

${ }^{*}$ Corresponding author.

Background and aims.- In response to the current crisis of the public social and health care services, hybrid governance models, in which public health bodies, local communities and economic actors work together to co-produce social services for mental health promotion, may increase system's sustainability. In this context, social farming (SF) -a term used to describe farming activities for social inclusion of people with mental disabilities - is an innovative practice that could link healthcare institutions and local social and economical contexts. Notwithstanding this potential, there is still very little evidence on the effects of SF in promoting mental health as well as social inclusion. Objective of this pilot study was to assess the effect of SF in ameliorating social and professional skills in a sample of adult with mental health issues.

Methods.- A sample of 25 individuals with a diagnosis of psychosis or intellectual disability were recruited and involved in SF activities (e.g., horticulture, food processing, selling of products, animal care). A questionnaire was developed to assess the effects of SF activities on participants' social and professional skills. Preliminary data were collected at the beginning, after a training period, and at the end of the project.

Results.- Results indicate improvements in different areas including social competence, autonomy, and motivation/engagement.

Conclusions.- So far, data clearly indicate that SF has the potential to address specific needs of mentally ill persons, including rehabilitation and sheltered employment. Open questions remain as to the training of professionals tutoring patients and long-term sustainability of the system.

Disclosure of interest.- The authors have not supplied a conflict of interest statement. 
E-PV0410

\section{The economic impact of mood disorders in romanian psychiatric patients}

C. Crisan ${ }^{1 *}$, M. Saftencu ${ }^{2}$, I. Miclutia ${ }^{1}$

${ }^{1}$ Iuliu Hatieganu University of Medicine and Pharmacy,

Neurosciences, Cluj-Napoca, Romania; ${ }^{2}$ Iuliu Hatieganu University of Medicine and Pharmacy, Faculty of Medicine, Cluj-Napoca, Romania

* Corresponding author.

Background and aims.- Mental health services are largely outpatient treatment, prescription medication, or a combination of both. Mood disorders place substantial clinical, social and economic burdens on individuals with the disorders, as well as on their families and wider society. The aim of this study is to evaluate the costs associated to psychiatric care in patients admitted to the Psychiatry Department of the Emergency County Hospital Cluj-Napoca and diagnosed with mood disorders.

Methods.- We performed a retrospective analysis of the total number of patients admitted in 2017 and diagnosed with mood disorders. Several variables (sex, age, ICD-10 diagnosis, history, length of the hospitalization, total cost/admission, comorbidities, suicide attempts and treatment) were taken into account.

Results.- $19.4 \%$ of a total of $n=3483$ patients were diagnosed with mood disorders. The highest rates for admissions were in May and September. The total health care cost for the aforementioned category was $4.5 \mathrm{M}$ RON. Medication and paraclinical tests generated only 5,84\% of total costs, the rest being allocated for food and accommodation. Patients with mood disorders had a mean hospitalization of 15.5 days/patient. For patients older than 65 years, the hospitalization period was significantly longer than for younger patients $(p=0.01)$, generating significantly higher costs $(p=0.02)$. Conclusions.- Mood disorders have a major impact on patients' functionality. An adequate treatment for out-patients and the improvement of therapeutic adherence for these severe psychiatric disorders could generate a better cost-efficiency rate. At the same time, the scarcity of healthcare resources necessitates the efficiency of alternative treatments to be examined.

Disclosure of interest.- The authors have not supplied a conflict of interest statement.

\section{E-PV0411}

\section{Specificity of stigmatization of mental disease in the professional community}

D. Dovbysh ${ }^{1,2^{*}}$, M. Bebtschuk ${ }^{1}$, V. Chub ${ }^{2}$

${ }^{1}$ Scientific-practical Children's and Adolescents Mental Health Center n.a. G. Sukhareva- Moscow Department of Health Care-MoscowRussia, Psychiatry, Moscow, Russia; ${ }^{2}$ Federal State Autonomous Educational Institution of Higher Education I.M. Sechenov First Moscow State Medical University of the Ministry of Health of the Russian Federation Sechenov University, Faculty of Higher Nursing Training- Psychology and Social Work, Moscow, Russia ${ }^{*}$ Corresponding author.

Background and aims.- The stigmatization of mental illnesses still remains one of the most serious reasons for not seeking help from specialists. According to the analysis of the literature, stigma exist not only among people who did not meet such a problem, but also among mental health workers.

To study specificity of stigmatization of people with mental illness in healthcare system in Russia

Methods.- Questionnaire (analysis of clinical examples and evaluation of personal experience of interactions with people, suffered from mental illness); Semantic differential (assessment of the image of a person with mental illness).

Results.- The formal level of knowledge about mental illness in both groups can be assessed as high enough: $96 \%$ correctly distinguish cases of mental illness, 79\% recommend a psychiatrist as one of the specialists to help. At the same time, for the majority of specialists it is typical to refuse personal contact with people with various mental disorders: 92\% would not use them as babysitters, $27 \%$ are not ready to maintain friendly, $48 \%$ would dissuade loved ones from getting married with ill person. Attention is drawn to the presence of maximum ranks in the following characteristics used to describe people with mental illnesses: aggressive, unpredictable, closed, hostile, dependent.

Conclusions.- The combination of a high level of formal knowledge and the desire to avoid personal contact with people suffering from mental illness in various fields, confronts the professional community with the task of further reflecting and developing destigmatization programs that focus not only on society as a whole, but also on specialists.

Disclosure of interest.- The authors have not supplied a conflict of interest statement.

\section{E-PV0412}

\section{Validation of the tripartite scale of self-conscious emotions in a sample of the Portuguese population}

H. Espírito-Santo*, C. Duarte, C. Sério, L. Lemos

Instituto Superior Miguel Torga, Psychology, Coimbra, Portugal

${ }^{*}$ Corresponding author.

Background and aims.- Self-conscious emotions are aroused by selfreflection and self-evaluation, and they aid in self-regulation. These types of emotions have a role in the development and maintenance of psychopathology. No instruments were found to measure shame, guilt, and pride separately with evidence of its validity with adults in the Portuguese context. Therefore, we intended to validate a new Tripartite Scale of Self-Conscious Emotionsin a sample of the Portuguese population based on the Test of Self-Conscious Affect-3. Methods.- 342 participants (age: $M \pm S D=25.25 \pm 7.87$ years; sex: $61.4 \%$ men) filled a sociodemographic questionnaire and the 15items Tripartite Scale of Self-Conscious Emotions inan internet survey. Instruction for the Scale consists of "Here are some phrases that may or may not describe how you are feeling now. Evaluate each sentence on the 5-point scale. Remember to evaluate each sentence based on how you are feeling at the moment". Items (e.g., "I feel the desire of crawling into a hole and disappear"), are rated ona 5 -point scale ( 1 = "I don't feel anything this way"; $5=$ "I feel $100 \%$ like this").

Results.- Cronbach's alpha was 0.72 , with a forced three-factor structure explaining $62.9 \%$ of the variance (Kaiser-MeyerOlkin $=0.88$; Bartlett's test of Sphericity: $p<.001$ ).

Conclusions.- The preliminary results indicate that the Tripartite Scale of Self-Conscious Emotionsis a valid instrument for the general population. Future directions include the analysis of further psychometric properties, a confirmatory factor analysis, and its study with clinical samples.

Disclosure of interest.- The authors have not supplied a conflict of interest statement.

\section{E-PV0413}

\section{Survey of psychiatrists' experience of working with primary care in the United Kingdom}

R. Faruqui ${ }^{1,2^{*}}$, S. Afghan ${ }^{3}$, C. Gerada ${ }^{4}$, P. Wilson ${ }^{2}$

${ }^{1}$ East Kent Neuropsychiatry Service- KMPT, Neuropsychiatry,

Ashford, United Kingdom; ${ }^{2}$ University of Kent, Primary Care Academic Unit- Centre for Health Services Studies, Canterbury, United Kingdom; ${ }^{3}$ Dudley \& Walsall Mental Health Partnership Trust $\mathcal{E}$ University of Wolverhampton- UK, Mental Health, Walsall, United Kingdom; ${ }^{4}$ Medical Director NHS Practitioners Health Programme $\mathcal{E}$ 
Former Chair RCGP, Practitioner Health Programme, London, United Kingdom

${ }^{*}$ Corresponding author.

Background and aims.- A majority of patients with mental health conditions are treated in the Primary Care. In this context it is important that mental health services are able to effectively collaborate with the primary care in delivering an effective specialist service.

Methods.- We conducted a survey of psychiatrists' experience of working with the primary care. We used an online survey tool and membership database of the Royal College of Psychiatrists to conduct a national survey in the UK

Results.- 536 respondents from England, Wales, Northern Ireland, and Scotland participated in the survey. $79 \%$ respondents reported working for Mental Health Trusts and 9\% with Community NHS Trusts. 5\% respondents reported working for Independent Sector. $33 \%$ respondents reported a high or very high level of satisfaction with their communication with the primary care. Only $27 \%$ reported a low or very low level of satisfaction on this variable. These results were proportional to reported satisfaction (31\%) and dissatisfaction (28\%) with time allowed by the employers to communicate effectively with the General Practice.

Only $6 \%$ of the sample reported high or very high level of satisfaction with the availability of joint CPD opportunities with the Primary Care. Over $15 \%$ of respondents reported high or very high level of satisfaction with availability of joint working opportunities with General Practice.

Conclusions.- The findings of the survey have implications for improving psychiatrists' experience of working with the primary care. Availability of joint CPD opportunities and co-working between the General Practitioners and Psychiatrists are likely to enhance mutual support and quality of care.

Disclosure of interest.- The authors have not supplied a conflict of interest statement.

\section{E-PV0414}

\section{EIP in a natural setting: preliminary \\ results}

I. Fernández ${ }^{1}$, I. Ramos ${ }^{2}$, A. Stoppa ${ }^{1}$

${ }^{1}$ Consorci sanitari de Terrassa, Psychiatry, Terrassa, Spain; ${ }^{2}$ Consorci sanitari de Terrassa, Psychology, Terrassa, Spain

${ }^{*}$ Corresponding author.

Background and aims.- The EIP was born in Australia aiming to reduce the hospitalization and severity of symptoms, improving life quality in first episode patients. Nowadays it is extended worldwide. Similarly to this, we implemented an EIP program following and mixing the Stand-Alone and Enhance community mental health care models. In our project we created a multidisciplinary team formed by a psychologist, a psychiatrist, a nurse and a social worker for each area of action. A diffusion was made to professionals from different areas to ease the detection of patients susceptible to suffer a psychotic disorder. Once the case is detected a response is given in $24-72 \mathrm{~h}$.

The main objectives of this program are the early detection of risk profiles and first episodes, reduction of damage and improve the prognosis. Specific objectives are to promote preventive interventions in risk profiles, offer comprehensive treatment, facilitate recovery, prevent relapse, improve adherence to psychosocial and pharmacological treatment and support the family.
Methods.- To describe our program and to present descriptive preliminary results and qualitative acceptance by patients and families.

The variables will be analyzed through basic statistics with the SPSS program

Results.- After 3 months of starting this program, we have a sample of 23 patients, mostly single men who have shown the first symptoms in the last two years. Almost all the subjects of the sample have been hospitalization before enter in the program.

Conclusions.- It is soon to conclude the efficiency of EIP, however, first analysis show promising results on its application.

Disclosure of interest.- The authors have not supplied a conflict of interest statement.

\section{E-PV0416}

\section{Service user experience of multidisciplinary team meetings on scarisbrick inpatient unit, ormskirk and district general hospital, lancashire care nhs foundation trust, UK}

M. Gani ${ }^{*}$, B. Naguib ${ }^{2}$, F. Devlin² ${ }^{2}$ D. Gurung ${ }^{2}$

${ }^{1}$ Greater Manchester Mental Health NHS Foundation Trust, Psychiatry - North Manchester General Hospital, Manchester, United Kingdom; ${ }^{2}$ Lancashire Care NHS Foundation Trust, Psychiatry Scarisbrick Unit- Ormskirk and District General Hospital, Ormskirk, United Kingdom

* Corresponding author.

Background and aims.- This evaluation is based on a NICE (National Institute for Health and Care Excellence) quality statement on service users' views being utilised to improve mental health services. On Scarisbrick ward, the Patient Centred MDT (multidisciplinary team) Meeting holds weekly.

Aims.-

- To assess Service User Experience of MDT meetings and use these findings to inform and develop an appropriate intervention.

To assess Service User Experience of implemented intervention Methods.- Participants: Admitted, willing adult patients between May and July 2018.

Process:

- Initial questionnaire developed with MDT members

- Questionnaire passed round patients

- Collection and collation of completed questionnaires.

- Results discussed with MDT

- Intervention chosen - information leaflet on the MDTs

- Information leaflet rolled out on ward over three weeks

- Another questionnaire passed around to obtain feedback.

Results.-

Initial questionnaire - $88 \%$ of respondents ranked the meetings as satisfactory or better. Post intervention questionnaire - $90 \%$ rated the meeting as satisfactory or higher. $30 \%$ found the leaflet had helped lower feelings of anxiety. About half the respondents felt more empowered and involved in their own care.

$80 \%$ recommend we continue to use the information leaflet for new patients (figure 1 ). 


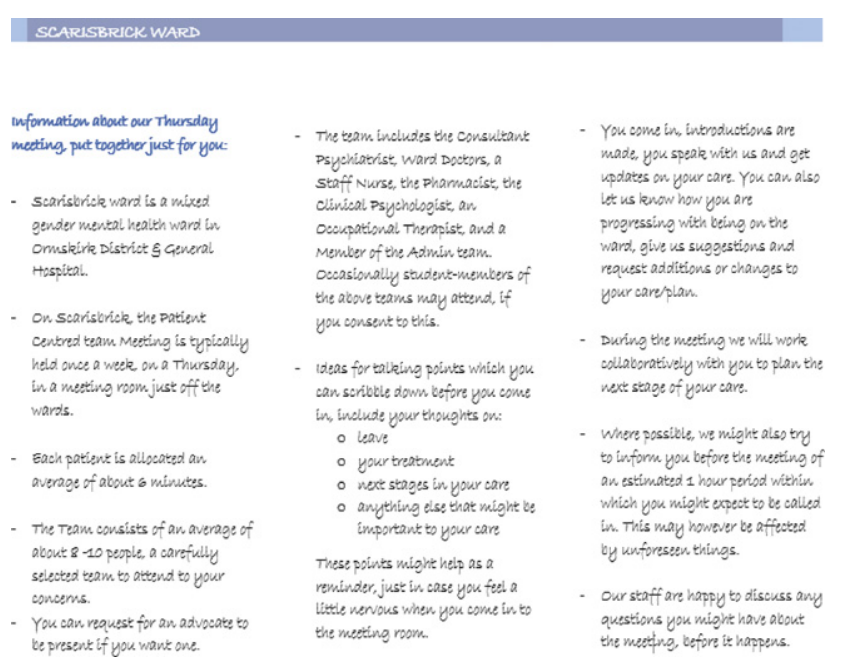

Figure 1. Scarisbrick ward.

Conclusions.- Service users views are invaluable in providing high quality, patient centred care; in keeping with the King's fund/ Richmond group reports. We hope this leaflet can be rolled out as 'standard'.

Disclosure of interest.- The authors have not supplied a conflict of interest statement.

\section{E-PV0418}

\section{Testing a new model, integrating mental health care with ips (individual placement and support), in common mental disorders: $1200+$ patients in a randomized controlled trial}

A. Hoff

Mental Health Services- Capital Region Denmark, Research UnitPsychiatric Centre Copenhagen, Copenhagen, Denmark

Corresponding author.

Background and aims.- Sick leave due to common mental disorders (CMD) has major negative influence on society because of the lost productivity, social benefits, and treatment costs, and OECD estimates that the cost is equivalent to $3,4 \%$ of the Danish gross national product.

The objective is to examine, whether integrating mental health care with vocational rehabilitation, conveys shorter return to work (RTW) time, compared to treatment as usual, after sick leave due to a CMD such as depression, anxiety, and stress-related disorders. The main aim of the intervention is to provide the shortest possible RTW time, which is the primary outcome.

Methods.- Patients are included in one of two randomized controlled studies (anxiety or depression in one RCT, and stress-related disorders in another), after sick leave for at least four weeks. Patients, in both trials, are randomized into one of three groups: a) control group, where they receive treatment as usual in primary care b) intervention group: mental health care (MHC) in the research project or c) intervention group: MHC in the research project, integrated with a special project occupational rehabilitation.

Results.- We will present baseline data of the entire inclusion sample, as well as 6 months outcome data: RTW and symptom levels (though blinded, since we break the blinding after analyses of primary outcome data at 12 months = May 2019).

Conclusions.- We have currently included the entire needed sample of at least 1206 participants, and hence have the needed power to present results in spring 2019 !
Disclosure of interest.- The authors have not supplied a conflict of interest statement.

\section{E-PV0420}

\section{Assessment of empathy and psychological characteristics of smokers}

G. Iatrou $^{1 *}$, K. Gourgoulianis ${ }^{1}$, E. Kotrotsiou ${ }^{2}$, M. Gouva ${ }^{3}$, S. Kotrotsiou $^{2}$, T. Paralikas ${ }^{2}$

${ }^{1}$ University of Thessaly, Medicine, Larissa, Greece; ${ }^{2}$ TEI of Thessaly, Nursing, Larissa, Greece; ${ }^{3}$ TEI of Ioannina, Nursing, Ioannina, Greece * Corresponding author.

Background and aims.-

Introduction.- It is well known that smoking causes at least 25 life-threatening diseases or groups of diseases. Smoking is also often associated with psychiatric symptoms such as high levels of anxiety disorders, depression, increased use of alcohol and other substances.

Damage however is caused by smoking also to those called "passive smokers". Empathy could help smokers control their desire to smoke in public places because they recognize that apart from the harmful effects on their body, they also harm people around them. Smokers must understand and respect the fact that people around them are not necessarily smokers themselves.

Aim.- To evaluate empathy and the psychological characteristics of Greek smokers.

Methods.- For research purposes we used questionnaires Toronto Empathy Questionnaire (TEQ), Symptom Check list 90-R, Experience of Shame Scale (EES) and Other As Shamer Scale- OAS.

Results.- A statistically significant difference has been observed in the TEQ at the factor 3, which measures the empathy in smokers and non smokers, and shows lower levels of empathy in smokers (Figure 1 and table 1 ).

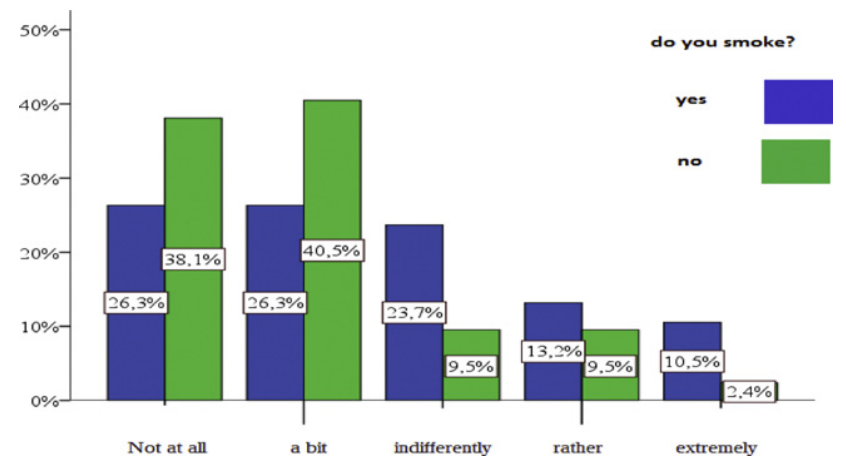

Figure 1. Do you smoke?

Table 1 Results: "do you smoke in public places?"

\begin{tabular}{llll}
\hline & $\mathrm{N}$ & mean & S.D. \\
\hline yes & 28 & 2.9 & 0.5 \\
no & 11 & 3.3 & 0.4
\end{tabular}

Conclusions.- The sample consisted of 81 subjects, 39 smokers and 42 non smokers $(48,15 \%$ and $51,85 \%)$. Of smokers, $71.7 \%$ stated that smoke in public areas. From the results of our research we observe that smokers exhibit moderate to low empathy and high levels of anxiety disorders. The educational level and gender of the participants do not seem to play a significant role in empathy and psychopathology of smokers.

Disclosure of interest.- The authors have not supplied a conflict of interest statement. 


\section{E-PV0421}

\section{Innovative "adolescent-friendly" health services for gender dysphoric youth in Greece}

A. Kanellopoulos ${ }^{*}$, A.K. Papakonstantinou, F. Bacopoulou Center for Adolescent Medicine and UNESCO Chair on Adolescent Health Care- First Department of Pediatrics- Medical SchoolNational and Kapodistrian University of the Athens- Aghia Sophia Children's Hospital- Athens- Greece, Center for Adolescent Medicine and UNESCO Chair on Adolescent Health Care- First Department of Pediatrics- Medical School- National and Kapodistrian University of the Athens- Aghia Sophia Children's Hospital- Athens- Greece, Athens, Greece

${ }^{*}$ Corresponding author.

Background and aims.- Sexuality is usually neglected during adolescent health maintenance visits. Adolescents are often reluctant to engage in sexuality talks whereas the majority of physicians are not proactive in addressing their sexuality issues. Even when sexuality is discussed, conversations are brief, andthe limited exchange is often inadequate to meet youth sexual needs.

Although adolescents are a heterogeneous group with different expectations and preferences, they all share a common need; they want to be treated with respect and be sure that theirconfidentiality is protected.

To make it easier for gender dysphoric youth and their families in Greece, to obtain the health services they need to protect and improve their health and well-being.

Methods.- The statutory Center for Adolescent Medicine (CAM) and UNESCO Chair on Adolescent Health Care of the First Department of Pediatrics, Medical School, National and Kapodistrian University of Athens at the Aghia Sophia Children's Hospital in Greece, is an innovative "adolescent-friendly" tertiary referral center for adolescents aged 10-21 years, recognized officially by the Hellenic Ministry of Health and Welfare. It operates within the WHO framework for "adolescent-friendly" health services, i.e. it is accessible, acceptable, equitable, appropriate and effective.

Results.- The CAM provides outpatient services to youth with gender dysphoria. A multidisciplinary team including a childadolescent psychiatrist, adult psychiatrist, adolescent medicine specialists, psychologists, provides quality care the meet the complex and delicate medical, mental and social issues of these adolescents.

Conclusions.- Provision of innovative youth-friendly health services may improve the care of gender dysphoric youth and their families. Disclosure of interest.- The authors have not supplied a conflict of interest statement.

\section{E-PV0422}

\section{Investigation of the levels of stress on 1 st and 3rd semester students of nursing department tei of thessaly Greece and strategies for stressful situations}

S. Kotrotsiou ${ }^{1 *}$, N. Apostolou ${ }^{2}$, T. Paralikas ${ }^{1}$, C. Barbouta ${ }^{2}$, A. Barbouta $^{3}$, A. Kalfoutzou ${ }^{4}$, E. Tsagalas ${ }^{5}$

${ }^{1}$ T.E.I of Thessaly Greece- Postgraduate program Mental healthResearch Laboratory Primary Health Care- T.E.I of Thessaly- LarissaGreece, Nursing, Larissa, Greece; ${ }^{2}$ T.E.I of Thessaly GreecePostgraduate program Mental health, Nursing, Larissa, Greece; ${ }^{3}$ University of York MSc Psychology in education, Education, York, United Kingdom; ${ }^{4}$ T.E.I of Thessaly Greece, Nursing, Larissa, Greece; ${ }^{5}$ University Hospital Larissa- Greece, Nursing, Larissa, Greece

${ }^{*}$ Corresponding author.

Background and aims.- The stress has been linked with students life.
Methods.- Studied Population is the undergraduate Nursing students of the first two years of study. The sample was a total of $n=119$ students of the 1st and 2nd year of study of Nursing Department TEI of Thessaly. The research tools used: a) State - Trait Anxiety Inventory, b) Ways of Coping (Lazarus R.S. and Folkman S., 1984). The statistical analysis was done using the SPSS 22.0.

Results.- According to the results, $86.6 \%$ of the students were women and $13.4 \%$ were men, with an average age of 22.8 years (a constant deviation of 8.05) and a range of 15 to 51 years. Investigating the correlation between the socio-demographic characteristics of nursing students and the Scale students has revealed a multitude of correlations. Among these was a statistically significant negative relationship of the "Positive Approach" strategy to all Nursing students' Anxiety Styles, a statistically significant positive relationship of the "Avoidance / Escape" strategy to the Permanent Anxiety $(r=0,177)$ of Nursing students and a statistically significant positive link of the "Problem" with the Nervous Transient Anxiety $(r=0.176)$ of Nursing students.

Conclusions.- The high level of stress on studying population and especially on nursing students appoint the need of intervention in order to secure the quality of students life. It is important the effort of stress decrease on the newentering students on academic place through systematic energies and social networks with student organizations and activities.

Disclosure of interest.- The authors have not supplied a conflict of interest statement.

\section{E-PV0423}

\section{Assessment of cultural knowledge of midwifes in greek public hospitals}

T. Paralikas ${ }^{1}$, E. Kotrotsiou ${ }^{1}$, S. Kotrotsiou ${ }^{2}$, E. Lahana ${ }^{1}$, A. Vaitsidou $^{1}$, M. Malliarou ${ }^{1}$, E. Tsagalas $^{3}$, G. Tsioumanis ${ }^{1}$

${ }^{1}$ University of Applied Sciences of Thessaly T.E.I. of Thessaly. Postgraduate Program in Mental Health, Laboratory of Care, Nursing, Larissa, Greece; ${ }^{2}$ T.E.I. of Thessaly, Nursing Department, Larissa, Greece; ${ }^{3}$ General University Hospital of Larissa, Nursing, Larissa, Greece

* Corresponding author.

Background and aims.- Cultural competence has involved many researchers. Adherence to rules on respect for cultural diversity is considered a basic principle for health professionals.

Aim of this study was to assess the level of cultural competence, especially of a cultural knowledge of midwives.

Methods.- The study sample consisted of midwives working at public hospitals in Greece. The collection of research material was done through a questionnaire namely Cultural Competence Assessment Tool (CCAT). The creation of the CCATool scale was based on the Papadopoulos, Tilki and Taylor Model. It consists of 40 declarations, which per ten refer to cultural Vigilance, Knowledge, Sensitivity and Practice.

Results.- The midwifery experience appears to have a marginal statistically significant relationship with cultural sensitivity $(p=0.069)$. Midwives who work for less than 5 years are more sensitive than others. The language, country of birth, age and service as a midwife do not seem to affect the cultural sensitivity of the participants. Cultural Sensitivity is statistically relevant to cultural Knowledge (positive relationship, $r=.502$ and $p=.000$ ), i.e. the greater the cultural knowledge, the greater the cultural sensitivity of the participants. Cultural Practice is statistically relevant to Cultural Knowledge (positive relationship, $r=.344$ and $p=.006$ ).

Conclusions: There is a great need of education in cultural competence. Curricula should include lessons of a cultural approach to health and illness and intercultural practice.

Conclusions.- There is a great need of education in cultural competence. Curricula should include lessons of a cultural approach to health and illness and intercultural practice. 
Disclosure of interest.- The authors have not supplied a conflict of interest statement.

\section{E-PV0425 \\ Evaluation of a district mental \\ healthcare plan in a low-resource \\ setting in Nepal: outcomes at community, facility and individual levels}

N.P. Luitel ${ }^{1^{*}}$, M.J.D. Jordans ${ }^{2}$, B.A. Kohrt ${ }^{3}$, S.D. Rathod ${ }^{4}$, E.C. Baron ${ }^{5}$, I.H. Komproe ${ }^{6}$, V. Patel ${ }^{7}$, C. Lund $^{5}$

${ }^{1}$ Transcultural Psychosocial Organization TPO-Nepal, Research, Kathmandu, Nepal; ${ }^{2}$ King's College London, Institute of PsychiatryPsychology and Neuroscience, London, United Kingdom; ${ }^{3}$ George Washington University, Department of Psychiatry, Washington DC, USA; ${ }^{4}$ London School of Hygiene and Tropical Medicine, Department of Population Health, London, United Kingdom; ${ }^{5}$ University of Cape Town, Alan J Flisher Centre for Public Mental Health- Department of Psychiatry and Mental Health, Cape Town, South Africa; ${ }^{6}$ Utrecht University, Faculty of Social and Behavioural Sciences, Utrecht, Netherlands Antilles; ${ }^{7}$ Harvard University, Harvard Medical School, Cambridge, USA

Corresponding author.

Background and aims.- In most low- and middle- income countries, care for persons with mental, neurological, and substance use (MNS) disorders is largely absent, especially in rural settings. To increase treatment coverage, integration of mental health services into community and primary health care settings is recommended. The aim of this study was to evaluate contact coverage, detection and treatment outcomes as a result of a complex multi-component district level mental health care program for adults in Nepal.

Methods.- Treatment coverage was evaluated through a community study $(N=4612)$ and through community surveillance data $(N=727)$, detection and initiation of minimally adequate treatment in primary care facilities was evaluated through a facility study $(N=3627)$ and the outcomes of patients receiving primarycare based mental health treatment was evaluated through cohort studies $(N=448)$.

Results.- The results demonstrated modest to large changes in contact coverage (ranging from $7.5 \%$ for alcohol use disorder to $50.2 \%$ for psychosis). Small to large effect sizes for change in health worker detection of mental illness (ranging from $d=0.3$ for depression after 12 months to $d=1.6$ for alcohol use disorder, post training). Small to medium effect sizes for clinical outcomes (ranging from $d=0.25$ for improved functioning among people with psychosis at 3 months to $d=0.59$ for reduction in symptoms for depression at 3 months). Conclusions.- These combined results make a strong case for feasibility and impact of community- and primary-care based services delivered through an integrated district mental health care plan in reducing the treatment gap and increasing effective coverage for MNS disorders.

Disclosure of interest.- The authors have not supplied a conflict of interest statement.

\section{E-PV0427 \\ Meditation and yoga as interventions for psychiatric disorders: ADHD and PTSD}

A. Mendrek ${ }^{1}{ }^{*}$, D. $\operatorname{Logan}^{1}$, T. Veronique ${ }^{1}$, H. Poissant ${ }^{2}$

${ }^{1}$ Bishop's University, Psychology, Sherbrooke, Canada; ${ }^{2}$ Universite du Quebec a Montreal UQAM, Pedagogy and Education, Montreal, Canada

* Corresponding author.
Background and aims.- Yoga and meditation have entered the mainstream culture and are being introduced to psychiatric hospitals and clinics. While the body of evidence supporting the effectiveness of yoga and meditation in the general population has been growing exponentially, the evidence from clinical trials is limited. Objectives.- In this review, we examine existing literature concerning use of meditation and yoga in two markedly different disorders in adults, in which non-pharmacological interventions may be particularly suitable: attention deficit hyperactivity disorder (ADHD) and post-traumatic stress disorder (PTSD).

Methods.- Using the terms "ADHD and meditation or mindfulness" and using a separate search term "PTSD and Yoga" we searched Pub Med and Psych INFO databases for clinical trials, prospective and follow-up studies, and selected 11 ADHD and 22 PTSD studies.

Results.- All ADHD studies showed improvement of symptoms. Six studies (55\%) reported improvement in measures related to emotion dysregulation (anxiety, depression, and stress) and six studies (55\%) reported amelioration of executive function. Twenty (90\%) PTSD studies showed positive effects. Thirteen studies $(60 \%)$ reported reduction in trauma-related symptoms (anxiety, hyper-arousal, re-experiencing, avoidance, depression, insomnia or dissociative symptoms) and seven (32\%) revealed improved well-being (relaxation, self-esteem, resilience, inner-peace, acceptance, empowerment, centeredness).

Conclusions.- While there is a great promise is employing yoga and meditation as treatment add-ons to available psychotherapy and/or pharmacotherapy in ADHD and PTSD, we need to proceed with caution and get knowledgeable about specific methods. The available literature is promising but variable in the methodology and no clear guidelines have been established.

Disclosure of interest.- The authors have not supplied a conflict of interest statement.

\section{E-PV0428}

\section{Medical and psychological support of soldiers and servicemen of the armed forces of Ukraine}

B. Mykhaylov ${ }^{1 *}$, R. Shevchenko ${ }^{2}$

${ }^{1}$ Kharkiv Medical Academy of Postgraduate Education,

Psychotherapy, Kharkiv, Ukraine; ${ }^{2}$ Odessa National Maritime

University, Practical Psychology, Odessa, Ukraine

"Corresponding author.

Background and aims.- The results are based on the data of a complex psychodiagnostic examination of 465 soldiers and servicemen of the Armed Forces of Ukraine with neurotic and psychosomatic disorders, who were undergoing hospital treatment in the Military Medical Clinical Center of the Southern Region, city of Odessa.

The first stage (2008-2014), the second stage (2014-2017).

Attention was paid to the study of the phenomenology of maladaptive reactions, and the optimization of psychocorrectional programs that are important for possible restoration of further professional activity.

Methods.- Two systems of support of servicemen at the hospital stage were developed. The first direction includes two stages: express-sorting and differentiated psychocorrectional intervention.

Results.- The second direction was developed for soldiers and includes three blocks: "Stabilizing", "Transformation", "Revaluation".

Conclusions.- The system of medical and psychological support of soldiers and servicemen of the Armed Forces of Ukraine at the hospital stage has been further improved, it is aimed at restoring of the social functioning and increasing the adaptive capacity based on a comprehensive interdisciplinary analysis of medical, psychological and socio-psychological characteristics of neurotic and psychosomatic disorders. 
Disclosure of interest.- The authors have not supplied a conflict of interest statement.

\section{E-PV0430 \\ Institutional violence and the impact of otherness in the traumatic process \\ F. Nyegue ${ }^{1^{*}}$, A. Fraud ${ }^{2}$ \\ ${ }^{1}$ Lirces/Universite de Nice Sophia-Antipolis, \\ Psychologie/Psychanalyse, Nice, France; ${ }^{2,}$ Lash, Nice, France \\ * Corresponding author.}

Background and aims.- Health systems are undergoing increasingly stringent ethical regulations and procedures, and economic issues are creating psychological and moral pressure on the health caregivers' side, we are witnessing the powerlessness of institutions and caregivers because of the violence of the patients and the exhaustion of the interveners.

We thought that the regulations of teams as well as the analyzes of the practices would solve the problems of care encountered by the teams. The reality shows the traumatic dimension of the sufferings observed in caregivers victims of violence from the patients. The institutionalization of delinquents and patients with real psychopathological problems is therefore a real challenge.

The aim is to consider how to organize health care methods adapted to a varied and multi-problems patients.

Methods.- Psychological assessments by projective tests (TAT Rorschach ...); non-directional interviews; observations on populations with various problems

Results.- In progress.

Conclusions.- The care of patients in prisons and in the judiciary is more oriented towards the mixing of subjects presenting problems of delinquency with real psychopathological. Because of the economic ssues, teams and care staff are forced to manage in inappropriate conditions. Managing issues of delinquency when we are caregivers becomes as complex as managing psychiatric issues when we are educational staff. Inadequate responses to inappropriate patient needs become a source of misunderstanding, conflict and violence. The symptoms because of the violence they induce then produces suffering and traumatisms on the side of the professionals.

Disclosure of interest.- The authors have not supplied a conflict of interest statement.

\section{E-PV0431 \\ Procrastination in students of clinical psychology department}

\section{F. Sultanova, E.I. Ognyannikova*, V.V. Barabanshchikova}

Lomonosov Moscow State University, Psychology, Moscow, Russia

* Corresponding author.

Background and aims.- Almost 90\% of examined students suffer from procrastination (Steel, P, 2007). The concept of this phenomenon and predictors are still complicated. Kuhl's action control theory mentions procrastination as a main source of unrealized intentions (Kuhl, J., 1994a). J. Ferrari defines procrastination as a tendency towards laziness (Ferrari, J. R., 2010). This study aims to combine theoretical background and previous research (Barabanshchikova V.V. at all., 2017), to examine the effect of action control and laziness on procrastination in students of clinical psychology department $(n=35)$.

Methods.- Diagnostic means included three questionnaires: "Action Control Scale» J. Kuhl (Kuhl J., 1986), (S. Shapkin, 1997), "Self-diagnostics of laziness» (D. A. Bogdanova and S. T. Posokhova (Ilyin, 2011)), "General Procrastination Scale (GPS) », (C. Lay, 1986), (Varvaricheva, 2010).

Results.- The results of regression analysis revealed that reduction of action control $(\beta=-0,421 ; p=0,038)$ is a predictor of procrastination $\left(r^{2}=0,603 ; p=0,0001\right)$. The unexpected result was no dependence between procrastination and laziness $(\beta=-0,247$; $p=0,117$ ).

Conclusions.- In case of procrastination, phenomenon an individual's ability to realize of the intended action has to be built on the principles of action control. Confirming the previous research, this analysis showed that laziness cannot be taken as a doubtless predictor of delay. Present study helps to identify predictors of procrastination's development and prospectively allows increasing a job performance efficiency.

Disclosure of interest.- The authors have not supplied a conflict of interest statement.

\section{E-PV0432}

\section{Working memory:repetition}

B. Prathanee ${ }^{1}$, N. Ooppanasak ${ }^{2 *} ;{ }^{1}$ Faculty of Medicine- Khon Kaen University, Department of Otorhinolaryngology, Khon KaenThailand- 40002, Thailand; ${ }^{2}$ Faculty of Medicine- Khon Kaen University, Department of Otorhinolaryngology, Khonkaen, Thailand

* Corresponding author.

Background and aims.- Repetition is one of memory span that has become a widely useful, scientifically fruitful construct. Memory span tasks have proven to be both reliable and valid measures of working memory capacity. The objective of this study was to find the average items of repetition of target words and phrases of color shapes in adults and aging people.

Methods.- Adults and aging people in community were recruited for study in project of community medical service, Khon Kaen University. One hundred and eight as well as 99 adults and aging people were recruited for average items in repetition test with criteria $80 \%$ and $100 \%$. Participants were asked to repeat words or phrases of color shapes (colors: red, white, black, yellow; shape: circle, triangle, and quadrangle). Assessors firstly told target words or phrases, then participants repeat. Assessors secondly told target words or phrases in case of participants could not repeat them within 5 second. Target words or phrases composed of 1-10 items (e.g. 1 item $=$ circle, 2 items $=$ red circle, $3=$ small red circle, 4 items $=$ red circle and yellow triangle etc.). Correct responses was recorded.

Results.- Average items of $80 \%$ and $100 \%$ of correct repetition were 5.18 and 4.82 items, (standard deviation $=1.06$ and 0.98 , respectively.

Conclusions.- Average items was a target level for promotion and eliciting working memory in adult to aging people or anyone who are at risk of memory deficits.

Disclosure of interest.- The authors have not supplied a conflict of interest statement.

\section{E-PV0433}

\section{Immediate memory span in}

\section{adults-aging people: comprehension}

B. Prathanee ${ }^{1^{*}}$, N. Ooppanasak ${ }^{2}$

${ }^{1}$ Faculty of Medicine-Khon Kane University, Department of Otorhinolaryngology, Khonkaen, Thailand; ${ }^{2}$ Faculty of MedicineKhon Kaen University, Department of Otorhinolaryngology, Khon Kane, Thailand

* Corresponding author.

Background and aims.- WM has become a widely useful, scientifically fruitful. One of measurement tools widely used for cognitive psychology is working memory span. The objective of this study was to investigate the average items of identification/comprehension of color shapes in adults and aging people. Methods.- Survey study recruited adults and aging people in a community (project of community medical service, Faculty of Medicine, Khon Kaen University). One hundred and two as well as 71 peo- 
ple were recruited for comprehension test with criteria of $80 \%$ and $100 \%$ of correct response. Participants were asked to repeat words or phrases of color shapes (colors: red, white, black, yellow; shape: circle, triangle and quadrangle), then, identify them. Evaluators firstly told target words or phrases, then participants repeat and point to pictures. Evaluators secondly told target words or phrases in case of participants could not correctly repeat or identify or point pictures within 5 seconds. Target pictures composed of $1-10$ items (e.g. 1 item = circle, 2 items = red circle, $3=$ small red circle, 4 items = red circle and yellow triangle etc.). Eighty percent and $100 \%$ of correct identification or comprehension ( 4 of 5 and 5 of 5 target words or phrases, respectively) were recorded.

Results.- Average items of $80 \%$ and $100 \%$ of correct identification or comprehension were 4.35 and 3.86 items ( standard deviation $=1.27$ and 1.26 , respectively).

Conclusions.- Average items of comprehension in normal adults and aging people can be used to be guideline for promotion the memory span.

Disclosure of interest.- The authors have not supplied a conflict of interest statement.

\section{E-PV0434 \\ Descriptors of adaptation resource in families where a patient with schizophrenia lives}

N. Pshuk*, L. Stukan, A. Kaminska

National Pirogov Memorial Medical University- Vinnytsya, Medical Psychology and Psychiatry Department with the Course of

Postgraduate Education, Vinnytsya, Ukraine

* Corresponding author.

Background and aims.- Social intelligence is an integral intellectual ability that determines success of communication and social adaptation, and allows us to assess level of interpersonal interaction in families. Taking into consideration the above, our research purpose was to determine social intelligence level of referential relatives in families where a patient with schizophrenia lives.

Methods.- Methods: psychological testing, social-demographic method. To study level of social intelligence J.P. Guilford and M. Sullivan social intelligence test was used. 168 family members of patients with paranoid schizophrenia (97 men and 71 women) were included into study. Control group included 55 individuals, in whose families there is no mentally sick person.

Results.- Received numerical data suggest that referential relatives of patients with schizophrenia cannot always clearly build their own behavioral strategy to achieve certain goals in treatment of patients, they misconstrue results of their actions and actions of the others, experience difficulties in assessing states and feelings according to non-verbal attributes in process of interpersonal communication, and are often guided by single statements of interlocutors rather than their facial expression, postures and gestures. Openness and friendliness are not always the key to success in communication; referential relatives have reduced sensitivity towards emotional states of others and not always appropriately assess their own emotional status, which are direct prerequisite for dysfunctionality in communication as sensitivity to non-verbal expression significantly enhances our ability to understand the others.

Conclusions.- Thus, referential relatives of patients with schizophrenia have reduced adaptation personality resources in interpersonal interaction compared to control group respondents. Disclosure of interest.- The authors have not supplied a conflict of interest statement.
E-PV0438

\section{Perception of social issues by the prisoners of the penitentiary system of russia in comparison with a control sample}

L. Shaigerova, F. Ushkov, Y. Zinchenko, O. Almazova, A. Dolgikh, R. Shilko , O. Vakhantseva

Lomonosov Moscow State University, Psychology, Moscow, Russia

Corresponding author.

Background and aims.- Detention in prison can affect mental health, subjective well-being, lead to identity transformations, and to changes in attitudes and values. A long separation from social life can change the perception of social issues and the integration into society. In the research we evaluated the impact of detention in prison on the perception of social issues.

Methods.- The research involved 119 prisoners aged 18 to 65 years $(\mathrm{Me}=32 ; \mathrm{SD}=8)$ (99 male) and 119 respondents of the control group. Participants were offered a list of social issues such as unemployment, immigration, drug abuse, alcohol abuse, poverty, terrorism, interethnic conflicts, crime level, etc. and were asked to what extent they are concerned about each of the problems.

Results.- Prisoners expressed greater concern for all of the problems in comparison to the control group ( $t$-test, $p<0,005$ ). Comparison with the control group showed that prisoners are significantly more concerned about the decline of birth rate, the impact of Internet on safety, alcoholism and changing family values. The participants from the control group were more concerned about the rise of prices, the infrastructure and quality of health care.

Conclusions.- Staying in places of detention increases anxiety about social problems in general and leads to a change in the perception of real social issues in comparison with the ordinary population. The lack of current need to worry about material security is expressed in prisoners by having less anxiety about physiological needs and greater anxiety about safety and belonging.

The research was supported by the Russian Science Foundation, with the Grant 15-18-00109.

Disclosure of interest.- The authors have not supplied a conflict of interest statement.

\section{E-PV0439}

\section{Alternative therapy in public health: librarytherapy or simply literapy \\ C.C. Silva*, L. Gurgel \\ Unifor, Psychology, Fortaleza, Brazil \\ Corresponding author.}

Background and aims.- Literapy consists of using carefully selected books (by the doctor and psychologist) readings to provide relief for patients with illnesses (who are hospitalized or followed up at basic health facilities). The goal is to join an alternative therapy together with classic drug therapy. This is the art of healing illness through reading. Not only in psychological ailments, but also in pains of the body. This therapy can be conceptualized as the prescription of reading materials with therapeutic function. The literature practice can be used as an important tool in the psychic recovery of individuals with emotional and physical disorders. She admits the possibility of therapy through reading, but also the additional comments to it, and proposes reading practices (interpretation of the text). Methods.- It consists of filling out a questionnaire with questions about reading preferences, along with others of a personal nature, such as the type of life, the main concerns. After this apply psychotherapy in patients suffering from psychic suffering, such as depression, anxiety disorders, panic and generalized and borderline anxiety. Dialog with the patient for a knowledge of their preferences to direct the therapy for a better performance of the technique. 
Results.- The project is underway and patients report improvement of mood and we are able to perform the weaning of benzodiazepines more quickly and efficiently with the combination of drug treatment with this alternative therapy

Conclusions.- Literapy is an alternative to complement medication treatment in psychic disorders since we noticed a more rapid improvement in patients treated with these two therapeutic compared to patients treated only with drugs.

Disclosure of interest.- The authors have not supplied a conflict of interest statement.

\section{E-PV0441}

\section{Mental health of PhD students at Polish universities - preliminary results}

M. Kowalczyk ${ }^{1}$, M. Talarowska ${ }^{1}$, P. Gałecki ${ }^{1}$, M. Karbownik ${ }^{2}$, E. Pabjańczyk-Wlazło 3

${ }^{1}$ Medical University of Lodz, Department of Adult Psychiatry, Lodz, Poland; ${ }^{2}$ Medical University of Lodz, Department of Pharmacology and Toxicology, Lodz, Poland; ${ }^{3}$ Polish National Representation of Doctoral Candidates, Krajowa Reprezentacja Doktorantów, Warsaw, Poland

* Corresponding author.

Background and aims.- The state of health of students taking doctoral studies in Poland is determined by obligatory preventive examinations performed by specialists in the field of occupational medicine. The results of these tests contain information about the admission of a given person or not to study.

In the available literature, there are no papers describing the mental health of polish doctoral students.

The aim of our own research was to assess the health of PhD students of Polish universities.

Methods.- The study used the GHQ 28 Questionnaire, D. Goldberg et al. (2001). Each questionnaire is accompanied by a note about, among others, age, sex, type of university, year of study, place of residence, marital status. PhD students from all universities associated in the National Representation of Doctoral Candidates were invited to the study. There were 830 volunteers. In return, 352 completed questionnaires were received.

Results.- It was found that depression is statistically more common in doctoral students who are not in any relationship with another person, and anxiety and insomnia are more common in women than in men and less often in doctoral students of general universities than Technical Universities. Approximately 5\% of PhD students receive a high point value (over 20) in particular points from scales $B, C$ and $D$, which may indicate a depressive mental health disorder with a co-occurring risk of committing a suicide attempt.

Conclusions.- There seems to be an urgent need to establish a care strategy for selected groups of students to improve their wellbeing.

Disclosure of interest.- The authors have not supplied a conflict of interest statement.

\section{E-PV0442}

\section{Romanian health care policy issues and familial resources of adults patients with intellectual and developmental disorders and behavioral problems}

R.O. Tipa*, A. Giurgiuca, C. Tudose

University of Medicine and Pharmacy "Carol Davila"- Bucharest, Psychiatry Department, Bucharest, Romania

${ }^{*}$ Corresponding author.
Background and aims.- Romania has been facing several difficulties in terms of resources, healthcare policy change and implementation regarding adult patients diagnosed with developmental disorders and behavioral problems. Having a child born with a disability is a permanent cause of stress and high vulnerability. Romanian caregivers (parents) are facing not only the burden of disease, but also barriers as systems' inabilities and failures. The aim of this paper was to examine specific familial variables like disease understanding, parental stress, adaptation, social support, personal burden as predictors of patients health status and health outcomes in a sample of adult patients diagnosed with developmental disorders during childhood.

Methods.- The sample consisted of 34 care-givers (parents) of adult patients diagnosed with developmental disorders during childhood, who were admitted to Clinical Psychiatric Hospital "Prof. Dr. Alexandru Obregia" Bucharest.

Results.- Developmental disorders have tremendous consequences for both individuals and their families as lack of adaptation, lack of understanding of the disease, lack of support, social isolation, hyper protection, disease denial. Higher rates of family hyper protection associated with lack of understanding of the disease were significantly associated with poor health outcomes.

Conclusions.- The burden of developmental disorders and behavioral issues has a significant impact on families. New strategies and policies should be developed in order to decrease the burden of the disease and to constantly help families to cope with the disability starting from early childhood.

Disclosure of interest.- The authors have not supplied a conflict of interest statement.

\section{E-PV0443}

\section{Recovery of patients with mental disorder in an era of turmoil}

C. Tsopelas ${ }^{1 *}$, G. Tzeferakos ${ }^{2}$, M. Dimitraka ${ }^{3}$, D. Petsas ${ }^{4}$, A. Gatos Gatopoulos $^{3}$, P. Papadimitriou ${ }^{3}$

${ }^{1}$ Psychiatric Hsopital of Attica, 5th Psychiatric Dept., Athens, Greece; 2 2nd Dept. of Psychiatry-Medical School- National University of Athens- Athens -Greece., 2nd Dept. of Psychiatry-Medical SchoolNational University of Athens- Athens -Greece., Athens, Greece; ${ }^{3}$ th Psychiatric Dept. Psychiatric Hospital of Attica, 5th Psychiatric Dept. Psychiatric Hospital of Attica, Athens, Greece; ${ }^{4}$ Psychiatric Hospital of Attica, 4th Psychiatric Dept., Athens, Greece

${ }^{*}$ Corresponding author.

Background and aims.- Mental Health Recovery is presented as a journey of healing and transformation for a person with mental health problems. Recovery has some elements linked primarily to the individual and others that are more deeply infused with the role of the community to provide resources and opportunities to individuals as they embark on a recovered journey. We tried to investigate the concept to real life conditions in developed countries Methods.- Using keywords as "mental health recovery", "psych\$" and "recovery" we did thorough research of the main databases, and web search engines such as Goggle, for relevant studies, and scrutinize them independently.

Results.- Beside the individual journey to recovery equal and more important part plays the culture of the community supporting this. Recovery is elusive to mentally ill patients who are guided to the penal system in an increasing rate. In six European countries (England, Germany, Italy, Netherlands, Spain, Sweden) there is a reduction in psychiatric beds from $15 \%$ to $65 \%$ but at the same time an overall increase in forensic beds of $10 \%$ to $143 \%$ and to prison population of $16 \%$ to $104 \%$.

Conclusions.- The benefits of the concept of recovery journey are obvious, we should not exceed the boundaries of common sense. We should take into account the socio-economical conditions of our era and allow people to use highly specialized services like long term domiciliary facilities or long term admissions to specialized 
hospitals. We should aim to treat persons and not cases, concepts or theories.

Disclosure of interest.- The authors have not supplied a conflict of interest statement.

\section{E-PV0445}

\section{Cognitive functions in children with short stature caused by hypopituitarism, treated with recombinant growth hormone}

K. Wiecheć* , E. Mojs, M. Stanislawska-Kubiak

Poznan University of Medical Sciences, Department of Clinical

Psychology, Poznań, Poland

* Corresponding author.

Background and aims.- Growth hormone deficiency caused by hypopituitarism is a rare cause of short stature. It also belongs to a group of rare diseases whose treatment with recombinant growth hormone ( $\mathrm{rhGH})$ is refunded. The procedure of inclusion in the treatment is quite troublesome and stressfull for parents and their children. Previous researches suggest a connection between treatment with growth hormone and an increase in some cognitive functions, especially in the field of long-term and short-term memory. The aim is to examine what is the relationship of short stature rhGH treatment, with the efficiency of selected cognitive functions in children and adolescents.

Methods.- The study group contain 40 children from 6 to 16 years old, both sexes, diagnosed with somatotropin deficiency caused by hypopituitarism and who are qualified for treatment with rhGH. Patients were tested twice by the Wechsler Intelligence Scale for Children. First measurement took place on the first visit in hospital, before the first dose of the hormone. Second measurement took place at the control visit after a year of treatment with rhGH.

Results.- According to literature authors expects to increase the efficiency of some cognitive functions during the treatment and the age of treatment will be related to the size of these changes.

Conclusions.- Results could not only be a source of knowledge about the effects of growth hormone therapy, but also could be an important voice in discussion about validity of funding for rhGH treatment.

Disclosure of interest.- The authors have not supplied a conflict of interest statement.

\section{E-PV0448}

\section{Job satisfaction among mental health professionals in a psychiatric hospital}

R. Maalej, Y. Zgueb ${ }^{*}$, U. Ouali, R. Jomli, F. Nacef

Razi Hospital Tunisia, Department of Psychiatry A, Manouba, Tunisia

Corresponding author.

Background and aims.- Introduction: Although there have been many studies about job satisfaction of healthcare workers over recent years, research focused essentially on general doctors and nurses.

Objectives.- The aim of this study is to explore the level of job satisfaction of mental health professionals and to exanimate the causes of dissatisfaction.

Methods.- Total of 200 mental health care workers in Razi hospital (including nurses, residents, psychologists, psychiatrists ...) were included in this study. Socio-demographic data form, and the job satisfaction survey (Paul E Spector) were used to evaluate the level of job satisfaction

Results.- The majority of the subjects were $30-39$ years old $(39,5 \%)$, were female (61\%), were married $60 \%$, and were nurses $(44,5 \%)$. The average score of the JSS was 115,8 . Only $9 \%$ of the mental health workers were satisfied with their jobs and $30 \%$ were dissatisfied. Job satisfaction was not significantly correlated to any socio-demographic data. Feeling that the patients are dissatisfied $(p=0,028)$, the bad organization of the work environment $(p=0,007)$ and the lack of resources $(p=0,032)$ contributed to the poor job satisfaction.

Conclusions.- Results indicate a low degree of satisfaction level among the mental health workers in Razi, thus we have to discuss recommendations to improve the nature of work and the work environment.

Disclosure of interest.- The authors have not supplied a conflict of interest statement.

\section{E-PV0449 \\ Burnout among healthcare professionals in a psychiatric hospital \\ R. Maalej, Y. Zgueb", U. Ouali, R. Jomli, F. Nacef \\ Razi Hospital, Department of Psychiatry A, Manouba, Tunisia \\ Corresponding author.}

Background and aims.- Over the last decades, there have been an increasing number of studies about burnout among doctors and nurses especially those working in the mental health departments. Objectives.- The aim of this study is to evaluate the prevalence of burnout experienced by healthcare professionals working in a mental health setting in Tunis (Razi) and to examine the causes.

Methods.- Total of 200 mental health care workers in Razi hospital (nurses, residents, psychologists, psychiatrists ...) were included in this study. Socio-demographic data form and the Maslash burnout score were used to evaluate the level of burnout.

Results.- In this study the majority of the subjects were 30-39 years old (39,5\%), were female (61\%), were married $60 \%$, and were nurses (44,5\%). Of the 200 respondents $43,5 \%$ experienced high level of emotional exhaustion, $44 \%$ experienced high level of depersonalization, and 65\% experienced low level of personal accomplishment. Emotional exhaustion and depersonalization were reported more frequently by nurses, the healthcare professionals with more experience in psychiatry department, and those who had more nightshifts. Healthcare professionals who were physically aggressed while working reported less personal accomplishment.

Conclusions.- The prevalence of burnout among staff working in psychiatric department is important, thus we have to try to prevent the predisposing factors to improve the mental health of the staff. Disclosure of interest.- The authors have not supplied a conflict of interest statement.

\section{E-PV0450}

\section{The relation between burnout and job satisfaction among healthcare professionals in a psychiatric hospital}

R. Maalej, Y. Zgueb ${ }^{*}$, U. Ouali, R. Jomli, F. Nacef

Razi Hospital, Department of Psychiatry A, Manouba, Tunisia

* Corresponding author.

Background and aims.- Burnout and job satisfaction in mental health community has been a subject of interest over the last decades.

Objectives.- The aim of this study is to evaluate the prevalence of burnout and job satisfaction among health care professional in the psychiatric hospital Razi and to identify the relationship between them.

Methods.- We have conducted a cross-sectional study and recruited a total of 200 mental health care workers in Razi hospital (nurses, residents, psychologists, psychiatrists ...). Socio-demographic data form, the Maslash burnout score and the job satisfaction survey (JSS) (Paul E Spector) were used.

Results.- The majority of the subjects were 30 to 39 years old $(39,5 \%)$, were female (61\%), were married $60 \%$, and were nurses 
(44,5\%). $49 \%$ of the respondents experienced moderate or high level of burnout, and only $9 \%$ of them were satisfied with their jobs. We found a statistically significant correlation between burnout and dissatisfaction $(p<0,0001)$. Emotional exhaustion was negatively correlated to Contingent Rewards $(p=0,02)$. Depersonalization was negatively correlated to communication $(p=0,016)$ and relation with coworkers $(p=0,037)$. Personal accomplishment was negatively correlated to fringe benefits $(p=0,029)$.

Conclusions.- Burnout and dissatisfaction at work are considered a real problem for mental health workers in Razi that's why we should find solutions to these problems to improve the quality of health care services.

Disclosure of interest.- The authors have not supplied a conflict of interest statement.

\section{E-PV0451}

\section{The relation between job satisfaction and human rights among healthcare professionals in a psychiatric hospital}

R. Maalej, Y. Zgueb ${ }^{*}$, U. Ouali, R. Jomli, F. Nacef

Razi Hospital, Department of Psychiatry A, Manouba, Tunisia

${ }^{*}$ Corresponding author.

Background and aims.- The respect of human rights is mandatory at work. It concerns both the staff and the users of the service.

Objectives.- The aim of this study is to determine the relationship between job satisfaction and the respect of the human rights of both the patients and the staff working in a mental health hospital in Tunisia.

Methods.- We have conducted a cross-sectional study and recruited a total of 200 mental health care workers in Razi hospital (nurses, residents, psychologists, psychiatrists). We have collected sociodemographic data, used the job satisfaction survey (Paul E Spector) and asked the participants 2 questions: how much do you think the human rights of the users of your service are respected and how much do you think the human rights of your staff are respected in your service ? (From 1 - nothing, to 6 - completely).

Results.- The majority of the subjects were 30 to 39 years old $(39,5 \%)$, were female $(61 \%)$, were married $(60 \%)$, and were nurses $(44,5 \%)$.

$30 \%$ of the respondents were insatisfied with their job, and $42,2 \%$ of them thought that the human rights of the staff were 'completely' 'not respected. However, we found no statistically significant correlation between job satisfaction and the respect of the human rights of the staff nor of the patients.

Conclusions.- The relationship between job satisfaction and the respect of the human rights of the staff and the patients are not well elucidated hence further studies are needed to explore it. Disclosure of interest.- The authors have not supplied a conflict of interest statement.

\section{E-Poster Viewing - 7-9 April: Mental Health Policies}

\section{E-PV0452}

\section{Effectiveness of community mental health teams in north west Ireland. Factors which can increase it}

\section{Adamis ${ }^{1 *}$, G.M. Krompa ${ }^{2}$, J. Tan ${ }^{1}$, E. O’Mahoney ${ }^{1}$}

${ }^{1}$ Sligo Mental Health Services, Psychiatry, Sligo, Ireland; ${ }^{2}$ Dublin

City University, Business School, Dublin, Ireland

${ }^{*}$ Corresponding author.
Background and aims.-

Introduction.- Community Mental Health Teams (CMHTs) have been introduced as an alternative to inpatient treatment. CMHTs are multidisciplinary, and they are organized around the needs of patients who live in a geographically defined area. However, CMHTs in Ireland are not well developed to provide adequate community support, with little multidisciplinary input and with significant regional variation.

Objectives.- To examine factors that can influence team effectiveness in CMHTs.

Methods.- Prospective cross-sectional study of eight CMHTs in Sligo / Leitrim Mental Health Services. Number of participants was $n=106$ (response rate $87 \%$ ). Data collected were: demographics (age, gender), discipline and years of experience, number of days of sick leave during the last year, length of time in the same team, simultaneously working in other teams, number of team members. Also, the following scales were administered: Team Effectiveness Scale (TES), Authentic Leadership Questionnaire (ALQ), and Team Cohesion Scale (TCS).

Results.- The following factors were found to have a significant impact on team effectiveness: authentic leadership $(p<0.001)$, team cohesion $(p<0.001)$, smaller size of the team $(p<0.036)$, the profession of doctor $(p=0.015)$, the profession of secretary $(p=0.043)$. After controlling for the examined variables (multilevel regression analysis) significant independent predictors of the team effectiveness were: team cohesion, authentic leadership, and the size of the team.

Conclusions.- To increase CMHT effectiveness, interventions are needed to those areas: a swift to make leadership styles more authentic, to improve team cohesion, and smaller team size.

Disclosure of interest.- The authors have not supplied a conflict of interest statement.

\section{E-PV0454}

\section{An exploration of the complexity of compensation \& pension assessments for service men \& women}

\section{S.O. Okpaku}

Center for Health-Culture and Society, Center for Health-Culture and Society, Nashville, USA

* Corresponding author.

Background and aims.-

1. Provide background to VA benefits of US service members

2. Address clinical strategies to improve the compensation \& pension examinations, reports

3. Highlight social cultural, spiritual, and family factors that may influence presentation and application for benefits

4. Various administrations have attempted providing adequate compensation for individuals who have served in armed forces. It's estimated that roughly $200,000-250,000$ annually leave the military since the post $9 / 11$ conflicts. Adjustment and transition to civilian life is not easy; for some, the only life they know is military. Marital stressors, divorces, unemployment, and associated social problems may occur. Some of these individuals may meet the diagnostic criteria for PTSD, TBI, MDD, GAD, and psychotic disorders. The teasing out of these diagnostic categories is not easy. Many of these Veterans fear being labeled and stigmatized with consequences for continuation of their services in the military.

Methods.- Review of 250 consecutive compensation examinations carried out by a single psychiatrist.

Results.- Assessment of individuals in addition is complicated by a variety of factors:

- Desire to be compensated for services to the nation.

- The inability of the VA to provide the assessments for these individuals 
Conclusions.- The need for a comprehensive assessment of these individuals and recommendation for services citing challenges for their rehabilitation. A discussion of the role of social cultural factors e.g. spirituality, attitudes and stigma, and family relationships in the complaints and adjustments of these individuals. The pattern of alcohol use in the immediate post deployment period is cited as well as the success of the military in controlling non-alcohol drug abuse.

Disclosure of interest.- The authors have not supplied a conflict of interest statement.

\section{E-Poster Viewing - 7-9 April: Migration and Mental health of Immigrants}

\section{E-PV0455}

\section{Clinical approaches to cultural diversity in mental health care. A literature review}

L. Carballeira Carrera ${ }^{1}$, M.R. Moro ${ }^{2}$, J. Curto Ramos ${ }^{3 *}$, I. López Álvarez ${ }^{4}$, J. Lachal ${ }^{5}$

${ }^{1}$ Alicia Koplowitz Foundation Short Term Fellowship, Maison des Adolescents de Solenn- Cochin Hospital- AP-HP, Paris, France; ${ }^{2}$ Descartes University, Maison des Adolescents de Solenn- Cochin Hospital- AP-HP, Paris, France; ${ }^{3}$ University Hospital La Paz, Psychiatry and Mental Health Department, Madrid, Spain; ${ }^{4}$ Alicia Koplowitz Foundation Short Term Fellowship, Tavistock and Portman NHS Foundation Trust. Gender Identity Development Service, London, United Kingdom; ${ }^{5}$ Descartes University, Maison des Adolescents Maison de Solenn- Cochin Hospital- AP-HP, Paris, France

* Corresponding author.

Background and aims.- Inequity on mental healthcare access is a main problem for migrants. In addition, migration-related factors may function as social determinants for mental health. It is necessary to adapt our services to the needs of this vulnerable population. This should be done taking into account that cultural factors have an impact on the way that mental illness and treatment are conceptualized.

The aim of this study is to gain a global vision of the different types of clinical approaches to migrants' mental healthcare that have been developed throughout the world.

Methods.- Literature review via scientific database (PubMed, PsycInfo) was conducted. The screening process resulted in a selection of 32 papers.

Results.- The adaptations to cultural diversity in mental health are mostly found in England, France, Canada, Australia and the US. In recent years, they have started spreading to other countries. Some of the adaptations found were: using interpreters and cultural brokers, professionals training and supervision, innovations on the therapeutical setting, cultural consultation and ethno-specific clinics. How these adaptations are developed is related to the country's migration patterns, as well as its citizenship model.

Conclusions.- There are many ways in which we can improve mental healthcare access for migrants. However, there is still a long way to get away from ethnocentric perspectives, treasuring multicultural pluralism.

Disclosure of interest.- The authors have not supplied a conflict of interest statement.

\section{E-PV0456}

\section{Humanitarian workers' mental health; state of art and considerations about development of mental health support program}

J. Douesnard

Université du Québec à Chicoutimi, Economic and Administrative Sciences- Laboratory on Critical Incidents in Workplace, Saguenay, Canada

* Corresponding author.

Background and aims.- Often working in unstable, dangerous context and witness to human suffering as well as atrocities, it is well known that the humanitarian workers work in difficult situations and are exposed to several stressors. This context is not without consequence on their mental health. It is urgent to decrease the mental illness engendered by their work, all the while increasing retention and re-engagement for future missions. What is the state of art on this subjet? What kind of research and interventions programs can we develop to decrease the mental impacts of working in traumatic situation?

This communication focuses to answering these questions by presenting the results of an exhaustive literature review (2010-2018). From this analysis, we present a classification of core topics related to the research area of humanitarian workers' mental health and, in addition to some up-to-date prevalences, identify the methodology and most used psychometric tools. Secondly, new avenues of research and interventions focused on these workers' mental health care will be explored. Furthermore, consideration concerning the follow-up post-intervention will be presented, to guide the development of mental health support program for humanitarian workers back from a mission. These elements will be articulated around the precise concerns bound to the mental health in emergency and trauma, in a context of migratory crisis which requires qualified and committed on the long-term workers, and also, around the understanding of the elements which allows them to remain mentally healthy when returning on mission.

Methods.- N/A.

Results.- N/A

Conclusions.- N/A.

Disclosure of interest.- The authors have not supplied a conflict of interest statement.

\section{E-PV0457}

\section{Divergences between portuguese and european mental health laws and their impact on a population of migrants: a review}

A. Miguel ${ }^{1 *}$, B. Ramos ${ }^{2}$, A. Samico ${ }^{1}$, E. Mendes ${ }^{1}$

${ }^{1}$ Centro Hospitalar Vila Nova de Gaia, Department of Psychiatry and Mental Health, Vila Nova de Gaia, Portugal; ${ }^{2}$ Centro Hospitalar São João, Department of Psychiatry and Mental Health, Porto, Portugal Corresponding author.

Background and aims.- Despite some common standard concepts there is still a plurality of systems regulating involuntary admissions and treatment of mental health patients in Europe. Portugal is the country in the European Union with the highest proportion of emigrants within its population, often with temporary stays abroad. This pertains to its physicians a necessity to deal with the migrant flow of mental health patients on a very common basis. Subsequently, we intend to compare and understand within its cultural and historical context the legal framework for involuntary admission/treatment of people with mental illness in the different European countries, focusing on the most common destinations for Portuguese migrants in contrast with Portugal. 
Methods.- Literary review of the mental health laws in Europe. Research on the cultural and historical context, recent advances/ongoing discussions regarding the topic.

Results.- The application of coercive measures in mental health patients still differs over some European countries: the revaluation time varies from 1 to 7 days and only seven countries have a clear distinction between compulsory admissions and treatment. The inclusion and exclusion criteria is also divergent between states.

Conclusions.- There is a divisive dynamic between a medical approach and a civil liberties approach that seems to be mostly determined by each country's cultural and historical context. Bearing this in mind and with the growing migration flow of the Portuguese population in our background, updated knowledge and an analysis of the impact of these differences emerges as of utmost importance as it may drive changes in treatment and follow up of patients.

Disclosure of interest.- The authors have not supplied a conflict of interest statement.

\section{E-PV0458}

\section{Le mal d'afrique. A case report}

R. Vecchio ${ }^{*}$ A. Corso, P. Cardinale, S. Penati, G. Serafini, M. Amore Università degli Studi di Genova, Psychiatry Department

Neurosciences-Rehabilitation-Ophthalmology-Genetics-Maternal and Child Health, Genova, Italy

* Corresponding author.

\section{Background and aims.-}

Introduction.- The migratory processes put the doctors in front of other types of patients that they don't know; often they are not ready to take care of them. This sometimes leads to superficial and "dangerous" treatment decisions for patients.

Objectives.- It is present the clinical case of a twenty year old Gambian man suffering from epilepsy since the age of ten and has been immigrated in Italy in 2016. The patient presented behavioral anomalies and episodes of psychomotor agitation, during which he expressed thoughts that seemed delusional to an European listener. He occasionally suffered from chest pain. He was initially considered a psychotic patient with amplifying behaviors and therefore he was treated with monthly depot injection therapy for three months. He was admitted to the psychiatric clinic of Genoa, where doctors made radical changes in diagnosis and drug therapy.

Aims.- Highlighting the risk of ignoring important clinical features due to linguistic and cultural barriers.

Methods.- Clinical case observation; X-ray and C.T. scan investigations; recourse to a cultural mediator; involvement of a multidisciplinary team (medical and surgical).

Results.- Although some physical and psychic symptoms (perplexity, high fever, flexibilitas cerea) have been reduced by the suspension of therapy, doctors have further investigated the clinical picture involving the cultural mediator and making several clinical and radiological examinations to identify the correct diagnosis. The mediastinoscopy with biopsy demonstrated the presence of a reactivated tuberculosis outbreak.

Conclusions.- It is important to take into consideration the cultural aspects and the endemic diseases of the native lands.

Disclosure of interest.- The authors have not supplied a conflict of interest statement.

\section{E-Poster Viewing - 7-9 April: Neuroimaging}

\section{E-PV0459 \\ Orbitofrontal syndrome in 18-year old \\ female -a case report}

K. Bliźniewska-Kowalska*, A. Orzechowska, M. Talarowska, P.

Gałecki

Department of Adult Psychiatry, Medical University of Lodz, Łódź, Poland

* Corresponding author.

Background and aims.- Orbitofrontal syndrome is a variant of frontal lobe syndrome in which behavioural disturbances are dominant. Patients with orbitofrontal syndrome usually present disinhibited, impulsive behawior, inappropriate jocular affect, euphoria, emotional lability, distractibility, poor judgment and insight.

Methods.- In spite of severe behavioural disturbances cognitive functions are often intact. Orbitofrontal syndrome may be confused with other psychiatric disorders such as mania /hypomania or antisocial personality disorder.

Results.- This poster presents a case report of orbitofrontal syndrome in 18-year-old female after a serious car accident. Computer tomography revealed area of extensive injury in the right frontal lobe and numerous fractures of the skull bones. Patient underwent several neurosurgical and reconstructive surgeries. Due to her dysforic mood patient was presented to psychiatrists. Psychological tests evaluating her personality and cognitive functioning were performed twice. Patient received pharmacotherapy. She is attending follow-up visits. She is functioning well.

Conclusions.- Clinical features and possible modes of presentation of this syndrome are discussed in this poster.

Disclosure of interest.- The authors have not supplied a conflict of interest statement.

\section{E-PV0460}

\section{First-episode psychosis: the role of neuroimaging}

L. Castanheira ${ }^{1}$, T. Duarte ${ }^{1 *}$, C. Leite Rodrigues ${ }^{1}$, R. Santos ${ }^{1}$, E. Fernandes $^{1}$, M.J. Gonçalves ${ }^{1}$, R. Saraiva ${ }^{1}$, T. Cavaco ${ }^{1}$, D. Silva ${ }^{2}$, M. Rosário $^{2}$, S. Reimão ${ }^{3}$

${ }^{1}$ Centro Hospitalar Lisboa Norte - Hospital Santa Maria, Psychiatry and Mental Health, Lisbon, Portugal; ${ }^{2}$ Centro Hospitalar Lisboa Norte - Hospital Santa Maria, Neurology, Lisbon, Portugal; ${ }^{3}$ Centro Hospitalar Lisboa Norte - Hospital Santa Maria, Neuroimaging, Lisbon, Portugal

* Corresponding author.

Background and aims.- The prevalence of psychotic disorders in the general population is approximately $3 \%$, and $0.21 \%$ in medical conditions. The psychopathological examination does not always allow the differential diagnosis between "primary" psychoses, without identified organic cause, and "secondary", originated from an established organic cause. Thus, it is necessary to perform complementary diagnostic tests, such as imaging tests. Between 5 and $17 \%$ of patients with first psychotic episode (FEP) present changes in imaging tests, most of them neurodevelopmental changes. The aim is to review the most recent literature on the psychoses with an organic cause and to present the clinical cases diagnosed at Santa Maria Hospital, emphasizing the importance of neuroimaging tests in the differential diagnosis of psychoses.

Methods.- The Pubmed database was searched and the clinical processes were consulted.

Results.- Computed Tomography and Magnetic Resonance Imaging (MRI) are the most frequently requested neuroimaging tests when approaching patients with FEP. Among the organic causes that can 
cause psychosis with neuroimaging changes are: cerebrovascular diseases, trauma, occupying diseases of space, autoimmune diseases, demyelinating diseases, metabolic diseases, degenerative diseases and infectious diseases. The guidelines are divergent regarding neuroimaging in the $\mathrm{FEP}$, so neuroimaging should be requested only in the presence of neurological symptoms or signs, atypical pictures, symptoms suggestive of delirium/organicity (visual hallucinations, disorientation, memory loss) and in patients over 50 years old.

Conclusions.- The neuroimaging tests help the differential diagnosis and should be performed only in situations that present, clinically, with sign/symptoms suggestive of "secondary" psychosis. In these, MRI is the method of choice.

Disclosure of interest.- The authors have not supplied a conflict of interest statement.

\section{E-Poster Viewing - 7-9 April: Neuroscience in Psychiatry}

\section{E-PV0463}

\section{Musical anhedonia: a review of the science to date}

F. Bernardini ${ }^{1 *}$, L. Attademo ${ }^{2}$, G. Loas $^{3}$

${ }^{1}$ CSM 24 h Area delle Dolomiti Friulane - REMS- AAS5 "Friuli

Occidentale", Department of Mental Health, Pordenone, Italy; ${ }^{2}$ SPDC Potenza ASP Basilicata, Department of Mental Health, Potenza, Italy;

${ }^{3}$ Hopital Erasme ULB, Department of Psychiatry, Anderlecht, Belgium ${ }^{*}$ Corresponding author.

Background and aims.- Anhedonia, or the inability or the loss of the capacity to experience pleasure, is a psychopathological feature of several types of psychiatric disorders. Different types of anhedonia have been identified. Hedonic processing is a complex of both anticipatory and consummatory components. Music is considered among the highest sources of pleasure and has been present in all human cultures since prehistory. Neuroimaging studies show that the reward system of the human brain is central to the experience of pleasure from music. Musical anhedonia is defined as a selective impairment of emotional experience only in listening to music.

Methods.- We searched the Pubmed electronic database for all articles up to September 2017 with the search term "musical anhedonia". The search included all languages. We compiled articles that (1) were published in English, Italian or French (2) provided empirical data (as opposed to reviews or commentaries)

Results.- Some 14 articles were identified. We excluded two articles that were unrelated to the topic, one comment, one review article, one article in Japanese, and one article presenting the translation and the validation of a questionnaire for the assessment of musical anhedonia. In addition to the PubMed search, two other relevant clinical case report of patients presenting musical anhedonia were identified based on bibliographies. A final set of 10 articles, five original research articles and five clinical case report, comprised the set of studies reviewed.

Conclusions.- Specific musical anhedonia is rare and bears further investigations, as it may better clarify the function and dysfunction of the reward system

Disclosure of interest.- The authors have not supplied a conflict of interest statement.

\section{E-PV0465}

\section{The rare differential diagnosis \\ between eating disorders and}

\section{intermittent porphyrias: a case report}

M.L. De Falco*, L. Peroni de Jesus, N. Haddad, D. Tavares, T.E. Abreu

Silva, M.B. Cordeiro, P. Teguan

Fmabc Brazil, Psychiatry, São Paulo, Brazil

Corresponding author.

Background and aims.- In this case report we aim to discuss the case of a 20-year-old female patient, no psychiatric history, who was admitted by induced vomiting with her hands and feet due to diffuse and intense referred abdominal pain. She was emaciated, with an ataxic gait. We are here demonstrating what led us to the differential diagnosis between bulimia and AIP.

Methods.- Follow-up of the patient's hospitalization in the psychiatric ward of HEMC Hospital, Santo André SP.

Results.- Were denied symptoms of anorexia, bulimia or body dysmorphic disorder. There was never obvious concern with body image, binge eating or purging. The condition started three months earlier, after gastric discomfort during a copious meal. There was atrophy of the fingers and difficulty of walking due to a thalamic and peripheral neuropathy and evidence of executive and cognitive impairment. MRI revealed significant atrophy, fourth ventricle dilation and periventricular gliosis areas, with involution in relation to 3 months earlier MRI.CT evidenced aspiration pneumonia and hepatosplenomegaly. UD endoscopy showed no alterations. The patient evolved with anemic core, and two red blood cells were transfused on CTI and then referred to the psychiatric ward with Bulimia HD. Nasoenteral catheter and hydroelectrolytic correction was necessary. Pain was administered with Tylex. Quetiapine, Olanzapine, topiramate and anti-emetic medications were tried unsuccessfully.

Conclusions.- Given the diagnostic challenge, uroporfibilinogenic was increased. Were initiated for IAP dietary measures, hyperglycemia, withdrawal of medications possibly triggering, clorpromazine for psychic and emetic conditions and tramadol. There was improvement of the case, demonstrating the importance of rare differential diagnosis between cases of eating disorders and porphyrious episode.

Disclosure of interest.- The authors have not supplied a conflict of interest statement.

\section{E-PV0466}

The rare differential diagnosis on an eating disorder case: a discussion

M.L. De Falco ${ }^{*}$, L. Peroni ${ }^{2}$, H. Natalia ${ }^{2}$, D. Tavares ${ }^{2}$, M.B. Cordeiro ${ }^{2}$, P. Teguan ${ }^{2}$, T.E. Abreu Silva ${ }^{2}$

1 fmabc Brazil, Psychiatry, São Paulo, Brazil; ${ }^{2}$ Fmabc, Psychiatry, São Paulo, Brazil

* Corresponding author.

Background and aims.- In this case report we aim to discuss the case of a 20-year-old female patient, no psychiatric history, who was admitted by induced vomiting with her hands and feet due to diffuse and intense referred abdominal pain. She was emaciated, with an ataxic gait. We are here demonstrating what led us to the differential diagnosis between bulimia and AIP.

Methods.- Follow-up of the patient's hospitalization in the psychiatric ward of HEMC Hospital, Santo André SP.

Results.- Were denied symptoms of anorexia, bulimia or body dysmorphic disorder. There was never obvious concern with body image, binge eating or purging. The condition started three months earlier, after gastric discomfort during a copious meal. There was atrophy of the fingers and difficulty of walking due to a thalamic and peripheral neuropathy and evidence of executive and cognitive impairment. MRI revealed significant atrophy, fourth ventricle 
dilation and periventricular gliosis areas, with involution in relation to 3 months earlier MRI.CT evidenced aspiration pneumonia and hepatosplenomegaly. UD endoscopy showed no alterations. The patient evolved with anemic core, and two red blood cells were transfused on CTI and then referred to the psychiatric ward with Bulimia HD. Nasoenteral catheter and hydroelectrolytic correction was necessary. Pain was administered with Tylex. Quetiapine, Olanzapine, topiramate and anti-emetic medications were tried unsuccessfully.

Conclusions.- Given the diagnostic challenge, uroporfibilinogenic was increased. Were initiated for IAP dietary measures, hyperglycemia, withdrawal of medications possibly triggering, clorpromazine for psychic and emetic conditions and tramadol. There was improvement of the case, demonstrating the importance of rare differential diagnosis between cases of eating disorders and porphyrious episode.

Disclosure of interest.- The authors have not supplied a conflict of interest statement.

\section{E-PV0468 \\ Late hormone-mediated effects of cerebral irradiation from the radioactive iodine}

A. Kaminskyi ${ }^{1}$, K. Loganovsky ${ }^{2}$, V. Talko ${ }^{3}$, D. Afanasyev ${ }^{1}, \mathrm{~T}$. Loganovska $^{2}$, G. Lavrenchuk ${ }^{3}$, O. Kopylova ${ }^{1}$, I. Chikalova ${ }^{1}$, I. Muraviova $^{1}$, Y. Teplaya ${ }^{1}$, I. Kiselova ${ }^{1}$, N. Dombrovskaya ${ }^{1}$

${ }^{1}$ State Institution "National Research Center for Radiation Medicine of National Academy of Medical Sciences of Ukraine", Radiation Endocrinology, Kyiv, Ukraine; ${ }^{2}$ State Institution "National Research Center for Radiation Medicine of National Academy of Medical Sciences of Ukraine", Radiation Psychoneurology, Kyiv, Ukraine; ${ }^{3}$ State Institution "National Research Center for Radiation Medicine of National Academy of Medical Sciences of Ukraine", Radiobiology,

Kyiv, Ukraine

* Corresponding author.

Background and aims.- Radioactive iodine was the leading source of human radiation exposure after the Chornobyl catastrophe (1986). The objectives are evaluation of the effects of accidental irradiation from iodine isotopes on the development of hormone-mediated CNS disorders.

Methods.- Clinical neuropsychiatric and endocrinologic methods, hormonal assays, biochemical tests, instrumental research sessions and psychophysiological testing.

Results.- There is an increased prevalence of mental and neuropsychiatric disorders in the remote period in the Chornobyl catastrophe survivors. Dysfunction of hypothalamic and pituitary centers involved in regulation of the alpha-melanocyte-stimulating hormone (alpha-MSH) synthesis and secretion is recognized nowadays as a new aspect of the radio-induced cerebral effects in human. As a result the suppression of alpha-MSH synthesis leads to a significant $(p \leq 0.05)$ increase in obesity incidence with comorbid depression in the exposed individuals. Such a kind of central disorders result in abnormal integral regulation of the energy balance and eating behavior disorders in the Chornobyl catastrophe survivors. Decreased concentrations of serotonin and melatonin are revealed at that. Radiation damage of parathyroid glands emerged as another late effect of exposure to radioactive iodine followed by the disorders of calcium and phosphorus metabolism that makes an impact in its turn on the cerebral functions.

Conclusions.- Accidental radiation exposure from radioactive iodine of the certain cerebral structures and parathyroid glands and radioactive iodine application in healthcare within diagnostic and radiotherapy procedures leads to emergence of the late direct and indirect hormone-mediated CNS dysfunction.

Disclosure of interest.- The authors have not supplied a conflict of interest statement.

\section{E-PV0472 \\ EEG based easy-to-use monitor of sustained attention: a supporting tool for psychiatric rehabilitation}

L. Lipskaya-Velikovsky ${ }^{1 *}$, E.V. Harel $^{2}$, G. Shahaf ${ }^{3}$

${ }^{1}$ Tel Aviv University, Sackler Faculty of Medicine- Department of Occupational Therapy, Tel Aviv, Israel; ${ }^{2}$ Beer Yaakov-Ness Ziona Mental Heath Center, Psychiatry, Beer Yaakov, Israel; ${ }^{3}$ Reuth Rehabilitation Hospital, Reuth Research and Development Institute, Tel Aviv, Israel

* Corresponding author.

Background and aims.- Introduction: Psychosocial and cognitive rehabilitation are important intervention modalities in the landscape of psychiatric services. However, rehabilitation outcomes depend, to a large extent, on the clients' engagement. There are various ways to conceptualize and measure engagement in an intervention, among others, based on parameters of sustained attention (SA). Indeed, it was demonstrated that allocation of attention resources to an intervention is critical for success of the process. However, there are no established procedures to monitor brain activity parameters of SA in real-time during the rehabilitation process.

Objectives.- To approve feasibility of a new, easy-to use, real-time electroencephalographic (EEG) activity based marker for SA - the Brain Engagement Index (BEI) for psychiatric rehabilitation.

Methods.- Three clinical case studies (persons with schizophrenia, major depression and general anxiety disorder) of psychosocial and cognitive rehabilitation supported by the BEI monitoring were completed. BEI was recorded in real time using wireless singlechannel headset with one dry frontal electrode and one reference electrode on the earlobe. The EEG data was transferred to computer, automatically interpreted and presented in real-time on the screen in an easy-to-read format.

Results.- BEI usage feasibility for clinical practice was approved. Electro-physiological patterns of attentional processes were detected using BEI among people with mental health disorders. Moreover, we found that there are strategies for improvement of brain engagement and SA allocation in real time during rehabilitation to enhance the results.

Conclusions.- BEI usage following strategies for SA improvement may enhance rehabilitation outcomes. BEI clinical applications should be further supported by empirical evidence.

Disclosure of interest.- The authors have not supplied a conflict of interest statement.

\section{E-PV0473}

\section{Comparing social and nonsocial stimuli in accessing visual awareness}

S. C. Soares ${ }^{1}$, S. Pinheiro ${ }^{1}$, S. Silva ${ }^{2}$, F. Silva ${ }^{3}$, N. Gomes ${ }^{3}$, S. Magano $^{4^{*}}$, N. Madeira ${ }^{5}$

${ }^{1}$ University of Aveiro, CINTESIS.UA- Department of Education and Psychology, Aveiro, Portugal; ${ }^{2}$ University of Aveiro, Department of Electronics- Telecommunication and Informatics DETI/Institute of Electronics and Informatics Engineering IEETA, Aveiro, Portugal; ${ }^{3}$ ISPA, William James Center for Research- ISPA, Lisboa, Portugal; 4 Centro Hospitalar e Universitário de Coimbra, Psychiatry, Coimbra, Portugal; ${ }^{5}$ Centro Hospitalar e Universitário de Coimbra,

Department of Psychiatry, Coimbra, Portugal

* Corresponding author.

Background and aims.- Social and nonsocial stimuli signaling danger (e.g., angry faces, snakes) selectively activate the fear module, regardless of visual awareness (VA). Understanding whether abnormal functioning of this module is related to the type of stimuli presented and its awareness levels, poses a relevant question for the comprehension of psychiatric disorders, aiding to their diagnosis. 
However, no study has yet directly assessed the difference between these two types of stimuli in accessing VA, even in non-clinical samples.

The present study investigates the differences between social and non-social stimuli with distinct threat relevance in accessing VA, in a non-clinical sample, serving as a baseline for future studies addressing clinical groups.

Methods.- For 37 university students, we measured RTs for social (angry, neutral faces) and non-social (snakes, caterpillars) stimuli to access VA, adopting a breaking Continuous Flash Suppression paradigm. Given their heterogeneity, all stimuli were equalized for area, spectral and pixel properties using SHINE.

Results.- Social stimuli were faster to access VA, although no differences between threatening and neutral categories were observed. Conclusions.- The findings suggest faster processing of social stimuli with the lack of differences among emotional categories being explained by the equalization of image properties (notably spectral), as proposed by recent literature. This emphasizes the significant impact of highly controlled stimuli sets for this research area. Provided that some studies showed that social stimuli processing is impaired in some psychiatry disorders (e.g., schizophrenia), the current research might serve as a baseline for future comparisons with clinical samples.

Disclosure of interest.- The authors have not supplied a conflict of interest statement.

\section{E-PV0475}

\section{A case of moderate depression with somatic symptoms and incidental finding of retrocerebellar arachnoid cyst}

S. Mukherjee

Calcutta National Medical College, PSYCHIATRY, Kolkata, India

* Corresponding author.

\section{Background and aims.-}

Introduction.- Arachnoid cysts are benign space-occupying brain lesions that contain cerebrospinal fluid. Most cases are congenital in origin, caused by failed fusion of the arachnoid membrane early in fetal development. Cases are mostly detected incidentally on neuro-imaging and neuropsychiatric manifestations are less common and under-recognized. Arachnoid cyst sometimes may be associated with seizure, headache, cranial nerve deficit, hydrocephalus etc.

Methods.- Case Report: A 23 year old married female from rural India presented with headache, insomnia, low mood, lethargy, persisting for last 6 months without any obvious stress factors. She also informed that she had few episodes of unresponsiveness without any features like tongue biting, confusion, injury or involuntary micturition and defecation. Her EEG report was within normal limit and MRI report confirmed the presence of Retro-cerebellar Arachnoid cyst in and around midline. Detail neurological and ophthalmological examination was done and findings were within normal limit. She was treated with Amitriptyline, Pregabalin and Paracetamol on when required basis. Psychotherapy was initiated for adequate management of her depressive symptoms and responded well.

Results.- Not applicable as it is a case report.

Conclusions.- Conclusion: It can be emphasized that patients who are reporting with symptoms like headache a detail evaluation should be done to find out organic etiology. Depressive and cognitive symptoms and it's association with arachnoid cyst may depend upon the location of the lesion and further research is necessary to establish it's causal relationship.

Disclosure of interest.- The authors have not supplied a conflict of interest statement.

\section{E-PV0476}

The role of theory of mind and executive functions in creative performance of people with schizophrenia

A. Sampedro Calvete ${ }^{1 *}$, J. Peña ${ }^{1}$, N. Ibarretxe-Bilbao ${ }^{1}$, P. Sánchez ${ }^{2}$, E. Elizagarate ${ }^{2}$, N. Iriarte ${ }^{2}$, C. Pavón ${ }^{2}$, I. Hervella ${ }^{2}$, G. García ${ }^{2}$, A. Ortiz de Zárate ${ }^{2}$, N. Ojeda ${ }^{1}$

${ }^{1}$ University of Deusto, Department of Methods and Experimental Psychology, Bilbao, Spain; ${ }^{2}$ Osakidetza, Mental Health Service,

Vitoria-Gasteiz, Spain

* Corresponding author.

Background and aims.- Impairment in executive functions has been suggested to influence a worse creative performance in people with schizophrenia. However, it is unknown whether an impairment in theory of mind (ToM) could also lead to a lower creative capacity in this disease.

The aim of this study was to analyze differences in creative performance in patients with schizophrenia compared to healthy controls as well as to explore the potential role of executive functions and ToM as mediators of this relationship.

Methods.- Forty-five patients with schizophrenia and 45 healthy controls underwent a neuropsychological assessment including executive functions, ToM, and verbal and figural creativity.

Results.- Analysis of covariance showed that patients with schizophrenia obtained lower scores in creativity, working memory, inhibition, and ToM compared to healthy controls. Path analysis showed firstly, that ToM played a mediatory role in the relationship between group (being a patient with schizophrenia or a healthy control) and both figural $(Z=2.075, p=.037)$ and verbal creativity $(Z=2.570, p=.010)$. Secondly, working memory mediated between group and figural creativity $(Z=2.034, p=.041)$ and had a tendency to significance in verbal creativity $(Z=1.930, p=.053)$. Thirdly, inhibition mediated between group and figural creativity $(Z=2.454, p=.014)$

Conclusions.- Results suggest that the low performance seen in verbal and figural creativity among patients with schizophrenia is partly due to an impairment in executive functioning and ToM. These results partially support the shared vulnerability model. Disclosure of interest.- The authors have not supplied a conflict of interest statement.

\section{E-PV0478}

\section{Emotional disorders and pituitary}

\section{tumors}

Y. Sidneva ${ }^{*}$, L. Astafyeva, O. Zaitsev, P. Kalinin, M. Kutin, I. Klochkova

N.N. Burdenko National Medical Research Center of Neurosurgery, Neurosurgery, Moscow, Russia

* Corresponding author.

Background and aims.- Among tumors of the diencephalic region there are: pituitary adenomas, craniopharyngiomas, gliomas, meningiomas and others. Emotional disorders in the clinic of tumors of the diencephalic region are revealed in $2-100 \%$.

Methods. -260 patients (18-65 years old, mean age $38+2$ ): pituitary adenomas - 150 (58\%), craniopharyngiomas - 110 (42\%). Methods: psychopathological, data from endocrinological, neurological, neuroimaging methods.

Results.- 1. Pituitary adenomas with excessive secretion of growth hormone - emotional disorders are in $60 \%$. 2. Pituitary adenomas gland with excessive secretion of adrenocorticotropic hormone:1) Cushing's disease - changeable mood, depression, apathy, sleep disturbance, with visceral symptoms (tachycardia, fluctuations in blood pressure) are in 50\%.2) Nelson's syndrome - a decrease in emotional reactions and motor activity. Patients are apathetic, 
monotonous, poor in mimic manifestations. 3. Pituitary adenomas with excessive secretion of prolactin - emotional disorders, sleep disturbance are in $30 \%$. Nonspecific symptoms of the asthenia in almost a quarter of patients.4. Pituitary adenomas with excessive secretion of thyroid-stimulating hormone - increased emotionality, excitability, changeable mood, with frequent "panic attacks" are in $40 \% .5$. Non-functioning pituitary adenomas:a) With hormoneinactive tumors of the pituitary psychopathology is present in $6 \%$. There are violations of sleep, changeable mood, weakness, decreased memory.b) In craniopharyngiomas emotional and personality disorders was in $67 \%$. This is combined with cognitive, motivational and other impairments.

Conclusions.- violations of emotions in the defeat of the diencephalic region are caused by the localization of the tumor with the involvement of the corresponding brain structures in the pathological process.

Disclosure of interest.- The authors have not supplied a conflict of interest statement.

\section{E-PV0481}

\section{Rapid remission from mild to severe, traumatic brain injury induced acute psychosis and cognitive decline, after prolonged osmotherapy and low dosage risperidone. $A$ case report}

G. Thomaidis ${ }^{1}$, C. Krasanaki ${ }^{2}$, E. Aifantis ${ }^{3}$, I. Anastasiadis ${ }^{4}$, T. Siozos ${ }^{5}$, A. Vlachaki ${ }^{*}$

${ }^{1}$ Papanikolaou General Hospital, Department of Psychiatry, Thessaloniki, Greece; ${ }^{2}$ Aristotle University of Thessaloniki, Department of Psychology, Thessaloniki, Greece; ${ }^{3}$ Aristotle University of Thessaloniki, School of Engineering, Thessaloniki, Greece; ${ }^{4}$ Katerini General Hospital, Department of Psychiatry, Katerini, Greece; ${ }^{5}$ Papanikolaou General Hospital, Department of Neurosurgery, Thessaloniki, Greece

${ }^{*}$ Corresponding author.

Background and aims.- Introduction: Traumatic Brain Injury (TBI), complicated by permeating edema and resulting abnormal cortical deformation is a known predictor of cognitive decline and psychosis. Response to antipsychotics, has been linked to normal gyrification, in first-episode psychosis. An application of prolonged and combined osmotherapy for the acceleration and optimization of antipsychotic treatment is presented.

Objective.- To test and control the effect of osmotherapy, on the antipsychotic treatment response rate, on a patient with mild to severe TBI induced psychosis, disorientation, dissociation, and memory loss.

Methods.- The patient had personality traits predisposing to psychosis, completed first line osmotherapy in a neurosurgical department (2CT-scans), showed persisting disorientation and memory gaps during the 10-day rehabilitation period (1CT-scan) and was transferred at the psychiatric department after acute onset psychosis. There, two new cycles of osmotherapy were completed, using mannitol (cycle 1) and acetazolamide (cycle 2), for days D1-D12, plus low dose risperidone. Vitamin B12 plus folic acid supplementation were added at D15. Symptoms were monitored daily. New CT-scan was done at D5.

Results.- Edema and hematoma were significantly absorbed. Psychotic symptomatology ceased until D12, Orientation (space+time), insight and short term memory were restored until D16, long term memory until D25, the memory gap around the time of injury showed gradual improvement until D30.

Conclusions.- Prolonged osmotherapy, after the completion of standard osmotherapy protocols, could be used for acceleration of the convergence of deformed cortical morphology to the normal pattern, and subsequently support the optimization of antipsychotic treatment strategy and cognitive restoration in certain TBI cases.
Disclosure of interest.- The authors have not supplied a conflict of interest statement.

\section{E-Poster Viewing - 7-9 April: Obsessive-Compulsive Disorder}

\section{E-PV0482 \\ OCD symptoms induced by antipsychotics}

R.A. Baena Mures ${ }^{*}$, I. Alberdi Paramo, G. Montero Hernandez, J. Ibañez Vicioso, B. Rodado

Hospital Clinico San Carlos, Psychiatry, Madrid, Spain

* Corresponding author.

Background and aims.- OCD symptoms are more common in patients with antisychotics treatment. Specially if treatment has a significant anti-serotoninergic action. In 1992 clozapine was the first antipsychotic that noticed OCD induced symptons in schizofrenia patients. In clinical practice we have problems to determinate delussions or obssesion specially in patients who have positive symptoms. There are a lot of studies that try to determinate the influence of neuroleptic treatment in the begining and maintenance of OCD in psychotic patient.

Methods.- We try to make a bibliographic review and update clinicians about OCD induced symptons in patient who take antipsychotic treatment for psychotic disorder.

Results.- There are a lot of contradiction in the reviews. The role of clozapine in the appeareance of OCD induced symptons is still inconclusive. However olanzapine and clozapine are the most reported in the apperarence of this symptoms. Lenght of clozapine treatment is a risk factor. Compulssions and obsessions has been replicated in studies. A plausible mechanism for the persistence of this symptoms would be serotoninergic effects of clozapine's antagonism in 5HT2a y 5 HT2c receptors.

Conclusions.- There are evidence about the apperaence and genesis of OCD induced symptoms produced by atypic antipsychotics. We need to do more studies to determinate variables associated to this symptons and kind of psychotic disorder or OCD. There are some hypothesis about the role of 5HT receptors in the genesis of OCD symptoms.

Disclosure of interest.- The authors have not supplied a conflict of interest statement.

\section{E-PV0483}

\section{The various management of compulsive obsessive disorder in children}

M. Bitar ${ }^{1^{*}}$, L. Achour Achour ${ }^{2}$, F. El Jebbouri El Jebbouri ${ }^{2}$, H. Kisra Kisra $^{3}$

${ }^{1}$ Dr, Hopital Arrazi, Rabat, Morocco; ${ }^{2}$ Arrazi, Emergency, Rabat, Morocco; ${ }^{3}$ Arrazi, Pedopsychiatry, Rabat, Morocco

"Corresponding author.

Background and aims.- Obsessive-compulsive disorder (OCD) is an invasive psychiatric condition that causes suffering or interferes with the patient's life. OCD in children is a common disorder whose clinical expression is similar to that of adults and whose spontaneous evolution tends towards chronicity. The management of OCD in children uses various therapies. However, cognitivebehavioral therapy remains the most recommended and the most effective.

The objective of our study is to update the different methods of treatment of obsessive compulsive disorder in children. 
Methods.- Cross-sectional study conducted among residents and psychiatrists practicing in Ar-razi de Salé Hospital using a questionnaire assessing the therapeutic attitudes of physicians in the management of obsessive-compulsive disorder in children.

Results.- In our sample, $76 \%$ of our patients have an average age of between 11 and 14 years.76\% had no family history. Among those found are: OCD, major depressive disorder and schizophrenia.60\% of no patients had no comorbidities. Those found are: major depressive episode, eating disorders, secondary enuresis, post-traumatic stress disorder, generalized anxiety disorder and social anxiety disorder.48\% of all our patients have obsessions like Idéïques and 32\% have compulsions type checks. In $68 \%$ of our patients, management was based on the combination of cognitive behavioral therapy (CBT) and SSRIs other than sertraline. $32 \%$ were on sertraline alone. Conclusions.- The literature shows quite clearly that CBT is the most successful form of intervention to date. It is also important to learn more about the effectiveness of pharmacotherapy, used alone or in combination with CBT.

Disclosure of interest.- The authors have not supplied a conflict of interest statement.

\section{E-PV0485}

\section{Glutamate modulators as emerging therapies for obsessive-compulsive disorder}

\author{
A. Carapucinha* , A. Barcelos \\ Hospital Garcia de Orta, Psychiatry, Almada, Portugal \\ Corresponding author.
}

Background and aims.- Obsessive-compulsive disorder (OCD) is a neuropsychiatric disorder characterized by the presence of obsessions and/or compulsions. With a typical early onset and a lifetime prevalence of $1 \%$ to $3 \%$, OCD is associated with significant impairment. Early recognition and treatment may improve outcomes, but OCD is characteristically chronic. Selective serotonin reuptake inhibitors (SSRIs) provide the most effective treatment and along with cognitive behavior therapy constitute the first-line treatments. However, as much as $40-60 \%$ of patients do not respond to antidepressants therapy. Of the emerging alternative approaches, one promising therapy seems to be the use of glutamate modulators.

Methods.- The authors performed a non-systematic review of the literature on the evidence for glutamate modulators use in treatment-resistant OCD. A search through PubMed has been made using the key words "refractory", "resistant", "obsessivecompulsive disorder", "treatment" and "glutamate modulator".

Results.- In view of the common resistance to antidepressants, attention has shifted towards other neurotransmitter systems and associated neuroanatomical structures, showing a key role of glutamate pathways upregulation within the cortico-striatalthalamo-cortical circuitry and temporal lobes in OCD pathogenesis. Preliminary data supports the use of glutamate modulators such as riluzole, memantine, ketamine, topiramate, lamotrigine and dcycloserine as alternative pharmacologic options for OCD in select cases.

Conclusions.- Given the importance of the glutamatergic pathways disturbance in OCD genesis, glutamate modulators may be an effective alternative treatment for OCD. Despite the emerging evidence favoring their use, further investigation is still needed to establish their benefit.

Disclosure of interest.- The authors have not supplied a conflict of interest statement.

\section{E-PV0486 \\ Compulsive sexual behavior: a case report}

M. Belarbi, L. Jouini*, N. M'Hadhebi, U. Ouali, R. Jomli, F. Nacef

Razi hospital, Psychiatric A, Manouba, Tunisia

Corresponding author.

Background and aims.- Compulsive sexual behavior (CSB), is characterized by repetitive and intense preoccupations with sexual fantasies, urges, and behaviors that are distressing to the individual and/or result in psychosocial impairment. The problematic posed by CSB is described through our clinical case.

Methods.- From the clinical file of the patient, we collected the data concerning his socio-demographic and clinical profile.

Results.- Mrs N.B is 39 years old, divorced for 20 years and has no children. Her story begins after her divorce. Mrs. N.B has had multiple partners. She admitted to me that she had at least 40 sexual partners, knowing that she had a lasting relationship with only 2 of them. she happens to seduce young men and even teenagers, to go out in the evening for the sole purpose of sleeping with strangers. The story has spread. Everyone in his city knew it, my brother kept repeating, "You have dishonored our family, you are useless, it would have been better if you had died!" "I lost a lot, my family, my dignity, but her impulses were very difficult to control, it's as soon as I find myself alone that it takes me, it scares me because I know that my impulses overwhelm me [...]".

Conclusions.- There is an ongoing discussion about the proper categorization of CSB. With the exclusion of CSB from the DSM-5 and the ongoing debate about its classification, new research will most likely begin to emerge in an attempt to find its correct position within psychiatric disorders.

Disclosure of interest.- The authors have not supplied a conflict of interest statement.

\section{E-PV0487}

\section{The korean version of family accommodation scale for obsessive-compulsive disorder}

C.H. Kim ${ }^{1 *}$, J.G. Chang ${ }^{2}$, S.J. Kim ${ }^{1}$

${ }^{1}$ Yonsei University College of Medicine, Psychiatry, Seoul, Republic of Korea; ${ }^{2}$ Myungi Hospiyal, Psychiatry, Seoul, Republic of Korea

Corresponding author.

Background and aims.- Family responses related to patient's obsessive-compulsive (OC) symptoms have been linked to treatment response, symptom severity, and levels of functioning. The Family Accommodation Scale (FAS) is the most widely used measure of accommodation behavior in obsessive compulsive disorder (OCD) patients. The present study was to develop a Korean version of the FAS-Self-Rated Version (FAS-SR), to examine its reliability and validity, and to study the clinical correlates of family accommodation in OCD.

Methods.- We preliminarily examined the psychometric properties of the Korean version of the FAS-SR in a sample of 21 Korean OCD patients and 21 relatives. The Korean version of FASSR was developed through translation and back-translation of FAS-SR.

Results.- Korean version of FAS-SR demonstrated good internal consistency (Cronbach's $\alpha=0.898$ ). For the convergent validity between the Korean version of FAS-SR and other measures completed by the patient, severity of OC symptoms (Y-BOCS; $r=0.797$, $p<0.01$ ), functional impairment (GAF; $r=-0.730, p<0.01$ ), psychological distress $(\mathrm{K} 6,-0.655, p<0.01)$ showed significant correlations but impulsivity didn't. Additionally, family accommodation behavior was correlated with family member's anxiety sensitivity (ASI, $r=0.542, p<0.01$ ). 
Conclusions.- Korean version of the FAS-SR has acceptable psychometric properties for assessing family accommodation in Korean patients with OCD in both research and clinical settings.

Disclosure of interest.- The authors have not supplied a conflict of interest statement.

\section{E-PV0488}

\section{Schizo-obsessive disorder: a case report}

J.J. Lopez Castillo*, H.L. Leyre, R.N. manuel

Virgen de las Nieves University Hospital, Mental Health Therapeutic Community, GRANADA, Spain

${ }^{*}$ Corresponding author.

Background and aims.- The high prevalence and comorbility in schizophrenia and Obsessive-Compulsive Disorder have impelled many authors to report about a differenciated diagnostic category: the Schizo-Obssesive Disorder, with its own clinical characteristics and important implications on its treatment and development. We describe a schizophrenic 45 years old patient who started at 18 with a psychotic outbreak, to which lately added obsessive-compulsive symptoms. After several hospitalizations in mental health acute units and the impossibility of managing symptoms with outpatient attention, he is finally referred to our full hospitalization program. Methods.- We want to highlight memory and attention alterations, anhedonia, and subdepressive mood state. Thought disorders as persecutory delusions with affective and behavioural response, and obsessive thoughts. Compulsive rituals which take so much time they become highly disabling.

Results.- Many multidisciplinary treatment interventions have been required for succeding in reaching discharge from our unit. Treatment with Clozapine allowed initially to control positive symptoms, but induced obssesive-compulsive symptoms. When reduced to a low dose, combined with Paliperidone injection and fuvoxamine, has demonstrated the desired therapeutic effect in diminishing the most disabling symptoms and allowing the patient to live in the community.

Conclusions.- This patient could be considered as part of this increasingly reported in literature subtype of shizo-obsessive patients. This profile would feature a specific clinical presentation: combined psychotic and obsessive symptoms, worse response to ordinary treatment, impaired social functioning, neuropsychological signs and poor prognosis. This findings support the need for further longitudinal studies in order to identify and stablish valid and reliable criteria to describe this new diagnostic category.

Disclosure of interest.- The authors have not supplied a conflict of interest statement.

\section{E-PV0489}

\section{Structural variants of compulsive personality}

A. Nirestean ${ }^{1}$, E. Lukacs ${ }^{2}$

${ }^{1}$ University of Medicine and Pharmacy, Psichiatric Clinic No.II, Tirgu Mures, Romania; ${ }^{2}$ University of Medicine and Pharmacy, Psichiatry, Tirgu Mures, Romania

* Corresponding author.

Background and aims.- Within the scope of personality disorders, the obsessive personality has defining features of distinct severity. The obsessive traits can have, alternatively or simultaneously, an adaptive character, respectively a maladaptive one.

Methods.- These attributes are conditioned by the self-esteem level and by the hypertrophy or hypotrophy of the Superego. There are also associated the affective and attitudinal rigidity, the ambivalence and ambitendency level, the one of anticipatory anxiety, the presence of one's own truth feeling, the responsibility and devotion in the professional role, the quality of relational abilities.

Results.- We propose a comparative description of two sub-variants of the obsessive personality - typical obsessive, respectively the psychashtenic type - approached according to the common dominant attributes described. This allows highlighting the structural differences according to the adaptive capacities and vulnerability, for Axis I disorders. In reference to how they relate to action, the two sub-variants are similar from the perspective of deliberation, but they are different according to their activity development and efficiency.

Conclusions.- Under boundary circumstances, the insight of the typical obsessive guides him to an identity reshuffle with an exacerbated rigorousness and perfectionism, and under the same circumstances, the psychashtenic cannot reform himself, instead he falls deeper into uncertainty and identity dissociation.

Disclosure of interest.- The authors have not supplied a conflict of interest statement.

\section{E-Poster Viewing - 7-9 April: Old Age Psychiatry}

\section{E-PV0492}

\section{Do not confuse depression with fronto-temporal dementia}

C. Alario Ruiz ${ }^{* *}$, C. Fernández Rodríguez ${ }^{1}$, P. Herrera Gener ${ }^{1}$, A. Royuela Rico ${ }^{1}$, A. Pascual Alonso ${ }^{2}$

${ }^{1}$ Psychiatry, Psychiatry, Palencia, Spain; ${ }^{2}$ Psychiatry, Psycology, Palencia, Spain

* Corresponding author.

Background and aims.- Frontotemporal Dementia (FTD) is a multiethiological syndrome comprising three clinical subtypes: behavioral variant (FTD-BV), primary progressive aphasia, and semantic dementia.

From the psychiatric point of view, the most important variant is the first one. The clinical diagnosis is complemented with neuropsychological tests. Apathy-abulia is the most common neurobehavioral disorder and may be confused with a depressive syndrome. However, these patients do not present anhedonia, nor do they report low or depressive mood.

Objectives.- Review diagnostic criteria of FTD.

Review differential diagnoses with depressive syndromes.

Methods.- It is presented a case report and a bibliographic review of the Frontotemporal Dementia (diagnosis and treatment).

Results.- The consensus diagnostic criteria of FTD have been an important step in the phenomenological delineation of FTD, but only one third of patients are diagnosed in their earliest stages, since only a minority of patients with FTD-BV meet all the central diagnostic criteria in their earliest stages. These clinical features have little to do with the typical cognitive alterations of other dementias, so confusion with depressive conditions is common. There is currently no FDA- or EMA-approved treatment for FTD-BV. Trazodone may improve some behavioral problems such as irritability, agitation, depressive symptoms, and eating disorders.

Conclusions.- In patients over 50 years of age, when faced with apathy in patients with no history of depression, it would be advisable to include FTD-BV in the differential diagnosis. Alterations in the ability to make moral judgments, loss of social tact and behavioral changes make it necessary to think about FTD-BV.

Disclosure of interest.- The authors have not supplied a conflict of interest statement. 
E-PV0493

\section{Delirium as a mortality predictor in elder patients after scheduled operations}

P. Argitis ${ }^{1}$, C.M. Platsa ${ }^{1}$, S. Karavia ${ }^{1}$, A. Kourti ${ }^{1}$, K. Paschalidis ${ }^{2}$, M. Poulou $^{3}$, M. Fasoula ${ }^{1}$, A. Papatsoris ${ }^{1}$, T. Tilemachou ${ }^{1}$, P. Dalli ${ }^{1}$, Z. Chaviaras $^{1}$

${ }^{1}$ General Hospital of Corfu, Psychiatric, Corfu, Greece; ${ }^{2}$ Psychiatric Hospital of Thessaloniki, Psychiatric, Thessaloniki, Greece; ${ }^{3}$ General Hospital of Kymi, Anesthesiology, Kymi, Greece

${ }^{*}$ Corresponding author.

Background and aims.- Delirium is a condition that psychiatrists are often called to deal with in clinical practice. It has been observed that is common in surgical departments. The aim of this study is to investigate whether delirium is a mortality predictor for elder patients, undergone a surgical intervention.

Methods.- The research was conducted in patients, older that 60years-old, hospitalized in the General Hospital of Corfu, due to their admission in the Surgical Department (brain and thoracic surgery excluded), who met the criteria of delirium. Aiming to a more extensive monitoring, the assessment continued at intervals of 3, 6 and 12 months. The patients did not suffer from any other psychiatric disorder of the psychotic spectrum in the past and have not previously preceded anesthesia in the context of physical disease. Results.- According to the data of the study, 54 patients met the criteria of the research. One patient died during hospitalization. The death ratio of the initially hospitalized patients in the first 3 months after diagnosis was $1,62 \%$. Respectively, in 6 months after hospitalization the percentage was $3,78 \%$, while in the end of the first year $4,32 \%$ of the initial patients had died.

Conclusions.- The data analysis shows that delirium is not a significant mortality predicting factor for the elder patients, undergone a scheduled operation and these data do not differentiate from the sources of the Hellenic Statistical Authority.

Disclosure of interest.- The authors have not supplied a conflict of interest statement.

\section{E-PV0494 \\ Is leukocyte telomere length a marker of physical frailty in late-life depression?}

M.H.L. Arts ${ }^{1}$, R. Collard ${ }^{2}$, H. Comijs ${ }^{3}$, L. de Jonge ${ }^{4}$, B. Penninx ${ }^{3}$, P. Naarding $^{5}$, R. Kok $^{6}$, R. Oude Voshaar ${ }^{7}$

${ }^{1}$ Mental Health Care -Western North Brabant GGZ-WNB, Department of Old Age Psychiatry and Neuropsychiatry, Halsteren, The Netherlands; ${ }^{2}$ Radboud University Medical Center, Department of Psychiatry, Nijmegen, The Netherlands; ${ }^{3}$ GGZinGeest/VU University Medical Center, Department of Psychiatry, Amsterdam, The Netherlands; ${ }^{4}$ University of Groningen- University Medical Center Groningen, Department of Psychiatry, Groningen, The Netherlands; ${ }^{5}$ GGNet, Department of Old Age Psychiatry, Apeldoorn, The Netherlands; ${ }^{6}$ Parnassia Psychiatric Institute, Department of Old Age Psychiatry, Den Haag, The Netherlands; ${ }^{7}$ University of Groningen- University Medical Center Groningen, University Center for Psychiatry, Groningen, The Netherlands

Corresponding author.

Background and aims.- Although average life-expectancy is still increasing worldwide, ageing processes markedly differ between individuals, which has stimulated the search for biomarkers of biological ageing. In this study we will explore to what extent leukocyte telomere length (LTL), a frequently used molecular marker of ageing, is associated with a clinically defined phenotype of biological ageing.
Objectives \& Aims.- Exploring the cross-sectional and longitudinal association between LTL as molecular marker of ageing and the physical frailty phenotype (PFP) as a clinical marker of ageing. To examine whether these associations are moderated by the presence of a depressive disorder, as depression can be considered a condition of accelerated ageing.

Methods.- Among 378 depressed older patients (according to DSMIV criteria) and 132 non-depressed older persons participating in the Netherlands Study of Depression in Older persons (NESDO), we have assessed the PFP and LTL. The PFP was defined according to Fried's criteria and its components were reassessed at two-year follow-up.

Results.- LTL was neither associated with the PFP at baseline by Spearman rank correlation tests, nor did it predict change in frailty parameters over a two-year follow-up using regression analyses adjusted for potential confounders.

Conclusions.- We found no association between LTL and PFP; neither in non-depressed nor in depressed older persons. As LTL and PFP appear to represent different aspects of ageing, they may complement each other in future studies.

Disclosure of interest.- The authors have not supplied a conflict of interest statement.

\section{E-PV0495}

\section{Late-life depression and mediterranean diet. A cross sectional study in East Attica, Greece}

K. Argyropoulos ${ }^{1}$, E. Machini ${ }^{2}$, A. Argyropoulou ${ }^{3}$, D. Avramidis ${ }^{4 *}$ E. Jelastopulu ${ }^{1}$

${ }^{1}$ School of Medicine- University of Patras- Greece, Public Health, Patras, Greece; ${ }^{2}$ Hellenic Open University, Social Sciences, Athens, Greece; ${ }^{3}$ Health Centre of Andravida, Family Medicine, Patras, Greece; ${ }^{4}$ University of Patras- Greece, School of Medicine, Patras, Greece

* Corresponding author.

Background and aims.- The rapid increase in the numbers of older adults worldwide makes a focus on mental disorders and aging both timely and imperative.

The aim of the present study was to estimate the prevalence of Late-Life Depression (LLD) of an urban area in Athens and to investigate associations with adherence to a Mediterranean-based dietary pattern and other risk factors.

Methods.- A cross-sectional study was conducted among the members of the open day-care centres for older people, in East-Attica, Greece. An anonymous questionnaire was developed to collect basic demographic data, the Geriatric Depression Scale (GDS-15) was applied to screen the elderly for depressive symptoms and the MedDietScore (MDS) for assessing adherence to Mediterranean diet. Statistics was processed with SPSS 24.0.

Results.- According to GDS-15, 24.7\% (21.4\% moderate and 3.2\% severe type) screened positive for depressive symptoms. Depression was more frequent in women than in men $(14.3 \%$ vs $3.2 \%$, $p=0.034)$, in lower-educated $(p=0.012)$, in participants with lower monthly income $(p=0.003)$, and in older people with comorbidity such as, musculoskeletal disorders $(p=0.026)$, anxiety $(p<0.001)$, depression $(p<0.001)$ and respiratory problems $(p=0.001)$. Although MDS is not significantly associated with GDS-15 ( $p=0.051$ ), or other demographics parameters, greater consumption of a dietary pattern that was higher in vegetables and lower in poultry and alcohol was associated with decreased likelihood of developing LLD.

Conclusions.- Our results support that depression in older adults is common and strongly associated with several risk factors. Adherence to a Mediterranean diet may protect against the development of depressive symptoms in older age.

Disclosure of interest.- The authors have not supplied a conflict of interest statement. 


\section{E-PV0496}

\section{Anxiety as symptom of presentation of subdural hematoma. Case report}

N.M. Casado Espada*, R. De Alarcón Gómez, J.I. De La Iglesia Larrad, Á. Pérez Laureano, M.T. Lozano López, R.S. Gamonal Limcaoco, P. Andrés Oliveira, A. Zambrana

University of Salamanca Health Care Complex- Psychiatry-

Salamanca- Spain, Psychiatry, Salamanca, Spain

${ }^{*}$ Corresponding author.

Background and aims.- A subdural hematoma is usually associated with traumatic brain injury. If the bleeds are large enough to put pressure on the brain, signs of increased intracranial pressure or damage to part of the brain will be present; however, anxiety is an uncommon clinical manifestation of subdural hematoma.

The aim of this case report is to show an uncommon etiology of anxiety and to emphasize the importance of differential diagnosis in this syndrome.

Methods.- Collection of data on the patient's clinical history and bibliographic review

Results.- We present a 68 years old male patient, with no prior personal or familiar psychiatric records, who presented an anxious syndrome as the main clinical manifestation of a subdural hematoma.

Conclusions.- Anxiety is a very common syndrome in the general population, present in many diseases of organic nature, so it is essential to make a good differential diagnosis. In the elderly, this becomes even more important due to the greater prevalence of pluripathology in this population, especially if there is no psychiatric personal history. Psychiatrists must pay attention to the signs of organicity and carry out an adequate organic screening for each clinical case.

Disclosure of interest.- The authors have not supplied a conflict of interest statement.

\section{E-PV0497}

\section{Influence of comorbidities (high blood pressure and diabetes) on the length of hospitalization in elderly people with depression}

A. Ciubara ${ }^{1 *}$, A.B. Ciubara ${ }^{2}$, F. Sarbu ${ }^{3}$

${ }^{1}$ Faculty of Medicine and Pharmacy-University "Dunarea de Jos"-“Elisabeta Doamna" Hospital, Psychiatry, Galati, Romania; ${ }^{2}$ University "Dunarea de Jos"-"The Holy Apostle Andrew" Hospital, Orthopedic Surgeon-Traumatology, Galati, Romania; ${ }^{3}$ Faculty of Medicine and Pharmacy- University "Dunarea de Jos", Psychiatry, Galati, Romania

* Corresponding author.

Background and aims.- Elderly people are at an increased risk of developing depression, both because of their vulnerability due to the physiological process of aging and chronic, sometimes disabling chronic diseases. Depressive elderly patients who are associated with comorbidities are fragile patients with low biological and non-compliant resources due to complex treatment schemes prescribed for somatic disorders.

Assessing how hospitalization is influenced by high blood pressure and diabetes associated with depression.

Methods.- This survey included 83 participants, all over 60 years of age, hospitalized with the diagnosis of depressive disorder at "Elisabeta Doamna" Psychiatric Hospital in Galaţi, Romania, from March to April 2018.

Results.- Of the total number of people with depression, 10 people associated hypertension (12\%) with an average hospital time of 11 days, 12 subjects diagnosed with ADO type 2 diabetes (15\%) and average hospital time of 12 days, 2 subjects had insulin-dependent type 1 diabetes (2\%) and 19-day hospital time.
Conclusions.- In the elderly, depression can be masked or associated with somatic disease symptoms, and the subjects reach psychiatric counseling when the condition is aggravated or chronically diagnosed. The length of hospitalization in depressive elderly patients associating with comorbidities is increased compared to depressed people without chronic illness, especially in those with insulin-dependent type 1 diabetes who have reduced compliance with prescribed treatment and accepting hypoglycemic diets.

Disclosure of interest.- The authors have not supplied a conflict of interest statement.

\section{E-PV0498}

\section{The many faces of dementia - similarities and differences between two early onset cases of alzheimer's and frontotemporal dementia}

V. Dionisie*, M. Manea, A. Craciun

"Prof Alexandru Obregia" Clinical Psychiatric Hospital, IVth Clinical Department, Bucharest, Romania

* Corresponding author.

Background and aims.- Dementia is a major public health issue due to increasing prevalence. Alzhemier's Dementia (AD) is the most common type, accounting for $50-60 \%$ of all cases and rarely is younger-onset. Frontotemporal dementia (FTD) accounts for about $13 \%$ of cases. AD and FTD differ greatly regarding pathophysiology and treatment.

Methods.- We present two case studies: a case of AD caracterised by memory loss, impairment in concentration and planning, disorientation in time and place, insight into illness, aphasia and agnosia along with depressive features and a case of behavioral variant of FTD, initially misdiagnosed as manic episode, and distinguished by personality change and cognitive deficit (behavior disinhibition with socially inadequacy, loss of manners, stereotypy of speech, elevated mood, memory deficit, partially oriented in time and space, moderate impairment in executive functions and lack of insight).

Results.- Even thought the two cases of dementia share a core symptoms of impaired mental functions and the age of onset, the debut of symptoms is different - with a personality change simulating a manic episode in FTD and - with cognitive deficit in AD. Also, the impaired mental functions differ, meaning that visuospatial and episodic memory are relatively preserved and a moderate deficit in executive functions for the patient with FTD as against the AD. In terms of mood, in our case, AD is characterized by depression, as a symptom or as prodromal stage while the FTD by an elevated mood and cheerfulness.

Conclusions.- We will also discuss possible treatment options, common mistakes in differential diagnostic and recent pathophysiological discoveries.

Disclosure of interest.- The authors have not supplied a conflict of interest statement.

\section{E-PV0499}

\section{Vitamin D supplementation promotes correction of mood disturbances in the old postmenopausal women}

\section{J. Fedotova*, S. Pivina}

I.P. Pavlov Institute of Physiology RASci, Neuroendocrinology, Saint-Petersburg, Russia

* Corresponding author.

Background and aims.- Menopause is characterized by a dramatic development of affective-related disorders and different psychoemotional pathologies (Santoro et al., 2015; Maki et al., 2010). 
Considering the wide use of complementary and alternative medications such as vitamin supplements in menopausal patients and our insufficient knowledge about the interaction between HRT and vitamin supplements, investigating the subject from a clinical studies seems very beneficial.

Methods.- This study evaluated the effect of Vitamin D3 supplementation (cholecalciferol) in several doses (20000, 40000 and $60000 \mathrm{IU}$, per os) alone or in a combination with standard hormonal menopausal therapy (HRT) on anxiety and depression scores, monoamines and hormonal levels for the old (55-63 years old) postmenopausal women.

Results.- The old menopausal women treated with Vitamin D3 at a dose of $20000 \mathrm{IU}$ alone or in a combination with HRT had greater reduction in anxiety and depression scores than the control group $(p<0.05)$. The vitamin D3 treated groups of the old postmenopausal women had significantly higher 25-hydroxyvitamin D3 levels as compared to the control group $(p<0.05)$. Also, we found restoration of gonadal hormones levels in the blood and monoamines turnover in the daily urine of the old menopausal women treated with Conclusions.- This work promotes more effective creating of the novel therapeutic targets and strategies for affective-related state treatment in postmenopausal women. We suggest further clinical trials in old women who are at risk for postmenopausal mood disorders.

The reported study was funded by Russian Science Foundation (RSF) accordingly to the research project № 16-15-10053.

Disclosure of interest.- The authors have not supplied a conflict of interest statement.

\section{E-PV0500}

\section{Glutamine supplementation on systemic inflammation, mood and cognitive status in elders: a secondary analysis from a randomized clinical trial (RCT) \\ S.S.M. Fernandez ${ }^{*}$, A.A. Bastos, S.M. Lima Ribeiro}

University of São Paulo, School of Public Health, São Paulo, Brazil

* Corresponding author.

Background and aims.-

Introduction.- Gut leakage, typical of aging, is thought to be associated to a number of outcomes, including cognitive decline and mood disorders. In turn, glutamine is essential to the maintenance of tight junction of the enterocytes. We hypothesize that glutamine supplementation can reduce the gut leakage in elders, reducing, consequently, the systemic inflammation and slowing down the cognitive decline and mood disorders.

Aims.- to identify the effect of glutamine supplementation on systemic inflammation, cognitive status and depressive symptoms in apparently healthy elderly.

Methods.- Double-blind RCT (REBEC; RBR-6qr9xx), six months intervention. Forty-nine elders (60+years old, both genders), assigned into two groups, according to supplementation (both receiving $12 \mathrm{~g}$ substance/day): glutamine or placebo. Before and after the supplementation protocol, we analysed:-cognitive status (by Mini-mental status examination; MMSE); presence of depressive symptoms (by geriatric depression scale, GDS-15); biomarkers of pro- and anti-inflammatory status (TNF-a, IL-6 and IL-10). We investigated the modification in MMSE and GDS-15 according to groups (chi-square), and performed logistic binomial multiple regressions, investigating inflammatory status, age and sex as covariables (SPSS Software ${ }^{\circledR}$ ).

Results.- We did not find differences between differences in MMSE and GDS-15, either in time or groups (X2 to MMSE $=0,600 ; p=0.44$; $\mathrm{X} 2$ to GDS-15 $=0,159 ; p=0,69$. The regression models did not point any significant effect of inflammation, age or sex (R2 (Cox\& Snell) to $\mathrm{MMSE}=0,176$; and $=0,083$ to GDS- 15 .
Conclusions.- Our results pointed that apparently healthy elders did not improve either their cognitive or mood status, as well as inflammatory status, with glutamine supplementation.

Disclosure of interest.- The authors have not supplied a conflict of interest statement.

\section{E-PV0501 \\ Delusion of pregnancy: an unusual case in frontotemporal dementia}

L. Ferreira $^{1 *}$, C. Ferreira ${ }^{2}$, N. Santos ${ }^{1}$, V. Gonçalves ${ }^{1}$

${ }^{1}$ Hospital Distrital de Santarém, Departamento de Psiquiatria e

Saúde Mental, Santarém, Portugal; ${ }^{2}$ Centro Hospitalar Lisboa Norte Hospital Santa Maria, Psiquiatria, Lisboa, Portugal

${ }^{*}$ Corresponding author.

Background and aims.- Delusions can complicate practically all brain disorders. An example is the delusion of pregnancy. Delusions of pregnancy have been reported in a wide variety of functional and organic psychiatric conditions but rarely with dementia. Psychotic phenomena such as delusions and hallucinations are rare in frontotemporal dementia syndrome but have been recognised as a feature in some cases of frontotemporal lobar degeneration. Our aim is to discuss the findings from previous studies about the phenomenon of delusion of pregnancy, in the context of dementia and correlate with the clinical case.

Methods.- We reviewed the literature on the topic and the information regarding the clinical case was obtained by personal interview and by consulting the patient's clinical file.

Results.- We describe the clinical case of a 53-year-old woman admitted with frontotemporal dementia. She complained that she was pregnant, so decided to stop all medication because that could be harmful for the baby. Her delusion of pregnancy persisted for five days. There was no impairment of consciousness and she didn't have others features of delirium.

Conclusions.- Although delusions are a common feature of dementia, delusions of pregnancy has rarely been reported. Delusion of pregnancy is a bizarre form of delusion due to organic, functional or drug-induced causes. It's reported in delusional procreation syndrome that consists of delusions in the human procreation cycle. This case of frontotemporal dementia with delusion of pregnancy is presented to illustrate the need to consider neurodegenerative disease as well as primary psychiatric disorder as the underlying cause of this striking symptom.

Disclosure of interest.- The authors have not supplied a conflict of interest statement.

\section{E-PV0502}

\section{Benzodiacepines in the elderly}

M.J. Gordillo Montaño*, I. Moreno Encabo, J. Iglesias Lopez, S.V. Boned Torres, M. de Amuedo Rincon, S. Latorre, M. Guisado Rico Hospital Can Misses, Psychiatry, Eivissa, Spain

* Corresponding author.

Background and aims.- The drugs consumption has increased in the last two decades in the industrialized countries in a striking way affecting mainly to the elderly population. It constitutes an increasing population group and they are the ones that require most of the medication and medical attention. They are characterized by the high degree of polypharmacy, and by the appearance of side effects. Polypharmacy worries the medical authorities and is of interest in the scientific, family and social environment as they lead to an increase in number of hospitalizations, serious complications and even death. We intend to reach the largest amount of public and try to raise awareness about the problem.

Methods.- We conducted a literature review to clear up this clinical suspicion. 
Results.- The global consumption of drugs in the elderly in industrialized countries is characterized by two basic facts:

- a great increase: 4.2-8 drugs / person / day

- the type of drugs, not all indicated for their pathologies, estimating that $97 \%$ of the institutionalized elderly and $61 \%$ of those living at their own home consume an inappropriate or inadequate drug. The group of tranquillizers occupies the third place. Benzodiazepines have become one of the most prescribed drugs: in general medical practice $2.5 \%$ of the population uses benzodiazepines regularly. $5 \%$ of primary care consultations can end with the prescription of benzodiazepines.

Conclusions.- The treatment with benzodiazepines should be very limited in time, and in very specific situations. If a prescription is needed in the elderly: half of the dose than in young adults, slow dose escalation, short periods.

Disclosure of interest.- The authors have not supplied a conflict of interest statement.

\section{E-PV0503}

\section{Mental disorders' treatment of elderly patients with polymorbidity}

O. Khaustova ${ }^{1 *}$, O. Osuchovska ${ }^{2}$, I. Kalugin ${ }^{3}$

${ }^{1}$ Bogomolets National Medical University, medical psychologypsychosomatic medicine and psychotherapy, Kiyv, Ukraine; ${ }^{2}$

Ukrainian research institute of psychiatry, clinical narcology, Киев, Ukraine; ${ }^{3}$ Mariupol psychoneurological hospital, gerontopsychiatry, Киев, Ukraine

${ }^{*}$ Corresponding author.

Background and aims.- The way to increase the effectiveness of mental disorders' treatment of elderly patients with polymorbidity may be the therapeutic complex' optimization on the basis of studying quality of life and satisfaction of medical care.

Methods.- A total number of 325 patients at the age of 60 and more years participated in the study. The main group (MG) of 238 people received standard treatment and psychotherapeutic intervention. A comparison group (CG) of 87 people had only standard treatment. Results.- According to the research results, elderly patients had a high level of polymorbidity according to the CIRS-G scale. The average number of concomitant diseases in the MG was 3.7; in CG 3 ,4. The average amount of daily taken drugs in MG - 3,7; in CG - 3.0. The severity and number of concomitant diseases increased with age and was associated with a deterioration in life quality. The tendency of life quality's decrease was directly proportional to the increase in the number of concomitant diseases, daily and periodic volume of pharmacotherapy, to a greater extent was observed in men and with increasing age of patients. The psychotherapeutic programme based on CBT included a combination of psychosocial education, compliance therapy and pharmacomania prevention training.

Conclusions.- The analysis of the results of the conducted medical work made it possible to conclude that the use of such therapeutic complex contributed to increasing compliance, quality of life and satisfaction with medical care of patients with the specified pathology.

Disclosure of interest.- The authors have not supplied a conflict of interest statement.

\section{E-PV0504}

\section{Sleep in the elderly: a mixed methods approach to investigate subjective sleep disturbances in a population of older South Africans}

K. Laxton

University of Stellenbosch, Department of Psychiatry, Cape Town, South Africa

Corresponding author.

Background and aims.-

Introduction.- There's a growing ageing population in Sub-Saharan Africa. South Africa is under-prepared for this health burden. Little is known about this population, including depressive and anxiety symptoms and causes for insomnia related experiences. The magnitude of old age sleep disturbances is reportedly high with compensatory prescribing of Benzodiazepines by practitioners. There's an urgent need to investigate alternative and safer methods targeting sleep symptoms in the older person by gaining detailed subjective accounts of this experience.

Objectives.- To describe the South African ageing population, including demographics, secondary causes for insomnia, subthreshold anxiety and depressive illnesses and mild neurocognitive symptoms in a sample of functional participants between ages 65-80 years. Insomnia Disorder and associated compensatory behaviours and their effects on physical and mental health forms this study's core.

Methods.- This will be a cross-sectional, mixed methods study: a quantitative survey followed by semi-structured qualitative interviews of participants identified with Insomnia Disorder and who meet specific inclusion criteria.

Results.- Results will be quantitative (measurable) and qualitative in nature. The aim is to gain measurable information about the mental health of this population and then describe the sleep experience in qualitative depth.

Conclusions.- If an emphasis were to be placed on addressing particular needs in the ageing population it makes sense to gain insightful understanding of sleep disturbances, the various approaches to compensate for and/or treat insomnia as well as the consequences of disrupted sleep. With this knowledge such objectives may be achieved, with an emphasis placed on the individual and not the symptom alone.

Disclosure of interest.- The authors have not supplied a conflict of interest statement.

\section{E-PV0506}

\section{Disguised dementia}

I. Lokmic-Pekic ${ }^{*}$, L. Bradic ${ }^{2}$, D. Begic ${ }^{1}$, A. Muhamed ${ }^{2}$

${ }^{1}$ Psychiatric Hospital, Intensive Care Unit, Sarajevo, Bosnia,

Herzegovina; ${ }^{2}$ Psychiatric Hospital of Canton Sarajev, Men's

Psychatric Department, Sarajevo, Bosnia, Herzegovina

* Corresponding author.

Background and aims.- It is of highest importance to differentiate dementia from depression since in most of the cases, depression can be treated with successful outcome.

Methods.- Female patient, 64 years old, working in kindergarten. Worsening of the mental state started month before admission to the hospital; she was moody, anxious with weakening of cognitive functions, she wasn't able to perform tasks by herself, she seemed confused and distant. At admission, HAMD score was 25 and MMS score was 15 . We increased dose of antidepressant altogether wit antidementic medication which made her feel relaxed, she gained her appetite and sleep was stabilized. However, it became obvious that she was all time confused, disoriented, damaged memory for fresh events. Her verbal expression was non-specific, with confabulations and stereotypic answers. She never accepted her child's loss, 
which was extremely stressful for her, as a mother. We repeated MMS, score was 14 , which implicated severe cognitive deficit. CT neurocranium was done - there were corticoatrophic changes at the very beginning.

Results.- Considering results of diagnostics that was done, symptoms that were presented and the progress of the disease, patient was considered as presenile dementia F 00.0.

Conclusions.- We wanted to specify difficulties of forehand diagnostic of presenile dementia, as well as differentiation of dementia and depression since there is depressive symptomatology at the very beginning of the dementia disease. These can lead to serious dilemmas considering diagnosis of the patient, and latter, to inaccurate therapy, since depressive symptomatology can disguise dementia's symptoms.

Disclosure of interest.- The authors have not supplied a conflict of interest statement.

\section{E-PV0507}

\section{Ambivalent and hostile ageism}

F. Daniel, I. Massano Cardoso*, A. Galhardo, I. Barroso Instituto Superior Miguel Torga, Instituto Superior Miguel Torga, Coimbra, Portugal

* Corresponding author.

Background and aims.- According to the World Health Organization, ageism corresponds to the stereotyping, prejudice, and discrimination against people based on age. Benevolent and hostile attitudes towards individuals on the basis of their age are experienced by older people in multiple societies. The aims of the current study were to assess ageism and explore whether there is a relationship between this construct and age, as well the role of health status perceptions in that relationship.

Methods.- This cross-sectional study was conducted in a sample of 356 participants ( 135 males and 221 females), with a mean age of 53.51 years old $(S D=20.27)$. Participants completed the Ambivalent Ageism Scale (AAS) and a multidimensional social resource, physical and emotional health questionnaire.

Results.- Mean scores of the different subscale items ranged from $2.50 \pm 2.21$ and $6.84 \pm 0.58$. These results were significantly higher than the ones reported in the original version of the AAS $(p \leq .003)$. Age was positively correlated with 9 items [ 6 benevolent (items, 4 , 5, 6, 7, 8 and 9) and 3 hostile (items 10, 12 and 13)]. Results also showed that participants presenting better health perceptions are the ones showing higher levels of ageism.

Conclusions.- The differences found between our results and those of the AAS original study may be explained by characteristics (education, rural area, cultural issues, etc.) which may impact on ageism definition. Therefore, it is important to enhance a better understanding of this construct in order to decrease the harmful effects of hostile and benevolent ageism.

Disclosure of interest.- The authors have not supplied a conflict of interest statement.

\section{E-PV0509}

\section{Hashimoto encephalitis (HE): a rare disease}

L. Mellado Cuerno*, F.G. Nazaret, M.S.J. Ainoa, B.R. Belén, M.L. Jesús, B.O. María Fe, R.P. Víctor, P.H. Juan Manuel Hospital Universitario La Paz, Psiquiatría, Madrid, Spain

* Corresponding author.

Background and aims.- Hashimoto encephalitis (HE) or steroidresponsive encephalopathy clinically associated with Hashimoto thyroiditis is a rare disease, poorly known and underdiagnosed. HE's pahogenesis is not fully understood. There is not a lineal association between the symptoms and the level of antiperoxidase antibodies. It has been described in the literature that HE consists of a possible vasculitis in different cerebral areas; showing an unespecific neuroimaging. One of most specific signs of this disease is the response to corticoid therapy. It may present a variety of neuropsyquiatric symptoms like psychosis $(26,1 \%)$, depression $(23,9 \%)$ or dementia (10,9\%). Objectives: To review the demografic caracteristics, different symptoms, psychiatric presentations and therapeutic management of HE.

Methods.- Case presentation and bibliography review about demographic distribution, diagnosis and management of HE.

Results.- We present a 77 year old woman who might be diagnosed of HE, her management and therapeutic results.

Conclusions.- The presentation of HE can be very heterogenous. We must keep on mind her psychitrist symptoms, like psychosis, depression, and dementia. HE must be considered when a patient shows high blood levels of anti TPO, incongruent symptoms or lack of response to antidepressive or antipsychotic medication. The standard treatment is steroid therapy. We should also monitor clinical response.

Disclosure of interest.- The authors have not supplied a conflict of interest statement.

\section{E-PV0511 \\ Prediction of future cognitive impairment among the community elderly: a machine-learning based approach}

K.S. Na

Gachon University Gil Medical Center, Department of Psychiatry, Incheon, Republic of Korea

${ }^{*}$ Corresponding author.

Background and aims.- Early detection of cognitive impairment is a key issue among the elderly. Although neuroimaging, genetic, and cerebrospinal measurements show the promising results, the costs and invasiveness hinder their widespread use. Predicting cognitive impairment using easy-to-collect variables by non-invasive methods for community-dwelling elderly is useful prior to conducting a comprehensive evaluation. The author aimed to develop a machine learning-based predictive model that predicts cognitive impairment two years later.

Methods.- The author used the Korean Longitudinal Study of Aging data between 2014 and 2016. A total of 5,210 community elderly over the age of 55 were included. Artificial neural network (ANN) and support vector machine (SVM) were exploited to build the best predictive model.

Results.- The results indicated that the strength of the cognitively impaired group at the baseline was 1692 (32.5\%). ANN showed better performance $(F-$ measure $=0.655$, Sensitivity $=0.709$ ) as compared to SVM (F-measure $=0.628$, Sensitivity $=0.633$ ).

Conclusions.- This study demonstrates that a machine learningbased predictive model has the potential to fully utilize basic variables usually collected at community-based institutions. With efforts to enhance the predictive performance, such a machine learning-based approach can further contribute to predicting future cognitive impairment.

Disclosure of interest.- The authors have not supplied a conflict of interest statement.

\section{E-PV0516 \\ Relationship between loneliness and anxiety in the elderly - exploratory study}

M. Pinheiro ${ }^{1^{*}}$, J. Gomes ${ }^{2}$, L.F. Malheiro ${ }^{3}$, H. Firmino ${ }^{4}$

${ }^{1}$ Centro Hospitalar do Tâmega e Sousa, Departamento de Psiquiatria e Saúde Mental, Penafiel, Portugal; ${ }^{2}$ Centro Hospitalar do Algarve, Departamento de Psiquiatria e Saúde Mental, Portimão, Portugal; ${ }^{3}$ 
Centro Hospitalar de Leiria, Departamento de Psiquiatria e Saúde Mental, Leiria, Portugal; ${ }^{4}$ Centro Hospitalar e Universitário de Coimbra, Departamento de Psiquiatria e Saúde Mental, Coimbra, Portugal

* Corresponding author.

Background and aims.- Few studies investigate the relationship between loneliness and anxiety in the elderly, even thought it is common among older people, and can be associated with poor quality of life, reduced mental health, and greater need for care The objective of the study was to examine the prevalence of loneliness among elderly in day care center, and assess the associations between loneliness and anxiety.

Methods.- We evaluated elderly people in day centers, and gathered sociodemographic and clinical data. The UCLA loneliness scale and Geriatric Anxiety Inventory (GAI) were applied. Statistical analyses of the data were performed using IBM SPSS. We considered $p$-value $<0.05$ to be statistically significant in all analyses.

Results.- The forty-five included adults had a mean age of 80.8 years. The majority of participants were women, widows, and with four or less years of education. Even though most had a chronic disease, few had pain, mobility, hearing or visual difficulties. Three quarters had regular psychiatry consultation. A third had negative feelings of loneliness and $38 \%$ had severe anxiety. There was a significant association between UCLA loneliness scale and GAI. Elderly people who had negative feelings of loneliness had more severe anxiety symptoms than other participants. In contrast, $86 \%$ of people with no negative feelings of loneliness had no severe anxiety symptoms. Conclusions.- In our sample, elderly people are at risk for loneliness, which in turn can lead to high levels of anxiety. It is important to value the loneliness and to develop strategies to solve it in order to avoid a worsening of mental health.

Disclosure of interest.- The authors have not supplied a conflict of interest statement.

\section{E-PV0517}

\section{Disintegration algorithms of higher mental functions in dementias in later age \\ L. Pishchikova}

Charitable Fund “Gerontological protection”, Ministry of HealthRussia, Moscow, Russia

${ }^{*}$ Corresponding author.

Background and aims.- Understanding the general patterns of loss of higher mental functions, disintegration algorithms, is relevant for correct diagnosis and definition of the clinical form and stage of dementia, has antidiscriminational significance for patients of late age in psychiatry.

Methods.- Clinical and expert analysis of 235 late age patients who underwent forensic psychiatric examination in Serbsky Federal Research Center in criminal and civil cases, revealed: "non-dement" mental disorders - $45.5 \%$, psychosis - $7.7 \%$, dementia - $46.8 \%$ (dementia in Alzheimer's disease with early and late onset - 16.5\%; mixed dementia - $39.4 \%$; vascular dementia $-42.2 \%$; amnestic syndrome - 1,8\%).

Results.- We have identified the following algorithms for the disintegration: from the higher to the lower (evolutionarily earlier); from previously learned information and behaviors - to later ones; from emotionally neutral information - to emotionally more significant; from dysmnestic disorders (violations of reproduction, fixation) - to fixative amnesia - to progressive amnesia - to sensory aphasia - to agnosia - to motor aphasia - to apraxia; sequence of the stages of memory decline: from the later acquired information to the earlier one; from fixative amnesia - to progressive amnesia (retrograde) - to affective memory; from less hardened skills to more fixed ones; sequence of loss of orientation: disorientation in space (temporary, then constant) - in time - in place - in one's own personality.

Conclusions.- Despite the existence of general disintegration algorithms of higher mental functions, different types of dementia are characterized by a recognizable specific clinical picture and dynamics (syndromokinesis, progredness, cognitive deficiency structure). Disclosure of interest.- The authors have not supplied a conflict of interest statement.

\section{E-PV0518}

\section{Can repetitive transcranial magnetic stimulation be used as augmentation treatment in elderly depressed patients? two clinical cases}

R.P. Sant'angelo ${ }^{*}$, F. Ambrosini ${ }^{2}$, G. De Paoli ${ }^{1}$, M. Pacetti ${ }^{3}$, C. Ravani $^{4}$, G. Piraccini ${ }^{1}$

${ }^{1}$ Ausl Romagna- S.P.D.C.- Hospital Maurizio Bufalini, Mental Health, Cesena, Italy; ${ }^{2}$ University of Bologna, Faculty of Psychology, Cesena, Italy; ${ }^{3}$ Ausl Romagna- C.S.M. of Forli, Mental Health, Forli, Italy; ${ }^{4}$ Ausl Romagna, Mental Health, Forli, Italy

"Corresponding author.

Background and aims.- Repetitive Transcranial Magnetic Stimulation (rTMS) showed its efficacy as augmentation treatment in depressed adults who are refractory to pharmacological treatments. According to the literature, age is a predictive factor of response to rTMS therapy. Specifically, only young patients seem to have a good response to rTMS. We report the results of rTMS therapy in two patients over 60 years of age.

Methods.- Both the patients (61 and 68 years old) had recurrent depression. They underwent to EEG, CT scan, blood and urine tests, psychodiagnostic, symptomatologic and neuropsychological assessment. The rTMS therapy was carried out with a stimulator (MagVenture, MagPro R30 ${ }^{\circledR}$ ), with the butterfly coil (Cool-DB80) positioned over the prefrontal cortex $5.5 \mathrm{~cm}$ in front of the motor threshold site). The first dose of treatment was set at $80 \%$ and was progressively increased at $100 \%$ at the fourth session. Both the patients had 30 sessions (10-Hz stimulation) distributed in 6 days a week. At the end of each session the patients filled a VAS to rate the side-effects of the stimulation.

Results.- One of the patients showed an amelioration of depressive and anxious symptoms, achieving the complete remission at the fifth week of therapy, referring only sleepiness as side-effect during the sessions. The second patient showed an amelioration at the second week of rTMS therapy, reaching the complete remission at the fourth week and without reporting any side-effect.

Conclusions.- The clinical cases show that even in elderly it is possible to reach the symptomatologic remission using rTMS as augmentation therapy.

Disclosure of interest.- The authors have not supplied a conflict of interest statement.

\section{E-PV0519 \\ Interest of olfactory workshop in patients with korsakoff syndrome in elderly institution}

N. Shpak-Deschamps*, G. Romero, E. Musellec

Psychiatric Hospital Guillaume Regnier, Geronopole, Rennes, France * Corresponding author.

Background and aims.- The medical care of elderly people with Korsakoff syndrome is difficult. The majority of these patients are failing cognitive rehabilitation programs and cognitive-behavioral therapies. It's explained as well by their specific cognitive impairments and particular social profile as by important neuropsychiatric disorders. 
Methods.- We have proposed to 6 men aged 73 to 84, MMS average $17 / 30$, with Korsakoff syndrome an olfactory workshop led by a psychologist using a study kit of smells. Each patient benefited of 5 individual sessions with two weeks brake between each. Behavioral disorders were assessed by the NPI-ES scale.

Results.- Certain smells have triggered clear and personal memories and led to verbal communication between patients and the psychologist. The therapeutic effect was resulted almost immediate in following pathological symptoms: apathy, aggression-irritability of mood, depression and anxiety (score reduction from 3 to 9 points per symptom). Hyperactive symptoms have been reduced till the next session. Manifestation of affective symptoms and apathy has been less prolonged.

Conclusions.- Our observation shows an interest and therapeutic prospects of stimulating olfactory function in people with Korsakoff syndrome to reduce the psycho-behavioral symptoms of these patients, to improve quality of their life and their acceptance in institutions for the elderly.

Disclosure of interest.- The authors have not supplied a conflict of interest statement.

\section{E-PV0520}

\section{Study of general practitioners' referrals to memory service and timeline of the assessments \\ S. Siddiqui ${ }^{1 *}$, J. Raju ${ }^{2}$, U. Ahmed ${ }^{3}$ \\ ${ }^{1}$ NHS, Psychiatry, Dewsbury, United Kingdom; ${ }^{2}$ NWBH, Old Age Psychiatry, St Helens, United Kingdom, ${ }^{3}$ Mental Health, Liverpool, United Kingdom \\ ${ }^{*}$ Corresponding author.}

Background and aims.- There are specific standards contained in the Memory Service policy, for General Practitioners (GPs) to follow when referring patients to Memory Service.

The information remains essential in conducting an assessment, to decide appropriate interventions, avoids delays, and to allocate urgent/routine appointments.

Aims.- To study quality of GP referrals for compliance with the agreed standards to improve patient care and effectiveness of service delivery.

Methods.-

Objectives.- To study GP referrals to the memory service to ensure important standards compliance including physical status, relevant medical history, results of investigations, current medications, current presentation, functioning and risks.

To study timeline of the assessments conducted by memory service following GP referrals.

Methodology.- Data was collected retrospectively from the Service Users clinical paper notes. 45 cases were randomised using the Research Randomizer software program.

Results.- Overall compliance for standards was 59\%. More than $90 \%$ compliance was noted for three standards including, medical history, list of current medication and diagnosis made within 12 weeks of the referral. A compliance of more than $80 \%$ was achieved for five standards inclusive of documentation of the Full Blood Count, Urea \& Electrolytes, Thyroid Function test, Liver Function Test and relevant medical history by other Consultants. Compliance for the remaining 20 standards was less than $80 \%$.

Conclusions.- This study identified spheres of good practice and sections that need improvement. The findings were circulated to relevant professionals and a new referral form was agreed with the Primary care to simplify the documentation of information to enrich information exchange with the aim to increase effectiveness patient care in a timely manner.

Disclosure of interest.- The authors have not supplied a conflict of interest statement.
E-PV0521

\section{Cholinesterase inhibitors and memantine may have further clinical advantages besides their impact on cognition in alzheimer's disease: $a$ restrospective study}

H. Simião ${ }^{1 *}$, M. Nascimento ${ }^{1}$, L. Alves ${ }^{2}$

${ }^{1}$ Centro Hospitalar de Lisboa Ocidental, Psychiatry, Lisbon, Portugal;

2 Centro Hospitalar de Lisboa Ocidental, Neurology, Lisbon, Portugal

Corresponding author.

Background and aims.- Patients with Alzheimer's disease (AD) develop a gradual cognitive deficit that becomes disabling in advanced stages of the disease. One of the earliest and major neurobiological findings in $\mathrm{AD}$ is a deficit in cholinergic neurotransmission. To date, cholinesterase inhibitors (ChEIs) are the only drugs that have shown significant improvements in the cognitive functions of $\mathrm{AD}$ patients. Current evidence supports the use of ChEIs and memantine with significant benefits in cognition, function and global outcome. However, regarding the specific treatment of secondary symptoms, such as anxiety, mood changes, psychosis and sleep problems, no specific agent or dosage is unanimously accepted. The aim of our study is to understand the advantages and disadvantages of anti-dementia drugs regarding secondary symptoms in AD.

Methods.- Retrospective study of all patients with possible AD, observed in a specialized consult for dementia since 2005. Evaluation of different secondary variables: name of anti-dementia drug, differences of psychopathology before and after starting treatment (psychotic features, anxiety or changes in sleep / mood) and other psychoactive agents used.

Results.- The majority of patients with active psychopathology that started an anti-dementia drug also started another psychoactive drug, with a good therapeutic response. Preliminary data regarding the impact of anti-dementia drugs in monotherapy on psychopathology are inconclusive; no significant relapses, worsening or new secondary symptoms were observed and no significant adverse effects were registered.

Conclusions.- Anti-dementia drugs may be useful and well tolerated regarding treatment of secondary symptoms of AD.

Disclosure of interest.- The authors have not supplied a conflict of interest statement.

\section{E-PV0522 \\ Intergenerational relationships among older adults and adult children \\ S. Von Humboldt ${ }^{*}$, I. Leal \\ ISPA-Instituto Universitário, William James Center for Research, \\ Lisbon, Portugal \\ ${ }^{*}$ Corresponding author.}

Background and aims.- The relationship of older adults with their adult children and grandchildren involves great emotional complexity and the quality of these relationships is associated with older adults' well-being. This qualitative study aims to examine how older adults conceptualize intergenerational relationships with adult children.

Methods.- The present study on qualitative data collected from indepth interviews was conducted with English and Portuguese older adults living in the community, designed to address their perspectives on intergenerational relations with adult children. 316 older adults participated in our study. The mean age of this group was 71.2 years. $65.3 \%$ were women, and a majority ( $54.7 \%$ ) had a partner. Results.- Content analysis generated four themes: affection and integration; satisfaction in the relationship; privacy and boundaries; financial support. 
Conclusions.- Intergenerational relationships are experienced by older adults with ambivalence and and stress the contradictory expectations of older adults with adult children.

Disclosure of interest.- The authors have not supplied a conflict of interest statement.

\section{E-PV0523}

\section{Adjustment to aging in the perspective of Lesbian, gay and bisexual older adults}

\section{S. Von Humboldt ${ }^{*}$, F. Carneiro, I. Leal}

ISPA-Instituto Universitário, William James Center for Research,

Lisbon, Portugal

${ }^{*}$ Corresponding author.

Background and aims.- This study aimed to build a structural model to explore whether socio-demographic, health and lifestyle-related variables, are correlates of adjustment to aging (AtA) in a group of lesbian, gay and bisexual (LGB) older adults.

Methods.- The sample comprised 287 community-dwelling LGB older adults aged 75 years old and older. Convenience sampling was used to gather questionnaire data. Measures encompassed the Adjustment to Aging Scale, the Satisfaction with Life Scale, demographics (sex, age, professional and marital status, education, household, and family's annual income), and lifestyle and health-related characteristics (self-reported spirituality, leisure, physical activity, perceived health and medication). Structural equation modeling was used to explore a structural model of the self-reported AtA, comprising all the above variables.

Results.- The structural model indicated the following significant correlates: perceived health $(\beta=0.456 ; p<0.001)$, leisure $(\beta=0.378 ; p<0.001)$, income $(\beta=0.302 ; p<0.001)$, education $(\beta=0.299 ; \quad p=0.009)$, spirituality $(\beta=0.189 ; p<0.001)$, sex $(\beta=0.156 ; p<0.001)$, physical activity $(\beta=0.142 ; p<0.001)$, satisfaction with life $(\beta=0.126 ; p<0.001)$, and marital status $(\beta=0.114 ; p=0.008)$. The variables explain respectively $76.4 \%$ of the variability of AtA.

Conclusions.- The results of this study indicate that policy making and community interventions with LGB older adults may benefit of including variables, such as, perceived health and leisure, income, education, spirituality, sex, physical activity, satisfaction with life, and marital status.

Disclosure of interest.- The authors have not supplied a conflict of interest statement.

\section{E-PV0524}

\section{Physical and mental comorbidities - how they affect quality of life of the elderly}

V. Voros", A. Boda-Jorg, A. Kovacs, T. Tenyi, S. Fekete, P. Osvath University of Pecs, Dept. of Psychiatry and Psychotherapy, Pecs, Hungary

* Corresponding author.

Background and aims.- Elderly may have several physical and mental illnesses, and they may take several medications to treat these conditions. According to the literature, the presence of these disorders can significantly influence their quality of life as well. Our aim was to investigate these factors and to assess how they affect their quality of life.

Methods.- In the framework of the ICT4Life project, the sociodemographic characteristics, prior and current physical and mental disorders and medication usage were assessed with semistructured interviews and with brief screening tools in elderly people over the age of 65 .
Results.- Mental, but mainly physical illnesses were common among the elderly. Prior and current mental disorders, especially depression were associated with poorer quality of life in the elderly. Cognitive and physical illnesses has less effect on their quality of life. The number of medications elderly were taken was not significantly associated with their quality of life.

Conclusions.- Low sample size and the limited number of factors analysed in our model are the most important limitations of our study. However, our results strengthen literature data, that the recognition and the early treatment of comorbid physical and mental disorders are highly important in the elderly, as it may not only treat these conditions, but it may improve their quality of life as well.

Disclosure of interest.- The authors have not supplied a conflict of interest statement.

\section{E-PV0525 \\ Qualitative studies contributions to clinical manage in old age patients with subjective cognitive decline: a comprehensive integrative review}

A. Weber ${ }^{1 *}$, A.R. Malta ${ }^{1}$, L.F.B. Mella ${ }^{2}$, A.R. Luzardo ${ }^{3}$, E.R. Turato ${ }^{1}$

${ }^{1}$ University of Campinas, Laboratory of Clinical-Qualitative Research - LPCQ Campinas, Brazil; ${ }^{2}$ University of Campinas, Department of Psychiatry and Medical Psychology, Campinas, Brazil; ${ }^{3}$ Federal University of Southern Border, Department of Nursing, Chapecó, Brazil

* Corresponding author.

Background and aims.- Complaints of memory loss by older adults have been considered as great value for clinical management of subjective cognitive decline. Studies with qualitative methodological strategy make it possible to explore the subjective dimensions of patients in order to understand the emotional meanings found for certain phenomena. To construct an integrative review makes it possible to identify concepts generated by such researches and synthesize them for use in clinical practice.

This study aims to understand the contributions of qualitative studies to the exploration of the clinical value of subjectivity.

Methods.- Methodologically, original and systematic reviews articles were included. Medline, EMBASE, ProQuest, Web of Science, CINAHL, Pubmed, Scopus, Bireme / BVS, Cochrane Library were accessed, using the terms: subjective cognitive decline, subjective cognitive impairment, subjective memory impairment, subjective memory impairment, subjective memory complaints, qualitative research, qualitative study. Time publication was not established. The selection was made after reading titles and abstracts. As final selection eligibility criteria, the JBI-QARI Critical Appraisal Checklist for Interpretative \& Critical Research instrument was used.

Results.- In short, 129 records were identified, 20 full-text articles were assessed for eligibility. Emotional effects and "practical concerns". Relational and organizational challenges: the relationship of cognitive and behavioral aging values in an inclusive environment. Conclusions.- We considered that subjective aspects of memory decline in older adults are not yet enough understood. There are a number of relational and organizational challenges to be faced in maintaining and caring for cognitive health in many of their aspects. Disclosure of interest.- The authors have not supplied a conflict of interest statement. 
E-PV0526

"Take care of your memory": an experience of promotion program of attention and prevention in cognitive health for the staff of a Brazilian university

A. Weber $^{1 *}$, E.R. Turato ${ }^{2}$, A.R. Luzardo ${ }^{3}$, A.R. Malta ${ }^{2}$, L.F.B. Mella ${ }^{4}$

${ }^{1}$ University of Campinas, Psychiatry and Medical

Pshycology/Laboratory of Clinical-Qualitative Research LPCQ

Campinas, Brazil; ${ }^{2}$ University of Campinas, Laboratory of

Clinical-Qualitative Research LPCQ Campinas, Brazil; ${ }^{3}$ Federal

University of Southern Border, Department of Nursing, Chapecó,

Brazil; ${ }^{4}$ University of Campinas, Psychiatry and Medical Pshycology,

Campinas, Brazil

* Corresponding author.

Background and aims.- Approximately half of people over 60 complain of reduced memory and cognitive functions. These symptoms appear to signify the onset of a neurodegenerative disease such as Alzheimer's Disease. From this perspective, health monitoring is crucial.

This report aims to describe an experience of a care and support service destined to professors and workers of a Southeast Brazilian university with complaints of memory impairment and other cognitive functions.

Methods.- This presentation describes a psychiatric outpatient service that attends individuals with complaints of memory loss and other cognitive functions from the academic community that works at the State University of Campinas - UNICAMP, a public institution of São Paulo. The program is called "Take care of your memory". Results.- Those are the criteria to participate: professor or employee - in activity or retired; 55 years old or more; to present complaint of memory decline or other cognitive domains. The program has a team with psychiatrists, geriatrics and neurologists, as well as nurses and psychologists. The demand of patients is spontaneous. Clinical evaluations include medical consultations, neuropsychological testing, laboratory tests and neuroimaging. After establishing diagnosis, patients will see on semi-annual follow-up for continuous evaluation or scheduling if needed.

Conclusions.- We highlight the importance of an attention focused on preventive actions in the preclinical stages regarding possible neurodegenerative diseases, allowing so an early tracing, general guidance and preventive care for this specific population.

Disclosure of interest.- The authors have not supplied a conflict of interest statement.

\section{E-Poster Viewing - 7-9 April: Oncology and Psychiatry}

\section{E-PV0530}

\section{Resilience and depressive symptoms in patients with breast cancer treated with radiotherapy and psychotherapy with music elements}

C. Consol $^{1 *}$, C. Gramaglia ${ }^{1,2}$, L. Girardi ${ }^{1}$, C. Guerriero ${ }^{1}$, M. Martelli ${ }^{1}$, L. Lorenzini $^{1}$, E. Gambaro ${ }^{1}$, L. Deantonio ${ }^{3,4}$, V. Burgio ${ }^{3}$, E. Grossini $^{5}$, M. Krengli ${ }^{3,4}$, P. Zeppegno ${ }^{1,2}$

${ }^{1}$ Università degli Studi del Piemonte Orientale, Department of Translational Medicine, Novara, Italy; ${ }^{2}$ Maggiore della Carità Hospital, SC Psichiatria, Novara, Italy; ${ }^{3}$ Università degli Studi del Piemonte Orientale, Specialist Medical and Oncology Department, Novara, Italy; ${ }^{4}$ Maggiore della Carità Hospital, Radioterapia
Oncologica, Novara, Italy; ${ }^{5}$ Università degli Studi del Piemonte Orientale, Department of Physiology, Novara, Italy

Corresponding author.

Background and aims.- A meta-analysis estimates that $8-24 \%$ of patients treated for cancer may experience depressive symptoms, with a high mortality rate (25\%). Factors implied in the development of depression are not completely explored. Mounting evidence has shown that group music therapy (GMT) may help to prevent anxiety and depression in these patients.

Objectives.- To assess depressive symptoms and resilience in patients affected by breast cancer treated with radiotherapy and to compare these features in patients randomized into two groups: GMT (Psy) and treatment as usual (Tau).

Methods.- Since March 2018, a research and clinical project started, involving the Radiotherapy, Psychiatry, and Physiology Institute of the University Hospital Maggiore della Carità (Novara). Patients' inclusion criteria are: age $\geq 18$ years, Eastern Cooperative Oncology Group 0-1, breast cancer diagnosis, surgically treated and then eligible for radiation therapy. Patients are asked to sign written consent to participation, which has been approved by the local ethical committee. Clinical/anamnestic information is gathered; patients are assessed with the Montgomery-Asberg Depression Rating Scale and the Resilience Scale for Adults, at baseline and after completing radiotherapy. Data collection is ongoing.

Results.- To date, of the 30 patients recruited, 30\% have been randomized to GMT.

Conclusions.- Music therapy is an effective method of supporting cancer care for patients at various stages of the disease. The evidence suggests that GMT may reduce hopelessness, anxiety, depression and increase resilience in cancer patients more than traditional treatment. It therefore seems appropriate to promote techniques of music therapy and integrated cancer care.

Disclosure of interest.- The authors have not supplied a conflict of interest statement.

\section{E-PV0531}

\section{Clinical and genetic factors associated with cognitive function and sleep disorders in palliative care cancer patients: a cross-sectional study}

A. Hajj ${ }^{*}$, R. Khoury ${ }^{1}$, B. ElJebbawi ${ }^{2}$, T. Nehme ${ }^{2}$, A. Awad ${ }^{2}$, F. Nasr $^{3}$, G. Chahine ${ }^{3}$, J. Kattan ${ }^{3}$, L. Khabbaz ${ }^{1}$

1 Saint-Joseph University, Laboratory of Pharmacology-Clinical Pharmacy and Quality Control of Drugs- Faculty of Pharmacy, Beirut, Lebanon; ${ }^{2}$ Saint-Joseph University, Faculty of Pharmacy, Beirut, Lebanon; ${ }^{3}$ Hôtel-Dieu de France Hospital-Saint-Joseph UniversityFaculty of Medicine, Department of Hemato-Oncology-, Beirut, Lebanon

* Corresponding author.

Background and aims.- Cognitive impairment and sleep disorders are persistent symptoms in cancer patients, especially in the end-of-life setting with deleterious consequences such as physical/mental exhaustion with altered mood, concentration, and memory. Our aim was to explore the clinical and genetic factors affecting cognitive/sleep functions in a sample of palliative care cancer patients.

Methods.- A cross-sectional study was carried out between December 2017-June 2018, using a questionnaire and different validated scales to explore the cognitive function (Functionnal Assessment of Cancer Therapy-Cognitive Function; FACT-Cog), insomnia (Insomnia severity index -ISI), anxiety-depression (Hospital Anxiety and Depression Scale-HADS). The ethical committee approved the study and patients gave their written consent (Reference: CEHDF1016). DNA was obtained using a buccal swab $\left(\right.$ FTA $^{\circledR}$ technology) and genotyping for different genes (COMT, OPRM1, CRY2, CLOCK) using the Lightcycler ${ }^{\circledR}$ (Roche). 
Results.- A total of 11 cancer patients were included. Multivariate analysis taking the mean perceived cognitive impairment score as a dependent variable showed that patients with GG genotype for the polymorphism rs10838524; $\mathrm{A}>\mathrm{G}$ in CRY2 encoding the cryptochrome2 had significantly higher cognitive scores than patients with AA genotype (protective effect of the $G$ allele) (Beta $=2.963$; $p=0.031$ ), a result not previously reported in the literature. In addition, patients with higher ISI scores had significantly reduced cognitive function compared to patients with lower ISI scores (Beta $=-2.920 ; p=0.033$ ). Finally, insomnia was significantly and positively correlated to depression (Beta $=0.804 ; p=0.003$ ).

Conclusions.- Even with a small sample size, our preliminary results highlight the need to continue the inclusions and determine all factors associated with cognitive dysfunction and sleep disorders particularly in end-of life cancer patients.

Disclosure of interest.- The authors have not supplied a conflict of interest statement.

\section{E-PV0532}

\section{Competence, autonomy, relatedness and coping strategies in rehabilitation in patients with hematological malignancies}

S. Khrushchev ${ }^{1,2^{*}}$, D. Vybornykh ${ }^{1}$, E. Rasskazova ${ }^{2}$, A. Tkhostov ${ }^{2,3}$, A. Koroleva ${ }^{4}$, V. Zorenko ${ }^{4}$, L. Kuzmina ${ }^{4}$, E. Parovichnikova ${ }^{4}$, V. Savchenko ${ }^{4}$

${ }^{1}$ National Research Center for Hematology, Psychiatry and Neurology Department, Moscow, Russia; ${ }^{2}$ Lomonosov Moscow State University, Psychology Department, Moscow, Russia; ${ }^{3}$ Sechenov First Moscow State Medical University, Psychology Department, Moscow, Russia; ${ }^{4}$ National Research Center for Hematology, National Research Center for Hematology, Moscow, Russia

${ }^{*}$ Corresponding author.

Background and aims.- According to self-determination theory (SDT) L. Daci \& R. Ryan)) psychological needs are universal, innate and include the need for competence, autonomy and relatedness. These needs similarly appear in situation of severe disease and treatment. Patients perceive themselves to be autonomous when they experience with a sense of volition and choice. Internalization of a treatment and rehabilitation decision as one's own, rather than being acted upon by an external influence, which linked with coping strategies effect on patients' quality of life.

Methods.- $N=83$ patients with acute leukemia (ALL and AML) after bone marrow transplantation and hematopoietic stem cell transplantation. Male $=38$, female $=45$. Control groups: $N=31$ (hemophilia A) and $N=20$ (chronic kidney disease (G4-G5) male $=9$, female $=11$. . All groups patients' ECOG:1-2. M.a. $=37.2 \pm 18.1$.SPSS used for statistical analysis (Compare means, ANOVA Scheffe multiple comparison test). Clinical interview and questionnaires were used (illness/treatment and rehabilitation perception, BAI, BDI-II, COPE, CABAH, IPQ-R, compliance and disease locus of control). Results.- Multiple comparisons (Sheffe method) showed significant differences $(p<0.05$; according to COPE questionnaire and SDT questionnaire for rehabilitation) between groups:" Positive reinterpretation and growth" $\mathrm{L} / \mathrm{H}(p=0.046)$, «Religious coping» $\mathrm{L} / \mathrm{CKD}(p=0.002)$ «Behavioral disengagement» L/H $(p=0.005)$, «Substance use» L/CKD ( $p=0.024)$, «Acceptance» L/CKD $(p=0.011)$, «Suppression of competing activities» $\mathrm{L} / \mathrm{H}(p=0.049)$, "competence in rehabilitation" $\mathrm{L} / \mathrm{H}(p=0.011), \mathrm{L} / \mathrm{H}$ "SDTneeds" $(p=0.060)$.

Conclusions.- Treatment of leukemia and chronic kidney disease include a list of numerous obligatory procedures and protocols (for example, hemotherapy or program hemodialysis) which patients can perceive as rehabilitation procedures, however patients with hemophilia are in less dependence on hospital stay treatment (it could explain lower rates on competence in rehabilitation and STD needs). And because of the same reasons patients with leukemia and CKD use "behavioral disengagement" less and "positive reinterpretation" more that patient with hemophilia. (Project_Supported_By_Funding_Of_Russian_Foundation_for_Basic_ Research_18-013-00125_A).

Disclosure of interest.- The authors have not supplied a conflict of interest statement.

\section{E-PV0536 \\ Prevalence of anxiety and depression among breast cancer patients in a general hospital}

C. Romeromartin ${ }^{*}$, P. Padilla Romero ${ }^{2}$, T. López-Arteaga ${ }^{2}$, M.T. Perez Castellano ${ }^{2}$

${ }^{1}$ Hospital Nuestra Señora Del Prado, Hospital Pharmacy, Talavera de la Reina, Spain; ${ }^{2}$ Hospital Nuestra Señora Del Prado, Psychiatry,

Talavera de la Reina, Spain

* Corresponding author.

Background and aims.- Breast cancer is the most frequently diagnosed cancer in women worldwide. The prevalence of psychological distress among cancer patients is high, and they are at higher risk of developing severe anxiety, depression and other mood disorders. An adequate management of anxiety and depression is fundamental to ensure the quality of life of these patients Objectives.- The present study was aimed to identify the prevalence of anxiety and depressive disorders in breast cancer patients and to assess the use of psychiatric drugs among these patients Methods.- Restropective case-series descriptive study. We included patients diagnosed with breast cancer and undergoing active oral and intravenous chemotherapeutic treatment in september 2018. We select patients using a computer-based medication prescription system for dispensing oral and intravenous chemotherapy treatment (Farmatools ${ }^{\circledR}$ ). We obtained data of psychiatric drugs from the electronic prescription.

Results.- We included 65 women with active chemotherapeutic treatment, median age 58 [31-85]. The prevalence of use of anxiolytics/hypnotics was $50,7 \%(n=33)$, and that of antidepressants was $29,2 \%(n=19)$. Only $9.2 \%(n=6)$ of the patients had follow-up in psychiatry.

Conclusions.- We identified a high number of patients with anxiety and depression disorders and a wide use of anxiolytics. The low follow-up by psychiatry of these patients stands out being the majority of prescriptions made by Primary Care. More psychiatric care should be given to breast cancer patients as they are at high risk of anxiety and depression. We concluded that there should be a greater follow-up of oncological patients by psychiatry in our area. Disclosure of interest.- The authors have not supplied a conflict of interest statement.

\section{E-PV0537 \\ Differential diagnosis in depression- always a challenge \\ B.D. Suciu ${ }^{*}$, C. Crisan ${ }^{2}$, I. Miclutia ${ }^{2}$ \\ ${ }^{1}$ Children's Emergency Hospital, Psychiatry Clinic I, Cluj Napoca, Romania; ${ }^{2}$ Iuliu Hatieganu University of Medicine and Pharmacy, Department of Neurosciences, Cluj-Napoca, Romania Corresponding author.}

Background and aims.- Numerous patients are treated for a psychiatric illness before being diagnosed with an organic disorder. Objectives.- The presentation of a clinical case, that describes an unusual clinical picture for an adrenal gland tumor.

Methods.- A 37-year-old male, diagnosed with an organic mood disorder (according to ICD-10 and DSM-V) and myocardial infarction in the previous year presented with depressive symptoms for admission in a psychiatric hospital. Over the course of the illness, 
the clinical picture included Cushing's syndrome and a newly diagnosed type II diabetes for which an abdominal CT with contrast was done, showing a tumor in the left adrenal gland with possible secondary metastases in the liver and lungs and also an incomplete inferior vena cava thrombosis.

Results.- Considering the clinical picture the patient was transferred to an oncology hospital, where he died. The psychiatric treatment that was administrated consisted of antidepressants and benzodiazepines. As a particular aspect, the mood symptoms were the predominant complaints, appearing first without any pain, weight loss or other typical symptoms that are associated with cancer.

Conclusions.- In the future, clinical research should improve the understanding of interactions between affective disorders and endocrine diseases.

Disclosure of interest.- The authors have not supplied a conflict of interest statement.

\section{E-PV0539}

\section{Psychoeducational videos about \\ illness and treatment of head and neck cancer proposed from reported experiences by Brazilian patients}

A.R. Malta ${ }^{1}$, A. Weber $^{1 *}$, C.S.P. Lima ${ }^{2}$, E.R. Turato ${ }^{1}$

${ }^{1}$ University of Campinas, Laboratory of Clinical-Qualitative Research - LPCQ Campinas, Brazil; ${ }^{2}$ University of Campinas, Department of Medical Clinic, Campinas, Brazil

* Corresponding author.

Background and aims.- Patients with head and neck cancer (HNC) have little to say objectively about their experience with illness and treatment. The society has a social and scientific demand to "hear and recognize the voice" of these patients. In this sense, life experiences of patients with HNC during treatment can be pedagogically valued for a collaborative construction of knowledge, democratization of access to quality information, exchange of experiences, strengthening of shared clinical-therapeutic decisions and improvement of care practices.

To produce psychoeducational videos on topics related to illness and treatment of $\mathrm{HNC}$ from the experiences reported by patients followed up at the General Hospital at the University of Campinas, South-eastern Brazil.

Methods.- Semi-directed interviews with open-ended questions, in-depth, was recorded in audio, fully transcribed and treated by Content Qualititave Analysis. Thirteen patients have participated. The proposal is to produce psychoeducational videos containing: patient reports, simulations and scenes representing the treatment, information bases with relevant research evidence and adapted to an accessible language.

Results.- Expected to qualify the teaching of communication skills for professionals and students in the health area; strengthen support actions and integral care to the patient with HNC; stimulating patient self-management for the search and access to information in reliable sources; create a bank priority themes for future research; to stimulate patients and their families to jointly validate scientific evidence.

Conclusions.- We expect psychoeducational videos can bring comprehensive and individualized care at the oncology clinic for HNC, articulated to patients' needs and preferences, their social network, and shared decision making.

Disclosure of interest.- The authors have not supplied a conflict of interest statement.

\section{E-PV0540}

\section{The validity and reliability of turkish version of the experience of cognitive} intrusion of pain scale

N. Mercan ${ }^{1}$, D. Ayhan ${ }^{2}$, R. Dogan ${ }^{3}$, C. Yuksel ${ }^{4 *} ;{ }^{1}$ Health Sciences University- Gulhane Training and Research Hospital, Department of Mental Health and Diseases, Ankara, Turkey; ${ }^{2}$ Bandırma Onyedi Eylul University- Faculty of Health Sciences-, Department of Nursing, Balıkesir, Turkey; ${ }^{3}$ University of Health SciencesGulhane Faculty of Nursing, Psychiatric and Mental Health Nursing Department, Ankara, Turkey; ${ }^{4}$ University of Health Sciences, Psychiatric and Mental Health Nursing Department, Ankara, Turkey

* Corresponding author.

Background and aims.- Although cognitive intrusion is a critical characteristic of pain, less is known about it, and no specific measure designed to capture its effects. The aim of the study was to test the validity and reliability of Turkish version of the Experience of Cognitive Intrusion of Pain Scale (ECIPS) developed by Attridge et al. in 2015 (1).

Methods.- This methodological study conducted in the hematology clinic of an Educational Research Hospital between February 2018 and June 2018. The data were collected from 120 patients. with the Sociodemographic Characteristics Questionnaire, Pain Catastrophizing Scale (PCS) and ECIPS.

Results.- As all the differences between the mean scores obtained from the $27 \%$ upper and lower groups were significant and the itemtotal correlation coefficients of all the items ranged between 0.79 and 0.89 , no item was removed from the scale. The Cronbach's alpha coefficient of the scale was 0.96 , its split-half reliability coefficient and test-retest reliability coefficient were 0.94 and 1 respectively. The correlation coefficient used to determine the criterion validity was $0.835, p<0.001$. For the construct validity of the scale, the factor analysis was performed and the suitability of the data for the factor analysis was evaluated by the Kaiser-Meyer-Olkin (KMO) coefficient $(0.926)$ and the Bartlett's test of sphericity $(p<0.001)$. Factor loadings ranged from 0.82 to 0.91 and the scale had a one-factor structure.

Conclusions.- The analyses demonstrated that the Turkish version of the ECIPS was a valid and reliable tool and could be used to assess the level of cognitive intrusion of pain.

Disclosure of interest.- The authors have not supplied a conflict of interest statement.

\section{E-Poster Viewing - 7-9 April: Others}

\section{E-PV0541}

\section{Reducing anxiety, mood temper and specific language deficit in ADD/ autism through a multi therapeutic methodologies. Two study cases}

T. Ahmad ${ }^{1}$

Cosmomid Audiology \& ENT Clinic, Psychology \& Speech-Language

Pathology, Salmyia, Kuwait

* Corresponding author.

Background and aims.-

Introduction and Objectives.- As we know Children with ADD/Autism likely to have some behavioral features and as well language problem as remembering daily events, recalling previous occasions and with poor verbal organization to talk about where, when, why it happened. In addition, they cannot express their feelings especially autistic ones. 
Objectives.- We tried through this study to divide the therapeutic program between therapists and parent to solve the problem of anxiety, mood temper and expressive language defects using multi therapeutic methodologies to reduce anxiety, mood temper and expressive language deficit and to solve these problems.

Methods.- The study hired (2) different cases sex with ADD/Autism aged 5:6 Yrs. In Kindergarten bilingual Arabic/English American School. We focus in the multi therapeutic approach which basedevidence assessment and treatment process on seven intervention strategies both cases having difficulties to reduces anxiety, mood temper, focus, processing speed, modulating emotions, organizing ideas and recall events. Using variations of indoor and outdoor activities thru technique (picture your life) as auxiliary tool.

Results.- The outcomes of the multi therapeutic methodologies showed significant improvement in the both ADD/Autism children that involved in the program; both family members and schoolteachers notice sensible change in the child behavior and language use.

Conclusions.- The important finding from this research that collaboration between therapist \&parent by using variety of intensive traditional and untraditional multi therapeuticmethodologies provided during the psycho-speech -language sessions helped the children toovercome their problems and improve concentration, processing speed and recall daily events.

Disclosure of interest.- The authors have not supplied a conflict of interest statement.

\section{E-PV0543}

\section{Relation between self-efficacy and diabetes related distress among diabetic patient in Riyadh}

M. Almutairi ${ }^{*}$, A. Fayed ${ }^{1}$, A. Alobedollah ${ }^{2}, \mathrm{H}$. Alsomali ${ }^{1}, \mathrm{H}$. Alarfaj $^{1}$, R. Alqurashi ${ }^{1}$, S. Alotaibi ${ }^{1}$

${ }^{1}$ Princess Nora bint Abdul Rahman University, Collage of Medicine, Riyadh, Saudi Arabia; ${ }^{2}$ King Fahad Medical City, Obesity Endocrine and Metabolism Center, Riyadh, Saudi Arabia

${ }^{*}$ Corresponding author.

\section{Background and aims.-}

Introduction.- Effective control of diabetes is not based merely on simple adherence to treatment, but it needs dynamic behavior from diabetics and high level of self-efficacy almost on a daily basis.

Objective.- To estimate the level of self-efficacy among diabetics and its relation to diabetes distress.

Methods.- A cross sectional study of 100 diabetics was conducted. A validated questionnaire was used to collect data inquiring about diabetes related distress (DRD), self-efficacy, and other data related to sociodemographic and diabetes history.

Results.- Most of the studied sample aged between 31 and 60years and $62.5 \%$ were females. The level of self-efficacy was modest, the average score was 60.2 and SD was $14.9 \%$. Better self-efficacy was reported by younger age, patients with less complications and type1 diabetics. No significant association was detected between self-efficacy and economic level or marital status. Patients who reported healthy self-care behaviours as checking their blood glucose or following healthy diet had higher level of self-efficacy. DRD was prevalent among the studied sample especially regimenrelated distress (64\%). Self-efficacy was inversely related to DRD $(r=-0.4, p<0.05)$ and positively correlated to the level of adherence to treatment $(r=0.3, p<0.05)$. The Most commonly reported barriers to follow proper self-care were the patients' dissatisfaction about health services, and distrust in physicians.

Conclusions.- The level of self-efficacy among our sample was modest and ispositively related to diabetes related distress. Adopting self-care behaviors among diabetics is related to the level of selfefficacy and is affected by patients' satisfaction and trust in health care providers.
Disclosure of interest.- The authors have not supplied a conflict of interest statement.

\section{E-PV0545 \\ Higher education stress among medical studies - similarities and differences in domestic and international students. Personal reflection of developing and carrying out scientific research}

D. Budzyna-Dawidowski ${ }^{*}$, K. Hennessy

Uniwersytet Medyczny im Piastow Slaskich we Wroclawiu, Faculty of Medicine- English Division, Wroclaw, Poland

* Corresponding author.

Background and aims.- It is widely recognised that medical education is highly demanding and brings up significant stress. Students studying in their home country have demands related to the learning process. Students who are at foreign universities have the added stress of acclimatisation to a new culture, different living circumstances and differences in the approach to the curriculum. The current study analyses the experience of students at the Wroclaw Medical University.

Methods.- Using the Higher Education Stress Inventory (HESI) the current study aimed to compare foreign students with domestic students.

Results.- On the basis of the questionnaire factors (Worries about Future Endurance/Capacity, Non-supportive Climate, Faculty Shortcomings, Workload, Insufficient Feedback, Low Commitment, Financial Concerns) and own experience, the authors provide a narrative of a journey through years of medical studies.

Conclusions.- In conclusion the authors will analyse the results and reflect on their perosnal journey through medical studies as foreign students and comment on their experience of setting up and carrying out scientific research.

Disclosure of interest.- The authors have not supplied a conflict of interest statement.

\section{E-PV0546}

\section{Are affective disorders linked to noise pollution? a systematic review}

D.V. Buhai*, S.D. Bolboacă

Faculty of Medicine- "Iuliu Hațieganu" University of Medicine and

Pharmacy, Medical Informatics and Biostatistics, Cluj-Napoca,

Romania

* Corresponding author.

Background and aims.- Noise pollution is one of the components of urbanization suggested to influence affective disorders potentially through the chronic activation of the hypothalamic-pituitaryadrenal axis. The primary objective was to establish if a link exists between the development or worsening of affective disorders and the noise pollution. Secondarily, possible methods to evaluate this link were identified.

Methods.- A search in the PubMed and Cochrane databases was conducted in September 2018 with the following syntax: ("noise pollution" or "traffic noise" or "aircraft noise" or "railway traffic" or "road traffic noise" or "sound pollution" or "environmental noise" or "noises" or "noise annoyance") and ("depression" or "depressive disorder" or "mood disorder" or "mood disorders" or "affective disorder" or "bipolar disorder" or "anxiety disorder"). Only full-articles from the last ten years were included. Exclusion criteria were: other diseases (for example fibromyalgia), acoustic hallucinations, other species (mice), physiology of excitable systems, air pollution, eval- 
uation of psychotropic medication or sleep quality in relation to noise, psychotherapy, artificial intelligence in medicine.

Results.- A total of 57 articles were identified, out of which 13 were selected. An extra article found independently was added. All studies examined depression and/or anxiety symptoms. In ten studies the symptoms were collected through questionnaires, only two implied a clinical diagnosis and two other studies referred to suicide. Four studies lacked an objective method of noise measurement and assessed noise annoyance or sensitivity to noise. Nine studies had a cross-sectional design.

Conclusions.- Noise pollution is correlated with the existence of affective disorders.

Disclosure of interest.- The authors have not supplied a conflict of interest statement.

\section{E-PV0548}

\section{Perception of coercive practice among medical staff}

F. Cherif*, J. Ben Thabet, M. Turki, M. Maalej, S. Omri, R. Feki, N.

Zouari, L. Zouari, N. Charfi, M. Maalej

Hédi Chaker University Hospital, Psychiatry C, Sfax, Tunisia

"Corresponding author.

Background and aims.- Use of coercive practice in mental health care has to balance between different ethical issues, such as respect of the autonomy of patient, beneficence, non-maleficence and justice.

The aim: to identify if there is a disparity between perceptions among psychiatric medical staff and other department that could have implications for the future care of service users and stigmatization.

Methods.- This transversal study enrolled medical staff in Hedi Chaker Hospital in Sfax. Demographic information and the perception of restraint were assessed by self-reported questionnaire. We used an adapted version of the MacArthur admission experience (AES) interview to measure the caregiver's perspective of the service users' admission and calculate three sub-scores (perceived coercion, negative pressures and voice).

Results.- Thirty-four subjects participated in this study. The sex ratio was 0,62 . The mean age was 35,62 years old. Thirteen $(38,23 \%)$ were working in a psychiatric department. Almost two third of the sample $(63,6 \%)$ had participated in experiments of restraint. For $82,4 \%$, the restraint was an instrument of prevention. The majority $(72,7 \%)$ felt that there was a change in the caregiver-patient relationship after the episode of restraint. For $70,8 \%$ it changed in a negative way. The mean score of the AES was 5,32 $( \pm 2,4)$. The median pressure scale score was significantly higher among medical staff in psychiatric department $(0,68)$ compared to other department $(1,83)(p=0,41)$.

Conclusions.- The recognition of these pre-existing thought about the use of coercive methods and dealing with it may be a way to decrease stigma and improve the quality of care of mental patient. Disclosure of interest.- The authors have not supplied a conflict of interest statement.

\section{E-PV0550}

\section{Follow up on the connections \\ between neuropsychiatric and dental \\ disorders: perspectives, possible \\ relevance of oxidative stress, pain, music or irritable bowel syndrome and possible approaches}

A.S. Ciobica ${ }^{*}$, P. Manuela ${ }^{2}$, A. Iulia ${ }^{3}$, C. Roxana ${ }^{4}$, S. Cristinel $^{4}$, T. Mihoko $^{5}$

${ }^{1}$ Alexandru Ioan Cuza University Iasi, Research, Iasi, Romania; ${ }^{2}$ UMF Gr. T. Popa Iasi, Psychiatry, Iasi, Romania; ${ }^{3}$ UAIC, Biology, Iasi,
Romania; ${ }^{4}$ UMF Iasi, Psychiatry, Iasi, Romania; ${ }^{5}$ Matsumoto Dental University, Social Dentistry, Shiojiri, Japan

Corresponding author.

Background and aims.- Although the connections between neuropsychiatric and dental disorders attracted the attention of some research groups for more than 50 years now, there is a general opinion in the literature that it remains a clearly understudied and underrated topic, with many unknowns and a multitude of challenges for the specialists working in both these areas of research Methods.- We selected 21 studies on this subject from the main research databases (Medline, Scopus, Elsevier, Wiley, Springer) by using keywords such: "neuropsychiatric and dental", "neuropsychiatric and stomatological" until 21 September 2018.

Results.- In this way, considering the previous experience of our groups in these individual matters which are hereby combined, we present/summarize in this mini-report the current status of knowledge on the connections between neuropsychiatric and dental manifestations, as well as some general ideas on how oxidative stress, pain, music therapy or even irritable bowel syndromerelated manifestations could be relevant in this current context and summarize some current approaches in this matter.

Conclusions.- Although it was clearly described before that most of the neuro-psychiatric patients could have an increased and varied stomatological risk, there is still a poor understanding for the connectivity between these two areas of research, as well as between the specialists working in these two fields. There is definitely also a need for an individualized specific approach for these patients, with complex preventive and rehabilitation programs in this direction. Disclosure of interest.- The authors have not supplied a conflict of interest statement.

\section{E-PV0551}

\section{The role of the psychiatrist in the multidisciplinary approach of chronic neurological disease}

E.I. Davidescu*, L. Iftene

University of Medicine and Pharmacy Carol Davila, Clinical

Neurosciences, Bucharest, Romania

Corresponding author.

Background and aims.- Multiple sclerosis (MS) is a chronic demyelinating and degenerative neurological disease affecting young people (20-40 years old) with huge direct and indirect costs, being considered a public health problem.

Assessing fatigability, mood disorders (anxiety, depression) and sleepiness in patients with MS treated with disease modifying therapies (DMT)

Methods.- A lot of 45 patients with MS (76\% women and 24\% men), mean age $=40(+/-2)$ years old, mean Expanded Disability Status Scale score of 3.5, mean time of evolution of the disease $=10$ years, treated with different DMT (mean time of treatment $=7.5$ years) were assessed with questionnaires: Fatigue Severity Scale, Beck Depression Index, Hamilton Anxiety Scale, Epworth Sleepiness Scale. Almost 54\% of the patients were retired.

Results.- Fatigability (35.71\% in our lot) is a strong predictor of impairment of quality of life, especially among active patients. Mood disorders are a natural phenomenon of chronic disease: $52.38 \%$ of our patients experienced depression and $71.43 \%$ anxiety, in lightest to moderate forms. Sleep disturbances should not be minimized as they are a useful predictor for patient's quality of life: $52.38 \%$ had daytime sleepiness.

Conclusions.- Symptomatic treatments (for depression, anxiety, sleep disorders) as well as behavioral cognitive counseling are as important as the establishment and maintenance of pathogenic treatment with DMT, especially for reasons related to the prolongation of professional activity in young patients. Efficient, multidisciplinary management of mood swings, sleep and fatigue 
is a good predictor of adherence to treatment, influencing patient's quality of life, so psychiatrists can play an important role in these patients' risk assessment.

Disclosure of interest.- The authors have not supplied a conflict of interest statement.

\section{E-PV0555 \\ Relationship of difficulties in emotion regulation with attention deficit/hyperactivity disorder among young adults}

C. Evren ${ }^{1 *}$, B. Evren ${ }^{2}$, E. Dalbudak ${ }^{3}$, M. Topcu ${ }^{4}$, N. Kutlu ${ }^{5}$

${ }^{1}$ Bakirkoy Training and Research Hospital for Psychiatry Neurology

and Neurosurgery, Research-Treatment and Training Center for

Alcohol and Substance Dependence AMATEM, Istanbul, Turkey; ${ }^{2}$

Baltalimani State Hospital for Muskuloskeletal Disorders,

Department of Psychiatry, Istanbul, Turkey; ${ }^{3}$ Private practice, Private practice, Ankara, Turkey; ${ }^{4}$ Cankaya University- Ankara- Turkey, Department of Psychology, Ankara, Turkey; ${ }^{5}$ Baltalimani State Hospital for Muskuloskeletal Disorders, Department of Psychology, Istanbul, Turkey

* Corresponding author.

Background and aims.- The aim of the present study was to evaluate relationship of difficulties in emotion regulation with attention deficit/hyperactivity disorder (ADHD) among young adults.

Methods.- The study was conducted with online survey among 1010 volunteered university students in Ankara, people who play games on the Internet and who are in the e-mail database of a company located in Istanbul that organizes e-sports tournaments and Turkish gamers from gaming forums. Participants were evaluated by applying the Difficulties in Emotion Regulation Scale (DERS) and the Adult ADHD Self-Report Scale (ASRS).

Results.- Age and gender did not differ between those with probable ADHD ( $n=190,18.8 \%)$ and those without probable ADHD $(n=820,81.2 \%)$. DERS scores were higher among those with probable ADHD. Among dimensions of DERS, goals (Difficulty engaging in goal-directed behavior) and clarity (Lack of emotional clarity) predicted the presence of probable ADHD in logistic regression analysis, whereas goals, impulse (Impulse control difficulties) and clarity predicted the severity of ADHD symptoms in linear regression analysis. Finally, in MANCOVA, goals and clarity predicted inattentiveness dimension of ADHD, whereas goals and impulse predicted hyperactivity/impulsivity dimension of ADHD.

Conclusions.- These findings suggest that the severity of ADHD symptoms related with the severity of difficulties in emotion regulation, particularly with goals, clarity and impulse dimensions, among young adults.

Disclosure of interest.- The authors have not supplied a conflict of interest statement.

\section{E-PV0556}

\section{Anxiety radical: nonverbal behavior features in the deception situation}

\section{T. Folomeeva, S. Fedotova}

Lomonosov Moscow State University, Faculty of psychology, Moscow, Russia

${ }^{*}$ Corresponding author.

Background and aims.- The accentuation anxiety radical as a personal characteristic determines nonverbal behavior. The situation (for example deception) can also lead to an anxiety increase. The aim of this study is to identify the nonverbal behavior features among people with an accentuated anxiety radical in a deception situation, as well as to identify expressive behavior elements for further quantitative analysis.
Methods.- For respondents selection V. Norakidze and C. Spielberger tests were applied ( 8 people with an anxiety radical accentuation, 7 respondents without accentuation (age from 16 to 26; 7 - male, 8 - female)). Two videos were recorded with each respondents who told the truth and a lie. The data was collected through expert interviews, and then was analyzed with qualitative content analysis with data triangulation. Estimates for five expressive systems (facial expressions, gaze, gestures, postures, tone) were taken into account in the pre-developed categorical grid.

Results.- Many differences between anxious and non-anxious persons were found in the autonomic nervous system manifestations (redness, tremor, sweating). The preliminary portrait of an anxiety person's expressive behavior in the deception situation is immobile facial expressions, long eye contact, few gestures, expressionless speech, etc.

Conclusions.- The anxiety radical prevents different patterns' usage of nonverbal behavior, including tones' variety; in a deception situation strong anxiety does not allow behavior control. It is expressed in the "extra" manifestations controlling ability (anxiety and fear obvious signs) but such persons have no possibility to natural behavior in this situation. The quantitative analysis of the anxious and non-anxious persons' non-verbal behavior differences is planning to conduct.

Disclosure of interest.- The authors have not supplied a conflict of interest statement.

\section{E-PV0557}

\section{Food addiction: obesity as an} impulsive-compulsive disorder

\section{Ferreira ${ }^{1 *}$, L.P. Ferreira ${ }^{2}$}

${ }^{1}$ Hospital de Santa Maria, Serviço de Psiquiatria e Saúde Mental, Lisboa, Portugal; ${ }^{2}$ Hospital Distrital de Santarém, Serviço de Psiquiatria, Santarém, Portugal

* Corresponding author.

Background and aims.- Obesity and its health and economical consequences have been a major public health concern over the last decades in developed economies and have become an emerging problem in the rest of the world. Our aim was to discuss the whether food can be addictive and it's construct as an impulsive-compulsive disorder.

Methods.- Literature research conducted using "PubMed" database andsearch equation built using the MeSH key-words: "food addiction", "behavioral addictions", "impulsive compulsive disorder", "obesity" and selected the significant articles published on the topic.

Results.- An increasing number of studies demonstrated that drug addiction and food addiction shares some similar clinical, neurobiological and psychopathological and sociocultural risk factors. Recent evidence examines changes in the mesolimbic dopaminergic circuit associated with exposure to highly palatable foods and obesity. It is only those forms of obesity apparently driven by excessive motivational drive for food and mediated by reward circuitry that might be considered impulsive compulsive disorders. It's also known that appetite/motivation for eating and the actual amount of food consumed can both be influenced by centrally psychopharmacological agents. The majority of obesity studies in animals have not measured addictive-like behaviors, but reports of such behaviors have been restricted to experiments using models of binge eating. Conclusions.- No clear consensus has yet emerged on the validity of the concept of food addiction and whether the neurobiological findings can corroborate it as a clinical entity, but the importance and impact that it's possible treatment would have in public health system makes it a worthy subject to keep on investigating. Disclosure of interest.- The authors have not supplied a conflict of interest statement. 
E-PV0558

\section{Anti-NMDAR autoimmune encephalitis induced by ovarian teratoma case report}

S. Gamonal Limcaoco*, N.M. Casado Espada, M.T. Lozano López, R. De la Mata Hidalgo, J. De la Iglesia Larrad, R. De Alarcon Gómez, I. Valriberas Herrero, P. Andres Olivera, J. Pérez Rodriguez, A. Maciá, A. Bullon Saez

University of Salamanca Health Care Complex, Psychiatry, Salamanca, Spain

${ }^{*}$ Corresponding author.

Background and aims.- Anti-NMDAR autoimmune encephalitis is an autoimmune disorder characterised by neuropsychiatric symptoms (anxiety, illusions, hallucinations, behavioural disorders, delirium and paranoia) preceded by nonspecific organic prodrome. We describe the case of a 31-year-old woman who was seen repeatedly by the psychiatric psychosomatic unit due to the neuropsychiatric symptoms she presented.

Methods.- The data from the patient's clinical history was collected together with a bibliographic review in this pathology in the main search engines: PUBMED and COCHRANE.

Results.- The diagnosis was reached after several cerebrospinal fluid examinations, search for infectious diseases, brain magnetic resonance imaging, computed tomography scans, repeated electroencephalograms and after ruling out other autoimmune encephalitis. During the follow-up by the Psychiatric Service, she was reviewed and treated for the anxious symptoms she presented as a result of her cognitive deficits, such as the difficulty to execute the language, inability to receive and remember new information, the hassle for her limb mobility and her vivid nightmares.

Conclusions.- The prompt diagnosis and treatment of anti-NMDAR encephalitis is a clinical challenge due to its unspecific manifestations, which include neuropsychiatric symptoms. We describe the details of a patient with this condition who was seen several times by many physicians who misdiagnosed and mistreated these symptoms at first. It is important to make a follow-up after due to the mood disorders this patients can have after they are diagnosed. Disclosure of interest.- The authors have not supplied a conflict of interest statement.

\section{E-PV0560}

\section{Myxedematous madness}

M.J. Gordillo Montaño*, I. Moreno Encabo, J. Iglesias Lopez, S.V. Boned Torres, L. Torres Guasch

Hospital Can Misses, Psychiatry, Eivissa, Spain

Corresponding author.

Background and aims.- Hypothyroidism is the most frequent hormonal alteration in daily medical practice. In addition to classical clinical signs and symptoms, it may show symptoms secondary to hormonal alteration, such as alterations in memory, attention, psychomotor retardation, visual-perceptual alterations or executive alterations. The association of neuropsychiatric manifestations with hypothyroidism is widely described, in the literature we find numerous cases of hypothyroidism that debut as psychosis.

Methods.- Case Report and literatura review.

Results.- A 35-year-old patient who came to the emergency room with confusion and hallucinations. This symptomatology begins two weeks before and will progressively worsen, eventually affecting psychomotricity. As a personal history, the endocrinological history stands out in an important way. We found a history of gestational DM, diabetic ketoacidosis 4 years later, which required admission to the ICU. There was no previous history in Mental Health. Days before admission, when the condition begins, antipsychotic treatment is instituted, which must subsequently be suspended due to excessive sleepiness, disorientation, bradypsychia and dysarthria. Control analysis was performed, finding TSH
(184,955), T4 (>0.4), HbA1c (10.5\%), CLT 460, LDL 358 and PRL11,44 altered. In coordination with Endocrinology, $200 \mathrm{mcg}$ of Levothyroxine are administered. From the day after the start of this treatment, progressive improvement of the level of consciousness to complete normalization at the cognitive level, being able to describe asthenia, somnolence and swelling, and completely disappearing hallucinatory andreferential clinic without the need of neuroleptic treatment.

Conclusions.- Before the atypical presentation of a psychotic picture we will have to keep in mind the neuropsychiatric manifestations that endocrine-metabolic alterations can generate.

Disclosure of interest.- The authors have not supplied a conflict of interest statement.

\section{E-PV0561}

\section{Sensorimotor correction as a method of development of self-regulation in children with dysarthria}

E. Sedova, T. Goryacheva*

Pirogov Russian National Research Medical University, Psychological-Social Faculty, Moscow, Russia

Corresponding author.

Background and aims.- The speech as a higher mental function has its specific role as it mediates other mental processes and integrates them into a single system. That is why a delay in the speech development leads to major developmental problems and along with speech difficulties a child with dysarthria demonstrates a lack of voluntary regulation and concentration as well as other behavioural problems.

The research aim was to increase the level of self-regulation of children with dysarthria.

Methods.- The sample consisted of 25 children of five years old, underwent hypoxic-ischemic encephalopathy at an early age (up to 1 year), with delayed speech development. The diagnostics included neuropsychological battery of tests in Glozman's adaptation. The intervention course consisted of 15 sessions of sensorimotor correction. Each session included breathing and oculomotor exercises, various kinds of crawling aimed at improving coordination and reciprocal coordination and some other physical exercises.

Results.- According to the results of the intervention program $89 \%$ of children showed improvement in the self-regulation level. There was no pronounced dynamics in $11 \%$ of the cases; we see the reason of it in a relatively short duration of the course.

Conclusions.- The positive effects of the course include normalization of muscle tone which also concerns vocal organs as well as improving concentration. Both factors provide the direct impact on the speech development. In addition, the frequency of manifestations of protest behavior decreased, the emotional background became smoother and more stable.

Disclosure of interest.- The authors have not supplied a conflict of interest statement.

\section{E-PV0562}

\section{Comparison of direct and indirect language learning strategies among English language students and non-English language students}

S. Shahriary, A. Homayouni*, M. Hoseiny, S. Sistani Department of Psychology, Bandargaz Branch, Islamic Azad University, Bandargaz, Iran

Corresponding author. 
Background and aims.- The aim of this study was to compare the direct and indirect language learning strategies among English language students and non-English language students.

Methods.- The research method was casual-comparative. In this research, 100 English language students and 100 non-English language students were selected from two universities and responded to the Oxford language learning strategies questionnaire. This questionnaire consists of six learning strategies and designed for using in non-English language environments. These strategies included three direct strategies (memorial, cognitive, compensatory), and an indirect strategies (emotional, metacognitive and social). The data were analyzed with independent $\mathrm{T}$ formula.

Results.- The results showed significant differences between two groups. English language students got more scores in direct and indirect language learning strategies than non-English language students, and the differences were statistically significant. These differences in cognitive and metacognitive strategies were much more especially rather than other language learning strategies. Conclusions.- According to the findings of the study, English language students in all of language learning strategies, especially cognitive and metacognitive strategies got more scores than nonEnglish students that it is caused their motivation and rate of English learning becomes higher than non-English students. Disclosure of interest.- The authors have not supplied a conflict of interest statement.

\section{E-PV0563}

\section{Clinical approaches to hyperprolactinemia secondary to atypical antipsychotics}

\section{P. Horta*, A.T. Pereira, J.D. Rodrigues, M. Araújo}

Centro Hospitalar Vila Nova de Gaia/Espinho EPE, Psiquiatria, Vila

Nova de Gaia, Portugal

* Corresponding author.

Background and aims.- Hyperprolactinemia is a highly prevalent adverse effect of antipsychotic agents, with potentially serious health consequences such as sexual dysfunction, menstrual irregularities, amenorrhea, galactorrhea, gynecomastia, infertility, osteoporosis, and breast cancer. The aim of this paper is to review and discuss current clinical approaches and existing evidence for managing antipsychotic-induced hyperprolactinemia.

Methods.- A PubMed/MEDLINE search was conducted on the topic of hyperprolactinemia caused by atypical antipsychotics and therapeutic options.

Results.- Some guidelines recommend against treating asymptomatic, antipsychotic-induced hyperprolactinemia and others recommend clear cut-off points of prolactin to determine whether to treat. The most recommended approach is to discontinue the prolactin raising agent and switch to a different antipsychotic as a first-line approach for hyperprolactinemia. Moreover, in a recent meta-analysis, the addition of aripiprazole at a dose of only $5 \mathrm{mg} /$ day was associated with a $79 \%$ normalization rate of prolactin levels. Adjunctive metformin was also associated with a significant decrease in serum prolactin levels across studies and was observed to be well-tolerated by patients.

Conclusions.- In this review we identified that, while some concordance between guidelines is evident, there remains a lack of consensus regarding management of hyperprolactinemia in adults who take antipsychotic medication. Further work is needed to clarify treatment for antipsychotic-induced hyperprolactinemia. Disclosure of interest.- The authors have not supplied a conflict of interest statement.

\section{E-PV0565 \\ Prevention of drug use on the Brazilian border}

J.A. Jaber Filho*, J. Verissimo Jr Filho, A. Hollanda Filho, P.C. Geraldes Filho

Clínica Jorge Jaber, Saúde Mental, Rio de Janeiro, Brazil

* Corresponding author.

Background and aims.- Introduction: Brazil is a country of continental dimensions and its air borders constitute important sites of entry of drugs. In this sense, the Brazilian Air Force FAB, created the project called Agate 5, which aims to curb the entry and movement of drugs in Brazil through the control and surveillance of the airspace at their borders. However, Agate 5, besides the work of repression of drug trafficking, prevention work included the use of drugs by inviting professionals from chemical dependency to act jointly in this operation, which was implemented in the cities of Porto Alegre, and Chui Foz do Iguaçu.

Methods.-

Methodology.- Lectures and dynamic guidance on issues related to addiction, treatment and prevention and healthy lifestyle habits. Results.- The work of guidance on actions to be taken towards preventing drug use was presented by group of professionals next to a group of managers in the city of Chui, southern Brazil. Likewise, lectures were developed with educators responsible for the, which is an institution of care to children in social risk in the city of Porto Alegre. In this same place, it was possible to interact with the children attending the institution and develop techniques play in structured activities aimed at promoting health.

Conclusions.- The actions of the group of professionals with the operation Agate 5 achieved the proposed objectives to promote scientific guidelines about addiction, and treatment of drug addicts, as well as providing a technical assistance for issues related to addiction.

Disclosure of interest.- The authors have not supplied a conflict of interest statement.

\section{E-PV0570 \\ Shared decision making in surgical versus non-surgical disorders: preliminary findings}

S. Nikolaou ${ }^{1}$, E. Theochari ${ }^{2}$, F. Louki $^{3}$, A. Giannoulis ${ }^{3}$, E. Tsaltas ${ }^{4}$, D Kontis $^{3^{*}}$

${ }^{1}$ Thriassion General Hospital, Internal Medicine Department, Elefsina, Greece; ${ }^{2}$ Asklipieion Voulas General Hospital, Psychiatric Department, Voula, Greece; ${ }^{3}$ Psychiatric Hospital of Attica, Cognitive Rehabilitation Unit- 4th Psychiatric Department, Athens, Greece; ${ }^{4}$ Athens University Medical School, Experimental Psychology Laboratory, 1st Department of Psychiatry, Athens, Greece * Corresponding author.

Background and aims.- During a pilot cross-sectional study, the 9Item Shared Decision Making Questionnaire (9SDMQ), which measures the participation of patients in medical decisions, and the 8-Item Client Satisfaction Questionnaire (8CSQ), which measures satisfaction from services, is being administered in two groups of patients, one $(N=60)$ suffering from surgical diseases and one $(N=60)$ from non-surgical diseases. We present preliminary findings based on the recruitment of the first 35 patients.

Methods.- The primary outcome is the difference on the 9SDMQ scores (transformed in a $1-100 \%$ scale) in patients suffering from surgical versus non-surgical disorders. One of the secondary outcomes is the correlation of 9SDMQ with 8CSQ scores.

Results.- A total of 35 patients (20 patients with non-surgical disorders and 15 with surgical disorders) with a mean age of 51.51 years (range: $15-87$ years, $S D=20.55$ years) were recruited. The two groups did not significantly differ in age or education. 
The mean transformed 9SDMQ scores for the groups of nonsurgical disorders and surgical disorders were $58.10(\mathrm{SD}=8.54)$ and $68.74(\mathrm{SD}=17.83)$, respectively. The difference in the mean 9SDMQ scores between the groups was significant $(t=-2.34$, $\mathrm{df}=33, p=0.025)$. The group of surgical disorders also scored better on the $8 \mathrm{CSQ}$ ( mean $=24.65, \mathrm{SD}=3.1$ versus mean $=27, \mathrm{SD}=2.85$, $t=-2.295, \mathrm{df}=33, p=0.028)$. The transformed 9SDMQ scores and the $8 \mathrm{CSQ}$ scores were significantly correlated (Pearson $r=0.444$, $p=0.008$ ).

Conclusions.- Patients with surgical disorders participate more on medical decisions compared with those with non-surgical medical disorders. Higher participation in shared decision making is associated with higher satisfaction from health services.

Disclosure of interest.- The authors have not supplied a conflict of interest statement.

\section{E-PV0573}

\section{Disseminating research on psychiatry and religion: a bibliometric analysis of the journal mental health, religion \& culture}

\author{
C.A. Lewis ${ }^{1,2^{*}}$, K.M. Loewenthal ${ }^{3,4,5}$, S. Dein ${ }^{6}$, K.I. Pargament ${ }^{7}$ \\ ${ }^{1}$ Bishop Grosseteste University, School of Social Sciences, Lincoln, \\ United Kingdom; ${ }^{2}$ University of Warwick, Centre for Education \\ Studies, Coventry, United Kingdom; ${ }^{3}$ Royal Holloway- University of \\ London, Psychology, London, United Kingdom; ${ }^{4}$ Glyndŵr University, \\ Psychology, Wrexham, United Kingdom; ${ }^{5}$ New York University- \\ London, Psychology, London, United Kingdom; ${ }^{6}$ Durham University, \\ Department of Theology and Religion, Durham, United Kingdom; ${ }^{7}$ \\ Bowling Green State University, Psychology, Bowling Green, USA \\ * Corresponding author.
}

Background and aims.- The journal Mental Health, Religion $\mathcal{E}$ Culture (MHRC) celebrates its $21^{\text {st }}$ anniversary, having commenced publishing in 1998. MHRC provides an authoritative forum and a single point of reference for the growing number of clinicians and academics working in the expanding field of mental health and religion. Over the course of the last two decades, well over 1,000 research articles have been published in MHRC. In acknowledgment of this anniversary, the aim of the present study was to provide a retrospective bibliometric assessment of MHRC over its initial 21 years of production.

Methods.- All previously published articles in MHRC (1998-2018) were empirically examined by two analytical tools: Google Scholar and SCImago Journal Rank (SJR indicator).

Results.- The data provided a wide range of findings. First, various scholarly metrics including impact factor, five-year impact factor, $h 5$-index, number of citations received, and self-citation rate were presented. Second, the most cited articles were identified and the common themes of these publications were explored. Third, the international diversity of the journal's author base was outlined. The analysis also presents the list of Special Issues published by MHRC.

Conclusions.- The findings are discussed with the aim of outlining common and popular themes within the literature published in MHRC, as well as trying to identify current gaps in the literature that could be examined in future articles, including Special Issues. The utility of MHRC for disseminating research on psychiatry and religion is highlighted.

Disclosure of interest.- The authors have not supplied a conflict of interest statement.

\section{E-PV0574}

\section{Neurocognitive alterations due to} antiretroviral treatments. Case report

M.T. Lozano Lopez ${ }^{*}$, S. Gamonal Limcaoco, A. Alvarez Pedrero, N. Casado Espada, R. De La Mata Hidalgo, J. De la Iglesia Larrad, R. De Alarcon Gomez, I. Valriberas Herrero, A. Macias Casas, A. Bullon

Saez, J. Matias Fernandez

Doctor, Psychiatry, Salamanca, Spain

* Corresponding author.

Background and aims.- Neurocognitive disorder in HIV- infected persons is characterized by a wide spectrum of behavioral, cognitive and motor dysfunctions, despite the success of combined antiretroviral therapy. It should be noted that the potential toxicity of some antiretroviral drugs remains remarkably underexplored. To illustrate, through a case report, the neurocognitive alterations due to antiretroviral therapy (Abacavir/Dolutegravir/Lamivudine), emphasizing its differential diagnosis, evolution and therapeutic possibilities.

Methods.- Retrospective collection of data from a patient's clinical history and bibliographic review in the main search engines: PUBMED and COCHRANE.

Results.- A 50-year-old HIV-positive male was hospitalized in the department of Psychiatry for cognitive disorders. A study of complementary tests was carried out and a clinical judgment of mild neurocognitive disorder induced by antiretroviral drugs was made, for which pharmacological adjustment was done; after which we observed the remission of the acute clinic.

Conclusions.- Recent findings indicate that the overall positive effect that combination of antiretroviral therapy on HIV-infected persons, despite its positive effects, could be accompanied by several detectable neurotoxic consequences. Given that some components of the neurocognitive disorder can be reversible, the nosological classification of this diagnostic entity is important due to the evolution and reversibility of the process that could be affected.

Disclosure of interest.- The authors have not supplied a conflict of interest statement.

\section{E-PV0576 \\ Neurocognitive functions in patients with carotid stenosis mesured in distant period}

I. Malenkova ${ }^{1}$, L. Medvedeva ${ }^{1}$, O. Zagorulko ${ }^{1}$, G. Shevtsova ${ }^{2}$

${ }^{1}$ National Research Centre of Surgery, Pain Department, Moscow,

Russia; ${ }^{2}$ First Moscow State Medical University, Department of

Nervous Diseases and Neurosurgery, Moscow, Russia

Corresponding author.

Background and aims.- The number of surgical interventions on the brachiocephalic arteries is increased progressively every year, whereas carotid endarterectomy is rated the second in most frequent operations in cardiovascular surgery.

Objective is to study the incidence of cognitive dysfunction in the long-term in patients with carotid stenosis who underwent carotid endarterectomy and who received only conservative treatment, evaluated with neurocognitive testing.

Methods.- The study included 80 patients (the main group) operated for hemodynamically significant atherosclerotic stenosis of the internal carotid arteries (ICA), as well as 20 patients with a similar lesion who refused surgical treatment (control group).

To detect cognitive dysfunction, all patients underwent complex cognitive testing using a battery of 3 tests:the Mini-Mental State Examination (MMSE), the Frontal Assessment Battery (FAB) of the Montreal Cognitive Assessment scale (MoCA). Testing in the main group was performed a day before the surgical intervention, and in the delayed (after 3 and 6 months) postoperative periods. In the 
control group, patients were tested at the time of enrollment, at 3 and 6 months of follow-up.

Results.- Prior to the operation cognitive tests of both groups patients were within normal limits. Patients operated on the ICA had lower levels of cognitive impairment after 3 months of followup than patients receiving only conservative treatment. Analysis of delayed test results showed lower values in the control group for the all scales after 6 months (MMSE $28.9 \pm 3, p=0.01$, FAB $15.3 \pm 3.8$, $p=0.03$, MoCA $28.4 \pm 3.4, p=0.01$ in the group of operated patients and $26.9 \pm 3.1, p=0.01,1.3 \pm 3.8, p=0.03,26.3 \pm 3.2$ in the control group respectively).

Conclusions.- ICA stenosis surgical treatment may benefit the patients' neurocognitive functions in the distant period.

Disclosure of interest.- The authors have not supplied a conflict of interest statement.

\section{E-PV0578}

\section{Total vitamin D levels in psychiatric inpatients}

S. Martinho*, A. Batista, F. Malheiro, M. Simões

Leiria Hospital Centre, Psychiatry and Mental Health, Leiria, Portugal

${ }^{*}$ Corresponding author.

Background and aims.- Recently, there has been a vivid scientific interest in vitamin $\mathrm{D}$ and its relationship with several pathologies. Psychiatry is no different, and vitamin D has already been associated with conditions such as depression and schizophrenia. With this study, we aim to give an account of vitamin D status in a sample of psychiatric inpatients.

Methods.- From January 2018 to August 2018, the measurement of 25 -Hydroxyvitamin D (25[OH]D) was performed in blood samples of patients whose assistant psychiatrist had already opted for blood work on clinical grounds. We included patients with 18 to 65 years of age. We utilized Mann-Whitney to ascertain differences between formed groups.

Results.- Of 165 patients, $83 \%$ presented with 25(OH)D levels below $20 \mathrm{ng} / \mathrm{mL}$ and $33.9 \%$ had levels below $10 \mathrm{ng} / \mathrm{mL}$. Mean level of $25(\mathrm{OH}) \mathrm{D}$ settled in $13.93 \mathrm{ng} / \mathrm{mL}$, with maximum and minimum levels of $41.24 \mathrm{ng} / \mathrm{mL}$ and $2.71 \mathrm{ng} / \mathrm{mL}$, respectively. There were no differences between gender $(p=.54)$. Patients who were admitted in the summertime (starting 21st July to 31th of August) had higher levels of $25(\mathrm{OH}) \mathrm{D}(p<.01)$.

Conclusions.- By several guidelines, the vast majority of our sample presented with total vitamin D levels below standard values. This result doesn't seem to be particular to psychiatric patients, as there's evidence of a large-scale vitamin D deficiency. However, it remains to be investigated what effect may hypovitaminosis D have in psychiatric illness.

Disclosure of interest.- The authors have not supplied a conflict of interest statement.

\section{E-PV0579}

\section{“Don't close the door": a tale of anxiety}

S. Mohammad

Mayo Clinic, Psychiatry and Psychology, Rochester, USA

${ }^{*}$ Corresponding author.

Background and aims.- Somatic Symptom and Related Disorders are disorders unified by the presence of physical symptoms and/or excessive concern regarding medical illnesses/symptoms. The DSM - 5 shifted the emphasis on the absence of a medical explanation serving as an organizing principle for these disorders and instead focused on the associated distressing somatic symptoms and abnormal thoughts, feelings, and behaviors. With this change, individuals formerly diagnosed as Hypochondriasis are now diagnosed as Somatic Symptom Disorder (those with somatic symptoms) and Illness Anxiety Disorder (primarily an illness anxiety in the absence of somatic symptoms).

Methods.- Ms. X is a 52-year-old African American female with chief complaint, "don't close the door". She is married but separated and is living with her sister and son. On her initial visit she was apprehensive and insisted on keeping the door open. She takes her time to scan the environment and then sits on a chair and said, "you don't know me". She is afraid she might die and said she has been having these thoughts and feelings "for long time since I had heart attack, since 2009".

Results.- Her maladaptive behaviors include: keeping doors open particularly bathroom door, isolating, not venturing out except for doctor's appointments, multiple phones (at least five cell phones), staying closer to hospitals, and sleep-wake reversal to counter her intrusive thoughts of illness and death.

Conclusions.- The patient in this case presentation has classic preoccupation of acquiring a serious undiagnosed medical condition and associated anxiety that is excessive and disproportionate, affecting her quality of life.

Disclosure of interest.- The authors have not supplied a conflict of interest statement.

\section{E-PV0580}

\section{Which are the risk factors of violence in mental illness? A scoping review}

M.C. Moreno-Calvete ${ }^{{ }^{*}}$, E. González-Fraile ${ }^{2}$, J.J. Uriarte-Uriarte ${ }^{1}$, I. Ruiz-Ibáñez ${ }^{1}$, B. Santos ${ }^{3}$, J. Ballesteros ${ }^{4}$

${ }^{1}$ Red de Salud Mental de Bizkaia RSMB, Biocruces, Osakidetza, Basque Health System, Bilbao, Spain; ${ }^{2}$ International University of La Rioja UNIR, Logroño, La Rioja, Spain; ${ }^{3}$ University of the Basque Country UPV/EHU, Department of Genetics, Physical Anthropology and Animal Physiology, Leioa, Spain; ${ }^{4}$ University of the Basque Country UPV/EHU, Department of Neuroscience-Psychiatry. Faculty of Medicine and Odontology, Leioa, Spain

* Corresponding author.

Background and aims.- Evidence shows an association between mental illnesses and violence. It is important to identify the risk factors and possible treatments to tackle this problem related to people with mental illness.

Aim.- To identify which risk factors can be related to the presence of violence behaviours in people with mental illness.

Methods.-This scoping review considered other systematic reviews and meta-analysis that included studies of risk factors for violence in adults with mental illness, without language or publication period restrictions for the studies. The databases Pubmed/Medline, Cochrane Library, Pubmed, PsycINFO, Epistemonikos, EMBASE and WOS were searched until August 2018.

Results.- 855 articles were examined and 8 reviews were identified. The risk factors of the systematic reviews assessed are diagnosis of schizophrenia, involuntary patients, substance abuse, impulsivity, history of violence, male etc. (Table 1 ). 


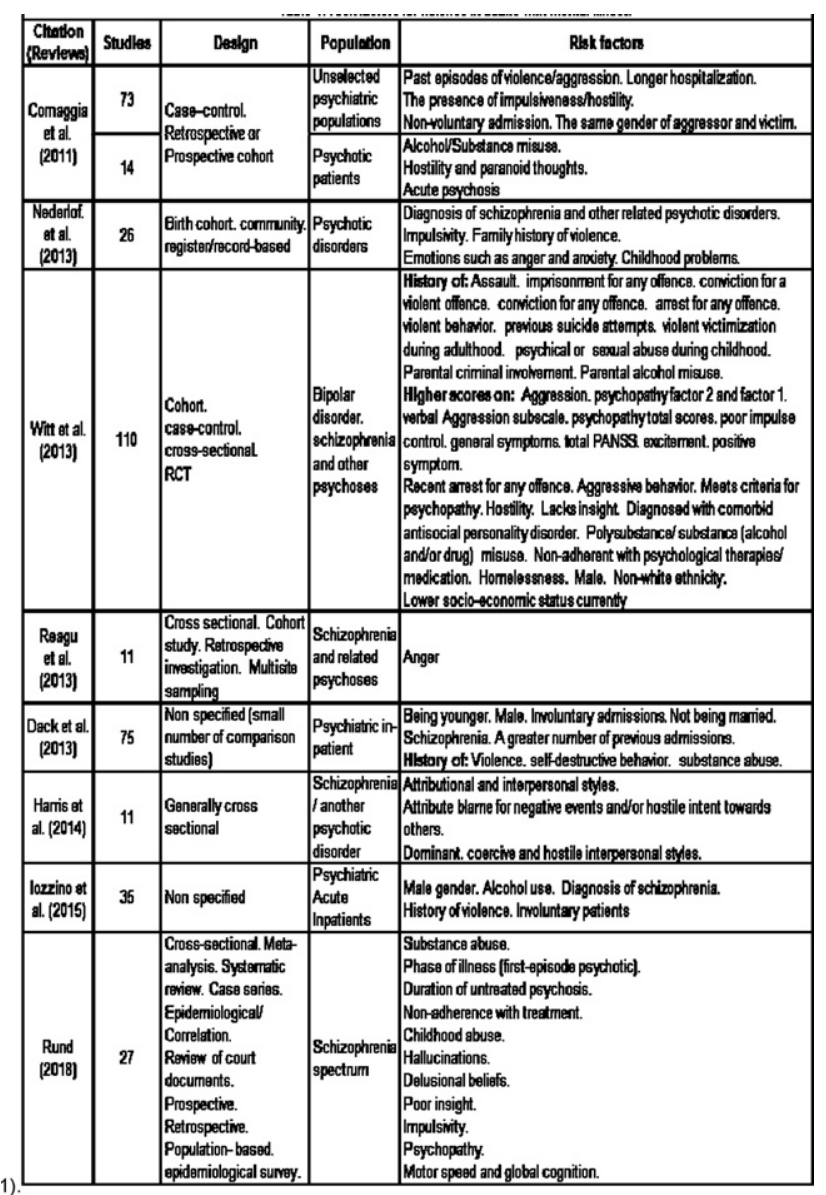

Table 1. Risk factors for violence in adults with mental illness.

Conclusions.- Some of the risk factors detected in the scoping review could be modifiable and suggest which strategies could be considered to prevent violence behaviour in people with mental illness.

Disclosure of interest.- The authors have not supplied a conflict of interest statement.

\section{E-PV0581}

\section{How do we manage unconscious conflicting information? Exploring the requirements for the emergence of unconscious conflict}

F. Silva ${ }^{1}$, S. Silva ${ }^{2}$, D. Moura ${ }^{3,4^{*}}$, N. Madeira ${ }^{3,4}$, S.C. Soares ${ }^{1,5}$

${ }^{1}$ Instituto Superior de Psicologia Aplicada ISPA, William James Research Center, Lisboa, Portugal; ${ }^{2}$ Institute of Electronics and Informatics Engineering- University of Aveiro, Department of Electronics, Aveiro, Portugal; ${ }^{3}$ Centro Hospitalar e Universitário de Coimbra CHUC- Portugal, Department of Psychiatry, Coimbra, Portugal; ${ }^{4}$ Faculty of Medicine, University of Coimbra, Institute of Psychological Medicine, Coimbra, Portugal; ${ }^{5}$ University of Aveiro, CINTESIS.UA, Department of Education and Psychology, Aveiro, Portugal

* Corresponding author.

Background and aims.- Although selective in what we experience, the information processed from our environment, far exceeds the information that we end up consciously attending. Having this in mind, recent research has proposed that information that is not consciously perceived might still have implications in our cognition. Nonetheless, the extent of this effect is still highly debatable. Considering the involvement of cognitive control deficits in a num- ber of psychiatric disorders (e.g., schizophrenia, OCD, depression), measuring if and how unconscious information impacts cognitive control is of crucial importance.

Our aim was to verify whether unconsciously processed information does yield effects on cognitive performance, as suggested by previous studies. Expanding on one of our prior studies that showed no conflict under unconscious conditions, we investigated how such components might be dependent on attention and temporal onset factors.

Methods.- We measured congruency effects caused by flanker arrow cues in an Eriksen Flanker task. This was performed with conscious and unconsciously presented flankers, using the Chromatic Flicker Fusion technique.

Results.- The initial results of this pilot study $(N=8)$ suggested that earlier presentation of unconscious flankers lead to conflict effects. Moreover, on further inspection, we also observed an additional impact when flankers were overlaid with the target.

Conclusions.- These preliminary results indicate that other factors might be necessary in order for conflict to occur under unaware conditions. We rely on future studies to further elucidate on the extent that unconscious conflict bears on cognition and how such might be tied with certain psychiatric disorders.

Disclosure of interest.- The authors have not supplied a conflict of interest statement.

\section{E-PV0582 \\ Investigating the efficacy of cognitive therapy-based perfectionism treatment on problem-solving skills and attribution style of adolescents} N. Nasertorabi ${ }^{1 *}$, A. Ghanbari Panah ${ }^{2}$

1 İslamic Azad University- Electronic campus, Psychology, Tehran, Iran; ${ }^{2}$ İslamic Azad University- Central Tehran Brach, Psychology, Tehran, Iran

* Corresponding author.

Background and aims.- Adolescence is a period of physical and emotional change and is also associated with concern and distress. Therefore, one needs to learn skills to pass through this phase safely. Distortions and cognitive qualities are likely to prevent the acquisition of these skills, why we need to recognize and correct them.

This study investigates the efficacy of cognitive therapy-based perfectionism treatment on problem-solving skills and attribution style of adolescents.

Methods.- The research method was quasi-experimental using a "pre-test"- "post- test" design with a control group. 450 adolescent girls aged 12-17 years old answered a questionnaire of a purpose full approach based on the score of the problem-solving skills (Hepner \& Patterson, 1982) and the attribution style (Patterson \& Seligman, 1983) in the pretest, where 210 of them scored low. 40 individuals of this group were selected randomly and were divide in 2 groups of 20 people for experimental and control group. The experimental group received 8 treatment sessions in-group, while the control group didn't receive any. Covariance analysis and SPSS software 23 were used to analyze data.

Results.- A comparison between the control group and the cognitive therapy-based perfectionism treatment group did show a significant improvement in problem-solving skills and the attribution styles of the adolescents participating in the study.

Conclusions.- A variety of psychological disorders in adolescence can be prevented by correct diagnose and psychological treatments of cognitive errors in problem solving and attribution style, which can be used to improve the mental health of general public.

Disclosure of interest.- The authors have not supplied a conflict of interest statement. 


\section{E-PV0583}

\section{Hoarding disorder on a 69 year old woman: a case report}

P.A. Pereira Vaz De Macedo*, J. Borges, G. Dias dos Santos, R. Almeida Leite, M. Almeida, A. Mesquita Figueiredo Centro Hospitalar do Baixo Vouga, Psychiatry, Aveiro, Portugal ${ }^{*}$ Corresponding author.

Background and aims.- Hoarding disorder is a recent separate diagnosis of the Diagnostic and Statistical Manual of Mental Disorders, fifth edition. There is an estimated prevalence of $1,5 \%$ of compulsive hoarding. The disorder consists of a persistent difficulty in discarding or parting with possessions, whether they are valuable or not. It's important that the possibility of discarding such possessions is associated with substantial distress. In severe cases, the disorganized accumulation of possessions, can lead to a range of health risks, including fire, falling and poor sanitation, with associated increase of the risk of death by multiple causes. Some neuropsychological testing associate this disorder with impaired decision-making, reduced attention and reduced memory confidence. There is a tendency for worsening of symptoms with age. In more than $50 \%$ of the cases, there is poor or no insight for the condition.

The aim of this study is to present a clinical case of hoarding disorder, it's treatment and follow up.

Methods.- The case report was sustained by literature included on scientific platforms.

Results.- In this case, treatment had to be assured by compulsive internment, due to the lack of insight. Significant health risks for the patient and surrounding population were prevented due to intervention.

Conclusions.- The patient showed a good response to treatment, but many difficulties arose from non-psychiatric interventions of the process, such as those related with cleaning process.

Disclosure of interest.- The authors have not supplied a conflict of interest statement.

\section{E-PV0584}

\section{Treatment of psychotic symptoms in Huntington's disease}

\section{A.T. Pereira*, P. Horta, M. Araújo, J.D. Rodrigues}

Vila Nova de Gaia/Espinho Healthcare Center Vila Nova de Gaia, Portugal, Department of Psychiatry and Mental Health, Vila Nova de Gaia, Portugal

${ }^{*}$ Corresponding author.

Background and aims.- Huntington's disease is a neurodegenerative disorder characterized by motor disturbances, progressive dementia and psychiatric features. Schizophrenia-like psychotic symptoms occur in Huntington's disease with an estimated prevalence of 6-25\%, varying both in their nature and time of occurrence over the course of the disease. To evaluate alternative therapeutic options for the treatment of Huntington's disease-associated psychosis.

Methods.- Non-systematic review of the literature (using PubMed/Medline) based on the terms "psychosis" and "Huntington's disease".

Results.- The treatment of psychosis in Huntington's disease is a controverse topic. One retrospective study demonstrated that risperidone was effective over the period of 1 year in reducing psychiatric symptoms. Case reports have also highlighted atypical antipsychotics in patients with Huntington's disease, including aripiprazole, quetiapine and olanzapine. Clozapine has been associated with a reduction in violent and aggressive behavior There is conflicting evidence on the antichoreatic properties of clozapine in Huntington's disease, with tolerability problems limiting its use. Some studies suggest beneficial effects of aripiprazole on motor and behavioral symptoms.

Conclusions.- Quetiapine, olanzapine, risperidone and clozapine can be used to manage psychosis and disruptive behavioral symptoms. Currently, limited information is available on the treatment of psychiatric symptoms and the safety of antipsychotic and antidepressives in Huntington's disease, therefore evidence-based guidelines are lacking and further research is needed.

Disclosure of interest.- The authors have not supplied a conflict of interest statement.

\section{E-PV0585}

\section{Collaborative circularity: a new paradigm}

C. Pires De Lima ${ }^{1,2^{*}}$, M. Figueiredo ${ }^{3,4}$, G. Cunha ${ }^{5,6}$, M. Bravo ${ }^{5}$ ${ }^{1}$ Psychology, Paredes de Coura, Portugal; ${ }^{2}$ CINTESIS - Center for Health Technology and Services Research, Psychology, Porto, Portugal; ${ }^{3}$ CINTESIS - Center for Health Technology and Services Research, Nursing, Porto, Portugal; ${ }^{4}$ Escola Superior de Enfermagem do Porto, Teacher, Porto, Portugal; ${ }^{5}$ Mediare, Conflict Medition, Porto, Portugal; ${ }^{6}$ Universidade Lusófona do Porto, Student, Porto, Portugal * Corresponding author.

Background and aims.- The social evolution resulting from globalization, from new health and social needs, coupled with the transformations of family configurations, have determined the emergence of new paradigms, in an attempt to give an integrated response to current problems. This integrative perspective, operating through complementarity between different areas of intervention, has as its primary purpose the training of families and individuals in the identification and mobilization of strategies for solving their problems and/or minimizing their needs.

Methods.- Examples of current situations that generate conflicts/difficulties in establishing effective communication patterns that imply dysfunctions in the maintenance of a healthy experience are the events associated with divorce, the regulation of parenthood, the maintenance of a positive identity after divorce and the establishing of adequate conditions for children and parents to adjust and adapt to daily living based on the evident fact of the existence of "two families". Other relational/behavioral problems require this complementarity of intervention, characterized by the contextual difficulty of establishing consensual standards regarding the functioning of the family system and/or its subsystems.

Results.- We intend to describe conceptual and interventional aspects regarding Family Mediation, Collaborative Law, Psychotherapy and Family Therapy, integrating Psychoeducational Therapy as a transversal strategy in what refers to the intervention areas mentioned.

Conclusions.- We emphasize the specificities of each area, which enables the referencing in a paradigm of collaborative circularity. On the other hand, we emphasize transdisciplinarity as a fundamental element in the maximization of the systems, selforganization, implying greater gains in health potential, greater social value and lower economic costs for citizens and society. Disclosure of interest.- The authors have not supplied a conflict of interest statement. 


\section{E-PV0588}

\section{Hyperprolactinemia and vortioxetine: a case report}

M. Robles Martínez ${ }^{1 *}$, M. Iglesias-González ${ }^{2}$, F. Palma-Álvarez ${ }^{3}$, S. Aguelo Rueda ${ }^{4}$, C. Rodríguez González ${ }^{4}$, R. Sauras ${ }^{5}$, F. Fonseca ${ }^{4}$, M. Torrens $^{6}$

${ }^{1}$ Institute of Neuropsychiatry and Addictions INAD. Parc de Salut Mar Barcelona, Spain, INAD, Barcelona, Spain; ${ }^{2}$ Parc Sanitari Sant Joan de Déu. Sant Boi de Llobregat, Barcelona, Spain; ${ }^{3}$ Addiction and Dual Diagnosis Unit, Vall Hebron University Hospital, CIBERSAM, Barcelona, Spain; ${ }^{4}$ Institute of Neuropsychiatry and Addictions INAD, Parc de Salut Mar Barcelona, Spain; ${ }^{5}$ Institute of Neuropsychiatry and Addictions INAD, Parc de Salut Mar Barcelona, Spain; ${ }^{6}$ Institute of Neuropsychiatry and Addictions INAD, Parc de Salut Mar Barcelona, Cataluña-España, Spain

* Corresponding author.

Background and aims.- Prolactin release is primarily regulated by tuberoinfundibular dopamine pathways, but it is also indirectly modulated by serotonin via the activation of 5-HT1C and 5-HT2 receptors ${ }^{1}$. Long-standing increases in peripheral prolactin levels are occasionally observed in patients using $\mathrm{ADs}^{2}$; hyperprolactinemia may have deleterious health consequences ${ }^{3}$.

Methods.- We present a clinical case.

Results.- 30 year old woman. Single, with no children, living with her partner. No known somatic diseases or previous interventions. No history of drug abuse. No current pharmacological treatment. She was admitted to psychiatry unit for presenting with depressive symptoms for the past 8 months. She had suffered several previous depressive episodes that were treated with SSRIs. She had presented many side effects: restlessness, insomnia, anxiety, dry mouth... and did not wish to undergo another SSRI treatment in this new depressive episode. She was started on vortioxetine $10 \mathrm{mg} /$ day with good but incomplete clinical improvement. We decided to increase vortioxetine to $20 \mathrm{mg}$ /day with total recovery and complete resolution of the symptoms. However, she reported mastalgia and an episode of galactorrhea. Analytical tests showed prolactin $56 \mathrm{mg} / \mathrm{dl}$. Vortioxetine was withdrawn slowly and 10 days after stopping the treatment a control analysis was scheduled showing rebalanced prolactin values.

Conclusions.- The temporal relationship between the initiation of treatment with vortioxetine and the onset of hyperprolactinemia, as well as normalisation of prolactine levels after pharmacological discontinuation of the drug, suggests a causal etiology. Vortioxetine causing hyperprolactinemia has never been reported before. Clinicians should be aware of this side effect as it can potentially affect treatment adherence.

Disclosure of interest.- The authors have not supplied a conflict of interest statement.

\section{E-PV0590}

\section{Predictors of impact factor in psychiatric publications}

H. Sandgreen ${ }^{1 *}$, B. Perron ${ }^{2}$, C. Sibbersen ${ }^{3}$, P. Munk-Jørgensen ${ }^{1}$

${ }^{1}$ Psychiatric Research academy, Odense University Hospital, Department of Psychiatry, Odense, Denmark; ${ }^{2}$ School of Social Work, University of Michigan, Ann Arbor, MI, USA; ${ }^{3}$ Odense University Hospital, Department of Psychiatry, Odense, Denmark

* Corresponding author.

Background and aims.- Impact factor (IF) is a commonly used bibliometric measure which indicates the average 2-year citation for articles published in a journal. Many researchers strive to be published in high IF journals.

The aim of the study was to identify correlations between bibliometric measures of publications from psychiatric departments and the IF of the publishing journal.
Methods.- This is a bibliometric study including all publications from the psychiatric departments in 6 hospitals in Denmark over a 4-year period. Bibliometric data e.g. number of authors, study type and international author affiliations, was collected and tested for correlation to the IF of the publishing journal in a linear regression model. Themes were conducted from full text scanning of titles and abstracts with omission of non-thematic words such as 'that' and 'patient'.

Results.- Oxford Level of Evidence (OLE) 3 and 4 studies, qualitative studies and non-OLE reviews, all had significantly lower IF compared to OLE 1 studies. Higher number of authors had a small positive correlation to higher IF. Articles with international author affiliations had a higher IF compared to local author groups. The themes 'Nationwide', 'Psychosis', 'Diabetes' and 'Death' all had a positive association to higher IF, and 'Danish' had a negative association.

Conclusions.- Higher impact factor is associated with higher level of evidence (Oxford Level of Evidence), more authors and international collaboration. High IF journals may tend to higher acceptance of studies including somatic specialties such as diabetology, large samples e.g. nationwide studies and definitive endpoints, for instance psychosis and death.

Disclosure of interest.- The authors have not supplied a conflict of interest statement.

\section{E-PV0592}

\section{Self-regulation development from childhood to youth: longitudinal research}

E. Sedova*, T. Goryacheva

Pirogov Russian National Research Medical University,

Psychological-Social Faculty, Moscow, Russia

* Corresponding author.

Background and aims.- The issues of self-regulation development remain in the focus of the research in the last decades as a matured system of self-regulation allows a person reaching success in various spheres of life whereas the defects of such system can lead to a destructive behaviour.

The aim of the current research is studying changes in the selfregulation level in the age of 7,11 and 21 years.

Methods.- The sample consists of 23 participants (10 male, 23 female) with normative mental development.

At the first two stages the level of the voluntary self-regulation has been diagnosed from the point of view of the neuropsychological approach. At the third stage the Morosanova's Questionnaire has been applied. The motivational level of self-regulation has been studied with the techniques of Guinsburg at the first stage; Grebenyuk's and Markova's at the second and Leontiev's SZhO Test at the third stage.

Results.- The research shows that only three persons demonstrate the same level of self-regulation at all the three stages (one- high, one- medium and one - low), in four cases a negative dynamics can be seen. The rest of participants have showed better results in the second follow-up (in the age of 21), than either at the first or at the second stage.

Conclusions.- We can conclude that the defects of self-regulation observed at the first two stages have compensated spontaneously but more precise analysis of the facts influencing this process is needed.

Disclosure of interest.- The authors have not supplied a conflict of interest statement. 


\section{E-PV0595}

\section{ADHD symptomatology and time perception}

M. Vňuková*, J. Raboch, R. Ptáček

First Faculty of Medicine, Charles University in Prague and General University Hospital in Prague, Psychiatry, Prague, Czech Republic

* Corresponding author.

Background and aims.- Attention Deficit Hyperactivity Disorder (ADHD) is a neuro-developmental disorder which significantly impacts entire life of affected individuals. Not much is known about the relationship between ADHD symptoms and time perception in adults although this association could be important for assessment and therapy.

Current project aims to gather information about the association between the intensity of ADHD symptoms and time perception as well as ascertain whether people with ADHD manifest specific styles of time perception.

Methods.- The data collection will apply combined methodology. In the first wave we will use standardized questionnaires for collections of ADHD symptomatology, time perception as well as lifestyle and demographic surveys. Based on the results of this analysis participants at risk will be invited for qualitative part of the research that will consist of interviews and in depth case studies of selected cohort.

Results.- Literature suggests that time perception that is significantly associated with ADHD symptomatology. ADHD patients seem to be stuck in the present, similar orientation has been observed in the wide array of people who suffer from dependencies which are very often linked to ADHD. Irrespective of diagnoses, the significance of balanced time perspective for a general satisfaction with life should not be underestimated.

Conclusions.- Although ADHD tends to be viewed as a disorder which generally well responds to behavioral therapy time perspective therapies are not being utilized. Only sporadic reports mention the usefulness of time perspective as a possible direction of treatment. We hope that our project will help to correct this blind spot. Disclosure of interest.- The authors have not supplied a conflict of interest statement.

\section{E-PV0597}

\section{The extent of direct coercion and related factors during hospitalizations in the psychiatric ward in a general \\ hospital}

U. Zaniewska-Chłopik ${ }^{1 *}$, M. Załuska ${ }^{2}$

${ }^{1}$ Community Treatment Team ul. Grebałowska 14, Bielanski Hospital, Warsaw, Poland; ${ }^{2}$ Centre of Postgraduate of Medical Education, Bielanski Hospital, Warsaw, Poland

${ }^{*}$ Corresponding author.

Background and aims.- The Mental Health Act (1994) specifies rules of use for direct coercion in Poland. Coercion (holding, forced medication and mechanical restraint) in psychiatric wards may improve the safety of patients and surroundings but influences compliance and satisfaction with treatment. This study aims to identify correlations between the extent of coercive measures and patient-related factors during hospitalization in a psychiatric ward.

Methods.- Analysis of hospitalizations of all patients admitted to the psychiatric ward in Bielanski Hospital in Warsaw $(N=318)$ over one year. Gathering data on coercive measures and identifying predictors of use of direct coercion. Patient's sociodemographic and clinical factors were tested in a multivariate logistic regression model. Frequency and length of coercion were compared between the working day and irregular hours (15.00 to 8.00 and weekends) (Wilcoxon test).
Results.- Coercion was used in $18 \%(N=58)$ of hospitalized patients. In patients received coercion: holding was used on $10 \%$, forced medication on $15 \%$ and mechanical restraint on $85 \%$. According to the regression model: patients with lower education, admitted without consent and with higher levels of disorientation (BPRS) had a higher risk of being coerced during hospitalization. Depression symptoms decreased risk of coercion. Coercive measures were used more often during irregular hours than during the working day. Conclusions.- The use of coercion depends on many factors. It is important to identify patients with a high likelihood of coercion and to design practices in the ward to minimize usage of coercion. Disclosure of interest.- The authors have not supplied a conflict of interest statement.

\section{E-PV0598}

\section{Is suicidality linked to prescription drug abuse in us adults with a history of chronic pain?}

M. Abdelghani ${ }^{1}$, S. Ibrahim Amin ${ }^{1}$, A. Said ${ }^{2}$

${ }^{1}$ Zagazig University, Psychiatry, Zagazig, Egypt; ${ }^{2}$ Zagazig University, Anesthesiology, Zagazig, Egypt

Corresponding author.

Background and aims.- Suicide is one of the most leading causes of death worldwide. Individuals with chronic pain are at higher risk for suicide than other population. Moreover, the potential for prescription drug abuse enormously exists. Little is known about the relationship between prescription drug abuse and suicide among those individuals. The study aimed to estimate the prevalence and associated correlates of suicidality and determine its relation to prescription drug abuse, among US adults with a history of chronic pain.

Methods.- Data were obtained from the Collaborative Psychiatric Epidemiology Surveys (CPES 2001-2003). This analysis was limited to US respondents who had a history of chronic pain $(N=5289)$ Multivariable logistic regression was used to determine the association between suicidality and abuse of prescription drugs among US adults with a history of chronic pain.

Results.- Among respondents with a history of chronic pain, there was a strong positive association between prescription drug abuse and suicidal ideation and attempts $(P$-value $<0.001,=0.006$, respectively). Logistic regression analysis adjusted for sociodemographic variables, lifetime mood, anxiety, and other substance use disorders, showed that those who abused prescription drugs had 1.5 times greater likelihood of suicidal ideation relative to those not abusing prescription drugs (95\% CI 1.18-1.82).

Conclusions.- There is a robust relationship between prescription drug abuse and suicidality in individuals with a history of chronic pain. Further longitudinal studies need to investigate the causal relationship between prescription drugs and other substance use disorders, and suicidality among those populations.

Disclosure of interest.- The authors have not supplied a conflict of interest statement.

\section{E-PV0599}

\section{Evaluation of the experience of patients with chronic pain after participation in group therapies. Design of a qualitative study using focus groups}

L. Nocete Navarro ${ }^{1}$, Á. Palao Tarrero ${ }^{1}$, M. Torrijos Zarcero ${ }^{1}, \mathrm{C}$. Rocamora González ${ }^{1}$, M. Bermúdez Castro ${ }^{2}$, D.H. Calle ${ }^{1^{*}}$, E. García Higuera $^{3}$, B. Rodríguez Vega ${ }^{1}$

${ }^{1}$ Hospital Universitario La Paz, Psychiatry and Mental Health, Madrid, Spain; ${ }^{2}$ Universidad Autónoma, Psychology, Madrid, Spain; 
${ }^{3}$ Hospital La Paz, Anesthesiology, Perioperative and Pain Medicine, Madrid, Spain

* Corresponding author.

Background and aims.- Chronic pain is a complex biological, psychological and social experience, involving many different factors in its genesis, maintenance, aggravation or improvement. In our country between $10-23 \%$ of the population suffer from chronic pain, which impairs their physical, emotional and social functioning and thus also their life quality. There are numerous meta-analyses on psychological interventions in patients with chronic pain that show positive, but modest, outcomes. The scales designed to assess the efficacy of chronic pain interventions are useful, but they are not capable of capturing subjective experience under the process of change. Aims: to present the design of a study that will allow us to understand, from a phenomenological/hermeneutic perspective, the experience of people with chronic pain who attended a therapeutic group.

Methods.- A review of the literature and presentation of the design of a qualitative study of focus groups with patients who participated in group treatments through Mindfulness-Self Compassion based therapy or Cognitive-Behavioural interventions in our center. Results.- In our context, few research groups regularly use qualitative methodology to obtain a more complete and complex view of the experience of patients with chronic pain.

Conclusions.- Through the analysis of the discourses of patients with chronic pain, a qualitative study with focus groups will allow us to infer the meanings that underlie the subjective perception of pain and their relation with it, as well as to understand the changes experienced after the group interventions.

Disclosure of interest.- The authors have not supplied a conflict of interest statement.

\section{E-PV0600 \\ Self-compassion in a chronic pain patient sample}

Á. Palao Tarrero, M. Torrijos Zarcero, B. Rodríguez Vega, E. Fernández Jiménez ${ }^{*}$, J. Curto Ramos, C. Rocamora González Hospital Universitario La Paz, Psychiatry, Clinical Psychology and Mental Health, Madrid, Spain

* Corresponding author.

Background and aims.- Chronic pain is associated with significant emotional distress and self-criticism.

"Self-Compassion" construct is gaining attention due to its strong link to health. As Gilbert pointed, promoting self-compassion could help to cultivate self-care.

Neff and Germer developed a program to enhance self-compassion (Mindful Self-Compassion, MSC) that could be helpful in DM, HIV and also Chronic Pain.

Methods.- We collected data of 144 patients with chronic pain at Hospital Universitario La Paz.

We measured Self-Compassion through Self-Compassion Scale (SCS, range 1-5), and conducted analysis to investigate how selfcompassive patients with chronic pain are.

We hypothesized that people with high self-compassion will show less anxiety and depression. We used the Hospital Anxiety and Depression Scale (HADS) to measure this symptomatology.

Results.- Our sample had a mean age of 48.86 years. Most of them were women $(89.4 \%)$, lived in couple $(68.1 \%)$, around a half were moderate-highly educated (58.1\%), and $18.2 \%$ were employed.

Mean self-compassion total score was 2,76; means of self-kindness, common humanity and mindfulness subscales of SCS were above 2.5 .

We found statistically significant correlations between selfcompassion and depression (Pearson correlation $=-0.343$, $p=0.000$ ); and anxiety (Pearson correlation $=-0,22, p=0.011$ ).
Conclusions.- According to Neff, these scores indicate a moderate level of global self-compassion and also regarding its components in our sample.

Self-compassion was negatively correlated with levels of anxiety and depression. These results are promising because we can then hypothesize that if we train patients in self-compassion, depression and anxiety will decrease. However, we must take these results carefully because there may be other factors that are mediating this relationship.

Disclosure of interest.- The authors have not supplied a conflict of interest statement.

\section{E-PV0601}

\section{Mental disorders in IDP's with somatic diseases and chronicity of pain syndrome \\ V. Korostiy \\ Kharkiv National Medical University, Psychiatry- Narcology and \\ Medical Psychology, Kharkiv, Ukraine \\ ${ }^{*}$ Corresponding author.}

Background and aims.- In the literature, a large amount of evidence of significant effects of emotions on pain perception. But depression does not develop in all patients with chronic pain.

The article presents the results of the observation of 169 IDPs after arriving in Kharkiv region, including the victims, who were in direct combat zone, during period of treatment in University hospital with different somatic diseases with pain. Control group -120 patients, residens of Kharkiv region.

Methods.- Clinical interview, "Questionnaire symptoms of PTSD forced migrants", Impact of Event Scale Revised (IES-R), Zung Self-Rating Depression Scale (ZDS), HADS; The McGill Pain Questionnaire (MPQ) The West Haven-Yale Multidimensional Pain Inventory (WHYMPI), Pain Catastrophising Scale (PCS).

Results.- The results showed that $32.0 \%$ of observed IDP's have anxiety disorders (13.5\%), depressive disorders (10.3\%), PTSD (8.2\%). No significant correlation between anxiety disorders, depressive disorders, PTSD and frequency of acute pain, but strong positive correlation present with chronic pain syndrome and mental disorders at all $(r=0,62, p \leq 0,01)$. Most significant correlation between catastrophising attitude of pain and chronicity of pain syndrome $(r=0,82, p \leq 0,005)$, and frequency of catastrophising attitude of pain match more higher in IDP's $(p \leq 0,01)$.

Conclusions.- High risk of chronicity of pain syndrome in IDP's with different somatic diseases associated with mental disorders, us well anxiety disorders, depressive disorders and PTSD, and have positive correlation with catastrophising attitude of pain.

Disclosure of interest.- The authors have not supplied a conflict of interest statement.

\section{E-PV0602}

\section{Beliefs about body and illness in patients with chronic pain: comparing headaches and dorsalgia}

M. Kovyazina ${ }^{1}$, E. Rasskazova ${ }^{2}$, J. Migunova ${ }^{2}$, A. Tkhostov ${ }^{2}$

${ }^{1}$ Moscow State University, Psychology, Moscow, Russia; ${ }^{2}$ Moscow

State University, Clinical psychology, Moscow, Russia

* Corresponding author.

Background and aims.- Research demonstrated the role of anxiety sensitivity (Ocanez et al., 2016), catastrophization (Holroyd et al., 2007), perception of pain (Mongini et al., 2009, Vowles et al., 2016) in chronic pain while cognitive behavioral therapy suggest strategies of effective pain management (McCracken, Turk, 2002). 
The aim was to reveal specific beliefs about body and illness related to poorer quality of life in patients with different pain localization and severity.

Methods.- 74 patients with chronic migraines and tension-type headaches and 38 patients with dorsalgia filled Migraine Disability Assessment Test (Stewart, 2001), revised version of Illness Perception Questionnaire (Moss-Morris et al., 2002), Cognitions About Body and Health Questionnaire (Rief et al., 1998) and a brief version of Quality of Life and Enjoyment Questionnaire (Ritsner et al., 2005).

Results.- Patients with chronic headaches comparing to dorsalgia reported more autonomic sensations, perceived their body as weak, didn't understand their illness, described that as having psychological reasons and were anxious about medication. Moderation analysis revealed that belief in psychological reasons in patients with low or moderate headache predicts poorer satisfaction with communication $(\beta=-0,41$ to $-0,40, p<0,05)$ while anxiety about medication predicts lower satisfaction with health $(\beta=-0,59$ to $-0,56, p<0,01)$. In patients with severe dorsalgia low satisfaction with leisure activities was related to catastrophization and anxiety about medication.

Conclusions.- Results support that for different pain localization there are different psychological factors related to poorer quality of life that should be addressed. Research is supported by the Russian Foundation for Basic Research, project No. 17-06-00849.

Disclosure of interest.- The authors have not supplied a conflict of interest statement.

\section{E-PV0603}

\section{Revision of mindfulness-based interventions for the treatment of opioid dependence in chronic pain}

\section{Á. Palao ${ }^{1}$, D. Mato ${ }^{2 *}$, M. Loreto ${ }^{1}$, N. Fernánde ${ }^{1}$}

${ }^{1}$ Hospital Unversitario La Paz, Psychiatry, Psychology and Mental Health, Madrid, Spain; ${ }^{2}$ Hospital Dr. R. Lafora, Psychiatry, Madrid, Spain

* Corresponding author.

Background and aims.- Around 10-23\% of people suffer from chronic pain. The use of opioids for pain management has been progressively increasing in recent decades. Misuse, abuse and dependence have been identified as prevalent complications in the prescription of opioids in the last years. Patients with chronic pain usually need long term treatments, with a high risk of developing dependence to opioids.

Cognitive-Behavioral therapy (CBT) has been the most used nonpharmacological chronic pain treatment up to date. Other kind of interventions are emerging (Mindfulness interventions) which are, at least, as effective as CBT. The aim of the study is to investigate the impact and mechanism of mindfulness-based interventions in chronic pain patients with opioid dependence.

Methods.- Review of mindfulness-based interventions for the management of opioid dependence in patients with chronic pain.

Results.- Mindfulness meditation has a potential role in managing the problem of excessive opioid prescription. Unregulated stress and poor management of negative emotions, both increased in chronic pain, leads to consumption of opioids as a way to calm them down immediately. The relief is not only of pain, but also a decrease of the fear and anxiety levels. Opioids calm the patient's thread system immediately, but not in the medium-long term. Conclusions.- Mindfulness training can strengthen the ability to regulate attention to conditioned stimuli associated with the use of substances in the past, counteracting attention bases by focusing it on neutral stimuli or health promoters. These results are promising in order to find effective interventions for this prevalent clinical problem.

Disclosure of interest.- The authors have not supplied a conflict of interest statement.

\section{E-PV0604}

\section{A case study of chronic pain, psychiatric comorbidity and opioid dependence}

\author{
D. Mato ${ }^{1 *}$, A. Palao ${ }^{2}$, P. Hervías Higueras ${ }^{3}$
}

${ }^{1}$ Hospital Dr. R. Lafora, Psiquiatría, Madrid, Spain; ${ }^{2}$ Hospital

Unversitario La Paz, Psychiatry, Psychology and Mental Health, Madrid, Spain; ${ }^{3}$ Hospital del Henares, Psychiatry, Madrid, Spain * Corresponding author.

Background and aims.- Chronic pain is an increasingly prevalent problem worldwide that affects 70 million in the US. Psychiatric patients suffering from chronic pain are more likely to develop dependence to opioids and also tend to have higher levels of anxiety and depressive symptoms.

Base on a clinical case we propose to carry out a bibliographic review on the comorbidity of pain and psychiatric disorders focusing in addiction problems.

Methods.- We present the case from the Pain Unit of Hospital Universitario La Paz in 2018, of a 78-year-old man with a history of gambling addiction who suffered from chronic abdominal pain and develop a fentanyl dependence.

Results.- Surveys show that some additions (tobacco, alcohol and opioids) are particularly prevalent in patients with chronic pain. The mechanisms of this link are multifactorial. Among neurobiologycal factors the brain reward and motivations system probably plays and important role. Both acute pain and euphoric drugs generate an increase in the transmission of dopamine in the brain reward circuit, on the other hand prolonged painful stimuli or prolonged consumption of drugs produce the opposite effect, reducing the motivational system of reward. This mechanism could explain the abuse of fast-acting substances as well as the addition to opioids as a way to regulate the motivation system.

Conclusions.- Psychiatric patients with chronic pain often associate fear, anxiety, depressive symptoms and dependence on substances such as tobacco, alcohol and opioids. It is important to investigate alternative therapies for pain in addition to analgesic drugs, which can reduce the levels of stress associated with pain.

Disclosure of interest.- The authors have not supplied a conflict of interest statement.

\section{E-PV0605 \\ Satisfaction with treatment in chronic pain patients who have participated in a mindful self-compassion program}

M. Torrijos Zarcero, Á. Palao Tarrero, B. Rodríguez Vega, L. Mellado Cuerno*, C. Rocamora González

Hospital Universitario La Paz, Psychiatry, Clinical Psychology and

Mental Health, Madrid, Spain

* Corresponding author.

Background and aims.- Chronic pain is a clinical condition that has impact in the life of patients. Psychological treatments for chronic pain have been developed since the 60's. Cognitive-behavioural treatments have been predominant in the last 30 years. In a previous study our group has demonstrated that Mindful SelfCompassion program is, at least, as effective as CBT in chronic pain. Up to now, research has not been focus on satisfaction of patients with treatments they go through. Nevertheless, IMMPACT recommends to asses such satisfaction.

Methods.- We conducted a MSC intervention. $N=35$ patients with chronic pain recruited in Hospital Universitario La Paz, Madrid. We collected measures of satisfaction with treatment after the intervention. We used a VAS (range from 0 to 10) and CRES-4 (range from 0 to 300$)$. 
We hypothesized that treatment adherence (number of sessions that a participant attends) and satisfaction with treatment were correlated.

Results.- 5,3\% were male and 94,7\% were female. Age mean: 48,34 years old. Satisfaction with treatment mean (VAS) was 8,67. Scores on CRES-4 mean was 213,68. Scores suggest that satisfaction with MSC was high.

We did not find a statistically significant correlation between adherence to MSC treatment and satisfaction with treatment. However, it is important to point that just 1 out of 35 patients of our sample left the treatment.

Conclusions.- MSC is an effective program in order to improve quality of life and to reduce anxiety and depression, as our group has found previously. Moreover, it is also an intervention that patients consider as satisfactory.

Disclosure of interest.- The authors have not supplied a conflict of interest statement.

\section{E-PV0606}

\section{Dependence of pain intensity on the level of anxiety and depression}

G. Shevtsova ${ }^{1 *}$, O. Zagorulko ${ }^{2}$, L. Medvedeva ${ }^{2}$, I. Malenkova ${ }^{2}$

${ }^{1}$ First Moscow State Medical University, Department of Nervous Diseases and Neurosurgery, Moscow, Russia; ${ }^{2}$ National Research Centre of Surgery, Pain Department, Moscow, Russia

${ }^{*}$ Corresponding author.

Background and aims.- The comorbidity of chronic pain and depression leads to negative consequences for the patient and society as a whole. In addition to depressive disorders, patients with chronic pain are characterized by anxious and somatoform disorders that transform and exacerbate the pain experience.

Aims.- Assess the dependence of pain intensity on the level of anxiety and depression on the example of patients with chronic headache (Chronic tension-type headache (CTTH) and chronic migraine $(\mathrm{CM})$ ).

Methods.- Prospective, randomized study included 20 healthy people (control group) and 40 patients with chronic headaches:20 with CTTH and 20 with CM). Hospital Anxiety and Depression Scale (HADS) was used to evaluate anxiety and depression, considering depression or anxiety if the score was $\geq 10$. The intensity of the pain was assessed using a Visual Analogue Scale.

Results.- The intensity of pain in the CTTH and CM groups was $5.3 \pm 0.8$ and $6.9 \pm 0.9$, respectively. In patients in both groups, anxiety and depression in testing were significantly higher than those in the control group and corresponded to clinically significant anxiety and depression. The HADS indices for anxiety and depression subscales in the control group were $2.9 \pm 0.4$ and $1.9 \pm 0.5$, while these values were significantly higher, reaching $14.1 \pm 1.6$ and $16.9 \pm 2.4$ points $(p=0.04)$ for patients with CTTH and CM in the subscale anxiety and $15.2 \pm 2.0$ and $13.1 \pm 1.8$ points $(p=0.03)$ for the depression subscale. The anxiety level was higher in the group of patients with migraine, and more severe depressive disorders were characteristic of patients with CTTH. In the presence of depression, patients experienced higher descriptors of pain at CTTH $(p=0.02)$ and CM $(p=0.04)$.

Conclusions.- Depressive disorders cause higher descriptors of pain in CTTH and CM.

Disclosure of interest.- The authors have not supplied a conflict of interest statement.

\section{E-Poster Viewing - 7-9 April: Personality and Personality Disorders}

\section{E-PV0607 \\ The factorial structure of psychoticism, agreeableness, and conscientiousness among Kuwaiti undergraduates \\ B. Alansari*, T. Alali \\ Kuwait University, Psychology, Kuwait, Kuwait \\ ${ }^{*}$ Corresponding author.}

Background and aims.- Introduction: Psychoticism is related negatively to agreeableness and conscientiousness. Researchers have suggested that psychoticism may be largely redundant with the agreeableness and conscientiousness constructs.

Objectives.- The current study investigated the factorial structure of psychoticism, agreeableness, and conscientiousness amongst undergraduates.

Methods.- Three samples were used to test the hypothesis. Sample 1 consisted of a revised Eysenck personality questionnaire (EPQR) and NEO Five-Factor Inventory - 3 (NEO-FFI-3) which were administered among a total of 921 Kuwait University undergraduates (652 females and 269 males). For sample 2, EPQ-R and Big Five Inventory 44-item (BFI) were administered among 745 Kuwait University undergraduates (412 females and 333 males). Sample 3 consisted of EPQ-R and NEO Personality Inventory-Revised (NEO-PI-R) which were administered among 637 Kuwait University undergraduates (368 females and 269 males). Exploratory factor analysis were calculated.

Results.- For sample 1, one bipolar factor solution explained $57.53 \%$ of the total variance with the loading (.81 for agreeableness, -.77 for psychoticism, .67 for conscientiousness) while sample 2 had one bipolar factor solution that explained $59.80 \%$ of the total variance with the loading (.93 for conscientiousness, .92 for agreeableness, -.26 for psychoticism). Sample 3, however, had one bipolar factor solution that explained $52.29 \%$ of the total variance with the loading (-.76 for psychoticism, .73 for agreeableness, .68 for conscientiousness).

Conclusions.- The results of both sample one and three indicated that psychoticism is merely the opposite of agreeableness and conscientiousness. However in sample two, psychoticism is not merely the opposite of agreeableness and conscientiousness, which warrants for further investigation.

Disclosure of interest.- The authors have not supplied a conflict of interest statement.

\section{E-PV0608}

\section{Further validation of the}

\section{psychological entitlement scale}

A. Araújo ${ }^{1 *}$, A. Pereira ${ }^{2}$, C. Cabaços ${ }^{2}$, C. Marques ${ }^{2}$, N. Madeira ${ }^{2}$, C. Roque $^{2}$, A. Macedo ${ }^{2}$

${ }^{1}$ Faculty of Medicine, University of Coimbra, Institute of

Psychological Medicine, Coimbra, Portugal; ${ }^{2}$ Institute of

Psychological Medicine, Faculty of Medicine, Coimbra University, Portugal, Coimbra, Portugal

Corresponding author.

Background and aims.- Psychological Entitlement Scale (PES; Campbell \& Bonacci, 2013) is a self-reported questionnaire composed of 9 items to evaluate dispositional psychological entitlement, conceived as the perception that one deserves or is entitled to more than others. In our preliminary psychometric study the Portuguese version of PES showed validity (exploratory factor analysis) and reliability as an unidimensional construct. Aims: To further investigate the psychometric properties of the Portuguese version of PES 
using data from a different sample: factor structure using confirmatory factor analysis (CFA), internal consistency, temporal stability and correlations with other potentially associated constructs.

Methods.- A community sample composed of 385 university students $(67.6 \%$ females; mean age $=21.50 \pm 2.382$; range: $17-34$ ) answered Portuguese versions of the following self-reported questionnaires: PES, Dirty Dozen (D12), Big Three Perfectionism Scale (BTPS), and HEXACO-60. To study temporal stability, 94 (65.1\% females) respondents re-answered the PES after approximately six weeks.

Results.- After seven errors were correlated, CFA indicated a good fit for the unidimensional model $(\mathrm{X} 2 / \mathrm{df}=2.382 ; \mathrm{CFI}=0.913$; $\mathrm{GFI}=0.978$, RMSEA $=0.050 ; P[\mathrm{RMSEA} \leq 0.01]<0.001)$. The PES' Cronbach alpha was "very good" $(\alpha=.743)$. The test-retest Pearson correlation coefficient was .782 $(p<.001)$. Pearson correlation coefficients of PES with Narcissism, Psychopathy and Machiavellianism from the D12 were all $r>.35$; with Narcissistic Perfectionism (from BTPS) was $r=.628$; with Honesty-Humility and Agreeableness (from HEXACO-60) was $r=-.484$ and $r=-.215$ (all $p<.01$ ). Conclusions.- PES has good construct and convergent-divergent validity as well as reliability (internal consistency and temporal stability). These results confirm the PES' adequacy for both clinical and research contexts.

Disclosure of interest.- The authors have not supplied a conflict of interest statement.

\section{E-PV0609}

\section{Gender identity disorder or a symptom of borderline personality disorder- a case report}

D. Bosnjak Kuharic ${ }^{*}$, T. Sabo ${ }^{2}$, P. Makaric ${ }^{2}$, Z.I. Surina Osmak ${ }^{3}$, I. Culo $^{1}$, T. Jendricko ${ }^{4}$

${ }^{1}$ University Psychiatric Hospital Vrapce, Department for Diagnostics and Intensive Care, Zagreb, Croatia; ${ }^{2}$ University Psychiatric Hospital Vrapce, Department for Affective Disorders, Zagreb, Croatia; ${ }^{3}$

Ministry of Defense, Croatia, Zagreb, Croatia; ${ }^{4}$ University Psychiatric Hospital Vrapce, Department for Psychotherapy, Zagreb, Croatia ${ }^{*}$ Corresponding author.

Background and aims.- Gender identity disorder (GID) is characterized by a strong and persistent identification with the other gender, followed by a continuous discomfort and dissatisfaction with current gender that can affect social, occupational and other aspects of functioning. Previous studies report high prevalence of comorbid personality disorders with GID, especially narcissistic and borderline personality disorders (BPD). Our aim was to discuss the diagnosis of GID in a patient with BPD and narcissistic traits. Methods.- We present a case report with detailed medical history and diagnostic work-up.

Results.- A 19-year-old female patient was admitted to a psychiatric clinic in Croatia due to psychotic decompensation with intense depersonalization phenomena, imperative auditory hallucinations, suicidality and frequent self-mutilating behavior. In a couple of days, she revealed her cross-gender identification accompanied by feelings of repulsion and discomfort towards her body and started to present as a male with a new, male name. During the treatment, characteristics of BPD with narcissistic elements and impulsive behavior came in the first plan, with frequent changes of therapists and psychiatric clinics later-on. Identification with male gender persisted despite the thoughts that she could never become a perfect man which was her goal concordant with the narcissistic traits of her personality.

Conclusions.- The case of our patient questions the line between identity disorder as a part of BPD and specific disorder in gender and sexual identity. Is it a symptom of BPD, or GID with comorbid BPD diagnosis?

Disclosure of interest.- The authors have not supplied a conflict of interest statement.

\section{E-PV0610}

\section{The influence pathways of hexaco personality traits on emotional intelligence}

C. Cabaços ${ }^{1 *}$, A.T. Pereira ${ }^{1}$, A. Araújo ${ }^{1,2}$, M.J. Brito ${ }^{1}$, N. Madeira ${ }^{1,2}$, A. Macedo ${ }^{1,2}$

${ }^{1}$ Institute of Psychological Medicine, Faculty of Medicine, University of Coimbra, Portugal; ${ }^{2}$ Psychiatry Department, Centro Hospitalar e Universitário de Coimbra, Coimbra, Portugal

* Corresponding author.

Background and aims.- Personality is a set of traits that represents differences among individuals in their tendency to behave, think or feel. The six-dimension personality structure of the HEXACO model includes: Honesty-Humility, Emotionality, Extraversion, Agreeableness, Conscientiousness and Openness-to-experience. The influence of these traits on several outcomes, namely in Emotional Intelligence (EI), is well defined.

EI is the ability to express, perceive and regulate emotions in a way that assists thought. Evidence points towards the existence of three dimensions: Perception and Understanding (PU), Expression and Labelling (EL) and Management and Regulation (MR) of emotion. The aim of this study is to investigate the influence pathways of HEXACO personality traits in MR.

Methods.- 623 university students $(68.4 \%$ females; mean age $=21.42$; range: $17-34$ ) answered the Portuguese versions of HEXACO-PI-60 and the 15-item-Emotional Skills and Competence Questionnaire (ESCQ). Correlation/mediation analyses were performed using SPSS/PROCESS.

Results.- The MR showed negative correlation with Emotionality $(r=-0.15)$ and positive correlation with Extroversion $(r=0.53)$ and Agreeableness $(r=0.21)$, all $p<0.01$. Low Extroversion was a full mediator in the relationship between Emotionality and MR.

Conclusions.- A surprising result was that Agreeableness correlated with MR while Honesty-Humility didn't. As Emotionality is a measure of kin altruism, it was also interesting to assess its negative correlation with MR. When put together in a model where low Extroversion worked as mediator, the direct effect of Emotionality on MR was not significant while total effect was strong and negative, meaning that Emotionality only works as a negative predictor of MR when low levels of Extroversion are present.

Disclosure of interest.- The authors have not supplied a conflict of interest statement.

\section{E-PV0611}

\section{Confirmatory factor analysis of the Portuguese version of the dirty dozen}

A. Macedo ${ }^{1,2}$, A. Araújo ${ }^{1,2}$, C. Cabaços ${ }^{1 *}$, C. Marques $^{1}, \mathrm{~N}$.

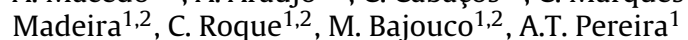

${ }^{1}$ Institute of Psychological Medicine, Faculty of Medicine, University of Coimbra, Coimbra, Portugal; ${ }^{2}$ Psychiatry Department, Coimbra Hospital and University Centre, Coimbra, Portugal

* Corresponding author.

Background and aims.- The Dark Triad refers to three socially aversive personalities - Narcissism, Psychopathy and Machiavellianism - characterized by a behavioral style oriented towards self-benefit through socially exploitative dispositions. Whereas these three constructs may be conceptualized as aspects of an unitary model ("unification theories"), there are specificities that aren't consistent among them ("discrimination theories"). Given an increased scientific interest on the dark triad, Jonason and Webster (2010) developed a 12-item-questionnaire to evaluate these three independent-yet-related constructs - the Dirty Dozen (D12). In our preliminary psychometric analysis, the Portuguese version of D12 showed good validity (exploratory factor analysis) and reliability. 
Aim.- to examine the factorial structure with confirmatory factor analysis (CFA) and divergent validity of the Portuguese version of the D12 in a different sample.

Methods.- 387 university students $(67.5 \%$ females; mean age $=21.50 \pm 2.375$; range: $17-34$ ) answered the Portuguese versions of the following self-reported questionnaires: Psychological Entitlement Scale (PES), D12, Big Three Perfectionism Scale (BTPS), and HEXACO-60.

Results.- CFA indicated a good fit for the second-order factor $\quad\left(X^{2} / \mathrm{df}=2.565 ; \quad\right.$ CFI $=0.937 ; \quad \mathrm{GFI}=0.945, \quad$ RMSEA $=0.064$; $P[$ RMSEA $\leq 0.05]<0.001)$. The D12's Cronbach alpha was "very good" $(\alpha=.743)$. Pearson correlation coefficients of D12 with PES was $r=.457$; with honesty-humility, agreeableness and conscientiousness (from HEXACO-60) were $r=-.541, r=-.321$ and $r=-.213$ (all $p<.01$ ), respectively; and with narcissistic perfectionism (from BTPS) was $r=.430$.

Conclusions.- Both the unidimensional and the three-dimensional models of the Portuguese version of the D12 presented good construct and convergent-divergent validity as well as reliability (internal consistency). Therefore, we consider that it would be clinically meaningful to analyze which levels of psychometric aggregation predict which specific outcomes.

Disclosure of interest.- The authors have not supplied a conflict of interest statement.

\section{E-PV0613}

\section{The child and adolescent mindfulness measure spanish adaptation (CAMM-S): internal consistency and retest reliability}

E. Fernández Jiménez ${ }^{1 *}$, V. Fernández Jiméne ${ }^{2}$

${ }^{1}$ Hospital Universitario La Paz, Servicio de Psiquiatría, Psicología

Clínica y Salud Mental, Madrid, Spain; ${ }^{2}$ PSYC Dissemination,

International Institute for the Dissemination of the Research in

Psychology, Córdoba, Spain

Corresponding author.

Background and aims.- Instruments that assess mindfulness are fundamental to examine if the outcomes after mindfulness-based interventions are attributable to changes in the ability to respond mindfully to the experiences, feelings and thoughts. Although there is a previous Spanish adaptation available, the present study aims to examine an improved Spanish version of the Child and Adolescent Mindfulness Measure (CAMM-S). This is the first study to assess the retest reliability of a Spanish version of the CAMM in an adolescent sample.

Objectives.- To examine the internal consistency and retest reliability of an improved Spanish version of the CAMM in a sample of non-clinical adolescents.

Methods.- A sample of 516 adolescents (52.7\% females) from the two high schools of Marchena (a town in the Province of Seville in Andalusia, Spain) was enrolled. Participants' age ranged from 14 to 19 years (mean age: 16.15; SD: 1.23 ). The CAMM was adapted to Spanish by using a forward and back translation process, under the guidance of the developers of the original version of the CAMM. The final Spanish adaptation of the scale was pilot tested to check if the items were correctly understood. To assess internal consistency reliability of the CAMM-S, alpha coefficient and mean inter-item correlation (MIC) were computed. Retest reliability was computed 12 months after the first administration.

Results.- Cronbach's alpha $=0.80$ and MIC $=0.28$, within the good and optimal range, respectively. Retest correlation $(n=340)$ was 0.57 .

Conclusions.- The CAMM-S is a reliable measure to assess unidimensionally trait mindfulness in adolescents.

Disclosure of interest.- The authors have not supplied a conflict of interest statement.
E-PV0614

\section{Borderline personality disorder and alcohol use - talking about a close} relationship

S. Dehanov, T. Ferreira*, J. Ramos, T. Maia

Hospital Prof. Doutor Fernando da Fonseca, Department of

Psychiatry, Amadora, Portugal

Corresponding author.

Background and aims.- According to the literature there is an important comorbidity between borderline personality disorder (BPD) and alcohol use disorder (AUD). Estimates suggest that approximately half of BDP patients meet criteria for a co-occurring AUD. Both genetic and individual specific environmental factors seem to contribute to this relationship, and some models have been proposed to explain this comorbidity. Our aim is to understand the relationship between BPD and AUD and the current treatment options.

Methods.- Review of the literature from Pubmed database.

Results.- Emotion dysregulation and impulsivity seem prominent features in the aetiology of both PBP and AUD, which may manifest common aetiological processes. According to the proposed models to date: there is evidence that the personality trait of impulsivity is associated with stress-reducing properties of alcohol (pharmacological vulnerability model); alcohol may be used to regulate negative affect (negative affect regulation model); impulsivity may lead to problems in socialization, which are associated with substance abuse (deviance proneness model). Despite the evidence of a high degree of comorbidity there are still few treatment options studied. Psychotherapies such as Dialectical Behavior Therapy-S (DBT-S), Dual Focus Schema Therapy (DFST) and Dynamic Deconstructive Psychotherapy (DDP), have been developed specifically for patients with this comorbidity. When it comes to pharmacotherapy, few drugs have been tested. Drugs such as nalmefene, disulfiram, topiramate, lamotrigine, olanzapine and aripiprazol may be benefitial.

Conclusions.- There is undoubtedly a relationship between PBP and AUD, which is still poorly understood despite its high prevalence. Comorbid models have been proposed, but treatment options need further investigation.

Disclosure of interest.- The authors have not supplied a conflict of interest statement.

\section{E-PV0615}

\section{Correlation between PSY-5/RORSCHACH indexes and disorganized attachment}

D. Galletta*, A.M. Mastrola, A. Cotena, V. Suarato

U.O. Federico II, Neuroscience and Reproductive and

Odontostomatological Sciences, Naples, Italy

${ }^{*}$ Corresponding author.

Background and aims.- Objective of the work is research any correlation between specific descriptive characteristic of the disorganized attachment model, as a specific dimension of the borderline personality disorder diagnosticate by SCID-II and other disorder in comorbidity.

Methods.- We extrapolated from a larger pilot study, eighteen (two male and sixteen famale) of most significant protocols. All patients, are subjected to a battery of tests as structured: MMPI-2, SCL-90-R, WAIS-R, RORSCHACH TEST.

We have focused on the elevation of clinical scales and content scales of MMPI-2, according to PSY-5 method, and some specific indices emerged by the analysis of RORSCHACH TEST as affective indices, impulsivity and self-control indexes, reality index. Results.- The analysis of Rorschach test reported an AFFECTIVITY INDEX oriented to closing, a TVI restricted, an index of significant 
impulsiveness, deficient mechanisms of self-control and structural rigidity, it could be associated with closing mechanisms, difficulty in relationship, use of mechanism of dissociation and other primitive defense mechanisms; While, in MMPI-2, significant elevation of Sc scale with specific Harries and Lingoes subscale, and clinic elevation of PSYC and NIEGE scale reported thought disorder, impulsivity and emotional discontrol, relational difficulties and a tendency to isolation characteristics that seem to converge with a disorganized style of attachment, structured in childhood.

Conclusions.- In conclusion, data show a correlation between test indexes and characteristics of disorganized attachment pattern, and open up further Hypothesis of correlation between that attachment pattern and other disorders in comorbidity.

Disclosure of interest.- The authors have not supplied a conflict of interest statement.

\section{E-PV0616}

\section{Security value is not related to decision-making in health dilemmas in youth \\ G. Arina, M. Iosifyan", L. Pechnikova, V. Nikolaeva}

Moscow State University, Department of Psychology, Moscow, Russia ${ }^{*}$ Corresponding author.

Background and aims.- The study investigated values as factors of decision-making in health dilemmas in youth. It was hypothesized that values are associated with decision-making in health dilemmas. A health dilemma is a conflict in which one has to choose between health or other valued outcomes. There were three types of health dilemmas: a conflict between (1) health and self-direction, (2) health and achievement, (3) health and benevolence. For example, one has to choose between curing a dangerous illness or gaining a significant career achievement.

Methods.- Participants ( $N=46$, age range $19-22, M=20.65)$ solved the dilemmas. They also performed Schwartz Value Survey which measured their values of security, achievement, benevolence and self-direction.

Results.- A regression analysis was performed, security values and antagonistic values (self-direction, benevolence and achievement) were independent variables, while decision in health dilemmas was a dependent variable. Age and sex were included as covariates. The model explained $18 \%$ of variance. Values of self-direction, benevolence and achievement were significantly related to decisions in health-dilemmas $(\beta=-.25, t(115)=-2.31, p=.023)$, while security value was not significantly related to them $(\beta=-.06, t(63)=-.33$, $p=.743$ ).

Conclusions.- The study shows that the choice between health and concurring valued outcomes (such as benevolence, self-direction and achievement) is not associated with personal importance of security values among young people. This choice is rather associated with personal importance of other values which are not related to health directly. These variables can be used as targets for future health preventive programs.

Disclosure of interest.- The authors have not supplied a conflict of interest statement.

\section{E-PV0619}

\section{Paradoxical effects of ssri antidepressants when treating mood dysregulation in borderline personality disorder}

\section{Lazzari}

International Centre for Healthcare and Medical Education ICHME, Department of Psychiatry, Wakefield, United Kingdom

${ }^{*}$ Corresponding author.
Background and aims.- Antidepressants of the SSRI (Serotonin System Reuptake Inhibitors) are frequently prescribed in primary and secondary care to address depression. SSRI antidepressants are also frequently prescribed in patients with a borderline personality disorder (BPD). However, the author noticed paradoxical effects in the combination SSRI-BPD.

Methods.- The aim of psychiatric teams in primary and secondary care is to stabilize the presentation and to reduce deliberate selfharm and suicidal ideation in patients with BPD. Patients with BPD have many mood dysregulations with dysthymia or cyclothymia. The BPD patients' narratives used for the study provided some evidence or paradoxical effects when SSRI antidepressants are used to address mood dysregulation in BPD.

Results.- Patients with BPD treated with SSRI antidepressants are at high risk of presenting with dose-related behavioral symptoms of serotonin activation including increased impulsivity, increased suicidal ideation and acts, increased violence towards others, increased deliberate self-harm, and increased agitation.

Conclusions.- The use of SSRI antidepressants in BPD should receive a warning reflection while the use of more sedative mood stabilizers should be considered instead.

Disclosure of interest.- The authors have not supplied a conflict of interest statement.

\section{E-PV0621}

\section{Attachment to parents and psychological distress: the mediating role of perfectionism}

B. Maia ${ }^{1 *}$, H. Moreira $^{2}$, M. Marques ${ }^{3}$, M.J. Soares ${ }^{4}$, A.T. Pereira ${ }^{4}$, A. Macedo 4

${ }^{1}$ The Catholic University of Portugal, Faculty of Philosophy and Social Sciences, Braga Regional Centre, Braga, Portugal; ${ }^{2}$ Faculty of Psychology and Educational Sciences, University of Coimbra, Center for Research in Neuropsychology and Cognitive-Behavioral Intervention, Coimbra, Portugal; ${ }^{3}$ Coimbra Hospital and University Centre, Coimbra Hospital and University Centre, Coimbra, Portugal; ${ }^{4}$ Faculty of Medicine University of Coimbra, Institute of Psychological Medicine, Coimbra, Portugal

* Corresponding author.

Background and aims.- Previous studies focused mainly on exploring, separately, the association between perfectionism and attachment and perfectionism and psychological distress. This study aims to explore the mediating role of perfectionism (selforiented, other-oriented, and socially prescribed) in the association between attachment orientations (anxiety and avoidance) to mother and to father and anxiety and depression.

Methods.- The sample was composed by 126 university students with a mean age of 20.07 years old $(S D=1.60$; range: $17-25)$; the majority was single (98.5\%) and female (74.6\%). Participants filled a sociodemographic questionnaire, the Portuguese versions of the Experiences in Close Relationships - Relationship Structures Questionnaire, the Hewitt and Flett Multidimensional Perfectionism Scale and the Depression Anxiety Stress Scale.

Results.- The examination of the indirect effects showed that only socially prescribed perfectionism mediated the association between attachment and depression. Specifically, socially prescribed perfectionism mediated the link between avoidance to mother and depression and between anxiety to father and depression. Moreover, socially prescribed perfectionism mediated the association between avoidance to mother and to father and anxiety. Conclusions.- Maladaptive perfectionism is an important mediator between adult attachment anxiety and avoidance and psychological distress. If our results are confirmed in future studies, psychological interventions may aim to reduce maladaptive perfectionism in situations in which it may not be feasible to intervene in attachment insecurities. 
Disclosure of interest.- The authors have not supplied a conflict of interest statement.

\section{E-PV0622}

\section{Dynamics of mental states' representations in conditions of experimental influence}

A. Nazarov", A. Prokhorov Oktyabrinovich, A. Chernov Valentinovich, M. Yusupov Gennadjevich, M. Valiullina Evgenjevna, A. Khalfieva Ramiljevna

Kazan Volga region Federal University, General Psychology, Kazan, Russia

Corresponding author.

Background and aims.- Mental representations of mental states, which are understood as internal structures formed in the process of a person's life, are under-researched in terms of their stability and changes. Understanding them is a prerequisite for an effective counseling therapy. The article presents the results of a study focused on the dynamics of evaluative, associative and imaginative characteristics.

The aim of the study was to study the changes in mental representations of mental states.

Methods.- The study was organized during lessons. Associative, evaluative and imaginative characteristics of mental representations were studied with the use the implicit associative test, the semantic differential method, and the mental state relief method developed by Prokhorov. Students entered a relaxed state with the use of the Schultz technique, Everly-Rosenfeld technique, original meditative techniques and the mobilization method developed by Alekseev. 27 people (age 18-19) took part in the experiment that lasted 6 months.

Results.- It was found that the results of relaxing or activating effects on mental states representations may depend on the nature of the technique used, as well as on the individual characteristics of participants: the Everly-Rosenfeld neuromuscular relaxation technique had a greater impact on physiological manifestations, whereas autogenously immersion had an effect on sensations, perceptions and memory, behavioral orientation, muscle tone, and made a mobilizing impact on imaginative characteristics.. After conducting meditations, the intensity of mental representations of all states among students reached optimal values.

Conclusions.- The changes that were found can be described as a tendency. The invariant and unstable characteristics of mental representations were identified.

Disclosure of interest.- The authors have not supplied a conflict of interest statement.

\section{E-PV0623}

\section{Dynamics of students' cognitive states with different level of reflection}

A. Nazarov ${ }^{*}$, A. Prokhorov Oktyabrinovich, A. Chernov Valentinovich, M. Yusupov Gennadjevich, M. Valiullina Evgenjevna, A. Khalfieva Ramiljevna

Kazan Volga region Federal University, General Psychology, Kazan, Russia

${ }^{*}$ Corresponding author.

Background and aims.- Reflection takes a special place in the process of educational activity, determining the manifestations of mental states. In the process of learning, reflection acts as a mechanism of self-knowledge, self-organization, self-determination and the basis for the independent activity of students. The formation of reflective abilities is especially necessary for productive educational activity. The purpose of the study is to identify the dynamics of cognitive states in the course of a lesson for students with different levels and focus of reflection.
Methods.- Three groups of students with different (high, medium and low) levels of reflection were formed. Using the questionnaire during the seminars, we studied the intensity and frequency of the cognitive states' manifestation in dynamics (in the beginning, in the middle and at the end of the session).

Results.- Were identified the most often experienced by students' cognitive states. Most of them are positive, absenteeism is presented from the negative. The general dynamics of cognitive states in the seminar is revealed. It is shown that the most optimal cognitive states are experienced by students with a low level of introspection and an average level of reflection of activity. The maximum intensity of cognitive states experienced by students is achieved by a combination of an average level of activity reflection and introspection.

Conclusions.- The obtained data will allow teachers to take into account the direction and level of expressiveness of reflectivity to achieve positive cognitive states during they work with students. Disclosure of interest.- The authors have not supplied a conflict of interest statement.

\section{E-PV0624}

\section{Psychosis or schizotypal personality disorder. Schizotypy is a term to be valued}

J. Pemán Rodríguez*, J.E. Ibáñez Vizoso, M. Villanueva Gallego, F.J. Gómez Beteta, L. Gallego Deike

Hospital Clínico San Carlos, Psychiatry, Madrid, Spain

* Corresponding author.

Background and aims.- Schizotypical personality disorder (PD) is difficult to diagnose due to the overlapping symptoms with other clinical entities.

Objectives.- To illustrate this difficulty with a clinical case and to recover the term schizotypy in a well-argued manner.

Methods.- We present the case of a 30-year-old male who consults for excessive social anxiety secondary to diffuse paranoid fears that make him have fantasies of escaping from his usual environment and that cause difficulties in interpersonal relationships and difficulty in getting a job once he has gotten rid of cannabis. Reactive to this anguish has had escapes to other cities or countries in the past that have meant for him to be maintained street situation for months or the need to prostitute himself consuming cannabis in high dose. He alternates with these escapes, incomes in therapeutic communities, cannabis consumption and hospitalizations by diffuse psychotic clinic and obsessive ruminations mainly of sexual content. The last admission was three years ago where he was diagnosed with polymorphous psychotic disorder and unspecified PD.

Results.- He is diagnosed of schizotypal PD and starts a treatment with olanzapine $2.5 \mathrm{mg}$ and being refer to a day hospital, where progressive improvement is observed. After 9 months he is asymptomatic and has recovered the family bond and works.

Conclusions.- The differential diagnosis of psychotic symptoms is not always simple but has prognostic implications. Schizotypy, a disused term, raises schizophrenia and schizotypal PD as part of a continuum, which is closer to clinical practice.

Disclosure of interest.- The authors have not supplied a conflict of interest statement.

\section{E-PV0625}

Non-suicidal self-injury in patients with narcissistic personality disorder in adolescence

E. Krylova ${ }^{1}$, A. Beburishvili ${ }^{1}$, I. Pluzhnikov ${ }^{2}$

${ }^{1}$ The Mental Health Research Center MHRC, The Mental Health Research Center MHRC, Moscow, Russia; ${ }^{2}$ The Mental Health 
Research Center MHRC, Department of Young Adult Psychiatry, Moscow, Russia

${ }^{*}$ Corresponding author.

Background and aims.- The aim of the presented research is to determine NSSI clinical psychopathological features in patients with narcissistic personality disorder in adolescence.

Methods.- Clinical psychopathological interview. Sample $N=57$ males adolescents (age: 16-25) with:1. Adolescent depression in narcissistic personality disorder and 2. Episodes of Non-suicidal self-injury in narcissistic personality disorder.

Results.- NSSI in patients with narcissistic personality disorder in adolescence develops affective lability due to identity crisis, changes of social stereotypes, different environmental factors. NSSI data has clear age specificity - polymorphism, connections with fixed ideas and different comorbid psychopathological syndromes, and followed behavioral problems, existential or/and body dysmorphic fixations (overvalued ideas) and high rate of suicidal tendencies (68.5\%). Based on psychopathological differences, several types of NSSI in patients with narcissistic personality disorder in adolescence were described: "demonstrative» $(N=15,26$, $3 \%)$, «anxious» ( $N=14,24,6 \%)$, «depersonalization» $(N=11,19,3 \%)$, "compulsive" $(N=8,14,0 \%)$, "impulsive» $(N=9,15,8 \%)$. Several correlations between NSSI in patients with narcissistic personality disorder in adolescence and comorbide syndromes:

- «anxious» и «depersonalization» NSSI connected with depressive syndrome

- "demonstrative» and "impulsive» NSSI associated with pathological personality features such as: conviction of their own mightiness, egocentrism, the need for praise

- "compulsive» NSSI appear with pathological thinking phenomena - fixed ideas and obsessive reactions, often filled by adolescent existential and / or dysmorphic content.

Conclusions.- Conclusion: Observed and clincally described NSSI typology of patients with narcissistic personality disorder in adolescence. It encourage more accurate evaluation of related clinical conditions in order to develop more specific approach to psychopharmacotherapy. In addition mental healthcare professionals may produce more detailed prognostic profile.

Disclosure of interest.- The authors have not supplied a conflict of interest statement.

\section{E-PV0626 \\ Effects of psychopharmacological treatment versus psychological intervention, in patients with borderline personality disorder (bpd) during a crisis of emotional instability}

S.S. Sanchez ${ }^{*}$, L. Soldado R.

Hospitalario de Jaen, UGC Salud Mental, Jaen, Spain

${ }^{*}$ Corresponding author.

Background and aims.- Patients diagnosed with BPD are complex patients due to their own psychopathology (unstable personality) and a surrounding conflict environment. Emergency consultations, due to "anxiety" or "depression", usually respond to specific situations of daily life ("crisis"), which do not depend on medication to improve. These patients can be treated by several professionals, with a combination and high doses of psychotropic drugs, and added risks of self-medication, abuse or impulsive intake.

- To determine benzodiazepines effects and if it is comparable to psychological intervention in emergency care, avoiding changes in usual treatments during crisis.

Methods.- Sample of 20 patients BPD aged between 18 and 45 in medical consultation due to "crisis" situation.

- Use of Symptoms-Check-List-90-R scale (SCL-90), seven symptoms selected for anxiety and depression.
- Score: (0-9 points mild symptoms, 10-19 moderate, 20-28 severe).

- Psychotherapy techniques are applied to all patients.

- After the crisis, patients with $>20$ points are sent to their mental health center for attention and a new assestment of SCL-90 scale. Results.-

- Patients presented scores from 14 to 28 points (average of 20.9 points).

- Most of patients exceeded 20 points in the emergency room (60\%); only $10 \%$ after the crisis.

- About $40 \%$ of patients required to increase the dose of their usual treatment, or new treatments added to psychological intervention.

Conclusions.- As scores in SCL-90R decrease subjectively after the "crisis", symptoms do not always require a change of treatment in these patients. Psychotherapy techniques are considerated an useful method in Emergency Psychiatry, relieving the specific symptoms and allowing a more standardized evaluation of the patient by their reference specialist.

Disclosure of interest.- The authors have not supplied a conflict of interest statement.

\section{E-Poster Viewing - 7-9 April: Philosophy and Psychiatry}

\section{E-PV0629}

The death drive in suicidal terrorism

A. Albassam ${ }^{1 *}$, A. Tyagi ${ }^{2}$

${ }^{1}$ Bronxcare Health System, Psychiatry, New York, USA; ${ }^{2}$ Northwell

Health, Psychiatry, New York, USA

* Corresponding author.

Background and aims.- Although the Death Drive or the Thanatos has been a subject of debate among psychoanalysts during Freud's lifetime and after, many successors of Freud recognized the importance of the death drive in psychoanalysis. They developed their own theories for its application in practice. It has largely contributed to a new understanding of the psychopathology of sadomasochism, repetition compulsion, narcissistic and borderline personality disorder.

Methods.- In our presentation we discuss the applications of the death drive to modern suicidal terrorism and the mechanisms underlying the transformation process of the erotic drive into the death drive in that group.

Results.- By reviewing many examples of terrorist attacks we found a common process where the primitive impulses behind the destruction of self and others are triggered through a decathexized libido suspended in perpetual tension within the self until releasing its energy in the destructive act. The ensuing act of sadomasochistic annihilation would resolve all tensions of suppressed libido in the orgasmic act of destructive suicide.

Conclusions.- This theory and presentation on our part is an attempt to address modern suicide terrorism psychoanalytically where modern psychiatry, sociology and political science have fallen short of interpreting and integrating a phenomenon which has taken its toll on our modern world. This attempt is based on the Freudian concept of the death drive which was quite advanced and misunderstood for its time, yet it addresses a significant cultural challenge in this cataclysmic epoch of chaotic violence and disruptive global change.

Disclosure of interest.- The authors have not supplied a conflict of interest statement. 
E-PV0631

\section{Genetic determinism, psychiatry and place}

M. Gildersleeve* , A. Crowden

University of Queensland, Historical and Philosophical Inquiry, St

Lucia, Australia

${ }^{*}$ Corresponding author.

Background and aims.- We review genetic determinism and highlight how our earlier research on psychoanalysis and the philosophy of place (see Gildersleeve, Crowden, 2018) can contribute to a better understanding of its relationship to psychiatry.

Methods.- We show how integrating place with psychoanalysis can undermine any philosophy of genetic determinism. We achieve this by demonstrating the prevalence of genetic determinism in the media, education and in debates on genetic modification. We also utilise research on the film GATTACA to conceptualise how place and genetic determinism contrast and why place coupled with psychiatry are an important antagonist to the ideology of genetic determinism.

Results.- By using our philosophy of place, our investigation highlights how psychiatry can contribute to a call for humanity to turn toward a "collective being-at-home-in-the-world", instead of being estranged from place which the rhetoric of genetic determinism unconsciously but actively promotes.

Conclusions.- Our examination intends to integrate psychiatry into ongoing discussions about genetic determinism as they play out in the media, education and influence discrimination. We show something that has not been considered before. Specifically, our philosophy of place combined with psychoanalytic principles shows the potentially dangerous implications of genetic determinism. Fundamentally, we argue that genetic determinism leads to mental illness through the formation of psychopathological obstructive complexes because an individual is estranged from their authentic place in the world. We show the media and educational programs plays an important role in this and why scholars in these areas should consider place and genetic determinism alongside psychiatry in their future theoretical and practical endeavours. Disclosure of interest.- The authors have not supplied a conflict of interest statement.

\section{E-PV0632}

\section{Correlation between epistemological beliefs with 12 motivational selves system, foreign language anxiety and foreign language learning among girls students}

A. Homayouni ${ }^{1^{*}}$, M. Zaremarzoni ${ }^{2}$

${ }^{1}$ Department of Psychology, Bandargaz Branch, Islamic Azad University, Bandargaz, Iran; ${ }^{2}$ PHD in Educational Psychology, Iran

* Corresponding author.

Background and aims.- Learning a foreign language, especially English language, is an important subject in educational institutions and even all around the world. Also foreign language acquisition is one of complicated and influential concepts in studies on foreign language learning. So, the aim of this study was to investigate correlation between epistemological beliefs with L2 Motivational Selves System, foreign language anxiety and and foreign language learning among girls students.

Methods.- The research method was correlation. Statistical population consisted of high school girls students that 106 students were selected and answered to Schommer's epistemological beliefs inventory, Papi's L2 motivational selves system questionnaires and Horwitz's foreign language classroom scale anxiety. Also, the students' semester final exam English language scores were used as criterion for foreign language learning. The Pearson Correlation formula was used to analyze the data.

Results.- The findings showed that subscales of epistemological beliefs (simple/definite knowledge, and rapid/stable learning) had negatively and significantly correlation with L2 motivational selves system subscales (ought to self, ideal self, intended effort, and language learning experience), and had negatively and significantly correlation with foreign language learning. Also subscales of epistemological beliefs had positively and significantly correlation with subscales of foreign language anxiety (communication apprehension, test anxiety, fear of negative evaluation).

Conclusions.- According to results, it is advised that the educational administers and teachers should plan extra curriculum and training sessions to change and enhance the epistemological beliefs in order to increase motivation to learn foreign language and decrease foreign language anxiety for students that live in EFL settings and environments.

Disclosure of interest.- The authors have not supplied a conflict of interest statement.

\section{E-PV0633}

\author{
Unitarian psychosis theory. one \\ invincible defeat? \\ M.J. Juan José \\ Juan José Martínez Jambrina- Psychiatrist, Head of Mental Health \\ Service, Avilés, Spain \\ ${ }^{*}$ Corresponding author.
}

Background and aims.- The purpose of this research is to rethink the issue of unitary psychosis from a psychopathological view. Essential to perform this exercise are theoretical developments from Bartolome.

Llopis, an ancient spanish psychiatrist, who passed away 52 years ago, but which a very rich work mainly regarding to the unitarity of all the psychosis.

Methods.- A important trend in psychiatry is trying to avoid the Kraepelinian dichotomy schizophrenia vs. manic- depressive illness and using the label 'psychosis' to appoint the spectrum of severe mental disorders. Some investigators like G Stanghellini have shown that the characteristics of psychotic symptoms vary across different diagnostic categories. They have compared i.e. delusions in schizophrenia and major depression and demonstrated how these phenomena differ under these two psychopathological conditions. The identification of specific types of delusions is principally achieved through the differential description of subjective experiences.

Results.- While asserting the existence of a unitary psychosis is controversial with current available data, it is equally true that the assertion of a schizophrenia/bipolar disorder dichotomy is also difficult to sustain. However, the axial syn-drome common to all psychoses, Bartolomé Llopis's brainchild, re-mains a fertile and very interesting idea.

Conclusions.- Unitarian psychosis is one the main problems in the psychopathological's theories. We think that reading authors like Llopis, Rennet, Guislain and Janzarik may be useful to rethink theories to explain the differences between different types of psychosis. Disclosure of interest.- The authors have not supplied a conflict of interest statement. 


\section{E-PV0634}

\section{Polish scholars and thinkers: a critical celebration of their contributions to 20th century russian psychiatry and psychology}

V. Krasnov ${ }^{1 *}$, N. Semenova ${ }^{2}$

${ }^{1}$ Moscow Research Institute of Psychiatry - subsidiary The Serbsky Federal Research Center for Psychiatry and Narcology, Affective Disorders, Moscow, Russia; ${ }^{2}$ Moscow Research Institute of Psychiatry - subsidiary The Serbsky Federal Research Center for Psychiatry and Narcology, Outpatient Psychiatry, Moscow, Russia

${ }^{*}$ Corresponding author.

Background and aims.- Introduction: Polish scholars and thinkers have made fundamental contributions to methodology, theory and practice of psychiatry, and psychology in Russia and in the Soviet Union.

Objectives.- The paper brings together scholars from Poland to critically celebrate some of the many contributions they have made to the disciplines in Russia's historical and educational context.

Methods.- The present paper comprises seven essays of Polish scholars (Ivan Balinsky, Antoni Kempinski, Andrzej Yakubik, Stefan Leder, Kazimierz Obukhovsky, Janusz Reikowski, and Jerzy Mellibruda). The details of their original publications are provided. The contributions draw upon different theoretical perspectives and use various methodologies.

Results.- Key Points: The aim is to present how, following different theoretical paths and using different methods, Polish scholars have contributed into the understanding of the mental health issues as well as the curriculum re-design, e.g., in clinical psychology training. The results will highlight the common grounds as well as the diversities of the contributions and enlarge the debate inviting contributions from the audience.

Conclusions.- Conclusions: The historical importance and contemporary relevance of the work of Polish scholars and thinkers is made clear and their massive and multi-faceted contribution to the discipline celebrated. The paper illustrates their massive contributions to the disciplines of psychiatry and clinical psychology in the 20th century and the continuing relevance of their work for the $21 \mathrm{st}$ century.

Disclosure of interest.- The authors have not supplied a conflict of interest statement.

\section{E-PV0635}

\section{Phylosophical, psychological, medical aspects of a pilot's image}

N. Lyssakov", E. Lyssakova

Moscow Aviation Institute National Research University, Institute of Engineering Economics and Humanities, Monino, Russia

${ }^{*}$ Corresponding author.

\section{Background and aims.-}

Introduction.- A pilot's image is regarded as an interdisciplinary phenomenon. Philosophical aspect is connected with the cultural and historical assessments of aviators and aviation profession. Psychological aspect is first of all, study about the composition of pilots' professionally important qualities. Medical aspect is the analysis of pilots' attitudes to professional deformation, their physical and mental problems.

Objectives.- Identification of a pilot image features in our generation. Determination of its development directions in the history of aviation. Formulation of system recommendations.

Methods.- Research methods were used: students' interviews about a pilot, analysis of fiction and cinematography devoted to aviation. Respondents $(N=146)$ were asked: "What personal qualities do you think a civil aviation pilot has?" Comparative analysis of two films was conducted: both have the same title "Flight" (released in
1979 and its remake in 2016), where the pilots operated in emergency situations, was conducted. Pilots characteristics in fiction were studied.

Results.- There is strengthening and fixing dynamics of sciencebased characteristics in a pilot's image. Attitude to the aviation profession as a socially significant and responsible one is formed. However, opinion about a pilot as addicted to alcohol (student interviews) and as a superhero (analysis of cinematography) were discovered. These facts indicate a distortion of a pilot's image.

Conclusions.- Conclusions Possible rrecommendations are in the following areas: 1) prevention and correction of mental health in pilots; 2) aviation psychology; 3) professional orientation of schoolchildren; 4) organization of cultural and educational work with the population.

Disclosure of interest.- The authors have not supplied a conflict of interest statement.

\section{E-PV0636}

\section{Methodology of psychological} syndrome analysis (Vygotsky-Luria school) and biopsychosocial model of health and disease: prospects for mutually enriching relationships

E. Pervichko*, Y. Zinchenko

Lomonosov Moscow State University, Faculty of Psychology, Moscow, Russia

* Corresponding author.

Background and aims.- The Biopsychosocial model (Engel, 1977) has been seen as a powerful theoretical basis of clinical and health psychology, and psychosomatic medicine. It is acceptable to assume that the basic theoretical and methodological developments of the Psychological Syndrome Analysis (PSA) concept (Vygotsky-Luria School) can contribute to the operationalization of the Biopsychosocial model and further uncover its creative potential.

The purpose of this research was to demonstrate methodological readiness of the concept of PSA to go in tune with Biopsychosocial model.

Methods.- The main method of presented research was methodological reflection upon main points of G. Engel's biopsychosocial model and scientific legacy of L. Vygotsky and A. Luria.

Results.- PSA embrace the idea of a syndrome as a structure shaped by a constellation of causally-related, multilevel symptoms (Vygotsky, 1934; Luria, 1972). Our results argue that main provisions of the PSA concept correspond to the principles of postnonclassical model of scientific rationality. The PSA concept is highly efficient at the current state of the evolution of science due to the potential of the new methodological context of the Postnonclassical model of rationality and a comprehensive character of Cultural historical concept proposed by L.S. Vygotsky and A.R. Luria regarding the person and his/her mind as a self-developing open systems. Using these methodological principles we described the psychosomatic syndromes in patients with mitral valve prolapse and in patients with stress-induced hypertension (Zinchenko, Pervichko, 2014, 2016; Pervichko, 2016).

Conclusions.- The PSA concept go in tune with Biopsychosocial model and personalized care models. This opens up prospects for mutually enriching relationships.

Disclosure of interest.- The authors have not supplied a conflict of interest statement. 
E-PV0637

\section{Genealogy, lineage and death of the concept of schizophrenia}

P. Sousa Martins*, V. Pimenta

Unidade Local de Saúde do Nordeste, Departamento de Psiquiatria e

Saúde Mental, Bragança, Portugal

${ }^{*}$ Corresponding author.

Background and aims.- The concept of schizophrenia has remained almost immutable since Emil Krapelin first coined the term dementia praecox more than a century ago. It is, however, a concept that has gradually become obsolete in the clinical setting. Now that advancements in neuroimaging, epidemiology and epigenetics have reshaped the nosological entity encompassed by the concept of schizophrenia, the need for a linguistic redefinition is pressing. The aim of this intellectual exercise is to understand how the concept of schizophrenia first came to be, how it evolved over the course of the twentieth century and what its fate will be based on the current tenets of psychiatric thinking.

Methods.- A selective revision of literature taken from the data base PubMed was made using the keywords "schizophrenia", "psychotic spectrum", "classification", "concept" and "linguistics, complemented with the reading of classic psychiatry writings.

Results.- There is overwhelming evidence pointing to the fact that the concept of schizophrenia as it is classically defined is an outdated nosological entity and represents only a small portion of the multidimensional psychotic spectrum.

Conclusions.- An alliance between psychopathology, linguistics and philosophy will permit us to redefine and even rename what is encompassed by the term "schizophrenia" in order to make it clinical relevant in the current paradigm of psychiatric practice.

Disclosure of interest.- The authors have not supplied a conflict of interest statement.

\section{E-Poster Viewing - 7-9 April: Posttraumatic Stress Disorder}

\section{E-PV0638}

\section{Comparing occupational stressors and the mental health of male and female canadian correctional officers}

A. Bahji $1^{*}$, R. Ricciardelli ${ }^{2}$, N. Carleton ${ }^{3}$, D. Groll ${ }^{1}$

${ }^{1}$ Queen's University, Department of Psychiatry, Kingston, Canada; ${ }^{2}$ Memorial University of Newfoundland, Department of Sociology, St John's, Canada; ${ }^{3}$ University of Regina, Department of Psychology, Regina, Canada

${ }^{*}$ Corresponding author.

Background and aims.- Occupational stressors occur to some degree in all work environments, and if they are severe enough they may affect the mental health of the employees. The current study was designed to: (1) examine two elements of occupational stress (operational and organizational) in male and female Canadian correctional workers; and (2) examine whether operational and occupational stressors are associated with symptoms of poor mental health.

Methods.- Data were collected by the Canadian Institute for Public Safety Research and Treatment using a web-based survey procedure, and included 1,308 correctional officers. The survey tools included established self-report measures for occupational stressors (20 organizational and 20 operational), and various aspects of mental health. Analyses included descriptive and non-parametric bivariate statistics.

Results.- Females reported slightly lower mean organizational and significantly lower operational stress than males ( 2.8 vs 2.9 , and
1.9 vs 2.1, respectively). The three highest mean organizational stressors were the same for both male and female workers - inconsistent leadership style, bureaucratic red tape, and feeling that different rules apply to different people. Operational and organizational stress was significantly correlated with increased symptoms of PTSD, anxiety, depression, stress, anger, and lower resiliency in both males and females.

Conclusions.- Male and female Canadian correctional officers reported the same top four operational and three organizational stressors. Both operational and occupational stressors, are significantly associated with increased mental health symptoms. It is important for management and government officials to identify sources of stress in order to potentially improve the mental health of correctional officers in Canada.

Disclosure of interest.- The authors have not supplied a conflict of interest statement.

\section{E-PV0639}

\section{The post war: fighting against symptoms}

J.P. Becker ${ }^{1,2}$

${ }^{1}$ University of Coimbra, Faculty of Psychology and Education

Sciences, Coimbra, Portugal; ${ }^{2}$ University of Coimbra, Trauma Centre of the Centre for Social Studies, Coimbra, Portugal

${ }^{*}$ Corresponding author.

Background and aims.- The image of soldiers hides the person that society prefers to ignore. Warriors, strong, brave and indestructible are characteristics associated with young people sent to battlefields. However, when soldiers leave war, they cannot leave the memories. Multiple stressors, intrusive memories, decreased interest in social activities, repressed affect, sleep disturbance, survivor's guilt, impaired memory and concentration are some of the aspects observed in veterans who faced the battle or did not face it at all. Recognizing the scars of wars, this study aimed at investigating the obstacles faced by veterans to seek professional help and by therapists to establish a good alliance and help them in unveiling the feelings behind their symptoms.

Methods.- Through a literature review and 11 interviews with Colonial War veterans enrolled at the Center for Prevention and Treatment of Psychogenic Trauma of the Hospital of the University of Coimbra, the author re-tracked the steps of these men from post-war to their treatment.

Results.- As somatic patients, veterans usually seek proper care when their bodies fall ill. Fear of stigma, the shame, the guilt, and the attempts to forget the war are the main reasons for denial of the symptoms. Considering this context, the health professional needs to be attentive to symptoms and medical test outcomes, but also to social and cultural issues.

Conclusions.- It is possible that all therapies promote psychological well-being and symptomatic relief, depending only on the veterans' ability to recognize and accept the influence of psychological and social factors, and to understand the goals of treatment.

Disclosure of interest.- The authors have not supplied a conflict of interest statement.

\section{E-PV0642}

\section{PTSD and post-traumatic stress}

spectrum in 50 parental couples of pediatric patients: gender differences in the psychopatological reaction to trauma

M. Corsi ${ }^{1}$, C. Bertelloni ${ }^{1}$, V. Pedrinelli ${ }^{1}$, G. Davini ${ }^{1}$, F.M. Barberi ${ }^{1}$, G. Massimetti ${ }^{1}$, D. Peroni ${ }^{2}$, A. Bonuccelli ${ }^{2}$, A. Orsini ${ }^{2}$, L. Dell'Osso' ${ }^{1}$, C. Carmassi ${ }^{1}$ 
1 Psychiatric Clinic, University of Pisa, Via Roma 67, 56100 Pisa, Italy, Department of Clinical and Experimental Medicine, Pisa, Italy; ${ }^{2}$ Pediatric Clinic, University of Pisa, Via Roma 67, 56100 Pisa, Italy, Department of Clinical and Experimental Medicine, Pisa, Italy * Corresponding author.

Background and aims.- Caregivers of pediatric patients with severe chronic illness, especially characterized by acute, repeated and impredictable clinical features, still represent a neglected public health concern that affect bi-directionally both parents' and children's health. Consistently, this particular kind of non-clinical subjects, may provide an interesting model to investigate psychopatological reaction to stress in the parental couple expecially addressing gender differences in the patterns of illness course and manifestations.

In this regard, we explored post-traumatic stress symptoms in couples of parents of cronically ill children.

Methods.- 50 mothers and 50 fathers, paired for one's child, of children followed at the Pediatric Unit of a major Italian University Hospital with a diagnosis of epilepsy, diabetes mellitus and anaphylactic shock, were enrolled, screened by means of the SCID-5 for DSM-5 and filled the Trauma and Loss Spectrum Self-Report (TALS-SR), an international instrument to evaluate post-traumatic stress symptomatology.

Results.- 25\% of the total sample presented a diagnosis of PTSD with a statistically higher prevalence of mothers (36\% and $14 \%$, respectively; $p=.021$ ). Furthermore, $44 \%$ (48\% mothers and $40 \%$ fathers) presented a partial PTSD. Important gender differences emerged also for all cluster dimensions of the TALS-SR except for the Avoidance. Finally, the analysis of the single items of the TALSSR evidenced that in mothers subgroup prevail cognitive symptoms of fear and sadness as well as somatic manifestations.

Conclusions.- Our results point out the differences between mothers and fathers in trauma response and underline the need to develop gender targeted models of healthcare prevention and assistance.

Disclosure of interest.- The authors have not supplied a conflict of interest statement.

\section{E-PV0646}

\section{Acute stress disorder in cancer patients: a case report}

N. Ghazouani ${ }^{1}$, C. ben Cheikh ${ }^{2}$, R. Jouini ${ }^{1}$, R. Maamouri ${ }^{1}$, H. Kefi ${ }^{2}$, A. Oumaya ${ }^{2}$

${ }^{1}$ Medical School of Tunis, Psychiatry, Tunis, Tunisia; ${ }^{2}$ Military

University Hospital of Tunis, Psychiatry, Tunis, Tunisia

${ }^{*}$ Corresponding author.

Background and aims.- The fourth edition of the Diagnostic and Statistical Manual of Mental Disorders recognized for the first time that posttraumatic stress disorder (PTSD) may be precipitated by lifethreatening illness.

Acute stress disorder (ASD) was also introduced in DSM-IV to identify individuals at risk of developing PTSD.

The aim of this study was to showcase the symptoms of ASD in a lung cancer patient with the focus on the importance of the communication between the patient and his doctor.

Methods.- We report the case of $\mathrm{Mr} \mathrm{JM}$, a 53 year old man who was hospitalized in the pulmonary department of the military hospital of Tunis in order to explore a pulmonary mass.

Results.- The medical staff waited for an anatomopathological proof before announcing the diagnosis to the patient. Meanwhile, the patient started to suspect the malignant nature of the mass and developed behavioural disturbances in the form of agitation, irritability and refusal of any medical intervention. The psychiatric interview revealed symptoms of ASD including derealization, depersonalization, anxiety, increased arousal, avoidance and intrusive thoughts. Among the risk factors of acute stress disorder and
PTSD, we find the subjective perception of cancer as life threatening and the difficult interaction with the medical staff. Thus the practitioner should pay attention to how and when the diagnosis should be communicated and not to let the patient extrapolate facts about the disease and its severity.

Conclusions.- Understanding the factors that predispose individuals to ASD following a cancer diagnosis may enhance our understanding of the prevention of pathological trauma responses in this population.

Disclosure of interest.- The authors have not supplied a conflict of interest statement.

\section{E-PV0648}

\section{A study on the development of psychological inventory and support of psychological treatment for veterans of South Korea}

M.H. Hyun ${ }^{1}$, S.M. Bae ${ }^{2}$, Y.K. Cho ${ }^{1}$, M. Kang ${ }^{1}$, S. Jeong ${ }^{1}$, H. Cho ${ }^{1}$, B. $\mathrm{Choi}^{2}$, H. Kim ${ }^{2}$

${ }^{1}$ Chung-Ang University, Department of Psychology, Seoul, Republic of Korea; ${ }^{2}$ Dankook University, Department of Psychology,

Chungnam, Republic of Korea

* Corresponding author.

Background and aims.- There was a psychological, economic, and physical problems of Korean veterans, and unique needs of the veterans and the bereaved family. The objectives of this study was to develop a basic counselling questionnaire and a psychological inventory to identify psychological problems as well as psychological disorders related to social adaptation of the veterans.

Methods.- We conducted a semi-structured interviews and FGI (focused group interview) with 51 veterans and the bereaved families. A basic counselling questionnaire and preliminary questions of psychological inventory were developed through qualitative analysis and expert advice. Total number of 586 adults between 20s and 60s who reported psychological trauma completed an online survey.

Results.- As results of the factor analysis, the final 39 items were selected, and five factors were identified: anger, alcohol problem, depression, insomnia and re-experience. We then compared the individual characteristics and the levels by calculating the standard score of the subfactor scores, and the combined score with a computer program.

Conclusions.- The results from this psychological inventory may help to plan an appropriate psychotherapy based on types and severity of psychological disorders among veterans. Further research is needed to verify the reliability and the validity of this inventory using veteran population.

Disclosure of interest.- The authors have not supplied a conflict of interest statement.

\section{E-PV0649}

\section{Socio-demographic risk factors for post-traumatic stress disorder in military personnel: about 30 cases}

W. Kabtni", C. Bencheikh, A. Rebai, A. Baatout, M.W. Krir, H. El Kefi, A. Oumaya

Military Hospital, Psychiatry Department, Tunis, Tunisia

* Corresponding author.

Background and aims.- Post-traumatic stress disorder (PTSD) is a disabling stress-related condition that occurs following exposure to traumatic event, with a prevalence in military personnel of $20-30 \%$. Our study aims to describe the socio-demographic profile and to identify risk factors for post-traumatic stress disorder in military personnel. 
Methods.- It's a case-control study.

Thirty military patients with PTSD were recruited from the external consultation of the psychiatry department of the Military Hospital of Tunis Versus thirty cases of military personnel who have participated in traumatic events and who have not developed a PTSD according to DSM-5 criteria.

We have collected socio-demographic data of every case and results were analysed using SPSS.

Results.- The analyse of socio-demographic profile of PTSD patients revealed that:

All participants were male.

The median age was 30 years [19-43].

$53.33 \%$ were single, $30 \%$ were in relationship and $16.66 \%$ were separated.

The troop rank category was predominant with $53.33 \%$.

Regarding the educational status, $66.66 \%$ have a secondary school level.

$43.33 \%$ of them have a low socio-economic level.

The young age ( $<25$ years), having troop rank and the high socioeconomic level were identified as risk factors for PTSD.

Conclusions.- PTSD is a major concern to the military because it causes considerable impairment which have implications for the individual affected and for society in general. More research is needed to understand modifiable factors in the military environment that reduce the risk of PTSD.

Disclosure of interest.- The authors have not supplied a conflict of interest statement.

\section{E-PV0650}

\section{Clinical and psychological characteristics of maladaptive states in the civilian population in conditions of macrosocial stress and hybrid warfare}

\author{
H. Kozhyna $^{1 *}$, A. Markov², M. Markova ${ }^{1}$, M. Khaustov ${ }^{1}$, K. \\ Zelenska $^{1}$ \\ ${ }^{1}$ Kharkiv National Medical University, Department of Psychiatry, \\ Narcology and Medical Psychology, Kharkiv, Ukraine; ${ }^{2}$ Kharkiv \\ Medical Academy of Postgraduate Education, Postgraduate \\ Education, Kharkiv, Ukraine \\ ${ }^{*}$ Corresponding author.
}

Background and aims.- Since 2014, Ukraine has been in the state of hybrid warfare, the key point of which is massive brainwashing the population using means of information-psychological war. Purpose: allocate the clinical specificity of the maladaptive states in the civilian population under the influence of macro-social stress, depending on the vulnerability to the action of information and psychological technologies, as part of the hybrid warfare.

Methods.- 221 patients of general-somatic profile were examined with manifestations of psychological maladaptation. The main group: 112 people with high involvement in the use of TV, Internet or periodicals, and the comparison group -109 patients with the low level of using information-psychological war means. Clinicalpsychological and psycho-diagnostic methods included a clinical interview and psychodiagnosis were used.

Results.- Patients in the primary medical network who are exposed to destructive information and psychological influences due to excessive involvement in watching TV, Internet use and reading of periodicals, have a greater severity of pathopsychological symptoms of maladaptive states compared with those with a safe level of TV and Internet use $(p<0.01)$. They are characterized by a high level of severity of psychosocial stress, formed (38.4\%) or expressed (37.5\%) neuro-psychological maladaptation, with the predominance of anxiety and depressive manifestations of the clinically significant level. The leading pathopsychological phenomenon in them is pathological anxiety, which is realized in the form of dom- inance of anxious-depressive (33.0\%) or anxious-dysphoric (38.4\%) constellations.

Conclusions.- The obtained data confirm the presence of negative influence of the information-psychological war on the state of mental health of the civilian population.

Disclosure of interest.- The authors have not supplied a conflict of interest statement.

\section{E-PV0651}

\section{Impact of comorbid depression on smoking behavior in trauma victims with post-traumatic stress disorder}

R. Lansari ${ }^{*}$, M.W. Krir ${ }^{2}$, O. Zerriaa ${ }^{1}$, H. el Kefi ${ }^{1}$, A. Oumaya ${ }^{1}$

${ }^{1}$ Principal Military Hospital of Instruction of Tunis, Psychiatry

Department, Tunis, Tunisia; ${ }^{2}$ Principal Military Hospital of

Instruction of Tunis, Psychiatry Department, Tunis, Tunisia

${ }^{*}$ Corresponding author.

Background and aims.- Research has highlighted the strong relationship between Post-Traumatic Stress Disorder (PTSD) and cigarette smoking. Individuals with PTSD are more likely to smoke more heavily. They have increased prevalence of nicotine dependence, and have close to the worst quit rates in comparison with patients with other psychiatric conditions.

Our main objective was an evaluation of the prevalence of cigarette smoking, nicotine dependence and the impact of comorbid depression on smoking behavior among military members treated for PTSD.

Methods.- To achieve our goal, a cross-sectional correlational study of a sample of 30 military members treated for PTSD in the principal military hospital was conducted. The PTSD checklist for DSM-5 (PCL5), Hospital anxiety and depression scale (HAD), and the fagerstrom test of nicotine dependence were administered for patients. Results.- All the patients assessed were male. Eighty per cent of patients were smokers. Roughly half of participants were dependent on nicotine. Sixteen per cent of patients show high levels of nicotine dependence. Severity of nicotine was positively associated with: Total PTSD symptoms, Hyperarousal symptoms, Avoidance symptoms and Celibacy. Fagerstrom score was significantly associated with HAD total scores.

Conclusions.- The results of this study illustrate the high prevalence of cigarette smoking and nicotine dependence among individuals with PTSD. Anxiety and depression may be related to smoking through a separate pathway from PTSD symptoms.

Disclosure of interest.- The authors have not supplied a conflict of interest statement.

\section{E-PV0652}

\section{Hopelessness as a risk factor of developing depression among military members with post-traumatic stress disorder}

R. Lansari", M.W. Krir, H. Ben mustapha, H. El Kefi, A. Oumaya Principal Military Hospital of Instruction of Tunis, Psychiatry

Department, Tunis, Tunisia

* Corresponding author.

Background and aims.- Post-traumatic stress disorder (PTSD) often co-occurs with depression and they may share common clinical features. Approximately half of people with PTSD also suffer from Major Depressive Disorder (MDD). Hopelessness may underlie the development of depression in trauma victims with PTSD.

This current study aimed to examine the association between hopelessness, the presence of comorbid depression in military members with a PTSD diagnosis. 
Methods.- Transversal descriptive study of a sample of 30 patients treated for PTSD in the principal military hospital was conducted to evaluate hopelessness and its relationship with depression. To achieve our aim we administered PTSD Checklist for DSM-5 (PCL5), the beck hopelessness scale questionnaire, and Hospital Anxiety and Depression Scale (HADS) for patients.

Results.- All patients assessed were male military members. The mean age of the sample was 29, 9 years. PCL-5 scores ranged from 19 to 46 with a mean of 30,8 . Severe PTSD (PCL5 > 33) was found in 16 patients. Depression was associated with PTSD in 54, $8 \%$ of the cases with a mean score of HADS $=11,84$. Hopelessness scores ranged from 0 to 18 with a mean of 5,65 . Hopelessness was positively correlated with HADS score $(r=0,727, p<0,001)$. Hopelessness was associated both with severe PTSD and depression. Conclusions.- Support was found for a relation between Hopelessness and depression in military members with PTSD. These findings have several potential implications, both for a better understanding of the nature and detection of depressive symptoms in trauma victims with PTSD.

Disclosure of interest.- The authors have not supplied a conflict of interest statement.

\section{E-PV0653}

\section{The effect of childhood maltreatment on the persistence of post-traumatic stress disorder}

H. Ben Mustapha, W. Krir, R. Lansari*, O. Zerriaa, H. Kefi, O. Abdel Aziz

Military Hospital, Psychiatry, Tunis, Tunisia

* Corresponding author.

Background and aims.- Childhood abuse increases the risk of mental health problems in adulthood, including post-traumatic stress disorder. The prevalence of PTSD in survivors of child maltreatment ranges from $21 \%$ to $74 \%$. The aim was to determine the effect of childhood trauma or abuse on the persistance of post-traumatic stress disorder in adulthood.

Methods.- This is a cross-sectional study conducted in the Department of Psychiatry of the Military Hospital of Tunis, including all consulting patients having PTSD according to DSM-5 (after a minimum of 11 months of follow-up) from first July 2018 to September the 18th 2018. Two ChildHood Trauma Questionnaire (CTQ) and PTSD self-questionnaires Checklist for DSM-5 (PCL-5) translated into Arabic were filled by these patients.

Results.- We have identified 30 patients with a male predominance with sex ratio $M / F=14$. The average age of patients was 35 years. We found $57 \%$ of our patients were single. All the patients were military soldiers except one. During the interrogation, we found personal and family histories of depression. Ten percent of patients had a traumatic event during childhood. In our series we found a high percentage of moderate to severe emotional abuse (33,3\%), emotional neglect (30\%) and physical neglect (70\%). In patients with persistent PTSD we also found a high pourcentage of moderate to severe emotional abuse (33\%) and physical neglect (40\%).

Conclusions.- Early detection of history of childhood abuse would be desirable, as well as spousal abuse experienced in adulthood. This screening would provide treatment services to victims of abuse and reduce potential consequences in the short or long term. Disclosure of interest.- The authors have not supplied a conflict of interest statement.

\section{E-PV0654 \\ Interpersonal psychotherapy for post traumatic stress disorder with comorbid major depressive disorder:} a case study

R. Lansari*, C. Ben cheikh, O. Zerriaa, H. El Kefi, A. Oumaya Principal Military Hospital of Instruction of Tunis, Psychiatry Department, Tunis, Tunisia

* Corresponding author.

Background and aims.- Interpersonal Psychotherapy (IPT) is a time-limited treatment that centers on resolving interpersonal problems. It's empirically validated mainly for major depressive disorder (MDD). IPT may be adapted for the treatment of post traumatic stress disorder (PTSD) and offers a non-exposure-based approach to PTSD.

This case report aims to demonstrate the efficacy of IPT as treatment for PTSD with comorbid MDD.

Methods.- We report the case of Mr. M, a young military member aged 34 years old who has been repeatedly traumatized after terrorist attacks in which he has lost many of his good friends and colleagues. He develops PTSD with comorbid MDD. IPT was adapted for the treatment and focused on the area of Grief or Complicated Bereavement. We describe the session-by-session IPT protocol, illustrating how to formulate the case, help the patient identify and address problematic affects and interpersonal functioning, and to monitor treatment response.

Results.- IPT has been successfully used as psychotherapy for PTSD with comorbid MDD. A significant improvement on the patient functioning and a decrease of the depressive symptoms were noted with subsequent improvement on PTSD symptoms.

Conclusions.- IPT acknowledges the impact of trauma on the patient's interpersonal functioning and may offer an alternative for patients who refuse or do not respond to exposure-based approaches.

Disclosure of interest.- The authors have not supplied a conflict of interest statement.

\section{E-PV0656}

\section{The impact of stress on the daily functioning in patients with post-traumatic stress disorder}

R. Maamouri ${ }^{*}$, A. Rebai ${ }^{1}$, C. Ben Cheikh² ${ }^{2}$ M.W. $\mathrm{Krir}^{2}, \mathrm{H}^{2} \mathrm{Kefi}^{2}, \mathrm{~A}$. Oumaya $^{2}$

${ }^{1}$ Medical School of Tunis, Psychiatry, Tunis, Tunisia; ${ }^{2}$ Military

Hospital of Tunis, Psychiatry, Tunis, Tunisia

* Corresponding author.

Background and aims.- Post-Traumatic Stress Disorder (PTSD) is associated with emotional dysfunction. People with PTSD respond differently to everyday situations and are more prone to stress leading to an impaired social functioning.

The aim of our study was to evaluate the impact of PTSD on the daily functioning of patients and to search for a correlation between the severity of post-traumatic stress symptoms and the level of social impact.

Methods.- It's a Cross-sectional descriptive study of 22 patients treated for PTSD at the outpatient psychiatry department of the military hospital of Tunis. Patients responded to 2 questionnaires: a self-administered 'Post Traumatic Stress Disorder Check List' (PCL) questionnaire to assess the severity of PTSD symptoms and a stress symptoms rating scale questionnaire.

The analysis was made by SPSS statistics 22 .

For the analysis of correlations and statistical links, we used linear regression.

Results.- The mean age of our sample was 34.05 years ( $\mathrm{SD}=9.183)$. The average PCL score was $38,86(\mathrm{SD}=12,985)$. 
The average stress symptoms rating scale score was 188,55 $(\mathrm{SD}=100,463)$.

There was no statistical link between the military rank and the stress symptoms rating scale score (BETA $=0,108 ; p=0,681>0,05$ ), but we noticed a significant correlation between PCL score and the severity of stress symptoms (BETA $=0.633 ; p=0,02<0,05$ ).

Conclusions.- A significant alteration of the socio-professional functioning caused by stress was reported. Professional help becomes important when a person with PTSD sees his general functioning affected linked to stress which is frequently described in this population.

Disclosure of interest.- The authors have not supplied a conflict of interest statement.

\section{E-PV0657}

\section{Post-traumatic stress disorder and self-esteem}

A. Rebai ${ }^{1}$, C. Ben Cheikh ${ }^{2}$, S. Tlili ${ }^{3}$, R. Maamouri ${ }^{*}$, I. Bouzouita ${ }^{1}, \mathrm{H}$. Kefi $^{2}$, A. Oumaya ${ }^{2}$

${ }^{1}$ Medical School of Tunis, Psychiatry, Tunis, Tunisia; ${ }^{2}$ Military Hospital of Tunis, Psychiatry, Tunis, Tunisia; ${ }^{3}$ Medical School of Tunis, Family Medicine, Tunis, Tunisia

Corresponding author.

Background and aims.- Low self-esteem is a characteristic found in several psychiatric pathologies. Few studies have focused on the impact of post-traumatic stress disorder (PTSD) on self-esteem and their possible interactions.

Methods.- Cross-sectional study including 23 military patients followed for PTSD at the outpatient psychiatry consultation of the Military Hospital of Tunis. Patients responded to two questionnaires. The first one was a self-assessment questionnaire: the Post Traumatic Stress Disorder Check-List Military (PCL-M). The second one was a self-assessment questionnaire to assess self-esteem: Rosenberg's Self-Esteem Scale.

Data analysis was performed by the SPSS software (IBM SPSS STATISTICS 22). A multiple linear regression was performed to search for correlations or statistical links between the dependent variable and the independent variables.

Results.- All patients were men. The mean age was 34,3 (SD =9,29). The mean PCL-M score was 38 ( $S D=12,62)$. The average Rosenberg Scale score was $15,61(\mathrm{SD}=6,88)$. In our sample, $47.8 \%$ of patients had low self-esteem, $43.5 \%$ had normal self-esteem and $8.7 \%$ had high self-esteem. We didn't find correlation between PCL-M score and Rosenberg Scale score $(\beta=-0.322, r=-0.322, p=0.144)$. There was not statically link between military grade and Rosenberg Scale score $(\beta=-0.049, r=-0.051, p=0.82)$.

Conclusions.- A few studies have suggested that PTSD is associated with low self-esteem, depending on the severity of post-traumatic symptoms (PTS). In our study, we did not find a correlation between the severity of PTS and low self-esteem. Further studies with larger samples are needed to support these results.

Disclosure of interest.- The authors have not supplied a conflict of interest statement.

\section{E-PV0658}

\section{The rigidity of the main arteries in young men of uzbek and Kyrgyz ethnicities eight years passed after the civil unrest \\ E. Molchanova ${ }^{1 *}$, L. Tsoy Molchanova ${ }^{2}$ \\ ${ }^{1}$ American University in Central Asia, Psychology, Bishkek, Kyrgyzstan; ${ }^{2}$ Kyrgyz State Medical Academy, Cardiology, Bishkek, Kyrgyzstan \\ * Corresponding author.}

Background and aims.- In Osh events of 2010, called "Uzbek-Kyrgyz three-day war" led to severe consequences for both mental and physical health among the residents of the city. The Uzbeks citizens were considered as "losers", and the Kyrgyz citizens - as "winners". Numerous studies have repeatedly shown the severity of chronic PTSD in adults, increased level of domestic violence, gender-based violence, suicidal attempts, along with raising interest in Islam and traditional spiritual practices in Osh and Jalabad regions, were the cruelest actions took place.

The aim of this research is to study objective physiological indicators of traumatic stress for those, who in 2010 were of 9-11 years old, and in 2018 was, respectively, reached 19 years - 21 years.

Methods.- Participants: 50 young Kyrgyz and 50 young Uzbek men 19-21 years old, witnesses of Osh events in 2010. Informed consent was presented in written form and signed by each participant Instruments.- The rigidity of the main arteries in young men was measured with the help of photoplethysmography on the apparatus "Angioscan-01M". The method also allows to measure additional parameters, such as VA - vascular age, augmentation index, reflection index.

Results.- There have been discovered a statistically significant $(p<0.001)$ difference between rigidity of main arteries among Uzbeks and Kyrgyz. Interestingly enough, the level of rigidity is higher in young Kyrgyz men - the result we've not expected.

Conclusions.- Long-term consequences of the disaster, even not clinically detected in mentally and physically healthy young people, can be determined by additional methods.

Disclosure of interest.- The authors have not supplied a conflict of interest statement.

\section{E-PV0659}

\section{Sleep problems within ptsd in firefighters: a screening program from the portuguese red cross}

R. Ferreira ${ }^{1}$, C. Pires De Lima ${ }^{1,2,3^{*}}$, P. Correia-Santos ${ }^{1,4}$, L. Dias ${ }^{1}$, M. Moreira $^{1}$, A. Oliveira ${ }^{1}$, D. Pereira ${ }^{1}$, A. Reis ${ }^{1,5}$, S. Silva ${ }^{1}$, R. Teles $^{1}$

${ }^{1}$ Portuguese Red Cross, Emergency Psychology, Vila Nova de Gaia, Portugal; ${ }^{2}$ ForAll- Desenvolvimento Pessoal e Bem-Estar- Lda, Psychology, Paredes de Coura, Portugal; ${ }^{3}$ CINTESIS - Center for Health Technology and Services Research, Psychology, Porto, Portugal; ${ }^{4}$ Universidade do Minho, Psychology, Braga, Portugal; 5 Escola Superior de Saúde de Santa Maria, Psychology, Porto, Portugal ${ }^{*}$ Corresponding author.

Background and aims.- First Responders (FR's) are often exposed to critical events. Although most FR's are resilient (e.g., Cunha et al., 2017), among the consequences of their activity are PTSD, depression, suicidality, anxiety, alcohol use, and sleep disturbances (Jones, 2017). The timing, amount, and poor quality of sleep associated to the job can also result in distress and impairments. Sleep problems in FR's can contribute to cognitive impairments, mood disturbances, and decreased quality of life (de Barros, Martins, Saitz, Bastos, \& Ronzani, 2013).

Objetive.- Screening for traumatic symptoms/ PTSD in FR's, with focus on sleep problems.

Methods.- Participants were 42 firefighters, most of them male (66.7\%), aged between $18-54$ years old $(M=32.07 ; S D=10.99)$, and on average with 11.2 years of experience $(S D=8.1)$. Data was collected from traumatic symptoms/ PTSD (PSSI-5 - Foa et al., 2015) and risk factors (based on Vogt, King, \& King, 2014).

Results.- The PSSI-5 item which assess sleep problems presented the highest average value $(M=1,03 ; \mathrm{SD}=1,51$; Range $=1-5)$, and the results also showed that more than half of the firefighters $(61,8 \%)$ have this symptom present $(\geq 1)$. Furthermore, the firefighters who report peritraumatic experiences appear to have more sleep problems $(M=2,17 ; \mathrm{SD}=1.60)$ than their peers, $t(32)=-2.15, p<.050$, $d=0.92)$. 
Conclusions.- These results underline the importance of assessing sleep problems in firefighters when evaluating PTSD, especially in those who report peritraumatic experiences.

Disclosure of interest.- The authors have not supplied a conflict of interest statement.

\section{E-PV0660}

\section{Sick-leave and the process of rehabilitation among patients with ptsd diagnosis in a psychiatric outpatient clinic in Sweden}

I. Sarajlic Vukovic ${ }^{1 *}$, M. Johansson Reg. psychologist ${ }^{1}$, A. Gonzales $^{2}$, E. Durakovic ${ }^{1}$, S. Steingrimsson ${ }^{2}$

${ }^{1}$ Sahlgrenska University Hospital, Gamlestaden Psychiatric Unit, Gothenburg, Sweden; ${ }^{2}$ Sahlgrenska University Hospital, Affective Psychiatry, Gothenburg, Sweden

${ }^{*}$ Corresponding author.

Aim.- To show the process of sick-leave and work-related rehabilitation within the group of patients treated in a specialized PTSD team in Gothenburg. This model is one of the existing possibilities in public health care. The goal is to utilize and optimize the existing resources and to maintain cost benefit.

Background.- Most of the patients in need for specialized treatment for PTSD are in working age (18-64). The goal in every society is to engage its citizens in the labor market. It enhances quality of life, promotes health and provides the public sector with necessary economical resources. The goal of every health system is reasonable cost benefit and preserving quality of health care in secondary and tertiary prevention. Guidelines for PTSD treatment in the West Gotaland region includes medical treatment and social interventions. This group of patients have high comorbidity and low functional level. Our interest is to monitor the process of sick-leave and work rehabilitation and to explore possibilities of optimization. Methods.- With descriptive statistical method show the process regarding the need for sick leave of the observed group in the period from 2014 to 2018 . The data will be displayed numerically and graphically.

Results.- Further objective: Demonstrate present situation in the PTSD team, which is the only PTSD-specialized psychiatric team in the region. To explore needs of optimizing current resources regarding team-work and other resources in the community. Conclusions.- There is a strong need to explore further development and the utility of this model.

Disclosure of interest.- The authors have not supplied a conflict of interest statement.

\section{E-Poster Viewing - 7-9 April: Prevention of Mental Disorders}

\section{E-PV0662}

\section{Starting early intervention programme for first episode psychosis patients in rural region of Latvia}

L. Berze ${ }^{1^{*}}$, S. Civcisa ${ }^{2}$, I. Krone ${ }^{3}$, S. Kikuste $^{4}$, I. Sapele ${ }^{4}$, E. Rancans ${ }^{2}$ ${ }^{1}$ Riga Stradins University, Department of Doctoral Studies, Riga, Latvia; ${ }^{2}$ Riga Stradins University, Department of Psychiatry and Addiction Disorders, Riga, Latvia; ${ }^{3}$ Latvian Association of Cognitive Behavioural therapy, Cognitive Behevioual therapy, Riga, Latvia; ${ }^{4}$ Daugavpils Psychoneurological Hospital, Department of Mental health care, Daugavpils, Latvia

${ }^{*}$ Corresponding author.
Background and aims.- All patients with first psychosis episode should receive a full range of pharmacological, psychological, social, occupational, and educational interventions (NICE, 2014). It is already known that early intervention during first episode is crucial for secondary prevention of illness progression. This is the first early intervention programme in Latvia, our aim is to improve mental healthcare for first psychosis patients.

Methods.- Daugavpils Psychoneurological hospital (DPNH) is the biggest regional hospital of Latvia, its catchment area is 250000 inhabitants. On 01.10.2017. we started to form a team and develop a programme for early intervention, it included a case manager, psychiatrist, psychologist, and nurse. The programmes' length is 6 months with appointments every week. The psychological intervention includes elements of acceptance and commitment therapy and mindfulness exercises. For each case the programme was tailored for patients' needs and to reach high availability. The study was approved by the RSU Ethics committee.

Results.- In the first 6 months of the programme, from all consecutive 27 psychosis patients admitted in DPNH, 20 were non-affective FEP, 18 agreed to join the programme. 4 dropped out at various stages. An intervention team was formed and successfully integrated in out-patient services. We received good feedback about the intervention process in family sessions and individual patient evaluations. We are continuing the programme's work and the first evaluation will be conducted in March 2018.

Conclusions.- This experience should be seen as a pilot project for intervention as a routine treatment option for out-patient care of first time psychosis patients.

Disclosure of interest.- The authors have not supplied a conflict of interest statement.

\section{E-PV0663}

\section{Youth perceptions towards psychiatry}

L. Luca ${ }^{1}$, A. Ciubara ${ }^{1,2^{*}}$, A.B. Ciubara ${ }^{1}$, A.C. Chirosca ${ }^{3}$, F. Sarbu ${ }^{1}$

${ }^{1}$ University "Dunarea de Jos", Medicine, Galati, Romania; ${ }^{2}$ University "Dunarea de Jos", "Elisabeta Doamna” Hospital, Faculty of Medicine and Farmacy, Galati, Romania; ${ }^{3}$ University of Medicine and Farmacy “Carol Davila”, Medicine, Bucharest, Romania "Corresponding author.

Background and aims.-

Introduction.- Most people associate the issue of mental health with stereotypes and fears, therefore the overall attitude is negative. One of the possible explanations of people's attitudes about mental disorders is that the process of stigmatization and the negative emphasis on differences are influenced by cultural mechanisms. Objectives.-

- youth perceptions concerning the stigmatization of psychiatry

- assessing the level of knowledge about mental health

- finding a correlation between the tendency of stigmatization and the level of psychoeducation of the investigated subjects

Methods.- This survey investigated 120 students, 19-24-year-old. Results.-

- $40 \%$ of subjects never used psychotherapy

- $75 \%$ know people who went to the psychologist

- $35 \%$ were reluctant to appeal to mental health specialists

- $30 \%$ of subjects would feel stigmatized if it ever got out that they were in psychotherapy / would not want anyone to find out that they were in psychotherapy

- $90 \%$ of those investigated consider mental health services to be beneficial

Conclusions.-

- increasing the addressability to mental health services could improve the quality of life

- the need to organize a campaign to improve mental health services

Disclosure of interest.- The authors have not supplied a conflict of interest statement. 


\section{E-PV0664}

\section{Mid-studying crisis in medical} students

A. Kaminska*, I. Pototska, G. Rudenko

National Pirogov Memorial Medical University, Vinnytsya, Medical

Psychology and Psychiatry Department with the Course of

Postgraduate Education, Vinnytsya, Ukraine

"Corresponding author.

Background and aims.- While studying at the university, each student undergoes a variety of crises. Mid-studying crisis is interesting to investigate. The most common symptom of this crisis is disappointment - in education, in chosen profession and in future prospects and opportunities. Emotional burnout is a major component of mid-studying crisis, since students feel exhausted and unmotivated.

Aim of this study was to investigate psychological components of mid-studying crisis in medical students.

Methods.- The study was conducted among 152 third-year students of National Pirogov Memorial Medical University, Vinnytsya. Emotional burnout of students was measured by "Diagnostics of emotional burnout" test by V. Boyko. Intellectual sphere was studied by Raven progressive matrices.

Results.- Emotional burnout study showed that "exhaustion" stage is formed in $18 \%$ of students, while the "formation" stage is present in $32 \%$. Specificity of studying at medical universities is high intellectual and psychological burden caused by large amount of information and high demands on students. In $38 \%$ of respondents the "resistance" stage was revealed. High level of intelligence is characteristic for $5 \%$, above average - $37 \%$, normal - $49 \%$, average level $-5 \%$ and below average - $4 \%$ of respondents. Surveying students has shown that $12 \%$ of medical students have medical job experience.

Conclusions.- Results of correlation analysis and data processing has proven direct correlation between high level of emotional burnout in students and their intelligence $(+0.7)$. Also, connection between work experience in medical field and emotional burnout was revealed. Students who had experience of working in medical sphere were more resistant towards emotional burnout.

Disclosure of interest.- The authors have not supplied a conflict of interest statement.

\section{E-PV0666}

\section{Psychological well-being and specific distressing symptoms in dentists}

E. Nikolaev*, D. Hartfelder

Ulianov Chuvash State University, Social and Clinical Psychology

Department, Cheboksary, Russia

* Corresponding author.

Background and aims.- Professional activity of dentists demands much of their mental health and psychological well-being.

Objectives.- To find out if subjectively experienced complaints intensity concerning specific distressing symptoms correlates with indices of psychological well-being in healthy dentists.

Methods.- Using SCL-90R and Ryff's Psychological Well-Being Scales (PWB), we have studied 122 healthy dentists (57 males, 65 females). The difference in symptoms manifestation was revealed by means of ANOVA.

Results.- ANOVA findings showed that only two SCL-90-R item scores (Overeating and Awakening in the Early Morning) had little influence on PWB indices. The rest SCL-90-R items and PWB scales and factors proved to have the following connections: Poor Appetite symptom, Environmental Mastery scale $(F=3.15 ; p=0.04)$ and Self-acceptance $(F=3.63 ; p=0.03)$; Trouble Falling Asleep symptom and Meaningfulness of Life factor $(F=2.53 ; p=0.04)$; Thoughts of Death or Dying symptom, Selfacceptance scale $(F=3.52 ; p=0.00)$ and Balance of Affect factor
$(F=3.59 ; p=0.00)$; Sleep that is Restless or Disturbed symptom and Balance of Affect factor $(F=2.96 ; p=0.03)$; Feelings of Guilt symptom, Autonomy scale $(F=4.73 ; p=0.00)$, Environmental Mastery $(F=6.28 ; p=0.00)$, Purpose in Life $(F=2.87 ; p=0.03)$, Self-acceptance $(F=7.76 ; p=0,00)$, Balance of Affect factor $(F=7.25$; $p=0.00)$, Meaningfulness of Life $(F=3.21 ; p=0.02)$ and Autonomy scale $(F=2.69 ; p=0.03)$.

Conclusions.- Specific distressing symptoms in dentists can manifest themselves in poor appetite and bad sleeping, and can be accompanied by anti-vital experiences. Feeling of guilt is connected with psychological well-being indices most of all and it is caused by personal problems of existential nature, which need to be discussed with a psychiatrist and psychotherapist.

Disclosure of interest.- The authors have not supplied a conflict of interest statement.

\section{E-PV0668}

\section{Bullying and cyberbullying in youth and adults: relationship to anxiety} and aggression

E. Rasskazova ${ }^{1}$, G. Soldatova ${ }^{2}$

${ }^{1}$ Moscow State University, Clinical Psychology, Moscow, Russia; ${ }^{2}$

Moscow State University, Personality Psychology, Moscow, Russia

Corresponding author.

Background and aims.- Internet has become an important part of life for contemporary youth and adults so that it could affect their psychological condition including mental health. Cyberbullying refers to aggressive activity using communication technologies (Grigg, 2010, Zych et al., 2016) and is differentiated from other contexts of bullying - at school, at work etc.

The aim was to reveal an effect of bullying in different settings on anxiety and aggression in youth and adults.

Methods.- 736 youth (18-30 years) and 1105 adults (30-65 years) from 8 Federal regions in Russia appraised whether they have experience of bullying at school, at the university, at work, in the Internet, filled Anxiety and Interpersonal Sensitivity scales from SCL-90R (Derogatis, 1994), Aggression Questionnaire (Buss, Perry, 1992).

Results.- In youth $67.1 \%$ reported experience of bullying at school, $23.4 \%$ at the university, $20.1 \%$ at work, $52.0 \%$ in the Internet. For adults these frequencies were $46.6 \%, 19.9 \%, 24.9 \%, 22.2 \%$, consequently. Experience of bullying at school predicts higher anxiety and hostility in both groups $\left(F=4.25-13.10, \eta^{2}=.01-.02\right.$, $p<.05)$, interpersonal sensitivity, anger and physical aggression in youth $\left(F=7.68-8.85, \eta^{2}=.01, p<.01\right)$. Cyberbullying predicts interpersonal sensitivity and hostility in both groups $(F=4.20-15.27$, $\left.\eta^{2}=.01-.02, p<.05\right)$, higher anxiety in youth $\left(F=5.85, \eta^{2}=.01\right.$, $p<.01)$, anger in adults $\left(F=9.85, \eta^{2}=.01, p<.01\right)$. Bullying at work is related to physical aggression in adults $\left(F=4.19, \eta^{2}=.01, p<.05\right)$. Conclusions.- Results indicate that not only experience of bullying at school but also cyberbullying are related to hostility, anxiety, anger and interpersonal sensitivity in both youth and adults. Study is supported by the Russian Science Foundation, project 18-1800365.

Disclosure of interest.- The authors have not supplied a conflict of interest statement.

\section{E-PV0671}

\section{Burnout syndrome and chronic fatigue prevention by means of human functional states' self-regulation}

M. Titova ${ }^{1 *}$, A. Kuznetsova ${ }^{2}$

${ }^{1}$ Lomonosov Moscow State University, Department of Work and Organizational Psychology Faculty of Psychology, Moscow, Russia; ${ }^{2}$ 
Lomonosov Moscow State University, Department of Work and Organizational Psychology, Faculty of Psychology, Moscow, Russia ${ }^{*}$ Corresponding author.

Background and aims.- The human ability to manage functional states in tensed working condition could be viewed as a key professional competence in various occupations, and as a factor of burnout and chronic fatigue syndromes effective prevention (Cartwright, Cooper, 2005; Friedman, 2003; Teichman, Leonova, 2014).

The aim of the study is to reveal functional state self-regulation means as effective ways of burnout and chronic fatigue prevention. A conceptual model of functional state self-regulation efficiency investigation was developed. The model was tested in different samples: school teachers, HR-specialists, IT-specialists, operators of power grid system $(N=420)$.

Methods.- The methods included: the job stress survey (Ch. Spielberger); the Russian variant of burnout inventory, developed on the base of $\mathrm{Ch}$. Maslach burnout syndrome model (Ch. Maslach, S. Jenkins, 1981; N. Vodopianova, E. Starchenkova, 2003); the strategic approach to coping scale ( $\mathrm{S}$. Hobfoll), the scale of the proactive coping (E.R. Greenglass, R. Schwarzer, S. Taubert), chronic fatigue scale (A. Leonova).

Results.- The research revealed the typical means of successful specialists' functional state self-regulation. The means of functional states self-regulation associated with social support and related to activation of self-confidence are typical for teachers without burnout and chronic fatigue syndromes. Orientation on self-development of effective functional state self-regulation is considered as a key predictor of professional success and the way of burnout and fatigue prevention ( $p=0,049 ; \beta=0,394)$.

Conclusions.- Orientation on the development of effective functional states' self-regulation means and high level of communicative competence allow to prevent fixation of destructive coping ways which may cause burnout and chronic fatigue syndromes and interfere professional success achievement.

Disclosure of interest.- The authors have not supplied a conflict of interest statement.

\section{E-PV0672}

\section{Risk assessment in primary attention for depression and anxiety in the elderly population}

M.C. Vastag Ribeiro De Oliveira*, N. Orso, E. da Silva

University of Western São Paulo, Faculty of Nursing, Presidente

Prudente, Brazil

${ }^{*}$ Corresponding author.

Background and aims.- Life expectancy is a universal phenomenon, along with the prevalence of psychiatric illnesses such as depression and anxiety. These have been related to isolation, increased dependence on care and reduced quality of life. Performing interventions to promote mental health through the early identification of signs and symptoms and their mental health factors enables and directs the development of competencies as empowerment, autonomy and self-efficacy. We evaluated risk for depression and anxiety in the elderly population that participates in the trekking project in Presidente Prudente.

Methods.- Cross-sectional quantitative prevalence study with Mini-International Neuropsychiatric Interview (MINI) and Beck anxiety and depression scales. Recruited 50 volunteers participating in a walking project and the female population was the predominant one. The data were collected through an individual interview and analyzed by paired and submitted to simple statistical treatment.

Results.- Fifteen (30\%)volunteers from the sample without depression, 32 (64\%) volunteers presented mild depression, 3 (6\%)presented moderate depression and no volunteers had severe depression. The Anxiety scale 41 (82\%)volunteers of the sample with minimum anxiety, $09(18 \%)$ presented mild anxiety. The prevalence of anxiety disorders was greater than depression. In addition to impairing quality of life,the presence of unidentified psychiatric diseases combined with pathophysiological changes, the aging process in the elderly population contributes to an increased risk of disability and mortality in this population. Conclusions.- Health screening measures become relevant and of great value for the early identification of signs and symptoms for depressive and anxious disorders, and should be performed during the multidimensional evaluation of the elderly, directing to specific interventions that are consistent with the reality of this population. Disclosure of interest.- The authors have not supplied a conflict of interest statement.

\section{E-Poster Viewing - 7-9 April: Promotion of Mental Health}

E-PV0674

\section{Stigma related to mental illness among community of Imam Abdulrahman Bin Faisal University}

N. Almuhanna*, A. Rasheed, A. Abdullah

Immam Abdulrahman Bin Faisal unversity, Counseling Center, Dammam, Saudi Arabia

* Corresponding author.

Background.- Stigma related to mental illness is an essential issue that must be addressed to overcome the barriers to seek help. It has an impact to many aspects of people's lives, the way they Perceive themselves, how the Media promulgated mental illness, how Social perceive people labeled with mental health illness, and how the Cultural background play a role in prejudicial attitude and discriminating behaviour. Mental health services in Saudi Arabia have dramatic changes. However, prejudice, discrimination, social distance existed result in relucted to seek treatment. To our knowledge, no previous community-based studies focused on the stigma in Saudi Arabia.

Aim.- To evaluate the level of the stigma of the university community within different factor to suggested avenues to reduce the stigma related mental illness in the community.

Methods.- We designed a new stigma scale questionnaire after reviewing previous scales. Therefore, We consider cover four dimensions including personal, social, media and culture/faith belief. The final form consists of 28 items, all of the self-report type, which individuals respond to in the light of 5-point Likert-Type scale.

Results.- The SS questionnaire distributed as pilot sample to 55 participants. In general, it shows a stigmatizing attitude (Fig. 1).

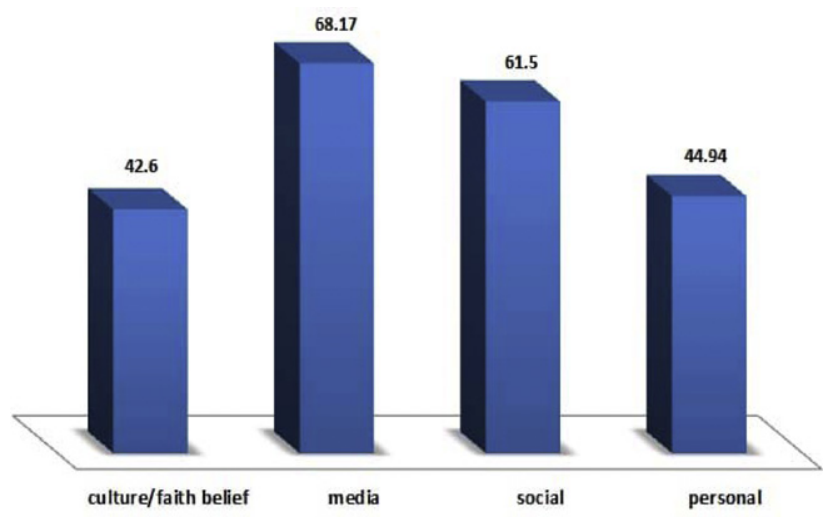


Fig. 1. Difference between the dimension of stigma with the sample in general.

Conclusions.- Stigma is high among participant. Therefore, its necessary to conduct a community-based study to identify the factors behind the stigma. Since the media was the highest impact on promulgated stigmatizing massages followed by social, It the time to change and anti-stigma interventions must be prompted by media platform.

Disclosure of interest.- The authors have not supplied a conflict of interest statement.

\section{E-PV0677}

\section{Through the lenses of threat: attachment to the others and to the self}

F. Carvalho*, C. Vitorino, A. Xavier, H. Moreira, P. Castilho

Centro de Investigação em Neuropsicologia e Intervenção

Cognitivo-Comportamental, Universidade de Coimbra, Coimbra,

Portugal

* Corresponding author.

Background and aims.- Throughout the lifespan, people develop interpersonal patterns of relating (i.e., attachment orientations), that are the outcome of the accumulation of experiences with attachment figures. These attachment orientations are associated with implicit cognitive-emotional strategies which intend to secure and maintain intra- and interpersonal safety and security. Studies suggest an association between external shame, selfcompassion, mindfulness, depression and insecure attachment styles.

The present investigation intends to explore the impact of external shame, self-compassion and mindfulness in anxious and avoidant attachment, controlling the effect of depressive symptoms.

Methods.- A battery of self-report instruments was applied to measure the variables being studied to a non-clinical sample of 375 women, university students.

Results.- The results of the study of correlational nature were expectable, with statistical significance. The comparison of the participants with low and high levels of anxious and avoidant attachment, through $t$-Student tests, showed that women with higher levels of anxious and avoidant attachment report more external shame, more depressive symptoms, less self-compassion and less mindfulness. The mediational models tested revealed significant results, explaining $26 \%$ and $23 \%$ of the dependent variable (anxious and avoidant attachment, respectively).

Conclusions.- Globally, the results suggest that self-compassion and mindfulness represent adaptive fundamental competences for the development of quality and secure adult attachment styles.

Disclosure of interest.- The authors have not supplied a conflict of interest statement.

\section{E-PV0678}

\section{Quality of life ( $Q 0 L)$ in persons with spinal cord injuries $(\mathrm{SCI})$ in Western Greece: first results of pilot study prior to intervention}

\section{E. Gkintoni* , A. Kyriakidis, A. Tzanos, E. Panagiotopoulos}

University of Patras, Spinal Cord Injuries Rehabilitation Department, Rio-Patras, Greece

* Corresponding author.

\section{Background and aims.-}

Introduction.- People with SCI face major health-related QoL challenges, especially in Greece, due to the lack of infrastructure and support networks.

Objectives.- To evaluate QoL of 30 persons with SCI living in the community who had more than one year after the completion of their rehabilitation program. The current pilot study aims to determine QoL of individuals with SCI after implementing a program of integrative care. The program includes sessions of physical activity/body empowerment, psychological support and psychoeducation via Information and Communication Technologies (ICTs). This project is funded by a research European program in order to promote QoL in community persons with SCI and to enhance scientific collaboration among young researchers.

Methods.- Thirty individuals with SCI living in Western Greece cooperated for the implementation of the current scientific project. World Health Organization Quality of Life BREF (WHOQOL-BREF) questionnaire was administered along with clinical measurements and a demographic questionnaire.

Results.- The mean age was $45.7 \pm 10.3$ years, the mean time since the injury was $12.6 \pm 11.8$ years and $90 \%$ of the participants were male. They were primarily graduates of primary school (43.3\%) and single parents (53.3). The mean QoL parameters were 53,3 \pm 14 (physical health),65.4 \pm 15 (mental health), 64,7 $\pm 24,1$ (social relationships), 65,9 $\pm 17,5$ (environment).

Conclusions.- The study documents relatively high rates of QoL in the population of individuals with SCI in West Greece. This could be interpreted by the easily accessible follow up health care services, the outpatient consulting hours by the multidisciplinary team, the peer teaching and support, and the participation in leisure activities.

Disclosure of interest.- The authors have not supplied a conflict of interest statement.

\section{E-PV0680}

\section{Age related correlates of self-esteem and healthy behavior in university students \\ E. Nikolaev \\ Ulianov Chuvash State University, Social and Clinical Psychology \\ Department, Cheboksary, Russia \\ * Corresponding author.}

Background and aims.- Modern students' lifestyle determines a physical and mental state of future society. How popular is healthy behavior with university students during their studies and how is it connected with some of their self-esteem indices?

Objectives.- To find age related correlates of self-esteem indices and healthy behavior in university students.

Methods.- Our survey covered 318 male and female students of different Russian universities aged 17-29. It contained questions about lifestyle and 11 self-esteem parameters. Our analysis considered statistically significant correlates with $p<0.05$.

Results.- The age of the students positively correlated with their body weight, the amount of their expenses, and the duration of their daily physical exercising. However, the students' age did not correlate with the level of stress, frequency of their physical activity and the amount of daily-consumed vegetables and fruit. Older students showed correlation of their age with a more positive self-esteem of their health, attractiveness, interestingness in communication, confidence, intellect and successfulness, but no correlation with the self-esteem of their happiness, well-being, cheerfulness, sportiveness and religiousness. Overall, a higher selfesteem of health correlates with a higher self-esteem of happiness and well-being.

Conclusions.- While studying, students become more mature and improve their financial status and, partially, mental health, which can be regarded as a natural phenomenon. Older students do not become happier, more cheerful or successful. Growing older, students do not acquire significant positive patterns of lifestyle related to their eating habits and frequency of their physical activity, which raises risks to their physical and mental health.

Disclosure of interest.- The authors have not supplied a conflict of interest statement. 


\section{E-PV0681}

\section{Socio-demographic variables explaining the variability in the structure and functioning of war victims' social support networks}

I. Ramos-Vidal ${ }^{1}$, A. Uribe ${ }^{2}$, V. Ilse ${ }^{2}$

${ }^{1}$ University of Seville and Universidad Pontificia Bolivariana

Montería, Colombia, Department of Social Psychology, Seville, Spain;

2 Universidad Pontificia Bolivariana, Psychology, Monteria, Colombia ${ }^{*}$ Corresponding author.

Background and aims.- Empirical evidence suggests that the structure and the patterns of interaction of the victims' social network are abruptly altered as a result of exposure to war violence. The aims of this study are to examine the effects of age, length of residence in the community and educational level on: (a) network density, (b) homophily and (c) of social support multiplicity of war victims. Methods.- A cross-sectional exploratory study was carried out to achieve the study objectives. The participants are 106 residents (86 female, $81.1 \%$ ) of a rural community. The mean age of the participants is 42.5 years $(S D=16.4)$ and on average they has been living in the community for 28.8 years $(S D=18.7)$. Most of the participants have primary education studies (47.2\%). An egocentric networks design was developed to evaluate the structure and function of social support networks.

Results.- The participants' networks are highly cohesive (Density $=96.1 \%$ ), presents moderate homophily based on place of residence $(-.52)$ and receives 1.4 types of social support from each alter $(S D=.82)$. Figure 1 shows results.

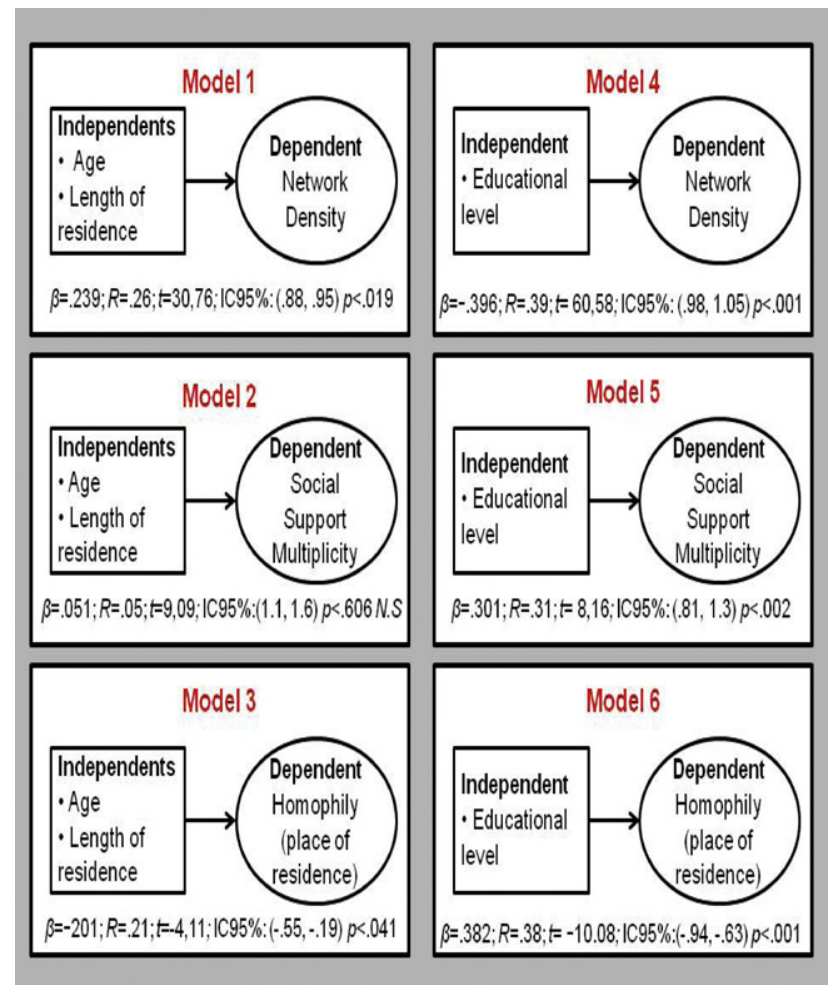

Fig. 1. Results of six models.

Conclusions.- The structure and internal dynamics of support networks are conditioned by socio-demographic variables as age and educational level. Older people are immersed in denser social structures but these networks are exhausted for providing different kinds of social support. Educational level is a key factor explaining social support diversification.

Disclosure of interest.- The authors have not supplied a conflict of interest statement.

\section{E-PV0682}

\section{Feelings of guilt and resentment in victims of land dispossession in Cordoba, Colombia}

M. Arcos ${ }^{1}$, M. Munoz ${ }^{1}$, A. Uribe ${ }^{1}$, I. Villamil ${ }^{1}$, I. Ramos-Vidal ${ }^{2 *}$

${ }^{1}$ Universidad Pontificia Bolivariana, Psychology, Monteria, Colombia;

2 University of Seville and Universidad Pontificia Bolivariana

Montería, Colombia, Department of Social Psychology, Seville, Spain

* Corresponding author.

Background and aims.- The Department of Cordoba allocated in the north of Colombia represents a social space where armed groups took huge land extensions during the armed conflict. This brought some consequences like population displacement, human rights violations and land dispossession. These kinds of events provoke severe consequences on mental health. When tragic events such as forced disappearances happened, people tend to experience sentiments as guilt and helplessness. From a theoretical perspective, sentiments of guilty are understood as a negative evaluation associated with the transgression of principles for the resentment. On the other hand, resentment is an affective disposition characterized by the need for revenge against aggressors. Both are constructs that should be investigated in the Colombian post-conflict context. The aim of this research is to analyze the relationship between guilt feelings and social resentment in victims of land dispossession. Methods.- A cross-sectional exploratory study was developed. Participants were forty subjects (52.5\% male) victims of land dispossession. The Capability Scale of Forgiveness [CAPER] developed by Rosales, Rivera and García (2017) was applied. CAPER instrument includes two subscales[Usuario2]: (1) a guilt subscale (Chrobachs' Alpha $=.632$ ) and a social resentment subscale (Chrobachs' Alpha =.741).

Results.- A positive and significant bilateral correlation is identified among the study variables $(p<.01)$. This represents a promising finding to understand the psychosocial effects of victims of displacement and land dispossession in the post-conflict era.

Conclusions.- Displacement and land dispossession are triggering negative effects on war victims' mental health. Social intervention programs should be aimed for reducing feelings of guilt and resentment.

Disclosure of interest.- The authors have not supplied a conflict of interest statement.

\section{E-PV0684}

\section{Emotional expectations of nursing personnel: what will replace the work? A qualitative study with Brazilian professionals with scheduled retirement in a university hospital}

H.O. Santos Santos, E.R. Turato*

State University of Campinas, Laboratory of Clinical-Qualittative

Research LPCQ Campinas, Brazil

${ }^{*}$ Corresponding author.

Background and aims.- In Brazil, in 2030, the number of over 60 years will be higher than that of individuals with up to 14 years. We wanted to know personal experiences of transition to retirement of a special health team: those people have helped to organize a hospital that has just completed three decades.

To explore emotional meanings brought by professionals that is going through the vital process of the retirement, after long employment - and affective - bond with the General Hospital, the State University of Campinas, Brazil.

Methods.- The sample construction was intentional, with information from the institution. Inclusion criteria: (1) long employment at General Hospital (two decades, at least); (2) scheduled retirement as referred by the invited persons. Instruments of data collection: 
semi-directed interview with open-ended questions, in depth; field notes with observations and self-observations. After full transcription of material, the treatment was made by Qualitative Content Analysis.

Results.- The investigation resulted in a group of six interviewees, who accepted the invitation to participate. All of them was constituted from nursing staff. Sample was closed by information saturation. Emergent categories were: (1) fantasies about what will symbolically replace of work (2) date marked felt as 'an elephant taken from me; (3) irreplaceability at work: perception if it is a myth or a truth; (4) real expectations of retirement as a strong rite of passage.

Conclusions.- The symbolic of retirement after long bond with health institution organizes the 'life turn' in emotional aspects that goes beyond certain objective organization in a new biographical phase.

Disclosure of interest.- The authors have not supplied a conflict of interest statement.

\section{E-Poster Viewing - 7-9 April: Psychoneuroimmunology}

\section{E-PV0685}

\section{Challenges in the workup of catatonia: a case report of nmda receptor encephalitis}

D. Bieber ${ }^{1^{*}}$, E. Bieber ${ }^{2}$, H. Betcher ${ }^{2}$, A. McKean ${ }^{2}$

${ }^{1}$ Mayo Clinic, Neurology, Rochester, USA; ${ }^{2}$ Mayo Clinic, Psychiatry \& Psychology, Rochester, USA

* Corresponding author.

Background and aims.- Despite being most often associated with affective disorders, catatonia due to a medical condition is responsible for up to $17 \%$ of child, and $25 \%$ of adult presentations. Because symptoms of either etiology are very similar, deciding when to initiate medical work up is challenging. Our aim was to present a case of NMDA receptor encephalitis, and explore feature of catatonia which may suggest a medical etiology

Methods.- Ms. S's chart was reviewed and literature review.

Results.- Ms. S, a 17-year-old young woman with no medical or psychiatric history presented with acute onset of falls and fever. Symptoms rapidly progressed to include alterations in mental status, bizarre behavior, perceptual disturbances, mutism, orolingual dyskinesia, autonomic instability, and finally seizures. A benzodiazepine challenge resulted in limited improvement of communication with moaning and pointing, but brought worsening agitation. Medical work-up included a normal magnetic resonance imaging (MRI) of the brain, and positive N-methyl-D-aspartate (NMDA) receptor antibodies in cerebrospinal fluid (CSF). Treatment included methylprednisolone and intravenous immunoglobulin (IVIG). Improvement was slow but progressive. At 2 months she was able to converse appropriately, recall discussions and events, and was future oriented. She returned to full days of school by 3.5 months after presentation, with minor cognitive deficits.

Conclusions.- Our case represents a classic presentation of NMDA receptor encephalitis. However on review of catatonia, currently there are no distinct features which would reliably separate medical from psychiatric etiologies of catatonia. Medical causes, including NMDA receptor encephalitis should remain high on the differential, as early specific intervention leads to better outcomes. Disclosure of interest.- The authors have not supplied a conflict of interest statement.

\section{E-Poster Viewing - 7-9 April: Psychopathology}

\section{E-PV0687}

\section{Concerns of parents and identification of early signs of autism: a study conducted in Algeria}

S.R. Bendiouis ${ }^{1 *}$, H. Yamina ${ }^{1}$, A. Asma ${ }^{1}$, B. Lotfi ${ }^{2}$, F. Borsali ${ }^{1}$, A. Mecherbet $^{1}$, A. Hamhami ${ }^{1}$

${ }^{1}$ University of Tlemcen, Hennaya, Tlemcen, Algeria; ${ }^{2}$ 05, Rue de l'indépendance, Tlemcen, Algeria

Corresponding author.

Background and aims.- Autism is an early onset disorder that affects the development of the child. More and more studies are moving towards the question of identifying the first signs of ASD. This orientation is largely explained by the issue of identification and early diagnosis in the implementation of support projects and appropriate interventions. Our research aims to study the variations in the age of detection of the first anomalies by the parents, but also the nature of the first signs appeared. We also try to verify if these anomalies depend on certain characteristics such as the socio-economic level of the parents.

Methods.- Our work focuses on the analysis of data collected in the context ofa prospective study based on assessment files made during consultations with parents who suspected an autistic disorder.140 files were examined. The variables used are: age of identification of the first signs by the parents, the nature of the signs observed and the socio-economic level of the parents Results.- Statistical analysis was performed using SPSS software. Preliminary results were obtained: The average age of identification of the first signs turns around 19 months; $41 \%$ of parents have concerns about delayed speech. However, we found no significant relationship between the age of first sign detection and the socioeconomic level of parents.

Conclusions.- In this work, we studied the average age of detection of autistic signs by Algerian parents as well as the nature of the signs identified. These results go into its literary data on this issue. Disclosure of interest.- The authors have not supplied a conflict of interest statement.

\section{E-PV0688}

\section{Depersonalization and psychosis-like experiences in the general population}

A. Bermejo Pastor ${ }^{*}$, M. Sánchez Amores, V. Perez Navarro, J. Pemán Rodríguez, P.L. Marta, Á. Huidobro Pérez-Villamil

Hospital Clínico San Carlos., Institute of Psychiaty and Mental Health, Madrid, Spain

Corresponding author.

Background and aims.- Depersonalization is a psychopathological phenomenon inwhich the individual experiences a sensation of unreality and detachment from himself, while judge of reality remains intact.

Psychosis-like experiences are attenuated symptoms similar to those found in psychosis.

Regarding such complex and prevalent phenomena, we aim to study whether they are correlated and how.

The aim of this poster is to review what is the prevalence of depersonalization and psychosis-like experiences and find how they are correlated

Methods.- A literature review of scientific papers in Pubmed and Medline. We considered only papers in English.

Results.- The prevalence of depersonalization in general population is $70 \%$.

The prevalence of depersonalization in the general population is $70 \%$, and the prevalence of psychosis-like experiences in the 
general population is $20-90 \%$. Some papers have found a link between depersonalization and full psychotic symptoms as well as psychosis-like experiences in the general population. The literature is heterogeneous: while some authors consider both phenomena indistinguishable in most cases, others see them as completely different entities. Despite different positions in this issue, many authors propose that depersonalization could be a contributing factor to the development of psychotic experiences. Moreover, studying the relationship between depersonalization and schizotypy, some authors have attempted to explain the link between both entities through three factors: cognitive failures, fantasy proneness, and childhood trauma.

Conclusions.- -Depersonalization and psychosis-like experiences are very prevalent phenomena in the general population and both experiences are statistically correlated.

- Depersonalization might play a role in the development of psychotic experiences.

Disclosure of interest.- The authors have not supplied a conflict of interest statement.

\section{E-PV0689}

\section{Delusional perception and kraepelin's paraphrenia - can they co-exist?}

L.A. C. Fernandes*, M.J. Santos, D. Almeida, J. Santos, N. Borja

Santos, T. Maia

Hospital Prof. Doutor Fernando Fonseca, Psychiatry, Lisbon, Portugal

${ }^{*}$ Corresponding author.

Background and aims.- Delusional perception is one of Schneider's schizophrenia first rank symptoms and perceived by this author as highly specific for its diagnosis. The present work aims to discuss the concept of delusional perception in the context of a Kraepelin's systematic paraphrenia case.

Methods.- Case report and brief literature review, using the keywords "paraphrenia", "systematic paraphrenia" and "delusional perception".

Results.- A 55-year old woman was admitted to our acute ward with psychotic symptoms. She had a long psychiatric history, with the first hospital admission due to psychotic symptoms, including delusional perception, at the age of 37 . By then she showed other positive symptoms - such as grandiose and persecutory delusions, delusion of control, and thought insertion - but no apparent disturbance of affects, volition, nor cognition. At the time, she was married and worked as teacher. After discharge she had several hospital admissions, due to poor medication compliance. Afterwards the patient maintained a steady follow-up, with presumed remission of all symptoms in the following 13 years. She continued to be functional at her job, without any relevant cognitive impairment. In the year prior to the present admission she abandoned her medication, with symptomatic recurrence.

Conclusions.- We illustrate a case of a psychotic disorder with long follow-up, closely resembling Kraepelin's description of systematic paraphrenia. This entity encompassed a cluster of dementia praecox patients with more benign longitudinal course, characterized by the absence of cognitive and negative symptoms. The present case shows that delusional perception, albeit highly specific of schizophrenia, can also be found in paraphrenic patients.

Disclosure of interest.- The authors have not supplied a conflict of interest statement.

\section{E-PV0690}

\section{Conversion catatonia. A case report of a rare cause of catatonia syndrome}

D.H. Calle*, M.B. Bardon Rivera, A. Muñoz San Jose, J. Marin

Lozano, M.F. Bravo-Ortiz

Hospital Universitario La Paz, Psychiatry, Madrid, Spain

${ }^{*}$ Corresponding author.
Background and aims.- Catatonia is a non-specific syndrome with many causes. Although rare, conversion disorder has been described as one of them. The inability to make an interview, the paucity of literature and the wide differential diagnosis makes the management of this condition a difficult task. The aim of this report is to outline the importance of conversion reactions in the differential diagnosis of catatonia.

Methods.- We report a case of a 34-year-old man who presented six months of complete mutism and hypoactivity that was admitted to the hospital after three days of refusal to eat and drink by voluntarily closing his mouth. He suffered three similar episodes, all precipitated by conflicts with his parents. Between episodes, although he presented moderate food restriction and selective mutism, he continued working as a teacher.

Results.- Although catatonia was initially diagnosed, several facts were incongruent with it: (1) Symptoms fluctuated with the examiner, not moving when ask by the psychiatrist but being collaborative with a complete exam by the neurologist. (2) Nor catalepsy, echopraxia, stereotypy or paratonia were present. (3) Mutism, hypoactivity and anorexia did not start simultaneously, as it happens in catatonia. (4) After the resolution of previous episodes he expressed the voluntary control of symptomology as a way of avoiding familiar conflicts. (5) Benzodiazepines and ECT were not effective. After excluding a neurologic aetiology, the most accurate diagnosis was a conversion syndrome.

Conclusions.- Conversion disorder is a possible cause of catatonia syndrome. An incongruent clinical picture and a possible secondary gain should alert of its possibility.

Disclosure of interest.- The authors have not supplied a conflict of interest statement.

\section{E-PV0692}

\section{The image of god and religiosity in patients with mental disorders}

J. Prusak ${ }^{1}$, D. Dudek ${ }^{2}$, K. Cyranka ${ }^{2 *}$, M. Siwek ${ }^{3}$, A. Michalska ${ }^{2}$

${ }^{1}$ Institute of Psychology, Department of Psychopathology and Preventive Psychology Jesuit University Ignatianum, Krakow, Poland; 2 Adult Psychiatry Clinic, Department of Psychiatry Jagiellonian University Medical College, Krakow, Poland; ${ }^{3}$ Institute of Affective Disorders, Deparment of Psychiatry Jagiellonian University Medical College, Krakow, Poland

Corresponding author.

Background and aims.- The aim of the study was to determine the image of God and religiosity in patients with various mental disorders (bipolar disorder, neurotic disorders, personality disorders) and to identify coping strategies in stressful situations in relation to religion and the image of God in people with mental disorders. Methods.- The study involved 30 patients treated for mental disorders (according to ICD), both in outpatient and stationary mode. Inclusion criteria.-

diagnosis from category F according to ICD-10: F20-F29; F31-F34; F40-F43; F-60.

Exclusion criteria.-

Active addiction to psychoactive substances

CNS organic disorders

no consent to participate in the study

The control group consisted of 30 people who were not in treatment due to mental disorders.

Persons from the studied group and from the control group were examined by psychiatrists and psychologists included in the research team with the use of a set of diagnostic tools: Brief RCope - measure of religious coping with major life stressors, Attitude to God Questionnaire, Sociodemographic Questionnaire, O'LIFE, TEMPS-A, CISS, HCL, MDQ, GHQ, Qualitative questions questionnaire designed by the authors of the study. The research was carried out anonymously in the Clinic of Adult Psychiatry. 
Results.- The study brings important observations about the attitude to God and to religiosity of patients various mental disorders in relation to their personality traits, coping strategies, level of anxiety and other parameters connected with patients' functioning.

Conclusions.- The study is important from clinical and practical perspective, suggesting also how the patients may project their attitudes towards authorities, doctors, psychotherapists, to the treatment and to the illness itself.

Disclosure of interest.- The authors have not supplied a conflict of interest statement.

\section{E-PV0694 \\ Is the praecox feeling a concept at risk of extinction?}

\author{
T. Ferreira ${ }^{*}$, I. Figueiredo ${ }^{1}$, S. Dehanov ${ }^{1}$, M. Melo ${ }^{2}$, N. Santos \\ Borja $^{1}$, T. Maia ${ }^{1}$ \\ ${ }^{1}$ Hospital Prof. Doutor Fernando da Fonseca, Department of \\ Psychiatry, Amadora, Portugal; ${ }^{2}$ Centro Hospitalar Psiquiátrico de \\ Lisboa, Psychogeriatric Unit, Lisboa, Portugal \\ * Corresponding author.
}

Background and aims.- The concept of praecox feeling (PF) was coined in 1941 by H.C. Rumke (1893-1967), who considered it highly specific to schizophrenia. However, it has been omitted in psychiatric semiology in recent years because it is considered too subjective and not useful as a diagnosis tool. Our aim is to define praecox feeling and understand its role in modern psychiatry.

Methods.- Review of literature from Pubmed database.

Results.- The PF, parallel to the Jasperian "radical incomprehensibility" and the Gestaltian "grasp of the whole clinical presentation", consists of a characteristic feeling of discomfort / bizarreness experienced early by the psychiatrist when in the presence of a person with schizophrenia. Impossibility of empathy, loss of affective resonance and instinctual rapport, and alterations in body expression and speech may contribute to that impression. Three studies with psychiatrists attempted to test its pertinence in diagnostic decision-making; the most recent one (Gozé, 2017) pointed to its presence in $90.1 \%$ of the respondents, being deemed reliable in $74.1 \%$. Two other recent studies with patients attempted to compare the accuracy of FP versus current diagnostic criteria, with disparate results.

Conclusions.- Although PF can not be considered for the reliable diagnosis of schizophrenia, this does not preclude its existence in clinical practice and its historical importance. PF could be useful to explore the experiences of the schizophrenic patient, its difficulties in interpersonal communication, and the importance of affectivity and empathy within the psychopathology of schizophrenia. Nowadays, it could make us question the importance of the intersubjective encounter and phenomenology in nosology. Disclosure of interest.- The authors have not supplied a conflict of interest statement.

\section{E-PV0697}

\section{Formal thought disorders - a narrative review}

J. Jerónimo ${ }^{1 *}$, Q. Tiago ${ }^{1}$, E.C. Cheniaux ${ }^{2}$, D. Telles-Correia ${ }^{3}$

1 Centro Hospitalar Lisboa Norte, Serviço de Psiquiatria e Saúde

Mental, Lisboa, Portugal; ${ }^{2}$ Universidade do Estado do Rio de Janeiro, Faculdade de Ciências Médicas, Rio de Janeiro, Brazil; ${ }^{3}$ Universidade de Lisboa, Faculdade de Medicina da Universidade de Lisboa, Lisboa, Portugal

* Corresponding author.
Background and aims.- In this communication the authors intend to review in an intelligible and comprehensive way the historical roots of Formal Thought Disorders.

Methods.- Studies were searched from PubMed database with the following keywords: psychopathology, thought disorders, formal thought disorders. A comprehensive manual search, including search from the reference list of included articles, was also performed.

Results.- Early descriptions of thought disorders date back to the XIX century with Esquirol, but it was in the first half of the $\mathrm{XX}$ century that several authors introduced the main features of the actual concept of Formal Thought Disorders. Emil Kraepelin described akataphasia (inability to find the appropriate expression for a thought) in patients with dementia praecox (a term that some years later was replaced by schizophrenia). Bleuler and Kretschmer also identified in schizophrenic patients a generalized "loosening of associations" and Carl Schneider described several Formal Thought Disorders such as derailment, fusion, omission, suspension and drivelling. In the beginning of the XXI century Nancy Andreasen studied the classical descriptions regarding Formal Thought Disorders, reclassified them and also introduced a scale to assess them. Conclusions.- Although the specificity of these symptoms in schizophrenia and psychosis has been a source of controversy among the different authors, the importance given to their presence in these mental disorders is universal. We defend that it is crucial that these historical and conceptual elements are grasped in order to assess Formal Thought Disorders for clinical and research purposes.

Disclosure of interest.- The authors have not supplied a conflict of interest statement.

\section{E-PV0698 \\ Cognitive deficits in patients with a first psychotic episode}

E. Kucherova ${ }^{1}$, D. Tikhonov ${ }^{2}$, S. Khrushchev ${ }_{-}^{1,3^{*}}$, A.

Spivakovskaya $^{1}$, G. Rupchev ${ }^{1,2}$, V. Kaleda ${ }^{2}$

${ }^{1}$ Lomonosov Moscow State University, Psychology Department, Moscow, Russia; ${ }^{2}$ Mental Health Research Center, Mental Health Research Center, Moscow, Russia; ${ }^{3}$ National Research Center for Hematology, Psychiatry and Neurology Department, Moscow, Russia ${ }^{*}$ Corresponding author.

Background and aims.- The change in neurocognitive indices in patients with schizophrenia can warn about the imminent exacerbation of the disease, and it can also influence on the choice of drug therapy and show the effect of the treatment. Nowadays diagnosis of the neurocognitive deficiency also helps to predict its consequences (impairments of quality of life). Cognitive impairments in patients varies significantly, despite the same diagnosis. Such differences should be taken into account in the treatment and rehabilitation of patients along with positive and negative symptoms.

Objective:Cognitive deficits in the manifestation of schizophrenia. Aims.- To reveal cognitive impairments and their variability in patients first hospitalized with the diagnosis of "schizophrenia". Methods.- $(N=17)$ male patients (age: $16-32$ y.o. mean age: 20.6 y.o.) with a verified diagnosis of schizophrenia. Patients were examined with a brief assessment of cognitive function in patients with schizophrenia test (BACS),scores of functions associated with cognitive impairment in CogFU (Cognitive Functioning Scale), Rorschach ink stain test of the Exner system (Exner, 1991) Results.- 


\begin{tabular}{|c|c|c|c|c|c|c|c|}
\hline Composite Scores & $\begin{array}{c}\text { Verbal } \\
\text { Memor } \\
\mathbf{y}\end{array}$ & $\begin{array}{c}\text { Digit } \\
\text { Sequencin } \\
\mathbf{g}\end{array}$ & $\begin{array}{c}\text { Token } \\
\text { Motor } \\
\text { Task }\end{array}$ & $\begin{array}{c}\text { Symbo } \\
\mathbf{I} \\
\text { Coding }\end{array}$ & $\begin{array}{c}\text { Verbal } \\
\text { Eluenc } \\
\mathbf{y}\end{array}$ & $\begin{array}{c}\text { Tower } \\
\text { of } \\
\text { Londo } \\
\mathbf{n}\end{array}$ & $\begin{array}{c}\text { BACS } \\
\text { Composit } \\
\text { e T Score: }\end{array}$ \\
\hline $\begin{array}{c}\text { Average for healthy } \\
\text { people }\end{array}$ & 50 & 50 & 50 & 46 & 54 & 50 & 50 \\
\hline Average for patients & 28,76 & 38,41 & 39,88 & 33,76 & 31,06 & 40,53 & 42,88 \\
\hline Minimum & -6 & 15 & 19 & 3 & 8 & 20 & 3 \\
\hline Maximum & 51 & 53 & 59 & 72 & 53 & 64 & 62 \\
\hline Median & 29 & 41 & 45 & 32 & 33 & 39 & 47 \\
\hline Standard Deviation & 14,4 & 9,99 & 12,75 & 17,16 & 12,32 & 9,36 & 12,98 \\
\hline
\end{tabular}

Table 1. Comparison of composite patient scores with regulatory data.

Cognitive dysfunction in patients with schizophrenia affects all functions of the cognitive sphere - memory, attention,action planning functions, motor functions, visual-motor coordination, abstract thinking. However,such impairments severity can vary widely in contrast to the normative values. Composite T-scores of patients ranged from the maximum possible to negative values for all subtests conducted. In patients the cognitive resource was lower than in others, in dozens of times, which must be taken into account in the treatment and further adaptation of patients suffering from schizophrenia (Table 1 ).

Conclusions.- There is significant variability in cognitive impairments in patients in the manifestation of schizophrenia who had the same diagnosis and treatment. This data may be used in treatment and rehabilitation programs development.

Disclosure of interest.- The authors have not supplied a conflict of interest statement.

\section{E-PV0699}

\section{Does how to interpret our experience affect how to memorize? Effects of cognitive bias modification (CBM) in depression \\ J. Lee}

Kangwon National University, Department of Psychology,

Chuncheon, Republic of Korea

${ }^{*}$ Corresponding author.

Background and aims.- Cognitive model of depression suggests that information processing such as attention, interpretation and memory bias respectively affect depressive symptoms. The combined hypothesis indicating that interpretation, attention and memory biases does not operate in isolation but influence depressive symptoms by working together is also of special interest. The present study aimed to investigate whether positive interpretation biases generated by CBM training would be helpful in recalling positive memory.

Methods.- Seventy depressed graduate and undergraduate students were allocated to one of two groups (intervention group with CBM training vs. control group without CBM training). Four sessions of computer based CBM trainings were delivered to the intervention group over the two week periods. Both groups completed preand post-tests: an ambiguous interpretation bias task, memory task and self-reported mood measurements.

Results.- The CBM training group aiming to promoting positive interpretations reported decreased sadness and tension whereas the control group did not show such changes in mood. In addition, the CBM training group interpreted ambiguous stimuli in a more positive manner and recalled more positive memory, compared to the control group.

Conclusions.- CBM training for positive interpretation might be helpful to memorize what the depressed experience in daily life in a more positive way. Our results support the combined hypothesis indicating that interpretation biases affect memory biases. A future study warrants to confirm the opposite direction as well: memory biases would affect interpretation biases.
Disclosure of interest.- The authors have not supplied a conflict of interest statement.

\section{E-PV0700}

\section{Low frequency alpha $(8-10 \mathrm{~Hz})$ activity correlated with inhibitory behavior}

Y.W. Kim ${ }^{1}$, S. Kim ${ }^{1}$, M.J. Jin ${ }^{1}$, C.H. Im $^{2}$, S.H. Lee ${ }^{3 *}$

${ }^{1}$ Clinical Emotion and Cognition Research Laboratory, Inje University, Goyang, Republic of Korea; ${ }^{2}$ Department of Biomedical Engineering, Hanyang University, Seoul, Republic of Korea ${ }^{3}$ Inje University Ilsan Paik Hospital, Psychiatry, Goyang, Republic of Korea * Corresponding author.

Background and aims.- Alpha frequency of EEG and the default mode network (DMN) are predominantly activated during restingstate. Also, alpha frequency power is known to be related with inhibitory function. This study investigated the neuropsychological characteristics of alpha band power and alpha DMN at resting state.

Methods.- Resting-state EEG, go/nogo ERP/behavioral data, and psychological measures were examined in a total of 101 healthy individuals. Relative alpha $(8-12 \mathrm{~Hz})$, low-alpha $(8-10 \mathrm{~Hz})$, highalpha $(10-12 \mathrm{~Hz})$ powers were calculated from the resting-state EEG data. Source activations of 25 DMN regions and their global/nodal network measures (clustering coefficient (CC), path length (PL), efficiency, strength, and eigenvector centrality (EC)) were also calculated. Psychological measures included the Gray's behavioral inhibition/behavioral activation scale (BIS/BAS), the Barratt impulsivity scale (BIS), and the Conner's adult ADHD rating scale (CAARS). Individuals were divided into 3 groups (low, middle, high) based on the level of power of each total/low/high-alpha frequency band.

Results.- Significant group differences were found in low-alpha frequency power. The high group had significantly higher BIS score (behavior inhibition), significantly higher levels of global/nodal CC, efficiency, and strength, and a significantly lower PL at all region after Bonferroni correction compared to low and middle group. BIS inhibition was positively correlated with global/nodal CC, efficiency, and strength, and negatively correlated with global PL.

Conclusions.- Our study revealed that low frequency alpha power is specifically related with inhibitory function. The results also suggest that DMN of low alpha frequency band could be a potential candidate of biological marker of inhibitory function.

Disclosure of interest.- The authors have not supplied a conflict of interest statement.

\section{E-PV0702 \\ Insights into secondary delusions - regarding a clinical case}

B. Moura ${ }^{1}$, S. Ramos ${ }^{2}$, A.T. Pereira ${ }^{1}$, A. Miguel ${ }^{1}$, R. Ribeiro Silva ${ }^{1}$ ${ }^{1}$ Centro Hospitalar Vila Nova de Gaia/Espinho, Department of Psychiatry and Mental Health, Vila Nova de Gaia, Portugal; ${ }^{2}$ Unidade Local de Saúde da Guarda, Department of Psychiatry, Guarda, Portugal

* Corresponding author.

Background and aims.- To Jaspers, belief presentation to consciousness ranges from normal, to overvalued ideas, to primary or secondary delusions. The latter are defined by their understandability in light of the individual's previous experience and personality, and the presence of other psychopathological symptoms, such as altered states of humour, conscience or abnormalities of sensoperception (SP).

Cognitive processes in delusional individuals have been extensively studied, and there is evidence they may be preserved, when the delusion arises in the presence of erroneous information (such as altered SP). 
We aim to describe a clinical case of secondary delusions.

Methods.- Description of clinical case. Review of literature after search of clinical databases.

Results.- L., female, 39 years old. University Degrees in Education and Translation. Unemployed since 2016. Currently living with parents. No psychiatric history until 2013, when she starts having auditory hallucinations, voices that insult her, and that she interprets as coming from unknown people on the street. She develops a persecutory delusion based on the belief she was being defamed. Progressive worsening of thought and behaviour disorganization. In 2018, after pressing charges for defamation, a clinical psychiatrical evalutation is mandated by the court, after which she is commited involuntarily. There was complete remission of auditory hallucinations with antipsychotic medication. The patient gained full insight.

Conclusions.- Several mechanisms can contribute to the emergence of delusions. Case description seems compatible with the phenomenological definition of secondary delusions, with the preservation of the mechanisms of logical thought, that allowed revaluation of beliefs with a gain of insight after pharmacological and psycotherapeutic interventions.

Disclosure of interest.- The authors have not supplied a conflict of interest statement.

\section{E-PV0704}

\section{Complications in Parkinson's disease aggravated under antipsychotic treatment}

\section{S. Trifu ${ }^{*}$, E.G. Carp ${ }^{2}$, M. Dragoi ${ }^{3}$}

${ }^{1}$ University of Medicine and Pharmacy “Carol Davila”, Neurosciences - Psychiatry, Bucharest, Romania; ${ }^{2}$ Hospital for Psychiatry, Psychiatry, Sapunari, Romania; ${ }^{3}$ Hospital for Psychiatry “Alex. Obregia", Psychology, Bucharest, Romania

* Corresponding author.

Background and aims.- The superimposition of elements within the psychotic Parkinson's disease involves a particular therapeutic approach because of the antagonism between the D2 receptors on which means anti-Parkinson and antipsychotic medication. These paperwork shows the presentation of a patient aged 69 years, admitted to a psychiatric ward for dementia, which present a Parkinson's subclinical, which was aggravated during the psychiatric hospitalization, under AP medication, and augmented by a respiratory infection.

Methods.- Psychological and psychiatric evaluation at initial admission, cerebral CT scan, MRI brain after worsening symptoms, interdisciplinary consult (infectious diseases, neurology), under medical supervision, monitoring combined neurology, cardiology and psychiatric treatment.

Results.- The patient comes to the hospital for neuro-cognitive disorders corresponding to a mixed dementia stage $\mathrm{V}$ Reisberg, psychotic phenomena and misbehavior events went up to hetero-aggressivity. Under Risperidona treatment, suddenly presents apathy state with high fever, leukocytosis, elevated creatine-kinase, lumbar puncture with albumin in sanguine plasma (uncertain outcome). Combined antibiotic treatment is hereby established and antiviral, neurologic examination is identifying a Parkinson's disease aggravated, favorable results under treatment with Amantadine, Madopar and Romparkin, together with PEV and vitamin therapy.

Conclusions.- Mixed dementia can hide a Parkinson component tremor that is more difficult to identify if it exclusively evolves with hypertonic status, Parkinson's disease may be aggravated by intercurrent infection or by incisive psychiatric medications, even low doses.

Disclosure of interest.- The authors have not supplied a conflict of interest statement.

\section{E-Poster Viewing - 7-9 April: Psychopharmacology and Pharmacoeconomics}

\section{E-PV0707 \\ Safety of amitriptyline-perphenazine during breastfeeding}

P. Argitis ${ }^{1}$, C.M. Platsa ${ }^{1}$, S. Karavia ${ }^{1}$, A. Kourti ${ }^{1}$, P. Platari ${ }^{2}$, K.

Paschalidis $^{3}$, Z. Chaviaras $^{1}$

${ }^{1}$ General Hospital of Corfu, Psychiatric, Corfu, Greece; ${ }^{2}$ General Hospital of Kalamata, Psychiatric, Kalamata, Greece; ${ }^{3}$ Psychiatric Hospital of Thessaloniki, Psychiatric, Thessaloniki, Greece

${ }^{*}$ Corresponding author.

Background and aims.- Amitriptyline/perphenazine combination is a common drug used in Greece. The object is to determine if the above combination can be usedwith safety during breastfeeding. Methods.- We conducted a search on the existing literature (Pubmed, Lactancia), related to the drugs amitriptyline, perphenazine and their safety to the infant during nursing.

Results.- There is evidence that amitriptyline milk/plasma ratio do not exceed 1.1 , in a therapeutic dose of $25-175 \mathrm{mg}$. In all cases, the levels of the drug in the serum of the infantare almost undetectable. Developmental adverse effects are not displayed in any case, in a therapeutic dose of 75-175 mg of amitriptyline. A study presented a case of a nursing mother, using amitriptyline $10 \mathrm{mg}$ for insomnia, which caused sedation of the infant and discontinuation of the drug. Sleepiness was reported in a study, which was self-limited with no developmental effect. Perphenazine in a dose of $24 \mathrm{mg}$, has been reported to milk/plasma ratios of $<1$, without evidence of toxicity or developmental adverse effects. Ten reports have addressed a total of 28 infants' exposure to antipsychotic agents (including perphenazine) through breastfeeding. In the majority of cases, no adverse events were observed.

Conclusions.- The combination of amitriptyline/perphenazine seems to be safe during breastfeeding,although more research is needed.

Disclosure of interest.- The authors have not supplied a conflict of interest statement.

\section{E-PV0708}

\section{Amitriptyline and perphenazine in cronic pain: a literature review}

P. Argitis ${ }^{1 *}$, C.M. Platsa ${ }^{1}$, S. Karavia ${ }^{1}$, A. Kourti ${ }^{1}$, P. Platari ${ }^{2}$, M. Poulou $^{3}$, Z. Chaviaras ${ }^{1}$

${ }^{1}$ General Hospital of Corfu, Psychiatric, Corfu, Greece; ${ }^{2}$ General Hospital of Kalamata, Psychiatric, Kalamata, Greece; ${ }^{3}$ General Hospital of Kymi, Anesthesiology, Kymi, Greece

* Corresponding author.

Background and aims.- Pain cannot always be entirely resolved with current treatments. In these circumstances, pain continues and becomes chronic with challenging treatment options. Our aim is to report the possible effects from the combination of amitriptyline/perphenazine a common drug used in Greece.

Methods.- We followed an extensive review of the existing literature concerning the combination of amitriptyline/perphenazine in cronic pain.

Results.- Tricyclic antidepressants, including amitriptyline, are first-line treatment in neuropathic pain (NNT3.2-4.1). Amitriptyline reverses the attenuation of endogenous analgesia through effects on the descending noradrenergic system in rats. There is evidence that oral phenothiazines, including perphenazine, have analgesic effect in studies that depend upon clinical observation, withdrawal and re-challenge, but the mechanism is not well established. Evidence demonstrate the significance of the combination of TCAs with phenothiazines, in order to decrease surgical 
interventions in postherpetic neuralgia,as well as the benefits of amitriptyline/perphenazine's combination in the treatment of this specific condition. Amitriptyline $75 \mathrm{mg}$, administered with perphenazine $6 \mathrm{mg}$ is most effective in patients with postherpetic neuralgia and in those with post-operative scar pain. The percentage of patients with deterioration of chronic pain is low, while treated with amitriptyline/perphenazine in the same dosage. Data also shows that postherpetic neuralgia seems to respond better in treatment with amitriptyline/perphenazine among other chronic pain conditions.

Conclusions.- More research is needed, in order to determine the exact mechanism of action of these combined agents in chronic pain. The knowledge that a low dosage of perphenazine can be used in gastrointestinal disorders, such us nausea and vomiting,might be contributive to amitriptyline's adverse effects.

Disclosure of interest.- The authors have not supplied a conflict of interest statement.

\section{E-PV0709}

\section{Nalmefene induced psychotic episode.}

\section{A case report}

P. Argitis ${ }^{1 *}$, A. Kourti ${ }^{1}$, C.M. Platsa ${ }^{1}$, S. Karavia ${ }^{1}$, P. Bouras ${ }^{2}$, K. Paschalidis $^{3}$, A. Topitsas ${ }^{1}$, I. Moraiti ${ }^{1}$, Z. Chaviaras $^{1}$

${ }^{1}$ General Hospital of Corfu, Psychiatric, Corfu, Greece; ${ }^{2}$ General Hospital of Corfu, Internal medicine, Corfu, Greece; ${ }^{3}$ Psychiatric Hospital of Thessaloniki, Psychiatric, Thessaloniki, Greece ${ }^{*}$ Corresponding author.

Background and aims.- Nalmefene is an opiate receptor antagonist with a partial agonist effect on $\kappa$-receptors,approved for alcohol dependency treatment. Most of the medications' side effects are innocuous, but others could be alarming and lead to hospitalization. Methods.- We present you the case of a patient admitted to our unit with psychotic features after taking up nalmefene.

Results.- J.H, who was suffering from alcohol abuse, ceased drinking alcohol three days before her admission to our hospital. After contacting a psychiatrist, the day before her admission, she took up nalmafene. A few hours before her admission, she presented visual and auditory hallucinations, persecutory delusions and delusions of reference and appeared to be confused. Since no pathological cause for her condition was identified in the emergency department, she was admitted to our psychiatric clinic. The following days we performed the essential tests and excluded a cause from the CNS. The second day of her hospitalization she featured long-term memory disorders lasting two days. During her hospitalization she was medicated solely with thiamine and benzodiazepines, not antipsychotics, and did not present signs of alcohol withdrawal since her arrival to the hospital. The fourth day, no pathological findings or psychopathology were present and she returned to her preadmission functionality.

Conclusions.- Nalmefene might be approved to treat alcohol dependacy, however not all of its side effects have been examined thoroughly. Thus, more research is needed in order for the doctors' better information and vigilance while treating a patient with nalmefene.

Disclosure of interest.- The authors have not supplied a conflict of interest statement.

\section{E-PV0710}

\section{Nortriptylline-induced exacerbation of glaucoma: a case-report}

M.H.L. Arts ${ }^{1}$, L. de Jonge ${ }^{2}$, S. Petrykiv ${ }^{3}$

${ }^{1}$ Mental Health Care -Western North Brabant GGZ-WNB, Department of Old Age Psychiatry and Neuropsychiatry, Halsteren, The Netherlands; ${ }^{2}$ Leonardo Scientific Research Institute, Department of Neuropsychiatry, Groningen, The Netherlands; ${ }^{3}$
MUMC, Department of Clinical Pharmacy and Pharmacology, Maastricht, The Netherlands

* Corresponding author.

Background and aims.- Tricyclic antidepressants (TCA) have been extensively investigated and applied in late-life depression. Compared to other TCAs, nortriptylline appears to have lower sedative and anticholinergic effects and lower a risk for orthostatic hypotension. However, the anticholinergic properties of this drug should not be underestimated. Next to urinary retention, serious visual problems have been documented.

Objectives $\mathcal{E}$ aims.- To present a case report of a patient who developed serious ocular pain en visual problems three weeks after starting therapy with the TCA nortriptylline.

Methods.- An English language literature search was conducted using Pubmed and EMBASE searching for case reports and observational studies reporting visual problems in older adults using nortryptilline.

Results.- A 77-year-old woman was known with major depression since two months. Two weeks after initiation of therapy with nortriptylline $50 \mathrm{mg} /$ day (plasma level $85 \mathrm{ng} / \mathrm{mL}$ ), she experienced severe eye pain, "colored halos", and loss of visual acuity. General medical and neurological examination excluded any pathology. During ophthalmological examination, an increased intraocular pressure was found, and she was diagnosed with narrow-angle glaucoma.

Conclusions.- Nortriptylline can increase intra-ocular pressure. This may cause or exacerbate glaucoma in people who are already at risk. Especially frail older adults are more susceptible for adverse effects of TCAs. In addition to the examination of (orthostatic) blood pressure, electrocardiography, exclusion of urinary retention, an ophthalmological history should be taken into account before prescribing nortriptylline.

Disclosure of interest.- The authors have not supplied a conflict of interest statement.

\section{E-PV0712 \\ Pseudoparkinsonism and secondary suicidal ideation after 3-month paliperidone palmitate administration: a clinical case \\ L.A. C. Fernandes ${ }^{1 *}$, L. Serra ${ }^{2}$, M. Lages ${ }^{1}$, D. Almeida ${ }^{1}$, J. Flores ${ }^{1}$, C. Tomé $^{1}$, N. Borja-Santos ${ }^{1}$ \\ ${ }^{1}$ Hospital Prof. Doutor Fernando Fonseca, Psychiatry, Lisbon, Portugal; ${ }^{2}$ Centro Hospitalar do Algarve, Psychiatry, Faro, Portugal * Corresponding author.}

Background and aims.- To report a relevant adverse effect associated with 3-month long-acting paliperidone palmitate (3M-PP) not noted during oral and 1-month long-acting paliperidone trials (1M-PP).

Methods.- Case report and literature review.

Results.- A 43 years-old man was admitted to the emergency room (ER) due to suicidal ideation. His past medical record was relevant for organic schizophrenia-like psychotic disorder with documented brain lesions and several acute psychotic episodes; and HIV infection, with an irregular compliance to anti-retroviral therapy. He had recently initiated $3 \mathrm{M}$-PP treatment. The oral and $1 \mathrm{M}-\mathrm{PP}$ trials ( 4 injections of $100 \mathrm{mg}$ ) went on with no reported adverse effects. Immediately after the first 3M-PP injection $(350 \mathrm{mg})$ the patient reported psychomotor retardation, with functional impairment, which worsened after the second administration. Associated feelings of hopelessness and suicidal thoughts were present, bringing the patient to the ER. On observation, grossly symmetric parkinsonism was evident. Neither criteria for major depressive disorder or acute psychotic episode were met. HIV viral load was undetectable at the time. Anticholinergic medication was given, 
with improvement of extrapyramidal symptoms (EPS) and suicidal ideation.

Conclusions.- Although PLP-3M approval studies reported a low incidence of pseudoparkinsonism, there is lack of data concerning the emergence of these complaints exclusively after the administration of 3-month injection. The onset of EPS in this case is confounded by several factors (HIV infection; structural brain abnormalities; pharmacokinetic interactions). This case reminds us EPS' functional impact and a possible relation with suicide risk, thus the importance of evaluating them in patients taking dopamine D2 receptor antagonists, particularly long-acting formulations.

Disclosure of interest.- The authors have not supplied a conflict of interest statement.

\section{E-PV0715 \\ Effectiveness and tolerability of vortioxetine in affective disorders: a naturalistic study}

B.M. Grancini", V. De Carlo, M. Vismara, G. Cirnigliaro, L. Degoni, B. Dell'Osso

Fondazione IRCCS Ca' Granda, Ospedale Maggiore Policlinico,

University of Milan, Department of Psychiatry, Milano, Italy

${ }^{*}$ Corresponding author.

Background and aims.-

Introduction.- Vortioxetine is a novel antidepressant with multimodal activity [1]. In the last decade, it has been approved worldwide $[2,3]$.

Objectives.- The present naturalistic study aimed to characterize effectiveness and tolerability of vortioxetine in affective disorders in real clinical practice.

Methods.- Study total sample consisted of 48 outpatients, treated with vortioxetine and mainly with a primary diagnosis of Major Depressive Disorder/MDD, Bipolar Disorder/BD, Generalized Anxiety Disorder, Panic Disorder, Obsessive Compulsive Disorder, Adjustment Disorder. Collected variables included sociodemographic, clinical and three periodic psychometric evaluations (week 0, 4 and 12; including HAM-D, MADRS, HAM-A, YMRS, CGI, Dosage Record Treatment Emergent Symptom and Treatment Emergent Symptoms Write-In scales). Statistical analyses were performed.

Results.- Most common primary diagnoses were MDD (43.8\%) and BD (33.3\%), with an overall comorbidity rate of 52\%. Associated medications (stable across follow up) were present in $92 \%$ of patients and the mean vortioxetine dosage was $12.92 \pm 5.68 \mathrm{mg}$. Repeated measures ANOVA highlighted a significant improvement of HAM-D, MADRS, HAM-A, YMRS and CGI-efficacy index across time. The $33.3 \%$ of the total sample dropped out, of which mainly because of non efficacy (43.8\%) and side effects (37.5\%). Of the total sample, $16.6 \%$ reported gastrointestinal, $2.1 \%$ sexual and $8.3 \%$ combined side effects (gastrointestinal + other).

Conclusions.- Although limited study sample, the present report highlights vortioxetine good effectiveness on affective symptoms together with partial tolerability and moderate rate of discontinuation. Larger sample and further studies are warranted to better characterize vortioxetine effectiveness and tolerability in clinical practice.

Disclosure of interest.- The authors have not supplied a conflict of interest statement.

\section{E-PV0718}

\section{Pedal edema and atypical antipsychotic: case report and systematic review}

\section{S. Ben Ali ${ }^{1}$, Y. Zgueb ${ }^{2}$, R. Jomli ${ }^{2}$, U. Ouali ${ }^{2}$, F. Nacef ${ }^{2}$}

${ }^{1}$ Razi Hospital, Psychiatric A Departement, Ariana, Tunisia; ${ }^{2}$ Razi

Hospital, Psychiatric A Departement, Manouba, Tunisia

"Corresponding author.

Background and aims.- Despite their effectiveness, atypical antipsychotic have several undesirable effects. these effects are partly responsible for the poor therapeutic observance. Pedal edema is one of those undesirable effects largely reported by patients but rarely considered by the practitioner.

We propose to report a case of Quetiapine-induced pedal edema and a literature review of atypical antipsychotic-induced edema. Methods.- case report and literature review.

Results.- This study describes a 49 years-old female patient who was being managed for bipolar affective disorder for over 20 years. This patient had develloped a bothersome and painful incidence of pedal edema two weeks after the initiation of atypical antipsychotic regimen quetiapine. All hematological and biochemical profiles were found to be normal. By decreasing the dose of quetiapine, she started showing gradual improvement in pedal edema. Quetiapine was slowly discontinued.. Pedal edema was found to be resolved within weeks of dechallenge of the regimen. recurrence of pedal There was no recurrence of edema occurred at four-months follow-up

Conclusions.- Antipsychotic-induced edema is not an uncommon side-effect of atypical antipsychotics. Edema is embarassing to patients and usually requires a multiple examinations to determine its causes.

Disclosure of interest.- The authors have not supplied a conflict of interest statement.

\section{E-PV0720 \\ Risperidone-induced liver injury is reversed following paliperidone treatment}

S. Giannelos ${ }^{1}$, N. Kokras ${ }^{1,2^{*}}$, A.A. Politis ${ }^{1}$, E. Vasilopoulos ${ }^{1}$, A.M. Politis $^{1}$, C.C. Papageorgiou ${ }^{1}$

${ }^{1}$ Medical School- National and Kapodistrian University of Athens, First Department of Psychiatry, Athens, Greece; ${ }^{2}$ Medical School, National and Kapodistrian University of Athens, Department of Pharmacology, Athens, Greece

"Corresponding author.

Background and aims.- Risperidone-induced hepatotoxicity is an uncommon side-effect with very few published case reports. Paliperidone, risperidone's active metabolite, undergoes minimal metabolism and is not expected to increase alanine aminotransferase (ALT) and aspartate aminotransferase (AST). Therefore it is probably a safe switch following risperidone-induced hepatotoxicity.

Methods.- We report a case of a 38 years-old female, with a history of schizophrenia since the age of 27 , who was treated with $20 \mathrm{mg}$ of olanzapine, and was admitted due to a recent exacerbation of the disease. Upon admission, the medical examination was unremarkable and routine blood tests were normal.

Results.- During hospitalization because of the unsatisfactory response to olanzapine, a switch to risperidone was performed with gradual titration to $6 \mathrm{mg}$. As soon as the 12 th day of risperidone treatment, an increase in ALT, AST, and Creatine Kinase (CK) was noticed. Levels of alkaline phosphatase (ALP) and gammaglutamyltransferase $(\gamma-G T)$ were unaffected. ALT, AST, and CK peaked during the 24th day of treatment with a substantially increased AST/ALT ratio, indicative of liver injury. A thorough med- 
ical examination did not reveal other reasons for liver damage. Due to the temporal association with the initiation of risperidone, and the patient's good response to risperidone treatment, an abrupt switch from risperidone to $9 \mathrm{mg}$ paliperidone was performed. ALT, AST and CK declined rapidly within 10 days.

Conclusions.- In those rare cases of risperidone-induced hepatotoxicity, switching to paliperidone proves a safe strategy, as predicted by the pharmacokinetic properties of paliperidone. Initiation of risperidone treatment may require observation of liver LFTs during the first weeks of treatment.

Disclosure of interest.- The authors have not supplied a conflict of interest statement.

\section{E-PV0721}

\section{Clinicians' opinions on the future of psychopharmacology}

A. Dudek, M. Ciułkowicz, M. Kozłowska, K. Krysta*

Medical University of Silesia, Department of Rehabilitation

Psychiatry, Katowice, Poland

* Corresponding author.

Background and aims.- Even though brain science is well developed nowadays, finding cerebral mechanisms which can be the target for pharmacological treatments still remains a challenge. The aim of the study was to evaluate clinicians' attitudes to the future of psychopharmacology.

Methods.- The author's questionnaire was prepared. The questions regarded the respondents' opinion on classification systems. 109 psychiatrists, aged mainly 41-50 (31 men and 78 women), were interviewed during a national psychiatric conference. Responses were collected and analyzed.

Results.- Affective disorders, schizophrenia and anxiety disorder were most frequent diagnoses made by the doctors while antidepressants was the major pharmacological group they used in daily practice. Areas facing the biggest medical need to introduce new drugs were schizophrenia and dementia. Versions of existing drugs, but with fewer side-effects and completely new drugs were regarded as the most desired forms of pharmacology. As reasons for the insufficient development of new psychopharmacological solutions they listed lack of enough funding and legal difficulties. Conclusions.- $51.38 \%$ of respondents answered that the development of new pharmacological agents reached its peak in the last decade of XX century. On the other hand psychopharmacological treatment methods still seem to be unsatisfactory and $37.62 \%$ of respondents expect completely new solutions.

Disclosure of interest.- The authors have not supplied a conflict of interest statement.

\section{E-PV0722}

\section{Olanzapine induced rhabdomyolysis: \\ a case report}

H. Maatallah", R. Damak, I. Berrahal, N. Ghazouani, W. Cherif, M. Cheour

Razi Hospital, Psy E, Tunis, Tunisia

${ }^{*}$ Corresponding author.

Background and aims.- "Atypical” antipsychotics tend to replace traditional antipsychotics as first line therapy for psychotic disorders, due to their better side-effect profile with fewer extrapyramidal manifestations, allowing a better observance. Olanzapine is a widely used. Few reports on the association between olanzapine use and rhabdomyolysis have been published to date.
Objectives.- To report a case of olanzapine-induced rhabdomyolysis with massive increase in CK activity and discuss its possible mechanisms.

Methods.- Case report of olanzapine-induced rhabdomyolysis and a Literature Review.

Results.- A 59 -year-old woman, had a history of chronic schizophrenia,his first episode of auditory hallucination and persecutory delusion occurring at 20 years age. Between the ages of 40 and 53 years,the patient was repeatedly hospitalized due to psychotic exacerbations. At the last hospitalization she was put under olanzapine $15 \mathrm{mg} /$ day with clinical improvement and stabilization of psychotic disorders during 4 years. The patient was hospitalized for psychotic relapse due to the cessation of treatment and followup during 18 months. She was put under olanzapine $10 \mathrm{mg} /$ day. But after five days, she could not get up from the floor due to generalized weakness and paralysis. She was conscious, and had decreased muscle power but no extra-pyramidal symptoms. Creatine kinase (CK) level was high (1377 UI/L),other parameters were normal. Rhabdomyolysis was diagnosed. Due to its contribution to rhabdomyolysis,olanzapine was stopped. Following hydration, the patient's CK level fell to $220 \mathrm{UI} / \mathrm{L}$, ten days later. The patient was put under another antipsychotic:risperidone $2 \mathrm{mg} /$ day.

Conclusions.- This patient's presentation illustrates that olanzapine can cause rhabdomyolysis. The case report points to the necessity of serum CK monitoring when patients complain about mild muscular symptoms.

Disclosure of interest.- The authors have not supplied a conflict of interest statement.

\section{E-PV0723}

\section{Functionality index, a theoretical concept for all antipsychotics used in schizophrenia}

\section{G.C. Marinescu}

Emergency County Hospital Pitesti and Psychiatry Private CarePitesti- Arges- Romania, Psychiatry, Pitesti, Romania

* Corresponding author.

Background and aims.- In our current practice we don't have a theoretical concept in order to quantify the power of an antipsychotic to improve functionality of patients with schizophrenia. We tried to quantify what actions of antipsychotics (clinical, adverse events) may be "good" or "bad" for functionality and what receptors and actions are responsive for these actions.

Methods.- We defined functionality index as a concept that expresses in percent the pharmacodynamic capacity of an antipsychotic to improve patient functionality relative to a theoretical possible maximum. Each receptor or action received 1 point if it is at maximum possible or a proportional less if not, depending on their degree of receptor binding or action. We calculated the possible theoretical maximum and assigned to this $100 \%$. For each antipsychotic, we summed up the "good" receptors and actions scores, and then we lowered the "bad" receptors and actions scores. We reported each result to the maximum theoretically possible and then we calculated the percentage.

Results.- We can see functionality index results in the next table (Fig. 1). 


\begin{tabular}{|c|c|c|c|c|c|}
\hline & & $\begin{array}{l}\text { Sum of pro } \\
\text { functionality }\end{array}$ & $\begin{array}{l}\text { Sum of anti } \\
\text { functionality }\end{array}$ & $\begin{array}{l}\text { Difference } \\
\text { Good-Bad }\end{array}$ & $\begin{array}{l}\text { \% from maximum } \\
\text { functionality }\end{array}$ \\
\hline \multirow{5}{*}{ The Pines } & Clozapine & 4,5 & 2,25 & 2,25 & $17,31 \%$ \\
\hline & Olanzapine & 4,25 & 2,25 & 2,00 & $15,38 \%$ \\
\hline & Quetiapine & 3,5 & 2,25 & 1,25 & $9,62 \%$ \\
\hline & Asenapine & 8,25 & 2,25 & 6,00 & $46,15 \%$ \\
\hline & Zotepine & 6,50 & 2 & 4,50 & $34,62 \%$ \\
\hline \multirow{5}{*}{ The Dones } & Risperidone & 5 & 1,5 & 3,50 & $26,92 \%$ \\
\hline & Paliperidone & 5 & 1,75 & 3,25 & $25,00 \%$ \\
\hline & Zprasidone & 6,75 & 1,25 & 5,50 & $42,31 \%$ \\
\hline & lloperidone & 4,75 & 1,75 & 3,00 & $23,08 \%$ \\
\hline & Lurasidone & 4,5 & 0,5 & 4,00 & $30,77 \%$ \\
\hline \multirow{3}{*}{ The Pips and Rips } & Aripiprazole & 5,5 & 1 & 4,50 & $34,62 \%$ \\
\hline & Brexpiprazole & 5,5 & 1,5 & 4,00 & $30,77 \%$ \\
\hline & Cariprazine & 3,75 & 0,75 & 3,00 & $23,08 \%$ \\
\hline \multirow{3}{*}{ The others } & Amisulpride & 1,25 & 0 & 1,25 & $9,62 \%$ \\
\hline & Sertindole & 5,5 & 1,25 & 4,25 & $32,69 \%$ \\
\hline & Perospirone & 3,5 & 1 & 2,5 & $19,23 \%$ \\
\hline
\end{tabular}

Fig. 1. functionality index results.

The results showed a good functionality index for aripiprazole, brexpiprazole, ziprazidone, zotepine, asenapine and sertindole.

Conclusions.- Antipsychotics have a different functionality index. For future we will take into account antipsychotic formulations and administrations: oral (three/two/one time per day, immediate/extended release) and long acting injectable treatment (with two weeks/one month/three month time of administration) which can influence plasma concentration oscillations with impact on adverse events, patient comfort and functionality.

Disclosure of interest.- The authors have not supplied a conflict of interest statement.

\section{E-PV0724}

\section{Safety of aripiprazole in long QT syndrome \\ D. Mato ${ }^{*}$, P. Hervías Higueras ${ }^{2}$ \\ ${ }^{1}$ Hospital Dr. R. Lafora, Psiquiatría, Madrid, Spain; ${ }^{2}$ Hospital del \\ Henares, Coslada, Psiquiatría, Madrid, Spain \\ * Corresponding author.}

Background and aims.- QT interval represents the period from the beginning of depolarization to the completion of repolarization of the ventricular myocardium. The most popular method to calculate it is the Bazett formula, calculating the corrected QT interval: QTc. When the QTc interval is greater than $500 \mathrm{~ms}$, the patient will be at risk of developing torsades de pointes (TdP).

We present the clinical case of a 53-year-old women who suffers from TdP after receiving Olanzapine $10 \mathrm{mg}$ in the context of a psychotic episode.

Methods.- We propose to carry out a bibliographic review on the risk factors of presenting a prolongation of the QT interval and $\mathrm{TdP}$, focusing on the psychotropic drugs and on the therapeutic alternatives.

Results.- The risk factors for prolongation of the QT interval are multiple. When the risk of prolongation of the QT interval induced by drugs is considered high, a basal ECG should be performed as well as when the added drug reaches steady state. Aripiprazole stands out among the therapeutic alternatives in the group of antipsychotics, considering the antipsychotic that has less risk.

Conclusions.- The use of psychotropic drugs that affect myocardial repolarization cannot be entirely avoided. Nonetheless, every attempt should be made to avoid the combined use of multiple drugs that can prolong the QTc interval and elevate the risk of TdP. Disclosure of interest.- The authors have not supplied a conflict of interest statement.

\section{E-PV0725}

\section{Psychotropics and hyponatremia - a real threat to be aware of}

\section{Melo ${ }^{1 *}$, R. Fernandes ${ }^{2}$, T. Ferreira ${ }^{3}$, P. Branco ${ }^{1}$}

${ }^{1}$ Centro Hospitalar Psiquiátrico de Lisboa, Psychogeriatric Unit, Lisboa, Portugal; ${ }^{2}$ Centro Hospitalar Psiquiátrico de Lisboa, Clínica 1, Lisboa, Portugal; ${ }^{3}$ Hospital Prof. Doutor Fernando da Fonseca, Psychiatry Department, Amadora, Portugal

Corresponding author.

Background and aims.- The association between the use of psychotropic drugs and hyponatremia is not new: it is one of the most common electrolyte abnormalities encountered, not only in hospitalized patients, but also in routine psychiatric clinical practice. The impact of hyponatremia on the general health and well-being of an individual is significant, posing as life-threatening if left untreated. We aim to review the association between psychotropics and hyponatremia, its incidence, clinical presentation and risk factors, in order to raise awareness about this potentially lifethreatening side effect, so that timely steps can be taken to prevent psychotropic-associated hyponatremia.

Methods.- A non-systematic review of the literature from Pubmed database, using the Keywords: psychotropics, antidepressants, antipsychotics, hyponatremia.

Results.- Hyponatremia has been reported as a side effect of many psychotropic medications: antipsychotics, antidepressants, mood stabilizers and sedative/hypnotics, with its risk increasing with the use of several psychotropics. It is important to note that, in psychiatric patients, the symptomatology caused by hyponatremia may be confused with the mental illness itself, which will inevitably cause a delay in its diagnosis. Among its early symptoms are headaches, agitation, lethargy, nausea, vomits, confusion and weakness. Some of the risk factors identified so far are increasing age,female sex,presence of comorbid medical conditions,polypharmacy and history of hyponatremia.

Conclusions.- Despite its life-threatening potential,practicing clinicians often overlook hyponatremia due to lack of awareness about its incidence,clinical presentation and risk factors. This may lead us to believe that its incidence may be even higher than it is currently thought,with more studies being necessary on this field.

Disclosure of interest.- The authors have not supplied a conflict of interest statement.

\section{E-PV0726 \\ Evaluation of cardiovascular risk in patients with iatrogenic hyperprolactinemia by antipsychotics: preliminary data}

A.L. Montejo ${ }^{1 *}$, J.M. Acosta ${ }^{2}$, N. Prieto ${ }^{3}$, M.T. Notario ${ }^{3}$, T.

Martin-Pinto $^{3}$, S. Sanchez-Iglesias ${ }^{3}$, M.J. López ${ }^{3}$, B. Cortés ${ }^{3}$, B.

Bote $^{3}$, M.T. Gallego ${ }^{3}$, A. Martin ${ }^{3}$, B. Buch ${ }^{2}$

${ }^{1}$ University of Salamanca EUEF, Psychiatry, Salamanca, Spain; ${ }^{2}$

Instituto de Investigación Biomedicina de Salamanca IBSAL,

Neurociencias 2, Salamanca, Spain; ${ }^{3}$ Hospital Universitario de

Salamanca, Psychiatry, Salamanca, Spain

"Corresponding author.

Background and aims.- Long-term iatrogenic hyperprolactinemia due to antipsychotics (APS) is associated with physical issues such as osteoporosis, hypogonadism, dyslipidemia and increased cardiovascular risk due to an increased arteriosclerotic process. There are few studies on cardiovascular risk in the psychiatric population. Objective: To evaluate the association of Chronic Iatrogenic Hyperprolactinemia with vascular structure and function in patients treated with antipsychotics.

Methods.- A cross-sectional descriptive observational study. We recruited 27 patients (15 men, 12 women) aged 43.8 (9.6) years 
in treatment with antipsychotics. Vascular rigidity was evaluated by the "Cardio-Ankle-Vascular-Index" (CAVI) measuring the pulsearm-ankle wave velocity on both sides (R-CAVI and L-CAVI) using the VASERA VS- $1500^{\circledR}$ system which indicates the stiffness of the aorta, the femoral artery and the tibial artery.

Results.- The mean prolactin level was 35.77 (41.20) in males and 60.58 (76.19) in females. The mean values of R-CAVI and L-CAVI were equal (6.50). No relationship was found between prolactin levels and arterial age. There were significant differences between the real age and that estimated by the CAVI in both the R-CAVI $(p=0.023)$ and L-CAVI $(p=0.009)$ for males and females $(p=0.015$ and $p=0.021$ respectively). The lower arterial age estimated by the CAVI, compared with the real one, does not indicate arterial aging in this group of patients.

Conclusions.- Due to the small size of the sample, it is necessary to continue studies on the influence of PRL levels on the risk of arteriosclerosis.

Disclosure of interest.- The authors have not supplied a conflict of interest statement.

\section{E-PV0727}

\section{Vortioxetine. Clinical and demographic profile}

C. Muñoz , A. Ramirez, A. Perez, F. Palermo, I. Pans, F.J. Peralta Hospital Universitario Virgen Macarena, U.G.C Salud Mental, Sevilla, Spain

${ }^{*}$ Corresponding author.

Background and aims.- New antidepressant drugs are changing to increase the benefit of the patient, improving tolerability and therapeutic adherence.

The aim is to describe clinical and epidemiological characteristics of the patient with Vortioxetine.

Methods.- It's a descriptive observational study, non-random sample $(n=75)$, from outpatients units, where we analyse the establishment of Vortioxetine treatment. This establishment was decided using clinical criteria.

Results.- $48 \%$ were women, $52 \%$ were men. They had an average age of 51.3. The main diagnosis was major depressive episode (39\%), followed by minor depressive episode (28\%) and dysthymia (17\%). Regarding the employment situation, $27 \%$ were in a temporary disability compared to $19 \%$, in permanent disability. $27 \%$ kept their job. $84 \%$ started with $10 \mathrm{mg}$ of Vortioxetine followed by $8 \%$ that started with $20 \mathrm{mg}$. 47\% was previously treated with an SRI, Fluoxetine and Escitalopram (26\% each one).

Conclusions.- We observed that the main type of patient treated with Vortioxetine would be: a man with a mean of 51.3 years old and diagnosis of Major Depressive Episode, with a first therapeutic trial with SRI and starting dose of $10 \mathrm{mg}$.

References.-

- Jacobsen PL et al. A randomized, double-blind, placebo-controlled study of the efficacy and safety of vortioxetine $10 \mathrm{mg}$ and $20 \mathrm{mg}$ in adults with major depressive disorder. J Clin Psychiatry. 2015 May;76 (5):575-82.

- Pae CU et al. Vortioxetine, a multimodal antidepressant for generalized anxiety disorder: a systematic review and meta-analysis. J Psychiatr Res. 2015 May; 64:88-98.

- NICE. Depression Guideline: management of depression in primary and secondary care. National Clinical Practice Guideline 23. Disclosure of interest.- The authors have not supplied a conflict of interest statement.

\section{E-PV0728 \\ Restless legs syndrome induced by quetiapine: review of the literature}

C. Oliveira*, C. Vieira

Hospital Prof. Dr. Fernando Fonseca, Psychiatry, Lisboa, Portugal

* Corresponding author.

Background and aims.- Restless legs syndrome (RLS) is defined as an urge to move the legs, usually accompanied by unpleasant sensations in the legs; beginning or worsening during inactivity; relief by movement; preponderance in evening or night. RLS overlaps Psychiatry in three critical areas: co-morbid disorders, dopamine-induced akathisia, and medications exacerbating RLS. Secondary RLS remains underdiagnosed especially when associated with second-generation antipsychotics medication.

This review aims to update the scientific advances on the pathophysiology of RLS and explore the association with quetiapine.

Methods.- We performed a literature search in Medline and PubMed with keywords "restless legs syndrome; quetiapine" in September 2018. We present a non-systematic review of the literature that approach RLS pathophysiology and the RLS symptoms induced by quetiapine.

Results.- RLS prevalence is approximately $2.5 \%$ to $15 \%$ of the general population, increases with age and have a female preponderance. There is increasing evidence for the role of brain iron insufficiency, dopamine neurotransmission abnormalities and hypodopaminergic states in the nigrostriatal circuits, in the development of RLS. We found a total of 13 case reports that describe RLS symptoms associated with small quetiapine doses (50-250 mg at bedtime) in a dose-dependent way. Although quetiapine is known for its low antidopaminergic activity, RLS is describe even after a single low dose quetiapine administration.

Conclusions.- The exact mechanisms by which abnormalities in dopaminergic neurotransmission in dopamine metabolism result in the development of RLS remain unclear. Quetiapine seems to carry a particular risk for RLS although this association appears quite rare and in need of clarification.

Disclosure of interest.- The authors have not supplied a conflict of interest statement.

\section{E-PV0729}

\section{Antipsychotics treatment continuation in patients with QT interval prolongation}

P.A. Pereira Vaz De Macedo*, J. Borges, R. Leite, G. Dias dos Santos, M. Almeida, A. Mesquita Figueiredo

Centro Hospitalar do Baixo Vouga, Psychiatry, Aveiro, Portugal

* Corresponding author.

Background and aims.- A prolonged QT interval is an important risk factor linked with dangerous arrhythmias, especially torsades de pointes, and sudden cardiac death. The risk associated to this prolongation is multifactorial and patients have different levels of susceptibility to QT prolongation of iatrogenic aetiology. In our daily practice we are confronted with the necessity of adjusting therapy based on this limitation on antipsychotic prescription. Sometimes it's possible to stop the medication, but sometimes the possibility of decompensation of the psychiatric disease doesn't allow the cessation of the treatment. Risk and benefits of therapeutic continuum have to be measured.

The purpose of this work is to review literature findings on the risk of QT interval prolongation associated to antipsychotics and try to define those with lesser risk and more prone for treatment continuation.

Methods.- The author made a research in article database pubmed and similar sources, using terms such as QT prolongation, antipsychotic, and cardiovascular risk. 
Results.- Antipsychotics and other psychotropic drugs are associated with an increased risk of cardiovascular mortality events, like sudden death. The use of these drugs is crucial for most of psychiatric diseases. There is evidence of lesser risk for some antipsychotic treatments, and some measures like dose reduction can be adopted for treatment continuation.

Conclusions.- Although there are drugs associated with lesser risk for cardiovascular events, it seems important to always measure risk and benefits of antipsychotic drug continuation in a patient with a QT prolongation.

Disclosure of interest.- The authors have not supplied a conflict of interest statement.

\section{E-PV0730}

\section{Antipsychotics and seizure threshold - review and case report}

I. Pinto ${ }^{1}$, S. Vilas Boas Garcia ${ }^{1}$, M. Bernardo ${ }^{2}$, C. Pereira ${ }^{3}$, I. Caldas $^{3}$, C. Ferreira ${ }^{1}$, M.J. Avelino ${ }^{1}$

${ }^{1}$ Centro Hospitalar Psiquiatrico de Lisboa, Clinica 1, Lisboa, Portugal;

${ }^{2}$ Hospital Garcia de Orta, Psiquiatria, Almada, Portugal; ${ }^{3}$ Centro

Hospitalar Psiquiatrico de Lisboa, Clinica 2, Lisboa, Portugal

"Corresponding author.

\section{Background and aims.-}

Introduction.- Almost all antipsychotics have been associated with risk of seizures among other side effects well known and studied. Clozapine is one of the most reported therapeutics concerning the lowering of seizure threshold and is frequently associated with several patient as well as dose and titration factors.

Objectives.- Collect and review the most recent information on antipsychotics and their relation with lower threshold for seizures. Differences between the different drugs in terms of risk and precautions were also a purpose of this work. A clinical case with a seizure occurring in a patient with a high dose of clozapine is going to be described as well.

Methods.- A literature research was carried out on antipsychotics, convulsive threshold and specifically on clozapine related seizures and risk factors associated.

Results.- The case involves a 22-year-old man with a 2-year history of schizophrenia associated with use of psychoactive drugs and a poor therapy adherence. In 2017 he had an involuntary admission and clozapine was titrated $50 \mathrm{mg}$ per week until $500 \mathrm{mg}$, time when he had a generalized tonic-clonic seizure. The majority of the information available describes risk factors and possible explanations for the increased risk with this specific atypical antipsychotic as well as some measures to prevent the occurrence of these adverse effects.

Conclusions.- The risk of seizure associated with antipsychotic drugs is likely to be related to patient features that should always be thoroughly investigated in the medical and personal history. Cautions related to titration, dose and concomitant medication must also be observed.

Disclosure of interest.- The authors have not supplied a conflict of interest statement.

\section{E-PV0731}

\section{Prevalence of drug-drug interactions with psychiatric drugs in a HIV-infected community: a real problem}

C. Romeromartin ${ }^{1}{ }^{*}$, P. Padilla Romero ${ }^{2}$, T. López-Arteaga ${ }^{2}$, M.T. Perez Castellano ${ }^{2}$

${ }^{1}$ Hospital Nuestra Señora Del Prado, Hospital Pharmacy, Talavera de la Reina, Spain; ${ }^{2}$ Hospital Nuestra Señora Del Prado, Psychiatry, Talavera de la Reina, Spain

* Corresponding author.
Background and aims.-

Introduction.- The high prevalence of neuropsychiatric disorders in individuals infected with the human immunodeficiency virus (HIV), is associated with polypharmacy. For this reason is important to know in clinical practice the prevalence of potential drug-drug interactions (PDDIs) between the antiretroviral drugs and psychiatric drugs.

Objectives.- Study of use of psychiatric drugs in patients with HIV-infection in our community. We assessed the prevalence and seriousness of PDDIs among HIV-infected patients on psychiatric treatment.

Methods.- Retrospective, descriptive study. We included patients that take monthly HIV-treatment at the hospital pharmacy. A search was conducted in a computer-based medication prescription system for dispensing HIV drugs. Psychiatric treatment was available on patients electronic prescription. PDDIs were identified using the online University of Liverpool HIV drug interactions database. Types of drug interactions: potential weak interaction (no dosage adjustment recommended), potential interaction (may require a dosage adjustment or close monitoring) and do not coadminister.

Results.- We included 102 patients $(74,5 \%(n=76)$ men, average age $48[19-81]) .36,3 \%(n=37)$ were receiving any psychiatric drug: $16,7 \%(n=17)$ any anxiolytic/hypnotic, $11,8 \%(n=12)$ any antidepressant, 9,8\% (n=10) any antipsychotic and 7,8\% $(n=8)$ methadone. 32 interactions were detected, where $40,6 \%(n=13)$ are considered weak interaction, $53,1 \%(n=17)$ potential interaction and $(n=2)$ do not coadminister. Quetiapine was the drug most involved in severe drug-drug interactions: quetiapinedarunavir/cobicistat and quetiapine-lopinavir/ritonavir.

Conclusions.- Psychiatric drugs are very common among HIVinfected patients on antiretroviral treatment. The prevalence of PDDIs was high. Psychiatrists and hospital pharmacists may play a crucial role in their detection, management and early communication.

Disclosure of interest.- The authors have not supplied a conflict of interest statement.

\section{E-PV0733}

\section{To whom do we prescribe alprazolam? An outpatient mental health service descriptive study}

A. Trabsa Biskri $1,2,3^{*}$, D. Buezo 4 , S.T. Castillo ${ }^{5}$, X. Lara $^{5}$, E. Mur ${ }^{5}$, L.

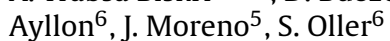

1 1-Institut Hospital del Mar d'Investigacions Mèdiques, IMIM, Parc de Salut Mar, Psychiatry, Barcelona, Spain; ${ }^{2}$ Institut Hospital del Mar d'Investigacions Mèdiques, IMIM, Parc de Salut Mar, Psychiatry, Barcelona, Spain; ${ }^{3}$ Universitat Autònoma de Barcelona, Psychiatry, Barcelona, Spain; ${ }^{4}$ ABS4 Sta Coloma de Gramanet, ICS Metropolitana Nord, Barcelona, Spain; ${ }^{5}$ Centre de Salut Mental D’Adult Martí $i$ Julià-Parc de Salut Mar, Psychiatry, Santa Coloma-Barcelona, Spain; ${ }^{6}$ Centre de Salut Mental D'Adult Martí i Julià-Parc de Salut Mar, Psychiatry, Barcelona, Spain

Corresponding author.

Background and aims.-

Introduction.- Alprazolam, with its short elimination half-life, causes a more severe withdrawal syndrome, higher physical dependence liability and more susceptibility of physical dependence than Benzodiazepines with longer elimination half-lives.

Identify individuals who have been prescribed alprazolam and they clinical and demographic characteristics could shed light on elements to head to a sensible prescription of alprazolam.

Objectives.- The aim of this study is to describe clinical, demographic characteristics and treatment dosages of patients who have been prescribed alprazolam in a mental health outcome service. Methods.- All patients who have been prescribed alprazolam at an Outpatient mental health service (CSMA Martí i Julià) were reg- 
istered in September 2018. Database information was completed with electronic medical records. Cross-sectional study was performed with IBM SPSS Statistics (Chicago INC).

Results.- From a total of 120.000 patients attended in CSMA Martí $\mathrm{i}$ Julià, 30 patients had alprazolam in their prescription. From these patients $65,5 \%$ were women and the mean age was 55 . From these patients $37.9 \%$ presented a depressive disorder, $24,1 \%$ anxiety disorder, $13,8 \%$ psychotic disorder $10,3 \%$ personality disorder and $13,7 \%$ other disorders. $75,9 \%$ of these patients do not present a substance use disorder. The mean prescribed dosage was $1,3 \mathrm{mg}$. Conclusions.- Profile of patients who were more prescribed alprazolam in this outcome mental health service were woman, middle aged, with depressive or anxiety disorders with no substance use disorder comorbidity, the mean dosage prescribed was high. We should identify these patients in order to consider other treatment options in long-term alprazolam patients users.

Disclosure of interest.- The authors have not supplied a conflict of interest statement.

\section{E-PV0734}

\section{Drug-related pneumonia due to clozapine treatment}

S. Tunc ${ }^{1 *}$, H.S. Basbug ${ }^{2}$

${ }^{1}$ Kafkas University, Department of Psychiatry, Kars, Turkey; ${ }^{2}$ Kafkas University, Department of Cardiovascular Surgery, Kars, Turkey

${ }^{*}$ Corresponding author.

Background and aims.- Drug-related pneumonia is common mainly due to acid reducing agents, antipsychotic drugs, glucocorticoids and sedatives. Clozapine is an extremely efficient antipsychotic and the single antipsychotic with an indication for suicidal behavior and treatment-resistant schizophrenia. However several side effects have been related to clozapine use. An increased risk of lower respiratory infections, including pneumonia was reported in individuals using atypical antipsychotics. Among the atypical antipsychotics, clozapine is related to the highest risk. We state that clozapine may cause aspiration pneumonia in schizophrenia patient.

Methods.- A 28-year-old female admitted with a purulent cough, fever, shortness of breath, and fatigue for the last eight days. In her drug history clozapine, $200 \mathrm{mg} /$ day usage was detected for the last two years with a diagnosis of schizophrenia. She had never experienced a pulmonary complication before. Her cough and fatigue were getting worse for the last three days. Biochemical test revealed an elevated white blood cells, erithrocyte sedimentation rate and Creactive protein. Chest computed tomography (CT) demonstrated multifocal infiltrates revealing for an infectious etiology. The sputum culture revealed a gram-positive bacteria.

Results.- She was hospitalized and given intravenous medication with the diagnosis of pneumonia. The clozapine dose was reduced to $100 \mathrm{mg} /$ day from $200 \mathrm{mg} /$ day. She recovered in two days. The general condition of the patient was improved.

Conclusions.- Clinicians should keep in mind that both the infection and the drug treatment of the infection by drug-drug interactions at CYP1A2 can cause elevated and toxic levels of serum clozapine that may lead to more troubles.

Disclosure of interest.- The authors have not supplied a conflict of interest statement.

\section{E-PV0735}

\section{An unusual case of urge incontinence due to aripiprazole}

S. Tunc ${ }^{1 *}$, E. Buyuksandalyaci ${ }^{2}$, H.S. Basbug ${ }^{3}$

${ }^{1}$ Kafkas University, Department of Psychiatry, Kars, Turkey; ${ }^{2}$

Bakirkoy Training and Research Hospital for Psychiatry, Neurology

and Neurosurgery, Department of Psychiatry, Istanbul, Turkey; ${ }^{3}$
Kafkas University, Department of Cardiovascular Surgery, Kars, Turkey

* Corresponding author.

Background and aims.- Urge incontinence is one of the adverse effects that can be observed after drug treatments. Urge incontinence is described as the involuntary loss of urine and can form hygiene or social trouble for affected patients. Aripiprazole is an atypical antipsychotic drug with antidepressant effects. Aripiprazole is partial agonist on dopamine D2 receptors and serotonin 5-HT1A receptors which have extraordinary features in contrast to other antipsychotic drugs. It is used in various psychiatric disorders including schizophrenia, bipolar disorders, unipolar depression, and anxiety disorders. In this paper, a rare case of aripiprazoleinduced urge incontinence in a schizophrenia patient was reported. Methods.- A 58-year-old female schizophrenia patient was admitted with the complaint of urinary incontinence after starting an antipsychotic aripiprazole $15 \mathrm{mg} /$ day one week ago for the first time.

Results.- The patient reported no complaint if she stopped the aripiprazole for trial purpose. She responded very well to anticholinergic drug trospium chloride $30 \mathrm{mg} /$ day which is used for urge incontinence. The aripiprazole treatment was thus discontinued. After the drug termination, urinary incontinence was interrupted.

Conclusions.- Clinicians should keep in mind that aripiprazole can cause urinary incontinence that may lead to more troubles as it may affect patients compliance with the prescription. Further studies should be done to delineate the mechanism and whether this complication is dose-related or not.

Disclosure of interest.- The authors have not supplied a conflict of interest statement.

\section{E-PV0736 \\ Population distribution analysis of pharmacodynamic genotypes of antischizophrenia drugs \\ Y. Zhang \\ Xi'an Mental Health Center, Pharmacy Lab, Xi'an, China \\ * Corresponding author.}

Background and aims.- objective to investigate the effect of different genotypes of dopamine d2 receptor (DRD2) and 5hydroxytryptamine receptor (HTR1A) on the actual therapeutic effect of schizophrenia patients and the population distribution of pharmacodynamic receptor genotypes of northwest in China.

Methods.- 239 cases of schizophrenia patients collected from the Xi'an mental health center, through panss evaluation in $30 \pm 10$ days get drug treatment effect, and collected DRD2 and HTR1A gene reports, get different drug response in different patient of pharmacophore receptor in the clinical.

Results.- the drug efficiency of DRD2 with genotype -141c del/del and $-141 \mathrm{c}$ int/del is higher drug response than that of genotype -141 cins/ins. The drug efficiency of HTR1A is affected by DRD2 receptor gene.

Conclusions.- schizophrenia drug response in DRD2 receptor and HTR1A receptors show different response, but also by phase, the influence of factors such as age, medication, schizophrenia drug resistance gene. So pharmacodynamic genotypes detection is of great significance to guide the clinical medication.

Disclosure of interest.- The authors have not supplied a conflict of interest statement. 


\section{E-Poster Viewing - 7-9 April: Psychophysiology}

\section{E-PV0737}

\section{Bridging neurophysiology and clinical neuropsychology through the RDOC}

\author{
A. Geraldo Geraldo ${ }^{1,2}$, A. Azeredo ${ }^{1}$, R. Pasion ${ }^{1}$, A.R. Dores ${ }^{1,2^{*}}$, F. \\ Barbosa $^{1}$ \\ ${ }^{1}$ Faculty of Psychology and Education Sciences, Laboratory of \\ Neuropsychophysiology, Porto, Portugal; ${ }^{2}$ School of Health- \\ Polytechnic Institute of Porto, Human and Social Sciences, Porto, \\ Portugal \\ " Corresponding author.
}

Background and aims.- Neuropsychological practices have been focused on promoting a deeper understanding of cognitive functioning and its relation to behavior and everyday functioning through a greater sophistication of the neuropsychological assessment process. A recent review of these advances has stated the importance of early detection of brain changes as a way of improving knowledge on brain-behavior relationships.

Our goal was to explore the advances in neuropsychological assessment under the aegis of the Research Domain Criteria (RDoC) framework, focusing on the use of neuropsychological tasks with EEG to extract Event Related Potentials (ERP) and examine the neural correlates of psychopathological and neurological conditions. Methods.- The RDoC Cognitive Systems Domain was reviewed considering the neuropsychological tests and neurophysiological correlates proposed by the framework. Potential applications were explored given the functional relevance of RDoC constructs to mental health disorders.

Results.- The RDoC framework adds value to neuropsychology by establishing neurophysiological correlates relevant to the early detection of psychopathological and neurological conditions, which may be obtained from consensually used neuropsychological tasks. Changes in specific neural correlates of persons with certain psychopathological and neurological conditions may be detected even in the absence of deficits in neuropsychological tasks.

Conclusions.- Considering the already established validity of the classical neuropsychological tasks, future advances in neuropsychological assessment might benefit from the integration of multiple levels of analysis to provide a more detailed neuropsychological characterization and early diagnoses.

Disclosure of interest.- The authors have not supplied a conflict of interest statement.

\section{E-PV0741 \\ Motor functions recovery after traumatic brain injury of mild severity in adolescents \\ S. Kovtoniuk \\ Moscow State University, Psychology, Moscow, Russia \\ * Corresponding author.}

Background and aims.- Restoration of motor functions in the longterm period of mTBI in adolescents in many cases is satisfactorily, however, in the course of a neurological diagnostics, even minor movement disorders are considered as pathological.

The objectives of the study included attempts to identify the features of the state of motor functions in adolescents after mTBI in the acute period and to assess the dynamics of recovery over half a year.

Methods.- The study is based on the original set of techniques designed by A.R. Luria.

31 patients with mTBI (mean age was $11,5+1,3$ ) and 20 healthy subjects (mean age was $12+1,5$ ) took part in the study.
Results.- Analysis of the results showed that the main component of motor disorders in the acute period after mTBI is a violation of the dynamic component, in the form of failures during the test, and also assimilation to inert stereotypes.

A month after the injury, the symptoms from the posterior regions were eliminated, as well as most of the frontal brain regions, which arose as irregularities in samples for reciprocal coordination, dynamic praxis, as well as a significant amount of impulsive errors when performing other motor tests.

Such indicators as the speed and efficiency of completing assignments, productivity, which do not decrease to the level of normative results even in a remote period, remain deficient to a large extent.

Conclusions.- Residual motor disoders affects not only the overall motor activity and success in sports, but also the attitude of the peers to the adolescent.

Disclosure of interest.- The authors have not supplied a conflict of interest statement.

\section{E-PV0742}

\section{Cognitive and somatic trait anxiety: subjective and psychophysiological responses}

F. Barros ${ }^{1}$, C. Costa $^{2}$, C. Figueiredo ${ }^{3}$, S. Brás ${ }^{1}$, J.M. Carvalho ${ }^{1}$, N. Madeira $^{4}$, S. Magano ${ }^{4 *}$, S.C. Soares ${ }^{1}$

${ }^{1}$ University of Aveiro, CINTESIS.UA-Department of Education and Psychology, Aveiro, Portugal; ${ }^{2}$ University of Aveiro, Department of Education and Psychology, Aveiro, Portugal; ${ }^{3}$ University of Aveiro, govcoop, Aveiro, Portugal; ${ }^{4}$ Centro Hospitalar e Universitário de Coimbra, Department of Psychiatry, Coimbra, Portugal

* Corresponding author.

Background and aims.- Anxiety is part of an emotional system that foster defensive behaviours towards non-specific stimuli perceived as potentially threatening, therefore able to jeopardize the organism. When anxiety is more persistent (as a trait) there is an increased risk of psychopathology. Because anxiety encompasses a set of physiologic, behavioural and cognitive responses, recent self-report measures have been developed as to reflect these different dimensions (specifically, cognitive and somatic anxiety). However, no study has yet investigated whether these dimensions are reflected in differentiated patterns of response (subjective reports and psychophysiology, respectively).

To assess the pattern of subjective and psychophysiological responses to the induction of emotions as a function of cognitive and somatic trait anxiety (high and low).

Methods.- A non-clinical sample of 37 participants watched three sets of videos inducing different emotions (fear, happy, neutral) while their cardiac response was recorded. Emotional state was reported before and after the emotional induction, using a visual analogical scale for happiness, anxiety, fear and stress.

Results.- The group with higher cognitive anxiety evidenced a less differentiated heart response in comparison with the group with low cognitive anxiety, than in neutral and happy conditions. Subjective data also showed differences between groups, but only in neutral condition, with a lower variation of anxiety reported by the group with high somatic anxiety.

Conclusions.- This study shows that high cognitive and somatic trait anxiety is differently related to variations in the psychophysiological and subjective responses towards non-threatening stimulation, reflecting a non-adaptive hyperarousal.

Disclosure of interest.- The authors have not supplied a conflict of interest statement. 


\section{E-PV0744}

\section{To opportunity of implicit measurement of positive mental health representations and psychological well-being}

R. Shilko*, A. Kiselnikov, L. Shaigerova, A. Dolgikh, O. Vakhantseva, A. Kipiani

Lomonosov Moscow State University, Psychology, Moscow, Russia ${ }^{*}$ Corresponding author.

Background and aims.- Positive mental health representations and psychological well-being evaluations are studied widely in explicit manner - through procedures of overt assessment using questionnaires and scales such as PANAS (Watson, Clark, Tellegen, 1988), SPWB (Ryff, Keyes, 1995), WEMWBS (Stewart-Brown, Janmohamed, 2008), SPANE (Diener, Biswas-Diener, 2009). In order to expand the research facilities in this area we propose to use perception-semantic association methodology (Kiselnikov et al., 2014) that was based on vector psychophysiological model (Sokolov, 2013) and successfully applied to mental health research (Arina et al., 2018; Kovyazina et al., 2018).

The aim of the research was to evaluate the opportunity of perception-semantic association methodology for implicit measurement of positive mental health representations and psychological well-being.

Methods.- Method of color-emotional semantic associations (Kiselnikov et al., 2014) was used. 7 males and 13 females as a subjects aged from 22 to 59 participated in the multidimensional scaling procedure with ten primary colors, ten names of basic emotions and some crucial semantic categories for positive mental health and psychological well-being (self, life, family, friends, work, independence, leisure, future, faith, wealth, psychologist, psychological help, stress, depression, insane).

Results.- It was found that semantic categories representing positive mental health and psychological well-being are located definitely on the surface of implicit two-dimensional Russel's affective circumplex (classical "Valence» and "Arousal» basic affective axes).

Conclusions.- Perception-semantic association methodology has a fruitful perspective for implicit measurement of positive mental health representations and psychological well-being.

The research was supported by Russian Foundation for Basic Research, with the Grant 17-29-02506.

Disclosure of interest.- The authors have not supplied a conflict of interest statement.

\section{E-PV0746}

\section{Psychiatric disorders and ABO blood}

\section{groups}

S. Vuk Pisk ${ }^{1^{*}}$, E. Ivezic ${ }^{1}$, K. Matic ${ }^{1}$, V. Grosic ${ }^{2}$, I. Filipcic ${ }^{3}$, T. Vuk ${ }^{4}$, I. Jukic $^{4}$

1 Psychiatric Hospital "Sveti Ivan", Acute female department- Faculty of Dental Medicine and Health University of Osijek, Zagreb, Croatia; ${ }^{2}$ Psychiatric Hospital "Sveti Ivan", Acute male Department, Faculty of Dental Medicine and Health University of Osijek, Zagreb, Croatia; ${ }^{3}$ Psychiatric Hospital "Sveti Ivan", Director of Psychiiatric Hospital "Sveti Ivan", Faculty of Dental Medicine and Health University of Osijek, Zagreb, Croatia; ${ }^{4}$ Croatian Institute for Transfusion Medicine, Croatian Institute for Transfusion Medicine, Zagreb, Croatia

${ }^{*}$ Corresponding author.

Background.- Previous research confirmed the correlation between the occurrence of some somatic diseases (most commonly referred to are cardiovascular, gastroduodenal and tumor diseases, and infections) with different genotypes of ABO blood groups. However, studies of association between blood groups and psychiatric disorders yielded contradictory results.
Aims.- The aim of this study was to examine whether there were significant differences in ABO blood groups between psychiatric patients and blood donors without diagnosed psychiatric disorder. Methods.- A total of 156 adult psychiatric patients (primary diagnoses: $38.7 \%$ psychotic disorder, $27.1 \%$ addiction, $26.5 \% \operatorname{mood}$ disorders, 7.7\% personality disorder) and 303 healthy blood donors participated in this study. Genomic DNA was isolated from blood on QIAcube device using QIAamp DNA Blood mini QIAcube kit (Qiagen, Germany). ABO genotyping on 5 basic $\mathrm{ABO}$ alleles was performed using allele-specific PCR.

Results.- The findings indicated a significant association between $A B$ blood group and psychiatric disorders: among psychiatric patients, there was a significantly higher proportion of those with AB blood group $\left(\chi^{2}=9.359, \mathrm{df}=3, P=0.025\right)$, when compared to healthy subjects. Also, the odds ratio showed that psychiatric disorders occur almost 3 times more frequently in people with $\mathrm{AB}$ blood group than in those with other blood groups.

Conclusions.- The results of this study support the hypothesis of the significant association between psychiatric disorders and $A B O$ blood groups. More specifically, it seems that psychiatric disorders are more likely to occur in carriers of $A B$ blood group.

Disclosure of interest.- The authors have not supplied a conflict of interest statement.

\section{E-Poster Viewing - 7-9 April: Psychosurgery \& Stimulation Methods (ECT, TMS, VNS, DBS)}

\section{E-PV0748}

\section{Electroconvulsive therapy response in depressed patients with comorbid personality disorder}

A. Carapucinha*, M. Bernardo

Hospital Garcia de Orta, Psychiatry, Almada, Portugal

"Corresponding author.

Background and aims.- Depression is one of the most common psychiatric conditions and an important cause of illness burden worldwide. Different pharmacological classes as well as psychotherapy have shown efficacy treating this condition alone. However, comorbid psychiatric disorders are frequent along with depression, with the prevalence of comorbid personality disorder (PD) rates ranging from $30 \%$ to $80 \%$. Such comorbidity often predisposes to lower treatment response and electroconvulsive therapy (ECT) might be an alternative treatment for these patients.

Methods.- The authors performed a non-systematic review of the literature on the role of ECT in depression with comorbid PD. A search through PubMed was made using the key words "electroconvulsive therapy", "depression" and "personality disorder".

Results.- Despite having potent antidepressant properties, ECT was shown to have a limited antidepressant efficacy, as there is an important clinical heterogeneity among depressed patients. Researchers have attempted to define the characteristics of this large group of patients that predict either positive or negative therapeutic outcomes for treatment with ECT, but there are few studies that have examined the effect of comorbid PD on the clinical outcome of ECT. Moreover, most of them focus exclusively on borderline personality and many have serious methodological limitations.

Conclusions.- There are limited and mixed results, but there seems to be preliminary evidence that depressed patients with a PD, especially borderline personality disorder, have a poorer outcome when treated with ECT.

Disclosure of interest.- The authors have not supplied a conflict of interest statement. 
E-PV0749

\author{
Can we keep the mind sharp? High \\ frequency repetitive transcranial \\ magnetic stimulation impact on \\ cognitive functions in schizophrenic \\ patients \\ W. Dębowska*, M. Debowska, A. Szulc \\ Faculty of Health Sciences of the Medical University of Warsaw, \\ Department of Psychiatry, Warsaw, Poland \\ ${ }^{*}$ Corresponding author.
}

Background and aims.- Schizophrenia is a serious mental illness which is characterized among other symptoms by deterioration of cognitive functions. Repetitive transcranial magnetic stimulation (rTMS) is a non-invasive method of inducing changes in cortical excitability that has been widely tested as a treatment method in psychiatric and neurological disorders. Here we study the effect of high-frequency rTMS on cognitive functioning of schizophrenia patients.

Methods.- Twelve participants ( 7 females; mean age $32.7 \pm 11.6$ ) diagnosed with schizophrenia received 10 sessions of $10 \mathrm{~Hz}$ rTMS of the left dorsolateral prefrontal cortex (DLPFC).

The level of cognitive deficits was measured with the Brief Assessment of Cognition in Schizophrenia (BACS) before and after the treatment. Significant improvement was found for verbal memory, psychomotor performance and attentional processes, as well as for the general level of cognitive functions.

Results.- The most significant difference was found for motor speed. Significant improvement was found for verbal memory, psychomotor performance and attentional processes, as well as for the general level of cognitive functions.

Conclusions.- We therefore conclude that rTMS shows promise as a therapy of cognitive deficits in patients with schizophrenia. However, the results need to be confirmed in further studies.

Disclosure of interest.- The authors have not supplied a conflict of interest statement.

\section{E-PV0751}

\section{Broken heart after ECT}

S. Medved ${ }^{1}$, Z. Ostojić ${ }^{2}$, H. Jurin ${ }^{2}$, V. Medved ${ }^{1}$

${ }^{1}$ Zagreb University Hospital Centre, Department of Psychiatry, Zagreb, Croatia; ${ }^{2}$ Zagreb University Hospital Centre, University Clinic of Cardiovascular Diseases, Zagreb, Croatia

* Corresponding author.

Background and aims.- We present the first case of postelectroconvulsive therapy (ECT) broken-heart syndrome in a male patient despite cardioprotective therapy. The broken-heart syndrome, known as Takostubo cardiomyopathy (TC), resembles acute coronary syndrome, but only coronary spasm occurs. Most of the evidence supports the catecholamine's hypothesis as a cause of spasm.

Methods.- Our patient, a middle-aged Caucasian man, severely nicotine and caffeine addicted, suffered from treatment-resistant schizophrenia. After two months of clozapine administration, the decision was reached to continue with ECT. Beta-blocker propranolol was added to the therapy. Ethical approval and inform consent were obtained. Pre-treatment evaluation showed no cardiological process, and bifrontotemporal stimulation was applied. Two hours later he presented with dyspnea and tests confirmed development of TC. He was transferred to the coronary care unit and was treated accordingly.

Results.- Psychosis is affiliated with catecholamine dysfunction, and male gender seems to show a greater autonomic response to stress. Clozapine, nicotine, and caffeine additionally elevate adrenalin and noradrenaline levels. Thereunto, ECT has a synergistic effect on the catecholamine blood levels.
Conclusions.- Until now, there have been sixteen cases reporting the development of TC after ECT, some of them describing retrials of ECT with beta blockade. Most of them, however, report TC in female patients suffering from depression, without any cardioprotective therapy. It seems that in our patient beta blockage was overcome by the catecholamine outburst due to psychosis, male gender, nicotine and caffeine consumption, clozapine and ECT use. Recognition of risk factors for developing TC could improve cardioprotective therapy ensuring safe protocols.

Disclosure of interest.- The authors have not supplied a conflict of interest statement.

\section{E-PV0752}

\section{Management of psychopharmaceutical previously to \\ ect sessions}

P. Padilla Romero ${ }^{1 *}$, C. Romeromartin ${ }^{2}$, T. López-Arteaga ${ }^{1}$, M.T.

Perez Castellano ${ }^{1}$

${ }^{1}$ Hospital Nuestra Señora Del Prado, Psychiatry, Talavera de la Reina, Spain; ${ }^{2}$ Hospital Nuestra Señora Del Prado, Hospital Pharmacy,

Talavera de la Reina, Spain

"Corresponding author.

Background and aims.-

Introduction.- Nowadays, electroconvulsive therapy (ECT) is still an important biological treatment with highly clinically tested efficiency. Its reduced use is partly due to the concern about possible interactions with psychopharmaceuticals or the necessity to interrupt the current treatment.

Objectives.- To propose some agreement on psychopharmaceuticals management previously to ECT sessions.

Methods.- Bibliographic review in Cochrane, clinical reference books and PubMed by introducing the following term: "electroconvulsive therapy" AND "psychotropic drugs".

Results.- Having limited the research outcome to results in adults, we obtained 50 different results. We selected two articles and the others were discarded as they referenced clinical aspects of ECT. Conclusions.- Antidepressants, antipsychotics and lithium can continue during ECT cycles due to their synergistic effect without compromising safety. Anticonvulsants and benzodiazepines often interfere (they decrease the duration of seizures) and they are recommended to be reduced, or even interrupted, previously to ECT. However, attention must be payed to the limitations found in the publications about this issue; particularly to reports of cases or observations on small size groups of patients.

There is not any consistent pattern regarding the combined use of psychopharmaceuticals during ECT. Moreover, experts recommend considering individual risks/benefits of each combination and precaution of their use.

Disclosure of interest.- The authors have not supplied a conflict of interest statement.

\section{E-Poster Viewing - 7-9 April: Psychotherapy}

\section{E-PV0753}

\section{Knowledge of and attitude towards computerized cognitive behavioral therapy in mental healthcare professionals in Saudi Arabia} A. Alhadi ${ }^{1,2^{*}}$, K. Alammari ${ }^{3}$, L. Alsiwat $^{3}$, N. Alhaidri ${ }^{3}$, N. Alabdulkarim $^{3}$, N. Altwaijri ${ }^{3}$, S. Alsohaily ${ }^{3}$

${ }^{1}$ King Saud University, Psychiatry Department- College of Medicine, Riyadh, Saudi Arabia; ${ }^{2}$ King Saud University, SABIC Psychological 
Health Research E Applications Chair, Riyadh, Saudi Arabia; ${ }^{3}$ King Saud University, College of Medicine, Riyadh, Saudi Arabia

${ }^{*}$ Corresponding author.

Background and aims.- We aim to measure the knowledge of computerized cognitive behavioral therapy (cCBT) in mental healthcare professionals in Saudi Arabia. In addition, we want to evaluate the attitude and preference of mental healthcare professionals towards computerized cognitive behavioral therapy in Saudi Arabia.

Methods.- The study is a quantitative observational cross-sectional with a convenient sample by selecting mental health care professional who work in the tertiary hospitals of Saudi Arabia. The participants received a self-administrated electronic questionnaire through data collectors measuring demographics, knowledge \& attitude of $\mathrm{CCBT}$ and the belief of using computers efficacy in therapy.

Results.- This study was applied to 132 mental healthcare professionals, most of which were males (59.5\%) and (40.5\%) were females. Most participants showed uncertainty and lack of knowledge regarding cCBT. However, most participants showed a positive attitude towards using computers in therapy. Participants clearly agreed with the principles of cCBT, believed in its efficacy and were generally confident in using computers. The comfort in using computers in therapy showed significantly higher results in males over females. It was significantly found that males were more comfortable using the internet than females. Other details will be discussed. Conclusions.- Mental health care professionals in Saudi Arabia need more education regarding CCBT and the usefulness of it, however their attitude towards it and their comfort in using computers in general show great promise. Further research should be done to assess acceptance of patients of CCBT in Saudi Arabia in addition to clinical trials measuring its effectiveness in the Saudi population. Disclosure of interest.- The authors have not supplied a conflict of interest statement.

\section{E-PV0754}

\section{Psychodynamic psychotherapy inside fire departments: a case study \\ J.P. Becker ${ }^{1 *}$, R. Paixão ${ }^{1}$, M.J. Quartilho ${ }^{2}$ \\ ${ }^{1}$ University of Coimbra, Faculty of Psychology and Education Sciences, Coimbra, Portugal; ${ }^{2}$ University of Coimbra, Faculty of Medicine, Coimbra, Portugal \\ ${ }^{*}$ Corresponding author.}

Background and aims.- Forest fires in Portugal are responsible for catastrophes that tend to be repeated annually, leading to dramatic consequences such as those that occurred in 2017, with the destruction of hundreds of houses and the deaths of dozens of people. Subsequently, the firefighters were exposed to these potentially traumatic events. Considering the context that firefighters face in their routines, Psychodynamic Psychotherapy (PDT) has been provided inside Fire Departments. The aim of this case study was to monitor the progress of two subjects with PTSD symptoms and the feasibility of implementing this intervention.

Methods.- The subjects (a female and a male) were selected with similar sociodemographic characteristics and PTSD symptom severity, and for verifying the applicability of the treatment to both genders/sexes. The PDT was 40 once-weekly 50 -min sessions. The symptom changes were assessed through a set of instruments (PHQ-15, PSQI, PCL-5, BSI, DASS and CALPAS-P) every three months. Two other subjects with the same characteristics but on a waiting list were analyzed and compared in the same time periods.

Results.- Qualitative analyses show that, despite reduced PTSD symptoms since the first assessment, anxiety symptoms increased in both patients in the first months. Since the focus of PDT is on raising awareness of unconscious thoughts and feelings related to traumatic events, they needed time to slow down the automatic processing of trauma stimuli and enhance emotion regulation in facing their experiences.

Conclusions.- The acceptability of firefighters, the treatment adherence, the therapeutic alliance and the reduction of PTSD symptoms suggest feasibility for implementing this intervention.

Disclosure of interest.- The authors have not supplied a conflict of interest statement.

\section{E-PV0755 \\ How doctors and artists can collaboratively lead creative writing-therapy in groups of people suffering from severe mental illness. Findings from a danish pilot study}

B. Bundesen ${ }^{1 *}$, B. Rosenbaum ${ }^{2}$

1 Mental health Care Center Amager, DPC team 1, Copenhagen, Denmark; ${ }^{2}$ Mental Health Care Center Copenhagen, Department of psychotherapy, Copenhagen, Denmark

Corresponding author.

Background and aims.- There is abundant evidence for the health promoting effects of therapeutic writing. In mental health care, "writing cures" have shown positive effects on numerous psychopathological measures. The research area has attracted attention due to the potential for cost-efficient adjuvant interventions. Several theories have been proposed to explain the positive health effects, including cognitive remediation, the relieving effect of disclosure, and the integrative effect from establishing a coherent life-narrative. So far, however, no consensus has been reached, and more research is needed to understand why and how therapeutic writing works in different diagnostic pictures.

Qualitative explorative investigation of four cases describing the effects of subjective experience of creative writing led by fiction writers in groups in outpatient mental health care treatment.

Methods.- Assessment of metacognition with MAS-A and qualitative interviews.

Results.- Conceptualization of the therapeutic effects of creative writing in groups with patients suffering from severe mental illness, led by fiction writers in mental health care. Focus is on 4 diagnostic categories: (1) psychosis (writing as reality check), (2) trauma-related states (writing as gentle disclosure of dissociated feelings), (3) borderline personality disorder (writing as emotion regulation), (4) depression (writing as generation of empowerment and hope). I will present a manual developed to be used in a new form of short term writing-group therapy inspired by short term psychodynamic therapy which focuses on stimulating the symbolization-function, interpersonal competence and egostrengthening through artistic practice.

Conclusions.- Collaboration between artists and psychotherapists focused on artistic practice and group-dynamics can be beneficial as complementary treatment in mental health care.

Disclosure of interest.- The authors have not supplied a conflict of interest statement.

\section{E-PV0756}

Under the mask, narrative storytelling in psychotherapy

\section{S. Chen", A. Ozdoba}

Montefiore Medical Center/Albert Einstein College of Medicine, Psychiatry, New York, USA

Corresponding author.

Background and aims.- Patients with Schizotypal Personality Disorder (SPD) rarely come into treatment and literature on psychotherapy for this population is sparse. Furthermore, exploration 
and developing a therapeutic alliance can be challenging given the frequency of difficulties with relationships and communication. The aim is to present a therapeutic intervention in a case of a patient with SPD.

Methods.- A male presented at the urging of his wife's friend. His affect, thought process, and speech were peculiar and vague. His clothes were peculiar and inappropriate. Patient's life had been shaped by multiple salient events. His mother bottle fed him into his teens and bathed him into adulthood. He did not have any friends and never experienced sexual intercourse. He would spend his days reading comic books and daydreaming. Exploration through typical therapeutic inquiry did not yield much information. It was quickly evident that the patient had a high level of suspiciousness and was not interested in any therapeutic goals. Once starting to utilize the comic books and imaginary stories it was discovered that under the peculiar façade lay a world of elaborate fantasy. Results.- After a shift to comic book narratives a therapeutic alliance was established and the patient allowed a glimpse into inner thought processes. His clothes and appearance also improved.

Conclusions.- Our case demonstrates the importance of creativity and narrative exploration in psychotherapy for patients with SPD. We will discuss use of narrative techniques as an effective method of obtaining history and implementing change.

Disclosure of interest.- The authors have not supplied a conflict of interest statement.

\section{E-PV0757}

\section{Remote working of a facilitation team: the experience of the 1st EPA MOOC on CBT}

T. Costa $1^{*}$, T. Gargot $2,3,4,5$, N. Arnaoutoglou ${ }^{3,6,7}$, O. Sidorova ${ }^{3,8}$, N. Liu-Thwaites $^{9}$, S. Moorey ${ }^{10,11}$, C. Hanon ${ }^{12,13}$

${ }^{1}$ Northumberland-Tyne and Wear NHS Foundation Trust, St. Nicholas Hospital, Newcastle upon Tyne, United Kingdom; ${ }^{2}$ TIM CHArt Laboratory - EA 4004, Paris 8 University, Saint-Denis, France; ${ }^{3}$ Psychotherapy Working Group, European Federation of Psychiatric Trainees, Brussels, France; ${ }^{4}$ Institut des Systèmes Intelligents et de Robotique, Sorbonne Université, Paris, France; ${ }^{5}$ Psychiatrie de l'Enfant et de l'Adolescent, Hôpitaux Universitaires, Pitié Salpêtrière, Charles Foix, Paris, France; ${ }^{6}$ Department of Psychiatry, University of Cambridge, Cambridge, United Kingdom; ${ }^{7}$ Windsor Research Unit, Cambridgeshire and Peterborough NHS Foundation Trust, Cambridge, United Kingdom; ${ }^{8}$ Department of Psychiatry and Addiction Disorders, Rīga Stradinš University, Riga, Latvia; ${ }^{9}$ South London and Maudsley NHS Foundation Trust, Medical Psychotherapy, London, United Kingdom; 10 South London and Maudsley NHS Foundation Trust, Psychotherapy Service, London, United Kingdom; ${ }^{11}$ Institute of Psychiatry, Psychology and Neuroscience, King's College, London, United Kingdom; ${ }^{12}$ Regional Resource Center of Old Age Psychiatry, Corentin Celton Academic Hospital, Paris, France; ${ }^{13}$ Secretary for Education, European Psychiatric Association, Strasbourg, France ${ }^{*}$ Corresponding author.

Background and aims.- Psychiatry trainees have a strong interest in psychotherapy, but available resources are scarce. To address this gap, the European Psychiatric Association (EPA) offered a Massive Open Online Course (MOOC), "Introduction to Cognitive Behavioural Therapy" (CBT) on April and May 2018. More than 7100 people subscribed, with 2000 completing it. In addition to traditional teaching materials, this e-learning course included an interactive component, facilitated by a team of 5 people. We will do a narrative report on the experience of this team.

Methods.- Narrative report on the experience of the facilitation team for the $1^{\text {st }}$ EPA MOOC on CBT.

Results.- All of the 5 moderators were psychiatry trainees or young consultants, assuming the responsibility on a voluntary basis and working remotely. They logged in to their accounts on a regular basis, according to a pre-set schedule, with hour long slots. The group communicated using mailing lists, tools for shared online documents and teleconference platforms. The main role was to support forum discussions amongst the participants, stimulating themed discussions with open questions and providing feedback on comments. This also included moderation and screening for abusive comments, perhaps the most challenging aspect of the role. They also produced a weekly report for the EPA Office, reviewed the course content and participated on the creation of the welcome and evaluation surveys.

Conclusions.- Remote facilitation is a flexible and low-cost option, which addresses one of the frequent criticisms of online courses, the lack of communication and involvement of the trainees, while fostering reflective practice and fuelling constructive discussion. Disclosure of interest.- The authors have not supplied a conflict of interest statement.

\section{E-PV0759}

\section{"A hidden request": the role of liaison psychiatry in working out a psychotherapy demand in primary care setting}

C. Derbel* , K. Tzartzas, T.S. Goncalves-Alvane, R. Marion-Veyron University of Lausanne, Department of Ambulatory Care and Community Medicine, Lausanne, Switzerland

Corresponding author.

Background and aims.- Primary care physicians (PCPs) are not only solicited for medical requests; social request such as medical certificates (MC) are usually a reason for consultation. In cases were PCPs find no organic reason to prescribe a MC, they feel out of his place, having usually to manage an underlying mental distress. Our aim is to demonstrate the role of consultation-liaison (CL) psychiatrist in investigating patient's underlying needs and proposing psychotherapy in primary care.

Methods.- Case-report of Mr. B, 19 years old man, who repeats his $1^{\text {st }}$ year of business studies. He doesn't inform his father to his first failure, being afraid of his reaction. Since a year, he consults a PCP, for persisting weight loss, initially attributed to gastritis. During the exam period he asks a MC attesting his inability to pass exams. PCP, being uncomfortable with this prescription refers to $\mathrm{CL}$, given the absence of clear somatic symptoms.

Results.- During a joint session, weight loss is linked to anxiety. The negative impacts that could have this failure on the relationship with his father and on the family balance immobilizes him. A brief CL intervention is proposed (4 sessions) during which we work around the hypothesis that anxiety is repressed, the patient expressing it by his body. Through our feedback, the PCP feels that a MC is justified. A systemic family psychotherapy is proposed to which the patient adheres.

Conclusions.- Social requests in primary care can be seen as an opportunity to provide a psychotherapeutic area, through working out the actual crisis.

Disclosure of interest.- The authors have not supplied a conflict of interest statement.

\section{E-PV0760 \\ Acceptance and commitment therapy for severe major depressive disorder: a single-case study}

E. Fernández Jiménez*, A. Hospital Moreno, B. Rodríguez Vega Hospital Universitario La Paz, Servicio de Psiquiatría- Psicología Clínica y Salud Mental, Madrid, Spain

Corresponding author.

Background and aims.- Acceptance and Commitment Therapy (ACT) is currently a probably efficacious treatment for depression which 
fosters psychological flexibility through: promoting the contact with one's negative inner experiences and working on personally meaningful values.

Objectives.- to examine the outcomes on a patient with major depressive disorder after undergoing several ACT sessions.

Methods.- The patient is a 53-year-old woman, divorced since 2011, without children, in sick leave because of her current depressive episode and with financial difficulties. She accepted ACT as psychotherapy plan and she is still under this therapy along with Desvenlafaxine. During the first six sessions, the following processes have been addressed: committed actions, self-as-context, mindfulness, acceptance and defusion. Mindfulness exercises were assigned as homework. At baseline (T1) were administered: the Beck Depression Inventory-II (BDI-II), State-Trait Anxiety Inventory (STAI), Five Facet Mindfulness Questionnaire (FFMQ) and Acceptance and Action Questionnaire-II (AAQ-II). Also, the BDI-II and the STAI-state were administered again biweekly (T2 and T3).

Results.- BDI-II (raw scores: T1 $=34 ; \mathrm{T} 2=40 ; \mathrm{T} 3=38$ ), STAItrait $(z=2.46)$, STAI-state $(z$-scores: $\mathrm{T} 1=2.45 ; \mathrm{T} 2=2.68$; T3 $=3.75)$, FFMQ ( $z$-scores: Observing =-1.93; Describing $=-1.06$; Acting with Awareness $=-1.77$; Non-Judgment $=-0.23$; Non-Reactivity $=-0.50$ ) and AAQ-II $(z=0.90)$. Her satisfaction about committed actions increased (0-10 range) from 3 to 6.5 points.

Conclusions.- Despite her severe depressive symptomatology and new negative life-events between therapy sessions, the patient is increasingly carrying out personally meaningful value-based actions and perceiving more satisfaction with these committed actions.

Disclosure of interest.- The authors have not supplied a conflict of interest statement.

\section{E-PV0761}

\section{Development of a novel therapy \\ workshop: “art therapy - yes, we can!"}

D. Goujon ${ }^{1 *}$, L. Berenguer ${ }^{2}$, F. Romann ${ }^{2}$

${ }^{1}$ Centre Hospitalier Spécialisé de l'Yonne, Psychiatrie Secteur 89G01, Sens, France; ${ }^{2}$ CHI Poissy, Psychiatry, Poissy, France

${ }^{*}$ Corresponding author.

\section{Background and aims.-}

Introduction.- Mental health therapies ought not to be resumed to pharmalogical treatments. There is a need for psychiatric services to provide diversity in the psychotherapy offered to patients.

Objectives.- We aimed to develop art therapy strategies across our two sectors in a climate of both financial and medical crisis. After three years, we succeeded in obtaining the agreement from a qualified "art-therapeute" in the service and secured funding for the training of a second "art-therapeute" whose role would be to conduct the therapy in the other sector.

Methods.- The art therapy project was originally created in 2015 by a Psychiatrist, two Psychiatric nurses and supported by the team Psychologists and the head consultant.

Results.- At CMP, an art workshop designed for a class of 8 adults has been active since 2016. The newly-qualified "art-therapeute" offers three 2-hour long workshops. Those are available to young adults between 16 and 25 years once a week and twice a week for adults. Future challenges are to expand the workshop to 6-10 and 10-14 year-olds. We also aim to spread this project across different services and run this as an inter-sector activity.

Conclusions.- Despite the logistical difficulties of lack of time and resources faced by modern Psychiatry, we have succeeded in acquiring the means to diversify therapies already in place. Our initial project has been enriched by the extension across sectors but also within our own sector as we have widened our patient population to include young adults. In this pessimistic climate we have proved that "Yes, we can!"

Disclosure of interest.- The authors have not supplied a conflict of interest statement.

\section{E-PV0762}

\section{Short-term cognitive therapy for survivors after traumatic incidents with suicidal behavior in the first week}

S. Lahutina

Bogomolets National Mediacal University, Medical Psychology-

Psychosomatic Medicine and Psychotherapy Department, Kyiv,

Ukraine

"Corresponding author.

Background and aims.- In the treatment of PTSD, CBT usually involves prolonged, imaginal exposure to the patient's memory of the trauma, and in vivo exposure to various reminders of the trauma, as well as cognitive restructuring and anxiety management techniques. There are as yet no studies of combined cognitive restructuring and anxiety management in the treatment of patients after traumatic incidents with suicidal behaviour in the first week after the incident. The main purpose of the study was to test the efficacy of a short-term cognitive therapy in survivors of traumatic incident with suicidal behavior in the first week.

Methods.- There were 20 participants met Diagnostic and Statistical Manual of Mental Disorders 4th Edition (DSM-IV) PTSD criterion A1 for a traumatic event. Participants received up to $12 \mathrm{~h}$ of cognitive therapy over a period of 10 working days, followed by 1 session a week later.

Results.- Combined cognitive restructuring and anxiety management were well tolerated and reduced PTSD symptoms in 73,6\% of participants. Combined short-term cognitive therapy had better results than comparable group treated by trauma-focused CBT, $73,6 \%$ versus $64,7 \%$. Also combined short-term cognitive therapy had a positive effect on depression and anxiety level. Treatment satisfaction was very good - 75\%, participant therapeutic alliance ratings were high - $92,1 \%$.

Conclusions.- This study showed that implementing combined cognitive restructuring and anxiety management could prevent PTSD symptoms as well as depression and anxiety. Short-term cognitive therapy may be especially beneficial in the first week after traumatic incident. Current results can help to develop effective early PTSD prevention.

Disclosure of interest.- The authors have not supplied a conflict of interest statement.

\section{E-PV0763}

\section{The defense mechanisms and a subjective perception of mental illness among the daily ward patients with schizophrenia and depressive disorders}

P. Lazewski-Banaszak ${ }^{*}$, K. Nitsch-Staszczuk, S. Kalinowska, B. Trześniowska-Drukała, J. Samochowiec

Pomeranian Medical University, Department of Psychiatry, Szczecin, Poland

* Corresponding author.

Background and aims.- A defense mechanism is a way of behaving or thinking which is unconscious and automatic reaction to unpleasant experiences or feelings. Illness perceptions are subjective and contain and organize the knowledge and beliefs people hold regarding their diseases. Illness acceptance mirrors quality of emotions and reactions related to a disease. The aim of the study was to assess a link between maturity level of defence mechanisms and a subjective perception of mental illness among patients of the daily ward of a mental health hospital. Inclusion criteria consisted of: age 18-60 years old, presence of depression disorder (F32, F33) or schizophrenia (F20) diagnosed according to ICD-10 manual, lack of severe medical condition, cognitive impairment or active addic- 
tion. Subjects were 30 patients with schizophrenia and 30 with depressive disorders.

Methods.- As the assessment tools we used Polish version of Defense Style Questionnaire 40 (DSQ-40), the Disease-Related Appraisals Scale (DRAS) and Acceptance of Illness Scale (AIS). Results.- The maturity level of defense mechanisms links to a subjective perception of mental illness, which is reflected by a level of the illness acceptance.

Conclusions.- The results highlight in what way selected features of patients with schizophrenia and depressive disorder influence their adaptation to own mental illness what translates into the clinical practice. It may call for more individualized approach to understand patients resources and indicates multidisciplinary care.

Disclosure of interest.- The authors have not supplied a conflict of interest statement.

\section{E-PV0764}

\section{Negative effects of group psychotherapy}

M. Linden ${ }^{1 *}$, B. Muschalla ${ }^{2}$

${ }^{1}$ Charité University Medicine Berlin, Psychosomatic Medicine, Berlin, Germany; ${ }^{2}$ University of Braunschweig, Insitute of Psychology,

Braunschweig, Germany

${ }^{*}$ Corresponding author.

Background and aims.- Group psychotherapy is a widely used mode of treatment with similar therapeutic effects as individual psychotherapy. The encounter with other patients can be therapeutically helpful, but also detrimental, as co-patients can make negative comments, start confrontational discussions, give wrong advice, or be a burden to others by inappropriate behavior. Objective of our study has been to study genuin and specific side effects of group psychotherapy

Methods.- Sixty two patients from a somatic rehabilitation hospital were randomized to cognitive behavioral group therapy or an unspecific group encounter and filled in the Unwanted Events in Group Therapy Scale (UE-G scale).

Results.- In the cognitive behavior therapy group $41.9 \%$ of the patients reported at least one severe burdensome effect, and $28.9 \%$ in the recreational group. Problems were an increase of anxiety and perception of work-problems, crowding in the room, the encounter with other patients, the content of discussions, worries in regard to confidentiality, and demoralization after observing the problems of others.

Conclusions.- Group psychotherapy regularly has frequent and severe side effects. There are burdens which are characteristic for all types of group therapy and others which are specific for the type of intervention. Therapists should be aware of this and patients should be carefully selected.

Disclosure of interest.- The authors have not supplied a conflict of interest statement.

\section{E-PV0765}

\section{Psychosocial assistance in the rehabilitation of patients with Alzheimer's disease}

S. Moroz ${ }^{1 *}$, I. Sislavskaya ${ }^{2}$

${ }^{1}$ CI "Dnipropetrovsk Region Clinical Hospital named after I.I. Mechnikov", Psychoneurology department, Dnipro, Ukraine; ${ }^{2}$ Dniprovsky City Psychoneurological Center, Psychiatric Department, Dnipro, Ukraine

${ }^{*}$ Corresponding author.

Background and aims.- Contemporary tendention of increase of Alzheimer's disease.

To help patients with Alzheimer's disease used: conversationmemories on photo and video materials and objects from the past patient; listening to the voice recordings of the patient's relatives; exercises to stimulate the senses; cognitive training.

Methods.- We to researched clinical structure of Alzheimer's disease in 108 patients. Psychosocial assistance is a therapy procedure wich we utilized for 59 patients. The number of session varied from 9 to 16 sessions for every patient.

Results.- Stabilization was obtained also for behavioral symptoms and amelioration of the MMSE score in $88,1 \%$ of cases. Psychosocial assistance should be used first of all as a targetoriented.

Conclusions.- Psychosocial assistance system were developed of patients with Alzheimer's disease. Psychosocial assistance system which represents no contraindication for the ages subjects or having cognitive disorders which she can stabilize even to improve. The proposed system demonstrated a significant improvement in $80 \%$ of patients.

Disclosure of interest.- The authors have not supplied a conflict of interest statement.

\section{E-PV0766}

\section{Transgenerational trauma and holistic treatment - case study of a family}

C. Moutafi $1^{*}$, P. Meintani ${ }^{2}$

${ }^{1}$ Dromokaiteio Hospital, Psychiatry, Athens, Greece; ${ }^{2}$ General

Hospital of Greek Air Force 251, Psychiatry, Athens, Greece

* Corresponding author.

Background and aims.- Transgenerational Trauma - failure to process trauma causes trauma to be unconsciously transferred to the next generations

Present the impact of the transgenerational trauma on the members of a 5-person family and the corresponding interventions (biological therapy and psychotherapy).

Methods.- The patient (20 years old girl) was hospitalized (psychiatric clinic) due to suicide attempt within Psychotic Depression. She received antipsychotic along with personal psychotherapy and Psychoeducation within family. Family intervention began during patient's hospitalization, all family members participating whatsoever.

The patient received also individual therapy supportive at the beginning, while later and after she was discharged from Clinic she received psychodynamic therapy (weekly).

Her mother participated in Group Therapy sessions of individuals related to patients suffering from Psychosis.

Results.- During interventions, it was ascertained that all family members were suffering from anxiety and depression, while receiving antidepressants. Mother was carrying a trauma since her childhood due to her father loss; mourning was transferred unresolved to other family members (to her daughter especially). The interventions held had to following favorable results:

1. They applied more effective methods to resolve their problems, while communication among them improved

2. They met their personal goals; young patient completed her university studies

3. Young patient's ego strengthened, her tolerance against cancellation enforced

4. Her mother's participation into Group sessions reduced her stress and feelings of guilt

Conclusions.- Interaction within a stable context, during and after hospitalization contributed to the comprehension of the dynamics within family, to the improvement of their communication, while recurrences were avoided

Disclosure of interest.- The authors have not supplied a conflict of interest statement. 


\section{E-PV0767}

\section{Attitude of polish psychiatrists to psychotherapy as a form of treatment in psychiatry}

S. Murawiec ${ }^{1}$, D. Litwin ${ }^{2}$, N. Seroka ${ }^{2}$, A. Kudlik ${ }^{3}$, M. Matuszczyk ${ }^{4}$

${ }^{1}$ Scientific Assocaition of Psychodynamic Psychotherapy, president, Warsaw, Poland; ${ }^{2}$ Warsaw Medical University, student, Warsaw, Poland; ${ }^{3}$ Dialogue Therapy Center, psychologist, Warsaw, Poland; 4 Mental Health Center Tychy, staff psychiatrist, Tychy, Poland

${ }^{*}$ Corresponding author.

Background and aims.- Psychotherapy is widely used method of treatment in psychiatry. The aim of our study was to evaluate the polish psychiatrists' attitude to this method of therapy of mental disorders.

Methods.- A special on-line questionnaire was prepared for the purpose of a series of such studies carried out in Poland every three years.

Results.- Study conducted in 2018 includes 196 psychiatrists and psychiatric trainees (women 62\%, man 38\%). Clinical experience in psychiatry varied from half a year to 54 years $(M=15, S D=10.50)$. Most of study group work in hospitals $(N=94,48 \%)$, in private outpatient centers $(N=43,22 \%)$ and in outpatient centers in public sector $(N=57,29 \%)$. Most of responders are not psychotherapists ( $n=145,74 \%)$ and 51 psychiatrists in study group work also as psychotherapists (26\%); 24\% respondents declared readiness to participate in psychotherapy course in the future and $31 \%$ in that group participated in psychotherapy training in the past. The biggest wish to improve skills declared responders who had participated in psychotherapy courses before. Psychiatrists who are also psychotherapists did not declared any neutrals or negative opinions about including psychotherapy in treatment $\left(\mathrm{Chi}^{2}=16,79\right.$, $p<0,001, V=0,29)$. They also more often recommend psychotherapy without initiating any form of pharmacotherapy to their patients $\left(\mathrm{Chi}^{2}=9,62, p=0,05, V=0,22\right)$.

Conclusions.- Psychiatrists who are also psychotherapists choose psychotherapy as the only form of treatment of mental disorders more often than psychiatrists without psychotherapeutic background..

Disclosure of interest.- The authors have not supplied a conflict of interest statement.

\section{E-PV0768}

\section{Towards new models: contemporary psychotherapy in Ukraine}

\section{B. Mykhaylov}

Kharkiv Medical Academy of Postgraduate Education, Psychotherapy, Kharkiv, Ukraine

${ }^{*}$ Corresponding author.

Background and aims.- The psychotherapeutic care in Ukraine is carried out in structure specialized psychiatric, psychoneurological and somatic hospitals and polyclinics of public health services. In 2011 parameters of mental illness and disturbances of behavior was 243,6 per 100 thousand population and in comparison with 2005 - 252,9 per 100 thousand population. In structure of parameters of mental illness and disturbances of behavior, in 2011 ( $71,6 \%$ or 174,4 per 100 thousand population) prevailed nonpsychotic. The basic organizational unit of the psychotherapeutic care is the cabinet of psychotherapy, which common number on 1.01 .2009 year was 317 , from them at the psychiatric network 132 (42\%), at the somatic 185 (that makes about 58\%). The psychotherapeutic care is carried out within the limits of medical and psychological model.

Methods.- Thus since 1997 the specialty medical psychology and a staff of the doctor-psychologist is entered. Now in a medical network there are medical psychologists with medical education (181 person) and psychological (559 person) education in parallel work.
Results.- The basic problem is defficiency posts of psychotherapists in specialized and somatic networks according to operating specifications. The general deficiency of staff makes 1000 persons. Conclusions.- The psychotherapeutic network requires immediate expansion, especially in somatic treatment-and-prophylactic hospitals and polyclinics. For these reason the psychological model of psychotherapy must by in priority and certain capacity of psychiatrist must be retrained in psychotherapy.

Disclosure of interest.- The authors have not supplied a conflict of interest statement.

\section{E-PV0769}

\section{On psychiatry and psychosomatics}

E. Neu ${ }^{1}$, M.C. Michailov ${ }^{1}$, H. Schumitz ${ }^{1}$, S. Molnar ${ }^{1}$, K. Molnar ${ }^{1}$, U. Welscher $^{1}$, H.W. Bauer ${ }^{2}$, A. Hofstetter ${ }^{3}$, E.R. Weissenbacher ${ }^{4}$, M. Schratz ${ }^{5}$, G. Weber ${ }^{6}$

${ }^{1}$ Institute Umweltmedizin c/o ICSD e.V., Pharmaco-Physiology, Munich, Germany; ${ }^{2}$ University München and Free University Berlin, Med. Fak., Munich, Germany; ${ }^{3}$ University München, Klinikum Grosshadern Dir. a.D., Munich, Germany; ${ }^{4}$ University München Med. Fak., Premium Med. Clinic Dir., Munich, Germany; ${ }^{5}$ University Innsbruck, School Education Dean, Innsbruck, Austria; ${ }^{6}$ University Lxbg. and Vienna, Fac. Psychology Dean, Vienna, Austria * Corresponding author.

Background and aims.-

Introduction.- New models for psychiatry incl. psycho-somatic (Th.v.UEXKÜLL) and somato-psychic theories (Y.IKEMI), appointed during opening-ceremony of ICPM-Congr. in Kobe by Emperor AKIHITO: "total symptoms of mind-body, seeking ways of holistic care" are to be discussed. Psychopathology in context of therapy\&prophylaxis includes high complex interaction of psychicphysiological-pharmacological-social factors.

Methods.- Psychosomatic practices of occidental/oriental medicine (patients/probands). Evaluation of psychic-"polar-attitudelist"/physiological-parameters: heart-rate, blood-pressure,etc. (ref.).

References.-

EPA-2018-Nice, Eur. Psychiatry 48/S1, S623\&567\&662. WPA-2018Mexico-City (in-press); 2011-Buenos-Aires, AB:PO1.200. ICPM2017-Beijing, AB:ID: 648493,648895,648749,648878; -2005-Kobe, J. Psychosom. Res. 58:85-86. IUPsyS-2012-Cape-Town, IntJPsychol 47:407; -2008-Berlin, 43/3-4:154,248,615,799; -2004 Beijing, AB:49,587. IUPS-2017-Rio-de-Janeiro, AB:No. 997,999,1001,1003: -2009-Kyoto. J. Physiol. Sci., 59/S1:168\&214\&447-8.

Results.- Observations about influence of music [1], respiratory [2], yoga-physical [3] therapies on psychosomatic parameters demonstrate strong positive effects. Items of categories psychophysiological (relaxed), emotional (tranquil/happy), cognitive (few/ordered-thoughts), voluntary (active/spontaneous), social (open/assertive), consciousness (clear/sleepy) are significantly positive changed $25-50 \%$. The 3 therapies have specific psychic effects, e.g. items "relaxed/tranquil" after respiratory- $(+45 / 50 \%) /$ musictherapy $(+20 / 5 \%)$, also "open" $(+25 \%)$ are positive, but negative after respiratory-therapy (-20\%). Items are stronger changed before then after gynecological-urological-surgical-intervention. Higher effects are observed for investigators\&children than of students of psychology. Psychic effects are correlated with positive physiological ones, e.g. heart/respiratory-frequency decreased $25-30 \%$, voluntary-apnoea prolonged 55\% ( $p<0.05-0.01, n=145)$.

Conclusions.- Integrative psychosomatic therapy incl. occidentaloriental (yoga/tai-chi/Zen,etc.) approaches could be applied for psychic disorders. Different methods are with preference, e.g. for depression is suitable respiratory/physical-training (activation) incl. for gynecological-urological patients, for mania: music-therapy (inhibitory-effect), for epilepsy: respiratory-therapy - hypo/hypercapnia: inhibitory/excitatory effects on CNS-structures. Systematic research about influence of psychosomatic therapies 
counteracting disturbances of psychiatric patients could support UNO-Agenda21 for better health-education,etc. on global level. Disclosure of interest.- The authors have not supplied a conflict of interest statement.

\section{E-PV0770}

\section{On the (re)search for the conjugal model: clinical case study}

M. Figueiredo ${ }^{1,2}$, C. Pires De Lima ${ }^{3,4^{*}}$, M. Cruz ${ }^{5}$, Z. Charepe $e^{2,6}$, G. Cunha ${ }^{7,8}$

${ }^{1}$ Escola Superior de Enfermagem do Porto Nursing School of Porto, Teacher, Porto, Portugal; ${ }^{2}$ CINTESIS - Center for Health Technology and Services Research, Nursing, Porto, Portugal; ${ }^{3}$ For AllDesenvolvimento Pessoal e Bem-Estar-Lda, Psicologia, Paredes de Coura, Portugal; ${ }^{4}$ CINTESIS - Center for Health Technology and Services Research, Psychology, Porto, Portugal; ${ }^{5}$ Escola Superior de Enfermagem do Porto Nursing School of Porto, Nursing, Porto, Portugal; ${ }^{6}$ Instituto de Ciências da Saúde da Universidade Católica Portuguesa, Nursing, Porto, Portugal; 7 Mediare, Conflict Medition, Porto, Portugal; ${ }^{8}$ Universidade Lusófona, Student, Porto, Portugal Corresponding author.

Background and aims.- The co-construction of conjugality influences the established interactions in the family as a whole, becoming the relational model in the expression of effectiveness and conflict management.

Clinical case report, in which the members of the couple perceive themselves as having marital problems, assuming these are due to conflicts with the family of origin.

Methods.- Exploratory-descriptive study, using the case study as an empirical approach of constructivist onto-epistemological matrix, centered on creativity and co-evolution. We used the technique of analysis of context of action, which emerged from the interactional reciprocity of the therapeutic system, sustained in the Appreciative Inquiry.

The case described refers to Couple Therapy, with ten sessions held in 2016. The members of the couple were present in all sessions. Results.- The family consists of the couple, male $T$ and female $\mathrm{B}$, and their son E. T and B have been married since 2008. About their marital relationship they state that it has been deteriorating because of the focus on the inherent functions as parents,and also because of the conflicts that have arisen between B and T's parents since E's birth in 2015. As far as the extended family is concerned, both elements of the couple come from nuclear families, with $\mathrm{T}$ being an only child and B having 2 siblings.

Conclusions.- We developed strategies which integrated the different theoretical and operational frameworks. The transformative change enabled the couple to take control of their relationship and allowed for the differentiation from the extended family,thus enabling them to decide the itinerary of their further development. Disclosure of interest.- The authors have not supplied a conflict of interest statement.

\section{E-PV0771}

\section{Group psychotherapy from the perspective of a psychiatrist}

\section{J. Poplawska}

Laboratorium Psychoedukacji, Psychotherapy, Warszawa, Poland

* Corresponding author.

Background and aims.- I will present my experiences of working as a group therapist with my psychiatric patients and I'll argue that combining pharmacology with group psychotherapy is the most efficient form of treatment when it comes to psychiatric patients. I will focus on the influence of my double role - of a psychiatrist and a psychotherapist - on the group process.
Methods.- In my presentation I will discuss the efficiency of group psychotherapy in psychodynamic approach for patients with broad spectrum of clinical symptoms. For more than 6 years (between 2012 and 2018) I have conducted 19 short-term psychotherapy groups lasting from 3,5 months (13 sessions) to one year (50 sessions). All were conducted in Warsaw in a Public Mental Health Clinic and in Laboratorium Psychoedukacji, a private psychotherapeutic centre. More than 200 patients have participated in these groups. Group therapy was recommended to patients to who were diagnosed with various psychological disorders and at the moment of being referred to the group were taking medication. All these group psychotherapy attendees were formerly my psychiatric patients, already established relationship of trust enabled us to build hope regarding the efficiency of this kind of treatment at the early stage of their psychotherapy.

Results.- In the long run it enables most patients to discontinue taking medication and reduce the frequency of their appointments with psychiatrist.

Conclusions.- I will try to show that a good relationship between doctor and patient facilitates group psychotherapy process and vastly contributes to reducing the number of patients falling out of the group.

Disclosure of interest.- The authors have not supplied a conflict of interest statement.

\section{E-PV0772}

\section{The role of mental images in the treatment of emotional dysregulation in borderline personality disorder: feasibility of a new short intervention}

Z. Sosic-Vasic ${ }^{*}$, C. Schaitz

University Clinic of Ulm, Department Psychiatry and Psychotherapy III, Ulm, Germany

Corresponding author.

Background and aims.- Mental imagery related to retrospective events play a role in the psychopathology of several mental disorders (e.g. posttraumatic stress disorder). However, recent studies have indicated a potential role of mental imagery related to prospective events (Holmes et al., 2007). Among borderline personality disorder (BPD), increased occurrence of aversive mental images associated with prospective dysfunctional emotional regulation strategies such as self-injury have been identified (Schaitz et al., 2018). This is the first psychotherapeutic attempt targeting prospective mental images related to self-harming behavior in patients with BPD by applying imagery rescripting and rehearsal techniques. Feasibility in terms of treatment acceptance and safety was evaluated.

Methods.- Seven women with BPD were treated during two sessions. Borderline symptoms (BSL-95), overall clinical improvement (CGI), emotion regulation (FEEL-E and ERQ), and self-harming behavior (emotional dysregulation behavior according to BSL-95) were recorded prior to treatment $(\mathrm{t} 1)$, post treatment $(\mathrm{t} 2)$ and at a 1-year-follow-up (t3).

Results.- No symptom exacerbation was recorded from t 1 to $t 2$, or even to $\mathrm{t} 3$ as indicated by a decrease in total BSL-95 score and an even mild CGI score improvement. No drop-out rates were recorded. Emotion regulation strategies (FEEL-E and ERQ) improved. Moreover rates of self-harm (BSL-95) decreased in our sample, however, not for all patients.

Conclusions.- Data from our feasibility study suggest that this shortterm intervention is save and acceptable for patients suffering from BPD. However, results are limited by the very small sample size, and the lack of a control group. Replication within a randomizedcontrolled trial is needed.

Disclosure of interest.- The authors have not supplied a conflict of interest statement. 


\section{E-Poster Viewing - 7-9 April: Quality Management}

\section{E-PV0774}

\section{Qualitative theme analysis of positive practice reports of joint mental health and primary care service innovations in the United Kingdom}

S. Afghan ${ }^{*}$, R. Faruqui ${ }^{2,3}$, P. Wilson ${ }^{2}$, C. Gerada ${ }^{4}$

${ }^{1}$ Dudley $\mathcal{E}$ Walsall Mental Health Partnership Trust E University of Wolverhampton, UK, Mental Health, Walsall, United Kingdom; ${ }^{2}$ University of Kent, Primary Care Academic Unit, Centre for Health Services Studies, Canterbury, United Kingdom; ${ }^{3}$ Kent $\mathcal{E}$ Medway NHS Partnership Trust, East Kent Neuropsychiatry Service, Ashford, United Kingdom; ${ }^{4}$ Medical Director NHS Practitioners Health Programme \& Former Chair RCGP, NHS Practitioner Health Programme, London, United Kingdom

${ }^{*}$ Corresponding author.

Background and aims.- Quality enhancement requires an on-going process of service innovation and reporting. We conducted a survey of UK Psychiatrists to identify service innovation areas enhancing joint working between primary care and mental health services. Methods.- We conducted an Online Survey for the Royal College of Psychiatrists. Survey respondents were invited to report on positive practice innovations between the secondary or tertiary care psychiatric services and primary care. A qualitative thematic analysis of 336 responses was conducted using line-by-line analysis technique and the grounded theory until a saturation point was reached.

Results.- Five core innovation themes were extracted:-

1. Teaching and Training: Examples include Dementia Masterclasses, Liaison Psychiatry Teaching in the General Hospital, and Joint CPD opportunities organized locally and nationally

2. Co-Working Pilots in Primary Care: Examples include Consultant Clinics in GP Surgeries, Joint Clinics for Medically Unexplained Symptoms, Joint CPA Clinics for People with Serious Mental Illness, Crisis Link through CRHT teams, and Community Matron Liaison for Older Adults' Admission Avoidance

3. Co-Working Pilots in Secondary and Tertiary Care Mental Health: Examples include Primary Care Liaison in Secure Care services and Acute Learning Disability Liaison

4. Workforce Innovations: Examples include deployment of Primary Care Mental Health Nurses, Primary Care Link Workers, Telephone Liaison and Advisory Service

5. Information Management System Innovations: Examples include joint access to investigations' record, and joint crisis management recording

Conclusions.- Our analysis has identified multiple areas of innovative practice and joint-working between the psychiatrists and primary care in the UK. Reported service innovations are likely to enhance quality of patient experience and staff training.

Disclosure of interest.- The authors have not supplied a conflict of interest statement.

\section{E-PV0776}

\section{Being a difficult patient brings more difficulties!}

F. García-Escribano*, G. Montero, M. Pérez Lombardo Hospital Clínico San Carlos, Psychiatry, Madrid, Spain

${ }^{*}$ Corresponding author.

Background and aims.- Patient security is a topic which is gaining importance, especially in mental health.

The aim of this bibliographic review is to check whether there is a relation between being a difficult patient (DP) and the susceptibility of adverse events (AE) taking place.

Methods.- Literature reviewed: white literature.
Searching method.- Medline (Pubmed).

Inclusion criteria.- English-spanish language; published in the last 5 years.

Search strategy (Fig. 1).

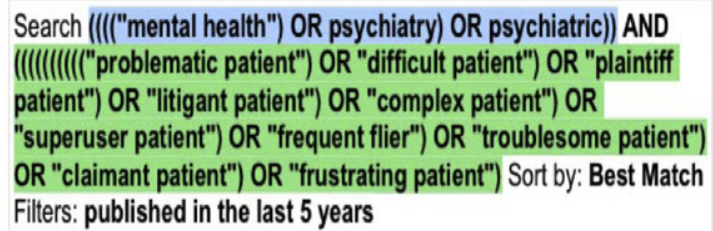

Fig. 1. Search strategy.

Exclusion criteria.- Literature not related to the main purpose of the review. Out of 70 articles, 61 were discarded upon exclusion criteria or not having the full text available. Only 9 articles remained.

Results.-

- DP may be more susceptible of being demedicalized (example: borderline personality disorder). Demedicalized pathologies are more stigmatized.

- Difficult clinical encounters are associated with: worsening of the symptoms and higher rates of healthcare utilization after the encounter.

- Poorer quality of healthcare towards DP may end up in worse prognosis.

- Healthcare professionals which are trained in mindfulness, cognitive-analytic therapy or dialectical behavior therapy, achieve better results in clinical encounters with DP.

Conclusions.-

- DP labeling may have a negative effect regarding contratransference and prognosis of the patient. Thus, we recommend being cautious upon using this label.

- Being a DP seems to be directly related to AE susceptibility. Disclosure of interest.- The authors have not supplied a conflict of interest statement.

\section{E-PV0778}

\section{The university of alberta hospital gender program: the process of establishing the evaluation of health outcomes for group therapy in \\ Edmonton, Alberta \\ M. Marshall ${ }^{1 *}$, B. Sperling ${ }^{2}$, P. Chue ${ }^{1}$ \\ ${ }^{1}$ University of Alberta, Psychiatry, Edmonton, Canada; ${ }^{2}$ Alberta \\ Health Services, Psychiatry, Edmonton, Canada \\ ${ }^{*}$ Corresponding author.}

Background and aims.- The University of Alberta Hospital Gender Program started in February, 2018. The aim of the Program is to provide good, compassionate care to the transgender and gender-diverse population of Edmonton and Northern Alberta. The Gender Program looked to offer group therapy to transgender individuals to manage psychiatric and psychological morbidity. The Program sought to measure specific parameters of wellbeing and mental health before and after 12 weeks of group therapy, but needed a short, targeted means to do so. The aim was to use the least resources to ask as few questions as possible to get the best outcome measurements, while minimizing survey fatigue and maximizing engagement.

Methods.- The bedrock of our approach was the maximization of utility for selected scales. Short and precise scales were selected over longer and more thorough scales when considering the balance of client engagement and survey fatigue. Age ranges, time points, survey length, statistical validity, cost, and primary source backing were all considered in selecting the best combination of scales to survey the health outcomes of clients in the Gender Program. 
Results.- Seven scales were chosen, covering cognitive fusion, mood, anxiety, coping strategies, cultural otherness, substance use and satisfaction to develop a unique survey package for the Gender Program. Each survey package took a maximum of $30-45$ min to complete.

Conclusions.- The transgender population in Edmonton and Northern Alberta has unique needs that need to be captured with applicable research tools that can be used in the future to evaluate success.

Disclosure of interest.- The authors have not supplied a conflict of interest statement.

\section{E-PV0780}

\section{Burn-out syndrome: how it infludes in the psychiatry department of an hospital in Cádiz (Spain)}

\author{
A. Pérez Morenilla ${ }^{1 *}$, P. Ortega Orihuela ${ }^{2}$ \\ ${ }^{1}$ Psychiatrist, Psychiatry and Mental Health, Cádiz, Spain; ${ }^{2}$ Puerto \\ Real University Hospital, Psychiatry, Cádiz, Spain \\ * Corresponding author.
}

Background and aims.- Burnout is defined as an inadequate response to chronic work stress, with cynical and depersonalized attitude to patients and feeling of inadequacy to perform tasks.

To evaluate burnout-syndrome in Mental-Health-Workers of Puerto Real Hospital (PRH; Spain), performing an evaluation according to professional categories, sex, work schedules.

Methods.- Descriptive cross-sectional study performed on all Mental Health professionals using self-administered MaslachBurnout-Inventory, consisting in 3 subscales: Emotional-Tiredness (ET), Depersonalization (DP) and Personal-Realization (PR).

Results.- $104 / 148$ workers participated (70.27\%), with a medium burnout level in ET (19.45), DP (6.14) and PR (38.68). According to professional categories, physicians showed higher level of burnout in ET (25.04, Sd:10.83), followed by administrative assistants (23.67, Sd:15.85); those with lower levels were hospital guards (15.4, Sd: 2.26). In DP, highest levels were presented by physicians (8.93, Sd: 7.36) followed by hospital guards (7, Sd: 4.79), and the lowest levels by occupational therapists. In PR, highest levels were presented by administrative-assistants (33.33, Sd: 8.02 ), followed by hospital guards and doctors (35.8, Sd: $11.5,36.22$, Sd: 6.02), the lowest levels for occupational therapists (47). Regarding sex, males had higher levels of burnout in ET (20.74Vs.19) and DP (6.66Vs.5.88), and lower in PR (39.69Vs.38.31). Regarding the employment situation, those hired showed higher level of burnout than permanent workers in ET, DP and PR. In terms of workinghours, tomorrow's workers have a higher burnout levels than those with variable hours.

Conclusions.- In our area, workers have a medium level of burnout, highlighting lower burnout in self-realization. It would be important to improve emplyers working conditions to minimize this syndrome with causes negative consequences on physical and psychological health, quality of life and professional efficiency, as well as negative consequences in the workplace for patients and for administration.

Disclosure of interest.- The authors have not supplied a conflict of interest statement.

\section{E-PV0781}

\section{Study of pre-leave risk assessment in in-patients in 4 secondary care adult psychiatric wards}

S. Siddiqui*, U. Ahmed

NHS, Psychiatry, Dewsbury, United Kingdom

${ }^{*}$ Corresponding author.
Background and aims.- Leave off the ward is a vital component in the management and recovery of in-patients. It is vital to ensure that only patients who are safe to be managed outside the ward environment are allowed to have a leave. Therefore a comprehensive risk assessment, is extremely important to contain the risks to the safety of the patient and others before any leave out of the ward is granted

Aims and objectives.- To study the current practice of documentation of pre-leave risk assessment in four ward in secondary care hospital.

Methods.- A retrospective study carried out to collect data for 40 patients (22 Male and 18 Female) from secondary care wards Results.- Leave was discussed and agreed in mutidisciplinary team meetings in $95 \%(n=38)$. Duration of leave was documented in $82.5 \%(n=33)$. Destination of leave was reported in $92.5 \%(n=37)$. Brief mental state of patient done in $87.5 \%(n=35)$. Risk to self in $80 \%(n=32)$ and Risk to others in $87.5 \%(n=35)$. Discussion what to do if distressed whilst on leave occurred in $17.5 \%(n=7)$ and there was discussion with $12.5 \%(n=5)$ about what to do if the patient cannot return to the ward on time.

Conclusions.- This study highlighted a number of areas to improve patients' safety. Recommendations were made to share this study findings with relevant professionls, ward staff and to give ward contact cards to pateints before leave

Disclosure of interest.- The authors have not supplied a conflict of interest statement.

\section{E-Poster Viewing - 7-9 April: Rehabilitation and psychoeducation}

\section{E-PV0782 \\ Efficacy of group psychotherapy in co-dependent relatives of drug-dependent patients of inpatient rehabilitation department}

\section{Z. Bereza}

Medical Center Bechterev, Psychiatry, Sain-Peterburg, Russia

${ }^{*}$ Corresponding author.

Background and aims.- The behavior of co-dependent relatives affects the effectiveness of treatment of drug addicts. Psychotherapeutic interventions for relatives of opioid addicted patients seems challenging owing to such factors as: lacks consensus about a definition, phenomenology and an etiologyof this phenomenon; underestimation of influenceof "burden» of an illness.

The research is devoted to the study of the phenomenon of codependence in the families of drug addicts and the influence of psychotherapeutic correction of co-dependence on the stability of participation of addicts in the program of complex medical rehabilitation.

Methods.- 61 co-dependent mothers of 61 opiod addicted patients admitted for inpatient rehabilitation treatment, were examined. They were divided into two groups: I- subjects who attended group and individual sessions $(n=37)$ II- subjects who refused to attend sessions $(n=24)$.

Clinico-anamnestic,clinical-psychopathological methods (MADRS, SCL-90), 6 month follow-up data.

Results.- Changes in psychological and clinical and psychopathological parameters in mothers of both groups indicate an improvement in most of the measured variables estimated on the basis of Wilcoxon criterion. Kaplan-Meier survival function confirms the significant differences in the duration of participation in the rehabilitation program of the drug addicted patients of two groups. 
Conclusions.- Participation of co- dependent relatives in the program of psychotherapeutic support had a positive effect on the treatment and rehabilitation program of opiod addicted patients. The in-patient rehabilitation program for drug addicts has a positive impact on the dynamics of the psychological characteristics of their mothers, regardless of the participation or non-participation of them in the program of psychotherapeutic support for codependent relatives.

Disclosure of interest.- The authors have not supplied a conflict of interest statement.

\section{E-PV0785}

\section{Art-therapy in rehabilitation program in affective disorders}

I. Leshchyna ${ }^{1 *}$, T. Mozgova ${ }^{1}$, L. Ovcharenko ${ }^{2}$

${ }^{1}$ Kharkiv National Medical University, Psychiatry- Narcology and Medical Psychology, Kharkiv, Ukraine; ${ }^{2}$ Kharkiv National Medical University, Kharkiv National Medical University, Kharkiv, Ukraine ${ }^{*}$ Corresponding author.

Background and aims.- Art-therapy has a significant rehabilitation potential in working with patients with mental diseases, in particular affective disorders. The purpose was to evaluate the effectiveness of art-therapy in the rehabilitation of patients with affective disorders.

Methods.- The clinical-psychopathological, psychodiagnostical (The Hamilton Depression Rating Scale, The Hamilton Anxiety Rating Scale, The Social Adaptation Self-evaluation Scale by Bosc M. et al., 1997.) methods were used. 94 female patients with affective disorders were examined. 34 patients were diagnosed with recurrent depressive disorder, 28 with bipolar affective disorder, 20 with depressive episode, 12 patients with organic affective disorder.

Results.- The main group (49 patients) received the complex treatment with combination of psychopharmacotherapy and arttherapy. The second group (45 patients) had standard therapy. There were no statistically significant differences of level of social functioning between groups. The average depression score on the HDRS was $21.3 \pm 3.1$ ball. The average anxiety score on the HARS was $19.1 \pm 2.2$ ball. Indicators of the subjective level of social adaptation in patients demonstrate a difficulty in social adaptation. The object of art-therapeutic influence were the states of maladaptation, caused by chronic mental disorders. Psychoeducation was also utilized.

Conclusions.- The analysis of the dynamics of indicators on the SASS showed the significant improvement in the social functioning, interest in communication, daily activity, family interaction and creativity in the main group. Art-therapy help to stabilize the mood of patients, to greater extent, by reducing the level of anxiety, increasing self-esteem, self-confidence, interpersonal interaction, development of positive attitude towards their illness and forming a willingness for further treatment.

Disclosure of interest.- The authors have not supplied a conflict of interest statement.

\section{E-PV0786}

\section{A unique working model in rehabilitation- integrative groups of individuals with serious mental illness in the community}

Y. Mazor ${ }^{1}$, I. Adler-Ben Dor ${ }^{2}$, N. Galor-Teperberg ${ }^{2}$, Y. Dor ${ }^{2}$

${ }^{1}$ Amitim program for adults- IACC, Amitim fot Adults, Tel-Aviv, Israel; ${ }^{2}$ Amitim program for adults- IACC, Mental health, Lod, Israel ${ }^{*}$ Corresponding author.

Background and aims.-

Introduction.- The stigma of mental illness as an additional burden for people with serious mental illness (SMI) has been researched in the last decades (Gaebel et al., 2006). Stigma is a major barrier to recovery for people with SMI; it disturbs with community living and attainment of rehabilitation resources and goals, as well as damages self-esteem and self-efficacy (Scheyett., 2005). The model of 'Amitim' integrative groups aims to decrease both public stigma, as well as increasing well-being and direct social bonds between people with SMI and people from the general community. The model we shall present is part of the Amitim program (by the Israeli Ministry of Health and the Israeli Association of Community Center), which offers social rehabilitation in the community for people with SMI, and the promotion of personal recovery and social change. Methods.-

Objective \& methods.- Amitim's 'integrative groups' is a unique intervention model in the community that aspires to create a direct and equal social bond between both sub-groups of participantspeople with SMI and non-SMI people from the community. This bond is based on similar interests such as drama, poetry, creative writing, music, etc. The integrative groups are based upon thus said interests, and participants take part in weekly meetings, where they work on joint projects within their interest arena (Orkibi et al., 2014). The integrative groups also aim to reduce social stigma against people with SMI.

Results.-

Conclusions.- We aim to delineate different formats for using this model, while discussing the dilemmas and providing examples. Disclosure of interest.- The authors have not supplied a conflict of interest statement.

\section{E-PV0787}

\section{Conflict management in the school context: revision on the practice}

S. Gonçalves ${ }^{1}$, C. Pires De Lima ${ }^{1,2^{*}}$, A. Salgueiro ${ }^{1}$, M. Silva $^{1}$, M. Figueiredo $^{3,4}$

${ }^{1}$ For All- Desenvolvimento Pessoal e Bem-Estar- Lda, Psychology, Paredes de Coura, Portugal; ${ }^{2}$ CINTESIS - Center for Health

Technology and Services Research, Psychology, Porto, Portugal; ${ }^{3}$ Escola Superior de Enfermagem do Porto Nursing School of Porto, Teacher, Porto, Portugal; ${ }^{4}$ CINTESIS - Center for Health Technology and Services Research, Nursing, Porto, Portugal

* Corresponding author.

Background and aims.- Conflict is perceived differently in the literature. On a negative light Bilhim (1996) presents conflict as a process in which subject $A$ tries to purposefully hinder subject $B$ 's efforts towards the achievement of their goals and the development of interests, using any way of opposition that results in subject B's frustration. On the other hand, Ferreira, Neves and Caetano (2011) understand conflict as necessary and positive, stimulating innovation and creativity in regards to behaviors, attitudes and cognitions. Methods.- Bibliographic review.

Results.- Torrego (2003) presents conflict mediation as a process in which a third person helps mediate the conflict resolution between two sides. In this way, and highlighting the advantages presented by Uranda (1998, cit. by Torrego 2003) in the school context, this poster summarizes strategies (Perrenoud, 2000), benefits and agents in this specific intervention area.

Based on Piaget's studies, Telma Vinha (1999, cit. by Leite \& Löhr, 2012) refers that conflict is present on daily life. When conflict arises in the classroom it gives us clues about what the students need to learn.

Conclusions.- Because of this it becomes relevant to ponder the strategies that we can use in this context, so that children experience conflict as an opportunity for reflection and self-improvement. Disclosure of interest.- The authors have not supplied a conflict of interest statement. 
E-PV0788

\section{Differences in physical capabilities between the mdd patients and the high-functioning elderly. a case-control study}

W. Rachel ${ }^{1}$, S. Krupnik ${ }^{2}$, W. Datka ${ }^{1}$, D. Dudek ${ }^{1}$, P. Kedziora ${ }^{3}$, M. $\mathrm{Zak}^{4}$

1 Jagiellonian University- Collegium Medicum, Department of Psychiatry, Cracow, Poland; ${ }^{2}$ University of Physical Education, Faculty of Phisical Education and Sport, Cracow, Poland; ${ }^{3}$ Regional Hospital- Busko-Zdroj, Rehabilitation, Busko-Zdroj, Poland; ${ }^{4}$ The Jan Kochanowski University- Faculty of Medicine and Health Sciences, Institute of Physiotherapy, Cracow, Poland

${ }^{*}$ Corresponding author.

Background and aims.- The elderly with diminished functional capabilities stand a $1.45 \mathrm{x}$ higher chance of developing Major Depressive Disorder (MDD).

Assessment of differences in balance and cognitive abilities between the MDDs and the high-functioning elderly (HFEs) without MDD symptoms.

Methods.- The study covered 21 MDD patients over 60, and 21 elderly (HFEs) of 6 fitness centres. Assessment of the paired-up subjects was pursued with GDS, MMSE, TUGT, TUG ${ }_{\text {MAN }}$, TUG COG, $_{\text {, }}$ 30sChS, SLS OP and SLS CL tests. Inclusion criteria were 60-70 years of age and severity of depressive symptoms (GDS $>5$ points).

Results.- None of the subjects in either group complied with all reference standards of functional capabilities. MDDs and HFEs, whose performance fell short of pertinent norms, differed significantly between groups for TUGT ( $43 \%$ vs. $100 \%$ ), TUGCOG, (0\% vs. $100 \%$ ), $30 \mathrm{ChS}$ ( $52.4 \%$ vs. $100 \%$ ), SLS OP (38.1\% vs. $85.7 \%)$ and SLS CL (0\% vs. $23.8 \%$ ). MDDs and HFEs also significantly differed in number of fall incidents sustained by 57.1 and $4.8 \%$, respectively. Participation of MDDs increased the odds ratio (OR) for a drop in functional capabilities (OR >30), TUGT (OR >30) 30sChS (OR >30), SLSOP (OR 9.7). An alteration by 1 point in GDS significantly modified the TUGT score by $0.6 \mathrm{~s}$ within a group, regardless of its type.

Conclusions.- (1) MDD modified functional capabilities in seniors. (2) MDD significantly increased OR for a failure to meet pertinent standards in the single-purpose and dual-purpose balance and muscle strength tests. (3) The GDS scores were significantly correlated with effective locomotion.

Disclosure of interest.- The authors have not supplied a conflict of interest statement.

\section{E-PV0789}

\section{Extending the theory of ls vygotsky: implications for psychological treatments in psychiatry}

N. Semenova ${ }^{1 *}$, M. Kulygina ${ }^{2}$

${ }^{1}$ Moscow Research Institute of Psychiatry MoH RF, Outpatient Psychiatry, Moscow, Russia; ${ }^{2}$ Moscow Research Institute of Psychiatry - subsidiary The Serbsky Federal Research Center for Psychiatry and Narcology, Prevention, Moscow, Russia * Corresponding author.

Background and aims.- The work of LS Vygotsky (1896-1934) is set to exert an increasing influence on psychosocial treatment research in the new century. We begin by considering how Vygotsky's 'zone of proximal development' idea, combined with his emphasis on the social origin and semiotic mediation of higher mental functions, has already led to profound advances in our understanding of mental functioning.
The aim of the paper is an illustration of Vygotsky's theory, in particular, his account of 'zone of proximal development', and its application to psychosocial treatments for schizophrenia.

Methods.- Within this framework, we will focus on the process of peer learning within the group format psychosocial treatments. Results.- We outline the key underlying psychological principles that contribute to this area of work and relates this to existing research finding. Vygotsky's method was anti-reductionist in its emphasis on inter-functional relations, an approach which has proved particularly useful in research into phenomena such as verbal self-regulation. Illustrations are provided from our recent work on psychosocial treatments for schizophrenia.

Conclusions.- The 'zone of proximal development' in Vygotsky's analysis of learning and instruction - provides a useful framework for exploring potentially unknown sources of patient's motivation. The findings imply that the 'zone of proximal development' approach is needed to ensure effective mental health interventions. Such an approach may help to increase the possibility that interventions are tailored to the patient's perspective which in turn may increase the likelihood of treatment success.

Disclosure of interest.- The authors have not supplied a conflict of interest statement.

\section{E-PV0790 \\ Ability to perceive social support in women whose husbands are diagnosed with schizophrenia}

\section{Stukan*, A. Kaminska, V. Herasymuk}

National Pirogov Memorial Medical University-Vinnytsya, Medical

Psychology and Psychiatry Department with the Course of

Postgraduate Education, Vinnytsya, Ukraine

Corresponding author.

Background and aims.- Ability to perceive social support indicates the ability of a person to identify the most priority relationships with other members of society (family, friends, significant others). It is precisely because of the ability to perceive or not to perceive social support that social and communicative activity is formed, and features of emotional and behavioral responses of the individual are determined.

Aim of our study was to reveal features of ability to perceive social support in women whose husbands are diagnosed with paranoid schizophrenia.

Methods.- 100 women aged 22 to 48 years, whose husbands were diagnosed with schizophrenia according to ICD-10 criteria, participated in the study - they were included into main group (MG). Control group (CG) included 40 women aged 21 to 45, whose husbands weren't diagnosed with any chronic somatic and mental illness. Perception of social support was assessed with the use of the Multidimensional Scale of Perceived Social Support (MSPSS)by G. D. Zimet [1998].

Results.- Total scale of perceived social support scored $6.0 \pm 2.0$ in MG, while in CG - 11.2 points. By "Family" subscale MG scored $2.3 \pm 0.7, C G-3.5 \pm 0.2$; by "Friends" subscale MG scored $1,1 \pm 0,2$, CG $-3,5 \pm 0,5$; by "Significant other" subscale MG $-1,9 \pm 0,1$ and CG $-3,2 \pm 0,8$ points.

Conclusions.- Obtained and analyzed data show that ability to perceive social support in women, whose husbands are diagnosed with schizophrenia, is lower than that of the control group respondents, and is mainly provided by the family.

Disclosure of interest.- The authors have not supplied a conflict of interest statement. 


\section{E-PV0791}

\section{Eating behaviour and obesity: experiences of a nutritionist working at a Brazilian University psychiatric outpatient service}

C.M. Vieira Turato, D.B. Faria-Schützer Turato, E.R. Turato* State University of Campinas, Laboratory of Clinical-Qualitative Research LPCQ Campinas, Brazil

${ }^{*}$ Corresponding author.

Background and aims.- Rapid weight gain is frequent among patients in psychiatric treatment due to certain medications, and nutrition monitoring is recommended to discourage patients from discontinuing their treatment prescribed. The interruption in psychiatric medication can cause relapse.

Aim.- To report the professional experience, during a post-doctoral research, of a nutritionist that have worked with the outpatient psychiatric team at the General Hospital at the University of Campinas, South-eastern Brazil.

Methods.- This report regarding the clinical experience was supported by a humanistic methodological perspective of listening freely to psychiatric patients about symbolic meanings attributed to the body, diet, illness, treatment, team-patient relationship; and notoriously on their ambiguities.

Results.- The nutritionist's experience at the clinical activity indicates that conversations with patients focused on their personal relationship developed in life with food and its habits is more effective for managing excessive weight gain than is the insistence on prescribing diets and giving food options. This clinical observation also showed that the patients' families should be involved in their proposals for changing eating habits.

Conclusions.- Insights into how patients interpret and symbolize their food and eating habits in a caring and treatment context showed the importance of dealing with how they see their diets psychologically and culturally. For chronic patients under psychiatric treatment this process includes dealing with their multiple reactions to excessive weight gain.

Disclosure of interest.- The authors have not supplied a conflict of interest statement.

\section{E-PV0793}

\section{Peer support for recovery: an experience of peer sponsorship}

H. Wallot

University of Quebec, Mental Health, Quebec City, Canada

${ }^{*}$ Corresponding author.

Background and aims.- For people with a mental health disorder, peer support (peer in the sense of people having recovered from such a disorder) is a great therapeutic value. AA (Alcoholics Anonymous), were the first to show this therapeutic value. Their approach has several elements of which, aside of the "meetings" and the concept of the "12 steps", a major element is the sponsorship of an individual by a peer. Since then appeared organizations based on peer groups, and also this new profession of "peer support helper". We present here a experience of peer support initiated by a psychiatric center and based mainly on sponsorship of a person suffering psychosis by a peer. Since its introduction, this experience starts to give some results. Also, it has a component of «meeting» and a component of « 12 steps".

Methods.- The center where works the author offers services of psychiatric rehabilitation for persons suffering of severe psychotic disorders and functional deficits. Inspired by the AA, the Center initiated a mentoring program based of such values as peer support, all-encompassing respect, equity between participants, and confidentiality.
Results.- The voluntary pairing unites a person who has recovered with one still struggling toward recovery. The mentoring can take various forms, as an evening out, phone conversations, email exchanges, or any other form of mutual aid.

Conclusions.- Interesting potential developments grace this young Center program. A "peer-helper" or better yet, an AA-type mentor could make a significant difference in the quality of life and on the recovery of those in need.

Disclosure of interest.- The authors have not supplied a conflict of interest statement.

\section{E-PV0795}

\section{The effect of education on emotional intelligence in methamphetamine users under methadone maintenance therapy \\ S.M. Zamir*, S.H. Ghafeleh Bashi, M. Khazaei, S.A.H. Mirzadeh Qazvin University of Medical Science- Qazvin-Iran, Psychiatry, Qazvin, Iran \\ Corresponding author.}

Background and aims.- Considering substance users are characterized by lower emotional intelligence than others in society, this study aims to determine the effectiveness of training on emotional intelligence of methamphetamine users who receive methadone maintenance treatment.

Methods.- This study was conducted through randomized clinical trial with includes pretest-post test control group design. In this regard, 70 methamphetamine users who received methadone maintenance treatment in Drop In Center (DIC, Qazvin, Iran) and scored higher than 80 in Wechsler intelligence scale, were selected. The users were randomly divided into intervention group and control group. The two groups gave Bar-On emotional quotient inventory test. The intervention group attended 4 sessions of emotional intelligence training which took 90 minutes per weak. In contrast, control group did not undergo any intervention. 3 months after last training sessions, Bar-On emotional quotient inventory was repeated for members of the two groups.

Results.- The program for training of emotional intelligence could comparatively increase emotional intelligence score of the intervention group in a significant manner $(P=0.03)$. However, the program did not contribute to reduction of methamphetamine use $(P=0.13)$.

Conclusions.- Training of emotional intelligence to methamphetamine users could add to their emotional intelligence but further studies and more effective methods will be required for reduction of methamphetamine use.

Disclosure of interest.- The authors have not supplied a conflict of interest statement.

\section{E-Poster Viewing - 7-9 April: Research Methodology}

\section{E-PV0797}

Traditional pathopsychological tests and their integrative modification: from objective cognitive assessment to examination of inner world

N. Burlakova ${ }^{1 *}$, V. Oleshkevich ${ }^{2}$

${ }^{1}$ Lomonosov Moscow State University- Faculty of Psychology, Department of Neuro- and Pathopsychology, Moscow, Russia; ${ }^{2}$ Scientific-Practical Children's and Adolescents Mental Health Center n.a. G. Sukhareva, Department of Health Care, Moscow, Russia * Corresponding author. 
Background and aims.- Since psychological tests were first introduced to psychiatry, not only the questions about their validity have arisen, of inevitable relevance has always been a problem, whether they are authentic to the reality examined with their help. Modern clinical psychological tests can be roughly divided into two groups: objective and phenomenological tests. At the same time both can be characterized by concrete goals they meant to achieve. Therefore, the system analysis of clinical psychological tests and examination of integration possibilities appears necessary.

Methods.- Main question of the research was whether it was possible to use objective tests phenomenologically, i.e. whether we could use one method both for objective assessment and examination of the patient's inner world. Authors analyzed tests used for pathopsychological assessment in the tradition of the Moscow Pathopsychological School (B. Zeigarnik, S. Rubenstein) and concluded that each of them had phenomenological potential, i.e. its results could be used for reconstruction of a patient's inner world. Results.- The potential of such an integrative approach was exemplified by the Ebbinghaus test. Its results were employed for phenomenological reconstruction of the self-awareness and of the mental disorders' inner dynamics of patients suffering from schizophrenia, schizoaffective disorder and borderline personality disorder.

Conclusions.- After being modified, the Ebbinghaus test could be used to achieve both goals, which allowed to broaden the possibilities of pathopsychological assessment without lengthening the procedure. The data obtained open new horizons in clinical psychological assessment and allow to understand a patient's condition and psychodynamics holistically.

Disclosure of interest.- The authors have not supplied a conflict of interest statement.

\section{E-PV0799}

\section{Agreement of the CGI-SCH scale with the panss positive and negative Marder's score}

J.M. Haro ${ }^{1}$, C. Domenech ${ }^{1}$, P.B. Jones ${ }^{2}$, S. Ochoa ${ }^{1}$

${ }_{1}$ Parc Sanitari Sant Joan de Déu- CIBERSAM- Universitat de

Narcelona, Research- Teaching and Innovation Department, Sant Boi de Llobregat- Barcelona, Spain; ${ }^{2}$ University of Cambridge, Department of Psychiatry, Cambridge, United Kingdom

* Corresponding author.

Background and aims.- The CGI-SCH scale was validated by comparing the evaluation of the scale with the PANSS Lindenmayer five component model. However, PANSS negative and positive Marder's scores are increasingly being used in clinical trials. The objective of this study is to compare the ratings of the Clinical Global Impression-Schizophrenia (CGI-SCH) scale with the Marder's PANSS positive and negative scores.

Methods.- A battery of instruments were administered to the outand in-patients with schizophrenia, which included a sociodemographic and clinical questionnaire, the CGI-SCH scales, the Positive and Negative Symptom Scale $(3,20)$ and the Global Assessment of Functioning Scale (GAF).

Results.- Symptoms were assessed in 114 patients. Mean (sd) ratings for the CGI-SCH positive and CGI-SCH negative scales were 3.94 (1.7) and 3.42 (3.4), respectively. Mean (SD) ratings for the PANSS positive and negative symptoms scale are 22.5 (8.7) and 17.8 (8.2), respectively. The correlation coefficients between the CGI-SCH and the PANSS positive and negative scores were 0.82 and 0.82 , respectively. There was a clear linear relationship between the CGI-SCH positive and negative scores and the mean PANSS positive and negative mean scores (see Fig. 1).

Conclusions.- The CGI-SCH positive and negative items have high concordance with the PANSS positive and negative scales, respectively. Given its simplicity, brevity and validity, the scale is appropriate for use in observational studies, clinical trials and routine clinical practice.

Disclosure of interest.- The authors have not supplied a conflict of interest statement.

\section{E-PV0801 \\ Self-determination theory in rehabilitation of patients with somatic and mental illnesses: validation of illness and treatment self-regulation questionnaire in the Russian neurological sample}

M. Kovyazina ${ }^{1}$, E. Rasskazova ${ }^{2}$, E. Prigorneva ${ }^{2}$, N. Varako ${ }^{2}$

${ }^{1}$ Moscow State University, Psychology, Moscow, Russia; ${ }^{2}$ Moscow

State University, Clinical psychology, Moscow, Russia

Corresponding author.

Background and aims.- According to self-determination theory (Deci, Ryan, 2000), unsatisfaction of needs in autonomy, competence and relatedness both in somatic and mental patients (Sheldon et al., 2003) is a negative prognostic factor for mental health and health-related quality of life. One of the key psychiatric problems in the rehabilitation of neurological patients is to reveal psychological factors unrelated to cognitive deficits and related to compliance and life satisfaction.

The aim was to develop and validate a questionnaire appraising satisfaction of basic needs in the rehabilitation process in different illnesses.

Methods.- 50 after-stroke patients ( 35 men, $18-79$ years old) with paresis not having prominent cognitive dysfunctions filled MOCA, Illness and Treatment Self-Regulation Questionnaire, Satisfaction With Life Scale (Diener et al., 1985), Morisky-Green Compliance Scale and appraised their satisfaction with treatment.

Results.- Factor analysis revealed 3-factor structure according to SDT model describing $75,06 \%$ of variance with factor loadings $0,41-0,94$. Cronbach's alpha was $0,65-0,76$. Independently of cognitive functioning (MOCA), competence satisfaction was related to subjective treatment effectiveness $(r=0,53, p<0,01)$ and life satisfaction $(r=0,40, p<0,05)$, relatedness satisfaction - to the importance of rehabilitation goals and autonomy satisfaction - to subjective compliance $(r=0,52, p<0,01)$.

Conclusions.- SDT-based measure of need satisfaction in somatic and mental illnesses is a reliable measure with theory-based factor structure in after-stroke patients. Results should be proved on samples on different mental illnesses to check the relationship of need-satisfaction with quality of life and compliance of mental patients. Research is supported by the Russian Foundation for Basic Research, project No. 17-29-02169.

Disclosure of interest.- The authors have not supplied a conflict of interest statement.

\section{E-PV0802}

\section{Improved clinical trial designs in} psychiatry: the future is now?

S. Petrykiv ${ }^{*}$, M. Arts ${ }^{2}$, L. de Jonge ${ }^{3}$

${ }^{1}$ Maastricht University Medical Center, Department of Clinical Pharmacy and Pharmacology, Maastricht, The Netherlands; ${ }^{2}$ Mental Health Care -Western North Brabant GGZ-WNB, Department of Old Age Psychiatry and Neuropsychiatry, Halsteren, The Netherlands; ${ }^{3}$ Leonardo Scientific Research Institute, Department of

Neuropsychiatry, Groningen, The Netherlands

* Corresponding author.

Background and aims.- Treatment with SSRI's shows a high poor-response rate. Hence there is an unmet need to unravel the mechanisms of therapy resistance and to identify potential respon- 
ders. A large amount of negative randomized controlled studies with SSRI's - which operated with averages rather than assessing interindividual variability - can be in our opinion explained by failing trial designs to a bigger extend than by failing drugs. Novel trial designs are needed to bring clinical studies to a new level.

Methods.- Discussion on methodology aimed to improve phase IV clinical trials in psychiatry. We put the focus on cross-over studies with conformation period and their subforms: umbrella and platform study designs. Additionally, we will discuss the pro's and contra's of the enrichment period as a valuable tool to identify potential responders to SSRI's prior to the treatment period.

Results.- Cross-over trials are the novel tool to explain interindividual variability; few cross-over trial with psychofarmaca have been recently published. As all of them have certain methodological limitations, the authors of current work suggest improvements within current concept of a clinical trial conduct in psychiatry, by applying the improved methodology.

Conclusions.- Personalized approach became a routine procedure in many fields of medicine but it is still new in psychiatry. Understanding of treatment outcomes to psychofarmaca between and within individuals is impossible without improved trial designs. Application of cross-over studies, next to biomarker studies, offers the unique possibility to unravel the mechanisms of therapy resistance and helps to identify subjects with potentially favorable response. Disclosure of interest.- The authors have not supplied a conflict of interest statement.

\section{E-PV0803}

\section{Assessing relationship-centred care skills and attitudes}

A. Virgolino ${ }^{1}{ }^{*}$, O. Santos ${ }^{2}$, A. Miranda ${ }^{1}$, A. Vaz-Carneiro ${ }^{3}$

${ }^{1}$ Instituto de Saúde Ambiental- Faculdade de Medicina-

Universidade de Lisboa, Instituto de Saúde Ambiental, Lisbon,

Portugal; ${ }^{2}$ Instituto de Saúde Ambiental, Faculdade de Medicina,

Universidade de Lisboa, Instituto de Saúde Ambiental, Lisboa,

Portugal; ${ }^{3}$ Faculdade de Medicina, Universidade de Lisboa, Instituto de Saúde Ambiental, Lisbon, Portugal

${ }^{*}$ Corresponding author.

Background and aims.- Building a relationship-centred care (RCC) context is not easy and in their daily practice medical doctors face constraints that drive them to act according to implicit (non-conscious) directive attitudes, aligned with the traditional biomedical approach. Assessing interpersonal skills and attitudes towards patients' negotiation is a complex though fundamental task, aiming to enhance collaborative patient-doctor relationships. The main aim of this study is to present a study protocol for the development and assessment of psychometric properties of an Implicit Association Test (IAT), suitable for the evaluation of medical doctors' RCC implicit attitudes.

Methods.- This study consists on a psychometric assessment of the IAT, including literature search and experts' consultancy for the choice of associative stimuli, evaluation of internal consistency, test-retest reliability, construct validity and sensitivity to changes in implicit attitudes, and on the acceptance and burden of the questionnaire. The study will follow a cross-sectional design (except for test-retest reliability, which requires a longitudinal design). The sample will be composed of physicians with active clinical practice with chronic patients, randomly selected from the association of general and family medicine doctors.

Results.- Main expected results are to develop an IAT test with sound psychometric qualities to characterize doctors' implicit RCC attitudes.

Conclusions.- The assessment tool here proposed will enhance medical education programs, in order to promote attitudes among medical doctors more aligned with RCC and fitting with the participatory healthcare paradigm. The assessment tool will enhance medical education programs, to promote attitudes among medical doctors more aligned with RCC and fitting with the participatory healthcare paradigm.

Disclosure of interest.- The authors have not supplied a conflict of interest statement.

\section{E-Poster Viewing - 7-9 April: Schizophrenia and other psychotic disorders}

\section{E-PV0804 \\ Service innovation - schizophrenia outreach in larkana (SOUL): programme development, implementation and outcomes after 6 years}

S. Afghan $1^{*}$, B. Junejo ${ }^{2}$, G.M. Soomro ${ }^{3}$, F. Soomro ${ }^{4}$, R. Faruqui ${ }^{5}$

${ }^{1}$ Dudley E Walsall Mental Health Partnership Trust \& University of Wolverhampton- UK, Mental Health, Walsall, United Kingdom; ${ }^{2}$ Shaheed Mohtarma Benazir Bhutto Medical University- LarkanaPakistan, Psychiatry, Larkano, Pakistan; ${ }^{3}$ St. James Hospital- Solent NHS Trust- Portsmouth, Mental Health, Portsmouth, United Kingdom; ${ }^{4}$ Shaheed Mohtarma Benazir Bhutto Medical University-, SOUL Programme, Larkano, Pakistan; ${ }^{5}$ University of Kent, Primary Care Academic Unit-Centre for Health Services Studies, Canterbury, United Kingdom

* Corresponding author.

Background and aims.- SOUL programme combines psychiatric outreach with culturally relevant work with patients and families through home based treatment. The key objectives include early recognition, treatment, family education and psychosocial support in order to maximize clinical and functional outcomes.

Methods.- Prospective Cohort with innovative service structure and culturally relevant open label intervention design and developed with local academic psychiatric unit in Larkano, Pakistan. The service design works on structured training of local psychiatrists and outreach nurses and identify intervention roles for professionals, process of assessment, diagnostic formulation, delivering care, and use of recognized clinical outcome measures for progress monitoring.

Results.- We report clinical outcomes of a cohort of 125 patients (males $N=85$, females $N=40$ ) recruited on a continual basis over a six year period. Our analysis demonstrates higher PBRS and lower GAF ratings for men in comparison to female cohort at the baseline (mean BPRS scores of 66 versus 57.3, mean GAF 41 versus 48.6).

Our six-year follow up has demonstrated statistically significant clinical improvement on BPRS, CGI and GAF. The mean differences recorded from the baseline for individual measures after 12 months of follow-up were BPRS (24.0), CGIS (0.84), CGII (0.43) and GAF (18.86). All measures have demonstrated a statistically significant improvement of $P \leq 0.05$

Conclusions.- SOUL Programme is a highly effective and costefficient intervention model for treatment of schizophrenia in the developing countries. Our 6-year follow up study confirms the feasibility and cultural value of this intervention model through close working with families of our patients.

Disclosure of interest.- The authors have not supplied a conflict of interest statement.

\section{E-PV0806}

\section{Vehicle driving and psychosis}

A. Alonso Sanchez ${ }^{1 *}$, M. Alonso Sanchez ${ }^{2}$, A. Alvarez Astorga ${ }^{1}, \mathrm{H}$. De la Red Gallego ${ }^{1}$, M. Gomez Garcia ${ }^{1}$, N. De Uribe Viloria ${ }^{1}$, M. De Lorenzo Calzon $^{1}$, C. Capella Meseguer ${ }^{1}$, E.M. Rodriguez Vazquez ${ }^{1}$, J.I. Gonçalves Cerejeira ${ }^{1}$, I.D.L.M. Santos Carrasco ${ }^{1}$, L. Rodriguez Andres ${ }^{1}$ 
${ }^{1}$ Hospital Clínico Universitario de Valladolid, Psychiatry, Valladolid, Spain; ${ }^{2}$ Hospital la Princesa, Psychiatry, Madrid, Spain

Corresponding author.

Background and aims.- Despite the growing number of public transport services in developed countries, many people still do not have enough alternatives to private transportation, especially those living in rural areas.

It is widely known that people with mental health conditions incur in more accidents than the average population.

Methods.- Case report and bibliographic review.

Results.- We report the case of a 44 years old male patient. He has been diagnosed with schizophrenia since he was 24 years old. Since mental health services first approached him, his evolution has been marked by a lack of awareness of the disease and a lack of antipsychotic adherence. As a result, the patient has been living in an isolated manner during two decades, breaking all the bonds with his close family.

He has been living on the money he raises selling own produced wine, which he sells delivering by car the bottles directly to some restaurants in the region where he lives.

During one of his relapses, we have treated him inwards, where he recognised to have had 24 traffic infractions. Although he had his driving license removed, he has kept driving during all this time. Conclusions.- In 2018 there are still many people driving with unstable mental conditions not being adequately treated. This may be potentially harmful to the patients or other people in the road. New policies should be approved by national governments to promote adequate mental health controls in vehicle drivers.

Disclosure of interest.- The authors have not supplied a conflict of interest statement.

\section{E-PV0807}

\section{Relation between smoking and formal thought disorder in schizophrenia}

K. Alptekin

Prof Dr, Psychiatry, Izmir, Turkey

"Corresponding author.

Background and aims.- Formal thought disorder (FTD) is one of the fundamental features of schizophrenia. FTD usually proceeds in a vague form throughout the illness and exacerbates in acute episodes, and might persist in remitted patients. It is one of the strongest predictors determining conversion from first-episode acute transient psychotic disorder to schizophrenia. Prevalance of smoking cigarettes is higher in patients with schizophrenia compared to normalpopulation Possible reasons are due to decrease the severity of side effects or to increase cognitive abilities. The aim of this study is to investigate the relation between smoking cigarettes and formal thought disorder in schizophrenia.

Methods.- This research was a retrospective study. Data regarding the patients with schizophrenia were obtained from two separate studies conducted before. Schizophrenia patients who had been assessed with Thought and Language Index (TLI), Positive and Negative Syndrome Scale (PANSS), and Sociodemographic Data Form in the two former studies were included in the present study. 150 patients with schizophrenia were included into the study.

Results.- Schizophrenia patients using more cigarettes have less TLI scores compared to patients using less.

Conclusions.-

Discussion.- Smoking cigarettes may be related to formal thought disorder. However this may be result of the efforts to increase thought abilities. Underlying mechanisms need to be investigated. Disclosure of interest.- The authors have not supplied a conflict of interest statement.
E-PV0808

\section{Cost-effectiveness in patients with severe mental illness treated with an long-acting injectable (LAI) antipsychotics}

A. Alvarez ${ }^{1 *}$, A. Zambrana ${ }^{2}$, C. Martín ${ }^{2}$, P. Andrés ${ }^{2}$, B. Bote ${ }^{2}$, C. Lorenzo $^{2}$, L. Sánchez ${ }^{2}$, J. Matias ${ }^{2}$

${ }^{1}$ Corporació Sanitària Parc Taulí, Psychiatry Departament, Sabadell, Spain; ${ }^{2}$ University Hospital of Salamanca, Psychiatry Departament, Salamanca, Spain

Corresponding author.

Background and aims.- In our usual clinical practice, we found that the majority of patients with severe mental disorder are frequently treated with various drugs concomitantly, mainly in the acute hospitalization units in relation to the existing psychopathological severity.

The aim of the present study is to evaluate whether treatment with an long-acting injectable (LAI) antipsychotic at hospital discharge favors monotherapy

Methods.- Descriptive analysis of a prospective naturalistic study of two years (2015 and 2016), in the acute care unit of patients with severe mental illness, who after hospital admission, have been treated with paliperidone palmitate monthly and aripiprazole depot, assessing the existing pharmacological therapy, as well as the re-entry rates until August 2017.

Results.- We obtained a sample of 113 patients: 68 patients $(60.17 \%)$ were treated with long-lasting paliperidone palmitate and 45 (39.82\%) with long-lasting aripiprazole.

Of the 68 patients with paliperidone palmitate, 10 (14.70\%) patients were on monotherapy. The remaining 58 patients received concomitant treatment, of which 28 (48.27\%) received another antipsychotic, mainly risperidone. Of the 45 patients treated with depot aripiprazole, $4(8.88 \%)$, were on monotherapy while the other $41(91.11 \%)$ required concomitant oral treatment, 28 of these patients with concomitant treatment received another oral antipsychotic, mainly risperidone

Conclusions.- Paliperidone palmitate has been shown to have a readmission rate lower than that of depot aripiprazole, in addition to having a lower need for concomitant antipsychotic therapy.

Long-acting injectable (LAI) antipsychotic are an optimal and costeffective treatment option, since they have been shown to decrease the rate of hospital readmission and costs of these pathologies.

Disclosure of interest.- The authors have not supplied a conflict of interest statement.

\section{E-PV0810}

\section{SCH therapy - the importance of ODT} and depot form

S. Anakiev ${ }^{*}$, G. Tasic ${ }^{2}$

${ }^{1}$ SBPB “Gornja Toponica", Prijemno musko, Nis, Serbia; ${ }^{2}$ SBPB

"Gornja Toponica", Psr, Nis, Serbia

"Corresponding author.

Background and aims.- The importance of regularity in therapy and achieving and maintaining the remissin results from complexity of illnes itself and the destructive consequences it has on an individual, family or society. one of the characteristics of psychosis is the loss of reality check and connected to that a weak compliance. there is a lack of insight into the condition so the taking of the medicine becomes questionable.

Methods.- This is the analysis of the use of the new generation antipsychotics in our hospital.

The new generatin neuroleptics, "atypical" neuroleptics,are available in forms that help with the application, stigmatization is missing and the remission is kept. One of the first "benefits" is ODT form (olanzapin, aripiprazol) which automatically desinte- 
grates when put in the mouth,so it is impossible to eject the whole pill. it can be taken dissolved in water. It has a pleasant taste so the patient does not have the feeling of actually taking the medication. Even more is acomplished by long acting forms of antipsychotics (paliperidon) which, given by injection,enable comfortable application of the medicine, which keeps the level of the curative substance in the blood for a month or three months.

Results.- It achieves control of the symptoms, that is of the illnes, and easier fuctioning of the ill in the conditions of the social enviroment.

Conclusions.- All mentioned above suggests that the optimal choice for treating such complicated and chronic disease like schizophrenia is a form of medication which provides permanent, easy and certain use. That means the use of odt form and, even better, the long acting injectables.

Disclosure of interest.- The authors have not supplied a conflict of interest statement.

\section{E-PV0811}

\section{The effects of olanzapine on gastrointestinal microflora in patients diagnosed with paranoid schizophrenia}

A. Bąba-Kubiś ${ }^{1 *}$, M. Wroński ${ }^{1}$, P. Liśkiewicz ${ }^{1}$, S. Jerzy ${ }^{1}$, I. Łoniewski ${ }^{2}$, J. Pełka- Wysiecka ${ }^{1}$

${ }^{1}$ Department of Psychiatry, Pomeranian Medical University in Szczecin, Department of Psychiatry, Pomeranian Medical University in Szczecin, Szczecin, Poland; ${ }^{2}$ Department of Biochemistry and Human Nutrition, Pomeranian Medical University in Szczecin, Department of Biochemistry and Human Nutrition, Pomeranian Medical University in Szczecin, Szczecin, Poland

${ }^{*}$ Corresponding author.

Background and aims.- Emerging reports on the existence of a relationship between schizophrenia and gastrointestinal microflora disorders suggest the need for more extensive research. Its results would allow to broaden knowledge on the pathogenesis of this disease and to create modern standards of diagnostic and therapeutic procedures.

The aim of the project was to determine whether treatment with olanzapine influences the composition of bacterial flora of the gastrointestinal tract in persons suffering from schizophrenia.

Methods.- The study included 20 somatically healthy patients diagnosed with paranoid schizophrenia according to the ICD-10.

Psychometric tests were performed before, and after 2, 4 and 6 weeks of treatment with olanzapine, the composition of bacterial intestinal microflora was assessed before and after 6 weeks of pharmacotherapy.

Results.- The study revealed changes in the bacterial composition of the intestinal microflora in patients diagnosed with paranoid schizophrenia after treatment with olanzapine.

Conclusions.- The results have been promising so far and they need further research.

Disclosure of interest.- The authors have not supplied a conflict of interest statement.

\section{E-PV0812}

\section{How to manage resistant schizophrenia: 33 clinical cases.}

M. Bitar ${ }^{*}$, F.E. El jebbouri El jebbouri' ${ }^{2}$, A. Ouanass Ouanass ${ }^{3}$

${ }^{1}$ Dr, Hopital Arrazi, Rabat, Morocco; ${ }^{2}$ Hopital arrazi, emergency, Rabat, Morocco; ${ }^{3}$ Arrazi, Emergency, Rabat, Morocco

${ }^{*}$ Corresponding author.

Background and aims.- Schizophrenia is a chronic and debilitating psychiatric pathology that constitutes a real public health problem.
Antipsychotics have changed the management of schizophrenia, but the cases of resistance are more and more important.

This is a study on the care arrangements for patients who suffered of resistant schizophrenia at Arrazi hospital in Sale.

Methods.- Retrospective descriptive study carried out on the files of patients with resistant schizophrenia hospitalized at Arrazi hospital during the period spread over 3 years, from 2014 to 2017 .

- Inclusion criteria:

- Cases of schizophrenia and schizoaffective-resistant disorder are defined according to DSM 5 criteria and Kane's resistance criteria.

- Exclusion criteria:

- Insufficient clinical informations

- Patients with a neurological disease or intellectual disability.

- Statistical tool: SPSS 20.

Results.- The average age is 33 years old. An age range between 19 and 53 years old. All our patients are male. In our sample of 33 patients, 64.3\% have problematic drug use (tobacco, cannabis ...). $92.9 \%$ of our patients are schizophrenic and $7.1 \%$ have a schizoaffective disorder. The age of onset of the disease is around 21 years old. Half of our patients had haloperidol as initial therapy. The diagnosis time of the disease resistance is on average 2 years. $78.6 \%$ of our patients were treated by only clozapine, $13.3 \%$ were treated by antipsychotic + clozapine $7.1 \%$ were treated by clozapine + mood stabilizer. In $41 \%$ of cases there was an improvement under these treatments and no response in $59 \%$ of patients.

Conclusions.- Early treatment with good adherence is the cornerstone of progression to resistance on schizophrenia.

Disclosure of interest.- The authors have not supplied a conflict of interest statement.

\section{E-PV0813}

\section{First episode psychosis in cannabis users: primary psychotic disorder or cannabis induced psychotic disorder? Literature review and a case-control study proposal}

S. Boi ${ }^{*}$, T. Gonzalez Salvador, B. Sanz-Aranguez Avila, P. Maguilla Franco, R. De Arce Cordon, E.M. Suarez Del Rio

Hospital Puerta de Hierro, Psychiatry, Majadahonda, Spain

* Corresponding author.

Background and aims.- In cannabis users patients a first-episode psychosis (FEP) could be the first symptom of a primary psychotic disorder (PPD) or a substance use induced psychotic disorder (SIPD). Differential diagnosis is a challenge since the regular abusers frequently do not have consumption free periods. Therefore the psychotic symptoms seen during the higher use periods could be a SIPD or clinical manifestations of a PPD triggered by the consumption. This makes the diagnostic stability in FEP in cannabis-user low.

The principal aim of our study is to evaluate the diagnostic stability 6 and 12 months after FEP in cannabis-users and compare it with a control group of non cannabis-users suffering FEP. An analysis of different clinical and sociodemographic variables, genetic and immunological markers in both groups will also be made. This could allow detect predictor factors to recognise patients with SIPD diagnosis and high risk to evolve to PPD.

Methods.- Longitudinal and prospective case-control study during a year. FEP patients with cannabis active use are identified as cases and FEP patients with no drug abuse as controls.

Results.- The factors that are more consistently associated with diagnosis change are extended psychotic symptomatology before the hospital admission, an inadequate premorbid functioning during adolescence, a long history of drug abuse and a low functioning level. 
Conclusions.- Clinical variables detection of PPD could allow fulfilling a specific therapeutic approach not just focused in drug withdrawal. It is especially important since the prognosis of those patients depends greatly in the therapeutic scheme made during the first year.

Disclosure of interest.- The authors have not supplied a conflict of interest statement.

\section{E-PV0815}

\section{Neurocognitive functions in descendants at familial high risk for schizophrenia: what's new?}

G. Boukhari*, L. Mnif, M. Chakroun, S. Derouiche, W. Abdelghaffar, W. Melki

Razi Hospital, Sfar Department, Tunis, Tunisia

* Corresponding author.

Background and aims.- Schizophrenia is associated with neurocognitive deficits (intelligence, processing speed, memory, attention, and executive function) that precede the onset of illness. Descendants of parents with schizophrenia are themselves at increased risk of severe mental illness, and therefore are an important population to study the pathogenesis of this disorder.

Methods.- Electronic search was carried out in September 2018. PubMed database has been used to find studies to introduce in this review. Keywords used in the search process were represented by schizophrenia AND (young relatives OR offspring OR children OR descendants). Ultimately, eight articles were included following abstract review.

Results.- The studies suggest that descendants of parents with schizophrenia do show distinct developmental patterns characterized by higher rates of obstetric complication, neurodevelopmental features such as motor and cognitive deficits, and distinctive social behavior.

IQ in infancy, verbal ability in early childhood, and visual-spatial skills in late childhood had the largest between-group differences. Familial High Risk (FHR) descendants produced less speech and had a number of distinct discursive features, such as less cohesion between ideas and unclear or ambiguous references to previous ideas.

Seven-year-old FHR children had a 2.7 points lower IQ.

Conclusions.- Neurocognitive impairments are widespread in descendants of parents with schizophrenia, supporting the notion of neurocognition as an endophenotype for schizophrenia and a target for intervention.

Disclosure of interest.- The authors have not supplied a conflict of interest statement.

\section{E-PV0816}

\section{Lipid profile in schizophrenia: a case-control study in Tunisia}

R. Boukhchina ${ }^{1}$, L. Ghanmi ${ }^{1}$, N. Rezig ${ }^{2}$, M. Romdhan ${ }^{2}$, M. Abbes ${ }^{1}$ K. Zitoun ${ }^{1}$, H. Denguir ${ }^{3}$

${ }^{1}$ Regional Hospital of Gabès, Psychiatry department, Gabès, Tunisia;

2 Regional Hospital of Gabès, Laboratory of Medical Analysis, Gabès,

Tunisia; ${ }^{3}$ Regional Hospital of Gabès, Department of Cadiology,

Gabès, Tunisia

* Corresponding author.

Background and aims.- Cardiovascular diseases are the main factors of high mortality in schizophrenia. Lipid disturbances are a major risk factor for these cardiovascular diseases. In this context,a study of lipid profile in people with schizophrenia seems interesting.

The aims of this study were to estimate the prevalence of dyslipidemia among patients with schizophrenia and to compare it with matched healthy controls.
Methods.- A descriptive and analytical case-control study conducted between April 2016 and Mars 2017 on 88 patients with schizophrenia (DSM-IV) and 63 healthy subjects in which blood samples were taken to study serum lipid parameters: cholesterol, triglycerides,HDL-C and LDL-C. We referred to the NCEP-ATP III guidelines for cholesterol testing and management.

Results.- The patient was a male (68\%) and had a mean age of 46,8 years. The mean duration of illness was 16,8 $\pm 9,6$ years. Low HDL-c levels, high TG levels, high Total Cholesterol levels, and high LDL-c levels were found within $56,8 \%, 31,8 \%, 35,2 \%$ and $48,9 \%$ of patients with schizophrenia, respectively. The comparative study has shown no significant difference in the prevalence of dyslipidemia between the two groups. The mean value of triglyceridemia was significantly higher among patients with schizophrenia $(p=0,035)$. The analytical study has shown that low concentrations of HDL-c are significantly associated with couple life (54,3 vs. $45,7 \%$; $p=0,014$; $R R=3$, IC95\% [1,23-7,35]). No significant association between doses,duration and type of antipsychotic treatment and lipid disturbance was found.

Conclusions.- Patients with schizophrenia are at greater risk of dyslipidemia. Clinical and biological monitoring,with regular monitoring of lipid and lifestyle recommendations could help reduce morbidity and mortality among patients with schizophrenia Disclosure of interest.- The authors have not supplied a conflict of interest statement.

\section{E-PV0818 \\ Maintenance electroconvulsive therapy and recurrent catatonia: M-ECT program hospital 12 de Octubre (Madrid-Spain)}

M. Caballero-Gonzalez ${ }^{*}$, I. Torio, D. Rentero, E.M. Sanchez-Morla,

R. Rodriguez-Jimenez

Psychiatrist, Department of Psychiatry, Madrid, Spain

Corresponding author.

Background and aims.- Catatonia is a syndrome which can have multiple etiologies both medical and psychiatric.

Electroconvulsive Therapy results effective in $80-100 \%$ of all form of catatonia, even after pharmacotherapy with benzodiazepines has failed.

Maintenance Electroconvulsive Therapy (M-ECT) is used to prevent relapse or recurrence in patients with severe mental illness.

The aim of the present study was to analyze two recurrent catatonia cases included in the Maintenance Electroconvulsive Program of 12 Octubre Hospital in Madrid since its development in 2011.

Methods.- We present two clinical cases of recurrent catatonia and their evolution after being included in the M-ECT Program.

We compared their attendance to the emergency department, and number and leght of hospital admission pre and post inclusion in the M-ECT Program.

Results.- The two patients present a decrease in the total number of attendance to emergency department, hospitalizations and leght of admission has happened in comparison before their inclusion in the program.

Conclusions.- The M-ECT Program of 12 Octubre Hospital has demostrated clinical utility for recurrent catatonia

Disclosure of interest.- The authors have not supplied a conflict of interest statement. 


\section{E-PV0819}

\section{Neurosyphilis presenting with}

psychotic and dementia symptoms:

when the patient refuses intravenous

antibiotherapy

A. Canhao*, C. Antunes

Centre Hospitalier Universitaire Vaudois, Psychiatry, Prangins,

Switzerland

${ }^{*}$ Corresponding author.

Background and aims.- Tertiary syphilis is not a frequent clinical condition in western countries. Nevertheless, this infection can originate complex clinical conditions difficult to diagnose and requiring a very specific treatment, that often is difficult to administrate

The aim of this poster presentation is to share the difficulties experienced in finding the diagnosis and mostly the numerous problems that we faced in the treatment approach because intravenous therapy is frequently painful and acutely decompensated patients were not able to accept this kind of side effects leading to a treatment refusal

Methods.- In this presentation, we describe two different situations that were committed to our psychiatric unit and all the procedures and difficulties that were necessary to achieve for a proper treatment for the two patients

Results.- At the end of the hospital stay, both patients were partially compensated and institutional placement was necessary. The clinical constellation had suffered a good evolution but some of the symptoms remain. Other pathologies were unveiled during hospitalization

Conclusions.- Neurosyphilis remains a diagnostic to keep in mind. Even if it is less frequent than in the past, it exists and needs a particular approach, at the same time that it poses considerable challenges

Disclosure of interest.- The authors have not supplied a conflict of interest statement.

\section{E-PV0821}

\section{Delusional parasitosis as an antipsychotic resistance pattern: a case report}

F. Cherif", N. Charfi, M. Maâlej Bouali, S. Omri, R. Feki, L. Zouari, J. Ben Thabet, M. Maâlej

Hédi Chaker University Hospital, Psychiatry C, Sfax, Tunisia

* Corresponding author.

Background and aims.- Delusional parasitosis (DP) also known as Ekbom syndrome is an uncommon psychiatric condition, in which the main symptom is the delusion of being infested with living organisms. Managing a patient with this DP can be a challenge to the psychiatrists.

Aims.- To describe uncommon presentation of psychotic patient with DP and to discuss therapeutic difficulties in dealing with such disease.

Methods.- Case report.

Results.- We report the case of Mr A.F, 41 years old man, diagnosed 20 years ago as having Schizo-Affective Disorder. Over the years, many antipsychotics were prescribed, associated to mood stabilizer and antidepressant. He had needed hospitalization in 2017 and 2018 because of suicidal ideation. He was very anxious. He feels the insects moving under his skin and trying to consume him. Those delusional ideas were disruptive. The physical exam was unremarkable. He presented a severe depressive symptomatology. He claimed to be incinerated after his death. He denied any psychiatric pathology and requested to be directed to the infectious diseases ward.
Risperidone and Haloperidol caused neurological side effects for the patient. Taking into account the depressive symptoms, the patient was treated by Quetiapine but there was no clinical improvement. The combination of Olanzapine, Aripirazole and Antidepressant was more effective in reducing depressive symptomatology and adherence to delusional ideas leading to a little better daily functioning.

Conclusions.- DP can manifests in patient with chronic psychotic disorder. It is considered as a reason of therapeutic resistance. Careful clinical assessment is needed to assess eventual coexistant depressive symptomatology and prescribe the adequate treatment. Disclosure of interest.- The authors have not supplied a conflict of interest statement.

\section{E-PV0822}

\section{Summer heat can imitate an infectious agent in schizophrenic patients - case report}

J. Coric ${ }^{*}$, K. Marusic ${ }^{2}$, A. Prosev ${ }^{1}$, M. Misevic ${ }^{1}$, V. Lesandric ${ }^{3}$, Z. Misevic $^{1}$, N. Bagic ${ }^{1}$, I. Prosev ${ }^{1}$, S. Majer ${ }^{1}$, A. Turkovic ${ }^{1}$, D. Barisic ${ }^{1}$, M. Smilovic ${ }^{1}$

${ }^{1}$ County Hospital Cakovec, Department of Psychiatry, Cakovec, Croatia; ${ }^{2}$ University Psychiatric Hospital Vrapce, Department of Addiction, Zagreb, Croatia; ${ }^{3}$ General County Hospital Vinkovci, Department of Psychiatry, Vinkovci, Croatia

"Corresponding author.

Background and aims.- The published literature indicate that people with schizophrenia have an impaired ability to compensate to heat stress among with other disturbences in thermoregulation. Our aim is to show that these disturbences can be a life-threatening factor for schizophrenic patients as well as a reason for many unnecessary somatic examinations.

Methods.- The 31-years-old male patient has been treated due to paranoid schizophrenia and was hospitalized in acute psychotic state. During the treatment he developed high febrility. Due to possible somatic complications, the treatment involved internists, neurologists and infectologists who all reported the onset of neuroleptic malignant syndrome (NMS) as a possibility of febrile condition, although our psychiatric consultation found that slightly possible. However, for a caution, the patient was still at minimal doses of clozapine. He was in apparent suffering and was mostly fixed to his bed. Extensive somatic examinations were performed with no indicators of any somatic illness. At that time, the outside air temperature reached to $38^{\circ} \mathrm{C}$ and in the patient's room up to $32^{\circ} \mathrm{C}$ because of the unfavourable conditions in our psychiatric department. On the sixth day of a patient's febrility, the outside temperature dropped to $25^{\circ} \mathrm{C}$ and within two hours the patient became afebrile.

Results.- Patient was no longer developing febrility.

Conclusions.- It can be assumed that high external air temperatures can be a life-threatening factor. Although it is important to exclude the somatic causes of high febrility, the most important is to provide our schizophrenic patients with adequate spatial and climatic conditions, regardless of "healthy body".

Disclosure of interest.- The authors have not supplied a conflict of interest statement.

\section{E-PV0823}

\section{Lyme disease: an atypical first presentation of schizophrenia or a clinical finding?}

A.L. Costa $^{1 *}$, A.I. Oliveira ${ }^{2}$, C.A. Pereira ${ }^{1}$, B. Melo ${ }^{1}$, D. Teixeira ${ }^{1}$, A. Marques $^{1}$, J. Humberto Silva ${ }^{3}$

${ }^{1}$ Resident of Psychiatry, Departament of Psychiatry of Tondela-Viseu Hospital Center, Viseu, Portugal; ${ }^{2}$ Graduate Assistent of Psychiatry, 
Departament of Psychiatry of Tondela-Viseu Hospital Center, Viseu, Portugal; ${ }^{3}$ Senior Assistent of Psychiatry and Director, Departament of Psychiatry of Tondela-Viseu Hospital Center, Viseu, Portugal * Corresponding author.

Background and aims.- Lyme disease (LD) is a multisystemic illness caused by the spirochete, Borrelia burgdorferi. In addition to dermatological, cardiac, articular, and neurologic manifestations, psychiatric disorders such as depression, panic atacks and schizophrenia-like psychosis can also arise.

The aim is to report a case with LD and schizophrenic psychosis and to familiarize psychiatrists with LD and its atypical manifestations. Methods.- Case report and non-systematic review of the literature. Results.- We reported a case of a 42-year-old man who was admitted with strange behaviour: alienation, perplexity, very defensive and tense contact and a slight evasive speech. He had two previous admissions on an inpatiente unit three years prior and was diagnosed with psychosis not otherwise specified. There was no history of a tick bite but the patient had had a long stay in a rural environment; so anti-body specific to Borrelia was ordered and came positive. It remains unknown how long the infection was present prior to detection. He received antibiotics but without significant clinical improvement. Indeed, intermittent illogical thinking and negative symptoms, poor insight remained so he was discharged with diagnosis of Hebephrenic Schizophrenia.

Conclusions.- Physicians need to be aware of neuropsychiatric symptoms of LD, given that they may occur months to years after the initial infection. They should consider LD in the differential diagnosis of any atypical psychiatric presentation because early antibiotic treatment will prevent permanent sequelae. Although there is treatment for LD many patients do not get better with antibiotic therapy, which can result in permanent lesions as shown in this case.

Disclosure of interest.- The authors have not supplied a conflict of interest statement.

\section{E-PV0825}

\section{Childhood trauma and cognitive biases associated with psychosis: a systematic review and meta-analysis \\ J. Croft ${ }^{*}$, J. Heron, S. Zammit \\ University of Bristol, Centre for Academic Mental Health, Bristol, United Kingdom \\ * Corresponding author.}

Background and aims.- Childhood trauma is an established risk factor for psychosis, however the possible mechanisms that may mediate this association have not been established. Theoretical models suggest that the likelihood of cognitive and perceptual biases may be increased by childhood trauma and contribute to psychotic symptoms. There have been no studies, as far as we are aware, that have comprehensively examined the literature to establish whether childhood trauma is associated with psychosisrelated cognitive and perceptual biases.

Methods.- A systematic review was conducted of studies that examined the association between childhood trauma and the following biases: external locus of control, external attribution bias, jumping to conclusions bias and external source misattributions. We conducted a meta-analysis where feasible, or a narrative synthesis otherwise, and studies were assessed for quality and potential for bias.

Results.- 3,465 studies were identified and 24 met inclusion criteria. Of these, 14 studies were included in the meta-analysis. Individuals exposed to childhood trauma reported a more external locus of control $(\mathrm{SMD}=.55,95 \% \mathrm{CI}=0.33-.78, \mathrm{I} 2=93 \%)$. There was weaker evidence of association between childhood trauma and other biases examined in the review.
Conclusions.- The results provide support for integrated biopsycho-social models of psychosis. These posit that trauma may contribute to the aetiology of psychotic symptoms via cognitive and perceptual biases. If cognitive biases were found to have a mediating role in the pathway from trauma to psychosis, interventions would need to focus on modifying these biases.

Disclosure of interest.- The authors have not supplied a conflict of interest statement.

\section{E-PV0826}

\section{Mortality associated to the misuse and prescription of benzodiazepines in psychotic patients: a review of the literature}

J. De La Iglesia*, N.M. Casado Espada, R. de Alarcón Gomez, R. de la Mata Hidalgo, I. Valrivberas Herrero, L. Al Chaal Marcos, S.

Gamonal Limcaoco, M.T. Lozano Lopez, A. Maciá, A. Bullon, L. Sanchez Pernas, C. Gonzalez Soria, A. Álvarez Pedrero, P. Andrés Olivera, C. Roncero Alonso

Salamanca University Healthcare Complex, Psichiatry, Salamanca, Spain

" Corresponding author.

Background and aims.-

Background.- Schizophrenia is a prevalent, chronic and severe mental illness which can usually be of an importantly invalidating nature. The heterogenic presentation of schizophrenia and the variety of its symptoms usually lead to the incapacitation of the patients. This situation leads often to polypharmacology, in which benzodiazepines play a prominent role.

Aims.- To carry out a review of the existing evidence about mortality linked to the consumption of benzodiacepines, specifically in patients with schizophrenia.

Methods.- A search was carried out through the databases of the Cochrane Collaboration Library and the Pubmed search engine providing access to the MEDLINE directory with the terms "Benzodiazepines" and "Schizophrenia" as MeSH terms, AND mortality as an additional keyword.

Results.- As a result of our search strategy, 65 articles were found in the MEDLINE database, of which only 10 were closely related to the topic of our review and compose the main body of evidence of our work.

Conclusions.- A high exposure to benzodiazepinic agents tends to increase the risk of mortality in schizophrenic patients to up to $70 \%$ compared to no exposure, where other psychotropic agents tend to diminish it. Volumetric cerebral changes seem to be associated to benzodiazepine consumption as well. The studies tend to advice caution with their prescription, which is part of the polypharmacy which is usually employed with these patients. There is, though, still a need of more studies and evidence related to this topic.

Disclosure of interest.- The authors have not supplied a conflict of interest statement.

\section{E-PV0828}

\section{Psychotic symptoms as prodomic presentation of lewys body dementia (LBD): case report}

P. Del Sol ${ }^{1 *}$, A. Izquierdo ${ }^{1}$, E. Gamo ${ }^{2}$, C. Iglesias ${ }^{1}$

${ }^{1}$ Hospital Universitario Puerta de Hierro Majadahonda, Day

Psychiatric Hospital, Madrid, Spain; ${ }^{2}$ Hospital Universitario Puerta

de Hierro Majadahonda, Neurology, Madrid, Spain

* Corresponding author.

Background and aims.- The aim of this case is to present a case of multiple psychotic episodes as a posible insidious presentation of neurological diseases such as LBD. 
Methods.- We introduce a 52 years old women treated with psychiatric drugs for 10 years due to anxiety related to marital conflicts. In 2017 she suffered from two pscyhotic episodes consisting on hipertimic mood, insomnia and injury delusional referring severe fear and anguish of being killed. She was treated with asenapine at first and long acting aripiprazol secondly. In both remission of the symptons presented was solved. Aftewards she started follow up at the Day Psychiatric Hospital to identify diagnosis, where bradikinesia, tremor, rigidity and failling in swinging her arms were observed. These got worse despite the adittion of anticholinergic medication and withdrawal of antipsychotic medication. Quetiapine was initiated with partial response of the motor symptoms. Neurological exploration was performed with DAT scan, MRI and Iodine-123 metaiodobenzylguanidine (MIBG) SPECT realization. She was diagnosed with Lewy Body Dementia.

Results.- It is seen that neurological diseases can appear at first with psychiatric symptoms, in some cases many years before the motor signes.

Conclusions.- When neuropsychiatric symptoms are presented in adults without previous psychiatric history is important to be aware of organic diseases related with the clinical presentation. This case shows a women diagnosed with Body Lewys dementia in wich visual allucinations are not presented.

Disclosure of interest.- The authors have not supplied a conflict of interest statement.

\section{E-PV0830}

\section{Schizo-obsessive disorder: clinical case and revision}

\section{Fernandes Santos}

Hospital Garcia de Orta, Department of Psychiatry and Mental Health, Almada, Portugal

${ }^{*}$ Corresponding author.

Background and aims.- Although schizophrenia and obsessivecompulsive disorder (OCD) constitute distinct nosological entities, their relationship has been increasingly studied. OCD is a common comorbid condition in schizophrenia: $25 \%$ of schizophrenia patients suffer from obsessive-compulsive symptoms (OCS) and $12 \%$ fulfill the diagnostic criteria of an OCD.

They present a substantial overlap not only with regard to demographic and clinical characteristics, but also to structural and functional brain abnormalities and neurotransmitter systems. A diagnostic subgroup of schizophrenia - 'schizo-obsessive disorder' - has been proposed as a clinical entity with distinct psychopathological and functioning profile.

The aim of this study is to present a clinical case illustrative of the association between schizophrenia and OCD, and to review its nature and the relevance of the schizo-obsessive subtype.

Methods.- The author has conducted an online search in PubMed/MEDLINE database with the term 'schizo-obsessive disorder', from 2008 until 2018. To describe the clinical case, medical records were used.

Results.- A 25-year-old man, diagnosed with schizophrenia for about two years, initiates obsessions of aggressive content, with high emotional and functional impact, leading to modifications in his therapeutic plan.

These patients present clinical, neuroanatomical, genetic and therapeutic peculiarities, notably early onset age, preponderance of negative symptoms, positive and negative symptoms refractory to psychopharmacological therapy, specific neurocognitive deficits, more depressive symptoms and suicide attempts, as well as poorer outcome.

Conclusions.- Patients with schizophrenia and OCD may represent a discrete group with a specific clinical profile. Further research is needed to provide diagnostic criteria for schizo-obsessive disorder, with implications for psychiatric classifications, and to delineate specific therapeutic interventions.
Disclosure of interest.- The authors have not supplied a conflict of interest statement.

\section{E-PV0832}

\section{The interrelationship between emotional intelligence and quality of life in schizophrenia and bipolar-I-disorder}

B. Frajo-Apor ${ }^{1 *}$, S. Pardeller ${ }^{1}$, G. Kemmler ${ }^{1}$, M. Mühlbacher ${ }^{2}$, A.S. Welte $^{1}$, C. Hoertnagl ${ }^{1}$, A. Hofer ${ }^{1}$

${ }^{1}$ Medical University Innsbruck, Department of Psychiatry, Psychotherapy \& Psychosomatics, Innsbruck, Austria; ${ }^{2}$ Paracelsus Private Medical University, Department of Psychiatry and Psychotherapy, Salzburg, Austria

"Corresponding author.

Background and aims.- Social cognitive impairments exert a major influence on psychosocial functioning and well-being of patients suffering from serious mental illnesses. The primary objective of this study was to investigate the interrelationship between Emotional Intelligence (EI) and Quality of Life (QoL) in a sample of clinically stable outpatients suffering from schizophrenia and bipolar I disorder.

Methods.- 123 patients suffering from either schizophrenia $(n=63)$ or bipolar I disorder $(n=60)$ as well as 80 healthy controls were included. EI and QoL were assessed by using the Mayer-SaloveyCaruso Emotional Intelligence Test and the German version of the Lancashire Quality of Life Profile, the Berliner Lebensqualitätsprofil, respectively.

Results.- The two patient groups indicated comparable levels of overall EI and both subjective and objective QoL but significantly lower levels of EI and QoL compared to healthy controls. A significant interrelationship between EI and objective QoL was particularly seen in schizophrenia, however, overall effect sizes were small.

Conclusions.- Our findings indicate a trans-diagnostic difference in the inter-correlation between EI and QoL and suggest that patients suffering from schizophrenia and bipolar I disorder may have different needs to achieve recovery. It will be critical to develop training programs targeting EI and to examine their impact on objective QoL.

Disclosure of interest.- The authors have not supplied a conflict of interest statement.

\section{E-PV0833 \\ Mechanisms of the relationship between childhood trauma and risk of psychosis: a review of the role of personality traits and cognitive biases}

\section{Ł. Gawęda}

Medical University of Warsaw, II Department of Psychiatry, Warsaw, Poland

* Corresponding author.

Background and aims.- The relationship between childhood trauma and a risk for developing psychosis is well established. Indeed, a consistent body of research suggests that being exposed to early negative life events may increase the likelihood of the occurrence of psychotic-like experiences, subclinical psychotic symptoms, i.e., risk states for psychosis and full-blown psychosis. Nevertheless, little is known about psychological mechanisms of how life adversities shape the risk of psychosis. Some authors, including our group, have suggested that the linkage between early life adversity and the risk for psychosis is mediated by dysfunctional cognitive processes as well as personality traits. Better understanding of potential cognitive biases and personality traits that may be mechanisms 
via traumatic life events have an impact on the risk of psychosis requires a detailed review of specific dysfunctional information processing biases and personality structure that may play a role. Methods.- A systematic review of studies that have focused on cognitive biases and personality traits in the context of its linkage to trauma and risk of psychosis will be presented.

Results.- Based on the systematic review, an integrated and dynamic model of (reciprocal) interaction between exposure to traumatic life-events, personality traits and cognitive biases that shapes the risk of psychosis will be introduced. In particular, the role of external attribution and attention to threat will be presented.

Conclusions.- Importantly, the model explaining how trauma shapes the risk of psychosis may be beneficial in the context of developing more effective psychological interventions for those patients at risk for psychosis who were exposed to trauma.

Disclosure of interest.- The authors have not supplied a conflict of interest statement.

\section{E-PV0834}

\section{The aripiprazole-clozapine association in treatment ultra resistant schizophrenia: a case report}

M. Gharmoul", J. Nakhli, A. Mtiraoui, S. Ben Nasr

Farhat Hached University Hospital, Psychiatry Department, Sousse, Tunisia

Corresponding author.

Background and aims.- Clozapine remains the reference treatment in resistant schizophrenia. However, its effectiveness is obtained only in 30 to $50 \%$ of cases.

In this clinical case report, we discuss effectiveness of the addition of Aripiprazole to Clozapine as a therapeutic alternative in the treatment of ultra-resistant schizophrenia.

Methods.- We report the case of Mr A.T, 44 years old. He suffers from paranoid resistant schizophrenia since the age of 30 years and was treated with $900 \mathrm{mg} /$ day Clozapine without side effects. To evaluate efficacy of treatment, we used the Brief Psychiatric Rating Scale (BPRS), the scale for the Assessment of Negative Symptom (SANS) and the scale for the Assessment of Positive Symptoms (SAPS). Plasma concentrations of Clozapine and the main metabolite Norclozapine were evaluated by High-Performance Liquid Chromatography method (HPLC).

Results.- Despite the administration of high doses of Clozapine (900 mg/day), a correct plasma Clozapine concentration and correct plasma Norclozapine concentration (respectively $824 \mathrm{ng} / \mathrm{ml}$ and $72 \mathrm{ng} / \mathrm{ml}$ ), we have achieved only $22 \%$ improvement in the BPRS. Finally, when associate Clozapine $900 \mathrm{mg} / \mathrm{day}$ to Aripiprazole at a dose of $10 \mathrm{mg} /$ day, we noted better improvement in positive symptoms with a marked decrease of anxiety and psychotic symptoms. In this association we found improvement of $48 \%$ in the SAPS, $42 \%$ in SANS and $40 \%$ in BPRS.

Conclusions.- The results of this clinical case suggest that the addition of Aripiprazole to Clozapine in treatment ultra resistant schizophrenia could be an effective therapeutic alternative. Double-blind studies are needed to confirm this ascertainment. Disclosure of interest.- The authors have not supplied a conflict of interest statement.

\section{E-PV0835}

\section{Possibility of assessing of aggressivity in patients with schizophrenia - comparison of the outcomes of selected diagnostic methods}

S. Godinova

Psychiatrická nemocnica Hronovce, Acute Male Department, Hronovce, Slovak Republic

Corresponding author.

Background and aims.- Tendency to aggressive activity should not be assessed just as autonomous personality feature. It is important to consider the complexity of this type of behavior and the impact of social and biological factors (Mehta, Beer, 2009).

Methods.- Our poster brings brief comparison of the quantitative outcomes of several selected diagnostic methods - projective method Hand test, self-rating questionnaire SHaN, objective assessment scale HCR-20 and biochemical measurement of the testosterone hormone level. Study group consisted of 48 male inpatients hospitalized in Psychiatric hospital of Hronovce, diagnosed with schizophrenia, age 18 to 61 . The aggressive group included 21 inpatients with history of aggressive behavior at baseline or prior to hospitalization, non-aggressive group included 44 inpatients without the history of aggression.

Results.- Our findings did not confirm statistically significant intergroup differences in the outcomes of the projective method Hand test, self-rating questionnaire SHaN, nor in the values of the testosterone hormone level, but did confirm statistically significant intergroup difference in the score of HCR-20 $(U=105,00 ; p=0,00)$. Conclusions.- Projective methods show the current state, which may no longer be consistent with the violent act. Behavior at the time of decompensation does not necessarily result from long-term personality traits. The objective scale HCR-20 reduces the interference of social desirability, impact of personality disintegration, and considers wide range of factors influencing the risk of aggression. Disclosure of interest.- The authors have not supplied a conflict of interest statement.

\section{E-PV0836}

\section{Schizoaffective disorder: how long does it takes to diagnose?: “Case report"}

M.J. Gonçalves", M. Croca, R. Saraiva, C. Ferreira, C. Sereijo, C. Leite Rodrigues

Centro Hospitalar Lisboa Norte- EPE, Psychiatry and Mental Health, Lisboa, Portugal

* Corresponding author.

Background and aims.- Psychiatric diagnosis faces the challenge of variability and subjectivity associated with the evolution of the psychiatric illness. We aim to report a case of a 57-year-old woman with multiple psychiatric hospitalizations (2010-2018), during which different diagnostic hypotheses and therapeutic were proposed.

Methods.- A literature review on Schizoaffective Disorder (SD) was conducted using the PubMed search database and description of the clinical case with information provided by the patient and by her clinical records.

Results.- After analyzing the patients clinical records the hospital's psychiatric medical team proposed the diagnosis of SD. SD presents a high risk of recurrent hospitalizations and high costs associated with therapeutic and follow-up withdrawal, but there is limited data to assess the post-discharge critical periods.

Conclusions.- The psychiatric diagnosis faces the challenge of subjectivity and variability among clinicians according to the evolution pattern of the disease over the years. In fact, a single patient may have different diagnoses made and different therapeutics ini- 
tiated. Patients with hospital discharge and SD present a high risk of re-hospitalization. However, there is limited data to assess the post-discharge critical periods, during which the risk of rehospitalization is significant. Thus, further research in this area is required in order to adopt effective psychotherapeutic strategies and interventions to reduce the probability of hospital admissions and improve prognosis, reducing the associated costs of SD.

Disclosure of interest.- The authors have not supplied a conflict of interest statement.

\section{E-PV0837}

\section{Motor and cognitive abnormalities in schizophrenia}

A. Hopulele-Petri*, M. Manea

"Carol Davila" University Of Medicine and Pharmacy, Psychiatry, Bucharest, Romania

${ }^{*}$ Corresponding author.

Background and aims.- Motor abnormalities have been signaled in schizophrenia, from neurological soft signs (NSS), to bradykinesia, with the former even suggested as an endophenotypic marker. Cognitive rigidity, demonstrated in cognitively perseverant schizophrenic patients, has also been found in other neurological, dopaminergic disorders, like Parkinson's disease whose symptoms share some overlap with the negative symptoms in schizophrenia. The primary aim of this study was investigating the relationship between NSS, motor speed, motor imagery, and cognitive flexibility in schizophrenic patients. Secondary aims included evaluating the relationships between these domains and (1) neuroleptic treatment (2) clinical symptoms, and (3) disease progression.

Methods.- Recruitment consisted of patients diagnosed with schizophrenia, according to DSM-V criteria. NSS were assessed using the Brief Motor scale (BMS). Motor speed and imagery were assessed using the TimeUpAndGo! Task, and the imaginary version. Cognitive flexibility was assessed using a short-form version of the Berg Card Sorting test.

Neuroleptic treatment was assessed using defined daily doses while side effects were evaluated using the Simpson Angus Scale, and the Barnes Akathisia Rating scale. Clinical symptoms were measured using the Positive and Negative Symptoms scale.

Statistical analysis was done in SPSS, using Pearson's two-tailed correlation coefficients.

Results.- Preliminary results reveal a significant correlation between the three domains. Multiple significant correlations were also determined between these domains and negative symptoms, neuroleptic treatment, and illness duration.

Conclusions.- While undoubtedly affected by antidopaminergic treatment, the motor symptoms remain a fruitful direction for future research regarding its relationship to cognitive dysfunction and negative symptoms in schizophrenia.

Disclosure of interest.- The authors have not supplied a conflict of interest statement.

\section{E-PV0838}

\section{Polytherapy vs monotherapy in the}

\section{treatment of first episode of psychosis}

A. Hrnjica ${ }^{*}$, S. Bise ${ }^{1}$, D. Begić ${ }^{2}$, I. Lokmić-Pekić ${ }^{2}$

${ }^{1}$ Psychiatric Hospital Sarajevo, Women Department, Sarajevo,

Bosnia, Herzegovina; ${ }^{2}$ Psychiatric Hospital Sarajevo, Intensive Care,

Sarajevo, Bosnia, Herzegovina

${ }^{*}$ Corresponding author.

Background and aims.- Antipsychotic (AP) polytherapy is the use of more than one AP at the same time. Duration of Untreated Psychosis (DUP) is time between onset of positive psychotic symptoms and first treatment.
We aimed to review AP prescription patterns, a frequency of polytherapy vs monotherapy, in the treatment of First Episode of Psychosis (FEP); to analyze relationship between AP therapy and DUP, duration of hospitalization and frequency of rehospitalizations in follow- up period (one year).

Methods.- Retrospective, descriptive study, utilizing the database of Psychiatric Hospital Sarajevo (PHS). Eligible patients: adults, in FEP, hospitalized in period of 15 months. Analyzed parameters: therapy algorithms; relationships between therapy and: DUP, duration of hospitalization and freqency of rehospitalizations. Statistical data was processed in SPSS.

Results.- Thirty-one patients; $12(38.70 \%)$ on monotherapy, 19 (61.30\%) polytherapy. In the polytherapy group, 18 (94.73\%) had 2 antipsychotics (APs), 1 (5.27\%), 3 APs. Patients on monotherapy had DUP, 144.08 days ( $\mathrm{Md}=17.5, n=12)$; on polytherapy DUP was 362.84 days ( $M d=180, n=19), U=58.5, \mathrm{z}=-2.28, r=0,002$. On monotherapy hospitaliztions lasted 41.08 days ( $M d=43, n=12$ ), on polytherapy 45.21 ( $\mathrm{Md}=48, n=19$ ), $U=113,0, z=-0,41, r=0004$. Of all patients, 7 (22.58\%) were rehospitalized; 4 (57.15\%) on polytherapy, 3 (42.85\%) on monotherapy. In polytherapy group, 3 (75\%) were rehospitalized due non-compliance, in monotherapy, 1 (33.34\%).

Conclusions.- Polytherapy in the treatment of FAP is more frequent; those patients have statistically significant longer DUP and poor adherence. We found no statistically significant difference in duration of hospitalizations between two groups.

Disclosure of interest.- The authors have not supplied a conflict of interest statement.

\section{E-PV0839 \\ Neurobiology of schizoaffective disorder: a window into the nosological debate}

J.E. Ibáñez Vizoso*, M. Villanueva Gallego, Í. Alberdi Páramo, F.J. Gómez Beteta, M. Pérez Lombardo, J. Pemán Rodríguez, G. Montero Hernández, A. Carabias Contreras

Hospital Clínico San Carlos, Instituto de Psiquiatría y Salud Mental, Madrid, Spain

* Corresponding author.

Background and aims.- Krapelin's famous dichotomous classification of functional psychoses into "dementia praecox" and "manic-depressive insanity" soon revealed several cases which did not fully correspond to either of both entities. Kraepelin himself stated it in 1920. Various terms were used for some of these conditions, such as "boufée delirante" (Magnan), "cycloid psychoses" (Kleist) and "Schizoaffective disorder" (Kasanin). This last term succeeded and eventually changed its meaning to englobe all those concepts. Nevertheless, the conceptualization of schizoaffective disorder, as well as its distinction from Bipolar Disorder and Schizophrenia, remains unclear. The kraepelinian 2-disorders model, the continuum model and the DSM 3-disorders model have all found some degree of support.

Methods.- A comprehensive literature search was conducted via pubmed using the keyword "schizoaffective disorder" and any of the following terms: "neurobiology", "neuroimaging", "morphology", "nosology".

Results.- The differences in genetics, functional and structural neuroimaging, GABA signaling, transcriptome and oxidative stress are discussed.

Conclusions.- The valididy of SAD has been tested using several approaches, such as phenomenology, neurobiology or longitudinal studies of illness course, each of them providing support for different models. There is a substantial need for a revision and unification of the current diagnostic concept of SAD.

Disclosure of interest.- The authors have not supplied a conflict of interest statement. 


\section{E-PV0840}

\section{Psychosis and epilepsy: report a case}

A. Izquierdo De La Puente ${ }^{*}$ P. del Sol Calderón

Hospital Universitario Puerta de Hierro de Majadahonda, Psychiatry, Madrid, Spain

* Corresponding author.

Background and aims.- The aim of this case is to perform a review of postictal psychosis.

Methods.- A 25-year-old male with a previous diagnosis of epilepsy that presented, after a tonic-clonic convulsive crisis, megalomaniacal and injury delusional, with insomnia and disorganized behavior of several days of evolution. In addition, he presented mnesic failures during the previous days. The patient was admitted to the hospitalization unit where he was diagnosed with epilepsy and postictal psychosis.

Results.- It has been observed that prolonged epileptic seizures can cause psychotic symptoms that remain over time. These symptoms are indistinguishable from psychoses of primary origin; however, they are usually accompanied by episodes of low level of consciousness and episodes of post-critical confusion. The psychoses that occur in the context of epilepsy can be preictal (as the aura of a crisis), ictal (during the crisis) and postictal (after them) that can even be prolonged in later days. The prevalence of this type of psychotic episodes is not known with certainty, however, it is believed by various studies that interictal hallucinations could be more frequent than is believed. For treatment, antiepileptic drugs should be used.

Conclusions.- Therefore, psychotic symptoms in epilepsy are relatively frequent. They do not differ from primary psychosis; however, they have a better prognosis and more specific treatment. For these reasons, it is essential to have this type of symptomatology in mind, when dealing with a patient with epilepsy.

Disclosure of interest.- The authors have not supplied a conflict of interest statement.

\section{E-PV0841}

\section{Functionality, depression and anxiety in first psychotic episodes: a case-control study}

A. Izquierdo De La Puente ${ }^{1 *}$, A. Junquera Blanco ${ }^{2}$, P. del Sol Calderon $^{1}$

${ }^{1}$ Hospital Universitario Puerta de Hierro de Majadahonda, Psychiatry, Madrid, Spain; ${ }^{2}$ Hospital Dr. Rodríguez Lafora, Psychiatry, Madrid, Spain

* Corresponding author.

Background and aims.- The aim of this study was to evaluate the current status in terms of functionality, depression and anxiety in patients diagnosed with first psychotic episode.

Methods.- A case- control study was performed using a sample composed of 20 patients from the Hospital Clínico San Carlos, who were diagnosed and treated after a first psychotic episode, and 26 controls randomly selected from the general population. Both groups completed a battery of tests that included the MontgomeryAsberg Depression Rating Scale (MADRS), a Brief Performance Evaluation Test (FAST) and the Hamilton Anxiety Rating Scale (HARS).

Results.- The average age of cases and controls were 33 and 34 years respectively. $60 \%$ of the cases presently anxiety compared to $26.9 \%$ of the controls $(p=0.011)$, as well as $60 \%$ of the cases suffered from depression compared to none of the controls $(p<0.01)$. In this sample, cognitive dysfunction was observed in the group of cases. This dysfunction affects several domains of functioning, especially the psychosocial areas, highlighting that these limitations were present even from early stages of the disease (mean of cases 22 versus 12.08 in controls, $p=0.019$ ).
Conclusions.- It was found that patients diagnosed with this disease suffer anxiety-depressive disorders and cognitive worsening more often than the general population.

Disclosure of interest.- The authors have not supplied a conflict of interest statement.

\section{E-PV0842 \\ Description of the leucopenia case in the course of paliperidone treatment}

B. Janota ${ }^{*}$, G. Roganovic ${ }^{2}$, P. Michalska ${ }^{3}$, A.

Szymanik-Kostrzewska ${ }^{3}$, K. Janota ${ }^{4}$

${ }^{1}$ Psychiatry, Department of Psychiatry -Psychotherapy and

Psychosomatik-Carl-Thiem-Clinic-Thiemstreet 111- 03048 CottbusGermany, Cottbus, Germany; ${ }^{2}$ Carl-Thiem-Clinic, Department of Psychiatry -Psychotherapy and Psychosomatik-, Cottbus, Germany; ${ }^{3}$ Psychology Institute- Kazimierz Wielki University in Bydgoszcz, Department of Human Development, Bydgoszcz, Poland; ${ }^{4}$ MVZ, Internal Medicine - Endocrinology and Diabetology, Cottbus, Germany

* Corresponding author.

Background and aims.- In recent days, continuous development in the field of pharmacological treatment has been observed in psychiatry. Especially, an important group are antipsychotic medicines administered in intramuscular injections.

Case of patient diagnosed with schizoaffective disorder, not following compliance. In the course of Paliperidone treatment, increasing leucopenia was observed. After changing the treatment method from Paliperidone to Olanzapine, in the form of Depot, symptoms of leucopenia have subsided.

Methods.- Case study of a 51 years old women, who has been treated for 13 years with diagnosed schizoaffective disorder, not complying with medical prescriptions, in whom leucopenia was observed during Paliperidone treatment.

Results.- During the first hospitalization, laboratory tests did not show any abnormalities on the day of admission. Initially, the patient was treated with Risperidone. After 7 days of hospitalization, the patient discharged despite medical advice at her own request. On the same day after a few hours, she returned to the emergency room herself, reporting severe anxiety and auditory hallucinations. During further hospitalization due to non-compliance, the decision was made to change the form of treatment with Risperidone to Paliperidone. In the course of further treatment, increasing leukopenia was observed.

Conclusions.- During the course of treatment, a decrease in leukocyte concentration after Paliperidone administration was observed. During the further stay, the drug was changed to Olanzapine in the form of Depo. Both the stationary and daily ward confirmed a good symptomatic response to the drug used. Concentrations of leukocytes gradually compensated, which confirms the drugrelated cause of previously observed disorders in the blood count. Disclosure of interest.- The authors have not supplied a conflict of interest statement.

\section{E-PV0843}

\section{Impact of physical health screening Tool (PHST) for people with severe mental illness}

A. Chaturvedi, A. Jemmott ${ }^{*}$

Camden and Islington NHS Foundation Trust, Integrated Practice

Unit, London, United Kingdom

${ }^{*}$ Corresponding author.

Background and aims.-

Introduction.- People with severe mental illnesses such as psychosis and bipolar disorders are at a greater risk of death due to physical illnesses (World Health Organisation, 2015). This risk 
equates to dying 10-20 years earlier than the general population due to physical health conditions among those with a diagnosis of schizophrenia (Laursen, 2011).

Common physical health concerns observed with severe mental illnesses include chronic obstructive pulmonary disorder, cardiovascular disease, diabetes, obesity. Many of these symptoms go undiagnosed as people with severe mental illnesses don't volunteer for physical health screenings, while physical health monitoring remains limited in the primary and secondary care services (Paton, 2004). This treatment gap is being reduced at the Camden and Islington NHS Foundation Trust through its Physical Health Screening Tool (PHST).

Objective. We are evaluating the effectiveness of the PHST to screen physical illnesses for people with severe mental illnesses.

Methods.- A cohort of 159 people (Female $=40 \%$ ) with diagnoses of severe mental illnesses was built and tracked for incidence of physical health conditions such as smoking status, blood pressure abnormality, diabetes and obesity, by PHST, comparing it with the incidence reported by traditional physical health screening tools. Results.- PHST was 18.7 times more likely to report smoking status; 7 times more likely to report cardiovascular diseases; 6.5 times more likely to report diabetes and 7 times more likely to report Obesity, compared to the traditional physical health measures. Conclusions.- PHST was more effective in screening people with severe mental illnesses for physical health conditions.

Disclosure of interest.- The authors have not supplied a conflict of interest statement.

\section{E-PV0844}

\section{Clinical predictors of clozapine response in patients with treatment resistant schizophrenia: literature review}

R. Jouini ${ }^{*}$, A. Aissa ${ }^{1}$, A. Rebai ${ }^{2}$, F. Ellouze ${ }^{3}$, A. Khelifa ${ }^{1}$, H. Ben

Ammar $^{1}$, Z. El Hechmi ${ }^{1}$

${ }^{1}$ Razi Hospital, Psychiatry F Department, Tunis, Tunisia; ${ }^{2}$ Razi

Hospital, Psychiatry A Department, Tunis, Tunisia; ${ }^{3}$ Military Hospital of Tunis, Psychiatry Departement, Tunis, Tunisia

${ }^{*}$ Corresponding author.

Background and aims.- Schizophrenia is a chronic mental illness that ordinarily becomes more difficult to treat over time. Despite progress in pharmacotherapy, $30 \%$ of patients with schizophrenia do not respond to treatment, leading to increased mortality, economic costs and poor quality of life. Clozapine is the drug of choice for the management of treatment resistant schizophrenia and its efficacy has been established in several clinical trials. The aim of our study is to investigate clinical predictors of clozapine response in patients with treatment resistant schizophrenia through a systematic review of literature in order to improve clozapine prescription in daily practice and thus to avoid potentially fatal adverse effects. Methods.- We searched MEDLINE/PubMed, PsyInfo and Cochrane using the terms schizophrenia, treatment-resistant, clozapine, response and outcome and by manual searches of reference lists of relevant publications. We searched the most recent reviews, updating the evidence by relevant studies published since 2008.

Results.- Earlier age of onset, non-paranoid subtype, longer duration of illness, poor premorbid functioning and serum clozapine level below $350 \mathrm{ng} / \mathrm{ml}$ have been reported as potential clinical predictors of non-response to clozapine. Findings concerning baseline negative symptoms and past history of neuroleptic induced extrapyramidal symptoms as clinical predictors were contradictory.

Conclusions.- Clinical predictors of clozapine response in patient with treatment resistant schizophrenia could increase targeted clozapine use and may identify more patients who are likely to benefit with clozapine, preventing the occurrence of serious side effects. Future studies should focus on biological and genetical factors to predict response to clozapine in patient with treatment resistant schizophrenia.

Disclosure of interest.- The authors have not supplied a conflict of interest statement.

\section{E-PV0846 \\ Cannabis use and clinical outcomes in patients with schizophrenia}

D. Khattech*, E. Khelifa

Razi Hospital, Psychiatry F, Mannouba, Tunisia

* Corresponding author.

Background and aims.- Cannabis use and schizophrenia are connected, but we still have a limited understanding of their relationship. As cannabis is used widely among patients with schizophrenia, it is of a growing interest to search for the role it might have in their prognosis. The primary aim of this study was to examine the impact of cannabis use on the clinical outcomes in patients with schizophrenia.

Methods.- This was a cross-sectional study among patients followed for schizophrenia or schizo-affective disorder according to DSM-V and stabilized under medication. We used the severity of Dependence Scale to assess cannabis use, Positive and Negative Syndrome Scale and medical history of hospitalizations to assess illness severity.

Results.- Cannabis users had higher levels of positive symptoms, were more often hospitalized than non-users and had higher doses of antipsychotic medication. In consequence, cannabis use was associated with more negative clinical outcomes.

Conclusions.- Cannabis use is common among patients followed for psychosis. This substance abuse was associated with more negative clinical outcomes. These findings bring attention on the importance of preventing and treating cannabis use among these patients.

Disclosure of interest.- The authors have not supplied a conflict of interest statement.

\section{E-PV0847}

\section{Nicotine dependence and clinical outcomes in patients with schizophrenia}

\section{Khattech*, E. Khelifa}

Razi Hospital, Psychiatry F, Mannouba, Tunisia

* Corresponding author.

Background and aims.- Patients with schizophrenia are markedly prone to smoke tobacco in comparison to the general population, as well as to patients with other psychiatric disorders. This study aimed to examine the impact of nicotine dependence on the clinical outcomes in patients with schizophrenia.

Methods.- This was a cross-sectional study among patients followed for schizophrenia or schizo-affective disorder according to DSM-V and stabilized under medication. We used the Fagerström Test for Nicotine Dependence, Positive and Negative Syndrome Scale and medical history of hospitalizations to assess illness severity.

Results.- Tobacco smokers had higher levels on the PANSS total score, were more often hospitalized than non smokers and therefore had more negative clinical outcomes.

Conclusions.- Tobacco smoking and the subsequent nicotine dependence is common among patients followed for schizophrenia. This substance abuse was associated with more negative clinical outcomes. Thus, integrating a therapeutic management of smoking in these patients could improve their illness severity.

Disclosure of interest.- The authors have not supplied a conflict of interest statement. 


\section{E-PV0848}

\section{Relaxation techniques for psychosis} treatment

C. Kieckhaefer ${ }^{*}$, K. Broer, C. Theisen

LVR-Hospital Düsseldorf- Heinrich-Heine-University, Department of Psychiatry, Düsseldorf, Germany

Corresponding author.

Background and aims.- Modern schizophrenia treatment is complex and requires multimodal therapy. Relaxation techniques play an increasing role in times of reduced compulsory interventions and empowerment of patients. The diversity of these complementary treatments raises the question on effectiveness and suitable use. As some studies described cases of psychotic exacerbation due to meditation and mindfulness intervention, a systematic overview is required in particular for schizophrenia patients.

We assessed effectiveness of complementary treatments for relaxation in schizophrenia patients for use in daily therapy by a systematic review of published quantitative studies.

Methods.- We identified randomized controlled trials and metaanalyses of the most relevant relaxation techniques (meditation, yoga, tai chi, progressive muscle relaxation, mindful exercise, mindfulness interventions) for people with schizophrenia, based on regular searches of PubMed, PsycINFO, Web of Science and Cochrane Library. We assessed effectiveness of the techniques for early-stage psychosis patients and chronic schizophrenia patients, separated for inpatient and outpatient treatment.

Results.- Different relaxation techniques showed significant additional effects to pharmacotherapy, psychotherapy and psychoeducation, especially for reduction of stress and negative symptoms.

Conclusions.- Relaxation techniques can be seen as a useful addition to multimodal therapy of schizophrenia. The simplicity of their realization and absence of side effects give relaxation techniques an additional benefit within the inpatient and outpatient treatment. More randomised studies are required, especially a direct comparison of different techniques.

Disclosure of interest.- The authors have not supplied a conflict of interest statement.

\section{E-PV0849}

\section{Towards neurophenomenological model of self-disturbances in first episode of psychosis in the spectrum of schizophrenia}

\section{J. Kielan", L. Gaweda}

Medical University of Warsaw, II Department of Psychiatry, Warsaw, Poland

* Corresponding author.

Background and aims.- Phenomenologically-oriented scientists believe that the core of psychosis in the spectrum of schizophrenia may be self-disturbances. Psychotic symptoms, such as delusions and hallucinations, are also accompanied by cognitive biases. Researchers combines the cognitive model with phenomenological analysis, indicating the relationship of aberrant salience with hyper-reflexivity and disturbances of intuitive social understanding and disturbances source monitoring with self-affection and presence. Our team observed connection of self-disturbances with cognitive biases in healthy group, but the is still lack of information how is it connect in first episode patient.

The aim of the study is to test the relationship of self-disturbances with cognitive biases in first episode of psychosis in the spectrum of schizophrenia.

Methods.- The sample will consist 50 first episode patients and 50 healthy control. Participants will be tested with the MINI test, PANSS and PSYRATS to assess psychopathological symptoms. To investigate the self-disturbances will be used: SQUEASE and IPASE questionnaires. Cognitive functions will be measured by DEBASCO questionnaire, experimental tasks: action memory and Fish task and Aberrant Salience Inventory. Additionally we plan 12 months follow-up for FEP group to find out if changes in self-disturbances will predict changes in cognitive biases.

Results.- The preliminary results will be presented. Relationship between self-disturbances with cognitive biases will be tested using the correlation method. Sociodemographic factors and 12 months follow up will be check by regression analysis.

Conclusions.- Understanding relationship of self-disturbances and cognitive biases may have potential for developing better therapeutic interventions for FEP.

Disclosure of interest.- The authors have not supplied a conflict of interest statement.

\section{E-PV0851}

\section{Homocysteine levels in schizophrenia are associated with longer duration of} illness

E. Theochari ${ }^{1}$, S. Nikolaou ${ }^{2}$, F. Louki ${ }^{3}$, A. Giannoulis ${ }^{3}$, D. Kipreos ${ }^{4}$, E. Tsaltas ${ }^{5}$, D. Kontis ${ }^{3 *}$

${ }^{1}$ Asklipieion Voulas General Hospital, Department of Psychiatry, Voula, Greece; ${ }^{2}$ Thriassion General Hospital, Internal Medicine Department, Elefsina, Greece; ${ }^{3}$ Psychiatric Hospital of Attica, Cognitive Rehabilitation Unit- 4th Psychiatric Department, Athens, Greece; ${ }^{4}$ Psychiatric Hospital of Attica, Microbiology and Biochemistry Laboratory, Athens, Greece; ${ }^{5}$ Athens University Medical School, Experimental Psychology Laboratory-1st Psychiatric Department, Athens, Greece

Corresponding author.

Background and aims.- Schizophrenia has been associated with elevated homocysteine levels. We investigated the relationship of plasma homocysteine concentrations in schizophrenia with duration of illness.

Methods.- 90 patients with schizophrenia (32 females, mean age $=42.91$ years, $S D=10.1$, illness duration $=17.84$ years, $\mathrm{SD}=10.51$ ) were recruited during an exploratory genetic crosssectional study. Total homocysteine serum levels were determined quantitatively by fluorescence-polarization immunoassay. Linear regression analysis examined the effect of duration of illness in years on plasma homocysteine levels in patients with schizophrenia and the effect of age.

Results.- Mean plasma homocysteine levels in patients were 17.44 $(\mathrm{SD}=9.46) \mu \mathrm{mol} / \mathrm{lt}$. Plasma homocysteine levels were significantly associated with illness duration ( $B=0.215,95 \% \mathrm{CI}: 0.24,0.405$, $t=2.243, \mathrm{df}=83, p=0.028$ ). A ten years increase in illness duration is associated with $2.15 \mu \mathrm{mol} / \mathrm{lt}$ in plasma homocysteine levels. Illness duration explains $5.7 \%$ of the variance in plasma homocysteine levels $(R$ Square $=0.057$ ). However, age was not significantly associated with homocysteine levels (ANOVA of the Regression Model was not significant).

Conclusions.- Illness duration is associated with homocysteine levels in schizophrenia.

Disclosure of interest.- The authors have not supplied a conflict of interest statement.

\section{E-PV0853}

\section{Time of response and social cognition in schizophrenia: a comparative study} A. Ruiz-Toca ${ }^{1}$, G. Lahera ${ }^{2 *}$

${ }^{1}$ Germans Trias i Pujol University Hospital, Psychiatry, Badalona Barcelona, Spain; ${ }^{2}$ University of Alcalá, Psychiatry, Alcalá-Madrid, Spain

Corresponding author. 
Background and aims.- Neurocognitive deficits in information processing are a global and primary aspect of schizophrenia that affects global functioning and social skills. However, there are still few studies that explain the influence of processing speed as a mediating variable in emotional processing.

Objectives.- To analyze the relationship between social cognition and processing speed in people with schizophrenia.

Methods.- A sample of 90 outpatients diagnosed with schizophrenia (DSM 5 critera) and 100 healthy subjects between 18 and 65 years old were recruited. Sociodemographic variables, clinical variables measured by: Positive and Negative Syndrome Scale (PANSS), cognitive variables as processing speed measured by Trail Making Test (TMT), symbol coding (BACS), verbal fluency (animals) and social cognition (SC) measured by Movie Assesment Social Cognition (MASC) were measured.

Results.- The group of patients with schizophrenia showed a poorer performance in social cognition and processing speed. Processing speed significantly improved hits in MASC test, with a marked group effect. The patients groups showed a shorter response time in MASC that was associated with more correct answers $(P=-2.24$; $p=0.028$ ). There were no significant effects of the symptoms.

Conclusions.- The relationship between SC and processing speed suggests that slowing down the processes to identify and integrate the available information may produce an inadequate social response that is not mediated by the effect of the symptoms. Future studies should replicate the findings of the present study.

Disclosure of interest.- The authors have not supplied a conflict of interest statement.

\section{E-PV0854 \\ Differential diagnosis of psychosis and self-mutilation, starting from a case study \\ J. Libuy \\ Pontificia Universidad Católica, Psiquiatría, Santiago, Chile \\ ${ }^{*}$ Corresponding author.}

Background and aims.- 63 years old male patient, without history of psychiatric disease, with medical comorbidity of hypertension and dyslipidemia. Presenting a year of evolution of psychotic and depressive symptoms characterized by numerous delusions, including ideas of incest, paranoid and nihilist; apathy, anhedonia, depressed mood, anorexia with loss of weight up to $16 \mathrm{kgs}$, insomnia, without suicidal ideation. No history of alcohol or drug use.

A diagnostic workup including complete hemogram, biochemistry, serum VDRL test and HIV, and a plain and contrast magnetic resonance imaging of the brain, all revealed no abnormalities.

Objective.- Assess differential diagnosis of patients with psychotic symptoms and self-mutilation.

Methods.- A literary review was made in PubMed, search of Mesh Terms: "Psychotic Disorders" and "Self Mutilation" in October 2018. Not limited by time of publication. Articles screened were written in English and Spanish, other language were excluded.

The abstracts of all articles were read and full texts of the relevant ones were obtained.

Results.- 126 studies were found, 20 articles responded the clinical question. 339 cases are described with psychotic symptoms and self-mutilation. 154 of 339 cases were diagnosed with schizophrenia and related disorders, 64 with organic disorder and 24 with substance use disorder. Most of the cases were genital amputation or enucleation, with few cases of other types of self-injury.

Conclusions.- In this review, most patients with psychosis and selfmutilation are diagnosed with schizophrenia and related disorders (45\%). Case reports are subject to publication bias, and valid inferences cannot be made from the observation of small numbers of cases.
Disclosure of interest.- The authors have not supplied a conflict of interest statement.

\section{E-PV0855 \\ Investigation of vitamin d profile in tunisian patients with schizophrenia}

W. Guidara ${ }^{1}$, M. Naifar ${ }^{2}$, K. Jmal ${ }^{2}$, M. Maalej ${ }^{3}$, A.S. Ellouze ${ }^{3}$, S. Omri $^{3}$, M. Maalej ${ }^{3}$, N. Charfi ${ }^{3}$, J. Ben Thabet ${ }^{3}$, F. Ayadi ${ }^{1,2}$

1 "Molecular Bases of Human Pathology", Faculty of Medicine of Sfax, Biochemistry, Sfax, Tunisia; ${ }^{2}$ Laboratory of Biochemistry CHU Habib Bourguiba Sfax, Biochemistry, Sfax, Tunisia; ${ }^{3}$ Psychiatry "C"

Department - CHU Hédi Chaker Sfax, Psychiatry "C" Department,

Sfax, Tunisia

${ }^{*}$ Corresponding author.

Background and aims.- Current classifications of psychiatric disorders are based primarily on clinical observation and rating scales, but lack peripheral biological validators. We aimed to evaluate the vitamin D profile in subjects with schizophrenia (SCZ) compared to controls.

Methods.- This is a case-control study involving subjects with SCZ. The diagnosis was selected based on DSM-5 criteria, Psychological symptoms were assessed using the Positive and Negative Syndrome Scale (PANSS). A vitamin D was automated by methods enzymatic tests on Cobas 6000 robots from Roche ${ }^{\circledR}$.

Results.- This study included 45 patients $\operatorname{SCZ}(37,38$ years \pm 11 , 28 ) and 100 controls $(33,09 \pm 9,48)$. Vitamin D levels of subjects with SCZ were increased but not a statistical difference compared with controls. Vitamin D was no correlated with the PANSS score $(r=-0,024 ; p=0,819)$.

Conclusions.- Vitamin D levels of subjects with SCZ were increased. This encourages us to deepen research on the links between vitamin D levels and other psychiatric disorders.

Disclosure of interest.- The authors have not supplied a conflict of interest statement.

\section{E-PV0856 \\ Noah syndrome and psychotic disorder: a case report}

H. Maatallah*, R. Damak, I. Berrahal, N. Ghazouani, W. Cherif, M. Cheour

Razi Hospital, Psy E, Tunis, Tunisia

${ }^{*}$ Corresponding author.

Background and aims.- Noah Syndrome is a variant of diogenes syndrome accompanied by animal hoarding practices. The current scientific literature presents a lack of empirical studies on the phenomenon of animal hoarding.

Objectives.- To Study the association between Noah syndrome and psychotic disorder. And define an adequate therapeutic management to this disorder.

Methods.- We describe a case of women suffering of Noah Syndrome which resolved after putting her into atypical antipsychotic associated to cognitive behavior therapy.

Results.- Miss. C.B, age 39,was admitted to an inpatient psychiatric unit for management of acute psychotic symptoms. She has lived in solitude for the past 10 years. The patient appeared malnourished. During the first contact,she presented poor personal hygiene,hostile affect. The patient's family showed us pictures of her house. She lived with 40 cats that were in poor health. The patient had no awareness of the health's problem. The patient had behaved normally until 12 years ago,after a sentimental failure and the death of her mother, she began to develop insidious behavioral changes: hoarding animals, social isolation, verbal and physical aggression toward family members,domestic abandonment,and self-neglecting. A CT scan of the brain and physical examination were normal. The patient's clinical case was monitored for one 
month by putting her into risperidone $4 \mathrm{mg}$ per day with CBT. Actually Miss CB is followed on in our outpatient unit. She accept to give all the cats to an animal protection association.

Conclusions.- Hoarding Disorder is considered a complex phenomenon that causes problems of public health and legal impasses. Further studies are needed to better explain their psychopathological profile.

Disclosure of interest.- The authors have not supplied a conflict of interest statement.

\section{E-PV0857}

\section{Biomarkers in schizophrenia: protocol for follow-up study of patients with first episode psychosis}

P. Makaric ${ }^{*}$, D. Bosnjak Kuharic ${ }^{2}$, I. Kekin ${ }^{3}$, Z. Madzarac ${ }^{3}$, A. Savic $^{2}$, A. Silic ${ }^{2}$, D. Ostojic ${ }^{2}$, M. Sagud ${ }^{3}$, P. Brecic ${ }^{1}$, V. Jukic ${ }^{4}$, M. Rojnic Kuzman ${ }^{3}$

${ }^{1}$ University Psychiatric Hospital Vrapce, Affective Disorders, Zagreb, Croatia; ${ }^{2}$ University Psychiatric Hospital Vrapce, Department for diagnostics and intensive care, Zagreb, Croatia; ${ }^{3}$ University Clinical Hospital Center Zagreb, Department for psychiatry, Zagreb, Croatia; ${ }^{4}$ University Psychiatric Hospital Vrapce, Department for Forensic Psychiatry, Zagreb, Croatia

Corresponding author.

Background and aims.- Huge efforts have been made to identify a biological correlate of schizophrenia in order to confirm the diagnosis and predict the outcome, but there are still no reliable biological markers. Our aim was to investigate possible clinical, functional and structural biomarkers of schizophrenia which are present during the first episode and monitor their changes during time.

Methods.- We have conducted a longitudinal study in patients with first episode of psychosis (FEP) and healthy controls (HC). Assessment included clinical interview, sociodemographic questionnaire, psychiatric rating scales and questionnaires and specific additional assessments that were divided into three work packages (WP). The first WP included neurocognitive and facial emotional recognition assessment with adequate tests, the second WP assessment of cerebral blood flow velocity with transcranial Doppler ultrasound, and the third WP assessment of regional cerebral perfusion with single photon emission computed tomography. Patients were assessed at baseline and after 18-month follow-up and HC at one time point. Results.- We shall: 1 . analyse the differences between FEP and HC, 2 . analyse the changes during the course of illness from baseline to the end of study, 3 . perform an exploratory analysis combining the results of different tests in order to identify potential disease phenotypes and their impact on treatment outcome.

Conclusions.- Identifying possible biological markers could help us in better understanding of illness and its course, as well as to tailor its treatment on individual basis.

Disclosure of interest.- The authors have not supplied a conflict of interest statement.

\section{E-PV0859}

\section{Serum BDNF levels, neurocognition, quality of life and clinical symptoms in schizophrenia}

I. Martin Villalba ${ }^{1}$, M. Primé ${ }^{1}$, R. Penadés ${ }^{1}$, R. Catalán ${ }^{1}$, B. Arias ${ }^{2}$

${ }^{1}$ Hospital Clinic of Barcelona, Department of Psychiatry and Psychology, Barcelona, Spain; ${ }^{2}$ University of Barcelona, Department of Evolutionary Biology, Ecology and Environmental Sciences, Barcelona, Spain

* Corresponding author.

Background and aims.- BDNF (Brain-derived neurotrophic factor) is one of the most widely studied growth factor possibly involved in the development of schizophrenia. Previous studies have shown that a lower cognitive performance in schizophrenia patients has been related to lower serum BDNF levels.

The aim of this study is to test if serum BDNF levels are correlated with clinical symptoms, neurocognitive performance and consequently the quality of life in schizophrenic patients.

Methods.- It was an exploratory, cross-sectional study based on a sample of 70 outpatients with schizophrenia disorder who were recruited in the Hospital Clinic of Barcelona. BDNF was measured in serum samples with the Quantikine ${ }^{\circledR}$ ELISA Human BDNF Immunoassay. Clinical symptoms were assessed using the PANSS (Positive and Negative Syndrome Scale), cognitive performance using an exhaustive battery of neuropsychological tests, and quality of life was assessed with the EQ-5D (EuroQol-5D). In order to study possible covariates, Pearson correlations were performed. Results.- When analyzing correlations between serum BDNF levels and clinical $\left(r^{2}=-0,088 ; p=0,481\right)$, neurocognitive $\left(r^{2}=-0,091\right.$; $p=0,476)$ and quality of life $\left(r^{2}=0,045 ; p=0,719\right)$ variables, no significant correlations were found.

Conclusions.- The understanding of the relationship between serum BDNF levels and other variables such as neurocognition, clinical symptoms and quality of life is far from complete in patients with schizophrenia disorder. Thus, the role of serum BDNF levels as a biomarker in schizophrenia is still premature.

Disclosure of interest.- The authors have not supplied a conflict of interest statement.

\section{E-PV0862}

\section{Cycloid psychosis revisited}

N. Moura ${ }^{1 *}$, H. Simião ${ }^{1}$, S. Pereira ${ }^{1}$, F. Prates ${ }^{1}$, R. Silva ${ }^{1,2}$

${ }_{1}^{1}$ Centro Hospitalar de Lisboa Ocidental, Psychiatry, Lisboa, Portugal;

2 Nova Medical School, Psychiatry, Lisboa, Portugal

Corresponding author.

Background and aims.- Cycloid psychoses (CP) differ from affective psychoses or schizophrenia concerning symptom profile, course, and prognosis and don't fit in any clear diagnostic category. The pattern of episodic nonaffective psychosis with fast and full recovery between episodes would be included in the brief psychotic disorders category in DSM-5, or the acute and transient psychotic disorders in ICD-11. This disorder was first described by Leonhard (1957) and Perris and Brockington (1985) set the first operational diagnostic criteria. Since CP currently has an uncertain nosological status it has never been studied in large population samples.

The aim of this work is to present one clinical case of $\mathrm{CP}$ and review the concept, nosological status, diagnostic features and treatment. Methods.- We consulted the patient's clinical process and we conducted a conventional literature review in PubMed.

Results.- Thirty-two-year-old woman, with no previous history of psychiatric hospitalizations. One week before being taken to our emergency department she developed behavioral changes: clinophilia, motor retardation, anorexia, total insomnia, marked anxiety, intense fear of death and persecutory delusions. Once there, her mental state examination revealed perplexity, complex motor stereotypies associated with severe psychomotor inhibition, flat affect, persecutory delusions, auditory verbal hallucinations and absence of insight. She was admitted to our psychiatric inpatient unit, began treatment with paliperidone $6 \mathrm{mg} /$ day and was discharged two weeks after the admission asymptomatic.

Conclusions.- CP is not a widely recognized psychotic illness, nonetheless it appears to be clinically and biologically distinct from both severe mood disorders and schizophrenia and requires a different treatment and follow-up.

Disclosure of interest.- The authors have not supplied a conflict of interest statement. 


\section{E-PV0865}

\section{Risk factors of self-injurious behavior in the general population: the role of psychotic-like experiences and self-disturbances}

R. Pionke ${ }^{1 *}$, M. Krężołek ${ }^{1}$, D. Frydecka ${ }^{2}$, A. Cechnicki ${ }^{3}$, Ł. Gawęda ${ }^{1}$

${ }^{1}$ Medical University of Warsaw, II Department of Psychiatry,

Warsaw, Poland; ${ }^{2}$ Wroclaw Medical University, Department of Psychiatry, Wroclaw, Poland; ${ }^{3}$ Jagiellonian University, Department of Community Psychiatry-Chair of Psychiatry-Medical College, Krakow, Poland

${ }^{*}$ Corresponding author.

Background and aims.- Recent studies show that psychotic-like experiences, along with depressive symptoms, traumatic life events and psychiatric diagnosis are associated with increased risk of self-injurious behavior. So far, the role of self-disturbances in predicting self-harming has not been studied. The aim of our study was to investigate potential risk factors for self-injuries with particular emphasis on self-disturbances and psychotic-like experiences.

Methods.- A total of 3168 (2024 females, mean age 26.67, SD =4.78) individuals in the general population (psychotic disorders and substance dependence were excluded) were assessed with self-report scales that measure self-disturbances (Inventory of PsychoticLike Anomalous Self-Experiences), trauma (Childhood Experience of Care and Abuse Questionnaire), psychotic-like experiences (Prodromal Questionnaire), depressive symptoms (Center for Epidemiologic Studies Depression Scale), individual and family history of psychiatric diagnosis, and self-injurious behaviors history. We performed multivariate regression analysis with odds ratio calculations.

Results.- Multivariate regression analysis showed that all predictors, controlled for each other, were significant. Higher levels of self-disturbances were related to 1.57 of increased risk of self-injuries. The risk of self-harming was 3.20 times higher in participants with individual history of psychiatric diagnosis and 1.79 times higher in those with family history of psychiatric diagnosis. Psychotic-like experiences were associated with the highest risk of self-injuries $(\mathrm{OR}=4.13)$. Depressive symptoms were associated with 1.64 of increased risk of self-harm. Participants who experienced five or six childhood traumas were 2.12 times more likely to endorse self-injurious behaviors.

Conclusions.- Self-disturbances, along with psychotic-like experiences, trauma, depressive symptoms, individual and family history of psychiatric diagnosis are associated with an increased risk of self-injurious behaviors.

Disclosure of interest.- The authors have not supplied a conflict of interest statement.

\section{E-PV0866}

\section{The relationship between}

\section{self-disturbances, psychopathology and cognitive functions among individuals prone to psychosis}

R. Pionke ${ }^{1 *}$, M. Krężołek ${ }^{1}$, A. Kaliszz ${ }^{2}$, P. Błądziński ${ }^{2}$, A. Cechnicki², B. Misiak ${ }^{3}$, K. Kotowicz ${ }^{4}$, B. Nelson ${ }^{5}$, D. Frydecka ${ }^{4}$, Ł. Gawęda ${ }^{1}$

${ }^{1}$ Medical University of Warsaw, II Department of Psychiatry,

Warsaw, Poland; ${ }^{2}$ Jagiellonian University, Department of Community Psychiatry, Chair of Psychiatry, Medical College, Krakow, Poland; ${ }^{3}$ Wroclaw Medical University, Department of Genetics, Wroclaw, Poland; ${ }^{4}$ Wroclaw Medical University, Department of Psychiatry, Wroclaw, Poland; ${ }^{5}$ The University of Melbourne, National Centre of Excellence in Youth Mental Health, Melbourne, Australia ${ }^{*}$ Corresponding author.

Background and aims.- Phenomenologically oriented researchers have proposed that disturbance of the basic sense of self is psy- chopathological trait marker of psychotic vulnerability, but still self-disturbances are poorly understood. The aim of our study was to investigate relationships between self-disturbances, psychopathology and cognitive functions.

Methods.- A sample of 6228 young adults, aged 18-35 years, were screened for psychosis proneness with Prodromal Questionnaire via internet platform. Further, a total of 94 ( 49 females, mean age 25.27, SD $=4.73$ ) individuals among $10 \%$ of top scores in Prodromal Questionnaire were recruited and underwent clinical assessment with Comprehensive Assessment of At-Risk Mental States and short version of Examination of Anomalous Self-Experience. Cognitive functions were measured by Brief Cognitive Assessment Tool for Schizophrenia, California Verbal Learning Test and D2 Test of Attention.

Results.- Cognition and Stream of Consciousness were significantly related to all scales of CAARMS, the highest correlation was with positive symptoms $(r=0,49, p<0.01)$. Self-Awareness and Presence showed significant relationships with all symptoms, especially with positive symptoms $(r=0,49, p<0.01)$, behavioural changes $(r=0,44, p<0.01)$ and general psychopathology $(r=0.47, p<0.01)$. Bodily Experiences were related to positive symptoms $(r=0.30$, $p<0.01)$, cognitive change $(r=0,29, p<0.01)$, emotional disturbance $(r=0.29, p<0.01)$, behavioral changes $(r=0.44, p<0.01)$ and general psychopathology $(r=0.27, p<0.01)$. Existential Reorientation showed significant relationship only with positive symptoms $(r=0.25, p<0.01)$. There was no significant correlation between self-disturbances and cognitive functioning.

Conclusions.- Self-disturbances are related to psychopathology, especially to positive symptoms, but not to cognitive functioning. Understanding this association has potential for developing better therapeutic interventions for people at risk of psychosis and diagnosed with schizophrenia.

Disclosure of interest.- The authors have not supplied a conflict of interest statement.

\section{E-PV0867}

\section{The white matter structure disorder assessment of patients with schizophrenia deficit syndrome in regards to prefrontal cortex disconnection theory}

P. Podwalski ${ }^{*}$, J. Samochowiec ${ }^{1}$, J. Pełka-Wysiecka ${ }^{1}$, L. Sagan ${ }^{2}$

${ }^{1}$ Pomeranian Medical University in Szczecin, Psychiatry, Szczecin, Poland; ${ }^{2}$ Pomeranian Medical University in Szczecin, Neurosurgery, Szczecin, Poland

* Corresponding author.

Background and aims.- One of the schizophrenia etiology concept is the disconnection theory. Perhaps due to the development of neuroimaging, which allows the white matter in vivo analysis, we will be able to answer the question whether the functional or anatomic changes of the connections between various brain structures exist. Some part of the ill patients suffer from deficit syndrome. For those patients the course of the illness is particularly unfavorable. It has a crucial influence on the planning and course of the treatment. The goal of the research is the assessment of the white matter structure difference of the patients with deficit syndrome and healthy people.

Methods.- For the research there have been chosen 15 people with diagnosed deficit syndrome, 15 people with recognized schizophrenia without deficit syndrome and randomized control group. All of the participants participated in psychiatric examination and diffusion tensor imaging (data acquisition will be held with the use of MRI, 25 diffusion directions, b-value 1000). Tractography of uncinated fasciculus and arcuatus fasciculus and analysis of fractional anisotropy and mean diffusivity values. 
Results.- The anticipated results of the conducted research are lowered diffusion and anisotropy indicators values of the patients suffering from paranoid schizophrenia in comparison to control group and severely lowered indicators values of the patients with deficit syndrome.

Conclusions.- These changes may indicate the disorder of nerve fibers integrity and the disorder of connection between prefrontal cortex and the remaining brain structures. The research results may explain the schizophrenia deficit syndrome psychopathology and contribute to finding an objective diagnostic tool.

Disclosure of interest.- The authors have not supplied a conflict of interest statement.

\section{E-PV0868}

\section{A case report of clozapine-induced myoclonus}

J. Portes Cruz ${ }^{1^{*}}$, I. Hervella Garcés ${ }^{2}$, U. Lertxundi Etxebarria ${ }^{3}, \mathrm{R}$. Hernández Palacios ${ }^{3}$

${ }^{1}$ Complejo Hospitalaria Universitario Santiago de Compostela, Alava Psychiatric Hospital, A Coruña, Spain; ${ }^{2}$ Red Salud Mental Araba, Hospital Psiquiátrico de Álava, Alava, Spain; ${ }^{3}$ Red Salud Mental Alava, Hospital Psiquiátrico de Álava, Alava, Spain

* Corresponding author.

Background and aims.- Clozapine treatment is associated with a dose-dependent risk of seizures. Compared to other second generation antipsychotics, it seems to have the highest risk of seizure induction. However, the risk of myoclonus (involuntary, brief, abrupt and sudden, shaking movements) associated with clozapine is less studied.

Methods.- We describe a case of a 38-year-old male with a diagnosis of treatment-resistant schizophrenia, with no previous history of epilepsy, who developed a generalized myoclonic jerks (positive and negative myoclonus) without alteration of consciousness 3 weeks after clozapine was initiated. Seizures were observed with dose of clozapine $300 \mathrm{mg} /$ day (last blood levels $145 \mathrm{ng} / \mathrm{ml}$ with dose $150 \mathrm{mg} /$ day). This side effect was resolved after reducing the dose and adding an antiepileptic medication (valproate). So far, the patient continues to take clozapine without reappearance of symptoms.

Results.- This side effect was reported to the Basque Pharmacovigilance Unit. Causality assessment performed with the modified Karch-Lasagna algorithm (official algorithm of the Spanish pharmacovigilance system) showed that the adverse reaction was probably caused by clozapine.

Conclusions.- Myoclonus is a rare side effect, but the possibility of being predictor of future seizures should put us on alert and we should treat it properly.

Disclosure of interest.- The authors have not supplied a conflict of interest statement.

\section{E-PV0869}

\section{Role of BDNF Val66Met polymorphism on neurocognition in schizophrenia}

\section{Primé ${ }^{1 *}$, I. Martín-Villalba ${ }^{1}$, B. Arias ${ }^{2}$, R. Catalán ${ }^{1}$, R. Penadés ${ }^{1}$}

${ }^{1}$ Hospital Clínic, Department of Psychiatry and Psychology,

Barcelona, Spain; ${ }^{2}$ University of Barcelona, Department of

Evolutionary Biology - Ecology and Environmental Sciences, Barcelona, Spain

* Corresponding author.

Background and aims.- Schizophrenia has a strong genetic component. Val66Met polymorphism has been described in the gene that codes for $B D N F$, a growth factor possibly involved in the development of schizophrenia, specifically on cognitive functions. Previous studies have reported that Met carriers show a lower cognitive performance than Val homozygotes. The aim of this study is to compare neurocognitive performance between groups of patients with schizophrenia according to the Val66Met BDNF polymorphism (Met carriers vs. Val/Val homozygote).

Methods.- An exploratory, cross-sectional study was carried out to compare patients with schizophrenia according to gene variants $(n=69)$. Allelic frequency showed that $64,8 \%$ were homozygous for Val and 32,4\% were Met carriers, which included Met homozygous and heterozygous. Patients' cognitive performance was assessed using an exhaustive battery of neuropsychological tests. DNA from peripheral blood cells was extracted using a standard genomic DNA Kit.

Results.- No significant differences between groups were found in the following cognitive performance domains: verbal comprehension ( $p=0,973)$; verbal memory $(p=0,287)$; non-verbal memory $(p=0,757)$; working memory $(p=0,614)$; attention/vigilance $(p=0,566)$; processing speed $(p=0,594)$; perceptual reasoning $(p=0,966)$; nor executive functions $(p=0,868)$. The results contrast with published literature regarding Met carriers as having worse neurocognition than Val homozygotes.

Conclusions.- The precise relationship between BDNF gene polymorphism and neurocognitive performance in persons with schizophrenia is still far from complete. More research is needed in order to understand better how neurocogntion is influenced by Val66Met BDNF polymorphism.

Disclosure of interest.- The authors have not supplied a conflict of interest statement.

\section{E-PV0870 \\ Characterization of a patient group suffering from schizophrenia attended in two mental health units}

M. Robles Martínez ${ }^{1 *}$, M.Á. García-Carretero ${ }^{2}$, L. Moreno Hierro ${ }^{3}$, M. Mateo Mateos ${ }^{4}$, M. Fernández Puerto $4^{5}$, M.Á. Jordán Quintero ${ }^{6}$ ${ }^{1}$ Institute of Neuropsychiatry and Addictions INAD, Parc de Salut Mar Barcelona, Spain, INAD, Barcelona, Spain; ${ }^{2}$ CTS-391 Multidisciplinary Group for the progress of Mental Health, Cadiz University, School of Nursing and Physiotherapy in Cadiz, Spain, Cádiz, Cádiz, Spain ${ }^{3}$ Cadiz University, Doctoral Programme, Spain, Cádiz, Cádiz, Spain; ${ }^{4}$ Mental Health Unit in San Fernando, Cadiz, Spain; ${ }^{5}$ Mental Health Unit in San Fernando, Cadiz, Spain, Mental Health Unit in San Fernando, Cadiz, Spain; ${ }^{6}$ Puerta del Mar University Hospital. Cadiz. Spain, Puerta del Mar University Hospital. Cadiz. Spain, Cádiz, Spain ${ }^{*}$ Corresponding author.

Background and aims.- Schizophrenia is a serious mental disorder affecting more than 21 million people in the world, around $1 \%$ of the general population.

Objective.- To characterize a patient group suffering from schizophrenia who receive treatment in two mental health units. Methods.- Descriptive cross-sectional study in 85 patients with primary diagnosis of schizophrenia, according to DSM-5, who are attended in Mental Health Units of Cádiz and San Fernando. The sociodemographic variables, the psychopathological state (PANSS), the global activity assessment (GAF) and the therapeutic compliance (SMAQ) were analyzed.

Results.- In a sample of 85 patients $(72.7 \%$ men) with a mean age of $48.9 \pm 9.8$ years, $85.5 \%$ are single and $5.5 \%$ are married. As regards familial coexistence, $50.9 \%$ live with relatives, $34.5 \%$ alone and $10.9 \%$ in Faisem supervised housing. Regarding the educational level, $38.8 \%$ have completed secondary education, $48.2 \%$ have no studies and 3.6\% have University studies. In terms of employment, $75.6 \%$ has never worked and $11.2 \%$ are unemployed. The mean age of onset of the disease was $21.1 \pm 4.7$ years. According to PANSS scale, $68 \%$ belong to the positive subtype and $32 \%$ to the negative one. $43.8 \%$ of patients have a GAF score higher than 50 points, indicative of a better level of activity. $38.4 \%$ have a good therapeutic compliance according to the SMAQ. 
Conclusions.- The therapeutic non-compliance high rate is evident as well as the low level of activity present in more than half of patients.

Disclosure of interest.- The authors have not supplied a conflict of interest statement.

\section{E-PV0871}

\section{From psychotic symptoms to digeorge syndrome: a case report}

C.M. Rodríguez Mercado ${ }^{*}$, L. García González ${ }^{2}$, L. Pérez Gómez ${ }^{3}$, M.E. Martínez Díaz-Monasterio-Guren ${ }^{1}$, R. Villa Diez ${ }^{1}$

${ }^{1}$ Hospital Universitario de Cabueñes. SESPA, Centro de Salud Mental II, Gijón, Spain; ${ }^{2}$ Hospital Universitario Central de Asturias. SESPA, Etac, Oviedo, Spain; ${ }^{3}$ Hospital Valle del Nalón. SESPA, Centro de Salud Mental, Langreo, Spain

${ }^{*}$ Corresponding author.

Background and aims.- Di George Syndrome (DS) is a common chromosome alteration with multisystemic affectation, including psychiatric manifestations. It is the highest genetic risk factor known for the development of schizophrenia. Psychiatric comorbidities are highly prevalent, affecting three quarters of all diagnosed individuals. These include schizophrenia spectrum disorders. About one third of individuals developed a psychotic disorder, most of them early in adult life. The DS may be an opportunity to study the aetiopathogenesis of schizophrenia. It is essential to carry out a comprehensive approach to perform an early diagnosis in these cases, and minimise the clinical impact.

Methods.- In this case report we analyze the clinic manifestations of a patient affected with DiGeorge Syndrome, with debut of early onset schizophrenia. As well as the complex therapeutic intervention required.

Results.- The DS is the strongest genetic risk factor known for schizophrenia. It is estimated that at the end of adolescence and the beginning of early adulthood, up to one third of all people who carry the 22q11.2 deletion developed Schizophrenia or schizoaffective disorder, reaching $1-2 \%$ of all cases of schizophrenia. The schizophrenia associated with this syndrome is clinically indistinguishable from schizophrenia in the general population with respect to the prodrome, age of onset and presentation.

Conclusions.- From the clinical-practical point of view, it is a possibly underdiagnosed syndrome, whose genetic evaluation should therefore be carried out in the presence of psychotic symptoms and anomalies suggestive of DS. Given the clinical complexity of patients, their therapeutic intervention is essential through a multidisciplinary team.

Disclosure of interest.- The authors have not supplied a conflict of interest statement.

\section{E-PV0872}

\section{A peculiar psychotic episode}

E. Rodríguez Vázquez*, C.M. Capella Meseguer, N. De Uribe Viloria, M.M. De Lorenzo Calzón, M. Gómez García, A. Alonso Sánchez, H. De la Red Gallego, A. Álvarez Astorga, P. Marqués Cabezas, L. Gallardo Borge

Hospital Clínico Univesitario de Valladolid, Psychiatry, Valladolid, Spain

${ }^{*}$ Corresponding author.

Background and aims.- Multiple chemical sensitivity is a rare medical condition characterized by symptoms of variable intensity that appear even when exposed to low levels of chemical substances.
Objective.- To present the diagnostic difficulty of this disease against possible psychotic episode.

Methods.- Descriptive study of a clinical case and literature review. Results.- Woman, 41 years old, married, two children. At the age of 26 , she presented a psychotic episode related to cannabis use. Afterwards, she presented 2 psychotic episodes more, one of them post-partum. She attended the consultation saying that some months ago she began to suffer side effects and allergies to the hairdressing products she works with and after receiving clothes for her son from a friend. In check-up, she referred that the sole manipulation of the product's packaging had produced nonspecific symptoms to her (redness, pruritus, precordial pain ...). Allergy tests: negative. She came back with insomnia, more irritable and with psychotic symptoms that she tried to hide. Olanzapine $10 \mathrm{mg}$ was prescribed, which she did not take and she changed by homeopathic compound which worsened her psychotic symptomatology. Improvement after antipsychotic treatment.

Conclusions.- At first the diagnosis between multiple chemical sensitivity and a psychotic episode is complex. Multiple chemical sensitivity is a diagnosis of exclusion, principally clinical, without any predefined criteria. However, the following tests are recommended: immune determinations, brain tomography, and neurological and psychiatric evaluations. A psychotic episode should be suspected in a patient with premorbid alterations and always after ruling out organic pathology.

Disclosure of interest.- The authors have not supplied a conflict of interest statement.

\section{E-PV0873}

\section{First-generation versus second-generation long-acting injectable antipsychotics in schizophrenia: cognitive impairment}

S.L. Romero Guillena ${ }^{*}$, B.O. Plasencia Garcia de Diego ${ }^{2}$, F. Gotor Sanchez-luengo ${ }^{3}$

${ }^{1}$ U.S.M.C Carmona. UGC Salud Mental Virgen Macarena, psychiatry, Sevilla, Spain; ${ }^{2}$ De la Merced Hospital, psychiatry, Osuna, Spain; ${ }^{3}$ Nuestra Señora de Valme Hospital, psychiatry, Sevilla, Spain * Corresponding author.

Background and aims.- According to the studies, atypical antipsychotics improve cognitive function in patients with schizophrenia significantly, as compared to traditional neuroleptics.

Aim.- To assess differences in cognitive impairment in patients with schizophrenia receiving first-generation long-acting injectable (FG-LAI) versus second-generation long-acting injectable (SG-LAI). Methods.- A descriptive, cross-sectional, multi-center study. Inclusion criteria.- Clinically stable patients with schizophrenia without any changes to their treatment in the last six months. Simple stratified sampling was performed to collect data from patients with schizophrenia receiving: a) FG-LAI $(n=12)$, b) Paliperidone Palmitate one-month formulation (PP1 M) $(n=12), c)$ Aripiprazole long-acting injectable (ALAI) $(n=12)$

Cognitive domains were evaluated based on the Brief Assessment of cognition in Schizophrenia (BACS).

Results.- SG-LAI groups showed a better cognitive function in all domains (except for motor speed and attention) than patients receiving FG-LAI. With SG-LAI, the most severely impaired cognitive domain was attention, whereas motor speed was barely affected. In contrast, verbal memory was most impaired, whereas motor speed was the least impaired cognitive domain in the group receiving FG-LAI (see Table 1 ) 


\begin{tabular}{|c|c|c|c|c|c|c|c|}
\hline & $\begin{array}{l}\text { Global } \\
\text { B.A.CSS }\end{array}$ & $\begin{array}{l}\text { Verbal } \\
\text { Memory }\end{array}$ & $\begin{array}{l}\text { Working } \\
\text { Memory }\end{array}$ & $\begin{array}{l}\text { Motor } \\
\text { speed }\end{array}$ & $\begin{array}{l}\text { Verbal } \\
\text { fluency }\end{array}$ & Altention & $\begin{array}{l}\text { Executive } \\
\text { function }\end{array}$ \\
\hline FG:LAI & $\begin{array}{c}-3.10 \\
( \pm 0,91) \\
\end{array}$ & $\begin{array}{c}-3.19 \\
( \pm 0.58) \\
\end{array}$ & $\begin{array}{r}.2 .76 \\
( \pm 1.13) \\
\end{array}$ & $\begin{array}{c}0.27 \\
( \pm 0.75)\end{array}$ & $\begin{array}{c}\cdot 1.97 \\
( \pm 0.54)\end{array}$ & $\begin{array}{c}-3.12 \\
( \pm 3.18) \\
\end{array}$ & $\begin{array}{c}.2 .67 \\
( \pm 1.26)\end{array}$ \\
\hline PP1M & $\begin{array}{c}.2 .47 \\
( \pm 1.23)^{*}\end{array}$ & $\begin{array}{c}2.28 \\
( \pm 1.04)^{*}\end{array}$ & $\begin{array}{c}\cdot 1.50 \\
( \pm 1.20)^{k *}\end{array}$ & $\begin{array}{c}0.05 \\
( \pm 0.74) \\
\end{array}$ & $\begin{array}{c}\cdot 1.39 \\
( \pm 0.53)^{14}\end{array}$ & $\begin{array}{r}.2 .70 \\
( \pm 0.57) \\
\end{array}$ & $\begin{array}{c}\cdot 1.45 \\
( \pm 1.67)^{*}\end{array}$ \\
\hline ALAI & $\begin{array}{c}.2,37 \\
( \pm 1,08)^{*}\end{array}$ & $\begin{array}{c}2.21 \\
( \pm 0.76)^{*}\end{array}$ & $\begin{array}{c}\cdot 1.45 \\
( \pm 1.02)^{* 4}\end{array}$ & $\begin{array}{c}0.07 \\
( \pm 0.70)\end{array}$ & $\begin{array}{c}.1 .28 \\
( \pm 0.48)^{* *}\end{array}$ & $\begin{array}{c}.2,65 \\
( \pm 2,77)\end{array}$ & $\begin{array}{c}.1,55 \\
( \pm 1.64)^{*}\end{array}$ \\
\hline
\end{tabular}

Table 1. Results in Brief Assessment of cognition in Schizophrenia (BACS) $\left({ }^{*} p<0.05 ;{ }^{* *} p<0.01\right)$.

Conclusions.- In our study, patients with schizophrenia receiving SG-LAI have better cognitive function than patients receiving FGLAI.

Disclosure of interest.- The authors have not supplied a conflict of interest statement.

\section{E-PV0874}

\section{Magical thinking impact on distortions of representations of self and other in paranoid schizophrenia}

E.T. Sokolova ${ }^{1}$, K. Andreyuk $^{1}$, I. Oleichik ${ }^{2}$, A. Ryzhov $^{1 *}$

${ }^{1}$ Lomonosov MSU, Psychology, Moscow, Russia; ${ }^{2}$ Mental Health Research Center, Department of Endogenous Mental Disorders and Affective States, Moscow, Russia

Corresponding author.

Background and aims.- Magical thinking phenomena attract researchers' attention due to their presence as an irrational form of thinking in lay conceptions, contributing to fantastically distorted representations of reality, social relationships and political events. In the case of psychiatric disorders magical thinking leads to unrealistic representations of one's illness, interpersonal relationships, methods of treatment and psychotherapy (Sokolova, 2014; 2017). Objectives of study were to trace the relationships between magical thinking, reasoning and complexity of representation of people. Methods.- Participants were 40 inpatients with paranoid schizophrenia and 40 controls. Magical thinking was assessed with SPQ-74 questionnaire (Enikolopov, Efremov, 2010). Cognitive processes were assessed with Sorting test. The complexity of representations of people was assessed with SCORS scale for Thematic Apperception Test (Westen, 2002).

Results.- Mean scores on SPQ-74 magical thinking were higher in inpatients groups $(U=341,000, p<0,001)$, and were related to cognitive distortions: circumstantial abstraction, loss of goal and illogical cognitive strategies driven by affects and autistic motives $(r=0,275, p<0,01)$. Moreover, there were differences in complexity of representations, with contradictory, chaotic and schematic representations in inpatients group contrasted to integrated and developed ones in control group $(U=127,000, p<0,001)$. Regression analysis pointed to causal relationships in the pattern of variables; complexity of representations, magical thinking $(\beta=-0,456$, $p<0,001)$ and difficulties of abstraction $(\beta=-0,320, p<0,01)$. Thus, generalizations accompanied by irrationality cause unidimensional representations of internal reality of Self and Other.

Conclusions.- Results indicate that magical thinking distorts interpersonal representations in direction of low differentiation, being mediated by deficiency of analysis and generalization.

Disclosure of interest.- The authors have not supplied a conflict of interest statement.

\section{E-PV0875}

\section{Schizotypal traits as predictors of understanding of social causality impairment}

\author{
E.T. Sokolova ${ }^{1}$, K. Andreyuk ${ }^{1}$, I. Oleichik ${ }^{2}$, A. Ryzhov ${ }^{1 *}$
}

${ }^{1}$ Lomonosov MSU, Psychology, Moscow, Russia; ${ }^{2}$ Mental Health

Research Center, Department of Endogenous Mental Disorders and

Affective States, Moscow, Russia

* Corresponding author.

Background and aims.- Understanding of Social Causality as one aspect of mentalization implies the ability to bind psychological intentions with manifest interpersonal behaviour. This metacognitive ability starts developing in early childhood provided there is a sensitive and containing attitude in mother-child dyad. Its deficiency in the context of marked clinical symptoms contributes to social maladjustment in personality disorders and reduces effectiveness of compliance and psychotherapy.

Objectives of study.- Analysis of relationships between schizotypal traits and understanding of social causality

Methods.- Participants were 40 inpatients with schizotypal disorder and 40 controls. Understanding of social causality was assessed by SCORS scale for Thematic Apperception Test (Westen, 2002), schizotypal traits were diagnosed with SPQ-74 questionnaire (Enikolopov, Efremov, 2010).

Results.- Between-groups differences were found in the level of understanding of social causality $(U=278,500, p<0,001)$. Logical faults and inconveniences, predominance of situational causality characterise inpatients group in contrast with complex appraisal of the role of psychic processes in social behaviour in control group. Regression analysis indicate causal relationships between understanding of social causality and blunted affect $(\beta=-0,380$, $p<0,001)$, bizarre behaviour $(\beta=-0,224, p<0,05)$ and magical thinking $(\beta=-0,225, p<0,05)$. The characteristics listed determine distanced and eccentric behavior with reasoning detached from reality. A person with such a traits fences from mental reality of people, incorrectly interprets their motives or ignores them and deprives himself from important resources of analysis of social situations.

Conclusions.- Schizotypal traits of blunted affect, bizarre behaviour and magical thinking result to be predictors of understanding of social causality impairment, distancing the person from othersand hampering communication.

Disclosure of interest.- The authors have not supplied a conflict of interest statement.

\section{E-PV0876}

\section{Tolerance of ambiguity as a predictor of cognitive complexity of mental representations in paranoid schizophrenia}

E.T. Sokolova, K. Andreyuk, L.S. Pechnikova, A. Ryzhov*

Lomonosov MSU, Psychology, Moscow, Russia

* Corresponding author.

Background and aims.- The Other's internal world is multivocal and dynamic. Relying on empathy and reflexivity a person constructs it 'on-line' under the conditions of epistemological ambiguity. Automatic repetition of fixed stereotypes deprives mental representations of cognitive and emotional complexity, blocks understanding of feelings and thoughts of significant others and impairs resolution of conflicts in close interpersonal relationships. Objectives.- to trace the relationships between tolerance of ambiguity and complexity of representations of people in paranoid schizophrenia.

Methods.- 40 inpatients diagnosed with paranoid schizophrenia were compared to 40 controls. Tolerance of ambiguity was assessed 
with the "Novel questionnaire of ambiguity tolerance-intolerance" (Kornilova, 2009), the complexity of representations of people was assessed by SCORS scale for Thematic Apperception Test (Westen, 2002).

Results.- Mean scores for complexity of representations of people were lower in inpatients $(U=127,000, p<0,001)$. Inpatients also scored lower in tolerance to ambiguity $(U=290,500, p<0,001)$. Regression analysis indicates causal relationships of complexity of representations and tolerance to ambiguity $(\beta=0,406, p<0,001)$. Tolerance to uncertainty of psychic space in interpersonal relationships activates resources for its exploration, while excessive anxiety from facing the new experience leads to the use of primitive defence mechanism of restraint to limits of habitual. The mental representations lose differentiation and integrity, hampering social communication.

Conclusions.- Attitude to ambiguity as current research shows constitute one of predictors of cognitive complexity of representations, with tolerance of ambiguity providing possibilities of multifocal analysis of personal dispositions and subjective experience while fly from ambiguity into the rigid limits of given leads to concreteness of representations.

Disclosure of interest.- The authors have not supplied a conflict of interest statement.

\section{E-PV0878}

\section{Psychosis in the elderly: “geppetto's syndrome"}

A. Sánchez Pavesi ", L. Povedano, R. Calles, L. Navarro, M. Alcántara, F. García, E. Hernández, M.R. Cejas Méndez

Hospital Universitario de Canarias, Psychiatry, Santa Cruz de

Tenerife, Spain

${ }^{*}$ Corresponding author.

Background and aims.- Psychosis and dementia has been reported extensively in elderly patients. Psychotic symptoms are present in over 50\% of Alzheimer's patients during the course of this progressive illness, and some authors have reported rates of behavioral disturbance up to $70 \%$.

Methods.- We present a case study of an 73-year-old woman, which was hospitalized due to behavioral disturbances related to the fact that, as reported by herself, she had been raising up a child (which was actually a child size doll) as her mother's child, whom had passed away more than 20 years ago, and upon her deathbed, had told her to take care of this child.

The patient had lost its complete perception of reality, and had clear onset symptoms of Alzheimer's type dementia. Nevertheless, she had a calm state of mind, and was hopeful to carry out her mother's task. The patient was unmarried and hadn't been able to conceive in her lifetime. This delusional symptom had initiated around a year ago.

Results.- After our exam, we decided to release this woman without any antipsychotic medication, due to the fact that, this patient had developed a clear relationship with its doll, she had finally developed her motherhood desires, and had an ongoing degenerative disease which was rapidly advancing.

Conclusions.- Elderly patients presenting with psychotic symptoms require social, behavioral, and environmental interventions that are necessary for their safety and reorientation. Given the likelihood of comorbid medical disorders and concomitant medications, the mere presence of delusions or hallucinations is not always an indication for additional medications.

Disclosure of interest.- The authors have not supplied a conflict of interest statement.

\section{E-PV0879 \\ Long-acting antipsychotics in delusional disorder: a case report}

E. Segura ${ }^{1^{*}}$, C. Rodriguez Gomez Carreño ${ }^{2}$, L. Asensio Aguerri² ${ }^{2}$ I. Gomez Jimenez ${ }^{2}$, L. Nuevo Fernandez ${ }^{2}$, A. Ramirez Garcia ${ }^{2}$

${ }^{1}$ Associate prof, Psychiatry, Ciudad real, Spain; ${ }^{2}$ Ciudad Real, Spain

* Corresponding author.

Background and aims.-

Introduction.- The delusional disorder is included in psychotic disorders, and is characterized by long-time and structured delusional ideas, that involve a field of the life of the patient. It is infradiagnosed, and with low response to pharmacological treatment in clinical practice.

Methods.- We analyze a literature review of the treatment of chronic delusional disorder, and we present the clinical case of a patient with long-term diagnosis and treatment with paliperidone palmitate quarterly.

Results.- A 56-year-old patient with a diagnosis of delusional disorder at the age of 30 , with a clinical characterized by structured delusions of prejudice. Throughout the evolution it has been treated with different antipsychotics, both oral and long-term. She had a tendency to relapse with frequent admissions and abandonment of treatment. Four years ago, we introduced monthly paliperidone palmitate, and for 18 months after, it was changed to the quarterly formulation. In spite of the fact that the delirious Clinic does not completely remit, it improves the adherence, with compliance with the treatment and monthly monthly visits that are maintained. There is also a functional improvement. Following this clinical case, we made a review of the treatment of delusional disorder. We found that there is not enough scientific evidence regarding the recommendations for approaching this disorder. Presumably, the recommendations for schizophrenia and other psychotic disorders could be applied, but the lack of studies does not make it possible. Conclusions.- The description of a clinical case does not provide scientific evidence, but the results encourage to promote studies with new long antipsychotics.

Disclosure of interest.- The authors have not supplied a conflict of interest statement.

\section{E-PV0880}

\section{Sexual impairment in schizophrenic patients \\ A.C. Serban*, R. Paunescu, I. Miclutia \\ University of Medicine and Pharmacy Cluj Napoca, Psychiatry, Cluj \\ Napoca, Romania \\ * Corresponding author.}

Background and aims.- Sexual dysfunctions in schizophrenic patients have a debilitating emotional impact on the quality of life, with an average onset at a young age. It seems that schizophrenia itself has a strong component in SD, explained mainly by the symptomatology and the chronicity. Another cause of SD is antipsychotic medication.

This paper aims to highlight that using Quetiapine as an elective drug in the treatment of schizophrenia could greatly reduce the occurrence of these adverse drug reactions among patients, thus improving their quality of life.

Methods.- Study searches were made using the terms "schizophrenia," "sexual dysfunction" and "quetiapine" in the Science Direct, Pumed, Medline, Cochrane, Embase and Oxford Journals databases. We included studies involving patients over the age of 18 , admitted during 2004-2015, diagnosed with any subtype of schizophrenia, having sexual dysfunction and treated with Quetiapine; we applied the Arizona Sexual Experience Scale.

We excluded studies involving patients with schizophrenia-like illnesses or other psychiatric illnesses, as well as trials for other 
antipsychotics, except for Quetiapine, and those not using the ASEX scale to assess sexual dysfunction.

Results.- We achieved an overall positive result indicating a higher rate of lowering secondary sexual dysfunction in patients treated with Quetiapine by about $58.71 \%$ compared to the rest of the antipsychotics (ranging from 0.003 to 1.058).

Conclusions.- Quetiapine appears to have a better effect on the improvement of sexual symptom compared to other antipsychotics, in schizophrenic patients. Switching from a classic or atypical antipsychotic to Quetiapine could significantly reduce the rate of sexual dysfunction and improve it if it exists.

Disclosure of interest.- The authors have not supplied a conflict of interest statement.

\section{E-PV0881}

\section{Emotional intelligence and clinical symptoms in schizophrenia}

V. Shemet ${ }^{1^{*}}$, U. But-Husaim ${ }^{2}$

${ }^{1}$ Grodno State Medical University, Department of Psychiatry and Addiction Medicine, Grodno, Belarus; ${ }^{2}$ Grodno State Medical University, Department of Medical Rehabilitation, Grodno, Belarus ${ }^{*}$ Corresponding author.

Background and aims.- Schizophrenia is a debilitating disorder associated with emotional processing deficits that are crucial for adaptive functioning.

The aim of this study was to explore relationships between emotional intelligence and clinical symptoms.

Methods.- Examination results of 97 subjects with schizophrenia (according to ICD- 10 criteria) were analyzed in this study. We employed the Mayer-Salovey-Caruso Emotional Intelligence Test (MSCEIT), the Scale for the Assessment of Negative Symptoms (SANS) and the Scale for the Assessment of Positive Symptoms (SAPS). The data obtained were analyzed by Spearman's correlation coefficient.

Results.- The results showed that there is a significant negative correlation for all of the components of the MSCEIT and the anhedonia-asociality. The Identifying Emotions, Understanding Emotions, Managing Emotions and MSCEIT total scores were also significantly negatively correlated with the affective flattening. In addition, the Identifying Emotions and MSCEIT total scores were significantly negatively related with the avolition-apathy subscales. Our research also shows significant association between components of the MSCEIT and a few positive symptoms: negative correlation for all of the MSCEIT components and bizarre behavior, Identifying Emotions, Managing Emotions with thought disorders; positive correlations for Identifying Emotions, Using Emotions, MSCEIT total scores with delusions.

Conclusions.- Further research is required for understanding the relationship between emotional intelligence and clinical symptoms in schizophrenia. In addition, specific intervention of emotional intelligence development programs may be useful in supporting people with schizophrenia to achieve more satisfying levels of recovery.

Disclosure of interest.- The authors have not supplied a conflict of interest statement.

\section{E-PV0882}

\section{Use of virtual reality in treatment of refractory auditory hallucinations in schizophrenia: a literature review}

T. Skoneczny ${ }^{1}{ }^{*}$, E. Tyburski ${ }^{2}$, J. Kucharska-Mazur ${ }^{1}$, J. Samochowiec ${ }^{1}$

${ }^{1}$ Pomeranian medical university, Departament of psychiatry, Szczecin, Poland; ${ }^{2}$ University of Szczecin- Institute of psychology, Department of clinical psychology, Szczecin, Poland

${ }^{*}$ Corresponding author.
Background and aims.- Despite pharmacotherapy, many schizophrenia patients still suffer from refractory auditory hallucinations. Novel treatment options are being investigated. To date, virtual reality (VR) enabling creation of a safe, controlled environment has been proven effective in treatment of various mental disorders. This article reviews currently available literature on the use of different forms of VR in therapy of persistent auditory verbal hallucinations $(\mathrm{AVH})$ in schizophrenia patients.

Methods.- The PUBMed, PMC, Scopus, ProQuest, Oxford Academic Journals database searches using the terms "Schizophrenia" AND "Hallucinations" AND "Virtual Reality" returned the total of 438 records. Included papers investigated clinical use of VR in treatment of refractory AVH in schizophrenia patients. All articles were available in English.

Results.- 6 articles were included in this review: two case reports, two single-blind, randomized controlled trials, a randomized, single blind, partial crossover trial and a randomized, partial crossover trial. All studies examined the use of therapist-controlled, 3-dimensional virtual AVATAR and voice transformer to closely resemble patient's hallucinations presented either on flat screen, or using immersive 3D goggles. All studies reported improvement in AVH severity, advantage in using VR vs. treatment-as-usual and all but one reported improvement in depressive symptoms. One trial reported advantage in using AVATAR therapy vs. standard cognitive-behavioral therapy, and one suggested that AVATAR presentation via immersive $3 \mathrm{D}$ goggles rather than flat screen yields even better results.

Conclusions.- Although limited, available sources propose promising new interventions for treatment of refractory AVH in schizophrenia. Further investigation is required.

Disclosure of interest.- The authors have not supplied a conflict of interest statement.

\section{E-PV0883 \\ Insight in schizophrenia: gender differences}

I. Torio*, M. Caballero-Gonzalez, E.M. Sanchez-Morla, D. Rentero, M. Dompablo, R. Rodriguez-Jimenez

Instituto de Investigación Hospital 12 de Octubre imas12,

Department of Psychiatry, Madrid, Spain

Corresponding author.

Background and aims. - Partial or total unawareness is present in 50$80 \%$ of patients with schizophrenia. Poor insight is associated with worse treatment compliance, more hospital admissions, poorer functional outcome, and a higher severity of illness. While gender differences on age of onset, prevalence, and outcomes have been widely studied on schizophrenia, scarce and mixed data exists on gender differences on insight (Pruß et al., 2012; Parellada et al., 2013). The Scale to Assess Unawareness of Mental Disorder (SUMD) is a widely accepted and validated scale that evaluates several insight domains (Amador et al., 1993). The aim of the present study was to study gender differences on insight in a sample of patients with schizophrenia.

Methods.- A cross-sectional study was conducted in a sample of 103 patients (31.1\% female). The mean age was 41.5 years (SD 8.5). Insight was measured with the three general items of the SUMD (awareness of mental disorder, awareness of need for treatment, and of social consequences of the disorder). T-student tests were performed with SPSS version 23.

Results.- A significant difference for gender was observed in awareness of having a mental disorder $(t=-2.770 ; p=.007)$, and the total attribution of symptoms item $(t=-2.207 ; p=.030)$. No significant differences were obtained for awareness of the effects of treatment, awareness of social consequences, and total awareness of symptoms.

Conclusions.- On our sample, gender differences on two insight domains were observed. While further studies are necessary to 
determine if gender differences exist on insight determinants, our results might help the development of gender-specific insight interventions.

Disclosure of interest.- The authors have not supplied a conflict of interest statement.

\section{E-PV0884 \\ Schizophrenia and electroconvulsive therapy: hospital 12 de Octubre (Madrid)}

I. Torio ${ }^{*}$, M. Caballero-Gonzalez, E.M. Sanchez-Morla, M. Dompablo, D. Rentero, R. Rodriguez-Jimenez

Instituto de Investigación Hospital 12 de Octubre imas12, Department of Psychiatry, Madrid, Spain

* Corresponding author.

Background and aims.- Electroconvulsive therapy (ECT) was first applied in Italy in 1938 for the treatment of psychosis. The following years the ECT had a variable and often controversial use. Recent studies show that ECT, combined with antipsychotics, may be a therapeutic option for patients with schizophrenia, specially when an immediate recovery is required, or when show limited response to medication (Tharyan P \& Adams CE, 2005). Despite this findings, the ECT use in schizophrenia remains controversial. The aim of the present study was to analyse patients with schizophrenia who received ECT treatment at the 12 de Octubre Hospital in Madrid.

Methods.- A naturalistic study was conducted by analysing a one year period of activity. Included patients and their characteristics are described below. Included patients were refractory to pharmacological treatment, with various and severe psycotic episodes with, in not few cases, high suicide risk.

Results.- A total of twelve patients with a diagnosis of schizophrenia received treatment with ECT on the year 2017. Patients mean age was 41.5 years (SD 12.5) and 33\% of patients were female. Of these, 8 patients received acute ECT, while four were included in the continuation/maintenance (C/M) ECT Program. Three of those who were on the $\mathrm{C} / \mathrm{M} \mathrm{ECT}$ treatment received the treatment on ambulatory basis, while the other required previos day admission. Conclusions.- For the last decade, ECT administration has been sistematized and its risks lessened. It is a technique with virtually no contraindication for its administration, and it is nowadays a safe and effective treatment for certain patients with schizophrenia. Disclosure of interest.- The authors have not supplied a conflict of interest statement.

\section{E-PV0885}

\section{Current situation of first episode psychotic patients}

I. Torio*, E.M. Sanchez-Morla, D. Rentero, M. Dompablo, M.

Caballero-Gonzalez, R. Rodriguez-Jimenez

Instituto de Investigación Hospital 12 de Octubre imas12,

Department of Psychiatry, Madrid, Spain

${ }^{*}$ Corresponding author.

Background and aims.- Important efforts are being made to provide an early and specialized care to first-episode psychosis patients. A successful development of this programs require an adequate understanding of the patients they are aiming at. Thus, we intend to analyse the characteristics of first-episode psychosis patients admitted to our psychiatry unit, and to evaluate the change on severity of symptoms.

Methods.- A retrospective study was conducted on the 31 firstepisode patients admitted during 2017. The mean age was 25.3 years (SD 4.3). 39\% were female. A t-test for related samples was conducted to analyse the change on severity of symptoms mea- sured with the Clinical General Impression Scale, severity subscale (CGI-s). Patients older than 40 years were excluded.

Results.- Duration of untreated psychosis (DUP) was 7.3 months (SD 9.1). $45 \%$ of patients $(n=14)$ had previous contact with a mental health professional. Of these, 4 had received an ADHD diagnosis, while 4 had recent contacts in mental health services related to anxiety and/or depressive symptoms. Only 5 patients (16\%) had previous mental health contacts related to psychotic symptoms. At admission 58\% $(n=18)$ had cannabis use disorder, and 32\% $(n=10)$ alcohol use disorder.

The mean CGI-s score at admission was 5.2 (SD 0.8), while at discharge was 2.9 (SD 0.8). The change on CGI was statistically significant $(\mathrm{t}=10.5 ; p=.00)$.

Conclusions.- First-episode patients require interdisciplinary and individualized intervention plans, including those aimed at decreasing drug abuse and favouring early diagnosis. In our hospital, a mean of three first episode patients were admitted per month, and substance abuse was frequent.

Disclosure of interest.- The authors have not supplied a conflict of interest statement.

\section{E-PV0887 \\ First episode of schizophrenia is followed by disturbances in serum albumin antioxidant function}

M. Uzbekov ${ }^{1}$, V. Brilliantova ${ }^{1}$, N. Smolina ${ }^{1}$, S. Shikhov ${ }^{1}$, G. Dobretsov $^{2}$, I. Strelkova ${ }^{1}$, T. Syrejshchikova ${ }^{3}$

${ }^{1}$ Moscow Research Institute of Psychiatry, Brain Pathology, Moscow, Russia; ${ }^{2}$ Research and Clinical Center of Physic-Chemical Medicine, medical biophysics, Moscow, Russia; ${ }^{3}$ Lebedev Physical Institute, High Energy, Moscow, Russia

* Corresponding author.

Background and aims.-

Introduction.- Early intervention at first episode of schizophrenia (FES) accelerates onset of remission and reduces social losses. Objectives.- Serum albumin molecule has one reduced SH (thiol)group at Cys-34 position. Albumin thiols compose up to $80 \%$ of all detectable high-molecular-weight antioxidant thiols of plasma. Aim.- To study changes in concentration and reactivity of albumin thiol groups in FES patients.

Methods.- There were investigated 26 patients with FES and 10 healthy controls.

Serum albumin SH-group concentration was determined in Ellman's reaction in presence of detergent; reactivity of albumin $\mathrm{SH}$ groups was evaluated by modified Ellman's reaction in absence of detergent.

Results.- Severity of disorder before treatment was $75.5 \pm 2.2$ according to PANSS score. Comparison of concentration of albumin thiol groups in healthy individuals and patients with FES showed a tendency to decrease (for 30\%, $p=0.366$ ) in group of patients. Kinetic coefficient Kv of thiol serum albumin was measured in reaction with thiol-specific reagent - dithiobisnitrobenzoic acid. Investigation of reactivity of $\mathrm{SH}$ groups, retained in albumin fraction of initial blood serum, has revealed a significant decrease in KV in FES in comparison with controls $0.17 \pm 0.02 \mathrm{~min}^{-1}$ vs. $0.25 \pm 0.03 \mathrm{~min}^{-1}$, respectively, (by24\%, $p=0.05$ ). Moreover, patients with FES can be divided into two groups - with high $\mathrm{Kv}(0.38 \pm 0.02 ; n=6)$ and with low Kv $(0.12 \pm 0.01 ; n=21)$ (both $p=0.05)$ in comparison with controls $(0.27 \pm 0.03 ; n=10)$.

Conclusions.- Group of patients with FES is heterogeneous, that may require new differentiated approaches to pharmacotherapy of FES. Disclosure of interest.- The authors have not supplied a conflict of interest statement. 


\section{E-PV0888}

\section{Schizophrenia and opioid use disorder dual diagnosis - a case series}

D. Vasile*, O. Vasiliu, D.G. Vasiliu, F. Vasile

University Emergency Central Military Hospital “Dr. Carol Davila”,

Psychiatry Department, Bucharest, Romania

Corresponding author.

Background and aims.- Schizophrenia is highly comorbid with substance use disorders, data in the literature reaching values of 60-70\% for this dual diagnosis. Opioid use disorder is a worldwide continuously developing health problem, affecting patients rate of survival through significant premature death and invalidating secondary somatic diseases.

The objective is to present the evolution of two patients diagnosed with opioid use disorder and schizophrenia during 12 months of psychotropic treatment.

Methods.- Two patients who were diagnosed with schizophrenia and opioid use disorder, according to the DSM-5 diagnosis criteria, were evaluated monthly during one year treatment with long acting atypical injectable antipsychotics and an opioid receptors antagonist.

Results.- The first patient was 30-year old and presented a baseline Positive and Negative Syndrome Scale (PANSS) score of 92, with a Global Assessment of Functioning Scale (GAF) score of 30. After stabilization with oral olanzapine and concomitant detoxification from heroin, he received treatment with olanzapine pamoate $300 \mathrm{mg}$ every 2 weeks and $50 \mathrm{mg}$ QD naltrexone. The second patient was 35-year old and presented initial PANSS score of 107 and a 25 GAF score. After detoxification and stabilization, he received paliperidone palmitate $100 \mathrm{mg}$ monthly and naltrexone $50 \mathrm{mg}$ QD. Both patients had a favourable evolution at 12-month, as the PANSS scores decreased with $54.3 \%$, and $59.8 \%$, respectively. The GAF scores also decreased with more than $50 \%$ in both cases.

Conclusions.- The evolution of patients diagnosed with both opioid use disorder and schizophrenia during long-acting injectable atypical antipsychotics and naltrexone was favourable during 12-month treatment.

Disclosure of interest.- The authors have not supplied a conflict of interest statement.

\section{E-PV0889}

\section{Treatment of schizophrenia in late life-clinical vignettes \\ O. Vasiliu \\ Dr. Carol Davila" University Central Military Hospital, Psychiatry, Bucharest, Romania \\ * Corresponding author.}

Background and aims.- Late-onset schizophrenia (LOS) is a controversial psychiatric disorder and, since there are no guidelines for this specific population, a case-by-case analysis including somatic comorbidities and pharmacological interactions should be made prior to the treatment initiation.

The objective was to evaluate the medium and long-term impact of the antipsychotic treatment on LOS-diagnosed patients.

Methods.- Three patients diagnosed with schizophrenia, according to the DSM-5 diagnosis criteria, which presented the onset of their disorder after age of 40, were evaluated monthly during 12 -months treatment, with a 24-month follow-up.

Results.- The first patient was 50-year old and he had also tobacco use disorder and COPD. His baseline Clinician-Rated Dimensions of Psychosis Symptom Severity (CRDPSS) score was 13, and Positive and Negative Syndrome Scale (PANSS) score was 89 . He received treatment with olanzapine $20 \mathrm{mg}$ QD and nicotine replacement therapy, which lead to significant improvements (72.2\% decline on CRDPSS and $70.6 \%$ on PANSS). The second patient was 62 -year old, she also had alcohol use disorder, and she received treatment with risperidone $6 \mathrm{mg}$ BID and naltrexone $50 \mathrm{mg}$ QD. Her evolution was good, but she relapsed after 20 weeks, when she was switched on quetiapine $600 \mathrm{mg}$ QD. The third patient was 48 -year old, and she had also the diagnosis of schizoid personality disorder. She was initiated on aripiprazole $30 \mathrm{mg}$ BID and after stabilization she received monthly-administered long-acting aripiprazole, with favourable evolution.

Conclusions.- The evolution of patients diagnosed with LOS during atypical antipsychotic treatment was favourable, with most of the psychotic symptoms remitting during the first 6 months.

Disclosure of interest.- The authors have not supplied a conflict of interest statement.

\section{E-PV0890}

\section{The disturbances in language in patients with schizophrenia: word association, verbal fluency and vocabulary}

M. Víchová

Department of Psychology- Faculty of Arts- Masaryk University in Brno, Departement of Psychology, Brno, Czech Republic

Corresponding author.

Background and aims.- In this poster we present the results of studies included in the first author's Dissertations Thesis, which focus on language in patients with schizophrenia. In three studies we emphasised the disturbances in the speech, language and thinking among Czech people with this psychiatric illness.

Methods.- We compare qualitatively and quantitatively the scores in three diagnostic tests: Word association test, Verbal fluency test and Vocabulary (from the Wechsler Adult Intelligence Scale) with nonclinical sample.

Results.- We found a specific way in the associations, clustering and switching in verbal fluency and verbal description ability of words which is different from people without schizophrenia.

Conclusions.- The results are in accordance with the previous studies in other languages. The main goal of this poster is to summarize the results of these separate studies and to describe the general conclusion.

Disclosure of interest.- The authors have not supplied a conflict of interest statement.

\section{E-PV0891 \\ Depression and neurocognitive impairment: evaluation of their role as predictors of auditory $\mathrm{N100}$ in schizophrenia}

A. Vignapiano ${ }^{1}$, A. Mucci ${ }^{1}$, G.M. Giordano ${ }^{1}$, G. Di Lorenzo ${ }^{2}, \mathrm{~F}$. Ferrentino $^{2}$, M. Altamura ${ }^{3}$, A. Bellomo ${ }^{3}$, S. Galderisi ${ }^{1}$

${ }^{1}$ University of Campania "Luigi Vanvitelli", of Psychiatry, Naples, Italy; ${ }^{2}$ University of Rome "Tor Vergata", of Systems Medicine, Rome, Italy; ${ }^{3}$ University of Foggia, of Clinical and Experimental Medicine,

Foggia, Italy

Corresponding author.

Background and aims.- In schizophrenia, N100 amplitude reduction, which is thought to reflect impairment in early auditory sensory processing and initial selective attention, and its relationship with positive symptoms have been reported in several studies. Instead, on N100 latency, few data have been described and the findings are not robust. Until now, no investigation focused on N100 latency and its relations with different psychopathology dimensions and neurocognitive impairment in subjects with schizophrenia (SCZ).

In the context of a multicenter study of the Italian Network for Research on Psychoses, we attempted to disambiguate the role of 
psychopathology and neurocognitive impairment on N100 latency in schizophrenia.

Methods.- During an auditory odd-ball task, ERPs were recorded in 115 chronic stabilized SCZ and 64 healthy controls (HC). State of art instruments were used to assess psychopathology, neurocognitive functions and extrapyramidal side effects.

Multiple stepwise linear regressions were used to establish the variables that predicted N100 latency for standard and target stimuli. As independent variables, depression, parkinsonism, positive and disorganization dimensions, neurocognitive composite score, avolition/apathy and expressive deficit domains, were included in the model.

Results.- We did not observe group differences with respect to N100 latency for both stimuli. Regression models revealed that N100 latency for standard tone was predicted by "Depression" $(b=0.256$, $p=0.010$ ), instead for target tone "Neurocognitive composite score" was the independent predictor $(b=-0.211, p=0.026)$.

Conclusions.- Our results revealed that depression and global cognitive impairment in SCZ affected differently the early processing of task-relevant and frequent stimuli, suggesting their contribution in the activation of complex network of the auditory cortical areas. Disclosure of interest.- The authors have not supplied a conflict of interest statement.

\section{E-PV0892}

\section{Endophenotypes of psychotic illness: a pilot study in patients with psychosis and their healthy siblings}

V. Voráčková1,2* ${ }^{*}$ F. Španiel ${ }^{1,2}$, M. Rodriguez ${ }^{1}$, P. Knytl $^{2,3}$, A. Dorazilová ${ }^{1}$, P. Šustová ${ }^{1}$, J. Renka ${ }^{2,3}$, P. Mohr ${ }^{2,3}$

${ }^{1}$ National Institute of Mental Health, Applied Neurosciences and Brain Imaging, Klecany, Czech Republic; ${ }^{2}$ Third Faculty of Medicine, Neuroscience, Prague, Czech Republic; ${ }^{3}$ National Institute of Mental Health, Diagnostics and Treatment of Mental Disorders, Klecany, Czech Republic

* Corresponding author.

Background and aims.- In recent years, there has been a surge of studies in patients with schizophrenia and their relatives aiming to identify endophenotypes of the disorder (Zheutlin et al., 2016). Cognitive performance/deficit is one of the most promising candidates; however, research studies were not able so far to pinpoint the weaknesses in specific cognitive domains. Underlying mechanisms of cognitive deficit in schizophrenia can be studied with neuroimaging techniques, e.g., investigating metabolic changes in the specific brain regions.

The primary aim of our study was to describe specific cognitive domains that can be perceived as schizophrenia endophenotypes. The secondary aim was to examine whether cognitive impairment in siblings of patients is associated with metabolic changes in the anterior cingulate cortex (ACC).

Methods.- The study included 84 subjects in 4 groups: (1) patients with the first episode of psychotic disorder; (2) siblings of patients; (3) and (4) control groups. All subjects were administered a battery of neuropsychological tests and they underwent a MRI examination.

Results.- The data showed attenuation in cognitive functions of patient's siblings compared to healthy controls, but we did not find any difference in the choline and $\mathrm{N}$ - acetylaspartate concentrations between siblings and controls.

Conclusions.- Our results confirm that cognitive deficit can be an endophenotype of psychotic illness, as its weakness is evident in siblings. MRI analysis did not revealed metabolic changes in the ACC in a smaller sample of siblings. The future research should expand a study sample. Supported by MH CR AZV 15-28998A; GAUK 1582517; MEYS NPU4NUDZ: LO1611.
Disclosure of interest.- The authors have not supplied a conflict of interest statement.

\section{E-PV0893 \\ Gender differences in the assessment of negative symptoms using second generation rating scales - BNSS and} SNS

P. Wójciak ${ }^{1 *}$, K. Górna² ${ }^{2}$ K. Domowicz ${ }^{1}$, K. Jaracz ${ }^{3}$, K.

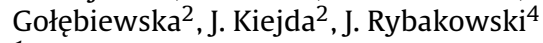

1 Poznań University of Medical Sciences, Department of Adult Psychiatry, Poznań, Poland; ${ }^{2}$ Poznań University of Medical Sciences, Department of Psychiatric Nursing, Poznań, Poland; ${ }^{3}$ Poznań University of Medical Sciences, Department of Neurological Nursing, Poznań, Poland; ${ }^{4}$ Poznań University of Medical Sciences, Department of Child and Adolescent Psychiatry, Poznań, Poland

* Corresponding author.

Background and aims.-

Introduction.- Recently, the novel rating scales for the assessment of negative symptoms of schizophrenia such as the Brief Negative Syndrome Scale (BNSS) and the Self-evaluation of Negative Symptoms (SNS) have been introduced. The Polish versions of both scales have been created.

Objectives.- To study possible gender differences in the assessment of negative symptoms by the BNSS and SNS scales.

Methods.- The study involved 40 patients (20 men and 20 women) with paranoid schizophrenia (ICD-10). They were in the period of symptomatic stabilization (total PANSS score $<80$ points) and had received unchanged pharmacological treatment during the last three months. The Polish versions of the BNSS and SNS were used. Results.- The patients did not show gender differences as to age and duration of the illness. The mean of total PANSS score was higher in men than in women (52 vs. 46 points). Negative symptoms evaluated by the BNSS clinical scale showed significantly greater scores in men compared to women in asociality (internal experience and total), avolition (internal experience and total) and alogia (spontaneous elaboration). On the other hand, no gender differences in the evaluation of negative symptoms by the self-assessment SNS were observed.

Conclusions.- The results suggest a significant gender effect in three subscales of negative symptoms, assessed by the clinical BNSS. The lack of such differences in the same patients performing the self-assessment indicates that clinicians may tend to assign higher scores on asociality, avolition and alogia to male schizophrenic patients compared to females

Disclosure of interest.- The authors have not supplied a conflict of interest statement.

\section{E-PV0894}

\section{Effects of second-generation} antipsychotics versus first-generation antipsychotics on quality of life in schizophrenia: a meta-analysis of clinical trials

P. Zagożdżon*, P. Waszak

Medical University of Gdansk, Department of Hygiene and

Epidemiology, Gdansk, Poland

* Corresponding author.

Background and aims.- It is not clear whether second-generation antipsychotics (SGAs) represent an advantage over first-generation antipsychotics (FGAs) in terms of quality of life improvement in of schizophrenia. We conducted a meta-analysis of randomized controlled trials examining the effect of different types of antipsychotics on the quality of life of patients with schizophrenia. 
Methods.- We searched the main health-related databases for relevant papers with randomized studies comparing the effect of SGAs on quality of life to FGAs or to clozapine. Then, we examined the titles and abstracts of the retrieved papers, applying exclusion criteria to filter out irrelevant papers; a more in-depth filtering process was then conducted by reading the full texts. Six studies remained at the end of this process. Next, we performed data extraction and assessed the methodological quality of the selected studies. This was followed by computation of effect sizes and the heterogeneity of the results, and then an assessment of the potential bias.

Results.- The systematic review found that only one trial in these six studies have a significant effect on quality of life. Quality of life scores were significantly higher in patients with schizophrenia given SGAs than in those treated with FGAs. There was no beneficial effects of SGAs when compared to clozapine.

Conclusions.- This meta-analysis provides evidence for the beneficial effects of SGAs treatment on the quality of life of schizophrenia patients. It is, however, suggested that further studies be conducted on this topic to assess the role of clozapine on quality of life outcomes when compared to other SGAs.

Disclosure of interest.- The authors have not supplied a conflict of interest statement.

\section{E-PV0895}

\section{Differences in psychopharmacological medication in the treatment of patients with schizophrenia, depending on the presence of aggressive behavior}

M. Zholob ${ }^{1^{*}}$, M. Zelman ${ }^{2}$

${ }^{1}$ Psychiatrická nemocnica Hronovce, Gerontopsychiatric Department, Hronovce, Slovak Republic; ${ }^{2}$ Psychiatrická nemocnica Hronovce, Acute Female Department, Hronovce, Slovak Republic Corresponding author.

Background and aims.- Aggressive behavior in patients with schizophrenia is often the main reason of their hospitalization and many times also one of the symptoms of relapse of psychotic disorder. If the intensity of violent behavior is not reducible nonmedically, we proceed to pharmacological therapy.

In our report, we bring the results of an intergroup comparison of the rate of classical and atypical antipsychotic medicaments in patients with schizophrenia, in dependence on the presence of aggressive behavior at baseline or prior to hospitalization to our hospital.

Methods.- Research was realized between the years 2016 and 2018 in patients hospitalized at the acute male ward of Psychiatric Hospital Hronovce. Our study group consisted of 65 patients hospitalized with a diagnosis of psychotic disorders without the comorbidity of substance abuse; this group was divided into 2 subgroups according to criteria of aggression. The medical treatment was monitored in 2 time intervals.

Results.- The group of aggressive patients included 21 men hospitalized with a history of aggressive behavior at baseline (age 18 to 61 years). Second, non-aggressive group included 44 hospitalized patients without a history of aggressive behavior (age 19 to 61 years). Drug groups, administration forms and daily doses were monitored at the beginning and at the end of hospitalization - when the patient was taken to outpatient care.

Conclusions.- Our findings show higher prevalence of indication of depot forms and also higher dosing of antipsychotics in the group of aggressive patients. Classic antipsychotics were used more often in the group of aggressive patients.

Disclosure of interest.- The authors have not supplied a conflict of interest statement.

\section{E-PV0896}

\section{Relationship of aggressive behavior and depressive symptoms in paranoid schizophrenia patients \\ K. Zhyvaho}

Bogomolets National Medical University, Psychiatry and Abuse, Kyiv, Ukraine

" Corresponding author.

Background and aims.- The problem of depression among patients with schizophrenia is actual question in the context of readaptation in social community and improving of the quality of life. Stigma, isolation and lack of social support reinforces the statement of that schizophrenia patients are aggressive. Not sufficiently investigated the interdependence of depressive symptoms and aggressive behavior in these patients.

Investigate relationship of depressive symptoms and aggressive behavior among paranoid schizophrenia patients.

Methods.- We examined 81 paranoid schizophrenia patients: 44 - with depressive symptoms and 37 - without it. Our investigation is based on a complex examination included: PANSS scale, The Calgary depression scale, RFL scale, Buss-Durkey Inventory and Boyko's Definition of integral forms of communicative aggression inventory.

Results.- Patients of both groups showed no differences in demonstration of oral or physical aggression. Also, inability to switch aggression on to inanimate objects was high in both groups. Differences were identified in self-aggressive subscale and were statistically significantly higher in group of patients with depressive symptoms. In this group also identified higher level of spontaneity of aggression and disability to stall it. These patients showed statistically significantly lower score of RFL scale with prevalence subscales: responsibility to family, child-related concerns, moral objections.

Conclusions.- Results showed that depressive symptoms facilitate increasing of the level of self-aggression among paranoid schizophrenia patients. Psychotherapeutic correction should be based on existing family values and aimed at strengthening of moral principles and belief in life. This direction requires further research and development of the effective methods of medical and psychotherapeutic correction.

Disclosure of interest.- The authors have not supplied a conflict of interest statement.

\section{E-PV0897}

\section{Psychotic disorder in post-traumatic brain injured patients: a case report}

Z. Boudali, S. Ellouze, F. Znaidi", N. Ghazouani, R. Ghachem

Razi Hospital, psychiatry B, Manouba, Tunisia

Corresponding author.

Background and aims.- People surviving a traumatic brain injury are at an increased risk of developing psychotic disorders. In this work, we propose to study, through a case presentation and a review of the literature, the mechanisms along with the clinical and evolutionary characteristics of psychoses following a brain injury.

Methods.- We report retrospectively the case of a patient who presented a psychotic disorder in the aftermath of a traumatic brain injury. A review of the literature was also conducted using the databases "PubMed" and "ScienceDirect" and the keywords "cranial trauma”, "psychosis", "schizophrenia”.

Results.- Mr L.N is 26 years old. He has no medical history. He was an English language student in a Tunisian university. His family describes him as a wise and sociable boy. In March 2014, the patient was a victim of a public road accident resulting in severe cranial trauma with loss of consciousness and was hospitalized in a resuscitation department. In 2016, about two years after his 
traumatic injury, he developed a delusional syndrome (persecution, bewitchment and filiation), auditory hallucinations and few dissociative elements. He was treated with neuroleptics with good clinical improvement.

Conclusions.- Psychoses emerging after a brain injury are not rare in psychiatry. The authors hypothesize that traumatic brain injuries lead to neuronal reshaping which could cause impairments in subjects vulnerable to schizophrenia. Nevertheless, many questions remain open to determine the role played by physical and emotional trauma as well as the complex interaction between pre-, perand post-traumatic factors.

Disclosure of interest.- The authors have not supplied a conflict of interest statement.

\section{E-Poster Viewing - 7-9 April: Sexual Medicine and Mental Health}

E-PV0898

\section{Shame traumatic memories and borderline personality traits in transgender people: the mediating role of self-disgust and self-compassion}

F. Carvalho ${ }^{1^{*}}$, M.J. Martins ${ }^{1}$, L. Fonseca ${ }^{2}$, G. Santos ${ }^{2}$, P. Castilho ${ }^{1}$

${ }^{1}$ Centro de Investigação em Neuropsicologia e Intervenção

Cognitivo-Comportamental, Universidade de Coimbra, Coimbra, Portugal; ${ }^{2}$ Centro Hospitalar e Universitário de Coimbra, Centro de Responsabilidade de Psiquiatria, Coimbra, Portugal

${ }^{*}$ Corresponding author.

Background and aims.- Shame traumatic memories are reference points for personal identity and meaning attribution to other experiences present in the individual's personal development. Literature suggests there is a an association between youth traumatic experiences, in the forms of shame, humiliation and rejection and the development of gender dysphoria in transsexual individuals. Moreover, despite the scarcity, some studies point out to some borderline symptomatology in this population.

This investigation intends to explore the relation between shame traumatic memories and borderline traits, exploring the mediating effect of self-disgust and self-compassion.

Methods.- In that sense, this investigation intends to explore the relation between shame traumatic memories and borderline traits, through the mediating effect of self-disgust and self-compassion. With that in mind, 42 patients with gender dysphoria filled out a protocol of self-report instruments that assess the variables being studied.

Results.- The results of the correlation analyses showed the hypothesized relations, with statistical significance. The comparison between the two stages of treatment which these patients engage (with or without hormonal therapy), revealed that transsexual individuals who have already initiated hormonal therapy present less self-disgust than those who have not done so. The tested mediation models proved to be significant, with the first model accounting for $53 \%$ of borderline self-image mediated by self-compassion, and the second one for $52 \%$ of affective instability mediated through self-disgust and self-compassion.

Conclusions.- Globally, these findings suggest that different types of internal auto-relation alongside shame traumatic experiences seem to contribute to maintaining borderline traits related with transsexuals with gender dysphoria self and emotional deregulation.

Disclosure of interest.- The authors have not supplied a conflict of interest statement.

\section{E-PV0899 \\ Fertility preservation and gender dysphoria. A literature review}

I. Lopez Alvarez ${ }^{1}$, J. Curto Ramos ${ }^{2,3^{*}}$, L. Carballeira Carrera ${ }^{4}$, A. Hospital Moreno ${ }^{2}$

${ }^{1}$ Alicia Koplowitz Scholarship, Tavistock and Portman NHS

Foundation Trust - Gender Identity Development Service, London, United Kingdom; ${ }^{2}$ University Hospital La Paz, Psychiatry and Mental Health Department, Madrid, Spain; ${ }^{3}$ NGO Apoyo Positivo, Psychiatry Chemsex Program, Madrid, Spain; ${ }^{4}$ Alicia Koplowitz Scholarship, Assistante publique - Hôpitaux de Paris AP-HP, Paris, France

* Corresponding author.

Background and aims.- Gender dysphoria rates among young people have increased largely in the last years. Current treatment includes hormone treatment, surgical interventions, speech therapy, and general medical care. Gender affirmative treatment is nowadays the validated treatment. However, long term effects of some of these interventions are not well known, although it is known that fertility may be affected and fertility preservation (FP) procedures are usually offered. We aim to find out the status of the issue through a review of the available literature in the main scientific data bases.

Methods.- 19 papers were selected for their relevance in scientific data bases PubMed and Google Scholar, using the key words $<$ gender dysphoria $>$ AND $<$ fertility $>$.

Results.- Most of papers reviewed show concerns about how fertility preservation is addressed for young people such as not offering adequate counselling or dilemmas like counselling about a desire not present in the moment which may become important in the future. Even in the cases where proper FP counselling with a specialist was offered, very few patients agreed to undergo FP procedures, which contrast with the not uncommon desire to have a child genetically related to them referred by transgender adults.

Conclusions.- There is little knowledge of the reasons for transgender population declining referral for FP. Financial reasons may be a reason in some countries, whereas not wanting to undergo the procedures required seems to be important in every country. More information and training about adequate FP counselling and FP alternative procedures should be offered in Gender Dysphoria Services.

Disclosure of interest.- The authors have not supplied a conflict of interest statement.

\section{E-PV0901}

\section{Sexualized drug use in the age of online social netwoks}

\section{Ferreira ${ }^{1 *}$, L.P. Ferreira ${ }^{2}$}

${ }^{1}$ Centro Hospitalar Lisboa Norte- Hospital de Santa Maria, Serviço de Psiquiatria e Saúde Mental, Lisboa, Portugal; ${ }^{2}$ Hospital Distrital de Santarém, Serviço de Psiquiatria, Santarém, Portugal

* Corresponding author.

Background and aims.- The use of both licit and illicit drugs and alcohol have been used in sexual contexts throughout history. However the emergence of new online social networks and platforms to buy those substances and meet new partners have reshaped it's prevalence and demography.

Our aim was to to discuss the influence of online apps on the use of substances within sexual contexts.

Methods.- We searched databases indexed at MEDLINE using the keywords "sexualized drug use", "chemsex", "nonmedical prescription drug use", "Internet network".

Results.- Apps are a way to date and seek sex and "Chems" have become a tool to potentiate it. Chemsex refers to the use of drugs to sustain, enhance or facilitate the sexual experience and has been linked to various health harms:they provide a particular sexually-disinhibiting "high", which represents a public health con- 
cern given that this sexualized drug use has been accompanied by higher-risk sexual activity than has ever been observed with any other kind of drug. Users of these drugs can feel invulnerable to harm, supremely confident, dismissive of consequences, sexually adventurous, experience a heightened sense of pleasure, and can possess a stamina and endurance that may keep them awake for many days, facilitating engagement in lengthy and condomless sex sessions with multiple partners often of unknown serostatus and unknown HIV treatment status.

Conclusions.- The use of these drugs in sexual contexts has potential implications for public health. Understanding the extent of the population at risk is essential for determining harms and developing a best clinical practice adapted to these new realities.

Disclosure of interest.- The authors have not supplied a conflict of interest statement.

\section{E-PV0902}

\section{Sexual health knowledge in first-episode psychosis patients}

M. Goujon", M. Burden, A. Littlejohn, J. Perez

Cambridgeshire and Peterborough NHS Foundation Trust, CAMEO

Early Intervention for Psychosis, Cambridge, United Kingdom

${ }^{*}$ Corresponding author.

Background and aims.- Physical health and mental health are closely intertwined. Regular physical health checks are a requirement in clinical guidelines for management of First-Episode Psychosis (FEP). However, there is very little emphasis on the sexual health component of the physical health assessment and on the needs for sexual health screenings, education or prevention in Early Intervention in Psychosis (EIP) community services in the UK.

The aim of this project is to find out the STI knowledge and risktaking behaviours in FEP patients and evaluate the need for regular sexual health screenings, education and providing simple barrier contraception during the Physical Health Clinic (PHC) of Cambridgeshire EIP community-based service, CAMEO.

Methods.- Based on previous studies reviewing sexual risktaking behaviours in FEP populations, we developed a 10-item self-complete questionnaire evaluating sexual behaviours and perception/knowledge about STI risk. Questionnaires were handed out in presence of a doctor during CAMEO PHC and, once completed, they were dropped in a sealed box amongst other questionnaires to ensure confidentiality.

Results.- To date, seventeen out of twenty-four patients seen consecutively in the CAMEO PHC completed the questionnaire. Preliminary data collected for this initial cohort revealed that $81 \%$ knew where to get sexual health checks. However, STI symptoms awareness dropped to 53\% and specific STI knowledge was variable. Conclusions.- Our preliminary results suggest that the methodology we employed to assess sexual knowledge and risk-taking behaviours in FEP patients is acceptable and well tolerated by this clinical population. A larger cohort is necessary to draw definite conclusions; hence, this project will be run throughout 2019.

Disclosure of interest.- The authors have not supplied a conflict of interest statement.

\section{E-PV0903}

\section{Transsexualism - Portuguese legislative evolution}

\section{J. Jerónimo ${ }^{1 *}$, P. Frade ${ }^{2}$, R. Xavier Vieira ${ }^{1}$}

${ }^{1}$ Centro Hospitalar Lisboa Norte, Serviço de Psiquiatria e Saúde Mental, Lisboa, Portugal; ${ }^{2}$ Centro Hospitalar do Oeste, Departamento de Psiquiatria e Saúde Mental, Lisboa, Portugal

* Corresponding author.

Background and aims.- Review the evolution of the Portuguese legislation regarding transsexualism and make a parallel with clinical practice, in order to understand the extent to which simplification or bureaucratization occurred in the process of sexual reattribution. Methods.- Studies were searched from PubMed, B-on, Elsevier databases.

Results.- According to the Parliamentary Assembly of the Council of Europe and the European Parliament Resolution recommendations of 1989, all member states must have legislation that recognizes transexual people the right of sexual reattribution, from the gender assigned to their gender identity. At that time the use of endocrine and surgical treatments and insertion in health and social services were recommended. Only in 1995, the Portuguese Medical Association (PMA) allowed such procedures to be carried out. It was mandatory a diagnosis validated by two psychiatrists and favorable opinion of an independent commission nominated by the PMA. In 2011, a new law allowed to change the sex and birth name in the civil registry by just delivering a report made by a clinical multidisciplinary team. In 2013, with the release of the Diagnostic and Statistical Manual of Mental Disorders, Gender Dysphoria was replaced to Gender Identity Disorder, emphasizing the distress and discomfort caused by gender incongruence.

Conclusions.- Legislation has evolved to facilitate the bureaucratic process inherent in gender reattribution and highlight the importance of rigorous evaluation, in order to protect transsexuals in the irreversible/advanced phases of the process. The reduction of time needed to change the sex and name, allowed combat social exclusion and the suffering of transsexual people.

Disclosure of interest.- The authors have not supplied a conflict of interest statement.

\section{E-PV0904 \\ Utilization of psycho-education to the husband/partner as a main treatment approach for sexual pain-penetration disorder (i.e vaginismus)}

\section{A. Kalboush}

General Secretariat of mental health and addiction treatment, psychiatry, cairo, Egypt

Corresponding author.

Background and aims.- Sexual pain-penetration disorder (formerly called vaginismus in the International Classification of Disease/ICD 10) is a challenging disorder in some cases, and the old name; vaginismus was misleading as it directed the therapeutic efforts to make patient's vagina penetrable, by using different methods, like using dilators, lubricants, muscle relaxants (mainly benzodiazepines). As any other type of specific phobias; Sexual pain-penetration disorder can respond well to psychotherapy through psycho-education provided to the husband/partner of the patient suffers from this disorder.

Methods.- By a thorough reviewing of the literature about Sexual pain-penetration disorder and its management and with conceptualizing the similarities of this disorder with different types of specific phobias, a novel treatment approach; depending on involving the husband/partner in the treatment plan as the main participant was tried to treat many cases of this disorder who differ greatly regarding the duration of illness which ranged from 10 days to 18 months.

Results.- A rapid and complete cure of Sexual pain-penetration disorder happened in most of cases who tried this new treatment approach which depends completely on the role of the husband/partner not the patient herself, regardless of the length of the duration of illness, the number and the type of unsuccessful methods tried before and the level distress present on starting the treatment.

Conclusions.- Understanding the Sexual pain-penetration disorder as a type of specific phobias can help to deal with it successfully 
with rapid response/cure, with lesser efforts and without any need for time consuming and exhausting methods to the patients. Disclosure of interest.- The authors have not supplied a conflict of interest statement.

\section{E-PV0905}

\section{Sexual dysfunction in men who have sex with men (MSM): a review}

L. Lopes

Centro Hospitalar de Vila Nova de Gaia/Espinho, Psiquiatria, Vila

Nova de Gaia, Portugal

${ }^{*}$ Corresponding author.

Background and aims.- Research and clinical data on male sexual dysfunctions mainly focus on heterosexual sexual practices. This study aims to explore and summarize existing data on sexual dysfunction in men with homosexual sexual practices.

Methods.- A non-systematic review of the available literature on sexual dysfunctions in men who have sex with men (MSM) was carried out.

Results.- Studies that looked at sexual dysfunctions in MSM reported a high degree of sexual concerns among this population. In fact, a higher frequency of sexual concerns seems to be related to the intensity of internalized homophobia.

Regarding erection difficulties studies have shown a higher frequency in MSM, being hypothesized that this population may suffer higher performance anxiety. Although no differences were reported on premature ejaculation assessment, ejaculatory problems seem to be more prevalent in MSM who engage in risky sexual behaviors or that live under significant stress.

Several studies present data on sexual dysfunctions after diseases such as prostate cancer. A lower stiffness of the obtained erection and a reduction of ejaculate volume seem to impact more negatively in patients with homosexual sexual practices.

Nonetheless, these works present methodological differences (participants' selection, definitions and assessing methods) that limit both interpretation and comparison among studies.

Conclusions.- It is essential to develop more research on this theme so we gain a better understanding of the impact of sexual dysfunction in the MSM population, so adequate and competent health care can be provided.

Disclosure of interest.- The authors have not supplied a conflict of interest statement.

\section{E-PV0906}

\section{Influence of menopause on sexuality}

K. Siala ${ }^{1}$, Y. Mejdoub ${ }^{2}$, L. Zouari ${ }^{3}$, J. Dammak ${ }^{2}$, M. Maalej ${ }^{3}$, M. Maalej ${ }^{*}$

${ }^{1}$ Ben Arous University Hospital, Department of Gynecologie, Tunis, Tunisia; ${ }^{2}$ Hedi Chaker University Hospital, Department of Community Medicine, Sfax, Tunisia; ${ }^{3}$ Hedi Chaker University Hospital, Department of Psychiatry "C", Sfax, Tunisia

${ }^{*}$ Corresponding author.

\section{Background and aims.-}

Introduction. Sexual health is important and does not stop with the end of the reproductive years, despite the changes associated with menopause.

Objectives. To study the sexuality of menopausal women and the factors correlated to sexual dysfunction

Methods.- We conducted a descriptive cross-sectional study among menopausal women, aged between 40 and 60. It concerned patients who consulted the Basic Health Centers of Sfax-Tunisia. We used the Female Sexual Function Index (FSFI) to assess sexuality, the Dyadic Scaling Scale in its 16 -items version to assess married life and the Hospital Anxiety and Depression Scale (HADS) to assess emotional state.
Results.- The study of sexuality in our sample showed that 58 (77.3\%) of menopausal women had sexual dysfunction. The most affected areas were: excitement (68\%), desire (60\%) and orgasm (49.3\%). The study of the emotional state of our sample showed an anxiety in $26(34.7 \%)$ cases and depression in 4 (5.3\%) cases. The overall FSFI score was correlated with the presence of particular psychological context ( $p=0.0017)$, participants' perception of their menopause $(0.005)$, overall DAS16 score $(<10-3)$ and the overall $\operatorname{HADS}$ score $(p=0.003)$. Indeed, it was found that the sexual arousal, orgasm and sexual satisfaction scores of the FSFI were inversely and significantly correlated with the HADS anxiety score and the HADS depression score.

Conclusions.- Our study shows that menopause leads to significant sexual dysfunction, which requires a collaborative approach between gynecologist and psychiatrist to improve the quality of life of menopausal women.

Disclosure of interest.- The authors have not supplied a conflict of interest statement.

\section{E-PV0910 \\ High frequency of sexual abuse in the church alerts adolescence desires that tried to be suppressed by joining religion but relapsed at later life}

\section{K. Pirkalani*, Z. Taleerad}

Mehr Medical Group, Internal Medicine/Psychiatry, Tehran, Iran

* Corresponding author.

Background and aims.-

Introduction.- In recent times large number of reports has been heard of about child abuse in the church from different ethnic groups. Clearly, the frequency outweighs its counterpart in the general population. This could not be explained by normal heterosexual desire frustrated for a long time as this would normally be translated into masturbation, adultery or covered relationships which are more convenient, less criminal and more routine. In fact child abuse is rather an explosive uncontrolled act which is suppressed for some time but becomes inevitable.

Objectives.- We tried to look more deeply into the psychopathology of child sexual abuse and its relation to the current Christian beliefs. Methods.- Though enough data are not available, we compared the reports from the church and general population of three major items i.e. pedophilia, adultery and homosexuality both with statistical and logical methods.

Results.- Sexual misconduct towards minorities is much more frequent (at least one log) in the church than any other ethnic or cultural group even compared to other religious backgrounds including Islam or Buddhism. The onset seems to be around the age of 30 (late twentieth) and lasts till late sixties and the frequency of real acts increase till the age of 45 .

Conclusions.- Pedophilic or homosexual desires begin early in the adolescence and are actively tried to be suppressed by choosing a religious job to cover it by altruism but it relapses by higher frequency as the clergyman attains personal and social legitimacy. Church must be cautious in accepting applicants for any new vacancy.

Disclosure of interest.- The authors have not supplied a conflict of interest statement.

\section{E-PV0911}

\section{Hypersexuality: a diagnosable entity? A case series}

\section{P. S R}

Clinical post-doctoral fellow, Centre for brain research-Indian institute of science IISE- Bangalore, Bangalore, India

* Corresponding author. 
Background and aims.- Definition, classification, and management of hypersexuality have been a problem despite research in this area. Formidable legal and ethical issues make the task of treating of hyper sexuality difficult. The present case series is an effort to highlight these challenges faced in day to day clinical situations. Methods.- Case summary: In this case series, we report three cases who presented with hypersexual behavior in the absence of diagnosable psychiatric illness with socio-occupational difficulty. Biochemical, radiological, pathological and psychological tests did not reveal any finding suggestive of organicity. Neurology and endocrinology opinion was sought and no abnormality detected. Results.- Absence of diagnosable illness.

Conclusions.- The evidence base for the treatment of hypersexuality disorder has few studies with no proper treatment guidelines, the future of these patients is uncertain. This report discusses problems regarding diagnosis, treatment, ethical issues, and rehabilitation. We also highlight the possible differential diagnosis, treatment, and outcome.

Disclosure of interest.- The authors have not supplied a conflict of interest statement.

\section{E-Poster Viewing - 7-9 April: Sleep Disorders \& Stress}

\section{E-PV0917}

\section{Acute effect of zingiber montanum essential oil aromatherapy on mood in healthy male volunteers}

W. Arpornchayanon ${ }^{1}$, S. Gomonchareonsiri ${ }^{2}$, S. Chansakaw ${ }^{3}, \mathrm{~N}$. Wongpakaran $^{4}$, T. Wongpakaran ${ }^{4}$

${ }^{1}$ Faculty of Medicine- Chiang Mai University, Department of Pharmacology, Chiang Mai, Thailand; ${ }^{2}$ Faculty of Medicine- Chiang Mai University, Department of Physiology, Chiang Mai, Thailand; ${ }^{3}$ Faculty of Pharmacy- Chiang Mai University, Department of Pharmaceutical Science, Chiang Mai, Thailand; ${ }^{4}$ Faculty of MedicineChiang Mai University, Department of Psychiatry, Chiang Mai, Thailand

* Corresponding author.

Background and aims.-

Introduction.- Inhalation of essential oils aromatherapy has been linked to the stimulation of olfactory cortex, results in serotonin release and modulation of mood status. Zingiber montanum or Plai contains high level of essential oils and flavonoids such as sabinene, terpenoids, butanoids, and curcuminoids. It is well known for analgesic property but its effect on mood is unclear.

Objectives.- This study aimed to investigate the acute effect of inhalation Zingiber montanum essential oil on mood in healthy volunteers.

Methods.- A prospective, randomized, double-blinded, controlled trial was conducted on 38 healthy male volunteers; 19 in Plai group and 19 in control group. Olive oil was used in the control group. Single inhalation was provided continuously for $15 \mathrm{~min}$ via hot steam. Mood status was assessed pre- and post-intervention using the Visual Analog Scales for Mood, Anxiety, Stress, and Alertness (MASA) and the Rorschach tests.

Results.- One-way ANCOVA was used to determine a statistically difference of mood tests between Plai and Olive on the mood variables controlling for pre-test scores. Both Plai and Olive increased the post-test scores of positive mood and reduced the scores of negative mood. However, no difference between groups was found, except on the Rorschach - stress. There was significantly different between Plai and Olive $(F(1,38)=6.486, p=0.015)$. The variances of change explained by the model ( $R$ square) were $32.8 \%$. The adjusted $R$ Squared was 0.290 .
Conclusions.- Inhalation aromatherapy of Zingiber montanum essential oil showed an immediate improvement of mood state, especially stress in healthy volunteers.

Disclosure of interest.- The authors have not supplied a conflict of interest statement.

\section{E-PV0919 \\ Sleep disorders in the elderly person (Moroccan context)}

M. Bitar ${ }^{1^{*}}$, M. Anwar Anwar ${ }^{2}$, F.E. El jebbouri El jebbouri ${ }^{2}$, A. Ouanass Ouanass ${ }^{2}$

${ }^{1}$ Dr, Hopital Arrazi, Rabat, Morocco; ${ }^{2}$ Arrazi, Emergency, Rabat, Morocco

* Corresponding author.

Background and aims.- Physiological aging is associated with changes in the structure of sleep. Sleep in the elderly is likely to be modified by multiple factors, diseases, drugs, changes in lifestyle, loss of social benchmarks.

The solutions are usually limited to changes in lifestyle, allowing the eviction of prescriptions most often against indicated.

Objective of our study.- Evaluate the main sleep disorders in the elderly and their prevalence, as well as their impact on these people. Methods.-

- Descriptive cross-sectional study in institutionalized elderly people.

- We use a questionnaire on socio-demographic parameters, antecedents and the sleep questionnaire.

- Criteria for inclusion:

- Subject aged over 65 years.

- Oral consent to participate in the study.

- Exclusion Criteria:

- Psychotic or insane subject.

- Statistical tool: SPSS 20.

Results.- The average age is 77 years old. An age range between 67 and 93 years old. In our sample of 50 patients, $90 \%$ have sleep disorders. Insomnia of falling asleep is in the foreground with a rate of $40 \%$, followed by the typical night-time waking disorder with a rate of $24 \%$ of the entire sample. In the statistical analysis, a statistically significant relationship was found between age and the presence of sleep disorders.

Conclusions.- When sleep disorders appear with age, we must look for an associated cause that can explain these disorders.

Disclosure of interest.- The authors have not supplied a conflict of interest statement.

\section{E-PV0920}

\section{Sleep paralysis: cultural interpretations and status of scientific literature}

T. Cavaco*, J. Rema, E. Reis, C. Leite Rodrigues, E. Fernandes, J.P. Lourenço, J. Jerónimo

Centro Hospitalar Lisboa Norte CHLN, Hospital de Santa Maria, Neurosciences Department, Psychiatry and Mental Health, Lisbon, Portugal

Corresponding author.

Background and aims.- While common and wide-spread, sleep paralysis (SP) is a poorly understood and acknowledged phenomenon. It is characterized by an inability to move upon going to sleep or waking up and is described as an unpleasant and difficult to comprehend experience as hallucinations and delusions can be present. Lack of physicians' awareness and of scientific grounding led to the foundation of several cultural-based interpretations of SP over time in an attempt to enlightenment.

The aim is to expose cultural interpretations to SP and to clarify the status of scientific literature on this disorder. 
Methods.- A literature review was conducted using PubMed search database.

Results.- SP has received great attention from the nonscientific field. From descriptions of a more spiritual framework to alien abduction reports and other supernatural beliefs many cultural specific explanations were given. The associated stigma and physicians' unfamiliarity with the condition led to a lack of report and approach. Only recently emphasis was given to the scientific research of SP but although limited, science-based assessment and management is possible.

Conclusions.- It is important to bring awareness to the concept and approach of SP to the physicians that are not sleep specialists and to the public in general. Further research on the subject is needed. Disclosure of interest.- The authors have not supplied a conflict of interest statement.

\section{E-PV0921}

\section{Restless legs syndrome and morningness-eveningness in the Korean high school students}

M.Y. Chung ${ }^{*}$, K.U. Lee ${ }^{2}$, S.H. Kang ${ }^{3}$

${ }^{1}$ Worker's Compensation Hospital/Yonsei Univ. College of Medicine, Dept. of Neuropsychiatry, Seoul, Republic of Korea; ${ }^{2}$ Catholic UniversityPsychiatry Affective Neuroscience Lab, Department of Psychiatry, Uijeongbu, Republic of Korea; ${ }^{3}$ Center for Sleep Medicine-Veterans Health Service Medical Center, Deprtment of Psychiatry, Seoul, Republic of Korea

${ }^{*}$ Corresponding author.

Background and aims.- Restless legs syndrome (RLS) lowers the quality of sleep, and is characterized by symptoms that follow a circadian pattern. The aim of this study was to determine the relationship between RLS and morning-eveningness in Korean adolescents.

Methods.- Of the 867 community-dwelling high school students, 590 subjects were included in this study. All participants completed self-report questionnaires, including demographic variables, particulars about menstruation, life style,body mass index (BMI) sleep duration, RLS severity, Composite scale (CS), Epworth sleepiness scale (ESS), Insomnia severity scale (ISI), Patient health questionnaire-9 (PHQ-9), Pittsburgh sleep quality index (PSQI) and Berlin questionnaire (BQ),.

Results.- Participants with RLS symptoms had a higher prevalence of depression, defined by a PHQ-9 score $\geq 10$ (OR 3.03, CI 1.11-8.26) and eveningness in chronotype (OR $1.95 \mathrm{CI} 1.15-6.43$ ) when adjusted for depression, excessive daytime sleepiness, insomnia symptom and morningness-eveningness. However, RLS symptoms had no association with excessive daytime sleepiness and insomnia, when adjusted for clinical factors.

Conclusions.- In Korean high school students, restless leg symptom may be preceded by depression and eveningness of chronotype. For healthy sleep lifestyle in the adolescents, an appropriate evaluation of RLS symptom as well as chronotype is recommended.

Disclosure of interest.- The authors have not supplied a conflict of interest statement.

\section{E-PV0925}

\section{Characteristic symptoms and hallucinations in isolated sleep paralysis: a two-site study \\ B. Sharpless ${ }^{1}$, M. Klikova ${ }^{2,3^{*}}$}

${ }^{1}$ American School of Professional Psychology- Argosy UniversityNorthern Virgninia, Clinical Psychology, Arlington, USA; ${ }^{2}$ Charles University, Third Faculty of Medicine, Prague, Czech Republic; ${ }^{3}$ National Institute of Mental Health, Sleep Medicine and Chronobiology, Klecany, Czech Republic

${ }^{*}$ Corresponding author.
Background and aims.- Isolated sleep paralysis (ISP) is a REM parasomnia characterized by the inability to move the trunk/limbs at sleep onset or when awakening. The paralysis is often accompanied by hallucinations, some of which may be frightening. In order to gain a better understanding of the symptoms and associated features of ISP, the primary goal of this study is to catalogue the frequency and relative severities of the symptoms and hallucinations. In addition, we will discern if the paralysis or the accompanying symptoms/hallucinations are associated with higher levels of fear and distress.

Methods.- 185 students with isolated sleep paralysis from different coasts of the USA were assessed by trained diagnosticians using the Fearful Isolated Sleep Paralysis Interview. This is a reliable measure which assesses 27 accompanying symptoms and also facilitates differential diagnosis.

Results.- Participants experienced a mean of 7.73 symptoms/hallucinations during typical ISP episodes. There was no significant difference between level of fear/distress cause by paralysis and symptoms/hallucinations, and both were of moderate, clinically-significant severity $(M=4.31$ and $M=4.46$ respectively on a $0-8$ scale). The most common symptoms during ISP episodes were numbness, attempting to speak but being unable to do so, and sensing a presence in the room. Of the hallucinations, visual hallucinations of other beings (e.g., ghosts, demons) and sensing a presence in the room were associated with the highest level of fear. Conclusions.- Sleep paralysis is typically accompanied by a multitude of symptoms and hallucinations that are associated with clinically-significant levels of fear.

Disclosure of interest.- The authors have not supplied a conflict of interest statement.

\section{E-PV0927 \\ Kleine-levin syndrome presenting as first-episode psychosis}

A.B. Medeiros ${ }^{1 *}$, B. Silva ${ }^{2,3}$, F. Ladeira ${ }^{4}$, P. Bugalho $0^{4,5}$

${ }^{1}$ Hospital do Divino Espírito Santo de Ponta Delgada, Psychiatry, Ponta Delgada, Portugal; ${ }^{2}$ Centro Hospitalar de Lisboa Ocidental, Psychiatry and Mental Health, Lisboa, Portugal; ${ }^{3}$ NOVA Medical School, Faculdade de Ciências Médicas, Universidade NOVA de Lisboa, Lisboa, Portugal; ${ }^{4}$ Centro Hospitalar de Lisboa Ocidental, Neurology, Lisboa, Portugal; ${ }^{5}$ CEDOC - NOVA Medical School, Faculdade de Ciências Médicas - Universidade NOVA de Lisboa, Lisboa, Portugal * Corresponding author.

Background and aims.- Klein-Levin Syndrome (KLS) is a rare, idiopathic sleep disorder characterized by recurrent episodes of hypersomnia, hyperphagia, hypersexuality and cognitive disturbances. The neuropsychiatric features are nonspecific. We report the case of a patient who presented with first-episode psychosis. Methods.- We reviewed the patient's clinical records.

Results.- An 18 year-old male presented to the emergency department with disruptive behavior and psychotic symptoms (paranoid ideation and verbal hallucinations). MRI and EEG were unremarkable. He was admitted to the inpatient psychiatry unit with a working diagnosis of Psychosis NOS. Hypersomnia, hyperphagia, depressed mood and bouts of aggressive behavior were noted, interpreted as atypical symptoms of depression. He was discharged with a diagnosis of Major Depressive Disorder. Behavioral and sleep symptoms remitted completely after one month. Three years later, he returned with a chief complaint of headaches, hyperphagia and hypersomnia (sleep duration of 18 to $24 \mathrm{~h}$ ). Three episodes of hypersomnia were registered, with three weeks duration separated by one week of total remission. A diagnosis of KLS was established, based on the presence of recurrent episodes of excessive sleepiness accompanied by cognitive dysfunction, altered perception, eating disorder (hyperphagia) and total remission of symptoms between episodes. Presently, he is being treated with fluoxetine $40 \mathrm{mg} /$ day 
and amantadine as needed. A decision was made to postpone treatment with lithium due to the low frequency of the episodes.

Conclusions.- KLS can mimic primary psychiatric disorders. Clinicians should be aware of its cardinal symptoms, as an early recognition can prevent misdiagnosis and ensure the correct management of this rare syndrome.

Disclosure of interest.- The authors have not supplied a conflict of interest statement.

\section{E-PV0928}

\section{The quality of life and insomnia after acute infections of the CNS-efficiacy of trazodone}

A. Miljatovic

Community Health Centre, Mental Health, Belgrade, Serbia

* Corresponding author.

Background and aims.- Infections in the central nervous system are caused by a wide range of microorganismus resulting in distinct clinical syndromes including meningitis,encephalitis, and pyogenic infections. The effects of acute infections of the CNS can be complete recovery,or partial recovery with consequences.

The aim of this study is to establish differences in the assessment of the components of life quality in relation to the outcome of the illness,separately for each of the three groups of the examinees: (1) CR-completely recovered, (2) RC-recovered with consequences and (3) C-control group; as well as to establish the differences between groups in relation to the assessment of life quality. We also wanted to point out the differences in the level of insomnia between these 3 groups.

Methods.- The study includes 110 examines that were treated at the clinic for infectious diseases from 2011-2017. All of the examines were diagnosed as suffering from acute CNS infection.

Medical Outcomes study short form 36 (SF 36) questionnaire was used as a source of information,while insomnia severity index (ISI) was used as a measuring instrument. Patients in all two groups who had a total score on the ISI scale greater than 14 were given trazodone. These patients were given $150 \mathrm{mg}$-daily of trazodone,a total of eight weeks.

Results.- 75\% of the examines from the CR group had no insomnia and 70\% felt calm and happy. There is a significant degree of differences in the level of insomnia between the examines two groups.

Conclusions.- The use of trazodone has led to a significant reduction in the level of insomnia.

Disclosure of interest.- The authors have not supplied a conflict of interest statement.

\section{E-PV0929}

\section{Personal accomplishment and propensity to burnout: empirical evidence from medical professionals}

L.F. Miranda Terraza ${ }^{*}$, J. Viloria Escobar ${ }^{2}$, L. Pedraza Álvarez ${ }^{3}, \mathrm{~K}$. Pérez Correa ${ }^{4}$

${ }^{1}$ University of Barcelona, MSc in Business Research, Barcelona, Spain; ${ }^{2}$ University of Magdalena, Faculty of Education, Santa Marta, Colombia; ${ }^{3}$ University of Magdalena, Faculty of Psychology, Santa Marta, Colombia; ${ }^{4}$ Cooperative University of Colombia, Faculty of Psychology, Santa Marta, Colombia

* Corresponding author.

Background and aims.- Excessive demands or pressures in the workplace, a poor job performance or complicated interpersonal relationships among other aspects, can generate a low professional achievement. That feeling of low achievement usually results in burnout which is considered a work disorder that causes physi- cal and mental exhaustion. Thus, the main objective of this study is to establish the relationship between the perceived personal accomplishment and propensity to burnout of a group of doctors from Santa Marta, Colombia. This research is relevant because that professionals' group is exposed to many factors that promote the development of high stress levels.

Methods.- For collecting data, the Maslach Burnout Inventory was applied to a sample of 59 medical professionals which were selected by a non-probabilistic sampling. This is a correlational study because the main purpose is to establish the relationship between two variables. For another hand, research design is nonexperimental. The correlation analysis was developed with the statistical package SPSS 25.

Results.- Results show that there is a high negative correlation between personal accomplishment and burnout propensity $(-0,72)$. A negative correlation means that there is an inverse relationship between the two variables.

Conclusions.- This exploratory analysis represents an important advance to know the nature of the relationship between these two variables and their implications for work life considering that the feeling of low personal accomplishment implies negative attitudes towards job. That situation can become in a poor meaning of work. We propose that future investigations propose and evaluate models with other variables that explain the main determinants of burnout. Disclosure of interest.- The authors have not supplied a conflict of interest statement.

\section{E-PV0930 \\ Occupacional stress in brazilian female shift workers}

J.C. Silva ${ }^{1}$, H. Theodoro ${ }^{2}$, M.T.A. Olinto ${ }^{1}$, A. Ludwig Neutzling ${ }^{1 *}$

${ }^{1}$ Universidade do Vale do Rio dos Sinos - UNISINOS, Postgraduate

Program in Collective Health, São Leopoldo, Brazil; ${ }^{2}$ Universidade de Caxias do Sul, Nutrition Course, Caxias do Sul, Brazil

* Corresponding author.

Background and aims.-

Introduction.- Occupational stress has been investigate as one of the health consequences of shift workers. The number of people exposed to the system of shifts work is increasing around the world. High psychological demand and low control at work defined Workrelated stress. Occupational stress has not considered an illness, but it is a risk factor to health problems.

Objectives.- To identify the prevalence of occupational stress in female shift workers, according to sociodemographic variables, lifestyle and Sleep Quality.

Methods.- Cross-sectional study, with data collected in the southern region of Brazil. Sample composed of 449 female shifts workers. "Job Stress Scale" was use for the definition of occupational stress in a reduced version, validated for the Brazilian population. The data analysis has performed through SPSS 21.0.

Results.- The prevalence of occupational stress is $37.4 \%$. The sample consisted of women with aged 31 to 40 years (34.7\%), white (69.3\%), married (54.4\%), with 9 to 11 years of schooling (76.8\%), with at least one gestation (72.2\%), non-smokers (73.8\%), workers of the day shift (76.2\%) and sleep disturbance (63.3\%). In the bivariate analysis, only schooling was associated with occupational stress ( $p$-value 0.044 ). Conclusions.- The analysis indicated a significant association for the schooling of the workers, that is, the lower the schooling the greater the frequency of occupational stress. However, the results shouldn't be considered conclusive, since there is scope to expand and investigate the associations in the absence of possible confounding variables.

Disclosure of interest.- The authors have not supplied a conflict of interest statement. 


\section{E-PV0931}

\section{Relationship between preschool children and caregivers on the use of internet}

Y. Oka ${ }^{*}$, N. Takata ${ }^{2}$, K. Itoh ${ }^{3}$

${ }^{1}$ Ehime University Hospital, Center for Sleep Medicine, Toon City, Japan; ${ }^{2}$ Shikoku University, School of Nursing, Tokushima, Japan; ${ }^{3}$ Ube Frontier College, Nursery Department, Yamaguchi, Japan ${ }^{*}$ Corresponding author.

Background and aims.- Overuse of internet is becoming a problem among small children. Parental use of internet may impact on the use of internet and other problems during childhood. The aim of the study was to elucidate the impact of parental internet use on children especially focusing on internet use and sleep habits of children.

Methods.- Preschool children attending kindergartens and nursery schools in Yamaguchi City, Japan were recruited for the study. Sleep Checklist was distributed to all caregivers of children between 4-5 years of age. Seven hundred and thirty seven responses were included in the analysis. Sleep habits of caregiver and their children, and the use of internet of their children were compared between the caregivers using internet more than two hours a day $(n=261)$ and less than two hours a day $(n=476)$.

Results.- Use of internet of children was twice as longer ( 0.2 vs $0.4 \mathrm{~h}$ on weekdays and $0.4 \mathrm{~h}$ vs $0.8 \mathrm{~h}$ on holidays) among children whose caregiver use internet more than two hours. As for the sleep habit of children, wake time was significantly later among children whose caregiver use internet more. Caregivers who use internet more than two hours showed disturbed sleep.

Conclusions.- Use of internet of caregivers significantly affect the use of internet of children and also impair sleep habit of both caregiver and children. Impact of internet use of caregiver on children was shown to present among preschool children, thus indicate the need for careful attention of caregiver's behavior over internet to prevent internet overuse in later life.

Disclosure of interest.- The authors have not supplied a conflict of interest statement.

\section{E-PV0932}

\section{Subjective complaints and reasons of sleep disturbances in patients with insomnia comparing to sleep apnea, parasomnia and healthy controls}

\section{E. Rasskazova ${ }^{*}$, G. Kovrov ${ }^{2}$ A. Machulina ${ }^{3}$}

${ }^{1}$ Moscow State University, Clinical Psychology, Moscow, Russia; ${ }^{2}$ I.M. Sechenov First Moscow State Medical University, Department of Pathology of Vegetatic Nervious System, Moscow, Russia; ${ }^{3}$ Brothers Bahrushins' Moscow Clinical Hospital, Neurology, Moscow, Russia ${ }^{*}$ Corresponding author.

Background and aims.- Polysomnography is expensive and typically not used in diagnostics of insomnia. Subjective complaints and descriptions of patients are the first and sometimes the only information that doctor consider.

The aim was to differentiate complaints and subjective reasons of sleep disturbances in patients with insomnia comparing to other sleep disorders and controls.

Methods.- 105 patients with chronic insomnia, 77 with sleep apnea, 15 with parasomnia (diagnoses verified by polysomnography) and 102 controls filled checklists of symptoms and reasons for sleep disturbances, Insomnia Severity Index (Morin, 1993), Hospital Anxiety and Depression Scale (Zigmond, Snaith, 1983), Epworth Sleepiness Scale (Johns, 1991).
Results.- Good sleepers reported events-related sleep disturbances in the past even more frequently than insomniacs $(p<.01)$. Although most of insomniacs reported longer sleep latency, frequent awakenings, early morning awakenings and sleepiness (59.0\%-79.0\%), the same reports emerged in 30.0\%-55.0\% of good sleepers and even more in apnea and parasomnias. The only sleeprelated complaint specific for insomnia was "absences of the night sleep at all" (36.2\% vs 0\%-3.9\% in other groups). Both patients with insomnia and controls attributed poor sleep to stress and life events (53.3\%-67.7\%) but insomniacs refused the negative role of behavioral factors (shift work, jet lag) and changes in mood $(6.7 \%-20.0 \%$ vs $43.4 \%-78.8 \%, p<.01)$. Clinical scales supported poorer subjective sleep, anxiety and depression in insomnia comparing to all other groups $(p<.01)$.

Conclusions.- Diagnostic of insomnia should include clinical scales while subjective report on symptoms is not specific in these patients. Research is supported by the Russian Foundation for Basic Research, project No. 17-06-00363.

Disclosure of interest.- The authors have not supplied a conflict of interest statement.

\section{E-PV0933 \\ Emotional faces processing in working memory related to coping strategies}

F. Sultanova*, B.B. Velichkovsky, G.E. Pupchev, A.A. Kachina, T.A. Zlokazova

Lomonosov Moscow State University, Psychology, Moscow, Russia * Corresponding author.

Background and aims.- Stress is omnipresent and a major source of mental health problems. Using an effective coping strategy is important for overcoming stress related issues. Preferred usage of a coping strategy can be reflected in objective cognitive measures. To relate the individual choice of a stress coping strategy to objective cognitive indicators of emotional faces processing in working memory.

Methods. $-N=60$ (44 female, mean age $27 \pm 7$ years). The participants completed a Russian version of the Coping Inventory for Stressful Situations (CISS), which identifies three coping strategies: task-oriented, emotion-oriented, and avoidant. The participants also completed a version of the Internal Shift Task (IST) with emotional and neutral faces as stimuli. The IST is a working memory updating task, where the subjects have to mentally count the male and female, or angry or neutral faces presented randomly. Reaction time while going from one face to the other was collected (IST is self-paced).

Results.- Pearson's correlations revealed that processing time for angry faces was negatively related to avoidant coping (specifically, to social distraction, $r=-0.52, p<0.01$ ), but only when processing emotional information was in the focus of attention $(p>0.1$ in the gender processing condition). It was also found that processing time for neutral stimuli was positively related to task-oriented coping $(r=+0.38, p<0.05)$ indicating a more thorough cognitive processing in the task-oriented coping strategy.

Conclusions.- Quick abandonment of angry faces processing and slowed processing of neutral stimuli can be used as objective indicators of coping strategies in pathology and normal population. Disclosure of interest.- The authors have not supplied a conflict of interest statement. 


\section{E-Poster Viewing - 7-9 April: Substance related and Addictive disorders}

\section{E-PV0934 \\ Gender disparities in tramadol misuse in a sample of Egyptian university students \\ M. Bassiouny, M. Abdelghani*, G. Salah El-Deen \\ Zagazig University, Psychiatry, Zagazig, Egypt \\ Corresponding author.}

Background and aims.- Tramadol abuse is an overwhelming problem in Egypt. Males and females who use tramadol differ in their sociodemographic and clinical profiles. This study aimed to identify gender differences in tramadol use and abuse in students at Zagazig University, Egypt.

Methods.- A cross-sectional study included a total of 1135 undergraduate students, aged 17-34 years, from both sexes; males ( $n=674,59.4 \%)$ and females $(n=461,40.6 \%)$, from ten colleges in Zagazig University. Participants were randomly selected and assessed for tramadol use and abuse using The Drug Use Disorders Identification Test (DUDIT) and The Drug Use Disorders Identification Test - Extended (DUDIT-E).

Results.- Tramadol use was more prevalent in male than female students (20.2\% vs $2.4 \%$ ). Smoking and alcohol use were shared predictive factors for tramadol use in both sexes. Mean years of education and cannabis use predicted tramadol use in males, while only mean age predicted tramadol use in females. Among tramadol users, males were more likely than females to be smokers, polysubstance users, using cannabis, and to subjectively perceive tramadol use as a helpful practice.

Conclusions.- Tramadol use was common among university students, with higher prevalence among males. Sex-related similarities and differences were present. Results can help local health policymakers develop appropriate management strategies. Future national population-based longitudinal studies need to investigate the causal relation between tramadol use and other substances in both sexes.

Disclosure of interest.- The authors have not supplied a conflict of interest statement.

\section{E-PV0935}

\section{A probable stimulant use disorder and withdrawal syndrome linked to modafinil: a case report}

\section{A.R. Hammad ${ }^{1}$, A. Eid El Aghory ${ }^{1}$, M. Abdelghani2 ${ }^{2}$}

${ }^{1}$ Ministry of Health, Abbassia Hospital for Mental Health, Cairo, Egypt; ${ }^{2}$ Zagazig University, Psychiatry, Zagazig, Egypt

Corresponding author.

Background and aims.- Modafinil is a non-amphetamine stimulant, that raises dopamine in the Nucleus Accumbens, a common final pathway for several psychoactive substances. Modafinil profile is initially assuring due to its mild dopaminergic effect and long half-life, so a weaker ability to induce a "rush," comparable to amphetamine stimulants. Few studies have assessed its addictive potency, despite the presence of preclinical data and label precaution for probable addiction liability. Nonetheless, Modafinil is still not controlled in many countries, including Egypt. Furthermore, it is widely accessed worldwide.

Methods.- Clinical description of a thirty-one-year-old Egyptian man, with self-use of modafinil to mitigate stress-related symptoms and enhance increasingly demanding work performance. A literature review was done for relevant cases in PubMed and ClincalKey.
Results.- Sufficient criteria were met for the DSM5 "severe stimulant use disorder," followed by insufficient stimulant withdrawal symptoms. Modafinil dose was self-increased to $1400-2000 \mathrm{mg} /$ day throughout one year. History of tramadol use, stress-related disorder and family history of Hashish use were present. Five cases are published till now for modafinil related addictive behaviors, and two studies for assessing its addictive potency.

Conclusions.- The current clinical findings support a growing body of literature on addictive behaviors associated with modafinil. To our knowledge, this is the first case to describe modafinil use disorder after stopping tramadol use and during the presence of a stress-related disorder. Accordingly, prescription of modafinil warrants clinical attention during the healthcare of patients with substance use disorders, patients with risk factors for addiction, or during stress-related disorders.

Disclosure of interest.- The authors have not supplied a conflict of interest statement.

\section{E-PV0937}

\section{Comorbidity of iatrogenic addiction to sedative-hypnotics and opioids. Integrated detoxification, driven by serum-BZD monitoring (sermonide) - the pilot study}

A. Basińska-Szafrańska ${ }^{*}$, U. Cieślak ${ }^{2}$

${ }_{1}^{1}$ Institute of Psychiatry and Neurology, Dept. of Prevention and Treatment of Addictions, Warsaw, Poland; ${ }^{2}$ Institute of Psychiatry and Neurology, 1st Dept. of Psychiatry, Warsaw, Poland

* Corresponding author.

Background and aims.- Parallel benzodiazepine (BZD) and opioid addiction is observed not only among polydrug users, but is common in basic practice. The resulting persisting withdrawal symptoms (many similar for both addictions) often dominate over primary ailments, but the former could be eliminated by detoxification. As sequential treatment significantly delay patient-perceived improvement, it was investigated whether the similarities between BZDs and opioids allow integration of both detoxifications into a single procedure.

Methods.- 26 BZD- and opioid-addicted patients (tramadol $100-2000 \mathrm{mg} / \mathrm{d}$ (med.757), or codeine $30-600 \mathrm{mg} / \mathrm{d}$ (med.140)), iatrogenic non-oncologic cases, underwent a serum-BZD monitored detoxification (SERMONIDE), originally developed for treatment of the BZD-addiction alone (described elsewhere). After switching to long-acting substitutes: diazepam (SERMONIDE standard) and tramadol slow-release (if needed), tramadol was tapered first, with serum-BZD kept stable, in some part utilizing the window of SERMONIDE accumulation-control stage. The treatment progressed then as the standard SERMONIDE BZD-elimination stage. Results.- Parallel to the BZD-satiation with diazepam, the additional substitution by tramadol slow-release, if applied, was well tolerated. The loading dose of tramadol was $0-700 \mathrm{mg} / \mathrm{d}$ (med.265 mg), with $9(35 \%)$ patients not demanding opioid substitution. Tramadol tapering lasted 4-20 (med.8) days, with light to moderate withdrawal symptoms. Total integrated treatment duration was 32-72 (med.51) days vs. 28-83 (med. 47) days for SERMONIDE in addicted to BZDs only.

Conclusions.- BZDs meaningfully alleviate opioid withdrawal symptoms, reducing the need/scale of opioid substitution. Presumed action pathways common for both drug groups allow the drug discontinuation in an integrated process.

Disclosure of interest.- The authors have not supplied a conflict of interest statement. 


\section{E-PV0938}

\section{Internet addiction among Tuzla university students}

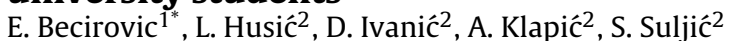

${ }^{1}$ UKC Tuzla, Klinika za psihijatriju, Tuzla, Bosnia, Herzegovina; ${ }^{2}$

Univerzitet u Tuzli, Medicinski fakultet, Tuzla, Bosnia, Herzegovina

${ }^{*}$ Corresponding author.

Background and aims.- Even Internet addiction is not recognized as a disorder in DSM 5 or ICD 11 there are lot of studies focusing it. International prevalence rates for Internet addiction range globally from $1.5 \%$ to $8.2 \%$. Aim was to establish prevalence of internet addiction among students of Tuzla university.

Methods.- Internet survey. Total number of 762 University students fulfilled three parts consisted survey: general data questionnaire, Young's Internet Addiction Test, Zung Self-Rating Depression Scale Results.- Survey analysis has shown that $18.8 \%$ of female and $20.7 \%$ of male students are internet addicts. The highest percentage (25\%). is reported among Faculty of economy students.

Conclusions.- High percentage of students at Tuzla University has problems with internet addiction.

Disclosure of interest.- The authors have not supplied a conflict of interest statement.

\section{E-PV0939 \\ Professional practices in benzodiazepines prescription among general practitioners in Tunisia}

\section{Ben Said*, H. Mami, M. Oumaya, R. Bouzid}

Mohamed Tahar Maamouri Hospital, Mental Health, Nabeul, Tunisia

* Corresponding author.

Background and aims.- Benzodiazepines are psychotropic drugs which have a sedative, hypnotic, anxiolytic, anticonvulsant, and muscle relaxant properties. Their pharmacological properties give them undeniable efficacy explaining their wide prescription by psychiatrists and general practitioners. Despite their good tolerance, benzodiazepines have a great potential for addiction. Thus, it is important to codify prescribing rules to which must conform any prescriber.

The aim of this study is to evaluate the professional practices in the prescription of benzodiazepines among general practitioners, and to compare them to general prescription rules in order to rationalize the use of these substances.

Methods.- Descriptive cross-sectional study conducted with 31 primary-care Tunisian physicians in the public sector, practicing in the governorate of Nabeul and having accepted to answer a preestablished and anonymous self-assessment questionnaire exploring knowledge and prescribing modalities of benzodiazepines.

Results.- Among the physicians surveyed, $81 \%$ have already prescribed benzodiazepines; $48 \%$ of them prescribed it in epilepsy treatment, 35\% in anxiety disorders treatment, $26 \%$ in the treatment of sleep disorders, and $19 \%$ for psychotic disorders. $61 \%$ of the surveyed physicians said they had not provided psycho-education informations about the risks that can be induced by benzodiazepines and $71 \%$ did not knew the recommended prescription period that must not be exceeded. $48 \%$ said they did not know how to assess benzodiazepine dependence.

Conclusions.- Benzodiazepine drug dependence is a multifactorial phenomenon. Non-compliance with the prescription rules remains the most common cause, but also the perfect field of intervention for prevention and awareness actions among all physicians.

Disclosure of interest.- The authors have not supplied a conflict of interest statement.

\section{E-PV0941}

\section{Prevalence of addictive behaviors among Tunisian patients with childhood trauma}

R. Boukhchina*, M.H. Aoun, K. Zitoun, M. Abbes, L. Ghanmi Regional Hospital of Gabès, Psychiatry Department, Gabès, Tunisia * Corresponding author.

Background and aims.- Childhood trauma increases the risk for Addictive Behaviors. Persons who had been neglected or abused during their childhood are doubtlessly eager to experience substance abuse such as tobacco, alcohol and psycho-active substances.

- To investigate the prevalence of Addictive behaviors among patients with Childhood Trauma

Methods.- A cross-sectional, descriptive and analytical study that focused on 68 patients followed at our psychiatry department. We used a questionnaire with three parts: the first part concerning the socio-demographic and clinical characteristics, the second part using the French version of the Childhood Trauma Questionnaire (CTQ) and the third part assessing the Addictive Behaviors, using the Fagerstrom Test to evaluate the level of Nicotine dependence and estimating alcohol and Psycho-Active Substances abuse.

Results.- 68 patients were included. They were males (58.8\%) and single (50\%). The mean age was 45 years. The three most frequent pathologies were Anxiety Disorder (42.6\%), Schizophrenia (29.4\%)and depression (29.4\%). The two most frequent forms of Childhood Trauma were emotional neglect (80\%) and physical neglect (70\%). $50 \%$ of the patients said to be smokers. Alcohol consumption was found among $11.8 \%$ and only $12.5 \%$ of Alcohol consumption was regular. The average age at the beginning of consumption was 21.7 years. The prevalence of Psycho-Active abuse was 3\% for Cannabis. Addictive behavior was significantly associated to male gender ( 77.5 vs. $10.7 \%, p \leq 10-3$ ), to schizophrenia ( 75 vs. $39.6 \%, p=0.008$ )and running away as a risky behavior ( 81.8 vs. $43.9 \%, p=0.02$ )

Conclusions.- This study demonstrates that Addictive behaviors are frequent among Psychiatry patients with Childhood Trauma. This aspect must be considered during the psychological evaluation especially when a treatment resistance occurs.

Disclosure of interest.- The authors have not supplied a conflict of interest statement.

\section{E-PV0943}

\section{Assessing the risk of hiv infection among injecting drug users: internal consistence of the risk assessment battery in Spanish population}

\section{F. Calvo ${ }^{1^{*}}$, X. Carbonell ${ }^{2}$, S. García-Ventura ${ }^{3}$}

${ }^{1}$ Institut d'Assistència Sanitària, Health, Girona, Spain; ${ }^{2}$ FPCEE

Blanquerna, Universitat Ramon Llull, Barcelona, Spain; ${ }^{3}$ CEU,

Universitat Abad Oliva, Barcelona, Spain

Corresponding author.

Background and aims.- Spain is the country of Western Europe with the highest prevalence of HIV among people who inject drugs (PWID). This situation requires tools to assess the level of risk of contagion to improve interventions. The Risk Assessment Battery $(\mathrm{RAB})$ is a self-administered scale designed to provide a fast method of assessing risk of HIV transmission. The 45 items of the RAB are organized in two subscales (drug risk items and sex behaviour items) or a global version.

In absence of alternative ways to establish the level of risk associated with drug consumption, the aim of this study is analyse the intern consistence of the RAB in Spanish population. 
Methods.- Participants were PWID of an ambulatory treatment and harm reduction centre. 127 complete observations of the RAB were collected and were analysed using Cronbach's Alpha.

Results.- Cronbach's Alpha analysis determined as acceptable the punctuations of the global scale and of the drug risk subscale and as low the punctuations of the sex risk subscale 3 (Table 1 ).

\begin{tabular}{|l|l|l|l|l|l|}
\hline & $N^{0}$ of variables & Mean & SD & Variance & Alpha \\
\hline \hline Drug risk subscale & 8 & .654 & .509 & .058 & .446 \\
\hline \hline Sex risk subscale & 9 & -757 & .421 & .069 & .721 \\
\hline \hline Total RAB & 17 & .708 & .356 & .063 & .714 \\
\hline
\end{tabular}

Table 1. Descriptive and internal consistency analysis of the Risk Assessment Battery.

Conclusions.- The feasibility level of RAB among Spanish population is lower than in the original context of application2but his acceptable level encourage us to continue with his use and data recruitment for posterior analysis.

Disclosure of interest.- The authors have not supplied a conflict of interest statement.

\section{E-PV0944}

\section{A particular case of abuse of non-psychoactive substances}

A. Canhao

Centre Hospitalier Universitaire Vaudois, Psychiatry, Prangins, Switzerland

${ }^{*}$ Corresponding author.

Background and aims.- As described in ICD -10, a wide variety of medicaments and folk remedies may be involved, but the particularly important groups are: psychotropic drugs that do not produce dependence, such as antidepressants, laxatives, and analgesics that may be purchased without medical prescription, such as aspirin and paracetamol.

The authors report a particular situation of substance abuse that does not fall in the most used substances and that filled a particular aim for the patient.

Methods.- The authors describe the case of a patient with a long story of dependence of loperamide hydrochloride, a synthetic antidiarrheal for oral use. In this specific situation, the patient needed a control over his intestinal transit and every little change in the rhythm that he had implemented was taken like a failure over bowel control or withdrawal symptoms, leading to an enormous difficulty to deal with the problem and help him to stop this kind of medication. The possible effect over the opioid receptors was not neglected.

Results.- The patient was hospitalized for the first time in 2015 and the second time three years later. In both occasions, the patient was taking daily doses of loperamide over 50 tablets a day. A classic intervention with anxiolytic therapy covering the classic symptoms of withdrawal did not work and the patient left the hospital with a smaller daily dose but still taking loperamide at doses not clinically recommended.

Conclusions.- Share their experience in dealing with this complicated and rare situation of substance abuse, even though the therapeutic results were not encouraging.

Disclosure of interest.- The authors have not supplied a conflict of interest statement.

\section{E-PV0945}

\section{Development and validation of the inventory assessing psychological risk} factors for drug use in adolescents

E. Rasskazova ${ }^{1}$, A. Tkhostov ${ }^{2}$, E. Demenko ${ }^{3}$, E. Bryun $^{3}$, V. Arshinova $^{3}$, R. Vlasovskikh ${ }^{3}$, A. Novakov ${ }^{3}$, A. Chepeliuk ${ }^{4}$

${ }^{1}$ Moscow State University- Mental Health Research Center, Clinical psychology, Moscow, Russia; ${ }^{2}$ Moscow State University- I.M. Sechenov First Moscow State Medical University, Clinical Psychology, Moscow, Russia; ${ }^{3}$ Moscow Research and Practical Center of Narcology, Addiction Medicine, Moscow, Russia; ${ }^{4}$ Moscow State University, Clinical Psycholgy, Moscow, Russia

* Corresponding author.

Background and aims.- Early diagnostic of drug use is highly important for adolescents (Burrow-Sanchez, 2006) demanding for brief instruments revealing risk group for further expanded clinicalpsychological consultations. Such instrument should have no direct items about drugs and take into account possible desire to hide this behavior. Based on existing research on regulatory functions, impulsivity, sensation seeking, aggression and relationships with parents in in addicts we created an item pool from different measures and used "empirical keying" approach (Tellegen et al., 2008) to choose items differentiating clinical and control groups.

The aim was to assess psychometric properties of the inventory. Methods.- 637 pupils of the upper grades of general education schools from 12 subjects of the Russian Federation and 108 pupils involved in the use of drugs filled the inventory.

Results.- Controls and addicts replied differently to 56 items. Based on expert appraisals items were combined to the next scales: technological addictions, antisocial tendencies, difficulties self-regulation, emotional control, novelty seeking, risky behavior, child-parent relations (for clinical sample Cronbach's $\alpha$ was .67-.91 except antisocial tendencies scale (.55). Pupils from clinical sample are characterized by a tendency to technological addictions, antisocial tendencies, difficulties in self-regulation and in childparent relations accompanied by overestimation of the own ability to control emotions, denying the interest for novelty, risky behavior $(p<.05)$.

Conclusions.- Inventory is a reliable measure that could be used to reveal possible risk group for drug use for further expanded diagnostic. Research is supported by the Russian Foundation for Basic Research, project No. 17-06-00271.

Disclosure of interest.- The authors have not supplied a conflict of interest statement.

\section{E-PV0946}

\section{Patterns of smoking pros and cons of adolescents reporting high and low level of smoking dependence}

M. Csibi ${ }^{1}$, S. Csibi ${ }^{2}$

${ }^{1}$ University of Medicine and Pharmacy Tg.Mures, Hygiene, Tg.Mures, Romania; ${ }^{2}$ University of Medicine and Pharmacy Tg.Mures, Ethics and Social Sciences, Tg.Mures, Romania

* Corresponding author.

Background and aims.- Adolescent smoking is the most significant risk behavior with impact on young and adult health. Our longitudinal study examines adolescents' smoking related cognitions and analyzes modification in the structure of smoking pros and cons after an educational smoking prevention.

Methods.- Participants were 1369 teenagers aged $15-16$ year old, from 16 secondary schools in Târgu-Mureş, Romania. A questionnaire package was used at baseline and after the implementation of the school-based smoking prevention program, with a follow-up assessment after 6-month. Instrumentation included the modified Fagerström Tolerance Questionnaire, the Decisional Balance 
Scale and the frequency of smoking assessed through the reported smoked cigarettes in the last 30 days and the number of smoked cigarettes per day.

Results.- Adolescents reporting high values on the modified Fagerström Tolerance Questionnaire exhibited higher rates of smoking pros and fewer smoking cons comparing to adolescents reporting low values on modified Fagerström Tolerance Questionnaire. Following the prevention program, adolescents' smoking related beliefs decreased among participants with high scores on modified Fagerström Tolerance Questionnaire, while their perception of smoking disadvantages significantly increased.

Conclusions.- The pattern of adolescents' smoking beliefs differs depending on the severity of smoking. After the program implementation, in the intervention group, the Pros values for smoking decreased and the Cons values increased significantly. The cigarette consumption of adolescents reporting high scores on the modified Fagerström Tolerance Questionnaire decreased considerably, thus the program show to be successful at prevention level in adolescent population.

Disclosure of interest.- The authors have not supplied a conflict of interest statement.

\section{E-PV0947 \\ Online porn addiction: an emerging pathology}

R. De Alarcón Gómez*, N. Casado Espada, J.I. de la Iglesia Larrad, R.S. Gamonal Limcaoco, M.T. Lozano López, A. Bullón Sáez, A.

Maciá Casas

Hospital Universitario Clínico de Salamanca, Psychiatry, Salamanca, Spain

${ }^{*}$ Corresponding author.

Background and aims.- Technology has widened the behavioral addiction spectrum. In the sexual sphere we find problematic online sexual behavior (also known as porn addiction), which counts with ever-growing data supporting it, given its current prevalence and comorbidity.

We We aimed to gather the available scientific evidence on this entity as a behavioral addiction and its place among hypersexuality disorder.

Methods.- We performed a bibliographic search on Pubmed with key words ("online porn* addiction", "internet porn* addiction", "cybersex addiction"), finding 147 relevant articles. After a large manual screening process we chose a number of 16 that summarized relevant information.

Results.- Its physiopathology appears to be related to dopaminergic reward circuits and several neural addictive processes that intervene in sensitization, desensitization and a executive functioning, responding to specific sexual stimuli. These alterations have clinical implications, originating sexual dysfunction, psychosexual insatisfaction and psychiatric comorbidty. These are proportional to the behavior and the exposure to these stimuli. The only validated diagnostic tool is the Problematic Pornography Consumption Scale (PPCS). Effects appear to be reversible once this behavior ceases. Conclusions.- The inclusion of "Compulsive sexual behavior disorder" in the ICD-11 is proof that the attention directed towards this entity and related sexual behavioral addictions is growing. Online porn addiction could very well be its online counterpart. However, despite relatively solid neurobiological evidence, we are in need of more quantity of proof availing it, in particular regarding concepts of tolerance and abstinence, to properly classify it as an addiction. Disclosure of interest.- The authors have not supplied a conflict of interest statement.

\section{E-PV0948 \\ Chemsex: when pleasure turns to harm}

S. De Lucia ${ }^{1}$, G. Bondolfi ${ }^{2}$, V. Pecoul ${ }^{3}$, A. Calmy ${ }^{3}$, B. Broers ${ }^{4}$

${ }^{1}$ Geneva University Hospital HUG, Psychiatry/Liaison Psychiatry, Geneva, Switzerland; ${ }^{2}$ Geneva University Hospital, PsychiatryLiaison psychiatry, Geneva, Switzerland; ${ }^{3}$ Geneva University Hospital, Infectiology, Geneva, Switzerland; ${ }^{4}$ Geneva University Hospital, Primary care- addiction disorders, Geneva, Switzerland * Corresponding author.

Background and aims.- The term "chemsex" is used for the voluntary intake of psychoactive and non psychoactive drugs to enhance sexual intercourses mostly among men who have sex with other men (MSM). The most common chemsex drugs are GHB, GBL, mephedrone, methamphetamine, phosphodiesterase inhibitors, and alkyl nitrites. Since the first reports of recreational chemsex in the early 2000', their use is increasing. There is a growing concern about the severe mental and physical, sometimes lethal complications, related to these drugs.

To discuss the medical and psychological issues related to the use of "chemsex"drugs

Methods.- Case reports of of 3 patients recently admitted in our hospital for severe side effects of chemsex drugs use (one accidental and one intentional overdose needing invasive ventilation; one case of severe addiction to 3MMC leading to profound psychological distress), and a review of the literature on this topic. We will highlight the hazards related to the use of these substances, and discuss the unresolved issues that health care providers have to face in dealing with abuse of theses substances.

Results.- Chemsex is increasing in large urban centers, as are severe, even life-threatening, side effects related to them. There is no consensus and a lack of competence for the recognition and management of this condition in most care settings

Conclusions.- Chemsex is a growing concern. There is a need to improve the preventive measures and the medical management of complications related to this emerging phenomenon, as well as the teaching of health professionals.

Disclosure of interest.- The authors have not supplied a conflict of interest statement.

\section{E-PV0949}

\section{Influence of substance abuse service on the alcohol related issues in the Nizhniy Novgorod region}

M. Denisenko ${ }^{1^{*}}$, A. Blagonravova ${ }^{2}$

${ }^{1}$ Nizhniy Novgorod Psychiary Clinic №1, Day clinic, Nizhniy

Novgorod, Russia; ${ }^{2}$ Privolzhsky Research Medical University, Department of Epidemiology-Microbiology and Evidence-Based

Medicine, Nizhniy Novgorod, Russia

* Corresponding author.

Background and aims.- There are several factors that influence on prevalence of alcoholism: social, demographical, cultural, biological, medical. Understanding the impact of each factor plays an important role for alcohol policy and related preventive measures. The aim of our research was to analyze the impact of the availability of drug beds and narcologists in the districts on the alcohol related issues in the Nizhniy Novgorod region.

Methods.- Prevalence and incidence of alcoholism and alcoholic psychoses and mortality due to fatal alcohol poisoning (FAP) were analyzed in 52 districts of the Nizhniy Novgorod region. Period study - 2009-2014. Districts with and without drug beds and with and without narcologists were compared. Statistical analysis (descriptive statistic, Mann-Whitney U test) was carried out in Statistica 7.0. 
Results.- No significant difference was found in prevalence of alcoholism between regions with and without narcologists: 1836,5 (1428-2542) [median (IQR)] and 1798,4 (1313-2320) per 100000 respectively, $p=0,51$. The same in districts with and without drug beds - 2164,9 (1733-2634) and 1729,9 (1428-2206) per 100000 respectively, $p=0,16$. Incidence of alcoholism and prevalence of alcoholic psychoses also did not differ in respect of narcologists and drug beds. Mortality due to FAP in the districts with narcologists also did not differ from districts without narcologists, $p=0,5$. Also no difference was found in FAP between regions with and without drug beds, $p=0,94$.

Conclusions.- Substance abuse service (drug beds and narcologists) do not influence significant to the alcohol related issues. The role of other factors should be analyzed in the region.

Disclosure of interest.- The authors have not supplied a conflict of interest statement.

\section{E-PV0950}

\section{Zolpidem abuse and its consequences - the review of evidence}

W. Dróżdż ${ }^{*}$, M. Skibicka², L. Giziński ${ }^{3}$, B. Kowalewska ${ }^{3}$

${ }^{1}$ IInd Clinic of Psychiatry, Psychiatry, Torun, Poland; ${ }^{2}$ IInd

Psychiatric Ward, Bieganski Regional Hospital, Grudziadz, Poland; ${ }^{3}$

IInd Clinic of Psychiatry, Psychiatry, Bydgoszcz, Poland

${ }^{*}$ Corresponding author.

Background and aims.- Reviews of prescribing practises indicates that Zolpidem misuse can be considered a worldwide problem. Zolpidem is one of the most popular treatments for insomnia and substantial numbers of patients take it chronically and in doses higher than recommended.

Methods.- The review of consequences of Zolpidem abuse and dependence reveals many serious concerns.

Results.- The whole-country USA population study revealed that long-term Zolpidem use constitutes a risk factor for suicide, in persons with or without mental disorders. Moreover, the higher the Zolpidem dose the greater the relative risk of suicide. Results of studies performed in Taiwan indicate that Zolpidem abuse significantly increases the odds ratio of stroke, initiation of Parkinson's disease, epilepsy, benign brain tumours and dementia in older persons. The standardised risk of development of any neoplastic disease is substantially higher in those who take Zolpidem inappropriately; among the most frequent are cancers of the oral cavity, kidney, oesophagus, colon, breasts, lungs, liver and gallbladder. Long-term Zolpidem ingestion is also associated with an increased risk of head injury or bone fracture. Zolpidem overuse elevates the odds ratio of glaucoma. The probability of causing a road accident is noticeably greater in people using higher Zolpidem doses. Obstetric complications associated with Zolpidem abuse include low birth weight and premature delivery. The relative risk of acute pancreatitis is especially pronounced in Zolpidem abusers in the presence of other comorbidities.

Conclusions.- The evidence favours a more restrictive approach to Zolpidem prescription.

Disclosure of interest.- The authors have not supplied a conflict of interest statement.

\section{E-PV0952}

\section{Video gaming correlates and video gamers profile}

H. Espírito-Santo*, C. Madeira-Sério, C. Duarte, J. Tomázio, L. Lemos Instituto Superior Miguel Torga, Psychology, Coimbra, Portugal

* Corresponding author.

Background and aims.- Video gaming can be a problem, with negative consequences for those who play excessively. So, we aimed to study the correlates of video games involvement (VGI) and the profile of video gamers

Methods.- 342 participants $(M \pm S D=25.25 \pm 7.87$ years old; $61.4 \%$ were men) filled, in an internet survey, a sociodemographic questionnaire, the new Scale of Video Games Involvement (14items/5-response options; Cronbach's alpha $=0.86$, a three-factor structure explained $53.77 \%$ of the variance), the new Tripartite Scale of Self-Conscious Emotions (15-items/4-response options; Cronbach's alpha $=0.72$; a forced three-factor structure explaining $62.9 \%$ of the variance), the Dissociative Experiences Scale - Distractibility subscale, and the Severity of Dependence Scale-General (SDS-G). Video gamers $(n=35 ; 10,2 \%)$ were identified by playing daily, and/or more than an hour a day and playing for at least 12 months.

Results.- VGI correlated with pride $(r=-0.44 ; p<.01)$, shame $(r=0.40 ; p<.05)$, guilt $(r=0.46 ; p<.01)$, distractibility $(r=0.44$; $p<.01$ ), and SDS-G. Video gamers were mostly male (65.7\%), with a college degree (45.7\%), no partner (74,3\%), a mean age of 26.69 years $(S D=9.34)$, and a mean education of 14.54 years $(S D=3.00)$. The majority drank (60.0\%), and smoked (54,3\%), and some used cannabis (40.0\%). They consider themselves as casual (34.3\%), social (5.7\%), specialists (34.3\%), or master players $(25.7 \%)$.

Conclusions.- These preliminary results have important implications for future research and for the treatment of video game addiction.

Disclosure of interest.- The authors have not supplied a conflict of interest statement.

\section{E-PV0953}

\section{Methadone treatment in pregnancy}

S. Fink ${ }^{*}$, E. Go

Bronx-Care Mount Sinai Health System, Addiction Psychiatry, Bronx, USA

* Corresponding author.

Background and aims.- Opioid Use in Pregnancy is one of the most challenging and complicated clinical scenarios to manage. Its prevalence is unknown but reportedly increased by $127 \%$, from 1.7 per 1,000 delivery admissions in 1998 to 3.9 per 1,000 delivery admissions in 2011 . More recently, almost $1 \%$ of pregnant women reported non-medical use of opioids in the past 30 days. The importance of this clinical situation is mainly due to high risks to the mother and unborn child as well as dosage management. There is high risk for medical, obstetric \& psychiatric complications in opioid use and pregnancy. There are also psychosocial factors that impacted the care of these pregnant patients. In the past 5 years, there were several pregnant women treated in our methadone clinic. We evaluated the treatment trend, dose adjustment and relapsing during their treatment course.

Methods.- This study is a retrospective case series study of deidentifiable pregnant women with opioid use who were pregnant from 2013 to 2018. Their charts will be reviewed for duration of their pregnancy, dosages, toxicology screening and psycho-social demographics.

Results.- 50\% required a higher dose of methadone.

Conclusions.- While opioid use in pregnant women may not be a common occurrence, it is important to learn the treatment strategies to safely manage them throughout their pregnancy. Clinicians should be aware of the potential risks of opioid use in pregnancy. By knowing these various risks, clinicians will also learn how to take extra important monitoring care, and dose management of pregnant women while in opioid treatment.

Disclosure of interest.- The authors have not supplied a conflict of interest statement. 


\section{E-PV0954}

\section{Social media disorder scale: portuguese version factor structure and psychometric characteristics in college students}

A. Galhardo ${ }^{*}$, E. Raimundo ${ }^{2}$, C. Ferreira ${ }^{3}$, I. Massano-Cardoso 4 ${ }^{1}$ Instituto Superior Miguel Torga and Faculty of Psychology and Educational Sciences of the University of Coimbra, Cineicc, Coimbra, Portugal; ${ }^{2}$ Instituto Superior Miguel Torga, Psychology, Coimbra, Portugal; ${ }^{3}$ Faculty of Psychology and Educational Sciences of the University of Coimbra, Cineicc, Coimbra, Portugal; ${ }^{4}$ Instituto Superior Miguel Torga and Faculty of Medicine of the University of Coimbra and Faculty of Economics of the University of Coimbra, Ceisuc, Coimbra, Portugal

* Corresponding author.

Background and aims.- Social media play an important role in society and its use has been rising both in number of users and time spent in their use. In this context, the Social Media Disorder Scale (SMD Scale) was developed and proved to be a valid and reliable measure of social media addiction. This study aimed to develop and test the fit of the Portuguese version of the Social Media Disorder Scale (SMD Scale-PT), and explore its psychometric properties in college students.

Methods.- A sample of 297 students, 205 women (69\%) and 92 men (31\%) completed online a set of self-report instruments: the SMD Scale-PT, the Depression, Anxiety and Stress Scales (DASS-21), the Internet Addiction Test (IAT) and the Nomophobia Questionnaire (NMP-Q-PT).

Results.- The SMD Scale-PT revealed a unidimensional factor structure, showing the following adjustment indicators: $\chi^{2} / g l=3.80$, $\mathrm{CFI}=.816, \mathrm{GFI}=.930, \mathrm{TLI}=.754, \mathrm{RMSEA}=.097, \mathrm{MECVI}=.473$. The Cronbach's alpha was .67 and composite reliability was .70 . The SMD Scale-PT was positively correlated with IAT and NMP-Q, which assess similar constructs. Regarding the association of SMD ScalePT with the anxiety, depressive and stress symptoms assessed by the DASS-21, there were also positive and statistically significant correlations.

Conclusions.- The Portuguese version of the SMD Scale-PT, although revealing some fragilities, showed similarities in factor structure and psychometric properties with the original and Turkish versions. This study also pointed out possible modifications that may contribute to this measure improvement.

Disclosure of interest.- The authors have not supplied a conflict of interest statement.

\section{E-PV0955}

\section{Behavioral addiction among medical students}

M. Gharmoul*, J. Nakhli, A. Souilem, S. Ben Nasr

Farhat Hached University Hospital, Psychiatry Department, Sousse, Tunisia

${ }^{*}$ Corresponding author.

Background and aims.- While addictions to substances such as alcohol, tobacco, and other drugs have been extensively investigated, interest has been growing in potential non-substance related addictive behaviors (BA) which the prevalence in the world varies between 1 and $8 \%$. The idea of BA is based in scientific knowledge, but the concept is still controversial.

Objectives.- Determine socio-demographic characteristics of BA among medical students and discuss on the relationship with depressive and anxiety disorders.

Methods.- Our study was a descriptive and cross-sectional study of 133 students enrolled in the Faculty of Medicine of Sousse in December 2016. Data collection was conducted using a questionnaire assessing socio-demographic characteristics. We have administered 4 validated BA scales (Young's IAT, Scale Addiction Face Book, Physical Activity Addiction Test, SOGS) and HAD scale to evaluate anxiety and depressive symptoms.

Results.- Face book addiction was the most common form (20.3\%), followed by gambling (12\%) and cyber addiction ( $0.8 \%)$. Problematic Internet use was noted in $18.2 \%$. Dependence to physical activity wasn't found. Face book and Internet addiction were predominant among females (24.8\%), unlike gambling. We have highlighted close relationships between behavioral and substance addiction and a strong associations between most BA and depressive and anxiety disorders which seem relatively non-specific.

Conclusions.- Face book addiction was the most prevalent behavioral addiction in medical students. All these behavioral addictions were associated with others substances addiction, depressive and anxiety disorders.

Disclosure of interest.- The authors have not supplied a conflict of interest statement.

\section{E-PV0956}

\section{Exploring the role of alcohol and nicotine addiction in first degree relatives of alcohol addicted women in Southern Bulgaria}

V. Giannouli $1^{*}$, D. Ivanova ${ }^{2}$

${ }^{1}$ Organisation Against Drugs, Okana, Athens, Greece; ${ }^{2}$ South-West University “Neofit Rilski”, Department of Psychology, Blagoevgrad, Bulgaria

* Corresponding author.

Background and aims.- Although there is a plethora of research regarding alcohol and opiate addicted parents and children, there is scarce research focusing on adult children coming from families were at least one parent has addiction problems. The aim of this study is to examine how and if having a first degree relative with alcohol and/or nicotine addiction(s) influences the Lesch typology in a sample of alcohol dependent women in Southern Bulgaria. Methods.- Fifty-three women with a diagnosis of alcohol dependence $\left(M_{\mathrm{age}}=43.84, \mathrm{SD}_{\mathrm{age}}=9.48 ; M_{\text {years of education }}=15.11\right.$, $\mathrm{SD}_{\text {years of education }}=3.21 ; \mathrm{M}_{\text {years of addiction }} 12, \mathrm{SD}_{\text {years of addiction }}=3.56$, all raised by their parents) were examined with the Bulgarian version of the Lesch Alcoholism Typology - Questionnaire (LAT), while data were collected with self-report questionnaires regarding their parents' drug use and abuse history. All participants came from the Municipal Council on Drug Addiction in Blagoevgrad.

Results.- Results indicated that following chi-square test of independence, no significant interaction was found $\left(x^{2}(2)=.696\right.$, $p=.706)$.

Conclusions.- According to the above results, women with alcohol dependence who have a parent with nicotine addiction and/or a parent with alcohol dependence is not more likely to suffer from a specific type of alcohol dependence according to Lesch's typology. This implies that other psychological, social and cultural factors may play a much stronger role in determining the Lesch types in alcohol use disorder.

Disclosure of interest.- The authors have not supplied a conflict of interest statement.

\section{E-PV0957}

\section{Loss of control, cognitive impairment and lesch typology in alcohol addicted women in Southern Bulgaria}

V. Giannouli ${ }^{*}$, D. Ivanova ${ }^{2}$

${ }^{1}$ Organisation Against Drugs, Okana, Athens, Greece; ${ }^{2}$ South-West University "Neofit Rilski”, Department of Psychology, Blagoevgrad, Bulgaria

Corresponding author. 
Background and aims.- Loss of control problems in patients suffering from alcohol dependence have been reported so far in numerous studies, but no systematic examination of the aforementioned problems with cognitive impairment and Lesch alcoholism typology has been made. The aim of this study is to determine associations between the Lesch typology, cognitive impairment and loss of control in a sample of alcohol dependent women in Southern Bulgaria.

Methods.- We recruited a sample of fifty-three women from the Municipal Council on Drug Addiction in Blagoevgrad. Participants had a diagnosis of alcohol dependence $\left(M_{\mathrm{age}}=43.84\right.$, $\mathrm{SD}_{\text {age }}=9.48 ; \quad \mathrm{M}_{\text {years of education }}=15.11, \quad \mathrm{SD}_{\text {years of education }}=3.21$; $\mathrm{M}_{\text {years of addiction } 12,} \mathrm{SD}_{\text {years of addiction }}=3.56$ ). They were evaluated with the Bulgarian version of the Lesch Alcoholism Typology Questionnaire (LAT), while data were collected regarding the severity of the cognitive deficits (none, slight, and severe) and the frequency of loss of control (none, occasionally, frequently, and permanently).

Results.- Results indicated that there is no association between Lesch typology, severity of cognitive impairment and the frequency of mentioned loss of control problems.

Conclusions.- The findings suggest that concerns about personal control do not have a clear influence on cognition or self-reported severity of cognitive deficits. Future research should further examine if specific aspects of cognitive control may have an influence on cognition in alcohol dependence by using objective measures and not only self-reports.

Disclosure of interest.- The authors have not supplied a conflict of interest statement.

\section{E-PV0958}

\section{Neurobiology and neurophysiology of internet addiction \\ D. Gorgoli}

General Hospital of Nea Ionia “Konstantopouleio", Psychiatric, Athens, Greece

${ }^{*}$ Corresponding author.

Background and aims.- Recent neurobiological and neurophysiological studies ave shown that there are certain prefrontal executive control functions, that are related to symptoms of Internet Addiction. These studies have also revealed that there are great similarities between "internet" regions and brain circuits of problematic behavior in substance abuse and behavioral addiction.

Methods.- One of the most interesting questions raised here, is whether these addiction areas had this particular structure before or after the internet/gambling/substance abuse: does the brain change over time so that it responds to this abuse in a specific way? How does this play its part in the loss of control in behavioral abuses?

Results.- What is found in these "internet-brains" is an overall change in the dopaminergic reward system: frontal-guided changes of glutaminergic innervation of the nucleus accumbens (Kalivas and Volkow, 2005) and parts of the dorsal striatum, along with limbic and para-limbic regions (eg. the orbitofrontal cortex) learn to habitually react on drug cues with craving (Bechara, 2005; Goldstein et al., 2009).

Conclusions.- Prefrontal control processes are reduced in individuals who are addicted to the internet and may be related to the patient's loss of behavioral control. So, we conclude that Internet Addiction should be classified as a separate diagnostic category in DSM-6 (DSM-5: only "Internet Gaming Addiction" is classified as a specific behavioral addiction). Last but not least, we try to make a connection further to be studied, between these internet addiction reward system processes and the recent findings, that link the vagus nerve with the reward system of the brain.

Disclosure of interest.- The authors have not supplied a conflict of interest statement.

\section{E-PV0960}

\section{Comparative investigation of alexithymia in addicted and non-addicted people}

A. Homayouni

Department of Psychology, Bandargaz Branch, Islamic Azad

University, Bandargaz, Iran

* Corresponding author.

Background and aims.- Alexithymia is a personality characteristic in which the individual is unable to identify and describe their emotions. The main feature of Alexithymia is an emotional unawareness, lack of social attachment, and poor interpersonal relating. Furthermore, those suffering from Alexithymia have difficulty recognizing and understanding the emotions of others. In medical definition, people with alexithymia typically display a lack of imaginative thought, have difficulty distinguishing between emotions and bodily sensations, and engage in logical externally oriented thought.

Methods.- The research method was causative - comparative. 50 addicted with history of substance abuse and 50 non addicted people were selected and responded to Toronto Alexithymia Scale (TAS-20). The TAS-20 measures three dimension of Alexithymia: difficulty identifying feelings and distinguishing them from bodily sensations; difficulty communicating or describing emotions to others; an externally oriented style of thinking. The data were analyzed with $\mathrm{T}$ independent formula.

Results.- The findings showed that there are significant differences in dimensions of Alexithymia between addicted and non-addicted people. Addicted people got higher scores of Alexithymia dimensions especially in difficulty identifying feelings and difficulty communicating than non-addicted people, but there are not significant differences in dimension of externally oriented style of thinking between two groups.

Conclusions.- Based on findings, it is suggested to plan educational programs in order to help the people for empowerment them to identify, describe, control and manage their feelings and emotions to decrease tendency to addictive behavior especially substance abuse.

Disclosure of interest.- The authors have not supplied a conflict of interest statement.

\section{E-PV0961 \\ Dual pathology on the model of alcohol dependence and polyneuropathy}

I. Sosin, O. Honcharova*, Y. Chuiev

Kharkiv Medical Academy of Postgraduate Education, Department of Narcology, Kharkiv, Ukraine

"Corresponding author.

Background and aims.-

Introduction.- Alcoholic polyneuropathy (APN) is considered a neuroscience issue of topical priority associated with therapeutic resistance, early incapacitation and disability. APN is a formal dual pathology, recognized in ICD-10 (International Statistical Classification of Diseases), code: G 62.1, that initiates interdisciplinary aspects of integrated therapy.

Objectives.- To develop a new effective way to treat alcoholic polyneuropathy in clinical addictology.

Methods.- Clinical and diagnostic identification of alcohol dependence, complicated by degenerative impairment of the peripheral nervous system, carried out by targeted and valid scales, tests, electroneuromyography, angiography examination.

Results.- Dual pathology treatment was tested by our method for treating alcoholic polyneuropathy (Patent 74888 UA Ukraine), its creative innovation essence combines complex pharmacologi- 
cal therapy (detoxification, vitamin therapy, vasotropic, stabilizing autonomic nervous system, antioxidant therapy) with Cocarnit injections and synchronous membrane plasmapheresis ( 1 session in 3 days, for course of $1-3$ sessions, with dosed blood plasma exfusion volume $600.0-1000.0 \mathrm{ml}$ ). Depending on APN clinical form, intravascular laser hemotherapy was used individually. Randomly selected 47 APN patients were examined and effectively treated with the proposed method. Complex tendency towards relieving APS manifestations was registered along with synchronously reduced pathological craving for alcohol, other psychological and behavioral disorders associated with alcohol withdrawal.

Conclusions.- The method for treating APN was statistically significantly more effective compared with conventional therapeutic approach.

Disclosure of interest.- The authors have not supplied a conflict of interest statement.

\section{E-PV0962}

\section{Method of integrated relieving of withdrawal syndrome at coaxil dependence}

I. Sosin, O. Honcharova*, Y. Chuiev

Kharkiv Medical Academy of Postgraduate Education, Department of Narcology, Kharkiv, Ukraine

${ }^{*}$ Corresponding author.

\section{Background and aims.-}

Introduction.- Antidepressant Coaxil (Tianeptine), abused as a psychoactive substance, presents an extremely malignant addiction. Pathogenic mechanism of the dependence is determined by direct agonistic (ligand) interaction of Coaxil with opioid receptors, combining psychoactive effects of opioids, heroin, ephedrone, cocaine, that explains pathological interest in it of the drug addicts. The problem arisen in treatment of Coaxil dependent (CD) patients results from severity of the withdrawal syndrome, present mental disorders (severe anxiety, agitation, nervousness), lack of conscious motivation for treatment. In fact, no remissions are observed in CD. Objectives.- Innovative improvement of relieving the withdrawal syndrome in $\mathrm{CD}$.

Methods.- MADRS, HADS, Hamilton, Spielberger-Khanin scales, clinician's global impression (CGI), Quality of life (QoL), tremor laser diagnosis (patent 29650, UA).

Results.- Innovative method of integrated relieving withdrawal syndrome at Coaxil dependence (Patent 56682, UA) is distinct by providing, along with conventional pharmacotherapy, membrane plasmapheresis, $1-3$ sessions at 3 day intervals with dosed blood plasma exfusion volume of $600.0-1000.0 \mathrm{ml}$; the pharmacotherapy is added with oral antidepressant Deprivox (Fluvoxamine) 50.0-100.0 mg once-twice daily, up to 3-month course; hepatoprotector Darsil (Silymarin) 1-2 tab $(3570 \mathrm{mg}), 3$ times daily, up to 3 months; bipolar transcerebral electrophoresis: Analgin (Metamizole sodium) 0.5\% solution, from cathode, and Dimedrol (Diphenhydramine) $2.5 \%$ solution, from anode, once per day, for 35 days, 25-30 min exposure, for 7-12-session course. The method was tested on 27 subject patients.

Conclusions.- The proposed method of relieving withdrawal syndrome provides targeted effect on pathogenic mechanisms of dependence, increases efficiency and reduces the term of treatment. Начало формы Конец формы

Disclosure of interest.- The authors have not supplied a conflict of interest statement.

\section{E-PV0965}

Premorbid peculiarities of persons suffering with combined abuse of hashish and tramadol

I. Khayredinova*, Z. Ashurov

Tashkent Medical Academy, psychiatry, Tashkent, Uzbekistan

* Corresponding author.

Background and aims.- The prevalence of the combination of hashish with tramadol abuse is still growing momentum day by day. Reviews, researches, clinical cases devoted to the study of the combined abuse of hashish with tramadol, are very scarce and contradictory.

Aim. Identify the relationship between the nature of the parental family, the resulting upbringing and character accentuation, suffering from poly dependence on hashish with tramadol.

Methods.- We observed 20 persons suffering from the combined abuse of hashish with tramadol - the main group and the control group - 18 persons with tramadol addiction. All patients are male, aged from 24 to 39 years. The survey was carried out using a specially designed questionnaire.

Results.- Patients in the main group were brought up in singleparent families with unfavourable psychological circumstances. The model of upbringing in the conditions of rigid relationships was more common in the main group - $60.0 \%$, while in the control group, education in the atmosphere of the cult of the disease prevailed-66.6\%. The first sample of cigarettes and hashish in the overwhelming majority (65.0\%) and (56.0\%) respectively, occurred were in adolescence. For the first time, $78.0 \%$ of them used cannabis in places of gathering - in clubs, bars and discos.

Conclusions.- the formation of inadequate characterological and behavioural reactions of the individual and the risk of early narcotization and the development of polydependence, are influenced not only by premorbid features but also by the unfavourable nature of family relations, improper upbringing.

Disclosure of interest.- The authors have not supplied a conflict of interest statement.

\section{E-PV0966}

\section{Specifics of clinical manifestations of alcohol addiction in people with different psychotraumatic experiences and levels of psychosocial \\ stress}

H. Kozhyna ${ }^{1 *}$, K. Gaponov ${ }^{2}$, M. Markova ${ }^{1}$

${ }^{1}$ Kharkiv National Medical University, Psychiatry- Narcology and Medical Psychology, Kharkiv, Ukraine; ${ }^{2}$ Kharkiv Medical Academy of Postgraduate Education, Narcology, Kharkiv, Ukraine

* Corresponding author.

Background and aims.- Despite the fact that the close correlation between alcohol addiction and social stress is established at both genetic and biochemical levels and at population levels, it is still unclear how the stress factor affects the style of alcohol use and the alcohol addiction clinic. The purpose of the work is to study the clinical and psychopathological specifics of alcohol addiction in people with different psychotraumatic experiences and levels of psychosocial stress, to understand the ways of modification of existing therapeutic and rehabilitation strategies, taking into account the aggravating effect of stress on the formation and the course of alcohol addiction.

Methods.- Clinical-psychopathological and psychodiagnostic methods, scales for assessing stress (L. Rider, PSM-25, IES-R) and severity of alcohol addiction (AUDIT, SADQ-C, CIWA-Ar) were used.

Results.- 312 men were examined:107 combatants, who had the experience of participating in military operations in the East of 
Ukraine as participants in the Anti-Terrorist Operation; 89 forced displaced persons from temporarily occupied districts of Donetsk and Lugansk region; 116 -residents of Kharkiv and Kharkiv region, who were not combatants or internally displaced persons.

Conclusions.- The regularities concerning the association of alcoholizationand psychosocial stress are revealed: the factor of severity of psychosocial stress most strongly affects the severity of alcohol addiction; the factor of participation in military operations and the factor of forced resettlement can be considered as actual factors of alcoholization; the factor of presence of psychotraumatic experience in itself, not associated with distinct response to stressful event, does not significantly affect the indicators of the severity of alcohol-related disorders.

Disclosure of interest.- The authors have not supplied a conflict of interest statement.

\section{E-PV0967}

\section{The diagnostic value of kynurenine as a marker of harmful ethanol use}

A. Kułak-Bejda ${ }^{1}$, K. Wilczyńska ${ }^{1}$, I. Ptaszyńska-Sarosiek ${ }^{2}$, A. Niemcunowicz-Janica ${ }^{2}$, A. Zalewska ${ }^{3}$, M. Maciejczyk ${ }^{4}, \mathrm{~N}$. Waszkiewicz ${ }^{1}$

${ }^{1}$ Medical University of Białystok, Department of Psychiatry, Choroszcz, Poland; ${ }^{2}$ Medical University of Biatystok, Department of Forensic Medicine, Białystok, Poland; ${ }^{3}$ Medical University of Bialystok, Department of Restorative Dentistry-, Białystok, Poland; ${ }^{4}$ Medical University of Biatystok, Department of Physiology, Białystok, Poland

* Corresponding author.

Background and aims.- L-kynurenine is a metabolite of the amino acid L-tryptophan which is used in the niacin's production. Kynurenine is synthesized by the tryptophan dioxygenase and indoleamine 2,3-dioxygenase. Kynurenine and its further products perform different biological functions such as regulating the immune response and dilation blood vessels during inflammation. The aim of the study was an evaluation of the usefulness assessment of kynurenine activity as the marker of ethanol abuse in people who died of ethanol intoxication as well as an attempt to determine the mechanism of their death.

Methods.- The research was conducted on two groups of the deceased: the first one consisted of 22 people who died due to ethanol intoxication (4-4.5\% in the blood serum and $4-6.1 \%$ o in the urine), the control group included 30 people in whose bodies no alcohol was found. Activity (pKat/mL) of kynurenine in supernatants of the serum, vitreous humor, cerebrospinal fluid and urine were determined.

Results.- Both in the control group and in the study group there were statistically significant $(p<0.001)$ higher level of kynurenine in the urine in relation to blood. In the control group, a higher level $(p<0.001)$ of kynurenine in the vitreous humor was demonstrated compared to cerebrospinal fluid. In the examined group these statistically significant differences were not as high as in the controls (kynurenine $p=0.005$ ).

Conclusions.- Our study showed that lower kynurenine levels, in people which died due to ethanol intoxication, might be valuable as a marker of harmfule ethanol use.

Disclosure of interest.- The authors have not supplied a conflict of interest statement.

\section{E-PV0968}

\section{The family pain in alcohol families}

A. Lutsenko*, A. Spivakovskaya

Moscow State University, Faculty of Psychology, Moscow, Russia

* Corresponding author.
Background and aims.- The problem of alcohol addiction is actively studied in the last decade in Europe. Most of the research on this topic was focused on the personality of the alcohol addicts, their cognitive and emotional traits. The relevance of the study is due to the wide prevalence of addictive behavior in Russian population and insufficient knowledge of the resource factors of the patient's family system.

Objectives.- To study the family pain experienced by adult people whose parents were alcohol addicts.

Methods.- The interviewees ( $n=52,41$ women, 11 men) were recruited among mentally healthy adults who did not suffer from addictions but whose parents were alcohol addicts. The members of the experimental group took part in the 12-step rehabilitation program. We used a phenomenological analysis of the 15 transcripts of the public meetings 12-step recovery program.

Results.- The state of family pain is transformed during the process of family members' participation in 12-step recovery program. It is possible to distinguish three stages of overcoming the state of family pain by relatives of alcoholics: the stage of awareness of the presence of family pain, the study of the peculiarities of experiencing family pain and its impact on family life, and the search for ways to work with family pain.

Conclusions.- Adult people whose parents were alcohol addicts feel the family pain. This feeling includes all negative emotions experienced by the family. It is associated with a sense of guilt and shame in the past and is not related to the actual state of the patient.

Disclosure of interest.- The authors have not supplied a conflict of interest statement.

\section{E-PV0971 \\ Practical problems in detoxification from benzodiazepines (BZD) - pharmacokinetic particularities (case report)}

U. Cieślak ${ }^{1}$, P. Mierzejewski ${ }^{*}$, A. Basińska-Szafrańska ${ }^{3}$

${ }^{1}$ Institute of Psychiatry and Neurology, 1st Department of Psychiatry, Warsaw, Poland; ${ }^{2}$ Institute of Psychiatry and Neurology, Department of Pharmacology and Physiology of the Nervous System, Warsaw, Poland; ${ }^{3}$ Institute of Psychiatry and Neurology, Department of Prevention and Treatment of Addictions, Warsaw, Poland ${ }^{*}$ Corresponding author.

Background and aims.- BZD detoxification usually starts by switching to a long-acting benzodiazepine. This presentation concerns an alternative approach after failure and complications of the standard one.

A 51-year-old male patient dependent on midazolam (1500 mg/d), transferred from the district hospital where he was admitted in a withdrawal status epilepticus. Thiopental and loading with diazepam $(700 \mathrm{mg}$ ) were used there. Upon transfer to the detoxification ward the patient was improved, without seizures, maintaining superficial and sometimes inadequate verbal contact. On the 1st day (serum-BZD 609ng/ml), in spite of continued diazepam ( $85 \mathrm{mg}$ ), his condition worsened again. On the 2nd day, consciousness disturbances persisted (serum-BZD 753ng/ml) and diazepam (next $45 \mathrm{mg}$ ) was followed by clorazepate $80 \mathrm{mg}$. On the 3rd day, at serum-BZD $496 \mathrm{ng} / \mathrm{ml}$, he lost contact.

Methods.- Analyzing previous evolution of symptoms, serum-BZD levels and succeeding drug doses, pharmacokinetics was considered for finding alternative loading pathways. Hypothesising ultra-rapid metabolism induced by chronically administered midazolam (megadoses) an attempt was made with lorazepam as an agent bypassing the metabolic routes typical for the above-listed BZDs (hydroxylation).

Results.- Within 1 day (total dose $24 \mathrm{mg}$, corresponding serum-BZD $157 \mathrm{ng} / \mathrm{ml}$ ) the patient regained orientation and contact. 
Conclusions.- Due to high tolerance, of both pharmacodynamic and pharmacokinetic nature, administration of diazepam (unless its megadoses were continued) and clorazepate did not enable nordiazepam accumulation and patient loading, which resulted in worsening of the symptoms. The different metabolism of lorazepam, bypassing hydroxylation typical for biotransformation of midazolam and both substitutes, made it possible to obtain the loading concentration.

Disclosure of interest.- The authors have not supplied a conflict of interest statement.

\section{E-PV0973}

\section{Determinants and biomarkers of alcohol dependence among male patients attending a tertiary care hospital in India}

S. Mukherjee ${ }^{1^{*}}$, S. Ghosh ${ }^{2}$

${ }^{1}$ Calcutta National Medical College- Kolkata, Psychiatry, Kolkata, India; ${ }^{2}$ N.R.S Medical College, Psychiatry, Kolkata, India

${ }^{*}$ Corresponding author.

\section{Background and aims.-}

Background.- Alcoholism is a major problem and a leading preventable cause of death. Research suggests that who have an early onset of alcohol use are more likely to develop severe dependenceAlcohol consumption is increasing with time alarmingly in countries like India mainly due to changes in socio-cultural and economic environment. Various international studies reported that individuals with family history of alcoholism have shown increased severity of alcohol dependence. Gamma-Glutamyl transferase, Mean Corpuscular volume are important biomarkers for alcohol dependence.

Objective.- All these determinants like social, occupational and biomarkers should be studied in detail to prevent alcohol dependence.

Methods.- It was a hospital based cross sectional observational study. 65 cases had been selected after interview by AUDIT scale and semi-structured questionnaire. Alcohol Dependence Scale was used to assess the severity of dependence. 65 healthy male controls were selected for biomarkers estimation and comparison with patients.

Results.- The mean age of onset (17.14 years) of alcohol drinking and family history, marital status, nature of work (unskilled) are significantly associated with severity of alcohol dependence ( $p$ value $<0.0001)$. Study suggests that mean value of GGT and MCV is significantly higher for cases $(101.95 \mathrm{U} / \mathrm{L}, 96.47 \mathrm{fL})$ than the controls (21.55 U/L, 88.16fL). Regarding evaluation of biomarkers GGT and MCV had shown significant association with severity of alcohol dependence.

Conclusions.- Alcohol intake in early period of life should be prevented by providing health education and biomarkers are to be used in suspected cases of alcohol dependence for diagnosis and for prognostic assessment.

Disclosure of interest.- The authors have not supplied a conflict of interest statement.

\section{E-PV0976 \\ Chemsex-induced psychosis, case report and literature review}

J.M. Pastor Haro*, V. Romero Pardo, J. Curto Ramos, N. Fernández Gómez, L. Mellado Cuerno, M.F. Bravo Ortiz University Hospital La Paz, Psychiatry and Mental Health Department-Madrid-Spain, Madrid, Spain

${ }^{*}$ Corresponding author.

Background and aims.- Chemsex is defined as the practice of using drugs intentionally during or before sexual intercourse, specially on men who have sex with men. During last years it has been a growing concern and it has been pointed out as a potential public health priority based on its consequences on substance dependence, HIV or HCV transmission. It involves the use of multiple psychoactive substances: principally mephedrone, crystal metanphetamine, gamma-hydroxybutyric acid, some of them having been linked with substance-induced psychosis.

Methods.- A case of substance-induced psychosis on a chemsex user of smoked metamphetamine is reported. A review on literature is conducted to understand the context of chemsex practice, to describe the link between the most used drugs and psychosis and to describe if chemsex pattern of drug use has repercussions on the treatment of psychosis on this kind of patients.

Results.- We describe a brief psychotic episode on a chemsex user with complete resolution within a week. To date several case reports have linked the use of crystal metamphetamine and mephedrone with psychosis; with gamma-hydroxybutyric acid being linked mostly with delusional symptoms during withdrawal but also with psychosis. More research is needed to describe potentially synergic effects of substance on psychosis induction.

Conclusions.- The pattern of drug use on chemsex vs. non-chemsex users has many differences. This differences make difficult the access of chemsex user to mental health network, therefore hindering the treatment of coexisting disorders. Specific mental health resources for chemsex users may be needed and harm-reduction strategies are encouraged.

Disclosure of interest.- The authors have not supplied a conflict of interest statement.

\section{E-PV0978 \\ The forbidden fruit: a complicated differential diagnosis of cannabinoid hyperemesis syndrome}

\section{Piotrkowicz ${ }^{1^{*}}$, M. Janoska-Jazdzik ${ }^{1}$, M. Wrobel ${ }^{2}$}

${ }^{1}$ Medical University of Warsaw, Faculty of Health Sciences, Warsaw, Poland; ${ }^{2}$ Mazowieckie Specjalistyczne Centrum Zdrowia, Psychiatry, Pruszkow, Poland

Corresponding author.

Background and aims.- Cannabinoid hyperemesis syndrome was described by Allen and al. in 2004 and is a rare condition linked to cannabis overuse. It is characterized by recurrent nausea, vomiting and abdominal pain, which can be relieved by hot showers, or by cannabinoids abstinence. Due to its rarity and patients reluctance to disclose full information about their cannabinoids use patterns, it can remain undiagnosed for long periods of time, leading to serious complications. Misdiagnosis may provoke unnecessary polytherapy. The case of a patient will be presented to show the complicated process of differential diagnosis.

Methods.- Medical documentation from psychiatric and internal diseases departments hospitalizations, as well as outpatient care unit was analyzed.

Results.- A 31-year old male patient was admitted to psychiatric hospital seventeen times between 2015-2018 due to recurrent nausea, vomiting, and abdominal pain, relieved by hot showers. First onset of symptoms occurred in 2010. Patient linked them to food poisoning with dirty apple during a holiday in Egypt. In the span of three years, he was diagnosed with various somatoform disorders, obsessive-compulsive disorder and mixed personality disorder. Patient confirmed occasional THC use in periods of time preceding symptoms onset. Due to severe somatic complications and ineffective pharmacotherapy consistent with diagnoses, he was subject to polytherapy and off-label treatment. Finally, during an outpatient visit, he admitted that he used cannabinoids on regular basis.

Conclusions.- Diagnosis delay due to lack of awareness of aforementioned syndromes symptoms and patients poor compliance led 
to numerous hospitalizations, polytherapy and somatic complications.

Disclosure of interest.- The authors have not supplied a conflict of interest statement.

\section{E-PV0979}

\section{Prevalence of psychotic symptoms amongst drug addiction treatment centre patients}

E. Pranskaityte ${ }^{1 *}$, V. Andrejauskiene ${ }^{2}$, R. Jurkyte ${ }^{1}$

${ }^{1}$ Vilnius University, Faculty of Medicine, Vilnius, Lithuania; ${ }^{2}$ Vilnius

Drug Addiction Treatment Centre, Department of Psychosocial

Rehabilitation, Vilnius, Lithuania

${ }^{*}$ Corresponding author.

Background and aims.- Substance-induced disorders, one of them being drug-induced psychosis, are a serious issue in modern psychiatry considering the problem of increased drug usage. It is important to know the prevalence of psychotic symptoms amongst patients treated from drug addiction disease in order to evaluate their risk for psychosis and improve the treatment. The aim of this research is to indicate the prevalence of psychotic symptoms amongst the multiple drugs taking patients who are being treated at drug addiction treatment centre.

Methods.- 50 Vilnius Drug Addiction Treatment Centre patients with a mean age of 32.9 years, who are using multiple drugs were asked to perform a 20 question psychosis screening test to assess psychotic symptoms.

Results.- 64\% of participants scored 7 or more points in the test when 7 was chosen as a cut off point for the total score of the psychosis screening test, indicating clinically significant psychotic symptoms. $18 \%$ of patients scored more than 14 out of 20 points. These results are listed in Fig. 1 . The mean of the all participants test results was 8.56 , which is significantly higher than the lowest positive score $(p>0.05)$.

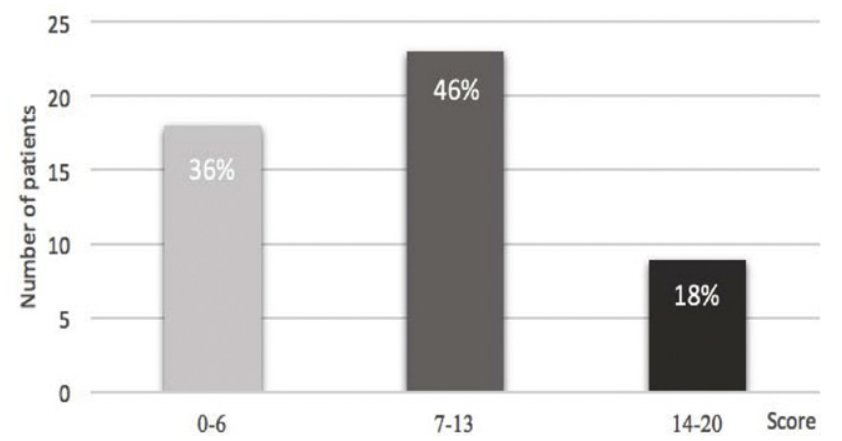

Fig. 1. Distribution of psychosis symptoms test scores.

Conclusions.- Psychotic symptoms amongst drug addiction centre patients are very common. Multiple drug users are at significantly high risk for psychosis.

Disclosure of interest.- The authors have not supplied a conflict of interest statement.

\section{E-PV0980}

\section{Internet addiction and its relation to anxiety and depression among Zagazig University students, Egypt}

N. Raafat ${ }^{*}$, H. Fouaad ${ }^{2}$, A. Shalanda ${ }^{3}$, A. Halima ${ }^{4}$

${ }^{1}$ Assistant prof, Psychiatry, Zagazig, Egypt; ${ }^{2}$ Lecturer, psychiatry, Zagazig, Egypt; ${ }^{3}$ Prof, psychiatry, Zagazig, Egypt; ${ }^{4}$ Assisstant lecturer, psychiatry, Zagazig, Egypt

* Corresponding author.
Background and aims.- Internet addiction (IA) is a growing problem among university students worldwide. There is lack of Egyptian national studies addressing this problem. We aimed to assess the prevalence of Internet addiction and its relation to anxiety and depression levels among Zagazig University students.

Methods.- Across sectional study was carried out on 1005 zagazig university students (768 females), 17-30 years old, recruited from 2 practical (faculty of medicine $\&$ faculty of science) \& 2 theoretical faculties (faculty of law and faculty of education). They were randomly chosen following multistage random sampling methodology. Students were asked to fill a questionnaire including demographic data, faculty type and the class. Internet addiction test (IAT) and the Hospital anxiety and depression scale (HADS) were used to assess internet addiction and anxiety and depression comorbidity.

Results.- About $4 \%$ of the studied students were internet addict while $32 \%$ were potentially internet addicted. Internet addiction was significantly higher among males, higher in practical faculties while the studying class had no effect on the internet addiction score. There was a significant direct positive correlation between HADS scores and IAT scores.

Conclusions.- This study revealed that Egyptian faculty students were at high risk for potential addiction and to lesser extent internet addiction but both groups were prone to anxiety and depression.

Disclosure of interest.- The authors have not supplied a conflict of interest statement.

\section{E-PV0981}

\section{Attention deficit hyperactivity disorder and substance use disorders: a case report}

L. Sahli ${ }^{1}$, S. Hela ${ }^{1}$, J. Rahma ${ }^{2 *}$, K. Wassim ${ }^{1}$, O. Abdelaziz ${ }^{1}$

${ }^{1}$ Military Hospital of Tunis, psychiatry department, Tunis, Tunisia; ${ }^{2}$ Razi Hospital, Psychiatry F Department, Tunis, Tunisia

Corresponding author.

Background and aims.- Adolescents with substance use disorders (SUD) and attention deficit/hyperactivity disorder (ADHD) are increasingly presenting in clinical practice. The overlap and role of treatment for these comorbid disorders remains unclear. Many clinicians are reluctant to prescribe stimulants to ADHD patients with SUD. Our objectives are to illustrate the complexity of the comorbidity of ADHD with SUD and to discuss treatment approaches for patients with comorbid ADHD and SUD.

Methods.- A clinical case of a 17-year-old male patient who was carried out in child psychiatry department for depression and cannabis addiction. Further interviews and the CONNERS scales revealed the diagnosis of ADHD. CBT was associated to the antidepressant treatment. Although he was recovering from the depressive symptomatology and his cannabis consumption decreased, the inattention symptoms were compromising his school education. The use of psychostimulant treatment was controversial for fear that he would use it as an addicitive substance. The decision was to put him on progressive doses of Methylphenidate. At followup visits, his inattentive symptoms reduced and had better school results under a single Methylphenidate medication. Cannabis consumption decreased significantly until stopping.

Results.- This case demonstrates the efficacy of single psychostimulant treatment Methylphenidate in treating comorbid ADHD and cannabis addiction. Methylphenidate improved both ADHD symptom and the SUD.

Conclusions.- With an increased awareness of the phenomenon of comorbid ADHD and SUD,there is an increased awareness of the potential benefit of ADHD treatment on substance abuse treatment outcome. Consideration of an individual's developmental relationship between attention deficit/hyperactivity symptoms 
and substance use can inform treatment planning among these patients.

Disclosure of interest.- The authors have not supplied a conflict of interest statement.

\section{E-PV0982}

\section{Dysregulation of positive emotions and gambling disorder: a empirical investigation}

G. Rogier ${ }^{1 *}$, S. Beomonte Zobel ${ }^{1}$, P. Velotti $^{2}$

${ }^{1}$ University of Rome- Sapienza, Department of Dynamic and Clinical Psychology, Rome, Italy; ${ }^{2}$ University of Genoa, Educational Sciences, Genoa, Italy

* Corresponding author.

Background and aims.- Several theoretical models underlined that the development or the maintenance of Gambling Disorder (GD) is undermined by difficulties in the regulation of negative emotions. Despite the fact that the role of positive emotions played in psychopathology has been widely neglected by the scientific community, their regulation appears a central feature of addictions as such as GD (Rogier \& Velotti, 2018). However, studies investigating this topic are limited.

Aim.- To investigate the role played by dysregulation of positive emotions in GD in a clinical sample of DGs.

Methods.- We administered to a sample of Disordered Gamblers (DGs) and a sample of community participants the South Oaks Gambling Screen (SOGS, Lesieur \& Blume, 1987) and the Difficulties in Emotion Regulation Scale Positive (DERS-P,Weiss et al., 2015).

Results.- Positive and significant correlations between the SOGS and the DERS-P were observed. Moreover, DGs scored higher on some of the subscales of the DERS-P compared to community participants.

Conclusions.- Dysregulation of positive emotions appears to be a central feature of GD. As such, future research should further investigate this issue. Also, clinical interventions have to assess and target the capacity of DGs to manage high levels of positive emotions. Finally, theoretical models should integrate the topic of dysregulation of positive emotions in the explanation of the development and maintenance of GD.

Disclosure of interest.- The authors have not supplied a conflict of interest statement.

\section{E-PV0983}

\section{Investigation of the role played by pathological personality, interpersonal problems and emotion dysregulation in facebook addiction}

G. Rogier ${ }^{1}$, S. Beomonte Zobel ${ }^{1}$, P. Velotti ${ }^{2}$

${ }^{1}$ University of Rome- Sapienza, Department of Dynamic and Clinical Psychology, Rome, Italy; ${ }^{2}$ University of Genoa, Educational Sciences, Genoa, Italy

${ }^{*}$ Corresponding author.

Background and aims.- Facebook Addiction is traditionally thought to be underlined by a difficulty in emotion regulation processes. Also, previous research showed that Facebook addicted may suffer from personality disorders and to present dysfunctional interpersonal styles. However, to our knowledge, the complex pattern of potential relationships between these variables and Facebook Addiction is still unexplored.

Objective.- To investigate the role played by pathological personality and emotional dysregulation in Facebook Addiction.

Methods.- We administered to a sample of community participants $(N=270)$ the Bergen Facebook Addiction Scale (BFAS, Andreassen et al., 2012), the Personality Inventory Index for DSM-V (PID-5,
Krueger et al., 2011), the Difficulties in Emotion Regulation Scale (DERS, Gratz \& Roemer, 2004) and the Interpersonal Problem Index47 (IIP-47, Lindberg et al., 2018).

Results.- Controlling for Age and Gender, we found that the IIP Aggression subscale, the Negative Affectivity domain of the PID-5 and the DERS Total score positively and significantly predicted BFAS scores. Moreover, results showed that emotion dysregulation partially mediated the relationship between pathological personality and Facebook addiction severity whereas interpersonal problems moderated the direct relationship between pathological personality and Facebook Addiction severity.

Conclusions.- Our results support previous studies showing the role played by pathological personality, emotion dysregulation and interpersonal problems in behavioral addictions. Moreover, the study shed light on the complex pattern of relationships between these variables. Clinical implications are outlined focusing on the importance of a dual focus, targeting both interpersonal competences and emotion regulation capacities. Future directions are discussed.

Disclosure of interest.- The authors have not supplied a conflict of interest statement.

\section{E-PV0985 \\ Effectiveness of group therapy based on the guided self-change treatment program (GSCT)}

V. Sanz Largo*, J.M. Chouza Pérez

Centro de Adicciones Bitarte, Red de Salud Mental de Gipuzkoa, San Sebastian, Spain

* Corresponding author.

Background and aims.- Problem drinkers (those who have identifiable life difficulties due to their drinking but are not severely dependant) actually constitute the majority of people who have trouble dealing with alcohol.

There is an increasing empirical evidence supporting the Guided self-change treatment (Sobell,M and Sobell, L 2005) as a highly effective approach to this problem. To this end, we intended to assess the effectiveness of this treatment in a group therapy among a Spanish sample of alcohol abusers,

Methods.- The current sample included 58 spanish speaking adults with DSM alcohol abuse diagnosis (41 men and 17 women), who attended an Addiction Center in 2017.

It is a cross-sectional study (two measurements) for pre and post interventions regarding: alcohol consumption, degree of alcohol dependance and negative consecuences of drinking, including mental wellbeing. They were measured, respectively, by using the timeline follow-back technique, the Short Alcohol Dependance Data (SADD) questionnaire, and the Spanish validated Edimburgo Mental wellbeing Scale.

Results.- Self-reported alcohol consumption was significantly reduced $(P<.0001)$ after treatment, as well as the consumption pattern, showing an increase in the days when they remain abstinent, and a reduction in both the number of drinks they consume in days drinking and heavy drinking occasions. The measures of alcohol dependance and negative consequences of drinking were also reduced with a significant improvement of the mental well being. Conclusions.- These results are similar to those reported in other countries using the same program and indicate the need to change public health policies to reflect the contemporary scientific understanding of the alcoholism disorder.

Disclosure of interest.- The authors have not supplied a conflict of interest statement. 


\section{E-PV0986}

\section{To the issue of controversial effectiveness of anti-drug public policy and services in women}

\section{Gabrilyants ${ }^{1}$, N. Semenova ${ }^{2}$}

${ }^{1}$ The 'Serbsky Federal Medical Research Centre for Psychiatry and Narcology' of the Ministry of Health of the Russian Federation, Administration, Moscow, Russia; ${ }^{2}$ The 'Serbsky Federal Medical Research Centre for Psychiatry and Narcology' of the Ministry of Health of the Russian Federation, Psychiatry, Moscow, Russia ${ }^{*}$ Corresponding author.

Background and aims.- This paper focuses on controversial effects of anti-drug public policy and service - with special reference to gender issues.

Methods.- We review the literature on the effectiveness of methadone maintenance treatment and other opioid replacement therapies, characterise current state of anti-drug public policy and service, as well as national campaigns approaches.

Results.- The data indicate that many services have treatment practices that are not effective according to the majority of studies. We present findings from qualitative approaches that explore a variety of messages, and discuss some gender issues.

Message 1: On the imposition of changes in legislation under the pretext of "vital necessity" of methadone for pregnant women. Methadone maintenance has been used for decades to treat opioiddependent pregnant women, but despite its long history of use, little is known about the long-term effects of methadone on the newborn.

Message 2: On comprehensive rehabilitation and re-socialization of drug users and countering "liberal" drug policy expansion. Even though this policy will help women users avoid illicit drug use, this does not guarantee that the policy will influence their drug use behavior and the psychosocial determinants of that behavior.

Message 3: On gender issues of the state anti-drug campaigns the messages perceived by women as ineffective are unlikely to prevent (and may actually facilitate) risky behavior in women. Conclusions.- Steps should be taken to change the treatment practices of methadone maintenance that could have a negative impact in women. Research is needed to fill gaps in our existing knowledge. Disclosure of interest.- The authors have not supplied a conflict of interest statement.

\section{E-PV0987}

\section{The importance of set and setting in psychedelic experiences: a review \\ F.G.M. Silva ${ }^{*}$, C. Bragança ${ }^{2}$}

${ }^{1}$ Hospital de Magalhães Lemos, Serviço C, Porto, Portugal; ${ }^{2}$ Unidade

Local de Saúde do Nordeste, Internamento, Bragança, Portugal

${ }^{*}$ Corresponding author.

Background and aims.- Psychedelics are capturing a renewed attention of both the mainstream culture and the scientific community, in what has been called the psychedelic renaissance.

Psychedelic experiences can range from blissful to traumatic and predicting individual responses remains difficult. Extrapharmacological factors have been proposed as important determinants of the quality of psychedelic experiences since the sixties. These factors include the set (one's state of mind prior to taking the psychedelic) and the setting (the physical conditions were the psychedelic experience occurs).

This study aims to review the scientific literature from the ongoing psychedelic renaissance about the impact of extra-pharmacologic factors in acute psychedelic experiences.

Methods.- A systematic review was conducted after a Medline search using the terms 'psychedelic' AND 'set' OR 'setting'. Only studies written in English and published after 1990 were consid- ered. References were independently selected by the authors and then compared.

Results.- Factors from the set predictive of positive psychedelic experiences include absortion (the personality trait of being susceptible to immersion in experiences), psychological wellness prior to taking the drug, clear intentions and expectations about the experience and being open to the experience. On the other hand, set factors like neuroticism, anticipatory anxiety and low trust in accompanying persons are associated with negative experiences. Regarding the setting, certain types of music seems to facilitate positive experiences.

Conclusions.- The current psychedelic renaissance is aware of the influence of extra-pharmacologic factors in the acute effects of psychedelic drugs, but scientific studies have only begun to emerge and are mainly observational.

Disclosure of interest.- The authors have not supplied a conflict of interest statement.

\section{E-PV0988 \\ Method for integrated therapy of comorbid alcohol and tobacco dependence}

I. Sosin*, O. Honcharova, Y. Chuiev

Kharkiv Medical Academy of Postgraduate Education, Department of narcology, Kharkiv, Ukraine

Corresponding author.

Background and aims.-

Introduction.- Alcohol and tobacco dependence, a kind of "addiction dyad", is the most common form of addiction. Such integration of addictions is based on common, same-type, intercomplicating pathogenetic mechanisms, clinical-syndromal, diagnostic and therapeutic essence. Meanwhile, addiction therapy is very short in simultaneous, combined, tandem complex treatment modalities for these two pathologies.

Objectives.- Search for integrated approaches to synergistic therapy of alcohol and nicotine dual addiction.

Methods.- Optimal pharmacological complex was searched technologically basing on analytical considerations: a) present-day concepts of clinical (symptomatic, syndromological, nosological) criteria of alcohol and nicotine dependence; b) subject information on adequate and typical drugs for predominant addiction conditions; c) scientific publications; d) recent clinical experience in addictology; e) targeted (pre-set) computer screening, selection and aggregation of integrative effects with available electronic version of the Drug encyclopedia.

Results.- Method for integrated therapy of comorbid alcohol and tobacco dependence (Patent 81199, UA) was grounded, developed and tested, which includes "pharmacological triad": oral daytime tranquilizer Adaptol (Mebicar) 0.3-0.5 g 2-3 times daily; anticonvulsant thymoleptic Carbamazepine daily 0.05-0.20 g; tranquilizer Noofen (Pheniubut) with antihypoxic and antiamnestic effect: $0.25-0.50 \mathrm{~g}$ three times a day, for 10-14 day treatment. Examination of 53 dual addicts revealed that pathogenetically and synergically the formed "pharmacological triad" increases effectiveness of treatment at minimal doses of medications and shortens the term of treatment.

Conclusions.- The proposed pharmacological method of integrated treatment (Mebicar, Carbamazepine, Phenibut) of dual addiction (alcohol and nicotine) is proven to be effective, safe and can be recommended for introduction in addiction care.

Disclosure of interest.- The authors have not supplied a conflict of interest statement. 


\section{E-PV0989}

\section{Apotemnophilia syndrome due to chronic cannabis use}

S. Tunc ${ }^{1 *}$, E. Buyuksandalyaci ${ }^{2}$, H.S. Basbug ${ }^{3}$

${ }^{1}$ Kafkas University, Department of Psychiatry, Kars, Turkey; ${ }^{2}$

Bakirkoy Training and Research Hospital for Psychiatry- Neurology and Neurosurgery, Department of Psychiatry, Istanbul, Turkey; ${ }^{3}$ Kafkas University, Department of Cardiovascular Surgery, Kars, Turkey

${ }^{*}$ Corresponding author.

Background and aims.- Apotemnophilia is an unusual condition which is defined as the strong and persistent desire to lose healthy extremities by amputation or seek to be paraplegic. It was first introduced by John Money in 1977. The etiology of Apotemnophilia is not yet wholly specified despite suggested several etiological mechanisms including psychiatric and neurological components. In this paper, a patient with apotemnophilia findings which is induced by using cannabis intensively for four years was reported.

Methods.-A 22-year-old male patient was referred with an extreme demand of amputation of his left hand from the admission of cardiovascular surgery (CVS) department where he was immediately sent to the psychiatry outpatient clinic. Although cannabis use was reported, no orthopedic or neurological abnormality was detected in the physical examination.

Results.- Cannabis use was stopped. Alprazolam $2 \mathrm{mg}$ /day and selective serotonin reuptake inhibitor (SSRI) (escitalopram, $5 \mathrm{mg}$ /day) was initially prescribed, and the dose was increased gradually (up to $20 \mathrm{mg} /$ day) in response to treatment. After ten days, overall symptomatic status was significantly improved. He almost never had an urge to amputation.

Conclusions.- This case of apotemnophilia is thought to be the cause of chronic and intensive cannabis use. Assuming that cannabis use has an impact on the chemistry or morphology of the temporal lobe, we aim to emphasize the need to support these findings with more comprehensive studies. Furthermore, investigating such curious conditions that stay in the hinterland between neurology and psychiatry may supply critical points into the question of how neural activity leads to mental problems.

Disclosure of interest.- The authors have not supplied a conflict of interest statement.

\section{E-PV0990}

\section{Combined pharmacological treatment for alcohol use disorders - review of current evidence \\ O. Vasiliu}

"Dr. Carol Davila" University Central Military Hospital, Psychiatry, Bucharest, Romania

${ }^{*}$ Corresponding author.

Background and aims.- Available treatments for alcohol use disorder (AUD) are focused on glutamatergic antagonism (acamprosate), opioid antagonism (naltrexone, nalmefene), and aldehyde dehydrogenase inhibition (disulfiram), but other relapse-prevention agents with different pharmacodynamic properties are also used. This review has as main objective to find evidence in the literature for combined treatment in AUD patients.

Methods.- A search of major electronic databases (Cochrane, PubMed, PsychInfo, EMBASE, CINAHL) was performed, using keywords "alcohol use disorder", "alcohol abuse", "alcohol dependence", "combined pharmacological treatment", and all the non-proprietary names of the main drugs used for AUD treatment.
Results.- Naltrexone plus acamprosate was compared in a large trial $(N=160)$ to either drug alone in patients with AUD and induced significantly lower relapse rates than placebo and acamprosate, but equal to the naltrexone monotherapy. The combination of naltrexone and disulfiram was investigated in patients $(N=208)$ diagnosed with AUD and cocaine dependence and induced higher rate of abstinence. Still another trial $(N=118)$ investigated in a double-blind manner the effect of acamprosate and disulfiram combination in AUD, and showed a better outcome on the cummulative abstinence duration in patients receiving both drugs. Addition of gabapentin to naltrexone improved drinking outcomes over naltrexone alone during the first six weeks after cessation of drinking, in a double blind randomized trial $(N=150)$. The combination of naltrexone and baclofen was investigated in a preclinical trial with positive results.

Conclusions.- Combining naltrexone with other anti-craving and relapse-preventing agents could be beneficial, and there are data to support its combination with acamprosate, baclofen, disulfiram or gabapentin in patients with AUD.

Disclosure of interest.- The authors have not supplied a conflict of interest statement.

\section{E-PV0991 \\ Efficacy and tolerability of pharmacological agents for internet gaming disorder- a systematic literature review \\ O. Vasiliu \\ "Dr. Carol Davila” University Central Military Hospital, Psychiatry, Bucharest, Romania \\ * Corresponding author.}

Background and aims.- Although very few good quality studies have been conducted on Internet gaming disorder (IGD), it worth mention that Internet addiction has been associated with high rates of psychiatric co-morbidities, like substance use disorders, attention deficit/hyperactivity disorder, depression, hostility, and social anxiety disorder. Therefore, the therapeutic management of patients diagnosed with IGD may be more complicated than it seems at a first glance. A review of the possible pharmacological treatments targeting core symptoms of IGD could be useful from the clinical perspective.

Methods.- A search of major electronic databases (Cochrane, PubMed, PsychInfo, EMBASE, CINAHL) was performed, using keywords "Internet gaming disorder", "Internet disorder", "pharmacotherapy", and all the non-proprietary names of the main drugs used for drug-related disorders treatment.

Results.- Only two trials were identified as corresponding to the inclusion and exclusion criteria. One was focused on IGD in 47 patients who received 6-month management program which included selective serotonin reuptake inhibitors. IGD symptoms improved significantly after 6 months of treatment, although the P300 indices were not significantly changed. Another trial evaluated the effect of atomoxetine and methylphenidate in adolescents with attention deficit hyperactivity disorder and problematic online gaming $(N=86)$, for 12 weeks. Patients who received atomoxetine had greater improvement in depressive symptoms than methylphenidate-treated group, and IGD symptoms and ADHD manifestations improved when compared to baseline values.

Conclusions.- Very few data exist about the efficacy and tolerability of medication in patients diagnosed with IGD. Larger, better designed studies seem granted for this indication in adolescents and young adults. 
Disclosure of interest.- The authors have not supplied a conflict of interest statement.

\section{E-PV0992}

\section{Dissociative experiences in opioid-addicted patients}

A. Zwierzchowska ${ }^{1}$, E.J. Gorzelańczyk ${ }^{2}$, P. Walecki ${ }^{3 *}$

${ }^{1}$ Jagiellonian University Medical College, Faculty of Medicine, Krakow, Poland; ${ }^{2}$ Nicolaus Copernicus University Collegium Medicum, Department of Theoretical Basis of Bio-Medical Sciences and Medical Informatics, Bydgoszcz, Poland; ${ }^{3}$ Jagiellonian University Medical College, Department of Bioinformatics and Telemedicine, Krakow, Poland

${ }^{*}$ Corresponding author.

Background and aims.- The aim of the study is to assess the occurrence of dissociative experiences in addicts (not under the influence of psychoactive substances) in comparison to healthy ones. Psychology defines dissociation as a separation of functions, which are normally integrated: consciousness, memory, identity, perception; disengagement of intentional and automatic processes.

Methods.- Twenty opioid-addicted patients ( 8 women, 12 men) from Med-Seven Substitution Treatment Clinic in Bydgoszcz and twenty-two healthy people were examined. The Dissociative Experience Scale (DES-II) and the NEO Five-Factor Personality Inventory (NEO-FFI) was used.

Results.- Opioid-addicted patients presented significantly $(p<0.001)$ higher DES Score $(22.21 \pm 13.01)$ compared to healthy ones $(4.99 \pm 2.63)$. Addicted patients has higher level of neuroticism $(7.20 \pm 1.94)$ compared to healthy ones $(3.27 \pm 1.83)$ and lower level of extraversion $(3.70 \pm 2.15)$ and conscientiousness $(4.00 \pm 1.89)$ compared to healthy ones $(6.14 \pm 2.29)$ and $(6.59 \pm 1.84)$. DES Score was positively correlated with the higher neuroticism level $(p<0.05)$. DES Score was also negative correlated with lower level of extraversion and lower level of conscientiousness $(p<0.05)$.

Conclusions.- The high level of dissociative experiences in people addicted to opioids was found. The results are suggesting common mechanisms responsible for dissociative experiences and using psychoactive substances, also genesis of addiction. The outcomes are encouraging to continue the researches to determine mechanisms which are connecting dissociation and addiction, and also to define the biological factors responsible for this phenomenon. Disclosure of interest.- The authors have not supplied a conflict of interest statement.

\section{E-PV0993}

\section{Application of infrared thermography to assess the change in body temperature after the administration of a therapeutic dose of methadone}

A. Zwierzchowska ${ }^{1}$, D. Pikor ${ }^{2}$, W. Lasoń ${ }^{3}$, E. Gorzelańczyk ${ }^{4}$, P. Walecki $^{*}$

${ }^{1}$ Jagiellonian University Medical College, SKN Telemedicine and Medical Informatics, Krakow, Poland; ${ }^{2}$ Jagiellonian University Medical College, Faculty of Medicine, Krakow, Poland; ${ }^{3}$ Jagiellonian University Medical College, Department of Bioinformatics and Telemedicine, Krakow, Poland; ${ }^{4}$ Nicolaus Copernicus University Collegium Medicum, Department of Theoretical Basis of Bio-Medical Sciences and Medical Informatics, Bydgoszcz, Poland

Corresponding author.

Background and aims.- An infrared thermography is used in medicine in order to assess the body temperature. Using the non-contact thermographic devices, we can quickly and precisely measure the temperature of various areas of the body. During the screening test we obtain two-dimensional maps of temperature distributions across the skin which can be used as a direct measure of the change in blood perfusion. During methadone maintenance treatment (Opioid Replacement Therapy - ORT), patients report different side effects such as "hot waves". The standard temperature measurement did not show any significant changes. To check whether this short-term sensation is subjective or objective, we can use the thermographic measurement in various areas of the face where the change in blood perfusion is high.

Methods.- The study involved 36 patients participating in methadone substitution therapy. A remote-sensing thermal imaging camera FLIR (thermal sensitivity $<0.15 \mathrm{DegC}$ ) to measure the nose and forehead temperature was used. Face thermal images were taken before methadone administration (BM) and 1 hour after methadone administration (AM).

Results.- The mean temperature of the forehead area BM was $31.56 \pm 1.11 \mathrm{DegC}$ and AM $32.25 \pm 1.46 \mathrm{DegC}$. The difference is statistically significant $t=-2.628$ and $p=0.013$. The mean temperature of the nose area $\mathrm{BM}$ was $29.49 \pm 2.64 \mathrm{DegC}$ and AM $30.60 \pm 3.10 \mathrm{DegC}$. The difference is statistically significant $t=-2.466$ and $p=0.019$.

Conclusions.- In the study, it was observed that the temperature measured in the face area increased after 1 hour after administration of methadone.

Disclosure of interest.- The authors have not supplied a conflict of interest statement.

\section{E-PV0994 \\ Patterns of synthetic ketones use and their impact on depression and oral behaviors}

G. Więckiewicz ${ }^{1 *}$, D. Danel ${ }^{2}$, T. Wieczorek ${ }^{3}$, J. Rymaszewska $^{3}$, J. Smardz ${ }^{4}$, N. Grychowska ${ }^{5}$, M. Więckiewicz ${ }^{4}$

${ }^{1}$ Faculty of Medicine, Medical University of Silesia, Department of Psychiatry, Tarnowskie Gory, Poland; ${ }^{2}$ Ludwik Hirszfeld Institute of Immunology and Experimental Therapy, Polish Academy of Sciences, Department of Anthropology, Wroclaw, Poland; ${ }^{3}$ Faculty of Medicine, Wroclaw Medical University, Department of Psychiatry, Wrocław, Poland; ${ }^{4}$ Faculty of Dentistry, Wroclaw Medical University, Department of Experimental Dentistry, Wroclaw, Poland; ${ }^{5}$ Faculty of Dentistry, Wroclaw Medical University, Department of Prosthetic Dentistry, Wroclaw, Poland

* Corresponding author.

Background and aims.- Synthetic ketones are novel psychoactive substances widely introduced to theglobal market during last 10 years as a replacement for illegal amphetamines. Because of easy accessibility, they becamepopular quickly and intoxications widely spread, some of which resulted with users' death. The objective of the study was to determine the impact of synthetic ketones usage on depression and oralbehaviors among recreational users.

Methods.- Users of online drugs-related forums were asked to fill in a questionnaire via Google FormsPlatform. The questionnaire contained questions about patterns of drugs use, Beck's Depression Inventory II, a self-reportinventory for measuring the severity of depression and Oral Behaviors Checklist (OBC). It was initially developed as achecklist in order to better determine the presence of parafunctional behaviors. Results were statistically analyzed andcompared to a control group $(p=0.05)$.

Results.- Seventy five users of synthetic ketones aged 15 to 28 years old filled in the questionnaire. All of the participantsused ketones and other psychoactive substances. Forty two participants used ketones during last month. Statistical analysisshowed correlation between amount of drugs types used and depression level for both groups, including control group aswell as a statistically significantly higher mean value of the $\mathrm{OBC}$ scores in a group of ketones users than controls. Correlation between depression and OBC is statistically significant for the entire study material. 
Conclusions.- Considering easy access to synthetic ketones, widely spread intoxications and young age of participants ofthis survey the subject needs to be widely researched. Psychoactive substances can predispose to development ofdepression and various forms of parafunctional oral behaviors.

Disclosure of interest.- The authors have not supplied a conflict of interest statement.

\section{E-Poster Viewing - 7-9 April: Suicidology and Suicide Prevention}

\section{E-PV0995}

\section{Non-suicidal self-injury: emotion regulation strategies in a sample of Italian undergraduate students}

S. Beomonte Zobel ${ }^{1}{ }^{*}, \mathrm{G}$. Rogier ${ }^{1}$, P. Velotti ${ }^{2}$

${ }^{1}$ La Sapienza Università di Roma, Dynamical and Clinical Psychology, Rome, Italy; ${ }^{2}$ University of Genoa, Educational Sciences, Genoa, Italy * Corresponding author.

Background and aims.- Several studies conducted all over the world claim that suicidality and non-suicidal self-injury (NSSI) are highly prevalent in college students. Research suggests that difficulties in emotion regulation may play a role in NSSI but the relationship between the two constructs is still uncertain as the discriminating factor between those who perform self-injury behaviors and those who do not remains unclear.

The purpose of our study is to examine the connection between NSSI, difficulties in emotion regulation and emotion regulation strategies. We hypothesized that even in non-clinical population individuals that report greater difficulty in regulating emotions would be more likely to present self-injury behaviors.

Methods.- Participants included 150 undergraduate students. Demographic information, including clinical history, were collected in a form. Non-suicidal self-injury was assessed using Deliberate Self-Harm Inventory (Gratz, 2001), difficulties in emotion regulation were assessed using Difficulties in Emotion Regulation Scale (DERS; Gratz \& Roemer, 2004), whereas emotion eegulation strategies were assessed with Emotion Regulation Questionnaire (ERQ; Gross \& John, 2003).

Results.- Our results show a correlation between NSSI, Emotion Dysregulation and emotion regulation strategies.
Conclusions.- The small size of our sample and its demographic characteristics may limit the generalizability of our findings. For the future, further researches should be conducted to investigate deeply the relationship between NSSI and emotion dysregulation. Disclosure of interest.- The authors have not supplied a conflict of interest statement.

\section{E-PV0997}

\section{Storytelling: a viable avenue for suicide prevention, mental health treatment}

A. Blackwood ${ }^{1 *}$, M. Hines ${ }^{1}$, A. Foreman ${ }^{2}$

${ }^{1}$ Kevin and Margaret Hines Foundation, Business Development, Atlanta, USA; ${ }^{2}$ Kevin and Margaret Hines Foundation, Chief Medical Officer, Atlanta, USA

* Corresponding author.

Background and aims.- Storytelling allows the human brain to "understand, to make sense, [and] to remember," which furthermore allows for the individual to create best possible outcomes for the situation sometimes without conscious recognition, using the "neural story net" (Haven, 2015). The stories of Kevin Hines and others invite a neurological response to positive help seeking behaviors before the emotional response is elicited. Once the emotional response is provoked, the listeners have then been doubly exposed to problem-solving thoughts, increasing likelihood for positive action in the listener before tools of suicide mitigation or recovery from suicidality or loss are explicitly communicated.

Aims.-

1. Explore the scope of existing research behind the efficacy of storytelling as a viable treatment option

2. Understand neurological processes behind the efficacy of storytelling and subsequent behavior change

3. Gain application methods for researchers and lived-expertise consultants to bridge research and storytelling, mutually increasing the impact of storytelling with regard to research, through collaboration

Methods.- A 9 item Pre-Post survey was given to audiences immediately before and after Kevin Hines shared his story of suicide attempt, survival, and recovery. See below for questions and results (Fig. 1). 

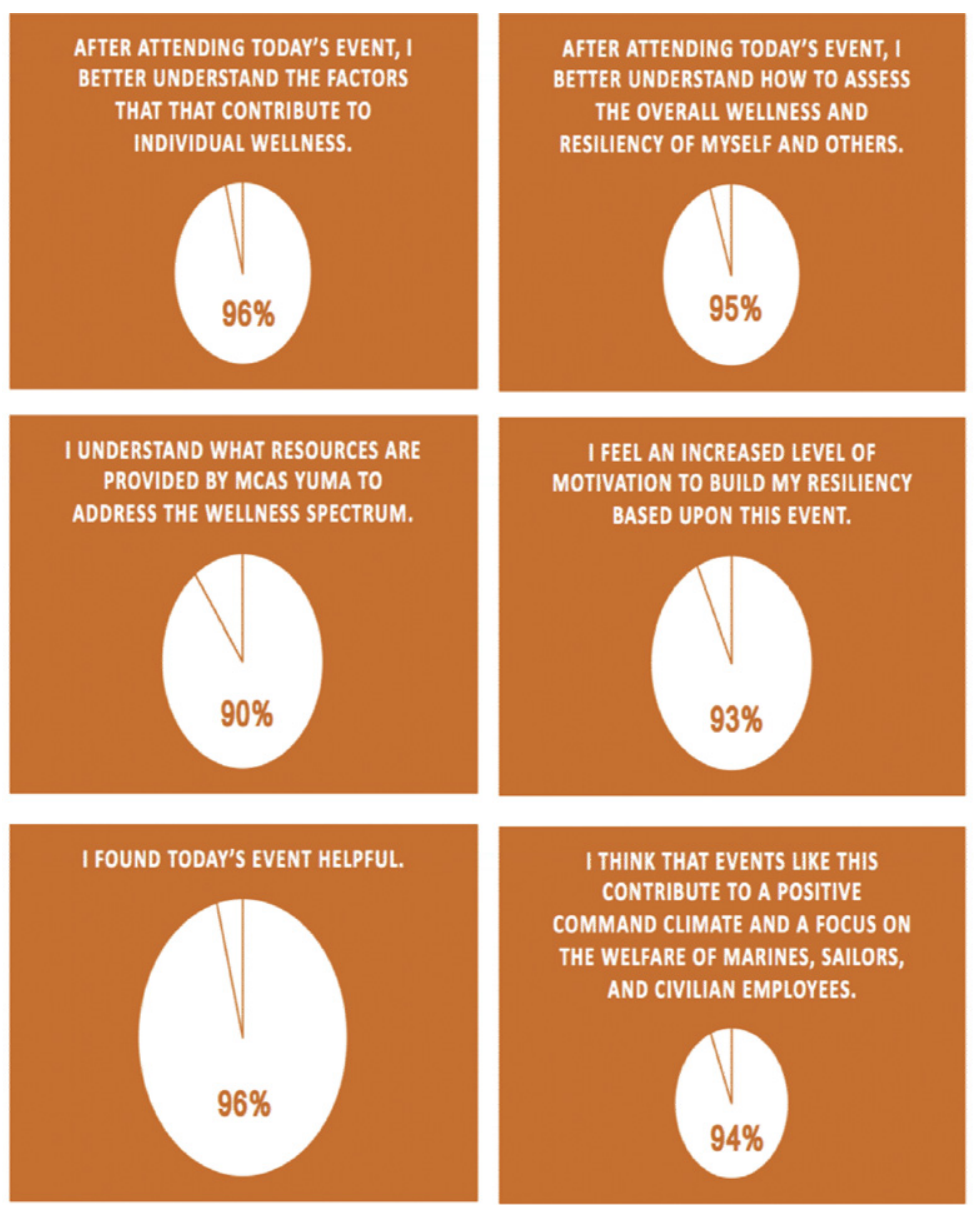

Fig. 1. Percentage of participants who agree or strongly agree.

Results.- A repeat measure paired t-test was conducted on prepost data. Statistical significance of $p<.01$ was found for each item. Cohn's d effect sizes ranged from .408 to 1.655 with six items about 8 .

Conclusions.- When stories of suicide that focus on hope, healing, and the possibility of recovery are shared, there is a neurological impact that increases likelihood of attitude shift, awareness, and behavior change, including help-seeking behaviours.

Disclosure of interest.- The authors have not supplied a conflict of interest statement.

\section{E-PV0998}

\section{Value and meaning orientations of borderline personality disorder patients}

L. Cherkasova ${ }^{1 *}$, A. Grigoryeva ${ }^{2}$

${ }^{1}$ Neurocentre of medical-psychological correction and rehabilitation, Department of clinical psychology, Moscow, Russia; ${ }^{2}$ Federal Medical Research Center of Psychiatry and Addiction, Department of prevention of drug use among young people, Moscow, Russia Corresponding author.

Background and aims.- Suicide risk for borderline personality disorder (BPD) patients vary from 3 to 10\% during lifetime (Paris, J., \& Zweig-Frank, H., 2001). Awareness of meaning in life (MiL) and values is a protective factor against non-suicidal self-harm \& suicide (García-Alandete, J. et al., 2014; Perez S. et al., 2015).

Objective.- is to reveal value-meaning orientations of BPD patients. Methods.- The sample was 52 individuals, over 18 years $(N=20$ - BPD; $N=32$ - healthy controls (HC)). The methods used were: Personal value questionnaire (Shwartz Sch. et al., 2012); Existence scale (Langle A. et al., 2000); Purpose in life test (Crumbaugh, J.C., \& Maholick, L.T., 1964); Personal belief questionnaire - borderline personality disorder (Butler A.C. et al., 2002).

Results.- Individual values Interpersonal Conformity, Universalism Concern, Benevolence Caring are significantly higher for BPD patients, whereas Tolerance is higher for HR. Self-distance is the lowest existential scale index for either BPD or HR.

Awareness of life and Locus of control-I are the lowest scales in Purpose in life test for BPD group (all scales are significantly lower for BPD).

For the BPD the borderline features are interconnected with the values of Hedonism, Power, Face and Self-Direction, for HR the value of Security.

In BPD group the feeling of helplessness and threats in interpersonal relationships is negatively correlated with responsibility and selfdistancing.

Conclusions.- BPD values are mainly looks like compensations for the sharp BPD interpersonal attitudes. The study of existential values will help us to go further in the MiL understanding of BPD patients.

Disclosure of interest.- The authors have not supplied a conflict of interest statement.

\section{E-PV1003}

\section{Relationship of difficulties in emotion regulation with history of suicide attempt among young adults}

C. Evren ${ }^{1 *}$, B. Evren ${ }^{2}$, E. Dalbudak ${ }^{3}$, M. Topcu ${ }^{4}$, N. Kutlu ${ }^{2}$

${ }^{1}$ Bakirkoy Training and Research Hospital for Psychiatry Neurology and Neurosurgery, Research-Treatment and Training Center for 
Alcohol and Substance Dependence AMATEM, Istanbul, Turkey; ${ }^{2}$ Baltalimani State Hospital for Muskuloskeletal Disorders,

Department of Psychiatry, Istanbul, Turkey; ${ }^{3}$ Private practice, Private practice, Ankara, Turkey; ${ }^{4}$ Cankaya University- Ankara- Turkey, Department of Psychology, Ankara, Turkey

${ }^{*}$ Corresponding author.

Background and aims.- The aim of the present study was to evaluate relationship of difficulties in emotion regulation with history of suicide attempt (HSA), while controlling the effect the depression among young adults.

Methods.- The study was conducted with online survey among 1010 volunteered university students in Ankara, people who play games on the Internet and who are in the e-mail database of a company located in Istanbul that organizes e-sports tournaments and Turkish gamers from gaming forums. Participants were evaluated by applying the Difficulties in Emotion Regulation Scale (DERS), and and the Beck Depression Inventory (BDI).

Results.- Age and gender did not differ between those with HSA ( $n=64,6.3 \%)$ and those without HSA $(n=946,93.7 \%)$. BDI and DERS scores were higher among those with HSA. In logistic regression analysis, severity of depressive symptoms predicted the presence of HSA, together with difficulties in emotion regulation, particularly "non-accept" and "strategies" dimensions.

Conclusions.- These findings suggest that the difficulties in emotion regulation, particularly "non-accept" and "strategies", mediates the relationship between the severity of depressive symptoms and HSA among young adults.

Disclosure of interest.- The authors have not supplied a conflict of interest statement.

\section{E-PV1004}

\section{Concealed suicides: what do they (not) \\ tell us?}

A. Paraschakis ${ }^{1}$, P. Ferentinos ${ }^{2 *}$

${ }^{1}$ Psychiatric Hospital of Attica "Dafni', Psychiatric Hospital of Attica "Dafni", Athens, Greece; ${ }^{2}$ National and Kapodistrian University of Athens- Medical School, 2nd Department of Psychiatry, Athens, Greece

${ }^{*}$ Corresponding author.

Background and aims.- Suicides are not uncommonly hidden among undetermined/other causes of death. Do concealed suicides represent an "elephant in the room" scenario for suicide research?

Methods.- Literature review (PubMed). Keywords: Suicide, classification, undetermined deaths, underreporting.

Results.- Seven articles closely matched our search. Suicide deaths appear underestimated by approximately $10 \%$. Underreporting could be due to the severe social stigma and the difficulty to categorize "suspect" deaths as suicides in the absence of witness(-es), suicide notes or known psychiatric history. Occasionally, forensic experts want to spare the family of the defunct from additional pain, particularly when younger people or children are involved. "Slow" suicides -conscious decision of protracted malnutrition, substance abuse or neglect of somatic therapies with a lethal intention- is another issue. Furthermore, deaths following hospitalization for a past suicide attempt are frequently attributed to the medical complications rather than to the suicidal act itself. Deaths by "active" methods -firearm use, cutting or hanging- are more easily attributed to suicide than "passive" ones, like poisoning or drowning. The latter appear more frequently chosen by females; hence, an underestimation of female suicides seems more probable. Finally, some deaths due to jumping from a height, being victim of fire or getting run over may conceal suicides unrecognized as such without reliable supportive evidence.

Conclusions.- Concealed suicides do not seem to fit the bill for an "elephant in the room" scenario. Nevertheless, better and constant training of forensic experts and continuous anti-stigma efforts are needed for further ameliorating the quality of suicide mortality statistics.

Disclosure of interest.- The authors have not supplied a conflict of interest statement.

\section{E-PV1007}

\section{Description of autolytic attempts during summer 2018 at hospital clinic Universitario of Valencia}

A. Pastor-Romero ${ }^{1}$, E. Guillot De Mergelina ${ }^{2 *}$, L. Álvarez ${ }^{1}$

${ }^{1}$ Hospital Clínico Valencia, Psychiatry, Valencia, Spain; ${ }^{2}$ Hospital

Clinico Valencia, Psychiatry, Valencia, Spain

* Corresponding author.

Background and aims.- We previously made a description by psychiatrists of autolytic attempts at the emergency department of the Hospital Clínico de Valencia during summer 2018. That analysis of the data did not count consummated suicides or those who, due to their severity, entered in the Intensive Care Unit or other services. Our objective was to analyse the characteristics of the population in our hospital who made autolytic attempts, in order to draft a prevention programme and improve patient care of this population in the future. Our goal was to try to reduce the number of autolytic attempts.

Methods.- A naturalistic descriptive study was carried out. The population was constituted by the patients who were treated in the emergency room due to an autolytic attempt. The attention to these patients was accompanied by a questionnaire filled by the emergency psychiatrists. This own questionnaire included sociodemographic and clinical variables and characteristics of the autolytic attempt.

Results.- Of the 63 cases seen in the emergency room, two thirds were women. The most common diagnoses were personality disorder (17.5\%) and depression (15.8\%). The most used method by far is the drug intake (79.33\%). Only $9.5 \%$ were planned and $17.4 \%$ had no rescue plan.

Conclusions.- Regarding the high number of impulsive attempts, we must bear in mind that the cases which were more serious were not examined by the emergency psychiatrists. These cases could have more planification. More studies and strategies on suicide prevention are needed since it is one of the most common causes of preventable death.

Disclosure of interest.- The authors have not supplied a conflict of interest statement.

\section{E-PV1008}

\section{The impact of loneliness on suicidal ideation among depressed patients: about 30 cases}

W. Kabtni", C. Bencheikh, O. Zeriaa, A. Baatout, M.W. Krir, H. El

Kefi, A. Oumaya

Military Hospital, Psychiatry Department, Tunis, Tunisia

* Corresponding author.

Background and aims.- Social relationships and social integration are crucial for emotional fulfilment and development over the life span. Then, loneliness, which is defined by feelings and thoughts of being isolated and disconnected from others, is considered as a risk factor for depression and specially for suicidal ideation.

Our study aims to identify loneliness as a risk factor for suicidal ideation in depressed patients.

Methods.- it's an analytic retrospective study about 50 depressed patients treated with serotonin reuptake inhibitors (SRI), recruited from the external consultation of the psychiatry department of the military hospital of Tunis. 
The diagnosis of depression was retained with the Patient Health Questionnaire [PHQ-2].

Patients answered the Patient Health Questionnaire (PHQ-9) to evaluate the severity of depression, the Depressive Symptom Index Suicidality Subscale (DSI-SS) and the UCLA loneliness scale.

We have compared the suicidal ideation in every level of depression (mild, moderate, moderately severe and severe) in the two groups: average Versus frequent to severe loneliness.

Results.- $90 \%$ of our patients were male.

$64 \%$ have at least moderately severe symptoms of depression (PHQ9 total score $\geq 10$ ) and $44 \%$ have positive screening for suicidal ideation (DSI-SS total score $\geq 3$ ).

$58 \%$ experienced frequent to severe loneliness.

Frequent to severe loneliness was identified as a risk factor for suicidal ideation, only in the group moderately severe depression. Conclusions.- Our findings support the view that loneliness poses a significant health problem with increased risks for suicidal ideation.

Then, systematic screening and level assessment of loneliness in depressed patients may prevent suicidal acts.

Disclosure of interest.- The authors have not supplied a conflict of interest statement.

\section{E-PV1009}

\section{Epidemiology and determinants of suicidal behaviors in patients hospitalized psychiatrically \\ T. Koweszko*, A. Szulc}

Medical University of Warsaw/Faculty of Health Sciences, Clinic of Psychiatry, Warsaw/Pruszków, Poland

* Corresponding author.

Background and aims.- The goal of this research project is the analysis of suicide behaviors frequency, identification of clinical and non-medical determinants of suicide behaviors and evaluation of the clinical usability of the Verbal Suicide Scale (VSS).

Methods.- This research project consists of five studies: the epidemiological analysis $(N=452)$, two retrospective study reports $(N=60, N=154)$ and two studies describing the VSS construction and validation process $(N=121, N=157)$. The following diagnostic tools were used: BPRS, ACL, C-SSRS, NGASR, SOC-29, WHOQOLBREF, VSS, SBQ-R and CSASS.

Results.- The patients diagnosed with F10-F19 (ICD-10) made $30 \%$ of all study population and of which $66 \%$ had suicide related behaviors. Low quality of life is substantially related with suicide behaviors, however this factor should be considered with patients gender taken into the account and the moment of life it refers to. The anticipated life satisfaction in future was negatively correlated with the threat of suicide for women and past life satisfaction was negatively correlated with suicide risk for men. In the VSS construction and validation process the Varimax factor analysis validated the three factor structure. Cronbach's alpha reliability for each factor was satisfactory.

Conclusions.- The subjects with F10-F19 diagnosis shows the strongest suicide inclinations. The non-medical factors are as much significant as psychopathological ones. The important suicide risk determinant is gender of a patient. Moreover the suicide risk assessment should consider the present situation and the life history of the patient. The achieved data confirms the validity of the VSS in terms of diagnosing risk factors related to volitional stage of suicide. Disclosure of interest.- The authors have not supplied a conflict of interest statement.
E-PV1010

Management of antidepressants in young patients with suicidal ideation

P. Padilla Romero ${ }^{*}$, C. Romeromartin ${ }^{2}$, M.T. Perez Castellano ${ }^{1}, \mathrm{~T}$. López-Arteaga ${ }^{1}$

${ }^{1}$ Hospital Nuestra Señora del Prado, Psychiatry, Talavera de la Reina, Spain; ${ }^{2}$ Hospital Nuestra Señora del Prado, Hospital Pharmacy, Talavera de la Reina, Spain

" Corresponding author.

Background and aims.- Introduction: Currently, there is still some controversy regarding the impact of antidepressants as triggers of suicidal tendencies in susceptible young people.

Objectives.- To assess the induction of suicidal thoughts after introduction of SSRIs antidepressant treatment of patients aged $<20$ with clinical depression. It was developed by clinical evaluation of 5 different cases.

Methods.- Case-series descriptive study. Clinical data were collected from 5 patients, 3 males and 2 females, aged 18-20, without psychiatric background. They went to Emergency Clinical Services experiencing clinical depression and were treated with SSRIs. Review of existing literature about management of antidepressants in young people with clinical depression and suicidal ideation.

Results.- In the 5 cases, patients were treated with an increase in dose of fluoxetine to $20 \mathrm{mg}$ /day. Patients were evaluated again after 6 weeks. De novo suicidal thoughts were only found in one of them. Conclusions.- Although some patients can develop de novo suicidal thoughts or self-injury with SSRIs, more recent analysis confirms this finding: SSRIs treatment decreases suicidal rates in young people and has a lower impact on the decrease of suicide in adults. In young people, suicidal tendencies which arise from antidepressant treatments can be related to a higher initial dose and fast dose-escalation. This supports the use of "slow initiation" approach. The majority of suicidal events among teenagers take place in the context of resistant depression, without evidence that medication induces behavioral activation.

Disclosure of interest.- The authors have not supplied a conflict of interest statement.

\section{E-PV1011}

\section{Suicide, post modernity and liquid} relationships

R.P. Parenti Freitas*, V. Garcia Caputo

Marilia Medical School, Psychiatry, Marília, Brazil

${ }^{*}$ Corresponding author.

Background and aims.- There has been an increase in suicidal behavior among young adults in Brazilian health services, the objective is analyze psychosocial risk factors for suicide and their relation with post modernity.

Methods.- Review of scientific literature on suicide epidemiology among Brazilian young adults and on post modernity and psychoanalysis concepts.

Results.- In Brazil, from 2000 to 2009, 14.7\% of suicides involved 10to- 14 -year-old children being $74 \%$ by hanging and $13 \%$ by firearms. From 2002 to 2012, the suicide rate increased 40\% in this age range. Among risk factors are family history of violence, lack of family and social support, inappropriate media, sleep deprivation, pathological internet use, and limited capacity to cope with frustrations.

Conclusions.- Data show an increase in suicide rate among young adults. An explanation for it may be demands of modernity such as: quick answers, immediate gratifications, happiness at any cost (reinforced by the media) and high productivity. This context weakens human bonds and favors the development of liquid relationships and narcissistic individuals. There does not seem to be the construction of a mental apparatus that is capable of tolerating frustrations, thus making the world frightening to them and reducing their resilience and ability to deal with pain. With archaic 
bonds, these young adults alone, are incapable of maintaining passion and meaning for their lives. Suicide emerges as an option to kill this empty part of their existence and disconnect them from their inner world which does not hold healthy dialogues with the outside world.

Disclosure of interest.- The authors have not supplied a conflict of interest statement.

\section{E-Poster Viewing - 7-9 April: Training in Psychiatry}

\section{E-PV1014}

\section{Rota in a foreign country: it really makes the difference}

A. Alonso Sanchez ${ }^{*}$, J.A. Blanco

Hospital Clínico Universitario de Valladolid, Psychiatry, Valladolid, Spain

* Corresponding author.

Background and aims.- Mental health problems are tackled differently all around the world and that is why spending some time abroad should be considered a cornerstone in the itinerary of a Psychiatry trainee.

Methods.- Report of a 4-month observership stance in an Early Intervention Service unit in London, UK.

Results.- Despite both British and Spanish mental health services are ruled by evidence-based policies, I discovered some differences which have widely enriched my training experience. Some of the examples are:

Psychosis in the Uk is understood not only as a medical issue but as a social issue. EIS centres guarantee fair attention to every psychotic patient in the UK. I was heavily surprised by the outcome and performance of those patients in the medium and long-term. Similar projects should be implemented in Spain within the next years.

Another massive difference concerned physical restraints with ties, as those are not allowed in the UK. This is possible thanks to the specific training for all the mental health workers and to an adequate patient/staff ratio. This is an important issue that should be discussed in Spain with the aim of reducing this traumatic experience to its minimum.

Conclusions.- learning from foreign psychiatrists and mental health workers and being in touch with other cultures widens the mind and leads to a better approach to mental health issues in the 21st century. Standardized exchange programmes should be developed all across Europe facilitating this experience to every Psychiatry trainee.

Disclosure of interest.- The authors have not supplied a conflict of interest statement.

\section{E-PV1015}

\section{The newest initiatives of the EFPT research working group}

M. Fellinger ${ }^{1}$, M. Alvarez-Mon ${ }^{2}$, A.R. Szczegielniak ${ }^{3}$, E.F.P.T. Research Working Group ${ }^{4}$, M. Asztalos ${ }^{5,6^{*}}$

${ }^{1}$ Medical University of Vienna, Department of Psychiatry and Psychotherapy-Clinical Division of Social Psychiatry, Wien, Austria; ${ }^{2}$ University of Navarra Hospital, University of Navarra Hospital, Pamplona, Spain; ${ }^{3}$ Medical University of Silesia, Department of Psychiatric Rehabilitation, Katowice, Poland; ${ }^{4}$ European Federation of Psychiatric Trainees, Research Working Group, Bruxelles, Belgium; ${ }^{5}$ Aalborg University Hospital, Department of Psychiatry, Aalborg, Denmark; ${ }^{6}$ Semmelweis University, School of PHD Studies, Budapest, Hungary

${ }^{*}$ Corresponding author.
Background and aims.-

Introduction.- The Research Working Group of the European Federation of Psychiatric Trainees (EFPT-RWG) investigates topics that concern trainees all around Europe. In 2018 the two new projects: "Evaluation of service user (SU) and family carer (FC) involvement throughout psychiatric training in Europe: experiences and personal views" (1) and "Empowering Twitter users for promotion of mental health $(\mathrm{MH})$ " (2) were initiated.

Objectives.- The goals are: (1) to assess and compare forms, extent and quality of SU\&FC involvement in the training period of psychiatric trainees and to evaluate their personal experiences and views, and (2) to facilitate the gathering and exchange of knowledge on addressing $\mathrm{MH}$ issues in Twitter and combat the lack of knowledge on $\mathrm{MH}$.

Methods.-

(1) An online survey, filled out by all psychiatric trainees of all European (EFPT) countries being distributed by national coordinators.

(2) An analysis of the official EFPT twitter account in terms of interaction of platform users with a published content

Results.- In the current stage of developing the survey we assume next to an underexposure of this crucial topic, huge national differences in the involvement of SU\&FC during psychiatric training. Twitter as a source of knowledge seems to be a strong tool for prevention and educational purposes and could be used more strategically.

Conclusions.- We hope that the current projects help us (1) to improve the quality of psychiatric training and to foster close collaborations with SU\&FC and (2) to promote MH in social media in a positive way throughout Europe.

Disclosure of interest.- The authors have not supplied a conflict of interest statement.

\section{E-PV1018}

\section{An attempt to get Bulgarian training programme in psychiatry out of the gray zone: a focus on psychotherapy}

K. Guevara ${ }^{*}$, D. Ignatova ${ }^{2}$, M. Stoyanova ${ }^{3}$, P.

Marinova-Djambazova ${ }^{4}$

${ }^{1}$ Military Medical Academy, Department of psychiatry, Sofia, Bulgaria; ${ }^{2}$ Medical University of Sofia, Department of psychiatry and clinical psychology, Sofia, Bulgaria; ${ }^{3}$ Mental health center "Prof. $N$. Shipkovenski", Outpatient department, Sofia, Bulgaria; ${ }^{4}$ Private practice “Orpheus", Psychiatry, Sofia, Bulgaria

"Corresponding author.

Background and aims.- Recent data from the literature show insufficiency of the information from training programmes in Psychiatry around Europe (1).

Methods.- Bulgarian Training Programme in Psychiatry (BTPP) was compared to the Training Requirements for the Speciality of Psychiatry (UEMS, 2017/08).

Results.- Our training programme generally complies with the UEMS recommendations with several exceptions, mainly concerning psychotherapy and supervision. While UEMS recommends "Programmes to include.. at least $120 \mathrm{~h}$ of theoretical teaching and $100 \mathrm{~h}$ of supervision of which at least $50 \mathrm{~h}$ should be individual", in the BTPP the words "psychotherapy" and "psychological treatment" were mentioned only in the following statements: 1 . "The programme aims to prepare specialist with... specific range of psychotherapeutic ... approaches"; 2 . "The programme covers the following subjects: ... psychotherapeutic interventions ...". 3. "Theoretical education, organized for 15 weeks with 2 hours per week ... each covering one of the following topics ... psychological treatment..." (i.e. only 30 hours of theoretical teaching). The only mentioning of supervision is: "Training is performed.... and includes clinical work under supervision..." and "The programme aims to prepare a specialist who... is able to use and perform supervision". 
The words "Supervisor" and "Chief of Psychotherapy Training" are not even mentioned, with no one assigned to perform the corresponding responsibilities.

Conclusions.- Further harmonization of the BTPP with focus on psychotherapy and supervision is needed. This is among the aims of the newly founded Bulgarian Young Psychiatrists' Association.

1 Mayer S. et al., 2014. EPA guidance on post-graduate psychiatric training in Europe. Eur Psy 29, 101-106.

Disclosure of interest.- The authors have not supplied a conflict of interest statement.

\section{E-PV1019}

\section{Psychosis, trauma and dissociation. a challenge for the psychiatry's trainee of the 21st century}

G. Hernandez-Santillan ${ }^{1 *}$, M.D. Picouto-Gonzalez ${ }^{2}$, F. Valencia-Agudo ${ }^{2}$

${ }^{1}$ Principe de Asturias University Hospital, Psychiatry, Madrid, Spain;

${ }^{2}$ Gregorio Marañon General University Hospital, Adolescent Psychiatry Unit, Madrid, Spain

* Corresponding author.

Background and aims.- Symptoms of dissociative disorders and positive symptoms of schizophrenia coincide frequently, leading to doubts in the diagnosis and therapeutic approach. Although, an early treatment can determine a better prognosis; If this is not well focused, it can also be iatrogenic.

Methods.- Case report and literature review.

Results.- A $17 \mathrm{Y}$ woman requires psychiatric admission due to psychotic decompensation. At 6Y, she started with experiences of abuse, neglect and reversal of roles. At $13 \mathrm{Y}$ she started with dissociative leakages. Until now, she have required more than ten psychiatric admissions during four years, although this is the second time in a adolescents unit. Currently, she has disorganized behaviour, hypervigilance, suspicion, inversion of sleep-wake rhythm, cenesthetopathies, depersonalization, and imperative voices ordering her to harm herself and others. She has received the following diagnoses: Schizophrenia, schizoaffective disorder, dissociative disorder, schizotypal disorder, personality diesmocional traits. He has received typical and atypical antipsychotics, without effective response and with side effects, such as obesity, hyperinsulinemia, hyperprolactinemia and galactorrhea. Clozapine is suspended on the third day due to dystonia, catatonia and probable neuroleptic malignant syndrome. Organicity was ruled out; Antipsychotics were suspended. Finally, she recovered with benzodiazepine at low doses and intensive psychotherapy. Conclusions.- Nowadays, training in psychiatry is scarce in historical knowledge, phenomenology and psychopathology, causing doubts in diagnostic and therapeutic mistakes to the detriment of patients. It is necessary to find points in common and new horizons in theories about dissociation and psychosis, returning to classics such as Bleuler, Janet, Schneider, and new ones as Van der-Hart, Moskowitz, among others.

Disclosure of interest.- The authors have not supplied a conflict of interest statement.

\section{E-PV1020}

\section{Stress and burnout in junior doctors: preventive considerations and interventions}

\section{Hernandez ${ }^{1 *}$, J.C. Mingote ${ }^{2}$, T. Palomo ${ }^{3}$}

${ }^{1}$ Royal College of Physicians Madrid, PAIME, Madrid, Spain; ${ }^{2}$ OMC, Psiquiatrist, Madrid, Spain; ${ }^{3}$ Universidad Complutense, Professor, Madrid, Spain

* Corresponding author.
Background and aims.- Medicine is among the five professions with higher psychiatric morbility: $10-12 \%$ of doctors will have mental health problems and/or addictions at some point in their lives.

Junior doctors are not immune to stress; following the initial "honey moon period" after qualifying, the stress inherent to the clinical practice and the lack of resources to cope with it may lead to emotional exhaustion, negative attitudes/behaviour towards the work, pateints, colleagues and/or relatives; it can harm self-image and result in burnout, absentism, depression, alcohol abuse... The suicide rate of male physicians is known to be higher than that of the general population while that of female physicians is clearly higher.

Methods.- A study by Mingote et al. measured different biological and psychological parameters in a group of doctors in training during their first days in hospital and six months later. From a gender perspective, women in the study had higher levels of psychosomatic stress than men leading to an inccrease in anxiety disorders and evitative responses while men were more susceptible to an active-agressive response. From a biopsychological perspective, there are other gender differences that are significant given the progressive feminization of the profession but also between specialties, clinical and non clinical work, organizational structures, cultures, modes of operation, etc...

Results.- The last part of the paper will describe the work carried out at PAIME- a Department of the Royal Colleges of Physicians in Spain that provides care for sick doctors - with references to post-graduate training and preventive interventions.

Conclusions.-

Disclosure of interest.- The authors have not supplied a conflict of interest statement.

\section{E-PV1021 \\ Attitudes of Tunisian medical students towards poeple with mental illness}

S. Taleb, Y. Zgueb, R. Jomli ${ }^{*}$, U. Ouali, F. Nacef

Razi hospital, "A" Psychiatric Department, La Manouba, Tunisia * Corresponding author.

Background and aims.- People with mental illness frequently encounter stigma, prejudice, and discrimination not only by the public, but also by the Medical practitioners. This negative attitude has an effect on the quality of service delivery as well as in their future choice of specialization in psychiatry.

The aim of the present study was to examine the attitude of medical students toward psychiatric patients and mental illness.

Methods.- The study was conducted in the Faculty of Medicine of Tunisia between April and May 2018. Fifth-year medical students responded to a questionnaire exploring their attitudes towards people with mental Illness. They were also asked for about the perception of society to the mentally ill.

Results.- 30 students responded to the questionnaire. No one has considered that the mental patient can be recognized by inspection. $80 \%$ consider that patients with mental illness are like other patients with medical illness. $30 \%$ of them think that the mental patient is dangerous. The perception of society to the mentally ill was improving according to student responses. Nearly one third of the students think that Society has archaic prejudices towards mental illness. The other opinions, on what society think of the mentally ill, are shared between: the mentally ill is dangerous, potentially criminal so must be avoided, and that Madness is scary that's why our society put them out of the field of view.

Conclusions.- Stigmatizing attitudes among medical students towards mental illness can be avoided by improving the quality of theoretical and practical training in psychiatry.

Disclosure of interest.- The authors have not supplied a conflict of interest statement. 


\section{E-PV1022}

\section{Adaptation of the communication skills attitude scale (CSAS) for medical students in Ukraine}

O. Khaustova*, O. Chaban, V. Omelyanovich, T. Abdryakhimova

Bogomolets National Medical University, Medical Psychology-

Psychosomatic Medicine and Psychotherapy, Kiyv, Ukraine

${ }^{*}$ Corresponding author.

Background and aims.- The medical students' training the specific features of communications in medical practice involves not only providing the necessary information and skills, assessing their mastery of the curriculum, but also feedback aimed at understanding the teacher degree of interest of students in mastering professional skills.

The study purpose was to adapt the Ukrainian version of "Communication Skills Attitude Scale" (CSAS), for further use in assessing the attitude of medical students in Ukraine.

Methods.- Study contingent consisted of 769 students of Bogomolets National Medical University, 230 of whom (29.91\%) belonged to male gender, and 539 people $(70.09 \%)$ to the female gender.

Following research methods were used: calculation of Cronbach's alpha Coefficient, Guttman Split-Half Coefficient, Equal-length Spearman - Brown, Ferguson's delta Coefficient, $\varphi^{*}$-test of Fisher's angular transformation, Spearman rank correlation coefficient, Pearson correlation coefficient, exploratory factor analysis.

Results.- The basic psychometric characteristics of the test were calculated: constructive, conceptual and prognostic validity, discrimination, consistency, retest and synchronous reliability. Due to the analysis of psychometric characteristics: the 13 and 16 statements of the original scale were deleted, and 22 statement were moved from the positive attitude subscale to the subscale of the negative attitude. After these changes, the Ukrainian adapted test - "CSAS-U" revealed a high level of all psychometric characteristics: construct validity (factor loads $>0,3$ ), conceptual validity and predictive validity $(p \leq 0,015)$, discriminativeness $(\delta$-Ferguson $=0,982)$, data consistency $(\alpha$-Cronbach $=0,86$; split-half $=0,84)$, test-retest reliability and internal consistency (Spearman-Brown prophecy coefficient $=0,476 ;(p \leq 0,045)$.

Conclusions.- Thus, Ukrainian adapted test "CSAS-U" can be used to assess the attitude of medical students in Ukraine to study of communicative skills.

Disclosure of interest.- The authors have not supplied a conflict of interest statement.

\section{E-PV1024}

\section{Integrating core knowledge into written assessments in psychiatry}

D. Mirfin ${ }^{1 *}$, M. Buccur ${ }^{2}$

${ }^{1}$ Surrey and Borders Partnership NHS Foundation Trust, Liaison Psychiatry, Chertsey, United Kingdom; ${ }^{2}$ Sussex Partnership NHS Foundation Trust, Linwood ATS, Haywards Heath, United Kingdom ${ }^{*}$ Corresponding author.

Background and aims.- Within a curriculum certain areas are core and essential to be understood in order to be proficient within the field. However existing assessment methodologies do not distinguish these and therefore we identified the concept of core knowledge and explored how this could be integrated into psychiatric written assessments within the MSc Psychiatry at BSMS. Our aim was to pilot this assessment method on a previous assessment to assess explore different strategies to take performance within certain questions (which cover core knowledge) into account and understand the impact this has on the overall performance within the assessment tool.

Methods.- We defined the questions in a previous assessment tool that would be defined as core knowledge and agreed this with con- sensus between two assessors within the course. These items were then scored separately from other questions and we utilised different methods to combine these scores including the use of cut off scores (where one failed if they did not meet either pass mark) and combined scores (where an average was taken of each score). These were then compared to the raw score and we compared if the scores were statistically significant and the overall impact this had on student grades within this assessment in MSc Psychiatry. Results.- There was an overall reduction in performance however the assessment was more discriminating of the overall performance within the course. The combined method was more effective at combining scores.

Conclusions.- Integrating Core Knowledge into assessment methods is feasible and valid and could improve the discriminatory power of assessments.

Disclosure of interest.- The authors have not supplied a conflict of interest statement.

\section{E-PV1025}

\section{New model of training for East-European psychiatrists}

\section{P. Morozov}

Russian State Medical Research University- Faculty of Advanced

Medical Studies, Psychiatry, Moscow, Russia

* Corresponding author.

Background and aims.- As part of the educational program of the WPA an East European Educational WPA- Servier Academy was established in 2013. It was decided to create a "bank" of our psychiatric journals of the Eastern Europe in order to improve the exchange of scientific information and to create additional possibilities for using new models and methods of training of young psychiatrists.

Methods.- On the basis of the recommendations of the National Societies 20 young researchers from different countries were selected (Russia, Belarus, Ukraine, Georgia, Armenia, Azerbaijan, Kazakhstan). All of them were equally fluent in Russian and English languages. Within following years, members of this group of young psychiatrists attended major European congresses -EPA and ECNP and were reviews of the most interesting issues, discussed at the Congresses. These reviews were passed at the regular meetings of the Presidents of the National Societies - chief- editors of psychiatric journals in Eastern Europe for future publications.

Results.- The works of WPA Academy published in 13 different psychiatric journals of the 7 countries of the Eastern Europe. The number of publications already incalculable, but the total amount has passed Figure 90 in the 6 years.

Conclusions.- At the final stage of training the best "academics" have already begun themselves to act as lecturers at various international conferences.

Disclosure of interest.- The authors have not supplied a conflict of interest statement.

\section{E-PV1026}

\section{The impact of a novel psychiatry training program on family medicine residents' attitudes towards psychiatry}

A.L.H. Peh ${ }^{1 *}$, M.S. Yan ${ }^{2}$, D.C.L. Teo ${ }^{1}$, E.K.M. Wuan ${ }^{1}$, A.T.S. Tay ${ }^{1}$, S.C.W. $\mathrm{Ho}^{3}$, S.E. Saffari ${ }^{4}$

${ }^{1}$ Changi General Hospital, Dept of Psychological Medicine, Singapore, Singapore; ${ }^{2}$ Duke-NUS Graduate Medical School-Singapore, Medical Student, Singapore, Singapore; ${ }^{3}$ Singhealth, Singhealth Polyclinics, Singapore, Singapore; ${ }^{4}$ Duke-NUS Graduate Medical SchoolSingapore, Office of Clinical Sciences, Singapore, Singapore

Corresponding author. 
Background and aims.- Singapore Health Services Family Medicine (FM) Residency developed a novel psychiatry training program that emphasizes education continuity, longitudinal exposure, and vocational, primary care setting as opposed to tertiary hospital psychiatry services. The primary aim of the study was to examine the impact of the novel psychiatry training on attitudes towards psychiatry amongst FM residents.

Methods.- All FM Residents ( $n=63$ ) were invited in this prospective study and completed modified Attitudes towards Psychiatry Scale (mAPS) pre- and post-training. Pre- and post-training mAPS scores were compared using paired t-test. A general questionnaire eliciting demographics was also administered at the start of the training. Regression analysis was carried out to determine background variables predictive of improvement in MAPS scores post-training. Results.- A total of 63 FM residents (100\% response rate) were included. All subjects completed the pre- and post-training surveys (100\% retention rate). There was significant improvement in overall mAPS scores (pre-training $3.20 \pm 0.25$, post-training $3.34 \pm 0.27$, $p<0.001$ ). Specifically, significant improvement was observed in 3 out of 4 mAPS domains: Domain 1 the merits of psychiatry as scientific medicine (pre-training $3.26 \pm 0.41$, post-training $3.40 \pm 0.35$, $p=0.008$ ), Domain 2 effectiveness of treatment (pre-training $3.18 \pm 0.45$, post-training $3.39 \pm 0.41, p=0.001$ ), Domain 4 inspiration from medical school (pre-training $3.07 \pm 0.37$, post-training $3.22 \pm 0.47, p=0.001$ ), but not Domain 3: stigma of psychiatry (pretraining $3.29 \pm 0.43$, post-training $3.35 \pm 0.49, p=0.25$ ). Regression models showed previous 3-month elective psychiatry inpatient posting was a significant negative predictor of improvement in mAPS scores $(p=0.036)$.

Conclusions.- The novel psychiatry training program significantly improved FM residents' attitudes towards psychiatry as shown by mAPS scores improvement post-training.

Disclosure of interest.- The authors have not supplied a conflict of interest statement.

\section{E-PV1027}

\section{EPA summer school 2018: a rare experience of diversity}

J.E. Pinzon Espinosa ${ }^{*}$, C. So ${ }^{2}$, E. Dashi ${ }^{3}$, Y. Rai ${ }^{4}$, S. Kocijančič ${ }^{5}$, R. Gonçalves $^{6}$, H. Yilmaz Kafali ${ }^{7}$, C. Noël ${ }^{8}$, G. Anmella ${ }^{9}$, B. Moura ${ }^{10}$, S. Mahabir $^{11}$, A. Haiduc ${ }^{12}$, L.D. Gherman ${ }^{12}$, I. Viltrakyte ${ }^{13}$, S.. Eray ${ }^{14}$, M.R. Malerba ${ }^{15}$, E.A. Carbone ${ }^{16}$, P. Makaric ${ }^{17}$, A. Juryk ${ }^{18}$, A. Kuzmanovic ${ }^{19}$

${ }^{1}$ European Federation of Psychiatric Trainees, Institute of Neurosciences, Hospital Clínic, Department of Adult Psychiatry and Psychology, Barcelona, Spain; ${ }^{2}$ European Federation of Psychiatric Trainees EFPT, University of Tours, Psychiatry, Tours, France; ${ }^{3}$ University Hospital Center "Mother Tereza", Department of Neuroscience, Tirane, Albania; ${ }^{4}$ Essex Partnership University Trust, Psychiatry, Essex, United Kingdom; ${ }^{5}$ General hospital Novo mesto, Adult Psychiatry, Novo mesto, Slovenia; ${ }^{6}$ Coimbra Hospital and University Center CHUC, Department of Child and Adolescent Psychiatry, Coimbra, Portugal; ${ }^{7}$ Ankara Children's Hematology Oncology Training and Research Hospital, Department of Child and Adolescent Psychiatry, Ankara, Turkey; ${ }^{8}$ World Psychiatric Association, Early Career Psychiatrists Section, Brussels, Belgium; ${ }^{9}$ Institute of Neurosciences. Hospital Clínic, Department of Adult Psychiatry and Psychology, Barcelona- Catalonia, Spain; ${ }^{10}$ Centro Hospitalar Vila Nova de Gaia/Espinho, Department of Psychiatry and Mental Health, Vila Nova de Gaia, Portugal; ${ }^{11} \mathrm{Cwm}$ Taf University Health Board, Outreach and Support Team-Department of Mental Health, Llantrisant, United Kingdom; ${ }^{12}$ Clinical Psychiatry Hospital "Prof. Dr. Al. Obregia", Psychiatry, Bucharest, Romania; ${ }^{13}$ Young Psychiatrists Association. Vilnius Centre for Psychotherapy and Psychoanalysis, Child and adolescent psychiatry, Vilnius, Lithuania;

${ }^{14}$ Uludag University Medical Faculty, Department of Child and Adolescent Psychiatry, Bursa, Turkey; ${ }^{15}$ University of Milano Bicocca,
General Adult Psychiatry- Department of Mental Health, Monsa, Italy; ${ }^{16}$ University of Campania "Luigi Vanvitelli", Department of Psychiatry, Naples, Italy; ${ }^{17}$ University Psychiatric Hospital Vrapce, Department for Affective Disorders, Zagreb, Croatia; ${ }^{18}$ Collegium Medicum Jagiellonian University, General Adult Psychiatry, Cracow, Poland; ${ }^{19}$ Clinic for Psychiatry- Psychotherapy and PsychosomaticsEmden Clinic, Psychiatry, Emdem, Germany

" Corresponding author.

Background and aims.- Every year, the European Psychiatric Association (EPA) organizes an exceptional training opportunity for trainees and early career psychiatrists. EPA Summer School offers a valuable programme from renowned professors. The 8th EPA Summer School took place in Strasbourg in September 2018, on the topic of "ABCs of Psychotherapy".

Aims.- The scholars were asked to get familiar with different psychotherapeutic approaches, improve psychiatric interviewing and communication skills, connect and share experiences.

Methods.- 27 selected scholars from 22 different countries participated in the Summer School. The faculty consisted of 7 prominent psychiatrists and a professional actor. Scholars attended lectures and took part in interactive discussions, workshops, role-plays and simulation sessions. Social events completed the three-day intensive educational programme.

Results.- All of the 27 scholars successfully survived the Summer School with no dropouts. Misconceptions significantly decreased in at least 3 types of psychotherapy. Participants are now familiar with the $\mathrm{ABC}$ of communication. Contact list significantly increased. However, many participants experienced adverse effects, included strain, exhaustion, early evening nap, lapsus, weird dreams, awkward selfies and sad goodbyes. Overall, participants unanimously enjoyed the programme and praised the professors' passionate teaching and availability for further discussion.

Conclusions.- The EPA Summer School is a demanding programme that challenges trainees and early career psychiatrist's practice and knowledge in psychotherapy. It is a very valuable experience. It offers a unique opportunity to embrace the diversity of psychotherapies, to meet outstanding teachers, and to settle an international network of enthusiast psychiatrists, opening possibilities for further collaboration.

Disclosure of interest.- The authors have not supplied a conflict of interest statement.

\section{E-PV1028 \\ Evaluation of the 26th European federation of psychiatric trainees' forum 2018 in Bristol, UK}

H. Ryland ${ }^{1 *}$, R. Harrison ${ }^{2}$, B. Wood ${ }^{3}$

${ }^{1}$ University of Oxford, Department of Psychiatry, London, United Kingdom; ${ }^{2}$ Avon and Wiltshire Mental Health NHS Partnership Trust, Core Training, Bristol, United Kingdom; ${ }^{3}$ North Bristol NHS Trust, Higher Specialist Training, Bristol, United Kingdom

* Corresponding author.

Background and aims.- The European Federation of Psychiatric Trainees (EFPT) is an umbrella association of 39 National Trainee Associations in 37 countries. The annual Forum includes the General Assembly, clinical visits, working groups and scientific sessions. The 2018 Forum took place in Bristol, UK, from 21-25 July. It included a training day on neuroscience and an optional psychotherapy training day on 26 July, both of which were also open to additional UK trainees. 114 EFPT delegates attended from 41 countries.

This evaluation sought to assess the impact of the Forum from the perspective of the delegates.

Methods.- All delegates were contacted following the event and invited to fill in an online feedback form about various aspects of the 
event, including the organisation, quality of the scientific sessions and usefulness of the various aspects of the programme.

Results.- 61 out of 106 delegates (excluding the 8 Local Organising Committee members present) responded, giving a response rate of $58 \%$. Respondents rated their enjoyment of the Forum $4.75 / 5$ overall and the Forum's usefulness $4.38 / 5$. 90\% said they would be 'very likely' to attend the Forum again and $90 \%$ of the respondents rated the organisation as excellent. All aspects of the Forum, including the neuroscience day, psychotherapy day, hospital visits and working groups were rated on average over 4 out of 5 . Suggestions for improvement, included more time for discussion and for delegates to submit proposals in advance.

Conclusions.- Overall, delegates rated all aspects of the Forum highly, indicating that the Forum is seen as a worthwhile activity by participants.

Disclosure of interest.- The authors have not supplied a conflict of interest statement.

\section{E-PV1029}

\section{Consultation - liaison joint interventions in a university setting: an "in-vivo" training for residents in general internal medicine}

K. Tzartzas ${ }^{*}$, A. Gouveia ${ }^{1}$, A. Linder ${ }^{2}$, R. Marion-Veyron ${ }^{1}$

${ }^{1}$ University of Lausanne, Department of Ambulatory Care and Community Medicine, Lausanne, Switzerland; ${ }^{2}$ University of Lausanne, Department of Psychiatry, Lausanne, Switzerland ${ }^{*}$ Corresponding author.

Background and aims.- More than half of patients consulting in primary care settings (PCS) suffer from a mental health disorder (MHD). Nevertheless, general practitioner's (GP's) psychiatric training is described as insufficient. Action is required to improve the diagnosis and treatment of MHDs. In the Department of Ambulatory Care and Community Medicine at the University of Lausanne (Switzerland), consultation-liaison (CL) psychiatrists are part of the GPs' team. We wanted to evaluate an "in-vivo" psychiatric training intervention proposed in PCS for GP residents.

Methods.- An informal exchange is brought forward for every psychiatric referral, focusing on doctor - patient relationship and proposing a first diagnosis hypothesis and psychotropic treatment consultation. When needed, a joint psychiatric intervention (JPI) is conducted (patient - doctor - psychiatrist). The psychiatrist performs a psychopathology and psychodynamic investigation in the presence of the GP. In the last minutes of the session, he comes up with a first diagnosis and actual crisis hypothesis, and proposes a treatment plan, introducing a biopsychosocial vision. Discussion takes place after the session around a posible psychiatric intervention. A focus group with residents was conducted in the end of their internship, investigating their lived experiences during CL interventions.

Results.- Residents appreciated psychiatric accessibility, continuous joint working and the possibility to ask a "quick question". Valuable psychiatric training was provided through this collaboration. They felt that JPIs increased diagnostic skills, helped decision-making and improved doctor-patient relationship.

Conclusions.- A close and continuous relationship between CL psychiatrists and GPs creates a fertile ground in which psychiatric training well adapted to PCS is offered.

Disclosure of interest.- The authors have not supplied a conflict of interest statement.

\section{E-PV1030}

\section{Non-cognitive effects of computer-based cognitive training (CT) in elderly group. Preliminary evaluation}

R. Wallner ${ }^{1}{ }^{*}$, A. Senczyszyn ${ }^{1}$, D. Szcześniak $1^{1}$, B. Tarnowska ${ }^{2}, \mathrm{~K}$. Urbanska $^{1}$, J. Rymaszewska ${ }^{1}$

${ }^{1}$ Wroclaw Medical University, Department of Psychiatry- Poland, Wroclaw, Poland; ${ }^{2}$ Silesian Public Library, Stowarzyszenie Organizacji Trenerów Pozarzadowych STO, Wrocław, Poland

* Corresponding author.

Background and aims.- Multidimensional cognitive trainings (CT) belong to the group of non-pharmacological methods aimed at enhancing cognitive performance. Besides, it's seem to affect also the so-called non-specific psychological factors, such as quality of life and general well-being.

The aim of the study was to evaluate the effects of nonpharmacological intervention on psychological variables in seniors participating in Computer-based CT “Academy of Mind ${ }^{\circledR}$ ".

Methods.- Of the 54 seniors (age $>65$ ) included in study, 40 finished the CT (F/M:30/10; age: $72.4 \pm \mathrm{SD}=7.3$; mean MoCA=23.6). CT lasted 9 weeks including one 90 minutes' session per week (individual computer tasks and group games focusing on specific cognitive functions) accompanied by a corresponding psychoeducational part (e.g. cognitive functions, training possibilities, compensation strategies), exercises completed by seniors at home. Sessions were led by a professional trainer. Psychological tests were performed before and after CT by following questionnaires: LOT-R, GSES, SWLS.

Results.- The analysis of data sets showed statistically significant improvement in $1 / 3$ participants CT $(n=14)$ of subjectively perceived sense of well-being, life satisfaction and dominance of positive feelings (SWLS, $p=0.02$ ), generalized optimistic anticipation (LOT-R, $p=0.001$ ), and self-efficacy (GSES, $p=0.0004$ ). After CT the rate of participants with high scores has doubled in SWLS/LOT-R (from $22.5 \%$ to $47.5 \%$ ), in GSES improved from $40 \%$ to $67.5 \%$.

Conclusions.- The results of the study confirm the positive effects of non-pharmacological intervention such as Computer-based CT on non-specific psychological variables of seniors. Further research is necessary to determine if level of cognitive deficits differentiate psychological factors (QoL, well-being) in seniors after Cognitive Training.

Disclosure of interest.- The authors have not supplied a conflict of interest statement.

\section{E-PV1031}

\section{Quality of the psychiatric interview of nurses in Tunisia and the different obstacles to the exchange}

Z. Boudali ${ }^{1}$, Y. Zgueb ${ }^{2}$, R. Jomli ${ }^{2}$, F. Znaidi ${ }^{*}{ }^{*}$, F. Nacef ${ }^{2}$

${ }^{1}$ Razi Hospital, psychiatry B, Manouba, Tunisia; ${ }^{2}$ Razi Hospital, psychiatry A, Manouba, Tunisia

Corresponding author.

Background and aims.- In psychiatry, nurses are part of the care team that accompanies the patient throughout his therapeutic process, from his hospitalization to his follow-up at the consultation. The aim of the study was to evaluate the quality of the psychiatric interview among our tunisian nurses and to study the possible obstacles to the exchange.

Methods.- We present a cross-sectional descriptive study on 30 nurses of the psychiatric department " $A$ " at the Razi hospital in Tunisia, from February to March 2018.

Results.- Results: Most nurses practice a psychiatric interview for patients (90\%). One hundred percent of the nurses practice interviews according to the mode of exercise and experience. Zero 
percent of the interviews are coded according to international standards and eighty percent of the nurses practice intake interviews. The minimum duration of the interview is 5 minutes, the maximum duration is 30 minutes. Only $33.3 \%$ provide continuity of care. The main obstacles to the exchange were: lack of training (90\%), lack of staff $(100 \%)$ and the difficult conditions of the service, notably lack of materials, (97\%).

Conclusions.- In Tunisia there is no specialized training in psychiatry, this allows us to understand why no nurses do a codified interview according to international standards.

Disclosure of interest.- The authors have not supplied a conflict of interest statement.

\section{E-Poster Viewing - 7-9 April: Women, Gender and Mental Health}

\section{E-PV1032}

\section{The association between dating violence victimization and psychiatric disorders in the Korean general population}

J. An ${ }^{1 *}$, C.S. Moon ${ }^{1}$, D.E. Kim ${ }^{1}$, S.Y. Lee-Tauler ${ }^{2}$, H.J. Jeon ${ }^{1}$, S.J. Cho ${ }^{3}$, S.J. Sung ${ }^{4}$, J.P. Hong ${ }^{1}$

${ }^{1}$ Samsung Medical Center, Psychiatry, Seoul, Republic of Korea; ${ }^{2}$ Johns Hopkins University, Mental Health, Baltimore, Maryland, USA;

${ }^{3}$ Gachon University of Medicine and Science, Psychiatry, Incheon, Republic of Korea; ${ }^{4}$ Kangdong Sacred Hospital, Psychiatry, Seoul, Republic of Korea

Corresponding author.

Background and aims.- This study aimed to assess the association between physical and sexual dating violence (DV) and mental health conditions among female victims in the general population in Korea.

Methods.- 3,160 South Korean female participants, age ranging from 18 to 74, responded to the Korean version of the WHOComposite International Diagnostic Interview (K-CIDI) version 2.1, and questionnaire of DV. Multiple logistic regression was used to examine the odds of psychiatric disorders associated with each type of DV.

Results.- Victimization of any DV was associated with significantly increased odds of experiencing any psychiatric disorders (OR 4.4, $95 \% \mathrm{CI} 2.4-8.0$ ). Of all types of DV, participants who experienced any sexual DV had the highest odds of having psychiatric disorders (OR 14.3, 95\% CI 4.1-54.8). Sexual DV experience among participants were associated with higher odds of major depressive disorder, anxiety disorder, OCD, PTSD, GAD, specific phobias, agoraphobia, and nicotine dependence. Alcohol use disorder was highly associated with any physical DV (OR 3.8, 95\% CI: 1.7-8.0). Among those female victims of DV, the youngest age group ranged from 18 to 35 years old $(2.6 \%, 95 \% \mathrm{CI} 1.4-3.8)$ and the never married group $(2.7 \%$, $95 \%$ CI 1.2-4.2) experienced higher proportion of any form of DV. Conclusions.- Psychiatric disorders are highly associated with the experience of DV among female victims in their lifetime and are most prevalent among those victims with experience of sexual DV. Further implication prevention of mental disorders in female DV victims should be provided early and specific to each type of DV. Disclosure of interest.- The authors have not supplied a conflict of interest statement.

\section{E-PV1034}

\section{Stigmatization towards transsexual people among psychiatry residents in Tunisia}

C. Ben Said", N. Bram, R. Maamouri, I. Ben Romdhane, W. Homri, R. Labbane

Razi hospital, Psychiatry C, Mannouba, Tunisia

" Corresponding author.

Background and aims.- Transgender individuals are the subject of little focused health research, mainly in non-developed countries, where their needs are not taken into consideration by health-care providers, policy makers or legislators. To date, few studies have addressed psychiatrists' attitude toward transgender individuals around the world, and no study has been conducted in Tunisia.

The aim of this study is to evaluate the attitude of psychiatry residents towards transgender individuals and to have an overview on the legal status of sexual minorities in Tunisia.

Methods.- 55 psychiatry residents among 61 in Razi Hospital accepted to respond to the Genderism and Transphobia scale (GTS) online.

Results.- $43.7 \%$ of the residents agree, at different degrees, that "god made two sexes and two sexes only" and $38.1 \%$ of them that "people are either men or women". $36.4 \%$ are disgusted by men who cross-dress for sexual pleasure. $27.3 \%$ think women who see themselves as men are abnormal and $31 \%$ would get violent if they found that their lover was the other sex. $23.6 \%$ agree with the item "sex change operations are morally wrong" and $21.8 \%$ remain neutral. The findings described here indicate preliminary trends that will be important to explore in further depth using larger samples of psychiatrists as well as other medical specialists.

Conclusions.- Psychiatry residents should approach subjects as gender and sexuality with fewer assumptions and be trained to provide services that are sensitive to transgender people's rights and responsive to their needs.

Disclosure of interest.- The authors have not supplied a conflict of interest statement.

\section{E-PV1036}

Psychiatrists as leaders: a comprehensive review of intimate partner violence and best practices

\section{E. Ekhause ${ }^{1}$, S. Chen ${ }^{1}$, A. Baluna ${ }^{2}$, A. Huh ${ }^{1}$, H. Raai ${ }^{1}$, M.}

Benavides $^{3}$, V. Pender ${ }^{3}$

${ }^{1}$ Montefiore Medical Center/Albert Einstein College of Medicine, Psychiatry, Bronx, USA; ${ }^{2}$ Montefiore Medical Center/Albert Einstein College of Medicine, Bronx, USA ${ }^{3}$ Columbia University Medical Center, Psychiatry, New York City, USA

${ }^{*}$ Corresponding author.

Background and aims.- Intimate Partner Violence (IPV) is a known and preventable phenomenon with consequences on the physical and mental health of women, children, and communities worldwide. IPV also has far reaching impacts on economies, policies, and culture. Participants will learn the various definitions of IPV and current epidemiologic data. They will learn the short and long term physical and mental health consequences of IPV and what the literature has learned about intergenerational and epigenetic impacts of IPV. Participants will better understand how IPV effects local and global economies. There will be a review of current evaluation, treatment and prevention strategies. Participants will feel prepared to advocate for policy and program implementation through a review of best practices and learn about a unique collaborative project in New York City working with families and survivors of IPV throughout the recovery process.

Methods.- A poster presentation which will review current literature on IPV. 
Results.- Viewers will gain a comprehensive knowledge of IPV essential for psychiatrists to become leaders in prevention, evaluation, and management of IPV and advocate for necessary IPV policies around the world.

Conclusions.- IPV is a worldwide phenomenon that has far reaching impacts on mental health and the field of psychiatry. Psychiatrists are important stake holders in advocacy. With a comprehensive understanding of IPV, psychiatrists will be able to be leaders for change in IPV policy and outcomes around the world.

Disclosure of interest.- The authors have not supplied a conflict of interest statement.

\section{E-PV1037}

\section{Girls and women on the autism spectrum: regarding a clinical case}

\section{Fernandes Santos}

Hospital Garcia de Orta, Department of Psychiatry and Mental

Health, Almada, Portugal

${ }^{*}$ Corresponding author.

Background and aims.- Autism Spectrum Disorder (ASD), first described in 1943 (Kanner), is a collective term for a constellation of neurodevelopmental conditions with heterogeneous etiologies, characterized by impairments in social interaction and communication, as well as restricted, repetitive and stereotyped patterns of behaviour and interests. One of the most consistent features of ASD is the predominance among males, with a male-to-female ratio of $4-5: 1$, increasing to $9: 1$ in the absence of comorbid intellectual impairment. The origin of this gender difference remains incompletely understood.

Regarding a clinical case, the author reviews the effects of gender on ASD prevalence and symptomatology, exploring the differences in behavioural symptoms and cognitive functioning between males and females, as well as potential genetic, hormonal and environmental underlying mechanisms.

Methods.- A search was conducted in PubMed/MEDLINE and ClinicalKey databases, using the keywords 'autism spectrum disorder' and 'gender difference', from 2010 until 2018. Clinical information was obtained through medical records.

Results.- ASD females may be under-identified or, at least, have different behavioural, cognitive, neuroanatomical and/or molecular expression patterns, imposing a diagnostic challenge, especially in cognitively able ones. Females have more communicative deficits, sleep problems, anxiety and depression. However, they have less restricted behaviours and show more interest in social relations, actively examining and imitating others, which results in a camouflage of autistic symptoms.

Conclusions.- Gender differences in ASD are vital to consider both in terms of research and clinical practice. Their understanding may lead to advancements in prevention, earlier diagnosis and treatment in girls and women on the autism spectrum.

Disclosure of interest.- The authors have not supplied a conflict of interest statement.

\section{E-PV1038}

\section{An explorative study to assess the psychosocial impact of infertility on female infertile patients}

\section{D.B. Ghost Dastidar}

R G Kar Medical College, Psychiatry, Kolkata, India

${ }^{*}$ Corresponding author.

Background and aims.-

1. To examine the relationship between infertility and depression, anxiety, quality of life, psychopathology: in both fertile and infertile female subjects.
2. To examine the relationship between psychological morbidity and socio demographic variables in both fertile and infertile population.

3. Psychological morbidity comparison within the infertile group on basis of etiology.

Methods.- 100 female fertile and infertile patients were selected by consecutive random sampling from the OPD of gynecology department of R G Kar Medical College.

Female infertile patients were administered SRQ for evidence of psychological morbidity. Subsequently both cases and controls were administered the following scales socio demographic proforma, BAI,BDI, SF 36, SCL 90 to assess anxiety, depression, quality of life and psychopathology.

Results.-

(1) Statistical analysis of data showed that there is significantly increased anxiety and depression level amongst infertile patients in comparison to the fertile patients group.

(2) There is statistically significant difference between the quality of life and psychopathology between the two groups.

Conclusions.- Infertility is a growing medical problem with 8-12 percent of couples suffering from this ailment world wide. In the South Asian context this poses a serious problem as majority of couples cannot avail expensive treatment such as IVF. Our study has showed significant levels of depression, anxiety and psychopathology in the infertile population group.

It is imperative that healthcare policy making institutions place greater emphasis on access of infertility treatment to general masses and to the development of social support systems to manage/treat the psychosocial aspects of infertility.

Disclosure of interest.- The authors have not supplied a conflict of interest statement.

\section{E-PV1040}

\section{Gender differences in opioid-user with chronic non-cancer pain}

T. López-Arteaga ${ }^{1 *}$, P. Padilla-Romero ${ }^{2}$

${ }^{1}$ Psychiatrist, Department of Psychiatry, Talavera de la Reina, Spain;

${ }^{2}$ Hospital Ntra. Sra. del Prado, Psychiatry, Talavera de la Reina, Spain * Corresponding author.

Background and aims.-

Introduction.- being a man, youth and history of substance use disorder (SUD) are factors associated to present opioid use disorder (OUD). However, when we talk about chronic non-cancer pain (CNCP) the patient profile can change.

Objetives.- determine whether gender is a risk factor for presenting CNCP. Determine whether gender is a risk factor for presenting OUS in this group and describe the characters of opioid-user in CNCP. Methods.- Prospective descriptive study. Sample: 187 patients evaluated by the department of psychiatry in the year 2017 derived from (PU) for psychopathological assessment.

Results.-

WOMEN: 80\%; Average age 61; History affective disorders 17,33\%; History of SUD 1,33\%; OUD 3,33\%; Daily dose $141 \mathrm{MME}$

MEN: 20\%; average age 46; history of affective disorder 2'7\%; history of SUD $10^{\prime} 81 \%$; OUD 64\%; daily dose $931^{\prime} 5 \mathrm{MME}$.

Conclusions.- Patients referred from PU to the department of psychiatry are usually women, 60 years old, without history of SUD, but with history of affective disorders. The percent of women with OUS is less than men, but they have a daily average of six times greater than women. There are more women with CNCP but men have more risk of reaching higher doses of opioids.

Disclosure of interest.- The authors have not supplied a conflict of interest statement. 
E-PV1041

\section{Epidemiological specificities of women with psychiatric disorders in Southern Tunisia}

M. Ben Hmida ${ }^{1}$, Y. Mejdoub ${ }^{1}$, L. Ghanmi ${ }^{2}$, M. Maalej ${ }^{3 *}$, J. Jdidi ${ }^{1}$, M. Trigui $^{1}$, M. Ben Jmaa ${ }^{1}$, R. Boukhchina ${ }^{2}$, M. Abbes ${ }^{2}$, K. Zitoun ${ }^{2}$, S. Yaich $^{1}$, L. Zouari ${ }^{3}$, J. Damak ${ }^{1}$, M. Maalej ${ }^{3}$

${ }^{1}$ Hédi Chaker University Hospital, Department of Community Medicine, Sfax, Tunisia; ${ }^{2}$ Regional Hospital of Gabes, Department of Psychiatry, Gabes, Tunisia; ${ }^{3}$ Hédi Chaker University Hospital, Department of Psychiatry “C”, Sfax, Tunisia

Corresponding author.

Background and aims.- Psychiatric disorders are responsible for a high death-rate and for an important morbidity resulting in a decrease of productivity, a poor quality of life and handicaps. However, the burden of psychiatric disorders especially among women in low-resource settings is not well documented. Our work aims to study the epidemiological specificities of women with psychiatric disorders in southern Tunisia.

Methods.- It was a descriptive cross-sectional study. It included all patients with first consultation in the psychiatric department of Southern Tunisia occurred between first January 2010 and 31 December 2013.

Results.- Of 1601 consulting patients in our department, women represented $51 \%(n=817)$. The average age of women patients was 36.93 years[6-99 years]. The socioeconomic level was low in 571 cases (74.7\%). Among investigated women, 405 patients (57.9\%) were housewives. Antecedent of attempted suicide was noted in 34 women (4.3\%) and 19 patients (2.4\%) had addictive behaviors. The average time between onset of symptoms and first psychiatric consultation was 1.2 years[ 0 days- 12 years]. The most common reasons for consultation were: anxiety (109 cases, $13.3 \%)$, behavioral disorder (84 cases, $10.3 \%$ ) and sleep disorder (82 cases, $10 \%$ ). The consultation after a suicide attempt was noted in 50 cases (6.1\%). Comparing with men, women were more likely to develop depression $(\mathrm{OR}=1.78, p=<10-3$ ) and adjustment disorder $(\mathrm{OR}=2.12$, $p \leq 10-3)$.

Conclusions.- Depressive disorder was the most diagnoses in women population. It merits further attention in the country's mental health policy and program planning.

Disclosure of interest.- The authors have not supplied a conflict of interest statement.

\section{E-PV1042}

\section{Association between common mental disorders and signs and symptoms of menopausal transition: population-based study in Southern Brazil}

A. Ludwig Neutzling ${ }^{1 *}$, M.T.A. Olinto ${ }^{1}$, F. Bairros ${ }^{2}$, J.S. Dias da Costa $^{1}$, H.M. Leite ${ }^{1}$, J.C. da Silva ${ }^{1}$, S.C. Schwendler ${ }^{1}$

${ }^{1}$ Universidade do Vale do Rio dos Sinos - UNISINOS, Postgraduate Program in Collective Health, São Leopoldo, Brazil; ${ }^{2}$ Universidade Federal do Rio Grande do Sul, Collective Health, Porto Alegre, Brazil * Corresponding author.

Background and aims.-

Introduction.- There is a high prevalence of common mental disorders (CMD) worldwide. During menopausal transition (MT), women show signs and symptoms that may be influenced by these disorders.

Objectives.- To investigate the association between CMD and the occurrence of MT signs and symptoms in women aged 40 to 69 years in southern Brazil.
Methods.- A population-based cross-sectional study with a representative sample of 400 women from a city in southern Brazil. The outcome signs and symptoms of MT were measured by the Menopause Rating Scale (MRS) and classified as 'none/mild' (08 ) and 'moderate/severe' (>9). The Self Reporting Questionnaire (SRQ-20) scale was used to check the presence of CMD (score>8). Unadjusted and adjusted Prevalence Ratios (PR) and Confidence Intervals of $95 \%(95 \% \mathrm{CI})$ were estimated by using the robust Poisson regression.

Results.- The prevalence of moderate/severe signs and symptoms of MT was 56.5\% (95\%CI, 51.5-61.4), and 34.5\% (95\%CI 29.8-39.4) of women had CMD. In the unadjustment analyses, women with zero to four years of study ( $p<0,001)$, physical inactivity $(p=0,005)$, five or more pregnancies $(p=0,035)$ and TMC $(p<0,001)$ presented higher prevalences of the outcome. After adjustment, women with CMD were twice as likely to present moderate/severe signs and symptoms compared to those without CMD (PR 2.23, 95\% CI 1.88$2.64, p<0.001$ ).

Conclusions.- There was a greater probability of moderate/severe signs and symptoms of MT in women with CMD. Other studies may help to clarify other aspects related to the mental health of women who experience MT.

Disclosure of interest.- The authors have not supplied a conflict of interest statement.

\section{E-PV1044}

\section{Body image in patients with mental disorders who have conflicted gender identity}

T. Piskareva

Mental Health Research Center, Medical Psychology, Moscow, Russia * Corresponding author.

Background and aims.- Several studies have shown that persons with conflicted gender identity display greater degrees of body dissatisfaction than controls. In such cases successful body alteration has been able to alleviate distress. However sex change is only permitted when mental disorder is excluded. There is lack of research on body image within persons, who suffer from mental disorders and have conflicted gender identity.

The purpose of the study is to describe the body image in women with endogenous mental disorders accompanied by conflicted gender identity.

Methods.- The sample consisted of 26 women aged 16-26 with mental illnesses who wanted to change their sex (EG1) or get rid of gender signs (EG2). The comparison group consisted of 29 women of the same age and with the same mental diseases, but without the desire to alter their sex. Some of them (17) had dysmorphophobia (CG1), the others did not (CG2). The probands were asked to estimate their body parts using the Skugarevsky Body Satisfaction Scale.

Results.- The results showed significant differences in body satisfaction between both clinical groups and CG2. Both control groups were less satisfied with gender related characteristics, then neutral ones. EG2 tended to overestimate significance of unsatisfactory body parts, like those with dysmorphophobia and unlike EG1.

Conclusions.- Patients with mental disorders willing get rid of gender signs show similar patterns to dysmorphophobia (high significance of the least satisfactory body parts). The desire to change sex was an attempt to acquire new identity with opposite qualities, including sex, in order to solve other psychological problems.

Disclosure of interest.- The authors have not supplied a conflict of interest statement. 